\title{
materials
}

\section{Recent Advances}

in Smart

Materials for the Built Environment

Edited by Cesare Sangiorgi, Filippo Ubertini and Anna Laura Pisello Printed Edition of the Special Issue Published in Materials 
Recent Advances in Smart Materials for the Built Environment 



\section{Recent Advances in Smart Materials for the Built Environment}

Special Issue Editors

Cesare Sangiorgi

Filippo Ubertini

Anna Laura Pisello 
Special Issue Editors

Cesare Sangiorgi

University of Bologna

Italy
Filippo Ubertini

University of Perugia

Italy
Anna Laura Pisello

University of Perugia

Italy

Editorial Office

MDPI

St. Alban-Anlage 66

4052 Basel, Switzerland

This is a reprint of articles from the Special Issue published online in the open access journal Materials (ISSN 1996-1944) from 2017 to 2018 (available at: https://www.mdpi.com/journal/materials/ special_issues/Smart_Built)

For citation purposes, cite each article independently as indicated on the article page online and as indicated below:

LastName, A.A.; LastName, B.B.; LastName, C.C. Article Title. Journal Name Year, Article Number, Page Range.

ISBN 978-3-03897-352-2 (Pbk)

ISBN 978-3-03897-353-9 (PDF)

Cover image courtesy of Anna Laura Pisello and Cesare Sangiorgi.

Articles in this volume are Open Access and distributed under the Creative Commons Attribution (CC BY) license, which allows users to download, copy and build upon published articles even for commercial purposes, as long as the author and publisher are properly credited, which ensures maximum dissemination and a wider impact of our publications. The book taken as a whole is (C) 2018 MDPI, Basel, Switzerland, distributed under the terms and conditions of the Creative Commons license CC BY-NC-ND (http:/ / creativecommons.org/licenses/by-nc-nd/4.0/). 


\section{Contents}

About the Special Issue Editors $\ldots \ldots \ldots \ldots \ldots \ldots$ vii

Preface to "Recent Advances in Smart Materials for the Built Environment" . . . . . . . ix

Branko Šavija

Smart Crack Control in Concrete through Use of Phase Change Materials (PCMs): A Review

Reprinted from: Materials 2018, 11, 654, doi:10.3390/ma11050654 . . . . . . . . . . . . . . 1

Claudia Fabiani, Anna Laura Pisello, Antonella D'Alessandro, Filippo Ubertini,

Luisa F. Cabeza and Franco Cotana

Effect of PCM on the Hydration Process of Cement-Based Mixtures: A Novel

Thermo-Mechanical Investigation

Reprinted from: Materials 2018, 11, doi:10.3390/ma11060871 . . . . . . . . . . . . . . 32

Anabel Palacios, Alvaro De Gracia, Laia Haurie, Luisa F. Cabeza, A. Inés Fernández and Camila Barreneche

Study of the Thermal Properties and the Fire Performance of Flame Retardant-Organic PCM in Bulk Form

Reprinted from: Materials 2018, 11, 117, doi:10.3390/ma11010117 . . . . . . . . . . . . . . 4

Xiangming Zhou, Gediminas Kastiukas, Claudio Lantieri, Piergiorgio Tataranni,

Rosolino Vaiana and Cesare Sangiorgi

Mechanical and Thermal Performance of Macro-Encapsulated Phase Change Materials for

Pavement Application

Reprinted from: Materials 2018, 11, 1398, doi:10.3390/ma11081398 _ . . . . . . . . . . . . 61

Piergiorgio Tataranni, Giulia Maria Besemer, Villiam Bortolotti and Cesare Sangiorgi

Preliminary Research on the Physical and Mechanical Properties of Alternative Lightweight

Aggregates Produced by Alkali-Activation of Waste Powders

Reprinted from: Materials 2018, 11, 1255, doi:10.3390/ma11071255 _ . . . . . . . . . . . 79

Marta Vila-Cortavitarte, Daniel Jato-Espino, Daniel Castro-Fresno and

Miguel Á. Calzada-Pérez

Self-Healing Capacity of Asphalt Mixtures Including By-Products Both as Aggregates and

Heating Inductors

Reprinted from: Materials 2018, 11, 800, doi:10.3390/ma11050800 . . . . . . . . . . . . 96

\section{Dessi A. Koleva}

An Innovative Approach to Control Steel Reinforcement Corrosion by Self-Healing

Reprinted from: Materials 2018, 11, 309, doi:10.3390/ma11020309 . . . . . . . . . . . . . . 110

Leyang Lv, Erik Schlangen and Feng Xing

Self-Sealing Cementitious Materials by Using Water-Swelling Rubber Particles

Reprinted from: Materials 2017, 10, 979, doi:10.3390/ma10080979 . . . . . . . . . . . . . 136

Marcin Górski, Rafał Krzywoń and Magdalena Borodeńko

Development of Self-Sensing Textile Strengthening System Based on High-Strength

Carbon Fiber

Reprinted from: Materials 2018, 11, 2062, doi:10.3390/ma11102062 . . . . . . . . . . . . 150 
Serena Andreotti, Elisa Franzoni and Paola Fabbri

Poly(hydroxyalkanoate)s-Based Hydrophobic Coatings for the Protection of Stone in Cultural Heritage

Reprinted from: Materials 2018, 11, 165, doi:10.3390/ma11010165 . . . . . . . . . . . . . . 160

Anna Al Sabouni-Zawadzka and Wojciech Gilewski

Smart Metamaterial Based on the Simplex Tensegrity Pattern

Reprinted from: Materials 2018, 11,673, doi:10.3390/ma11050673 . . . . . . . . . . . . . . . 186 


\section{About the Special Issue Editors}

Cesare Sangiorgi (PhD in Civil Engineering) is Associate Professor in the Department of Civil, Chemical, Environmental and Materials Engineering at the University of Bologna, Italy. He works in the Transportation Infrastructures section of the Department and addresses themes related to pavement materials and construction technology. The use of recycled materials and low-impact solutions are of primary importance in Dr. Sangiorgi's research, and the collaboration with renowned research institutes are the basis for the involvement of students at any level ( $\mathrm{PhD}$ and MSc). Since March 2018, Cesare Sangiorgi has been Coordinator of SAFERUP!, an H2020 MSCA-ITN project based on Sustainable, Accessible, Safe, Resilient, and Smart Urban Pavements.

Filippo Ubertini (PhD in Civil Engineering) is a Professor in the Department of Civil and Environmental Engineering at University of Perugia, Italy, where he teaches Advanced Structural Design. He graduated cum laude in Civil Engineering from University of Perugia in 2005, and received his PhD in Civil Engineering from the University of Pavia in 2009. He was visiting scholar at Columbia University in 2008. His research is mainly focused on structural health monitoring, with emphasis on smart materials and applications to earthquake engineering and cultural heritage structures. Currently, he is coordinating the research teams of the University of Perugia in two Horizon 2020 projects and he is coordinating a national PRIN project. He is an Editorial Board member of Shock and Vibration, Mathematical Problems in Engineering, and Sensors. He has been the recipient of prizes and awards.

Anna Laura Pisello (PhD in Energy Engineering) is an Assistant Professor in the Department of Engineering, University of Perugia, Italy, and she obtained the national professorship qualification as an Associate Professor in 2017. She is also visiting research collaborator at Princeton University, NJ, USA. She was formerly visiting scholar at Columbia University, Virginia Tech, and CUNY. She is the author of more than 90 international indexed publications and editor of five international journals about building physics and energy systems. She won six international academic research awards and eight European grants in the last 4 years for developing and analyzing new solutions for energy saving, microclimate optimization, and UHI mitigation. She coordinates an active research group under the framework of the Environmental Applied Physics Laboratory, and she coordinates the Interuniversity Cooperative Agreement between Princeton University and the University of Perugia. 



\section{Preface to "Recent Advances in Smart Materials for the Built Environment"}

The built environment of the future is expected to need novel smart and multifunctional construction materials that provide a variety of features in addition to strength and durability. In this view, many researchers worldwide are focusing on the development of such innovative and highly promising solutions, with the purpose of enhancing the construction material behavior in terms of environmental sustainability and technical performance, including the new frontiers of self-sensing, self-healing, energy harvesting, overheating control, noise reduction, microclimate mitigation, and other capabilities.

Society itself will be the first beneficiary of such scientific discussion, since it will take advantage of these characteristics that will allow savings in raw materials and energy, minimizing the externalities of construction, service and maintenance phases, and impact. Furthermore, those solutions are expected to transform the physical and mechanical behavior of structural and non-structural elements into precious sources of data, renewable energy, in addition to other functional advantages, such as noise and urban overheating reduction.

This book aims to present a number of high-quality up-to-date research contributions that have proven the benefits and feasibility of smart materials in the built environment at large. Technical papers, review contributions, and case histories are included.

Cesare Sangiorgi, Filippo Ubertini, Anna Laura Pisello Special Issue Editors 

Review

\title{
Smart Crack Control in Concrete through Use of Phase Change Materials (PCMs): A Review
}

\author{
Branko Šavija \\ Microlab, Delft University of Technology, 2628 CN Delft, The Netherlands; b.savija@tudelft.nl; \\ Tel.: +31-681-015-812
}

Received: 3 April 2018; Accepted: 19 April 2018; Published: 24 April 2018

\begin{abstract}
Cracks in concrete structures present a threat to their durability. Therefore, numerous research studies have been devoted to reducing concrete cracking. In recent years, a new approach has been proposed for controlling temperature related cracking-utilization of phase change materials (PCMs) in concrete. Through their ability to capture heat, PCMs can offset temperature changes and reduce gradients in concrete structures. Nevertheless, they can also influence concrete properties. This paper presents a comprehensive overview of the literature devoted to using PCMs to control temperature related cracking in concrete. First, types of PCMs and ways of incorporation in concrete are discussed. Then, possible uses of PCMs in concrete technology are discussed. Further, the influences of PCMs on concrete properties (fresh, hardened, durability) are discussed in detail. This is followed by a discussion of modelling techniques for PCM-concrete composites and their performance. Finally, a summary and the possible research directions for future work are given. This overview aims to assure the researchers and asset owners of the potential of this maturing technology and bring it one step closer to practical application.
\end{abstract}

Keywords: concrete durability; smart concrete; phase change materials; microencapsulation

\section{Introduction}

Reinforced concrete is a building material of choice for structures in challenging environments. Concrete is a highly durable building material and can have a long service life with little or no maintenance. When steel is embedded in concrete in order to take over tensile stresses, it is typically protected from corrosion by a passive film formed on its surface due to the high alkalinity of the concrete pore solution [1]. Under certain conditions, however, this passive film can break down, leading to reinforcement corrosion. Reinforcement corrosion can be caused by concrete carbonation [2] which leads to a drop in the $\mathrm{pH}$ value (below 9) and breaks down the passivation layer; or chloride ingress [3] which can locally break down the passive layer leading to pitting corrosion. Since steel corrosion is an expansive reaction, it will then lead to cracking and spalling of the concrete cover $[4,5]$. As a consequence, service life design guidelines require that the concrete cover to the reinforcement is of a certain dimension and quality [6,7]. These guidelines are, however, derived assuming that the concrete cover is uncracked. Reinforced concrete structures are, however, always cracked [8], due to a variety of reasons: mechanical loads, (restrained) shrinkage [9], thermal deformations [10], and freezing and thawing [11], among others. Most of these cracks do not pose a threat for the structural integrity of a structure. However, they may create durability issues: both chloride ingress [12] and carbonation [13] have been shown to advance much faster in cracked concrete, thereby leading to rapid steel depassivation and corrosion [14]. Consequently, there are numerous approaches proposed in the literature to minimize cracking in reinforced concrete structures.

Concrete is a quasi-brittle material that cracks at low strain levels [8]. One possibility for minimizing cracking is through the use of fiber reinforcement such as steel [15], polyvinyl alcohol 
(PVA) [16], or natural fibers [17]. If properly designed, these fibers are able to bridge cracks in concrete and reduce their width. A recent and more reactive approach is the use of self-healing concrete: once a crack forms, it is autonomously repaired by the material itself [18-20]. In this way, the durability of the structure is restored. In recent years, a new approach for reducing concrete cracking caused by thermal effects has been proposed: through incorporation of phase change materials (PCMs) in the concrete mix, temperature variations can be minimized thereby avoiding occurrence of thermal cracking. PCMs are combined (sensible- and latent) thermal storage materials that can store and dissipate energy in the form of heat [21]. In PCMs, heat is absorbed and released when the material changes its state from solid to liquid and vice versa. This is schematically shown in Figure 1.

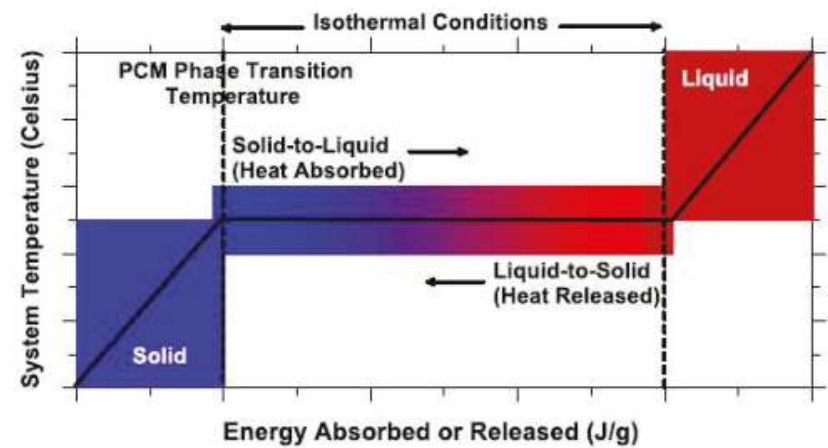

Figure 1. A schematic which illustrates the temperature-energy (heat) response of a phase change material (PCM) and the process therein [22].

During the heating pass of the phase change temperature, PCMs change from solid to liquid and capture heat, thereby reducing the temperature rise in the system. During the cooling pass of the phase change temperature, PCMs change from liquid to solid and release the stored energy, thereby reducing the cooling rate of the system. Therefore, PCMs can be used in concrete technology to reduce cracking caused by temperature effects [23]: early age cracking due to hydration of large concrete sections, and freeze/thaw damage in cold climates.

This paper aims to review the current state of the art of using PCMs for smart crack control in reinforced concrete, with the focus on the two aforementioned causes of cracking. The paper is organized as follows: first, different types of phase change materials and possible ways of application in concrete technology are discussed; then, studies regarding the use of PCMs to reduce cracking are reviewed; further, the influence of PCM addition on the properties of concrete is discussed; this is followed by a review of modelling approaches for PCMs in concrete; finally, conclusions and possible directions for future research are given.

\section{Phase Change Materials in Concrete Technology}

\subsection{Classification of Phase Change Materials}

A large variety of PCMs are available. In general, they can be divided in three categories (Figure 2): organic, inorganic, and eutectic PCMs. Organic PCMs are classified as paraffin and non-paraffin compounds [21,24,25]. Most organic PCM compounds are chemically stable, safe, and non-reactive [25]. Furthermore, organic PCMs in general do not suffer from phase segregation and crystallize with little or no supercooling [26]. Organic PCMs can be divided into two categories: paraffin compounds and non-paraffin compounds (such as fatty acids). Inorganic PCMs are classified as salt hydrates and metallics $[21,24,25]$. Inorganic PCMs may have potential applications in some types of building materials, because of their high volumetric heat storage capacity and good thermal conductivity [25]. 
In addition, they are relatively low-cost, readily available, and non-flammable [25]. However, most inorganic PCMs are corrosive to metals and undergo supercooling and phase decomposition [24]. A eutectic is a minimum-melting composition of two or more components, each of which melts and freezes congruently forming a mixture of the component crystals during crystallization [21]. This allows the design of organic/inorganic PCM mixtures to create optimum operating temperatures for specific applications [26]. Table 1 presents a comparison of the advantages and disadvantages of the three types of PCMs.

\section{Phase change material}

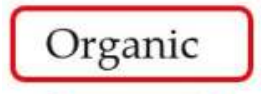

Paraffin compounds

Non-paraffin compounds

\section{Inorganic}

Salt

hydrates

Metallics

\section{Eutectics}

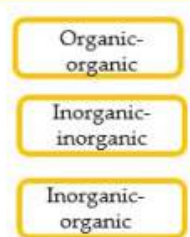

Figure 2. Classification of phase change materials (PCMs) (adapted from [21]).

Table 1. Advantages and drawbacks of various phase change material (PCM) types [26-28].

\begin{tabular}{|c|c|c|}
\hline Classification & Advantages & Drawbacks \\
\hline Organic PCMs & $\begin{array}{l}\text { 1. Availability in a large temperature range } \\
\text { 2. High heat of fusion } \\
\text { 3. No supercooling } \\
\text { 4. Chemically stable and recyclable } \\
\text { 5. Good compatibility with conventional } \\
\text { construction materials }\end{array}$ & $\begin{array}{l}\text { 1. Low thermal conductivity } \\
\text { 2. Relatively large volume change } \\
\text { 3. Flammable }\end{array}$ \\
\hline Inorganic PCMs & $\begin{array}{l}\text { 1. High heat of fusion } \\
\text { 2. High thermal conductivity } \\
\text { 3. Low volume change } \\
\text { 4. Low cost } \\
\text { 5. Sharp phase change } \\
\text { 6. Non-flammable }\end{array}$ & $\begin{array}{l}\text { 1. Corrosive to metals } \\
\text { 2. Supercooling }\end{array}$ \\
\hline Eutectics & $\begin{array}{l}\text { 1. Sharp melting point } \\
\text { 2. Properties can be tailored to match } \\
\text { specific requirements } \\
\text { 3. High volumetric thermal storage density }\end{array}$ & $\begin{array}{l}\text { 1. Limited data on } \\
\text { thermo-physical properties for } \\
\text { many combinations } \\
\text { 2. High cost }\end{array}$ \\
\hline
\end{tabular}

\subsection{Incorporation of PCMs in Concrete}

Most applications of phase change materials in construction are related to increasing the energy efficiency of the building envelope [24-26]. These applications have been a subject of numerous excellent reviews, and therefore are not the topic here. However, various techniques for incorporation of PCMs in building materials have been developed in these applications. The same techniques can be used when PCMs are utilized to control temperature variations within the material itself. The main approaches are:

- Using pipes filled with PCM incorporated in concrete. 
- Using porous carriers such as lightweight aggregates (LWAs) impregnated with PCMs.

- Using microencapsulated PCMs.

- Impregnating PCMs in the concrete pores from the surface.

These methods are schematically illustrated in Figure 3.

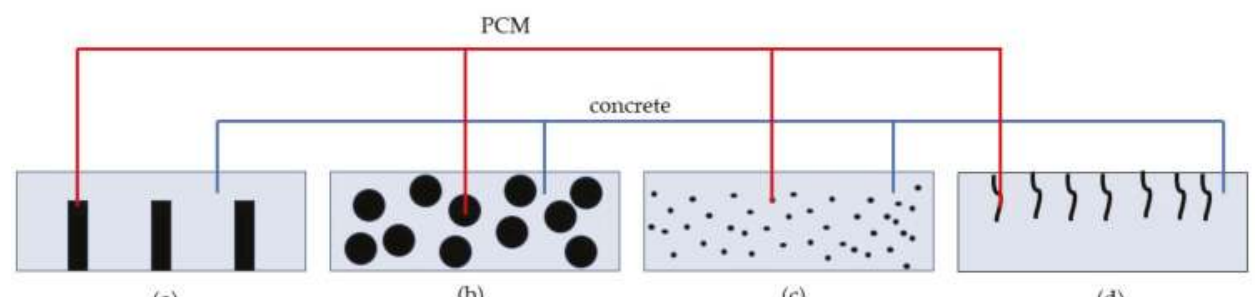

(a)

(b)

(c)

(d)

Figure 3. Methods for PCM incorporation in concrete: (a) using pipes filled with PCM; (b) using lightweight aggregate particles impregnated with PCM; (c) using microcapsules with PCM; (d) filling concrete surface voids via PCM absorption (adapted from [29]).

\subsubsection{Embedded Pipes}

For reducing temperature effects in massive concrete structures, the use of cooling pipes with running water is relatively common [30]. In order to increase the heat capacity of the embedded pipe systems, it has been suggested to fill them with PCMs with an appropriate phase change temperature. In general, a closed pipe system is used [29,31-33]. However, one study used a running system where water was replaced with PCM which was replaced every time a predetermined temperature was achieved, thereby increasing the rate of heat removal from the system [34]. A major advantage of the pipe system is that it is resistant to physical and chemical damage that it may undergo during casting and exploitation of concrete. Furthermore, leakage of PCM and its possible influence on fresh and hardened concrete properties are avoided when the pipe system is used. On the other hand, this approach has several drawbacks: first, the effect of the PCM is limited by the spacing of the pipe system; second, it is possible that the PCMs melt only partially due to their low thermal conductivity, resulting in an underutilized system; and finally, the system is as complicated as current cooling pipe systems.

\subsubsection{Lightweight Aggregates}

Another way of incorporating PCMs in concrete is through impregnation in lightweight aggregates. Water impregnated LWAs are commonly used for internal curing of concrete [35-37], providing additional water for hydration of high-strength concrete and thereby reducing its autogenous shrinkage. LWAs impregnated with bacteria and nutrients are also used in self-healing concrete [38,39]. Using the same approach, LWAs can be impregnated with suitable phase change materials, and then used in concrete (Figure 4).

Different types of LWAs have been used: expanded shale [23,29,32,40-42], expanded clay [40,43-46], perlite $[42,47,48]$, and others such as diatomite and vermiculite [49-52]. These LWAs are impregnated with (liquid) PCMs using either immersion/direct impregnation (e.g., [23,42-45,49]) or a vacuum impregnation procedure (e.g., $[29,32,46-48])$. Schematic representation of the vacuum impregnation procedure is given in Figure 5. Both of these procedures have advantages and disadvantages. While vacuum impregnation enables higher levels of saturation with PCM (see e.g., [47] for comparison), soaking under ambient conditions is easier to replicate in practice [42]. It has to be noted that the PCM absorption capacity of the LWA cannot be simply determined by water absorption measurements: due to the low viscosity of water, it is able to penetrate into the pore spaces of smaller diameter and hence occupy more pore space than the PCM [46]. Furthermore, using only porosity is 
also not useful in this respect: a combined mercury intrusion porosimetry (MIP) and PCM absorption study of Aguayo [42] showed that porous aggregates with larger pore sizes of comparable porosities have larger PCM absorption. In fact, for a rough estimation of the penetrating degree of liquid PCM into LWA, the cumulative pore volume curve needs to be known: Zhang et al. [40] estimated that the liquid PCM can penetrate pores larger than 1-2 $\mu \mathrm{m}$ under vacuum condition. Clearly, a larger threshold value holds if LWAs are immersed in a PCM solution under atmospheric pressure.

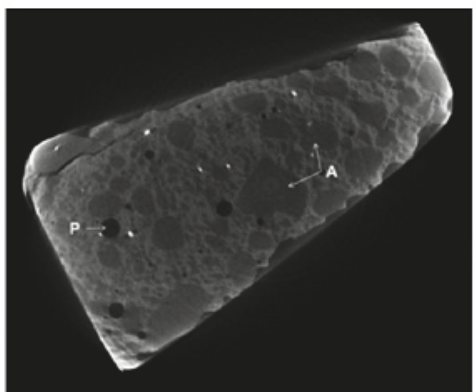

(a)

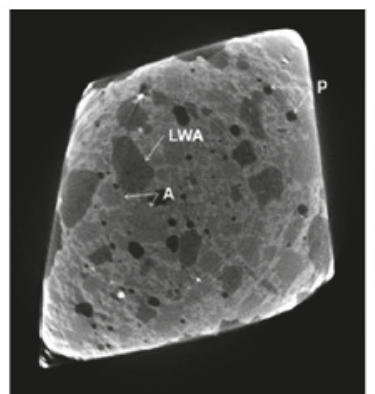

(b)

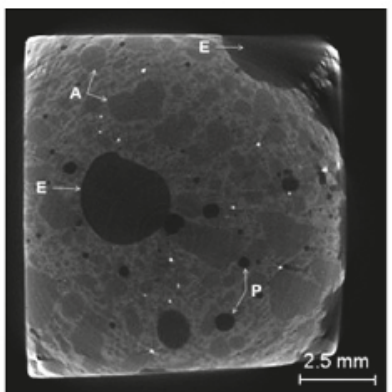

(c)

Figure 4. Microtomographs of investigated mortars: (a) control mortar without PCM, in which quartz aggregate (A) and pores/air voids (P) are easily visible; (b) mortar containing lightweight aggregate (LWA)-PCM, which appears similar to quartz aggregate but darker because of its lower density; and (c) mortar containing encapsulated PCM pellets (E) [43].

Compared to the method of embedded pipes, use of LWAs as PCM carriers provides a better spatial distribution of PCM. Especially in composites with relatively fine LWA the PCM will be well distributed, and the ability of heat to travel through the matrix and reach all corners of the system is not as important [44]. Essentially, thermal diffusivity of the cement matrix must be balanced by the spatial distribution of the PCM, similar to the concept of "protected paste volume" in internal curing studies [36]. When PCMs are less homogeneously distributed (such as the case when embedded pipes are used), increasing the thermal conductivity of the composite is important [43]. In some studies wherein coarse LWAs are used, this is addressed by utilizing graphite powder [46], multi-wall carbon nanotubes [50], or aluminum powder [53] which increase the thermal conductivity of the LWA/PCM particles.
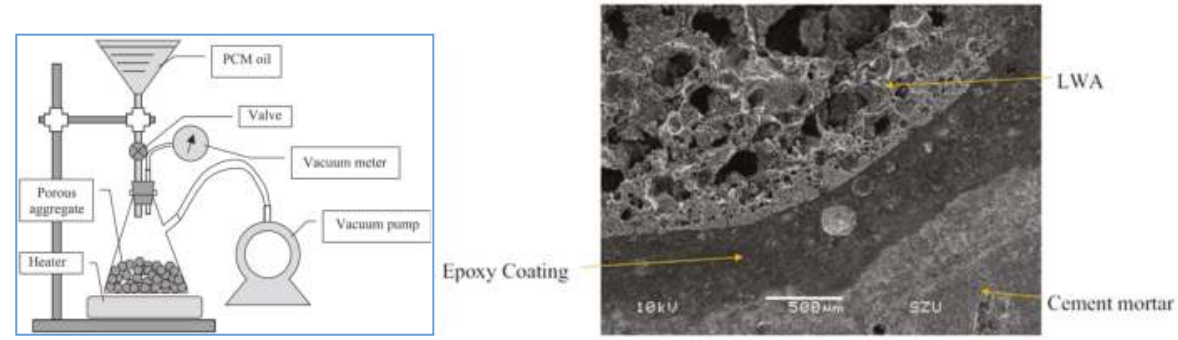

Figure 5. (left) Schematics of a vacuum impregnation procedure for LWA [40]; (right) scanning electron microscope (SEM) image of a PCM impregnated LWA particle coated with epoxy to prevent PCM leakage (scale bar given in the corner) [46].

Another major disadvantage of the LWA approach is possible leakage of the PCM during mixing and/or exploitation and its dissolution in the mixing water. If the ambient temperature is lower than 
the phase change temperature of the PCM, leakage during mixing will occur [47]. In fact, some PCMs such as polyethylene glycol (PEG) are known to retard cement hydration [23]. Furthermore, if the LWA exchanges PCM with water from the matrix, it will dehydrate the system and result in a lower degree of hydration [43]. It has been also suggested that PCMs could in these cases coat the cement particles, protecting them from reacting with water [43]. One study in which methyl laureate PCM impregnated in LWA was used showed that, in certain cases, an expansive chemical reaction can occur between the PCM and aluminate phases in the cementitious matrix, causing cracking [29]. On the other hand, PCM in surface pores of the LWA could impair the LWA/cement paste bond, thereby reducing the strength of the composite material [45]. In order to mitigate these issues, some authors suggested coating the PCM impregnated LWAs: Memon et al. [46] coated the particles with an epoxy resin (Figure 5), while Ramakrishnan et al. [47] used a hydrophobic agent. Although coating of the LWAs will ensure that no leakage occurs, it will increase the complexity and the price of the production process.

\subsubsection{Microencapsulation}

Microencapsulation is defined as a process in which tiny particles or droplets are surrounded by a coating to give small capsules with useful properties. In concrete technology, microencapsulation has been used for carrying self-healing agents [54-56] and corrosion inhibitors [57]. The primary advantage of microencapsulated PCMs are their chemically-inert nature (due to the polymer barrier between the PCM and any other material), the optimized heat transfer due to a high surface area-to-volume ratio, and their ability to be readily mixed into or coated onto other materials [58]. The shell needs to fulfil two major conditions: first, it needs to be able to sustain mixing and casting of concrete without damage and consequent leakage of the core material; and second, it needs to have long term stability in the highly alkaline environment in concrete.

In most studies, paraffin as the PCM core material is encapsulated in a polymeric shell. Most microencapsulated PCMs used in concrete have either a polymethyl methacrylate (PMMA) [59-65] or melamine formaldehyde (MF) shell [63,66-70]. In one study, urea formaldehyde was used as a shell material [71]. Typically, PCM microcapsules are relatively small, in the size range of 10s of $\mu \mathrm{m}$ (Figure 6). However, microcapsules in the size range of 200-500 $\mu \mathrm{m}$ have also been used [71]. The small particle size is optimal in terms of surface to volume ratio of the PCMs due to their low thermal conductivity. Furthermore, small particles can be better dispersed in the cement matrix compared to large or agglomerated particles (Figure 7).

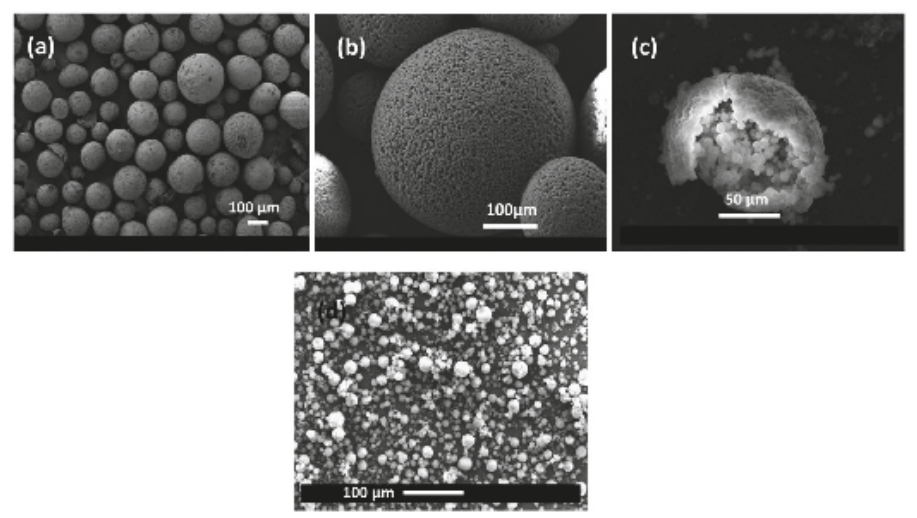

Figure 6. Micrographs of two types of PCM microcapsules: (a) PCM-A; (b) and (c) PCM-A showing smaller capsules that are agglomerated to form the larger capsule, and (d) PCM-B, which is composed of discrete particles [65]. 


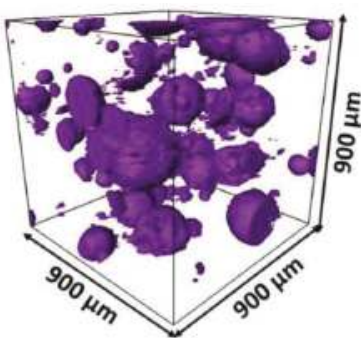

(a)

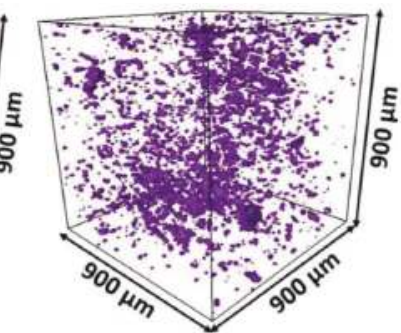

(b)

Figure 7. X-ray computed tomography scans showing distribution of two types of PCM microcapsules in cement paste: (a) PCM-A (agglomerated microcapsules, see Figure 6); and (b) PCM-B (discrete particles, see Figure 6) [65].

A major concern when using microencapsulated PCMs in concrete is their survivability and long-term durability. Survivability can be defined as the ability of microcapsules to survive the mixing process of concrete without rupture and leakage of the core material. Since this is a major concern, in most studies care is taken to minimize the exposure of the microcapsules to concrete mixing, for example by adding them as last ingredients [72-74]. Nevertheless, several studies have shown that a certain percentage of microcapsules does break during the mixing. For example, a large number of broken microcapsules were observed by Hunger et al. [72]. Other studies have also reported that a certain percentage of PCM microcapsules has ruptured during mixing [22,59,61].

On the other hand, in some studies it was emphasized that the microcapsules can survive the mixing process $[63,69,74]$. The issue then becomes their durability: the ability of microcapsules to maintain their integrity in the alkaline environment of the concrete pore solution while subjected to (mechanical) loading. Wei et al. [69] observed a 25\% reduction in enthalpy of the phase change of PCM microcapsules regardless of whether mechanical mixing was performed or not. This was attributed to a chemical reaction of the melamine formaldehyde shell with sulfate ions, causing the release of the core material and its reaction with the pore solution. The proposed mechanism is schematically described in Figure 8.

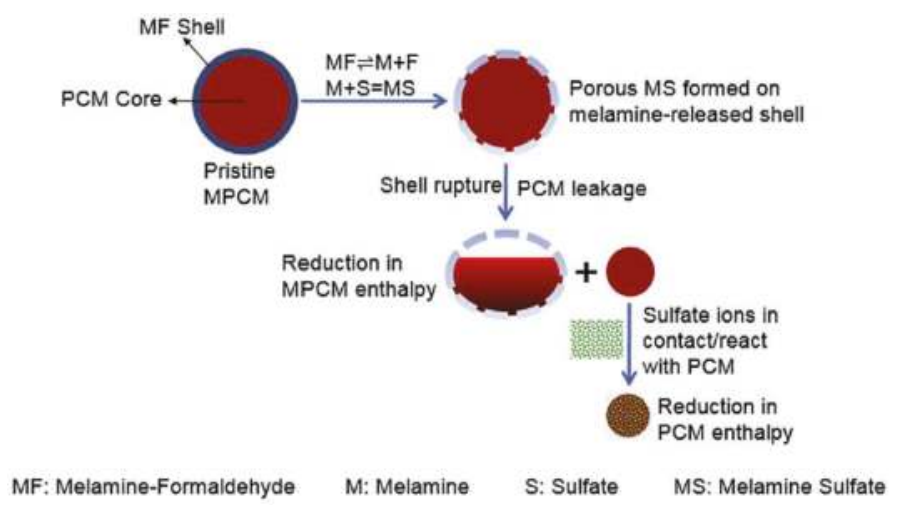

Figure 8. The proposed chemical interaction pathway which results in enthalpy reduction of the PCMs following exposure to caustic solutions containing sulfate ions [69].

Another possible cause of capsule rupture post-curing is mechanical loading. For example, Jayalath et al. [74] observed capsules that ruptured during cracking of the concrete. They suggested 
that the polymeric shell of the capsule is a soft material compared to the cementitious matrix. Consequently, the microcapsules act as crack initiation sites during loading, and the fracture propagates across the weakest point in the matrix, and this includes the PCM particle (Figure 9). This was also observed by others $[73,75]$. This is also in accordance with a study performed on a model gypsum plaster material containing controlled spherical porosity that acted as the initiation point for fracture [76]. Irrespective of the cause of the rupture, leakage of the core material may have consequences for the behavior of the composite. As already stated, it can reduce the enthalpy of the phase change, thereby reducing the thermal efficiency of the material $[59,61,69]$. Furthermore, if the leakage occurs during mixing, it can influence the cement hydration process. Eddhahak et al. [59] studied the effect of PCM leakage on the hydration process by intentionally damaging the capsules prior to mixing of the concrete. In their study, mortars with damaged PCMs exhibited a slight decrease in hydration heat (Figure 10) that was attributed to paraffin leakage that could have influenced the hydration process. Therefore, care should be taken when estimating thermal properties of cementitious materials with PCM microcapsules: if capsule breakage is neglected, material efficiency may be overestimated compared to reality.

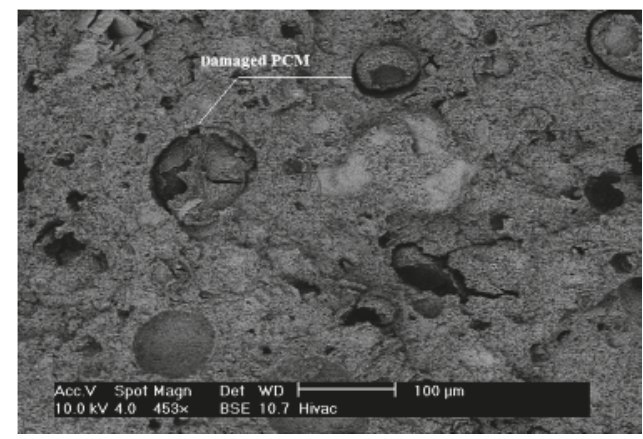

Figure 9. SEM micrograph (using back-scattered electron (BSE) detector) of concrete fracture surface showing damaged or collapsed PCM particles still occupying their original void [75].

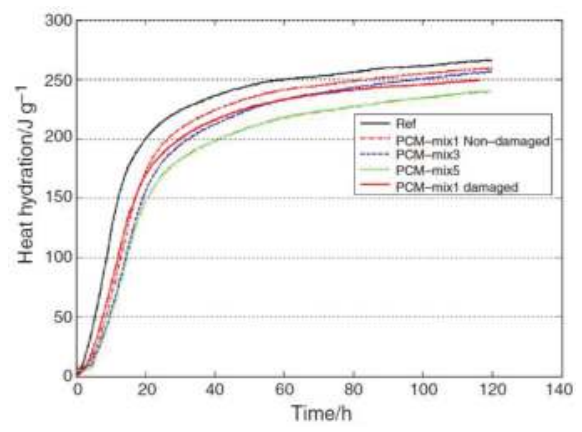

Figure 10. Heat release curves and comparison between damaged and non-damaged cases, showing lower hydration heat in the case when microcapsules are damaged [59].

\subsubsection{Surface Impregnation}

Wallboards with surface impregnated PCMs have been proposed for latent heat storage in buildings [77-79]. Furthermore, concrete blocks impregnated with PCMs were also produced [77]. 
However, since PCMs incorporated in this way are susceptible to leakage, surface impregnation has so far not been used for control of temperature related cracking in cementitious materials.

\section{Use of PCM to Reduce Concrete Cracking}

\subsection{Cracking of Concrete at Early Age Due to Hydration Heat Development}

The temperature in young concrete increases due to the exothermic nature of the chemical reactions occurring during cement hydration [80]. If a concrete element is not restrained, it will freely expand during heating and contract during subsequent cooling, without inducing stresses [81]. In practice, however, concrete is almost always restrained to a certain degree, either by adjoining structures (external restraint) or internally due to a temperature gradient in the structural element itself (internal restraint). Massive structures, such as dams, are prone to early age thermal cracking [82-84]. The concrete surface will cool down faster than the core, giving rise to a temperature gradient between different layers of the structure/element. Differences in thermal dilation between parts of the structure will cause occurrence of tensile stresses at the concrete surface; if these stresses are higher than the tensile strength of concrete, cracking will result $[85,86]$. Thermal cracking will depend on material, structural, and execution factors [87]. Common measures used in practice for tackling this problem include: (1) changes in concrete mix design (using blended cements or lower cement content); (2) modifications in structural design (such as additional reinforcement, pre-stressing, expansion joints); (3) adapting execution parameters (by using cold mix ingredients or built-in cooling pipes). Clearly, use of phase change materials with an appropriate phase change temperature could be of great use in reducing the risk of thermal cracking in young concrete [23].

The first study proposing use of PCMs for temperature control in early-age concrete was performed by Mihashi et al. [88], who added paraffin microcapsules to the mixture. Their study showed that the maximum achieved temperatures in semi-adiabatic conditions were significantly lower when PCMs were incorporated in the mix.

Two methods of incorporating PCM have been used for crack control in young concrete: using pipes filled with PCM and using PCM microcapsules (see Figure 3). Qian et al. [33] incorporated PCM (sodium sulfate decahydrate with a phase change temperature of $32.4{ }^{\circ} \mathrm{C}$ and a heat of fusion of $241 \mathrm{~J} / \mathrm{g}$ ) in pipes cast in concrete, which was left to hydrate in semi-adiabatic conditions. Varying quantities of PCM $(0 \%, 3 \%$, and $6 \%$ of cement mass) were incorporated in concrete. They showed that pipes with PCM can clearly reduce the peak temperature in semi-adiabatic conditions. Furthermore, they also delay the onset of the peak temperature.

In another study, Qian \& Gao [34] used an open circuit system: they replaced the water in the cooling system with a PCM suspension (Figure 11 left). The PCM suspension was replaced with a fresh one every time its temperature reached $25^{\circ} \mathrm{C}$. This enabled a significantly higher capacity to capture heat compared to a stationary PCM system. Furthermore, it was shown to be more efficient than using water as a cooling agent (Figure 11 right). Comparing to concrete with no cooling measure used, the temperature peak of concrete cooled by water and PCM suspension can be decreased to $76.1 \%$ and $84.9 \%$, respectively. Furthermore, the temperature gradient around the cooling pipe was less steep in the case where PCM suspension was used as a cooling liquid instead of water. Therefore, they concluded that such a system could additionally reduce the probability of temperature induced cracking. 

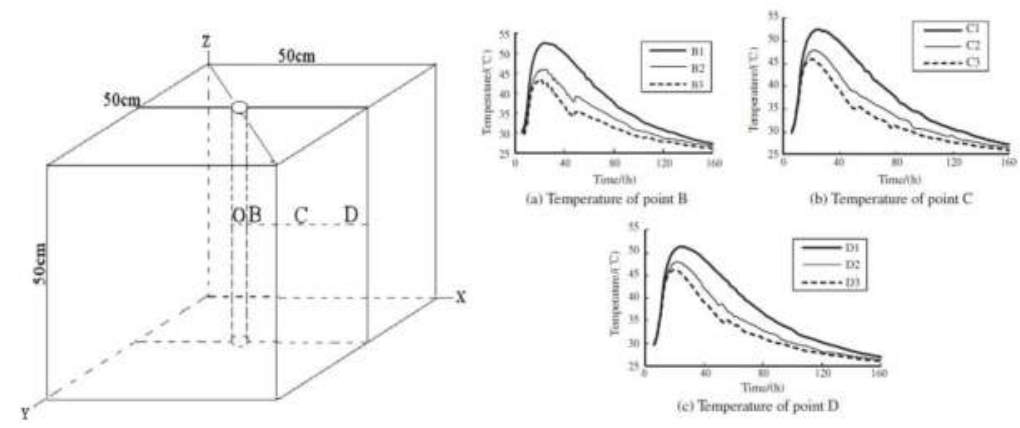

Figure 11. (left) Specimen geometry and points where temperature was measured; (right) temperature evolution in points B, C, and D (numbers 1, 2, and 3 mark cases where no cooling was used, water cooling was used, and PCM cooling was used, respectively) [34].

More commonly, microencapsulated PCMs have been used for temperature control in young concrete $[22,63,73,89,90]$. Hunger et al. [72] used commercially available PCMs with a phase change temperature of $23^{\circ} \mathrm{C}$ to reduce the temperature rise in hardening self-compacting concrete. They clearly showed that incorporation of microencapsulated PCM in the mixture caused a reduction in peak temperature in a semi-adiabatic test (Figure 12 left). Furthermore, a delay in the occurrence of maximum temperature was observed. The effectiveness of PCM microcapsule addition is proportional to the amount of PCM addition: the higher the PCM percentage, the lower the maximum temperature achieved and the later it occurs. Fernandes et al. [22] observed that, although there is a decrease in maximum temperature, the rate of temperature rise remains similar to the reference in mixtures incorporating PCMs. Snoeck et al. [63] showed that the effectiveness of PCMs in reducing the maximum temperature depends on the phase change temperature. In their study, when a PCM with a phase change temperature of $18^{\circ} \mathrm{C}$ was used, there was only a minor effect since the phase change temperature was below the initial testing temperature $\left(20^{\circ} \mathrm{C}\right)$. On the other hand, when the PCM with a phase change temperature of $28^{\circ} \mathrm{C}$ was used, the effect of encapsulated PCM could only start when the cement hydration reaction was already at "full speed", so the effectiveness was less pronounced. Their study stresses an important fact: the phase change temperature needs to be adjusted for different climatic conditions in order to be fully utilized. This was further addressed by Young et al. [90], who used a PCM with a phase change temperature of $40{ }^{\circ} \mathrm{C}$ in the warm climate in California, USA. It is also important to notice that the incorporation of microencapsulated PCMs reduces the rate of cooling, which could be even more critical for controlling early age cracking in concrete [22].

In addition, one study (by Kim et al. [28]) used barium based PCM which was directly added to the concrete mix. The PCM based mix showed excellent performance, with a semi-adiabatic temperature rise lower than the mixtures incorporating supplementary cementitious materials, which are commonly used to combat early age temperature rise.

In warm climates, incorporation of PCM has an additional advantage that goes beyond the early age behavior. Since PCMs typically remain active, they will go through a phase change every time their phase change temperature is passed. Therefore, they can contribute to reducing thermal fatigue in structures exposed to temperature variations, such as concrete pavements. Thiele et al. [89] showed that the addition of PCM microcapsules is able to "smooth" the temperature cycles in concrete (Figure 12 right). It has to be noted that the rate of temperature change has a significant effect on the rate of temperature change (as also observed by Fernandes et al. [22]): if the rate of temperature change is too fast, the benefits of heat absorption/release are overwhelmed as the concrete temperature is already higher/lower than temperature of phase change. In such cases, the thermal response of the concrete is governed by the sensible instead of the latent heat, which cannot stabilize the concrete temperature. 

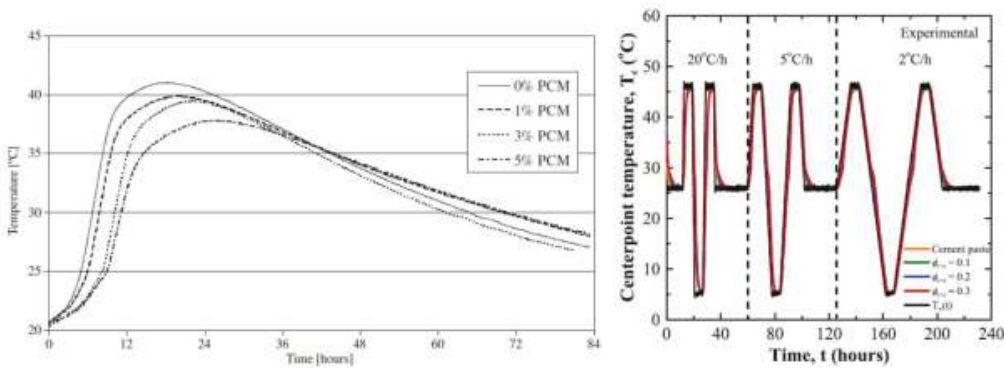

Figure 12. (left) Temperature development of four self-compacting mixes in a semi-adiabatic environment during the first 3.5 days after casting [72]; (right) Center point temperature as a function of time within cement paste specimens without and with microencapsulated PCM (phase change temperature $32{ }^{\circ} \mathrm{C}$ ) with a volume fraction of $0.1,0.2$, or 0.3 subjected to an imposed chamber temperature $\mathrm{T} \infty(\mathrm{t})$ varying at a ramp rate of 20,5 , and $2{ }^{\circ} \mathrm{C} / \mathrm{h}[89]$.

\subsection{Cracking of Concrete Due to Freeze/Thaw Cycles}

In cold climates, concrete may deteriorate due to freezing and thawing of water in the system $[80,91]$. Since the volume of ice is around $9 \%$ higher than the volume of water, expansive pressures are present in the concrete when freezing temperatures occur if there is not enough space to accommodate this expansion. If the water saturation level is higher than the critical value (theoretically $91 \%$, but in practice around $86 \%-88 \%$ [92]), damage may occur [93-96]. In practice, this is solved by air-entrainment, whereby air bubbles are intentionally introduced in the concrete by means of chemical additives [97]. If they are well distributed and properly spaced, they are able to accommodate the volumetric expansion without causing pressure or cracking [98].

The described approach essentially tries to avoid damage by accommodating the expansion. An alternative would be to avoid the cycles of freezing and thawing altogether. In the past few years, several studies have proposed the use of phase change materials to reduce the number of freeze/thaw cycles and improve concrete durability. The use of phase change materials in concrete technology for reducing freeze/thaw damage was first proposed by Bentz and Turpin in 2007 [23]. In this work, they performed numerical simulations in order to quantify the effect of PCM incorporation in concrete on the number of freeze/thaw cycles in twelve locations across the US, spanning a range of climates. They assumed that concrete contains $300 \mathrm{~kg} / \mathrm{m}^{3}$ of PCM with a phase change temperature of $5{ }^{\circ} \mathrm{C}$ and the enthalpy of phase change of $250 \mathrm{~J} / \mathrm{g}$. Since previous works showed that a greater number of freeze/thaw cycles can be expected in bridge decks compared to concrete pavements [99], a bridge deck was simulated. They concluded that the hypothetical concrete with PCM could reduce the number of freeze/thaw cycles by $30 \%$ on average across selected geographic locations in the US. However, they stated that the efficiency of PCMs will be strongly climate dependent-the ideal situation being a climate with numerous freeze/thaw cycles but without extremely cold weather.

Based on this, an additional study was performed by Sakulich and Bentz [43]. In the study, they performed more advanced simulations of freezing and thawing for various climates in the US (Figure 13). They concluded that the optimal range of phase change temperatures of PCMs for preventing freeze/thaw damage is between $3.5-6{ }^{\circ} \mathrm{C}$. This is because of the way bodies are cooled, which depends heavily on the thermal conductivity of the system. Simulations of Sakulich and Bentz [43] show that increasing the thermal conductivity of the system will result in fewer freeze/thaw cycles if the phase change temperature is in the optimal range. Cooling of a hot body will occur primarily due to convection or conduction. A greater thermal differential will lead to a more rapid cooling; a greater thermal conductivity will lead to a more rapid transfer of heat from the interior of the body to the surface and even cooling. In cases when thermal conductivity is low, heat cannot flow from the interior to the surface easily, so although the surface may cool rapidly, the interior will 
be maintained at a higher temperature and remain free of freeze/thaw damage. Therefore, in such applications, increasing the thermal conductivity of the composite will be important [43]. This is especially an issue when LWAs with PCM are used (see Section 2.2.2.).

(a)

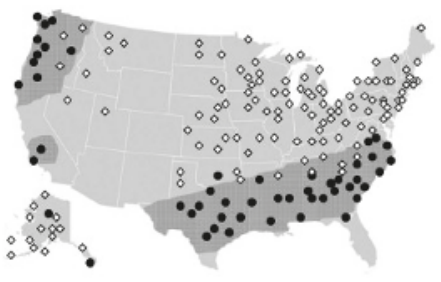

(b)

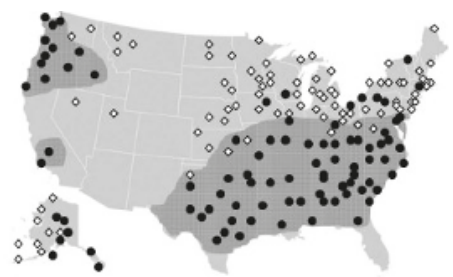

Figure 13. (a) US locations in which incorporation of $50 \mathrm{~kg} / \mathrm{m}^{3}$ of PCM increases bridge deck service life by less than 1 year (white diamond) or more than 1 year (black dot); and (b) US locations in which incorporation of the maximum $120 \mathrm{~kg} / \mathrm{m}^{3}$ of PCM increases bridge deck service life by less than 1 year (white diamond) or more than 1 year (black dot); shaded areas indicate a rough estimate of the regions in which PCM incorporation is practical for extending bridge deck service lives [43].

An additional benefit of using PCMs instead of air entrainment in this case is the ability of the material to "melt" ice and snow forming on its surface: because the concrete will remain warmer for a longer period of time, it will prevent snow from accumulating and ice from forming on the surface. This may be very beneficial for some applications, such as airport runway pavements. This possibility was explored in several studies. Farnam et al. [29] used two types of PCM (methyl laureate with a phase change temperature of $1.9^{\circ} \mathrm{C}$ and latent heat of fusion of $160.4 \mathrm{~J} / \mathrm{g}$; and paraffin oil with a phase change temperature of $2.9^{\circ} \mathrm{C}$ and latent heat of fusion of $129.4 \mathrm{~J} / \mathrm{g}$ ) either embedded in a tube system or incorporated in LWAs for melting snow on the concrete surface. Based on the energy released during the phase change and the volume of the concrete, they calculated the amount of ice that can be melted during the phase transition. In addition, they calculated the amount of heat released during the PCM phase transformation per cubic meter of concrete from a Low-Temperature Differential Scanning Calorimetry experiment. Both quantities are shown in Figure 14. It can be noted that concrete with ML (methyl laureate) impregnated in LWAs showed no improved performance, since a chemical reaction occurred between the PCM and cement hydrates. With PCM in embedded tubes, ML is able to melt a significant amount of snow. On the other hand, paraffin does a good job irrespective of whether it is impregnated in LWAs or embedded in tubes. Since ML has a higher heat of fusion, however, it performs the best in the case when it is embedded in a pipe system.

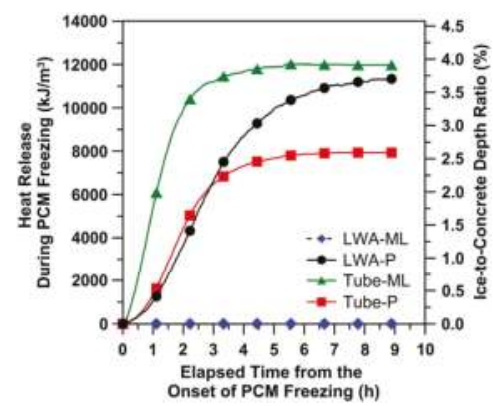

Figure 14. Released heat during PCM freezing in mortar specimen as a function of elapsed time from the onset of PCM freezing (notice that the quantity of PCM per volume of mortar sample varies for each case [29]. 
Based on these results, Farnam et al. [32] performed a follow-up study. In it, they prepared large scale slabs with (LWA and embedded tubes) and without PCMs. Their experimental setup is shown in Figure 15. In the two approaches, there is a slight difference in the amount of PCMs used: in the LWA system, there was $22 \%$ of PCM per volume, while in the embedded pipe system, there was $14 \%$ of PCM per volume of the specimen. Specimens were insulated on the sides in order to ensure 1D heat transfer. The slabs were then subjected to temperature cycling with and without (manmade) snow. An example of cycles and temperatures within the slabs is given in Figure 16. Their research showed that both methods (i.e., PCMs impregnated in LWAs and embedded pipes) are able to melt snow at the concrete surface. However, some differences between the two approaches have been reported: when the concrete slab was exposed to an ambient temperature above the freezing temperature of PCM before a snow event, a relatively rapid heat release was observed for the embedded pipe method during PCM phase transformation, while the heat release in the LWA method was more gradual. The rapid heat release in the PCM-PIPE slabs may be beneficial in melting ice and snow at a faster rate than the PCM-LWA slabs; however, the capability of snow melting in the PCM-PIPE slabs may noticeably decrease when the concrete slab is exposed to an ambient temperature near or below the freezing temperature of PCM before a snow event. Conversely, for the LWA method, the gradual heat release can be used over a wide range of temperature variation and the snow melting capability remains relatively beneficial.
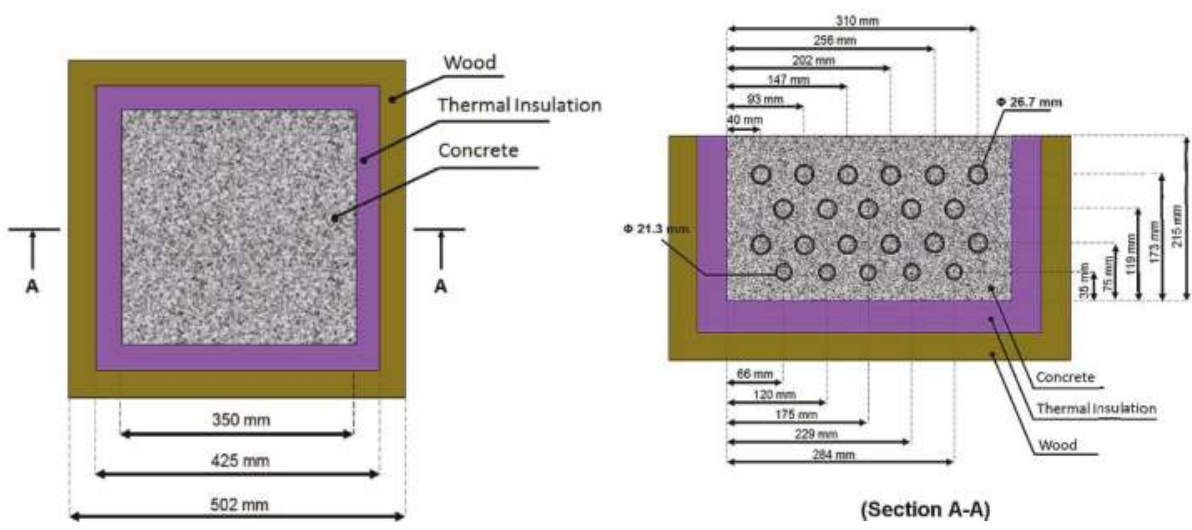

Figure 15. Design details for the large-scale concrete slab: (left) top view details of the mold and the concrete slab; (right) cross section details of the mold and the concrete slab with embedded pipes (to prevent heat transfer through metal pipes, additional outer thermal insulations were installed on both sides of the framework where pipes were out of the wood framework) [32]. 

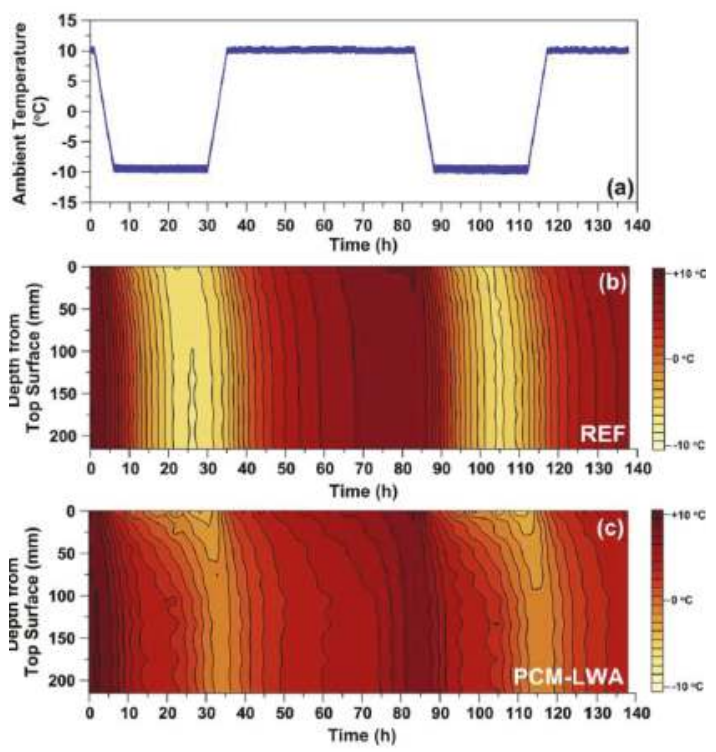

Figure 16. Thermal cycling of the concrete slab (a) ambient temperature applied in thermal cycling experiment; (b) temperature profile within the depth of reference slab as a function of time; and (c) temperature profile within the depth of PCM-LWA as a function of time [32].

Gao et al. [31] developed a composite bridge deck with an anti-freezing capability. It contains a phase change functional layer which consists of embedded steel pipes filled with PCM with the aim of reducing the amount of ice and snow that forms, similar to [29,32]. Their study confirms that the use of PCM filled pipes is a good way of keeping structures such as bridge decks ice-free (Figure 17).

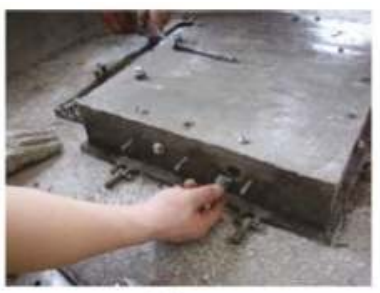

(a)

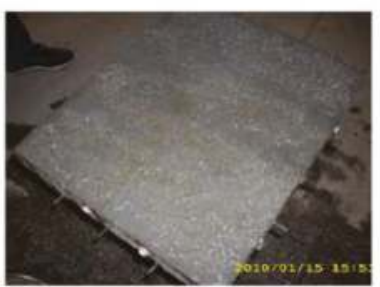

(d)

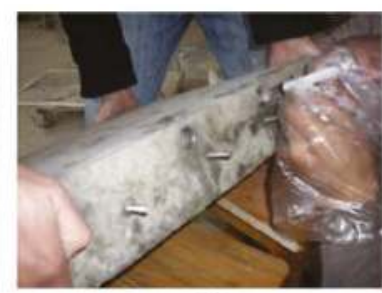

(b)

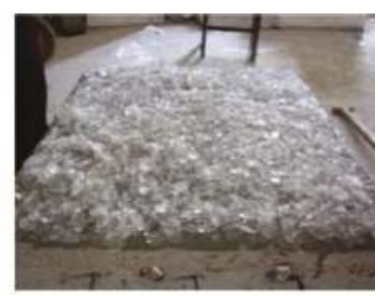

(c)

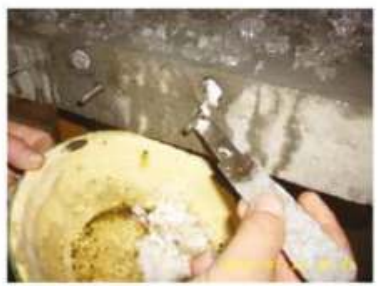

(e)

Figure 17. The simulation test is carried out under low temperature. (a) Injecting entrance design of PCM; (b) injecting PCM; (c) simulation of surface ice-snow condition; (d) melting ice conditions of surface; (e) phase change completion of PCM [31]. 


\section{Effects of PCM on Concrete Properties}

\subsection{Fresh Concrete Properties}

If PCMs are added to the mix as microcapsules or impregnated in LWAs, they may affect the properties of fresh concrete. If LWAs impregnated with PCMs are used, the following issue may occur: since PCMs are not able to penetrate into small pores of the LWA, these pores will remain air filled. If such LWA is placed in the mix, it may imbibe water from the concrete and thus dehydrate the system [100]. Sakulich and Bentz advised, therefore, that the LWAs be water saturated [43].

When microencapsulated PCMs are used, it has been shown that the workability of concrete may be affected due to their small size and, consequently, large surface area [62-64,72,101]. For example, Snoeck et al. [63] found that the addition of PCM microcapsules reduces the workability in proportional to the volume of PCM addition (Figure 18). The decrease in flow was observed irrespective of the way PCM microcapsules were added to the mix, i.e., by replacing a part of fine aggregates or by simply adding them to the mix. Due to these effects, Šavija et al. [73] needed a relatively high amount of superplasticizer for SHCC (strain hardening cementitious composite, an ultra-ductile cementitious material) mixes wherein large proportions of limestone powder were replaced by PCM. A drop in workability with PCM addition was also observed by Hunger et al. [72], but they were able to obtain concretes with good or partly excellent self-compacting properties.

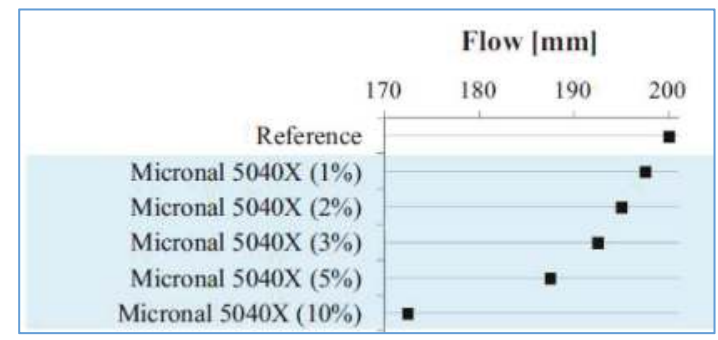

Figure 18. Flow test result for a mortar with a certain type of PCM microcapsules, showing a decrease in flow (workability) with increasing inclusion volume [63].

\subsection{Mechanical Properties}

Addition of PCM to the concrete mixture may affect its mechanical properties. When it comes to compressive strength, two technologies of PCM incorporation (i.e., PCM impregnated LWAs and microencapsulation) result in different effects.

When LWAs are used to replace a part of the aggregates in the concrete mix, a drop in compressive strength is expected [44]. The same behavior is commonly observed for PCM impregnated LWAs [44]. However, this is caused by the weak nature of LWA particles. In fact, the compressive strength of the material will be similar irrespective of whether LWAs are impregnated with water or with PCMs [42], provided PCMs are contained inside the LWA only. It has been observed that the presence of PCM on the LWA surface may impair the aggregate-paste bond and so reduce the compressive strength $[41,45]$. It has also been observed that some PCMs leaking out of LWAs may chemically interact with hydration products causing expansion and cracking [29].

When microencapsulated PCMs are used, in general two opposing effects on concrete strength occur [74]: first, replacement of sand particles with softer PCMs causes a reduction in strength; second, small PCM particles act as nucleation sites for cement hydration, thereby contributing to strength increase [65]. A balance between these two effects will determine which one is dominant. In general, however, there is a drop in compressive strength of cementitious materials 
proportional to the PCM inclusion percentage (e.g., as shown in Figure 19) $[22,60,62,63,68,72,73,75,102]$. Therefore, there is a limit to how much PCM may be added in structural concrete.

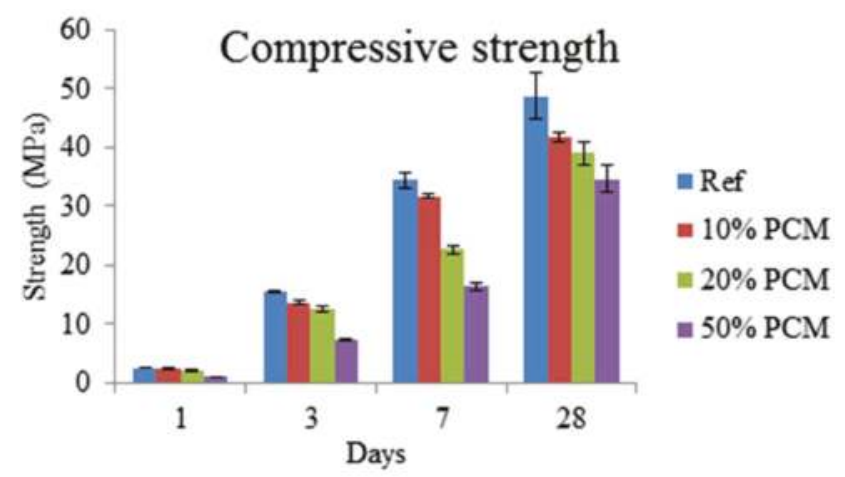

Figure 19. Compressive strength as a function of time for strain hardening cementitious composite (SHCC) mixtures containing different amounts of microencapsulated PCM (error bars indicate standard deviation) [73].

It has been observed that, in general, the drop in tensile/flexural strength with microencapsulated PCM addition is significantly less pronounced than the drop in compressive strength $[22,63,73,75,102]$. However, the influences of PCM additions on the fracture properties (fracture toughness and critical crack tip opening displacement) are less substantial, especially so for lower PCM dosages. This is attributed to the beneficial effects of crack blunting and twisting which are expected to emerge on the addition of compliant inclusions [22]. Similar effects can be achieved through use of mineral additives such as silica fume and fly ash [103-105]. For crack control, this is more important than the compressive strength.

It should be noted that the strength of concrete with PCMs may be, to a certain extent, dependent on the temperature (i.e., below or above the phase change temperature of PCMs). Šavija et al. [102] found that the force needed to rupture the microcapsules themselves was higher for temperatures lower than the phase change temperature (i.e., when PCM is solid) than for temperatures above the phase change temperature (i.e., when PCM is liquid). Pilehvar et al. [106] observed a temperature dependence in the compressive strength of geopolymer concrete incorporating PCM microcapsules. However, this dependence was not observed in other studies, and therefore more research is needed in this field.

\subsection{Thermal Properties}

Thermal conductivity of PCMs is relatively low compared to cementitious materials (Table 1). Therefore, it is expected that the thermal conductivity of PCM cementitious composites would be somewhat reduced. For concrete with PCM impregnated LWA, a reduction in thermal conductivity was reported in multiple studies [42-44,47]. Memon et al. [46] proposed adding graphite powder to increase thermal conductivity. When microencapsulated PCMs are added to the mix, thermal conductivity is found to decrease in proportion with the PCM addition $[60,64,71,72,74,75]$ (e.g., Figure 20). Since this decrease may be significant, it needs to be taken into account when designing PCM cementitious systems and assessing their efficiency. 


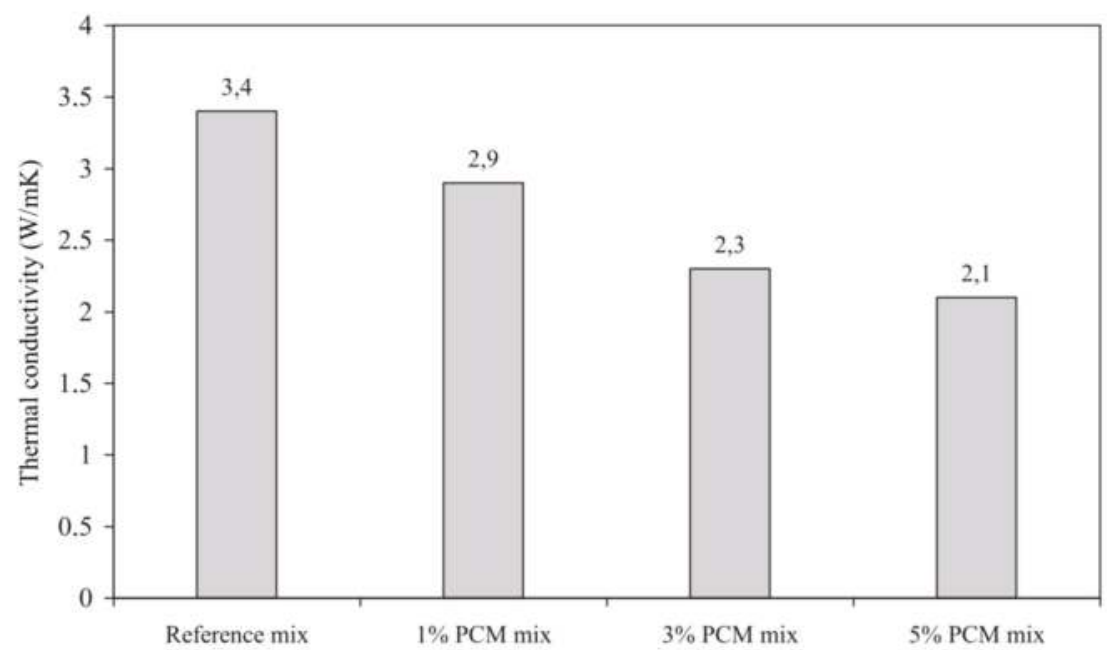

Figure 20. Thermal conductivity of the self-compacting concrete PCM mixes (weight\% of PCM) [72]

\subsection{Durability}

Durability of cement-based materials incorporating PCMs has been scarcely studied. When the long term behavior of such materials is considered, only shrinkage has received attention. It is expected that the addition of compliant inclusions, such as PCM microcapsules, into the mix will cause an increase in shrinkage deformation [80] compared to stiff inclusions (i.e., quartz sand). In fact, it has been observed that the drying shrinkage of cement paste with PCM microcapsules is higher than that of cement paste with the same volume of quartz sand [22]. This is because PCM microcapsules do not restrain paste shrinkage and fulfil a role similar to air voids (in the context of shrinkage) in the system [69]. In these cases, shrinkage is controlled by the paste response [22,68]. Shrinkage of cement pastes with PCM microcapsules is not different from shrinkage of plain cement paste. Based on this observation, and the fact that the strength of the material decreases with PCM addition, it would be expected that PCM-cement composites are more susceptible to restrained shrinkage cracking. In fact, it has been shown that they are more resistant (i.e., that cracking occurs at later age) compared to equivalent mortars with quartz sand [68] (Figure 21). This has been attributed to cracking blunting and deflection caused by soft PCM inclusions, and to a lesser extent to higher stress relaxation [68].

Transport properties of cement-based materials with PCMs have not been extensively studied. In the study by Wei et al. [69], it has been shown that the addition of PCM microcapsules reduces the water uptake of the material. Since the microcapsules are not porous, they act simply as inclusions in the matrix, increasing tortuosity and consequently slowing down the water transport. Such behavior is, in fact, expected, and has been observed in concrete as well [107-109]. Although other transport properties, such as chloride transport, have not been studied, it is expected that the addition of solid inclusions will have a similar effect on those $[110,111]$.

The effect of PCM addition on reinforcement corrosion has been studied by Cellat et al. [112]. In their study, no influence of eutectic microencapsulated PCM on corrosion of reinforcement was observed. Since not many studies on this have been performed, more research is needed. 


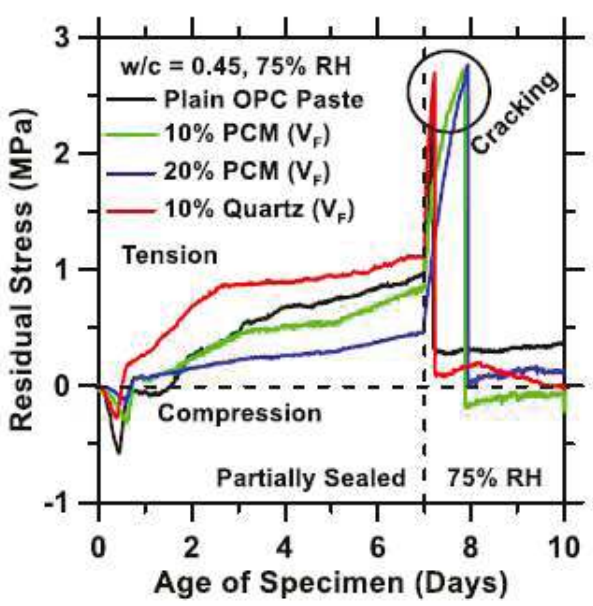

Figure 21. Residual stress development measured using the dual ring setup as a function of time for plain cement paste, and quartz and PCM inclusion dosed mixtures. After 7 days of partially sealed curing the specimens were dried symmetrically, i.e., from their top and bottom surfaces at $75 \%$ relative humidity (RH). The time at which the stress drops sharply indicates macroscopic damage localization (cracking), when a single-crack formed in the ring samples [68].

\section{Modeling of PCM Composites}

\subsection{Effective Properties}

As already described, properties that are most affected by the presence of PCM additions are (compressive) strength, elastic modulus, and thermal conductivity. Models developed to predict the effect of PCM addition on these properties are described.

\subsubsection{Mechanical Properties}

As previously discussed, many studies reported a drop in compressive strength with PCM addition. Based on their experimental findings, Fernandes et al. [22] proposed the following relationship between the inclusion (PCM microcapsule) volume and the compressive strength [113]:

$$
\sigma_{n}=100\left(1-\frac{V_{1}}{100}\right)^{y}
$$

where $\sigma_{n}$ is the compressive strength of any system normalized by the strength of the plain cement paste (unitless), $V_{I}$ is the volume fraction of inclusions in the system (\%), and $y$ is a fitting constant (unitless) that depends on the nature of the inclusion used. For microencapsulated PCMs and quartz sand used in their study, the following values of the fitting parameter y gave the best fit: $y=2.47$ or 3.22 for PCM mixtures for $\mathrm{w} / \mathrm{c}=0.35$ and $\mathrm{w} / \mathrm{c}=0.45$, and $y=-0.14$ for the quartz mixtures for $\mathrm{w} / \mathrm{c}=0.45$, respectively. A comparison between experiments and predictions is given in Figure 22. 


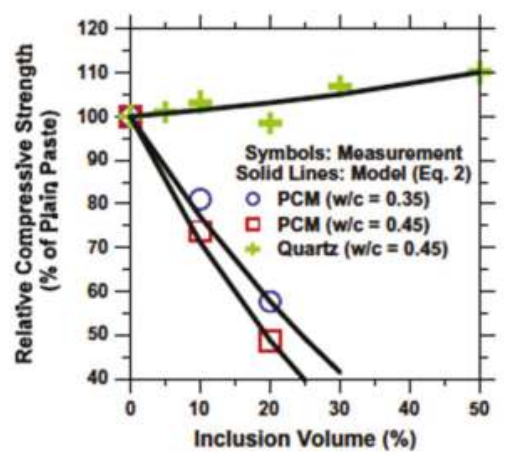

Figure 22. The relative compressive strength for mixtures containing different volume fractions of quartz and PCM inclusions [22].

In the case when PCM inclusions are combined with stiff inclusions (sand), it is not possible to use analytical expressions to predict the strength. For this purpose, Aguayo et al. [65] developed a finite element model wherein inclusions such as sand particles and PCM microcapsules can be explicitly modeled (Figure 23).

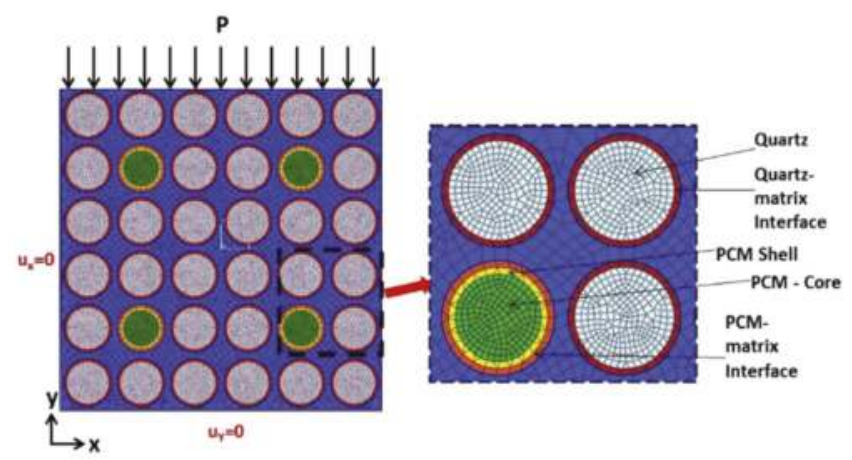

Figure 23. Finite element (FE) model showing the boundary conditions, applied compressive loading, and a magnified representation of the inclusions with the zones around them. The model contains $50 \%$ of inclusions (mortar with 10\% PCM-E particles replacing quartz particles) by volume (or area) [65].

The model showed that, when a combination of weak and soft inclusions (such as PCM microcapsules and quartz sand) is used, the influence of PCM addition on the compressive strength of mortars is more complex. The weaker paste-quartz interfacial elements resulted in the most likely failure path progressing around the inclusions. In systems where small amounts of quartz were replaced by PCM, localized stress concentrations were observed at the PCM-paste interface, but the PCMs being few and far between, the predicted failure region was still along the weaker paste-quartz interface. When the system contained a substantial amount of PCMs, the stress concentrations at the paste-PCM interfaces grew, and the likelihood of paste failure increased substantially. This was in accordance with their experiments [65], which showed somewhat higher compressive strength of systems with a small amount of PCM microcapsules compared to a reference OPC system. This is somewhat unexpected, because in a system where weak inclusions are added, a drop in strength proportional to the inclusion percentage is typically expected [76]. 
Similar approaches were proposed for determining the Young's modulus of the composite. Fernandes et al. [22] proposed to use the following equation [114]:

$$
E_{C}=E_{p}\left(\frac{(1-2 v)\left(1-V_{I}\right)}{\left(1-2 v_{p}\right)\left(1+V_{I}\right)}\right)
$$

Here, $E_{C}$ is the elastic modulus of the composite, $E_{p}$ the elastic modulus of plain cement paste, $v$ the Poisson's ratio of the composite (determined by averaging the Reuss-Voigt solution in proportion to the volume fractions of paste and inclusions in the composite and their properties), $v_{p}$ the Poisson's ratio of the plain cement paste, and $V_{I}$ volume fraction of inclusions/PCMs (\%). A more advanced approach based on micromechanics was proposed by $\mathrm{Xu}$ et al. [115]. In their framework, inclusions and their interfaces are considered explicitly (Figure 24). This way, systems containing both PCMs and quartz inclusions can be considered. The model showed a good agreement with experimental data.

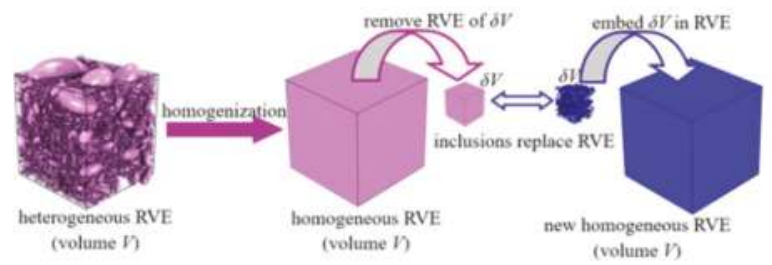

(A)

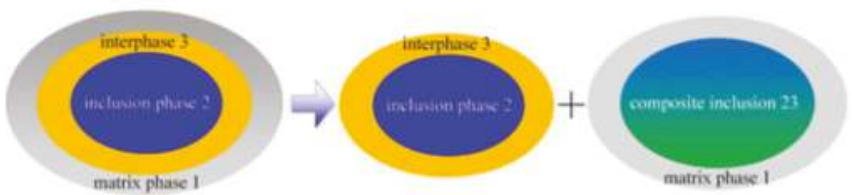

(B)

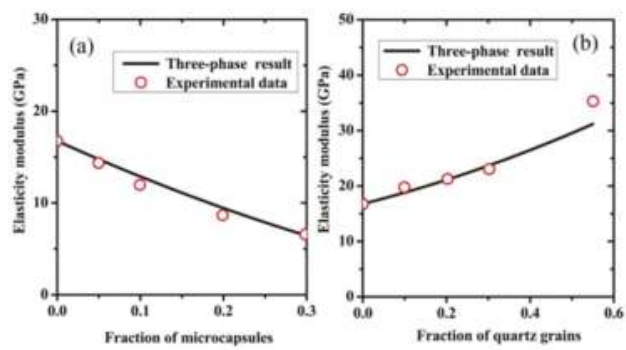

(C)

Figure 24. (A) Schematic view of incremental homogeneous of the differential effective medium (DEM) theory; (B) Schematic view of a three-phase representative volume element (RVE) divided into two two-phase cells. Aggregates (inclusion phase 2) and its adjacent interfacial transition zone (ITZ) (interphase 3) are mapped into a composite inclusion phase 23; (C) Comparisons of the predicted elastic moduli with the experimental data [115], (a) mortar containing microencapsulated PCMs; (b) mortar containing quartz inclusions.

\subsubsection{Thermal Conductivity}

As already described, the addition of PCMs has a profound influence on the thermal properties of the composite material due to PCMs lower thermal conductivity. To simulate the performance of PCM 
based concrete in elements and structures, it is therefore necessary to predict the thermal conductivity of the composite material depending on its constituents.

For composites incorporating microencapsulated PCMs, Ricklefs et al. [67] proposed the use of an equation based on Felske's model [116]:

$$
k_{e f f}=\frac{2 k_{m}\left(1-\phi_{C}-\phi_{S}\right)\left(3+2 \frac{\phi_{S}}{\phi_{C}}+\frac{\phi_{S}}{\phi_{C}} \frac{k_{C}}{k_{S}}\right)+\left(1+2 \phi_{C}+2 \phi_{S}\right)\left(\left(3+\frac{\phi_{S}}{\phi_{C}}\right) k_{C}+2 \frac{\phi_{S} k_{S}}{\phi_{C}}\right)}{\left(2+\phi_{C}+\phi_{S}\right)\left(3+2 \frac{\phi_{S}}{\phi_{C}}+\frac{\phi_{S} k_{C}}{\phi_{C} k_{S}}\right)+\left(1-\phi_{C}-\phi_{S}\right)\left[\left(3+\frac{\phi_{S}}{\phi_{C}}\right) \frac{k_{C}}{k_{m}}+2 \frac{\phi_{S} k_{S}}{\phi_{C} k_{C}}\right]}
$$

Here, $k$ and $\phi$ are the thermal conductivity and volume fraction and the subscripts $c, s$, and $m$ refer to the core, shell, and matrix, respectively. This model showed very good agreement with experimental data (Figure 25). Eddhahak et al. [60] tested four different homogenization approaches for predicting the effective thermal conductivity of the composite: Voigt and Reuss bounds, dilute scheme, and Mori-Tanaka homogenization. Except for the Voigt model, other models showed a good match with experience (i.e., experimental data) (Figure 25).
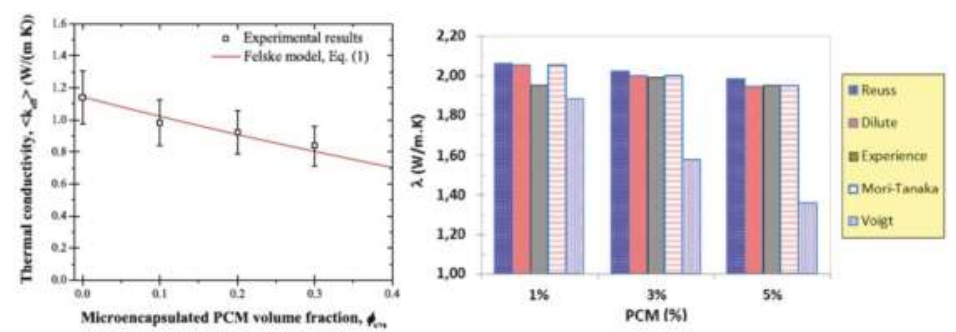

Figure 25. (left) Temperature-averaged effective thermal conductivity as a function of microencapsulated PCM addition, together with the predictions given by Equation (3) [67]; (right) Equivalent thermal conductivity calculated using four homogenization schemes, compared to experience [60].

The Mori-Tanaka homogenization scheme was also used by Aguayo et al. [42] to predict the thermal conductivity of concrete with LWA PCM. Their approach is outlined in Figure 26. According to Aguayo et al. [42], the application of Mori-Tanaka mean-field homogenization method that considered the known microstructural arrangement of the composite (i.e., a heterogeneous inclusion in a homogeneous matrix) resulted in a better predictive capability than the use of sequential homogenization based on thermal conductivity contrast of the different components in the composite.

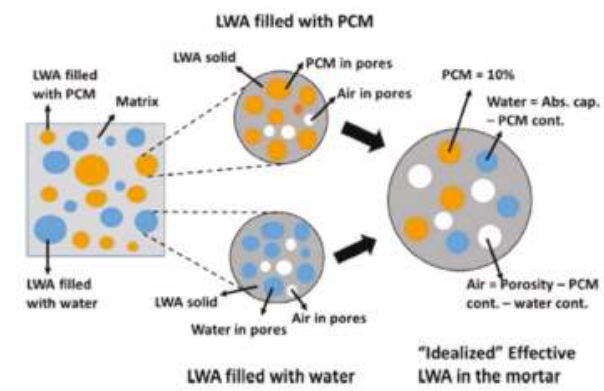

Figure 26. Idealized LWA for effective property determination. Some LWAs have PCM while some others have water to ensure desired PCM levels in the mortars. An "idealized" LWA is also shown, that is representative of the LWA phase [42]. 
For more complex cases, and also 3-dimensional analyses, Das et al. [117] proposed the use of a microstructure-guided numerical model based on the finite element method. An example of a 3D microstructure with randomly distributed PCMs is shown in Figure 27. According to their analyses, three-dimensional numerical homogenization shows better agreement with the experiments compared to a 2D homogenization and several analytical homogenization approaches (Figure 28).
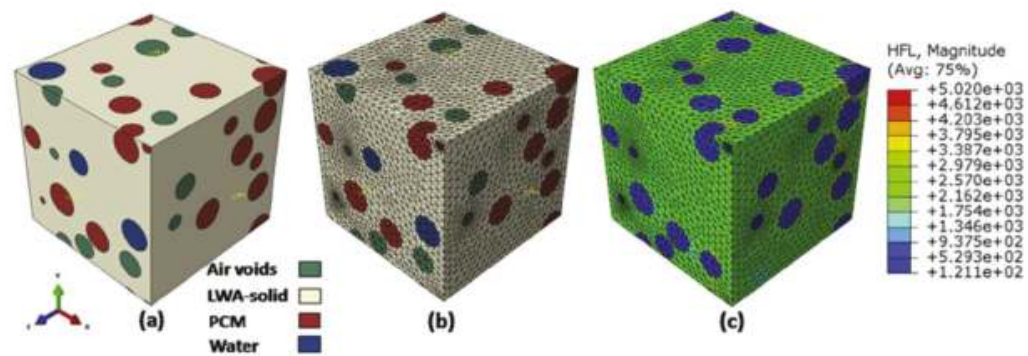

(b)

(c)

Figure 27. 3D Microstructure-guided numerical process to determine the effective thermal conductivity of PCM-incorporated LWAs: (a) FE model showing the water-filled, PCM-filled, and air-filled pores in the LWA inclusions (Pumice); (b) FE mesh; and (c) heat flux distribution (W/ $\mathrm{mm}^{2}$ ) under an imposed temperature difference of $15^{\circ} \mathrm{C}$ [117].

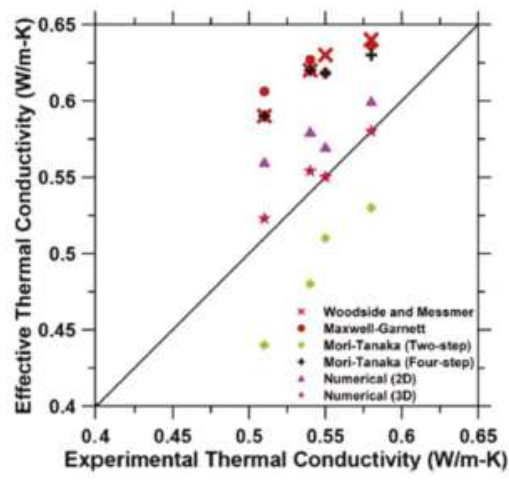

Figure 28. Predicted vs. experimental thermal conductivity of PCM-impregnated LWA mortars and comparison of different numerical and analytical techniques [117].

\subsection{Material and Structural Performance}

Time dependent heat transport can be described by the transient heat conduction equation for a stationary medium (in 1D) [118]:

$$
\rho c_{p} \frac{\partial T}{\partial t}=\frac{\partial}{\partial x}\left(k \frac{\partial T}{\partial x}\right)
$$

Here, $\rho$ is the density $\left(\mathrm{kg} / \mathrm{m}^{3}\right), c_{p}$ the specific heat capacity $(\mathrm{J} / \mathrm{kg} \mathrm{K}), k$ the thermal conductivity $(\mathrm{W} / \mathrm{mK}), T$ the temperature $(\mathrm{K}), t$ time $(\mathrm{s})$, and $x$ the spatial coordinate $(\mathrm{m})$. In case of PCMs, the sensible heat contribution (proportional to the mass and the specific heat capacity of the material) and the latent heat contribution (proportional to the mass and the enthalpy of phase change) both need to be considered. In the literature, latent heat stored during the phase change in the PCM microcapsules is mostly taken into account by using the heat capacity method [119]. In the heat capacity method, 
the contribution from the latent heat due to the phase change process is considered by using a piecewise temperature dependent function for the specific heat capacity of the PCM $[89,120,121]$ :

$$
\rho c_{p, c}(T)=\left\{\begin{array}{cl}
\rho c_{p, c} & \text { for } T<T_{P C}-\Delta T_{P C} / 2 \\
\rho c_{p, c}+h_{f} \frac{m_{p c m}}{\Delta T_{P C}} & \text { for } T_{P C}-\Delta T_{P C} / 2 \leq T \leq T_{P C}+\Delta T_{P C} / 2 \\
\rho c_{p, c} & \text { for } T>T_{P C}+\Delta T_{P C} / 2
\end{array}\right.
$$

Here, $c_{p, c}$ is the specific heat capacity of concrete $(\mathrm{J} / \mathrm{kg} \mathrm{K}), h_{f}$ the heat of fusion of the PCM, $m_{p c m}$ the quantity of PCM per cubic meter of concrete $(\mathrm{kg}), T_{P C}$ the phase change temperature, $\Delta T_{P C}$ the temperature window. Using this approach, the phase change can be simply simulated. Other approaches, such as the "Heat Transfer with Phase Change" approach, are seldom used [122,123].

Young et al. [90] developed a one-dimensional finite difference model for temperature development in early-age concrete pavements. The model showed that the addition of $10 \mathrm{vol} \%$ microencapsulated PCM within the pavement can induce noticeable reductions in the temperature developed within the first $24 \mathrm{~h}$ of placement. Furthermore, the addition of PCM also reduced local temperature gradients developed within the pavement section so long as the effective thermal conductivity of the pavement was not reduced. Arora et al. [124] further extended the finite-difference approach to take strength and stress development into account. As already discussed, the addition of PCM may have detrimental effects on the concrete strength, and these effects should be considered simultaneously with the temperature effects. They analyzed a concrete pavement exposed to the environment, as shown in Figure 29a. In Figure 29b, it can be seen that the addition of PCM microcapsules is able to reduce the peak temperature. Their analysis showed that, if properly designed (i.e., if the appropriate amount of PCMs with the appropriate phase change temperature are used), the PCM-concrete mix is able to reduce the stress during the hardening phase and thereby prevent formation of thermal cracking, as shown in Figure 29c.

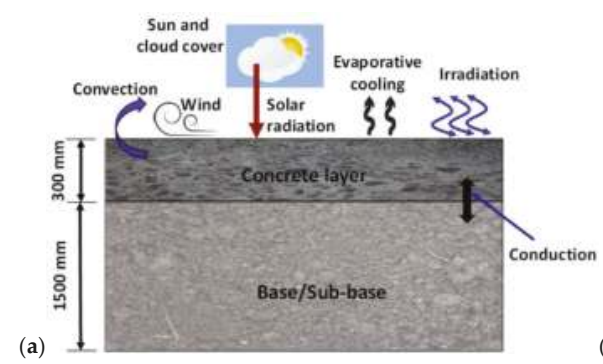

(b)
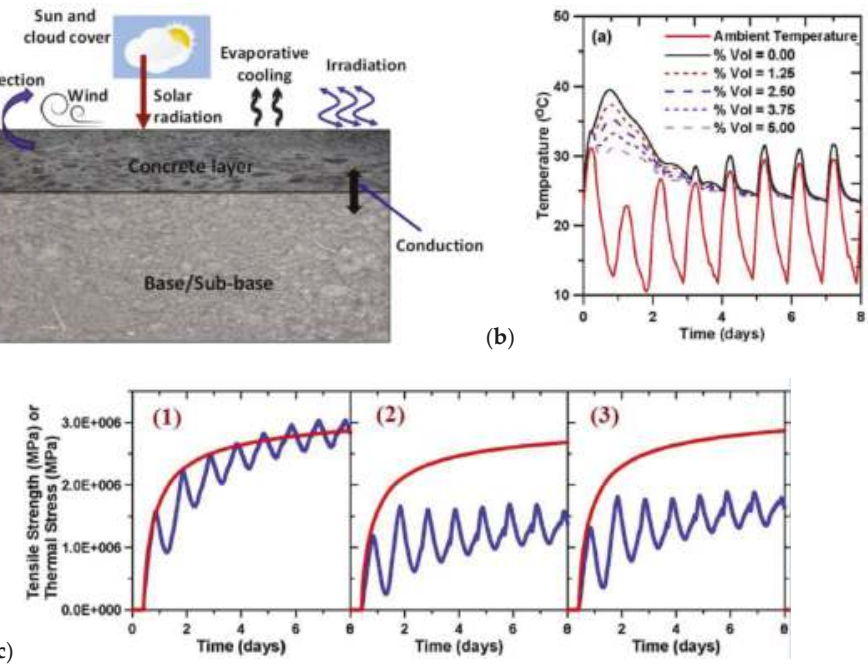

Figure 29. (a) Schematic model of a concrete pavement subjected to environmental effects at early ages; (b) comparison of maximum section temperatures for the case when PCM replaces a part of the cement paste; (c) critical thermal stress and tensile strength development for: (1) OPC concrete, (2) concrete with 10\% PCM replacing cement paste, and (3) concrete with 10\% PCM replacing fine aggregate. The monotonically increasing curve corresponds to the strength development. The pavement is placed in Phoenix, AZ in in the month of July, and the PCM has a phase transition temperature around $35{ }^{\circ} \mathrm{C}[124]$. 
Šavija and Schlangen [120] used a commercial FE package and the heat capacity method to simulate temperature and stress development in a hardening concrete wall cast on an existing slab (Figure 30). This model considers both the temperature and stress development and can take time dependent material properties into account. This enables the user to tailor their PCMs (in terms of quantity, phase change temperature which can be selected based on environmental conditions and heat of fusion) for the application.

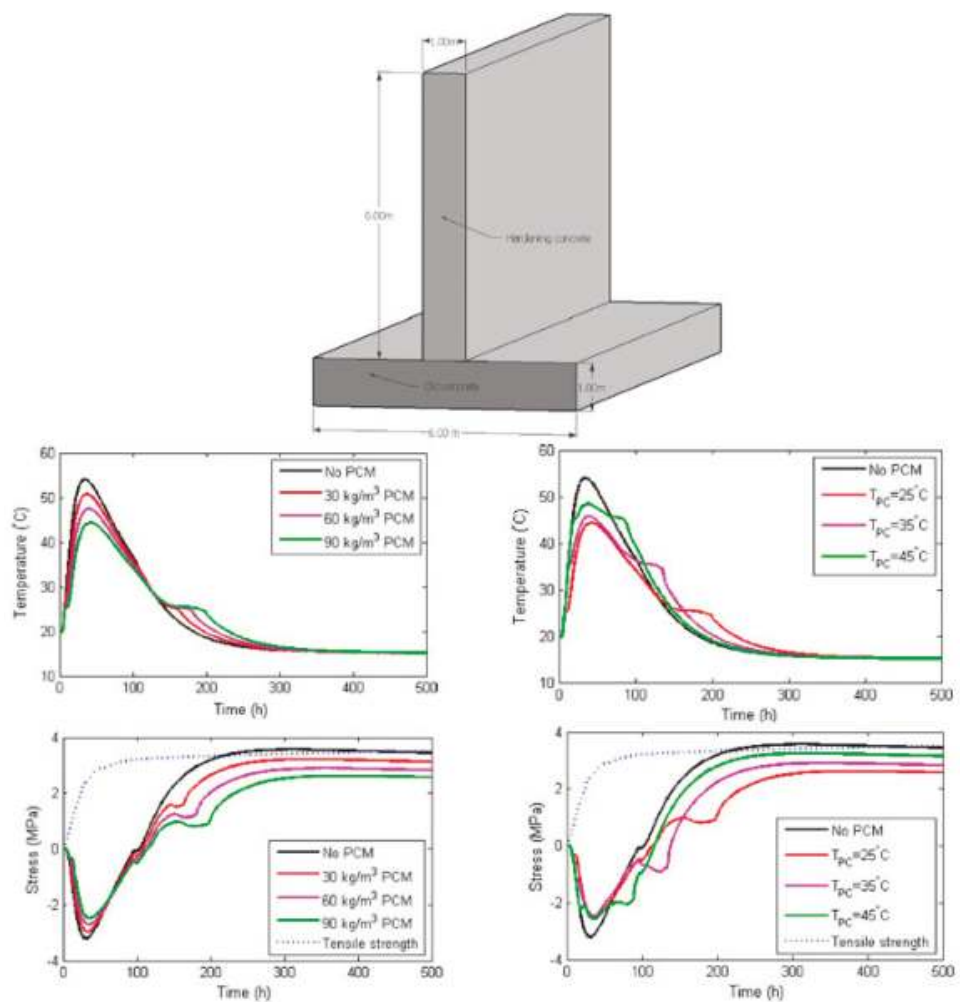

Figure 30. Wall-slab system considered in the analysis, together with temperature and stress developments for various parameters [120].

Based on an example structure analyzed in their study, they concluded that:

- In semi-adiabatic (i.e., field) conditions, the addition of PCM in hardening concrete has potential to delay the temperature rise, reduce the maximum tensile stress, and delay its occurrence. The maximum tensile stress is inversely proportional to the amount of PCM added to the mix (see Figure 30).

- In semi-adiabatic conditions, the phase change temperature does influence the maximum temperature developing in the structure (Figure 30).

- An increase in the latent heat of fusion serves the same purpose as an increase in PCM addition: it lowers the maximum temperature and maximum stress and delays their occurrence. Especially the cooling phase is prolonged. Therefore, a trade-off between the heat of fusion and quantity of PCM microcapsules is possible, where a smaller amount of PCMs with a higher heat of fusion can be used with the same (thermal) efficiency. This would be beneficial also in terms of mechanical properties of the concrete. 
Regarding performance of concrete with PCMs for reducing freeze/thaw damage, not many literature references are available. References [22,43] showed that PCMs can potentially be used for reducing the number of freezing/thawing cycles. The only numerical model dealing with this issue has recently been proposed by Esmaeeli et al. [123]. They developed a 1-dimensional finite difference model combined with homogenization techniques to study the influence of PCM impregnated LWAs on the number of freeze/thaw cycles. Their model showed that the transition temperatures of PCM during freezing and melting events are important properties that govern the effectiveness of PCM at reducing the impact of freeze-thaw cycles. In other words, they help control how quickly the temperature of the pavement drops below the freezing point of pore solution within concrete pores. The model results also showed that low transition temperatures were more effective. It has to be noted that the model of Esmaeeli et al. [123] focuses only on temperature effects: the effects of mechanical damage/cracking are not taken into account. Since, as already discussed, the addition of PCMs (especially microencapsulated PCMs) may result in a reduction of concrete strength, these effects need to be considered together with the temperature effects in order to fully assess the effectiveness of PCMs for reducing freeze/thaw damage.

\section{Summary and Future Research Needs}

In this paper, a systematic review of possible uses of phase change materials in concrete technology for smart (i.e., active) crack control is presented. Types of phase change materials and technologies for their use in concrete are described. Major fields of application, i.e., control of early age cracking and freeze/thaw damage in concrete structures, are reviewed. In addition, effects of PCMs on typical parameters of concrete, such as strength and workability, are reviewed. Finally, models for PCM based concrete are presented and discussed. It can be concluded that the use of PCMs for crack control in concrete has shown promising results so far.

Before the widespread application of this technology, however, several aspects deserve further study. First, although research studies so far have shown that the technology is promising, there have been no comparisons with methods used at the moment for crack control (such as e.g., air entrainment used for controlling freeze/thaw damage). In order for asset owners to be interested in the technology, it needs to be shown that it is advantageous compared to current practice. Second, no studies focusing on the long-term durability of the material are available. This information is necessary for performing life-cycle analyses. Finally, cost considerations (especially relatively high costs of microencapsulated PCMs) have been left out in the studies. Although it is expected that the materials would become cheaper if they were to be used on the large scale, it is important to keep in mind that concrete in itself is a relatively cheap building material and large increases in price are, for most contractors and owners, not acceptable.

The technology at the moment has reached a stage where field trials are needed to test its efficiency in practice. In the beginning, it is suggested to make simple structures such as concrete pavements or walls on slab, which bring relatively low risk. If crack control in these trail structures were to be successful, major applications of this technology could be for massive retaining walls (for hydration temperature control) and airport pavements (for reduction of freeze thaw damage and ice accumulation).

Author Contributions: The author performed the literature study, summarized the findings, and wrote the article.

Acknowledgments: A part of the study was funded by the European Union's Seventh Framework Programme for research, technological development and demonstration under the ERA-NET Plus Infravation programme, grant agreement no: 31109806.0001 . The costs for the open access publication were covered by Delft University of Technology, The Netherlands.

Conflicts of Interest: The author declares no conflict of interest. 


\section{References}

1. Bertolini, L.; Elsener, B.; Pedeferri, P.; Radaelli, E.; Polder, R.B. Corrosion of Steel in Concrete: Prevention, Diagnosis, Repair; John Wiley \& Sons: New York, NY, USA, 2013.

2. Revert, A.B.; De Weerdt, K.; Hornbostel, K.; Geiker, M.R. Carbonation-induced corrosion: Investigation of the corrosion onset. Constr. Build. Mater. 2018, 162, 847-856. [CrossRef]

3. Montemor, M.F.; Simoes, A.M.P.; Ferreira, M.G.S. Chloride-induced corrosion of reinforcing steel: From fundamentals to the monitoring techniques. Cem. Concr. Compos. 2003, 25, 491-502. [CrossRef]

4. Šavija, B.; Luković, M.; Hosseini, S.A.S.; Pacheco, J.; Schlangen, E. Corrosion induced cover cracking studied by X-ray computed tomography, nanoindentation, and energy dispersive X-ray spectrometry (EDS). Mater. Struct. 2015, 48, 2043-2062. [CrossRef]

5. Šavija, B.; Luković, M.; Pacheco, J.; Schlangen, E. Cracking of the concrete cover due to reinforcement corrosion: A two-dimensional lattice model study. Constr. Build. Mater. 2013, 44, 626-638. [CrossRef]

6. Helland, S. Design for service life: Implementation of fib Model Code 2010 rules in the operational code ISO 16204. Struct. Concr. 2013, 14, 10-18. [CrossRef]

7. Maage, M.; Helland, S.; Poulsen, E.; Vennesland, O.; Carl, J.E. Service life prediction of existing concrete structures exposed to marine environment. ACI Mater. J. 1996, 6, 602-608.

8. Van Mier, J.G.M. Fracture Processes of Concrete; CRC Press: Boca Raton, FL, USA, 1996.

9. Weiss, W.J.; Yang, W.; Shah, S.P. Shrinkage cracking of restrained concrete slabs. J. Eng. Mech. 1998, 124, 602-608. [CrossRef]

10. Yuan, Y.; Wan, Z.L. Prediction of cracking within early-age concrete due to thermal, drying and creep behavior. Cem. Concr. Res. 2002, 32, 1053-1059. [CrossRef]

11. Copuroglu, O.; Schlangen, E. Modeling of frost salt scaling. Cem. Concr. Res. 2008, 38, 27-39. [CrossRef]

12. Šavija, B. Experimental and Numerical Investigation of Chloride Ingress in Cracked Concrete. Ph.D. Thesis, Delft University of Technology, Delft, The Netherlands, 2014.

13. De Schutter, G. Quantification of the influence of cracks in concrete structures on carbonation and chloride penetration. Mag. Concr. Res. 1999, 51, 427-435. [CrossRef]

14. Blagojevic, A. The Influence of Cracks on the Durability and Service Life of Reinforced Concrete Structures in Relation to Reinforcement Corrosion. Ph.D. Thesis, Delft University of Technology, Delft, The Netherlands, 2016.

15. Barros, J.A.; Cunha, V.M.; Ribeiro, A.F.; Antunes, J.A.B. Post-cracking behaviour of steel fibre reinforced concrete. Mater. Struct. 2005, 38, 47-56. [CrossRef]

16. Li, V.C.; Wang, S.; Wu, C. Tensile strain-hardening behavior of polyvinyl alcohol engineered cementitious composite (PVA-ECC). ACI Mater. J. 2001, 98, 483-492.

17. Merta, I.; Tschegg, E.K. Fracture energy of natural fibre reinforced concrete. Constr. Build. Mater. 2013, 40, 991-997. [CrossRef]

18. Huang, H.; Ye, G.; Qian, C.; Schlangen, E. Self-healing in cementitious materials: Materials, methods and service conditions. Mater. Des. 2016, 92, 499-511. [CrossRef]

19. Van Tittelboom, K.; De Belie, N. Self-healing in cementitious materials-A review. Materials 2013, 6, 2182-2217. [CrossRef] [PubMed]

20. Šavija, B.; Feiteira, J.; Araujo, M.; Chatrabhuti, S.; Raquez, J.M.; Van Tittelboom, K.; Gruyaert, E.; De Belie, N.; Schlangen, E. Simulation-aided design of tubular polymeric capsules for self-healing concrete. Materials 2017, 10, 10. [CrossRef] [PubMed]

21. Sharma, A.; Tyagi, V.V.; Chen, C.R.; Buddhi, D. Review on thermal energy storage with phase change materials and applications. Renew. Sustain. Energy Rev. 2009, 13, 318-345. [CrossRef]

22. Fernandes, F.; Manari, S.; Aguayo, M.; Santos, K.; Oey, T.; Wei, Z.; Falzone, G.; Neithalath, N.; Sant, G. On the feasibility of using phase change materials (PCMs) to mitigate thermal cracking in cementitious materials. Cem. Concr. Compos. 2014, 51, 14-26. [CrossRef]

23. Bentz, D.P.; Turpin, R. Potential applications of phase change materials in concrete technology. Cem. Concr. Compos. 2007, 29, 527-532. [CrossRef]

24. Baetens, R.; Jelle, B.P.; Gustavsen, A. Phase change materials for building applications: A state-of-the-art-review. Energy Build. 2010, 42, 1361-1368. [CrossRef] 
25. Ling, T.C.; Poon, C.S. Use of phase change materials for thermal energy storage in concrete: An overview. Constr. Build. Mater. 2013, 45, 55-62. [CrossRef]

26. Kalnæs, S.E.; Jelle, B.P. Phase change materials and products for building applications: A state-of-the-art review and future research opportunities. Energy Build. 2015, 94, 150-176. [CrossRef]

27. Choi, W.C.; Khil, B.S.; Chae, Y.S.; Liang, Q.B.; Yun, H.D. Feasibility of using phase change materials to control the heat of hydration in massive concrete structures. Sci. World J. 2014. [CrossRef] [PubMed]

28. Kim, Y.R.; Khil, B.S.; Jang, S.J.; Choi, W.C.; Yun, H.D. Effect of barium based phase change material (PCM) on the mechanical properties of mass concrete. Thermochimica Acta 2015, 613, 100-107. [CrossRef]

29. Farnam, Y.; Krafcik, M.; Liston, L.; Washington, T.; Erk, K.; Tao, B.; Weiss, W.J. Evaluating the use of phase change materials in concrete pavement to melt ice and snow. J. Mater. Civ. Eng. 2015, 28. [CrossRef]

30. Kim, J.K.; Kim, K.H.; Yang, J.K. Thermal analysis of hydration heat in concrete structures with pipe-cooling system. Comput. Struct. 2001, 79, 163-171. [CrossRef]

31. Gao, Y.; Huang, L.; Zhang, H. Study on anti-freezing functional design of phase change and temperature control composite bridge decks. Constr. Build Mater. 2016, 122, 714-720. [CrossRef]

32. Farnam, Y.; Esmaeeli, H.S.; Zavattieri, P.D.; Haddock, J.; Weiss, W.J. Incorporating phase change materials in concrete pavement to melt snow and ice. Cem. Concr. Compos. 2017, 84, 134-145. [CrossRef]

33. Qian, C.; Gao, G.; Zhu, C.; Guo, Z. Influence of phase change materials on temperature rise caused by hydration heat evolution of cement-based materials. Mag. Concr. Res. 2010, 62, 789-794. [CrossRef]

34. Qian, C.; Gao, G. Reduction of interior temperature of mass concrete using suspension of phase change materials as cooling fluid. Constr. Build. Mater. 2012, 26, 527-531. [CrossRef]

35. Bentur, A.; Igarashi, S.I.; Kovler, K. Prevention of autogeneous shrinkage in high-strength concrete by internal curing using wet lightweight aggregates. Cem. Concr. Res. 2001, 29, 1863-1867. [CrossRef]

36. Bentz, D.P.; Snyder, K.A. Protected paste volume in concrete: Extension to internal curing using saturated lightweight fine aggregate. Cem. Concr. Res. 1999, 29, 1863-1867. [CrossRef]

37. Suzuki, M.; Meddah, M.S.; Sato, R. Use of porous ceramic waste aggregates for internal curing of high-performance concrete. Cem. Concr. Res. 2009, 39, 373-381. [CrossRef]

38. Wiktor, V.; Jonkers, H.M. Quantification of crack-healing in novel bacteria-based self-healing concrete. Cem. Concr. Compos. 2011, 33, 763-770. [CrossRef]

39. Tziviloglou, E.; Wiktor, V.; Jonkers, H.M.; Schlangen, E. Bacteria-based self-healing concrete to increase liquid tightness of cracks. Constr. Build. Mater. 2016, 122, 118-125. [CrossRef]

40. Zhang, D.; Li, Z.; Zhou, J.; Wu, K. Development of thermal energy storage concrete. Cem. Concr. Res. 2004, 34, 927-934. [CrossRef]

41. Sharifi, N.P.; Sakulich, A. Application of phase change materials to improve the thermal performance of cementitious material. Energy Build. 2015, 103, 83-95. [CrossRef]

42. Aguayo, M.; Das, S.; Castro, C.; Kabay, N.; Sant, G.; Neithalath, N. Porous inclusions as hosts for phase change materials in cementitious composites: Characterization, thermal performance, and analytical models. Constr. Build. Mater. 2017, 134, 574-584. [CrossRef]

43. Sakulich, A.R.; Bentz, D.P. Increasing the service life of bridge decks by incorporating phase-change materials to reduce freeze-thaw cycles. J. Mater. Civ. Eng. 2011, 24, 1034-1042. [CrossRef]

44. Sakulich, A.R.; Bentz, D.P. Incorporation of phase change materials in cementitious systems via fine lightweight aggregate. Constr. Build. Mater. 2012, 35, 483-490. [CrossRef]

45. Nepomuceno, M.C.; Silva, P.D. Experimental evaluation of cement mortars with phase change material incorporated via lightweight expanded clay aggregate. Constr. Build. Mater. 2014, 63, 89-96. [CrossRef]

46. Memon, S.A.; Cui, H.Z.; Zhang, H.; Xing, F. Utilization of macro encapsulated phase change materials for the development of thermal energy storage and structural lightweight aggregate concrete. Appl. Energy 2015, 139, 43-55. [CrossRef]

47. Ramakrishnan, S.; Sanjayan, J.; Wang, X.; Alam, M.; Wilson, J. A novel paraffin/expanded perlite composite phase change material for prevention of PCM leakage in cementitious composites. Appl. Energy 2015, 157, 85-94. [CrossRef]

48. Lu, Z.; Zhang, J.; Sun, G.; Xu, B.; Li, Z.; Gong, C. Effects of form-stable expanded perlite/paraffin composite on cement manufactured by extrusion technique. Energy 2015, 82, 43-53. [CrossRef]

49. Xu, B.; Li, Z. Paraffin/diatomite composite phase change material incorporated cement-based composite for thermal energy storage. Appl. Energy 2013, 105, 229-237. [CrossRef] 
50. Xu, B.; Li, Z. Paraffin/diatomite/multi-wall carbon nanotubes composite phase change material tailor-made for thermal energy storage cement-based composites. Energy 2014, 72, 371-380. [CrossRef]

51. Xu, B.; Li, Z. Performance of novel thermal energy storage engineered cementitious composites incorporating a paraffin/diatomite composite phase change material. Appl. Energy 2014, 121, 114-122. [CrossRef]

52. Xu, B.; Ma, H.; Lu, Z.; Li, Z. Paraffin/expanded vermiculite composite phase change material as aggregate for developing lightweight thermal energy storage cement-based composites. Appl. Energy 2015, 160, 358-367. [CrossRef]

53. Huang, J.; Lu, S.; Kong, X.; Liu, S. Form-stable phase change materials based on eutectic mixture of tetradecanol and fatty acids for building energy storage: Preparation and performance analysis. Materials 2013, 6, 4758-4775. [CrossRef] [PubMed]

54. Lv, L.Y.; Zhang, H.; Schlangen, E.; Yang, Z.; Xing, F. Experimental and numerical study of crack behaviour for capsule-based self-healing cementitious materials. Constr. Build. Mater. 2017, 156, 219-229. [CrossRef]

55. Lv, L.Y.; Yang, Z.; Chen, G.; Zhu, G.; Han, N.; Schlangen, E.; Xing, F. Synthesis and characterization of a new polymeric microcapsule and feasibility investigation in self-healing cementitious materials. Constr. Build. Mater. 2016, 105, 487-495. [CrossRef]

56. Beglarigale, A.; Seki, Y.; Demir, N.Y. Sodium silicate/polyurethane microcapsules used for self-healing in cementitious materials: Monomer optimization, characterization, and fracture behavior. Constr. Build. Mater. 2018, 162, 57-64. [CrossRef]

57. Xiong, W.; Tang, J.; Zhu, G.; Han, N.; Schlangen, E.; Dong, B.; Wang, X.; Xing, F. A novel capsule-based self-recovery system with a chloride ion trigger. Sci. Rep. UK 2015, 5, 10866. [CrossRef] [PubMed]

58. Norvell, C.; Sailor, D.J.; Dusicka, P. The effect of microencapsulated phase-change material on the compressive strength of structural concrete. J. Green Build. 2013, 8, 116-124. [CrossRef]

59. Eddhahak, A.; Drissi, S.; Colin, J.; Care, S.; Neji, J. Effect of phase change materials on the hydration reaction and kinetic of PCM-mortars. J. Therm. Anal. Calorim. 2014, 117, 537-545. [CrossRef]

60. Eddhahak-Ouni, A.; Drissi, S.; Colin, J.; Neji, J.; Care, S. Experimental and multi-scale analysis of the thermal properties of Portland cement concretes embedded with microencapsulated Phase Change Materials (PCMs). Appl. Therm. Eng. 2014, 64, 32-39. [CrossRef]

61. Drissi, S.; Eddhahak, A.; Care, J.; Neji, J. Thermal analysis by DSC of Phase Change Materials, study of the damage effect. J. Build. Eng. 2015, 1, 13-19. [CrossRef]

62. Figueiredo, A.; Lapa, J.; Vincente, R.; Cardoso, C. Mechanical and thermal characterization of concrete with incorporation of microencapsulated PCM for applications in thermally activated slabs. Constr. Build. Mater. 2016, 112, 639-647. [CrossRef]

63. Snoeck, D.; Priem, B.; Dubruel, P.; De Belie, N. Encaplsulated phase-change materials as additives in cementitious materials to promote thermal comfort in concrete constructions. Mater. Struct. 2016, 49, 225-239. [CrossRef]

64. Fenollera, M.; Miguez, J.L.; Goicoechea, I.; Lorenzo, J.; Angel-Alvarez, M. The influence of phase change materials on the properties of self-compacting concrete. Materials 2013, 6, 3530-3546. [CrossRef] [PubMed]

65. Aguayo, M.; Das, S.; Maroli, A.; Kabay, N.; Mertens, J.C.E.; Rajan, S.D.; Sant, G.; Chawla, N.; Neithalath, N. The influence of microencapsulated phase change material (PCM) characteristics on the microstructure and strength of cementitious composites: Experiments and finite element simulations. Cem. Concr. Compos. 2016, 73, 29-41. [CrossRef]

66. Lecompte, T.; Le Bideau, P.; Glouannec, P.; Nortershauser, D.; Le Masson, S. Mechanical and thermo-physical behaviour of concretes and mortars containing phase change material. Energy Build. 2015, 94, 52-60. [CrossRef]

67. Ricklefs, A.; Thiele, A.M.; Falzone, G.; Sant, G.; Pilon, L. Thermal conductivity of cementitious materials containing microencapsulated phase change materials. Int. J. Heat Mass Transf. 2017, 104, 71-82. [CrossRef]

68. Wei, Z.; Falzone, G.; Das, S.; Saklani, N.; Le Pape, Y.; Pilon, L.; Neithalath, N.; Sant, G. Restrained shrinkage cracking of cementitious composites containing soft PCM inclusions: A paste (matrix) controlled response. Mater. Des. 2017, 132, 367-374. [CrossRef]

69. Wei, Z.; Falzone, G.; Wang, B.; Thiele, A.; Puerta-Falla, G.; Pilon, L.; Neithelath, N.; Sant, G. The durability of cementitious composites containing microencapsulated phase change materials. Cem. Concr. Compos. 2017, 81, 66-76. [CrossRef] 
70. Wei, Z. Phase Change Materials and Clinkering-Free Cementation for Sustainable Building Materials. Ph.D. Thesis, UCLA (University of California at Los Angeles), Los Angeles, CA, USA, 2017.

71. Cui, H.; Liao, W.; Memon, S.A.; Dong, B.; Tang, W. Thermophysical and mechanical properties of hardened cement paste with microencapsulated phase change materials for energy storage. Materials 2014, 7, 8070-8087. [CrossRef] [PubMed]

72. Hunger, M.; Entrop, A.G.; Mandilaras, I.; Brouwers, H.J.H.; Founti, M. The behavior of self-compacting concrete containing micro-encapsulated phase change materials. Cem. Concr. Compos. 2009, 31, 731-743. [CrossRef]

73. Šavija, B.; Lukovic, M.; Kotteman, G.M.; Figuieredo, S.C.; de Mendica Filho, F.F.; Schlangen, E. Development of ductile cementitious composites incorporating microencapsulated phase change materials. Int. J. Adv. Eng. Sci. Appl. Math. 2017, 9, 169-180. [CrossRef]

74. Jayalath, A.; San Nicolas, R.; Sofi, M.; Shanks, R.; Ngo, T.; Aye, L.; Mendis, P. Properties of cementitious mortar and concrete containing micro-encapsulated phase change materials. Constr. Build. Mater. 2016, 120, 408-417. [CrossRef]

75. Dehdezi, P.K.; Hall, M.R.; Dawson, A.R.; Casey, S.P. Thermal, mechanical and microstructural analysis of concrete containing microencapsulated phase change materials. Int. J. Pavement Eng. 2013, 14, 449-462. [CrossRef]

76. Liu, D.; Šavija, B.; Smith, G.E.; Flewitt, P.E.J.; Lowe, T.; Schlangen, E. Towards understanding the influence of porosity on mechanical and fracture behaviour of quasi-brittle materials: Experiments and modelling. Int. J. Fract. 2017, 205, 57-72. [CrossRef]

77. Khudhair, A.M.; Farid, M.M. A review on energy conservation in building applications with thermal storage by latent heat using phase change materials. Energy Convers. Manag. 2004, 45, 263-275. [CrossRef]

78. Tyagi, V.V.; Buddhi, D. PCM thermal storage in buildings: A state of art. Renew Sust. Energ. Rev. 2007, 11, 1146-1166. [CrossRef]

79. Neeper, D.A. Thermal dynamics of wallboard with latent heat storage. Sol. Energy 2000, 68, 393-403. [CrossRef]

80. Mehta, P.K.; Monteiro, P.J.M. Concrete: Microstructure, Properties, and Materials; McGraw-Hill: New York, NY, USA, 2006

81. Emborg, M.; Bernander, S. Assessment of risk of thermal cracking in hardening concrete. J. Struct. Eng. 1994, 120, 2893-2912. [CrossRef]

82. Bofang, Z. Thermal Stresses and Temperature Control of Mass Concrete; Butterworth-Heinemann: Oxford, UK, 2013.

83. Benboudjema, F.; Torrenti, J.M. Early-age behaviour of concrete nuclear containments. Nucl. Eng. Des. 2008, 238, 2495-2506. [CrossRef]

84. De Schutter, G. Finite element simulation of thermal cracking in massive hardening concrete elements using degree of hydration based material laws. Comput. Struct. 2002, 80, 2035-2042. [CrossRef]

85. Pan, Y.; Prado, A.; Porras, R.; Hafez, O.M.; Bolander, J.E. Lattice modeling of early-age behavior of structural concrete. Materials 2017, 10, 231. [CrossRef] [PubMed]

86. Waller, V.; d'Aloia, L.; Cussigh, F.; Lecrux, S. Using the maturity method in concrete cracking control at early ages. Cem. Concr. Compos. 2004, 26, 287-296. [CrossRef]

87. Nobuhiro, M.; Kazuo, U. Nonlinear thermal stress analysis of a massive concrete structure. Comput Struct. 1987, 26, 287-296. [CrossRef]

88. Mihashi, H.; Nishiyama, N.; Kobayashi, T.; Hanada, M. Development of a smart material to mitigate thermal stress in early age concrete. In Control of Cracking in Early Age Concrete; Taylor \& Francis: Abingdon, UK, 2004; pp. 385-392.

89. Thiele, A.M.; Wei, Z.; Falzone, G.; Young, B.A.; Neithalath, N.; Sant, G.; Pilon, L. Figure of merit for the thermal performance of cementitious composites containing phase change materials. Cem. Concr. Compos. 2016, 65, 214-226. [CrossRef]

90. Young, B.A.; Falzone, G.; She, Z.; Thiele, A.M.; Wei, Z.; Neithalath, N.; Sant, G.; Pilon, L. Early-age temperature evolutions in concrete pavements containing microencapsulated phase change materials. Constr. Build. Mater. 2017, 147, 466-477. [CrossRef]

91. Sun, W.; Zhang, Y.M.; Yan, H.D.; Mu, R. Damage and damage resistance of high strength concrete under the action of load and freeze-thaw cycles. Cem. Concr. Res. 1999, 29, 1519-1523. [CrossRef] 
92. Li, W.; Pour-Ghaz, M.; Castro, J.; Weiss, W.J. Water absorption and critical degree of saturation relating to freeze-thaw damage in concrete pavement joints. J. Mater. Civ. Eng. 2011, 24, 299-307. [CrossRef]

93. Liu, L.; Ye, G.; Schlangen, E.; Chen, H.; Qian, Z.; Sun, W.; van Breugel, K. Modeling of the internal damage of saturated cement paste due to ice crystallization pressure during freezing. Cem. Concr. Compos. 2011, 33, 562-571. [CrossRef]

94. Liu, L.; Wu, S.; Chen, H.; Haitao, Z. Numerical investigation of the effects of freezing on micro-internal damage and macro-mechanical properties of cement pastes. Cold Reg. Sci. Technol. 2014, 106, 141-152. [CrossRef]

95. Cai, H.; Liu, X. Freeze-thaw durability of concrete: Ice formation process in pores. Cem. Concr. Res. 1998, 28, 1281-1287. [CrossRef]

96. Jacobsen, S.; Marchand, J.; Hornain, H. SEM observations of the microstructure of frost deteriorated and self-healed concretes. Cem. Concr. Res. 1995, 25, 1781-1790. [CrossRef]

97. Du, L.; Folliard, K.J. Mechanisms of air entrainment in concrete. Cem. Concr. Res. 2005, 35, 1463-1471. [CrossRef]

98. Attiogbe, E.K. Mean spacing of air voids in hardened concrete. ACI Mater. J. 1993, 90, 174-181

99. Bentz, D.P. A Computer Model to Predict the Surface Temperature and Time-of-Wetness of Concrete Pavements and Bridge decks. Available online: https://www.nist.gov/publications/computer-modelpredict-surface-temperature-and-time-wetness-concrete-pavements-and (accessed on 1 March 2018).

100. Lukovic, M.; Ye, G. Effect of moisture exchange on interface formation in the repair system studied by X-ray absorption. Materials 2015, 9, 2. [CrossRef] [PubMed]

101. Cunha, S.; Lima, M.; Aguiar, J.B. Influence of adding phase change materials on the physical and mechanical properties of cement mortars. Constr. Build. Mater. 2016, 127, 1-10. [CrossRef]

102. Šavija, B.; Zhang, H.; Schlangen, E. Influence of microencapsulated phase change material (PCM) addition on (micro) mechanical properties of cement paste. Materials 2017, 10, 863. [CrossRef] [PubMed]

103. Golewski, G.L. Generalized Fracture Toughness and Compressive Strength of Sustainable Concrete Including Low Calcium Fly Ash. Materials 2017, 10, 1393. [CrossRef] [PubMed]

104. Golewski, G.L. Improvement of fracture toughness of green concrete as a result of addition of coal fly ash. Characterization of fly ash microstructure. Mater. Charact. 2017, 134, 335-346. [CrossRef]

105. Golewski, G.L. Green concrete composite incorporating fly ash with high strength and fracture toughness. J. Clean. Prod. 2018, 172, 218-226. [CrossRef]

106. Pilehvar, S.; Cao, V.D.; Szczotok, A.M.; Valentini, L.; Salvioni, D.; Magistri, M.; Pamies, R.; Kjoniksen, A.-L. Mechanical properties and microscale changes of geopolymer concrete and Portland cement concrete containing micro-encapsulated phase change materials. Cem. Concr. Res. 2017, 100, 341-349. [CrossRef]

107. Lukovic, M.; Šavija, B.; Schlangen, E.; Ye, G.; van Breugel, K. A 3D lattice modelling study of drying shrinkage damage in concrete repair systems. Materials 2016, 9, 575. [CrossRef] [PubMed]

108. Wang, L.; Ueda, T. Mesoscale modeling of water penetration into concrete by capillary absorption. Ocean Eng. 2011, 38, 519-528. [CrossRef]

109. Bolander, J.E.; Berton, S. Simulation of shrinkage induced cracking in cement composite overlays. Cem. Concr. Compos. 2004, 26, 861-871. [CrossRef]

110. Šavija, B.; Pacheco, J.; Schlangen, E. Lattice modeling of chloride diffusion in sound and cracked concrete. Cem. Concr. Compos. 2013, 42, 30-40. [CrossRef]

111. Wang, L.; Ueda, T. Mesoscale modelling of chloride diffusion in cracks and cracked concrete. J. Adv. Concr. Technol. 2011, 9, 241-249. [CrossRef]

112. Cellat, K.; Tezcan, F.; Beyhan, B.; Kardas, G.; Paksov, H. A comparative study on corrosion behavior of rebar in concrete with fatty acid additive as phase change material. Constr. Build. Mater. 2017, 143, 490-500. [CrossRef]

113. Shin, K.J.; Bucher, B.; Weiss, W.J. Role of lightweight synthetic particles on the restrained shrinkage cracking behavior of mortar. J. Mater. Civ. Eng. 2010, 23, 597-605. [CrossRef]

114. Hobbs, D.W. The dependence of the bulk modulus, Young's modulus, creep, shrinkage and thermal expansion of concrete upon aggregate volume concentration. Materiaux et Constr. 1971, 4, 107-114. [CrossRef]

115. Xu, W.; Jia, M.; Zhu, Z.; Liu, M.; Lei, D.; Gou, X. n-Phase micromechanical framework for the conductivity and elastic modulus of particulate composites: Design to microencapsulated phase change materials (MPCMs)-cementitious composites. Mater. Des. 2018, 145, 108-115. [CrossRef] 
116. Felske, J.D. Effective thermal conductivity of composite spheres in a continuous medium with contact resistance. Int. J. Heat Mass Transf. 2004, 47, 3453-3461. [CrossRef]

117. Das, S.; Aguayo, M.; Rajan, S.D.; Sant, G.; Neithalath, N. Microstructure-guided numerical simulations to predict the thermal performance of a hierarchical cement-based composite material. Cem. Concr. Compos. 2018, 87, 20-28. [CrossRef]

118. Lewis, R.W.; Nithiarasu, P.; Seetharamu, K.N. Fundamentals of the Finite Element Method for Heat and Fluid Flow; John Wiley \& Sons: Hoboken, NJ, USA, 2004.

119. Lamberg, P.; Lehtiniemi, R.; Henell, A.M. Numerical and experimental investigation of melting and freezing processes in phase change material storage. Int. J. Therm. Sci. 2004, 43, 277-287. [CrossRef]

120. Šavija, B.; Schlangen, E. Use of phase change materials (PCMs) to mitigate early age thermal cracking in concrete: Theoretical considerations. Constr. Build. Mater. 2016, 126, 332-344. [CrossRef]

121. Thiele, A.M.; Sant, G.; Pilon, L. Diurnal thermal analysis of microencapsulated PCM-concrete composite walls. Energy Convers. Manag. 2015, 93, 215-227. [CrossRef]

122. Sharifi, N.P.; Freeman, G.E.; Sakulich, A.R. Using COMSOL modeling to investigate the efficiency of PCMs at modifying temperature changes in cementitious materials-Case study. Constr. Build. Mater. 2015, 101, 965-974. [CrossRef]

123. Esmaeeli, H.S.; Farnam, Y.; Haddock, J.E.; Zavattieri, P.D.; Weiss, W.J. Numerical analysis of the freeze-thaw performance of cementitious composites that contain phase change material (PCM). Mater. Des. 2018, 145, 74-87. [CrossRef]

124. Arora, A.; Sant, G.; Neithalath, N. Numerical simulations to quantify the influence of phase change materials (PCMs) on the early-and later-age thermal response of concrete pavements. Cem. Concr. Compos. 2017, 81, 11-24. [CrossRef]

(C) 2018 by the author. Licensee MDPI, Basel, Switzerland. This article is an open access article distributed under the terms and conditions of the Creative Commons Attribution (CC BY) license (http://creativecommons.org/licenses/by/4.0/). 


\title{
Article \\ Effect of PCM on the Hydration Process of Cement-Based Mixtures: A Novel Thermo-Mechanical Investigation
}

\author{
Claudia Fabiani ${ }^{1}$, Anna Laura Pisello ${ }^{1,2, *}$, Antonella D'Alessandro ${ }^{3}$, Filippo Ubertini ${ }^{3}$, Luisa F. \\ Cabeza ${ }^{4}$ and Franco Cotana ${ }^{1,2}$ \\ 1 CIRIAF-Interuniversity Research Center, University of Perugia, Via G. Duranti 63, 06125 Perugia, Italy; \\ fabiani@crbnet.it (C.F.); cotana@crbnet.it (F.C.) \\ 2 Department of Engineering, University of Perugia, Via G. Duranti 93, 06125 Perugia, Italy \\ 3 Department of Civil and Environmental Engineering, University of Perugia, Via G. Duranti 93, \\ 06125 Perugia, Italy; antonella.dalessandro@unipg.it (A.D.); filippo.ubertini@unipg.it (F.U.) \\ 4 GREiA Research Group, INSPIRES Research Centre, Universitat de Lleida, Pere de Cabrera s/n, \\ 25001 Lleida, Spain; lcabeza@diei.udl.cat \\ * Correspondence: anna.pisello@unipg.it; Tel.: +39-075-585-3563
}

Received: 26 April 2018; Accepted: 17 May 2018; Published: 23 May 2018

\begin{abstract}
The use of Phase Change Material (PCM) for improving building indoor thermal comfort and energy saving has been largely investigated in the literature in recent years, thus confirming PCM's capability to reduce indoor thermal fluctuation in both summer and winter conditions, according to their melting temperature and operation boundaries. Further to that, the present paper aims at investigating an innovative use of PCM for absorbing heat released by cement during its curing process, which typically contributes to micro-cracking of massive concrete elements, therefore compromising their mechanical performance during their service life. The experiments carried out in this work showed how PCM, even in small quantities (i.e., up to $1 \%$ in weight of cement) plays a non-negligible benefit in reducing differential thermal increases between core and surface and therefore mechanical stresses originating from differential thermal expansion, as demonstrated by thermal monitoring of cement-based cubes. Both PCM types analyzed in the study (with melting temperatures at 18 and $25^{\circ} \mathrm{C}$ ) were properly dispersed in the mix and were shown to be able to reduce the internal temperature of the cement paste by several degrees, i.e., around $5{ }^{\circ} \mathrm{C}$. Additionally, such small amount of PCM produced a reduction of the final density of the composite and an increase of the characteristic compressive strength with respect to the plain recipe.
\end{abstract}

Keywords: cement hydration; thermally efficient concretes; cement-based composites; concrete curing; phase change material; mechanical properties; thermal characterization; smart material

\section{Introduction}

Hydraulic binders are substances that harden independently and confer cohesion and resistance to a composite system. In the hardening process, which spontaneously takes place at room temperature, these substances chemically react with water and transform the plastic water-binder system into a solid matrix, with the ability to agglomerate other solid materials [1].

It is clear, then, that hydraulic binders, and particularly cement-based ones, play a crucial role in the constitution of concrete, whose production is about one ton per year for every human being on Earth, and can be considered as the most widely used material in the world [2]. From an environmental point of view, this is particularly significant, given that the production of one ton of the most common 
type of cement, i.e., the Portland one, is associated with the emission of about $600-1000 \mathrm{~kg}$ of $\mathrm{CO}_{2}$ in the atmosphere, accounting for at least $5 \%$ of man-made $\mathrm{CO}_{2}$ production worldwide [3]. Furthermore, the manufacturing of ordinary Portland cement (OPC) is a highly energivorous process depleting large quantities of precious raw materials and natural resources.

All this considered, in recent years, several research contributions focused on the reduction of the overall environmental impact of cement and consequently concrete, with the aim of producing greener products with comparable mechanical performance. In detail, two different approaches have so far been investigated by the research community. The former one consists of replacing part of the cement with more sustainable components such as waste materials, e.g., wheat straw ash and bamboo leaf ash $[4,5]$, or byproducts from the iron industry or coal combustion, e.g., slag, silica fume and fly ash [6-10]. Fly ash demonstrated being able to improve the overall manufacturing process with the aim of controlling the amount of sensible heat released by the cement during its hydration phase, and reducing the temperature-induced strains and stresses within the solid matrix [11,12]. This last approach allows for obtaining higher strength concretes by using the same amount of resources and would consequently reduce raw material depletion and green house gas emissions imputable to the cement industry.

In order to address this latter application, a better understanding of the kinetics of the hydration mechanism itself is still needed. For this reason, several researchers worldwide investigated the chemical processes occurring during the development of the cement-based solid micro-structure $[13,14]$. Unfortunately, these chemical processes may operate in parallel, in series or in even more complex combinations. For this reason, for the time being, it is not possible to have a complete picture of the reactions taking place during cement hydration. However, by studying the heat released during the process as a function of the hydration time, it is possible to divide such duration into five distinct stages with specific characteristics, i.e., (i) pre-induction; (ii) induction; (iii) acceleration; (iv) post-acceleration and (v) diffusion-limited reaction [1]. The highest amount of hydration heat is generally released during the acceleration and the post-acceleration phase, which usually take place between two and ten hours from the beginning of curing $[15,16]$.

In the context above, several studies have been investigating the effect of different additives or cement substitutes on the hydration heat evolution. Bouasker et al. for example, focused on the influence of limestone filler and granular inclusions on chemical shrinkage development and on the hydration degree of cementitious matrices. They found that the addition of the milestone filler causes an acceleration of both the hydration process and the chemical shrinkage, while the presence of the granular inclusions with diameters between 1 and $2 \mathrm{~mm}$ only has a weak influence on these factors [17]. Hu et al., on the other hand, investigated the effect of slag and fly ashes on cement-based mortars in terms of both hydration heat and early-age chemical and autogenous shrinkage [18]. They observed a linear reduction of the autogenous shrinkage with increasing slag and fly ash replacement levels and also found a good correlation with the amount of heat released during the process.

The relation between hydration heat and shrinkage-induced cracking in the early-age curing of cement-based composites has also been investigated by means of analytical and numerical modeling [19-22]. Zreiki et al. for example, developed a 3D thermo-chemo-mechanical model in order to assess the risk of cracking in massive concrete structures [19], while Yuan and Wan predicted the early-age cracking of concrete by using a numerical code based on micromechanical model and empirical formulas [20].

In general, all these contributions revealed an inverse correlation between the amount of heat released during the hydration process and the mechanical resistance of the investigated samples. Based on this evidence, in recent years, the attention of the scientific community has been focusing on the implementation of advanced techniques aimed at reducing the release of sensible energy during the chemical transformation of the cement-based composites. In particular, the implementation of a specific kind of latent heat storage material, i.e., phase change material (PCM), within the reacting cementitious matrices is receiving increasing consent among researchers worldwide. Phase change materials are substances capable of storing a large amount of heat in the form of phase change enthalpy, at a constant 
temperature. Until now, they have mostly been integrated in concrete mixtures with the aim of improving the thermal-energy behavior of a specific building component during the operative phase of the building itself. As a matter of fact, several contributions already testified their potentiality as passive regulator of the thermal fluctuations in the indoor built environment [23-25]. However, in the last few years, some contributions have also explored their potential benefit in reducing the amount of heat released during cement hydration [26-30].

In their study about the influence of two different kinds of paraffin-based microencapsulated PCMs on the microstructure and strength development of cementitious composites, Aguayo et al. [29] found a different behaviour of cement mortars and pastes. The former were, in fact, found to behave differently with respect to the specific considered PCM, while the latter decreased their mechanical performance consistently with increasing PCM volume fraction [29]. Fernandes et al. also used paraffin-based microencapsulated phase change materials to produce and mitigate thermal cracking of cement mortars [30]. They found that PCM inclusion did not influence the chemical reactions in the cementing material, but their presence was able to reduce the peak temperature during cement hydration. However, such reduction was relatively low due to dilution, and the final compressive strength of the composites was significantly lower than that of the reference product [30]. A consistent compressive strength reduction with increasing PCM content in concrete mixtures was also found by Cellat et al. [27].

Most of the above referenced studies considered the integration of relatively high PCM volume fractions in the original mix design of the cement-based composites, i.e., from $5 \%$ to $20 \%$, which in most cases was shown to cause a redistribution of the void structure and to produce weak interfacial bonds between the PCM and the cement grains. However, as reported in a previous study conducted by this research group, concretes with $1 \%$ weight content of PCM with respect to the final weight of the cement/water composite can be associated with a significant reduction in the statistical dispersion of the axial compressive strength with respect to classic concrete [25].

All this considered, this work aims at investigating both the heat storage potential during the hydration phase and the mechanical performance of cement pastes produced by adding $1 \%$ in weight of two different paraffin-based phase change materials. The final goal of this study is to produce a thermally enhanced composite capable of improving its mechanical performance by reducing the thermal stresses developed during the early-age hydration phase of the mixture.

\section{Materials and Methodology}

\subsection{PCM Selection}

Two different types of phase change materials were selected as additives for producing the cement pastes investigated in this work, i.e., the Micronal DS 5038X (Microtek, Dayton, OH, USA), and the Microtek mPCM18D micro-capsules (Microtek, Dayton, OH, USA).

A microencapsulated PCM consists of an external inert polymeric shell enclosing the inner latent heat storage medium in order to prevent the leakage phenomenon once the PCM is in its liquid form. Both the selected micro-capsules use paraffin-wax as the core material of the shell; however, the former is associated with a melting temperature of about $25^{\circ} \mathrm{C}$, while the latter of about $18{ }^{\circ} \mathrm{C}$. The PCM content in each capsule is about $85-90 \mathrm{wt} \%$ with respect to the whole weight. The capsules look like a white powder with a mean particle size of about 14-24 $\mu \mathrm{m}$, and 20-300 $\mu \mathrm{m}$ for the mPCM18D and the DS 5038X, respectively; however, the capsules from Micronal laboratories are an agglomeration of smaller shells with a diameter between 1 and $5 \mu \mathrm{m}$.

\subsection{Mix Design and Sample Preparation}

The selected PCM microcapsules were used to prepare two different cement pastes with a fixed amount of thermally enhanced additive, i.e., $1 \%$ with respect to the total cement weight. A standard 
cement paste without the addition of PCM was also prepared for comparative purposes (NP). Therefore, three different kinds of pastes were considered: a normal cement paste (NP), a paste additivated with $1 \%$ of microencapsulated phase change material with melting point at $18^{\circ} \mathrm{C}(\mathrm{PCM} 18 \mathrm{P})$, and a paste additivated with $1 \%$ of microencapsulated phase change material with melting point at $25^{\circ} \mathrm{C}(\mathrm{PCM} 25 \mathrm{P})$. The research focus was dedicated to the fundamental cement curing process. Therefore, no aggregates were considered because they might also affect the curing process and interact with PCM. In the PCM-doped composites, the additives were used in addition to the reference mix design, in order for them to have the same water/cement ratio of the original product and, consequently, comparable mechanical results. The three different mix designs are summarized in Table 1.

Table 1. Mix designs of the produced cement pastes, i.e., normal paste (NP), paste doped with microencapsulated phase change material with melting point at $18{ }^{\circ} \mathrm{C}(\mathrm{PCM} 18 \mathrm{P})$, and doped with microencapsulated PCM with melting point at $25^{\circ} \mathrm{C}$ (PCM25P).

\begin{tabular}{cccc}
\hline Components & NP & PCM18P & PCM25P \\
\hline Cement $(\mathrm{g})$ & 1277 & 1277 & 1277 \\
Water $(\mathrm{g})$ & 575 & 575 & 575 \\
PCM $(\mathrm{g})$ & - & 12.77 & 12.77 \\
\hline$w / c$ ratio & 0.45 & 0.45 & 0.45 \\
\hline
\end{tabular}

Fifteen cubic samples were produced in total, i.e., five samples per mix design, with a dimension of $10 \times 10 \times 10 \mathrm{~cm}^{3}$. The preparation of the five cement pastes was carried out by following the same procedure (Figure 1). First of all, the dry materials (cement and PCMs) were carefully manually merged in order to obtain an homogeneous powder mix. Consecutively, water was added to the compound, which was then carefully blended trying to preserve the capsules from brittle fractures due to the mechanical action. Finally, after reaching the required workability, the mixtures were poured into previously oiled molds and compacted in order to improve homogeneity and eliminate voids and air inclusions. Before the moulding of the samples, the workability was investigated through a modified Abram's cone test. The comparable and acceptable results avoided the use of any types of fluidifying additives. Finally, the samples were cured within the controlled environment of an empty, fully monitored test building, which was kept at the temperature of $16 \pm 0.5^{\circ} \mathrm{C}$ by using a built-in conditioning system during the whole curing process (28 days). After seven days, the specimens were unmolded within the same controlled environment where they were housed for the rest of the curing process.

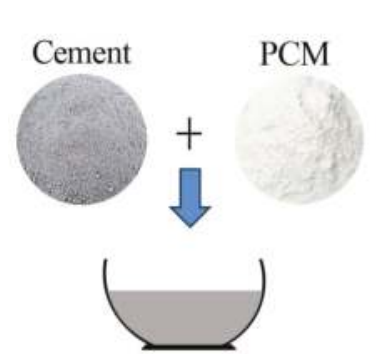

(a)

Mixing of the dry components

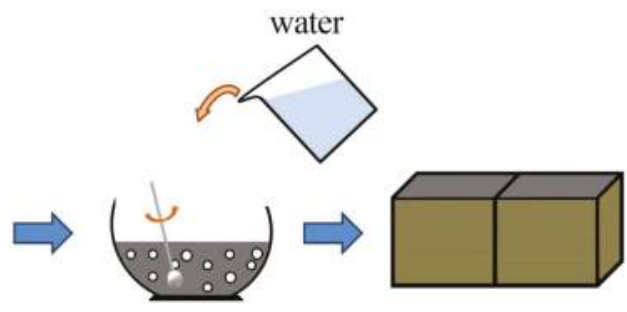

(b)

Blending (c)

Pouring into oiled molds

Figure 1. Samples preparation procedure: (a) assembly of the dry components; (b) addition of water and mixing; and (c) pouring into oiled molds. 


\section{Thermo-Mechanical Analyses}

Thermo-mechanical behaviour of the samples was analysed by means of a novel dynamic thermal procedure and acknowledged mechanical tests. From the thermal point of view, two different investigations were carried out with the aim of (i) monitoring the temperature evolution of the cementitious matrices during the hydration process and (ii) investigating the dispersion of the PCM characterizing the morphological features of the composite. From a mechanical point of view, both elastic modulus and compressive strength were evaluated. In addition, workability of the volume, weight, and density were measured.

\subsection{Thermal Characterization of the PCMs}

The thermal behaviour of both the microcapsules alone was analysed by means of the transient plane source (TPS) method at two different constant temperatures, i.e., $10^{\circ} \mathrm{C}$ and $35^{\circ} \mathrm{C}$. The capsules were placed in a specifically designed sample holder, equipped with a TPS sensor, and housed within the controlled environment of a climatic chamber. They were kept at the selected temperature for $36 \mathrm{~h}$ and five subsequent measurements were carried out (1 every $60 \mathrm{~min}$ ), during the last $4 \mathrm{~h}$ of the thermal stabilization.

Figure 2 shows the thermal conductivity, thermal diffusivity and volumetric specific heat of the PCM. By comparing the results achieved in both solid and liquid PCM configurations, different performance rates were achieved, e.g., both the capsules decrease their thermal conductivity and their volumetric specific heat with varying the test temperature from $10^{\circ} \mathrm{C}$ to $35^{\circ} \mathrm{C}$, while their thermal diffusivity increases. Results show that the DS 5038X PCM is associated with lower thermal conductivity and diffusivity, while it possesses higher volumetric specific heat, if compared to the mPCM18D. The whole PCM powder made by microcapsules was characterized by means of such method, since the microcapsules are radically smaller than the TPS probe detection capability specifically selected for this purpose. The TPS probe and method selection were driven by the purpose to characterize the whole hemispherical volume detected by the selected probe, whose diameter is of the order of magnitude of centimeters, i.e., $1.4 \mathrm{~cm}$. This selection was specifically carried out with the purpose to characterize the thermal properties of the whole powder itself, as typically done for any other additives responsible for thermal modification of the composite materials. Since both the selected PCM microspheres represent already commercial products with available technical sheets and proper documentation, the reader is kindly referred to [31,32] for further details such as henthalpy of transition phases. As previously mentioned, the whole microcapsules quantity was characterized at stabilized temperature, without interacting with the PCM transition phase itself. In this view, the TPS analyses refer to the whole volume occupied by the microcapsule, where the TPS is able to detect the hemispherical volume under its influence. This procedure may therefore produce non-consistent results compared to the ones about the PCM paraffin itself, but it has been considered as significant for investigating the role of such microcapsule volume included into the cement paste.

\subsection{Rheologial Behaviour of the Pastes}

As soon as the cement-based compound was produced, the fresh cement paste workability was investigated through a flow test carried out with a hollow truncated cone with a height of $90 \mathrm{~mm}$ and maximum and minimum diameters of $70 \mathrm{~mm}$ and $40 \mathrm{~mm}$, respectively. The value related to the workability was obtained by evaluating the average of orthogonal diameters of the flow on a livelled metallic table after the mold was removed. The results show very similar performance, index of similar consistency of the mix designs. In particular, NP, PCM18P and PCM25P demonstrated average diameters of 18.5, 18.2 and $18.3 \mathrm{~mm}$, respectively. This is particularly interesting, also considering that, as reported in Section 2.2, no plasticizer was added to any of the different mixes. 


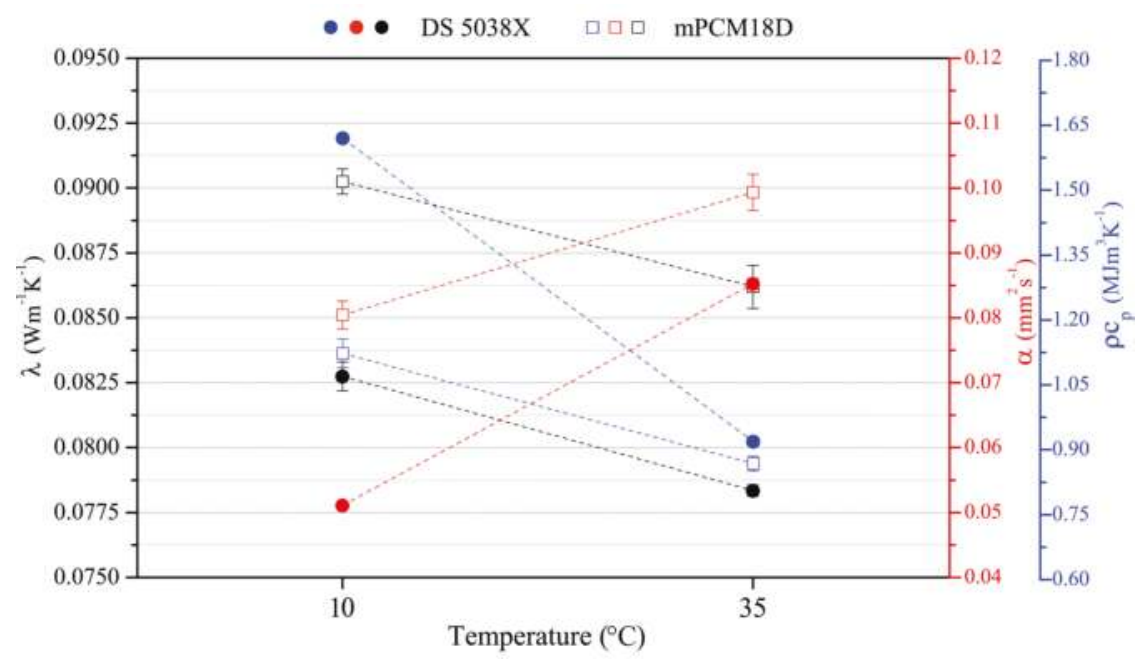

Figure 2. Thermal conductivity, thermal diffusivity and the volumetric specific heat of the DS 5038X and the mPCM18D microcapsules at 10 and $35^{\circ} \mathrm{C}$.

\subsection{Thermal Monitoring of the Hydration Process}

The thermal monitoring of the samples during the hydration process was carried out by using seven T-type thermocouples for each mix design. In more detail, six of these sensors were placed at the center of each samples' face while the last one was introduced within the main core of the specimen. In this way, it was possible to accurately register the temperature gradients produced throughout the whole volume of the samples during the overall extent of the hydration process (Figure 3).

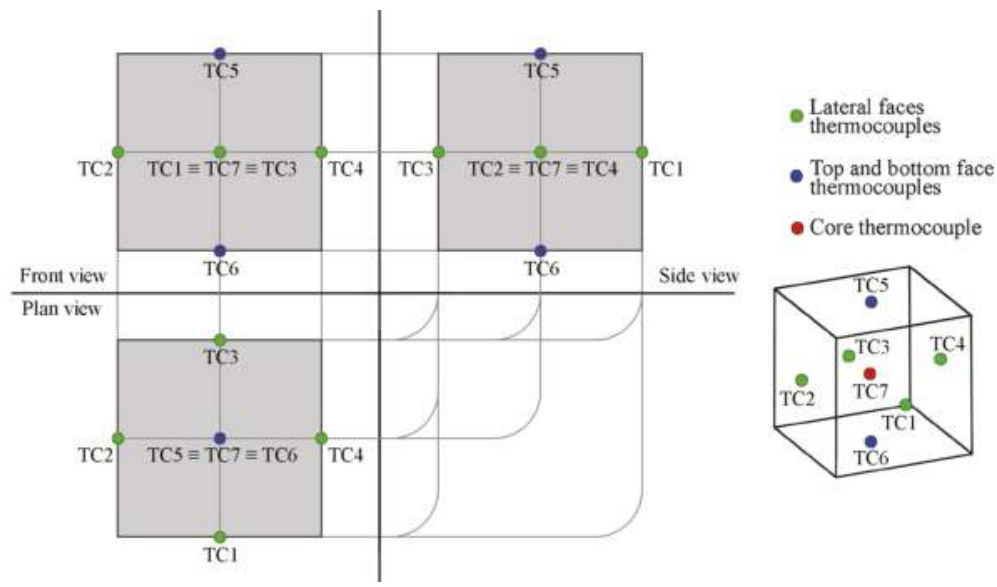

Figure 3. Thermocouples positioning within the cementitious samples.

The thermal analysis of the cement pastes was carried out inside polyurethane molds, with the same boundary conditions. This configuration was selected because, in cement-based materials, the critical exothermic reaction takes place in the first hours after casting and because insulating material that constitutes the formwork simulates the occurrence of more massive pourings. The thermocouples were connected to a data acquisition system model cDAQ-9184 equipped with two 
NI 9213 Spring slots (National Instruments, Austin, TX, USA), and programmed in order to read the sensors every $60 \mathrm{~s}$ during the next 28 days.

The samples were housed within the controlled environment of a test-building, where a conditioning system was used to keep the ambient temperature at $16 \pm 0.5{ }^{\circ} \mathrm{C}$. This particular temperature was chosen in order to allow both the selected PCM to eventually turn from solid to liquid and vice-versa due to the hydration heat released by the cementitious matrix.

\subsection{Thermal Behaviour and PCM Distribution Analysis}

As previously described in Section 2, the basic thermal properties of the PCM used as additive for the production of the composite cement pastes were firstly characterized by using the transient plane source (TPS) technique. This thermal analysis was carried by using a Hot Disk 2500S apparatus (Hot Disk AB, Göteborg, Sweden), which was also used to investigate the proper PCM dispersion in the final mixture, by means of a non-destructive experimental procedure.

The Hot Disk 2500S is an experimental piece of equipment able to implement a transient heat source to investigate the bulk thermal properties of a material $[33,34]$. In the general procedure, a certain heating power $P_{0}$ is provided by a double nickel spiral to the sample for a specific amount of time $t$. During the measurement, the resistance of the probe $R(t)$ is continuously monitored and finally related to thermal properties of the bulk material by using Equation (1):

$$
R(t)=R_{0}\left[1+a\left(\Delta T_{i}+\left(\frac{P_{0}}{\pi^{3 / 2} r \lambda}\right) D(\tau)\right)\right],
$$

where $t$ is the test time $[\mathrm{s}], R_{0}$ is the initial resistance of the sensor at time $t=0[\Omega], a$ is the temperature coefficient of resistivity (TCR) $[1 / \mathrm{K}], \Delta T_{i}$ is the temperature difference across the insulating layers of the probe $[\mathrm{K}], D(\tau)$ is the dimensionless shape function and $\tau$ is the dimensionless time, which is a function of sample thermal diffusivity $(\alpha)$.

Besides the classic bulk analysis, the TPS method has recently been applied for investigating the variation of thermal conductivity as a function of the probing depth in inhomogeneous materials [35]. As a matter of fact, in the TPS method, the thermal penetration depth can be determined as a function of the measurement time by means of Equation (2):

$$
d_{p}=2 \sqrt{\alpha t}
$$

where $d_{p}$ represents the experimental probing depth.

Equation (2) can be used to extend the mathematical model and approximate the thermal conductivity across the overall thickness of inhomogeneous materials. In order to do so, the fundamental fitting procedure of the TPS method is applied to smaller time windows, $\left[t_{i}, t_{i}+n \Delta t\right]$, where $n$ is the number of points in the time window itself, and $\Delta t$ is the sampling time step, producing locally averaged thermal conductivity and diffusivity values. In this way, by sliding the selected time window across the entire measurement time range, different thermal conductivity values are estimated along the probing depth of the sample.

In this work, both these analyses were used to define the average thermal properties of the considered mixtures and also indirectly identify the dispersion of the phase change material in the cement paste. This last application was possible thanks to (i) the huge contrast between the thermal properties of the cementitious matrix and the PCMs; and (ii) the highly different weight percentage of the two components (see Table 1). In detail, once the samples completed their curing process, they were placed within the controlled environment of a climatic chamber and kept at the fixed temperature of $10{ }^{\circ} \mathrm{C}$. Once the pastes reached a stationary temperature profile, they were analysed by means of the bulk and the structural double-sided module of the TPS method. The sensor was sandwiched between two identical samples of the same kind, the first time in direct contact with the two top surfaces of the samples, and the second time in direct contact with the bottom surfaces 
(see Figure 4). In this way, the thermal conductivity evolution was analysed through the overall thickness of the different samples. The same procedure was later repeated by conditioning the samples at $35^{\circ} \mathrm{C}$.

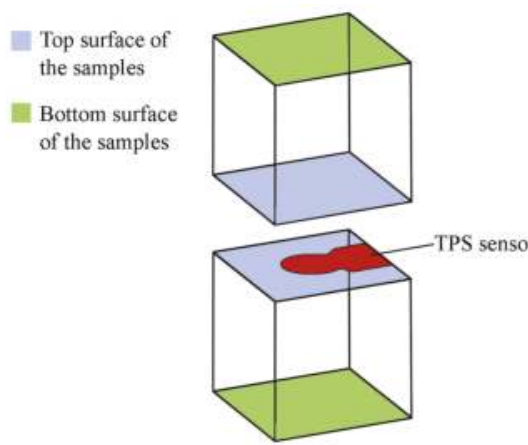

(a)
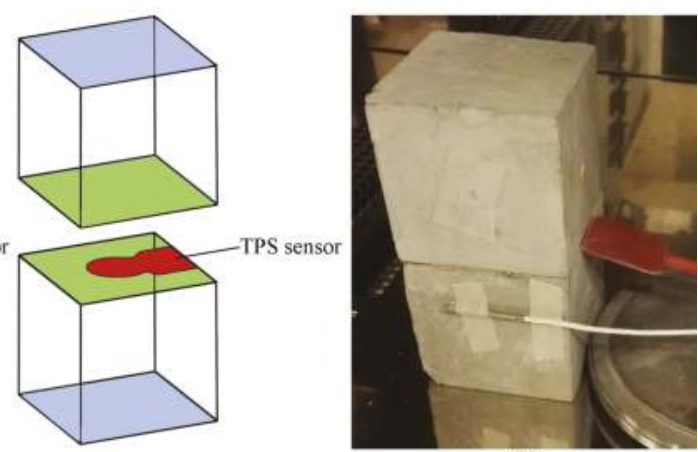

(b)

Figure 4. (a) schematic of the TPS (transient plane source) sensor and sample positioning; and (b) final experimental setup during the structural analyses using the Hot Disk 2500 S.

\subsection{Physico-Chemical and Mechanical Analysis}

After curing, all the samples were measured, weighed and observed in order to identify specific peculiarities and any defects. The elastic moduli were evaluated through cyclical compressive loads between 0.5 and $9 \mathrm{kN}$ applied with a speed of $1 \mathrm{kN} / \mathrm{s}$ using a servo-controlled pneumatic universal dynamic testing machine, model IPC Global UTM14P (IPC Global Pty Ltd., Boronia, Australia) with a controlled temperature chamber. Three samples of each type of paste were instrumented with two 2-cm long strain gauges placed on lateral opposite sides having a nominal resistance of $120 \Omega$ and Gauge Factor of 2. Compression tests up to the maximum strength were carried out using a Tecnotest compressive machine (Tecnotest, Marghera, Italy) with load control, according to UNI EN 12390-3 (UNI EN 1239-3 "Testing hardened concrete-Part 3: Compressive strength of test specimens" May 2009). The nominal speed was $5 \mathrm{kN} / \mathrm{s}$. The setup of the cyclical and ultimate compressive tests are reported in Figure 5.

1: DAQ strain gauges

2: Strain gauges connections

3 : Pneumatic compression testing machine

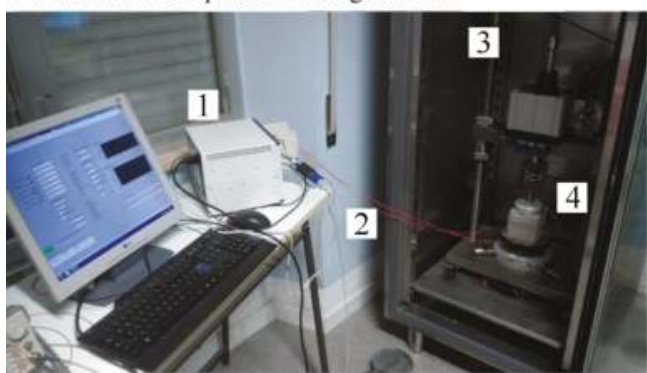

(a)

\section{5: Compression testing machine \\ 6: Cubic sample \\ 7 : Testing machine control unit}

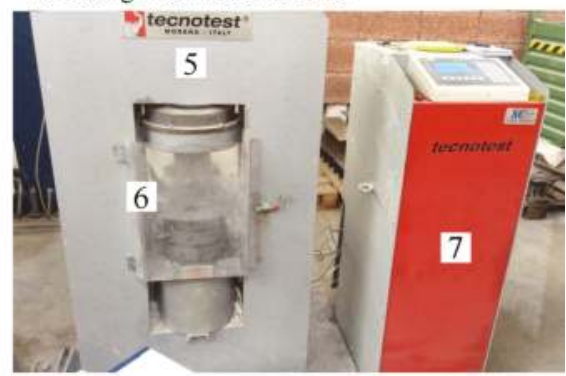

(b)

Figure 5. Experimental setup of the (a) cyclical compressive tests and (b) compressive tests up to break.

Average and characteristic compressive strength were calculated from the results. The characteristic values were obtained using the formula $R_{c k}=R_{m}-k \sigma$, where $R_{m}$ is the average strength, $\sigma$ the standard 
deviation of the compressive strength values of the tested samples, and $k$ is a coefficient depending on the number of tested samples, assumed equal to 3.4 [36]. The characteristic strength can be equivalently expressed as: $R_{C M}=R_{m}(1-k C O V)$, where $C O V$ is the coefficient of variation, i.e., the ratio between the standard deviation and the average strength.

\section{Results and Discussion}

\subsection{Thermal Characterization of the Hydration Process}

Results from the thermal monitoring of the first $72 \mathrm{~h}$ of the hydration process are shown in Figure 6, where the dot-dashed line represents the environmental forcing imposed by the conditioning system on the controlled air domain surrounding the samples.
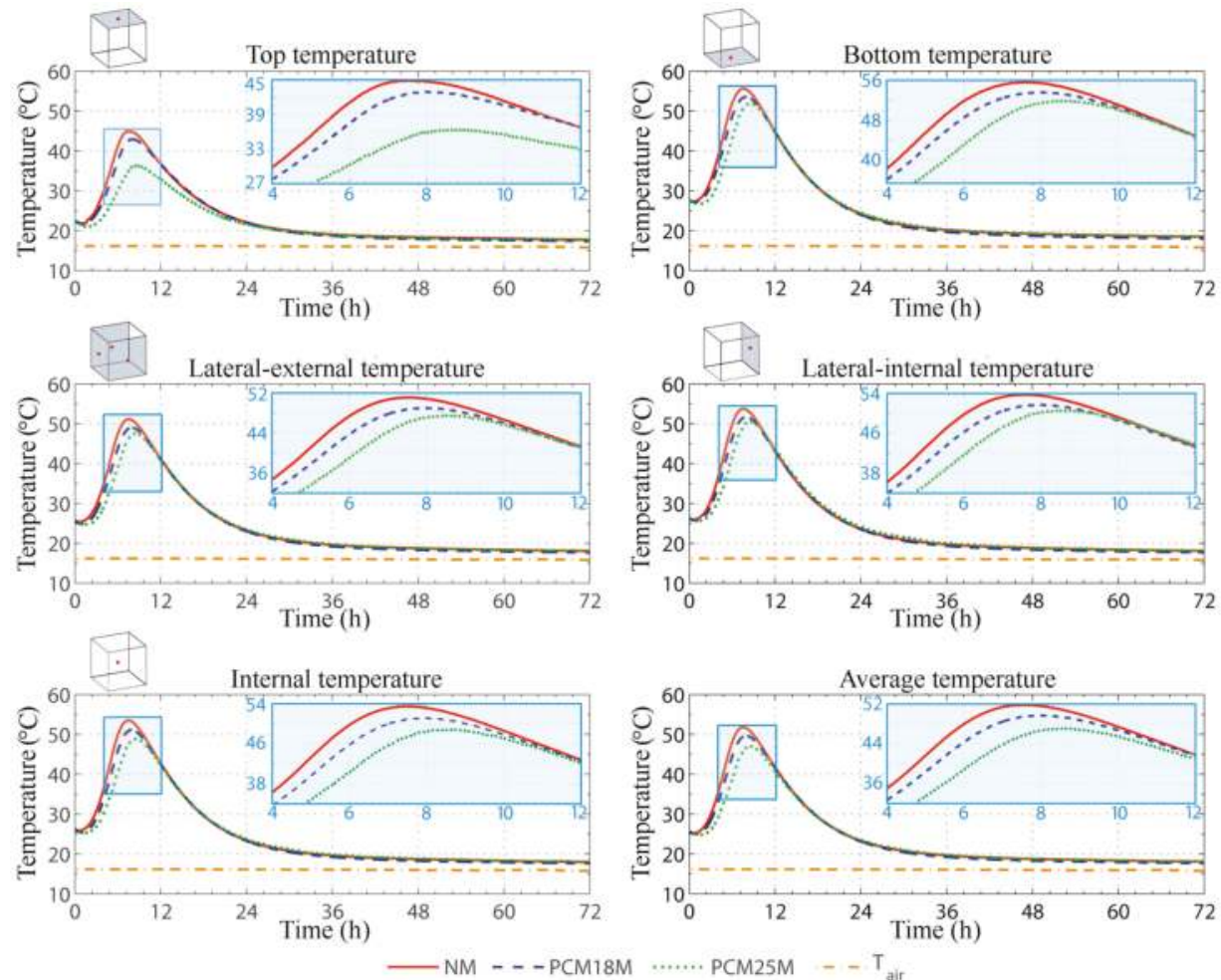

Figure 6. Monitored temperature waveforms of the NP (normal cement paste), the PCM18P (paste with mPCM18D) and the PCM25P (paste with DS 5038X) samples.

Every monitored temperature profile stabilizes at about $16{ }^{\circ} \mathrm{C}$ after the considered time frame, which fully embraces the exoenergetic reaction between cement and water; for this reason, here, only the temperature trends of the first three days are shown.

The graphs in Figure 6 display the temperature trend registered by the different thermocouples for each of the three kinds of pastes, i.e., the NP, the PCM18P and the PCM25P sample. The three lateral thermocouples directly exposed to the conditioned environment are reported here by considering their average value, while the fourth one, i.e., the one insisting on the side of the mold, which was in direct contact with another sample of the same kind, is separately reported. 
In general, Figure 6 shows a single temperature peak for every investigated sample. Such peak can be associated with the acceleration and the post acceleration period of the hydration process, since the actual monitoring of the samples started after 40 min after adding water to cement and PCM. By looking at the different temperature waveforms, the NP sample is always associated with the highest temperature values, always reaching peaks above $50{ }^{\circ} \mathrm{C}$, with the exception of the top surface, which is clearly associated with the highest heat flux through the local controlled environment. The PCM25P is the sample with the lowest amount of sensible heat released during the hydration process, while the PCM18P shows an intermediate trend, if compared with the two previous pastes.

It is noteworthy that the highest temperatures are always registered at the bottom and at the lateral internal surface of the samples and not in the main core of the cement pastes. This is probably due to two different factors: first of all, both these sensors are placed within the center of a cube face that is not directly exposed to the conditioned environment, secondly, the molds are made by a 20-mm thick PUR layer, which is a plastic material characterized by a fairly low thermal conductivity. As a consequence, these two thermocouples are in direct contact with a volume of cement, which cannot easily release heat, and is forced to store a higher amount of sensible energy in its matrix.

By taking a closer look to the average profiles defined for every analysed cement paste, it is possible to have a more detailed idea of the final effect that even a small amount of phase change materials can produce during the hydration reaction when they are used as additives for the production of thermally enhanced cement-based composite (see Figure 7).
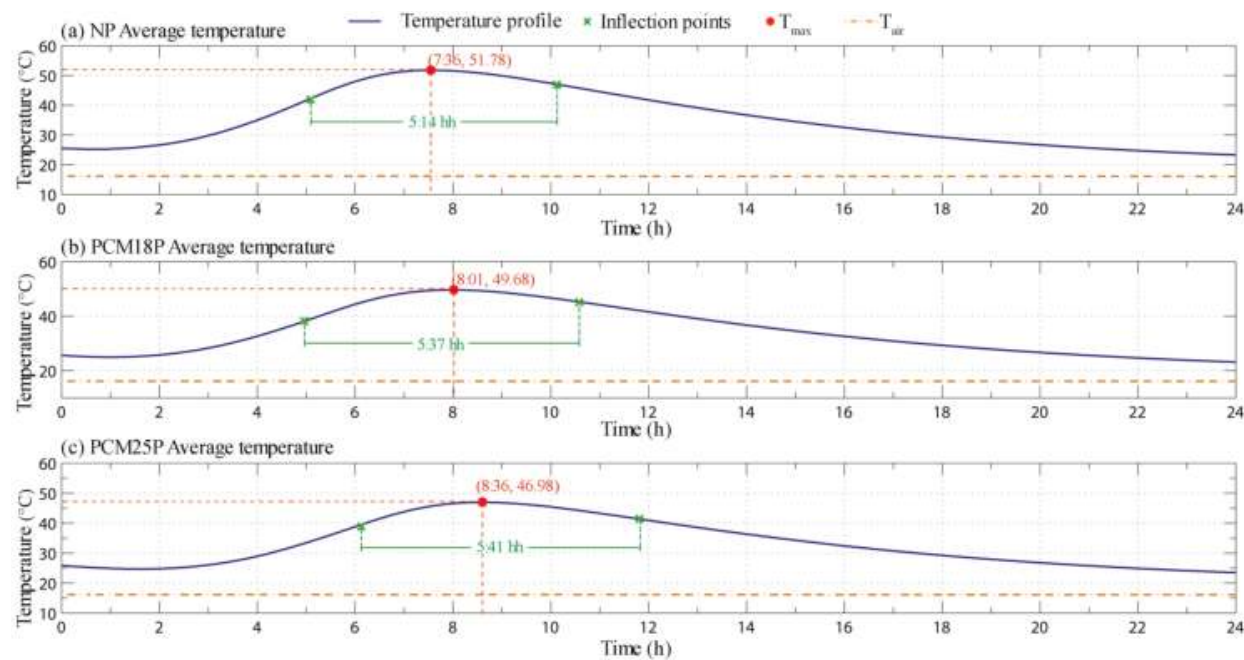

Figure 7. Average monitored temperature profiles for the first $24 \mathrm{~h}$ of the hydration process for (a) the $\mathrm{NP}$; (b) the PCM18P and (c) the PCM25P sample, with the respective maximum and inflection points.

The main temperature peak associated with the system reduces from $51.8^{\circ} \mathrm{C}$ in the NP sample to about $49.7^{\circ} \mathrm{C}$ and $47.0^{\circ} \mathrm{C}$ for the PCM18P and the PCM25P sample, respectively. From these data, the use of the PCM25P shows a higher effect on the actual reduction of the peak temperature registered during the observed exoenergetic reaction taking place in the mixture. This is probably due to the influence of the pre-induction and induction periods of the hydration process. At the beginning of the monitored period, in fact, all of the samples are associated with temperature values around $25^{\circ} \mathrm{C}$, thus well above the melting point of the mPCM18D, and, because of this, no phase transition could happen in the PCM18P paste at this stage of the curing.

However, the lower thermal conductivity and diffusivity of the paraffin microcapsules compared to those of the cementitious matrix (see Section 4.2) allows for releasing the heat produced during the 
hydration process at a slower rate. This slightly reduces the maximum peak temperature registered by the sensors, while producing a wider thermal wave. As a matter of fact, the time interval separating the two inflection points of the thermal waves increases of about 23 min with the addition of the mPCM18D microcapsules, i.e., from $5 \mathrm{~h}$ and $14 \mathrm{~min}$ to $5 \mathrm{~h}$ and $37 \mathrm{~min}$.

The same effect can also be seen when the temperature profile of the PCM25P paste is considered. However, in this case, the effect of the phase change also produces a time lag in the temperature trend. As it can be seen in Figure 7, the peak value of the monitored profile in this case is only reached after $8 \mathrm{~h}$ and $36 \mathrm{~min}$, which is about $1 \mathrm{~h}$ later than the NP paste and $35 \mathrm{~min}$ later than the PCM18P paste.

\subsection{Thermal Characterization and PCM Distribution Results}

As previously described in Section 3.4, in this work, the transient plane source method was used to define the basic thermal properties of the cured samples at two different temperatures, i.e., 10 and $35^{\circ} \mathrm{C}$, and also to investigate the actual PCM distribution in the final product. Table 2 shows the average values of thermal conductivity, thermal diffusivity and volumetric specific heat obtained using the classic bulk TPS analysis on the different samples. As can be seen, the introduction of the PCM in the cement-based mixture does not significantly influence the final thermal behaviour of the samples. However, every additivated paste is generally associated with lower volumetric specific heat and thermal diffusivity, and higher thermal conductivity. Additionally, the TPS denotes similar differences among comparable measurements carried out at different temperature conditions, particularly in the case of the PCM-doped recipes, i.e., PCM18P and PCM25P. As a matter of fact, the additivated samples always reduce their thermal conductivity and diffusivity with increasing temperature of the material, while their volumetric specific heat generally slightly increases.

Table 2. Thermal conductivity $(\lambda)$, thermal diffusivity $(\alpha)$ and volumetric specific heat $\left(\rho \mathrm{c}_{p}\right)$, with the relative standard deviation, i.e., $\sigma_{\lambda}, \sigma_{\alpha}$ and $\sigma_{\rho c_{p}}$, of three considered samples (NP, PCM18P, PCM25P), at different temperature $(\mathrm{T})$ conditions.

\begin{tabular}{cccccccc}
\hline Samples & $\begin{array}{c}\mathrm{T} \\
\left({ }^{\circ} \mathrm{C}\right)\end{array}$ & $\begin{array}{c}\lambda \\
\left(\frac{W}{m K}\right)\end{array}$ & $\begin{array}{c}\sigma_{\lambda} \\
\left(\frac{W}{m K}\right)\end{array}$ & $\begin{array}{c}\alpha \\
\left(\frac{m m^{2}}{s}\right)\end{array}$ & $\begin{array}{c}\sigma_{\lambda} \\
\left(\frac{m m^{2}}{s}\right)\end{array}$ & $\begin{array}{c}\rho c_{p} \\
\left(\frac{M J}{m^{3} K}\right)\end{array}$ & $\begin{array}{c}\sigma_{\rho c_{p}} \\
\left(\frac{M J}{m^{3} K}\right)\end{array}$ \\
\hline \multirow{2}{*}{$\mathrm{NP}$} & 10 & 0.794 & 0.001 & 0.378 & 0.001 & 2.102 & 0.019 \\
& 35 & 0.791 & 0.004 & 0.371 & 0.009 & 2.134 & 0.045 \\
\hline \multirow{2}{*}{ PCM18P } & 10 & 0.812 & 0.003 & 0.370 & 0.008 & 2.197 & 0.050 \\
& 35 & 0.810 & 0.003 & 0.368 & 0.004 & 2.204 & 0.016 \\
\hline \multirow{2}{*}{ PCM25P } & 10 & 0.813 & 0.011 & 0.382 & 0.147 & 2.131 & 0.063 \\
& 35 & 0.763 & 0.011 & 0.354 & 0.014 & 2.156 & 0.059 \\
\hline
\end{tabular}

As regarding the $\mathrm{PCM}$ distribution analysis, also in this case, the characterization procedure was repeated at 10 and $35^{\circ} \mathrm{C}$, however, and, as expected, no significant differences can be noticed in the two cases. For this reason, only the results at $35^{\circ} \mathrm{C}$ are directly reported in Figure 8, which shows the obtained thermal conductivity trends at a maximum distance of about $8 \mathrm{~mm}$ through the thickness of the cement-based pastes.

All the samples present similar profiles, which can be considered as a first indication about the relatively good dispersion of the PCMs in the mixtures. Smaller measurement times are always associated with a noisier signal which typically characterizes the portion of the pastes directly adjacent to the investigated surface, but can only penetrate short probing depths. With increasing measurement times, on the other hand, the thermal wave diffuses more deeply into the samples, thus the 2000 measurement points taken by the Hot Disk during the considered transient produce less accurate values in the superficial volume of the cubes, but allow for more deeply investigating the thermal conductivity evolution. 

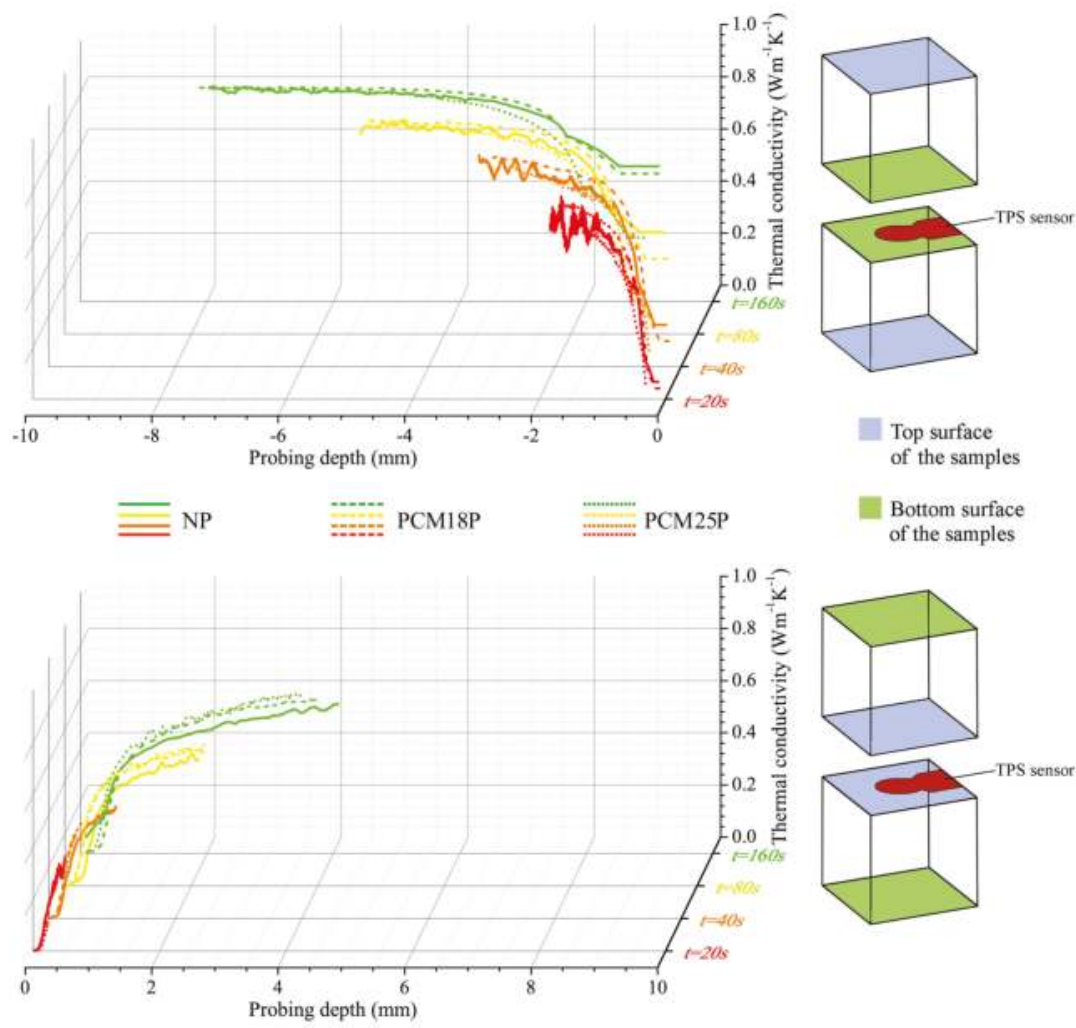

Top surface

of the samples

Bottom surface of the samples

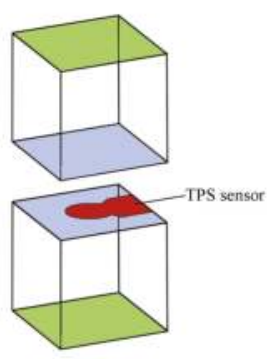

Figure 8. Thermal conductivity profiles as a function of the probing depth for the three different samples (NP, 18PCMP and 25PCMP) at $35^{\circ} \mathrm{C}$, considering four different probing times: 20, 40, 80 and $160 \mathrm{~s}$.

By comparing the thermal conductivity trends through the bottom surface of the different cement-based pastes, a huge and abrupt increase is generally found, and the thermal conductivity of the deepest points very closely approximates the average values obtained with the bulk analysis and listed in Table 2, for each of the considered specimens. Less sloped curves, typical of materials with higher insulation capability, on the other hand, characterize the signal recorded through the top surface of the pastes. Additionally, in this case, even using the largest measurement time, i.e., $160 \mathrm{~s}$, the obtained $\lambda$ value always remains well below the average results in Table 2. Such peculiar behaviour is detected in all the samples, and is even more pronounced when the normal paste is considered, so it can be safely stated that it is not an effect produced by a differential dispersion of the latent additive. On the contrary, this peculiar trend is most probably due to two different phenomena taking place simultaneously. The first one can be considered as an instrumental error, in fact, by sandwiching the TPS sensor between the two upper surfaces of the cement cubes, a higher contact resistance was detected by the double spiral. This is due to fact that the top-free surfaces of the samples are in direct contact with air during the overall duration of the curing process, and, for this reason, they develop a more irregular plane, with a higher number of voids, and, consequently, higher surface resistance. The second phenomenon can be associated with a sort of differential sedimentation occurring in the pure cement paste, which causes less dense components, particularly the air trapped in the cement paste during the blending procedure, to float in the mixture, and be mostly accumulated in the upper part of the sample. 
In general, the NP, PCM18P and PCM25P curves are similar; however, the bottom surface profiles show that (i) the NP sample produces the highest thermal noise; (ii) the PCM18P thermal conductivity trend is always the highest one; and (iii) the PCM25P always presents the lowest thermal conductivity. This is not true for $\lambda$ profiles obtained by interposing the TPS sensor between the top surfaces of the cement cubes. In this case, as a matter of fact, the normal paste (NP) is always associated with the lowest thermal conductivity trend.

\subsection{Physico-Thermal and Mechanical Characterization}

The analysis of the average density showed, as expected, minor values for mixes with PCM, expecially in the case of PCM25P. In addition, the coefficients of variation of doped cement pastes were relatively lower compared to normal pastes: 0.005 and 0.007 for PCM18P and PCM25P, respectively, against 0.027 of NP. Table 3 shows the physical characteristics of the 15 samples, i.e., the values of volume, weight, density and their standard deviation and coefficient of variation. Figure 9 shows the variation of density and its standard deviation of the three different cement-paste materials, together with their characteristic strength. Table 4 shows the elastic moduli obtained from cyclical loading tests together with the values of the average strain measured through the strain gauges. The results demonstrate that the PCM presence decreases the elastic moduli of the cementitious materials, especially in the case of PCM18P, which exhibits a Young modulus E $=8784 \mathrm{MPa}$, about one half the Young modulus of normal cement paste. The results of strength compressive tests are reported in Table 5. The table shows the results of all 15 samples, in terms of maximum force and resistance. Average compressive strength was calculated for each type of cement paste. The analysis was carried out without considering the first NP sample and the last PCM25P sample, which were clear outliers if compared to the others. The average strength of the normal samples was $34.94 \mathrm{MPa}$ while PCM18P and PCM25P exhibited values of 37.24 and $37.52 \mathrm{MPa}$, respectively, about 7\% higher than the NP.

Table 3. Measured volume $(V)$, weight $(w)$, density $(\rho)$, average weight $(\bar{w})$, average density $(\bar{\rho})$ and relative standard deviation $(\sigma)$ and coefficient of variance $(\mathrm{COV})$ of the $5 \mathrm{NP}, \mathrm{PCM} 18 \mathrm{P}$ and PCM25P samples.

\begin{tabular}{ccccccccccc}
\hline & Sample & $V\left(\mathbf{m m}^{3}\right)$ & $w(\mathrm{~g})$ & $\bar{w}(\mathrm{~g})$ & $\sigma_{w}$ & $\mathrm{COV}_{w}$ & $\left.\rho \mathbf{( k g} / \mathbf{m}^{3}\right)$ & $\bar{\rho}\left(\mathbf{k g} / \mathbf{m}^{3}\right)$ & $\sigma_{\rho}$ & $\mathrm{COV}_{\rho}$ \\
\hline \multirow{4}{*}{ NP } & 1 & $9.9091 \times 10^{5}$ & 1650 & & & & 1665 & & & \\
& 2 & $9.8049 \times 10^{5}$ & 1648 & & & & 1681 & & & \\
& 3 & $9.9570 \times 10^{5}$ & 1627 & 1672 & 41 & 0.024 & 1634 & 1694 & 46 & 0.027 \\
& 4 & $9.8288 \times 10^{5}$ & 1705 & & & & 1735 & & & \\
& 5 & $9.9013 \times 10^{5}$ & 1708 & & & & 1725 & & & \\
PCM18P & 1 & $9.8675 \times 10^{5}$ & 1647 & & & & 1669 & & & \\
& 2 & $9.9252 \times 10^{5}$ & 1671 & & & & 1684 & & & \\
& 3 & $9.9850 \times 10^{5}$ & 1680 & 1656.4 & 19 & 0.011 & 1683 & 1673 & 9 & 0.005 \\
& 4 & $9.9113 \times 10^{5}$ & 1649 & & & & 1664 & & & \\
PCM25P & 5 & $9.8067 \times 10^{5}$ & 1635 & & & & 1667 & & & \\
& 1 & $9.8586 \times 10^{5}$ & 1625 & & & & 1648 & & & \\
& 2 & $9.9431 \times 10^{5}$ & 1648 & & & & 1657 & & & \\
& 3 & $9.7761 \times 10^{5}$ & 1634 & 1633.8 & 9 & 0.006 & 1671 & 1664 & 12 & 0.007 \\
\hline
\end{tabular}

Table 4. Compressive load variation $(\Delta F)$, average sample surface (A), average strain $(\Delta \epsilon)$ and average elastic moduli (E) of the NP, the PCM18P and the PCM25C sample.

\begin{tabular}{ccccc}
\hline Sample & $\boldsymbol{\Delta} \boldsymbol{F}(\mathbf{k N})$ & $\mathrm{A}\left(\mathbf{m m}^{\mathbf{2}}\right)$ & $\boldsymbol{\Delta} \boldsymbol{E}$ & $\mathrm{E}(\mathbf{M P a})$ \\
\hline NP & 8.5 & 9949 & $4.866 \times 10^{-5}$ & 17,558 \\
PCM18P & 8.5 & 9945 & $9.730 \times 10^{-5}$ & 8784 \\
PCM25P & 8.5 & 9940 & $8.106 \times 10^{-5}$ & 10,549 \\
\hline
\end{tabular}


Table 5. Peak load (PL), strength $(\mathrm{R})$, average strength $\left(R_{m}\right)$ and standard deviation $\left(\sigma_{R_{m}}\right)$, characteristic strength $\left(R_{c k}\right)$ and covariance $(\mathrm{Cv})$ of the $5 \mathrm{NP}, \mathrm{PCM} 18 \mathrm{P}$ and PCM25P samples.

\begin{tabular}{|c|c|c|c|c|c|c|c|}
\hline & Sample & PL (kN) & $\mathrm{R}$ (MPa) & $R_{m}(\mathrm{MPa})$ & $\sigma_{R_{m}}$ & $R_{c k}(\mathrm{MPa})$ & $\mathrm{Cv}$ \\
\hline \multirow{5}{*}{$\mathrm{NP}$} & 1 & 513.6 & 51.42 & \multirow{5}{*}{34.94} & \multirow{5}{*}{2.7} & \multirow{5}{*}{25.60} & \multirow{5}{*}{0.079} \\
\hline & 2 & 322.2 & 32.43 & & & & \\
\hline & 3 & 340.2 & 34.19 & & & & \\
\hline & 4 & 340.8 & 34.29 & & & & \\
\hline & 5 & 386.6 & 38.86 & & & & \\
\hline \multirow{5}{*}{ PCM18P } & 1 & 397.6 & 39.96 & \multirow{5}{*}{37.24} & \multirow{5}{*}{2.8} & \multirow{5}{*}{27.77} & \multirow{5}{*}{0.075} \\
\hline & 2 & 396.3 & 39.85 & & & & \\
\hline & 3 & 336.1 & 33.73 & & & & \\
\hline & 4 & 348.8 & 35.13 & & & & \\
\hline & 5 & 373.7 & 37.53 & & & & \\
\hline \multirow{5}{*}{ PCM25P } & 1 & 382.1 & 38.44 & \multirow{5}{*}{37.52} & \multirow{5}{*}{2.5} & \multirow{5}{*}{29.18} & \multirow{5}{*}{0.065} \\
\hline & 2 & 375.7 & 37.78 & & & & \\
\hline & 3 & 395.6 & 39.79 & & & & \\
\hline & 4 & 338.3 & 34.06 & & & & \\
\hline & 5 & 302.8 & 30.41 & & & & \\
\hline
\end{tabular}

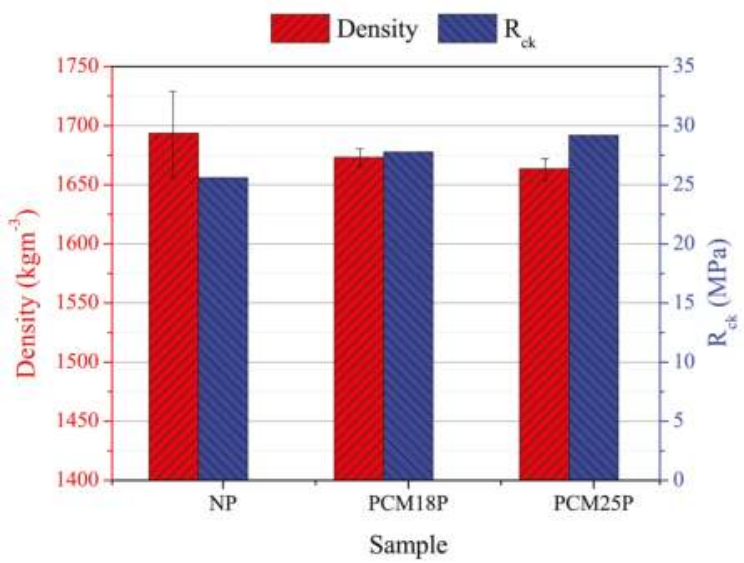

Figure 9. Average density and characteristic strength of the NP, PCM18P and PCM25P samples.

\subsection{Discussion of the Results}

Results from the thermo-mechanical characterization of the different cement pastes produced in this work can be used to draw some interesting conclusions about the use phase change materials within cement-based matrices. As a matter of fact, the thermal analyses carried out on the samples showed that the introduction of a small amount of PCM, i.e., $1 \%$ in weight of cement, can effectively reduce the peak temperature reached by the composites during the first $24 \mathrm{~h}$ of the curing process. Because of this, the PCM-doped matrices are exposed to lower temperature gradients throughout their volume and, consequently, to a more uniform thermal expansion, which, in turn, reduces the amount of strains and stresses that inevitably are generated in the solid volume as a consequence of the exoenergetic hydration reaction.

The non-destructive PCM-distribution analysis carried by using the TPS method, on the other hand, allowed for concluding that, despite the cement paste itself exhibiting an intrinsic dis-homogeneity, probably due to a small amount of differential sedimentation driven by density 
gradients in the paste, the latent additives were mostly well mixed during the production phase of the samples.

The promising thermal results were confirmed by the mechanical characterization of the samples in terms of density, and characteristic strength, although a notable reduction in Young moduli was made evident due to soft PCM inclusions. In particular, the introduction of $1 \%$ in weight of phase change material was able to increase its characteristic compressive strength by about $7 \%$ for both samples, while also reducing the mass density of the final composite. Characteristic compressive strength and COV are important values for structural applications: the first represents the strength that $5 \%$ of samples do not reach, while the COV is a measure of the reliability of the structural material. Both doped cement pastes exhibit higher characteristic strength and lower $\mathrm{COV}$, which demonstrate a good structural performance. In particular, NP, PCM18P and PCM25P showed Rck and COV of 25.60, $27.77,29.18 \mathrm{MPa}$ and $0.079,0.075$ and 0.065 , respectively.

\section{Conclusions}

In this work, a small amount of micro-capsulated PCM, i.e., $1 \%$ in weight, is introduced in a cement paste with the aim of improving its mechanical performance by reducing the sensible heat released during the hydration process and, consequently, the micro-cracking developed because of the thermally induced differential dilatation of the solid matrix. Two different paraffin-based latent additives are considered and compared in this study, i.e., the Micronal DS 5038X and the Microtek mPCM18D micro-capsules. These additives present similar thermophysical characteristics, but they have two different melting temperatures, i.e., 18 and $25^{\circ} \mathrm{C}$.

The thermal monitoring during the 28 days of curing showed how the latent nature of the PCM plays a significant role in reducing the sensible heat released during the first $24 \mathrm{~h}$ of the hydration process. In particular, the peak temperature registered for the standard cement paste was lowered by about $4{ }^{\circ} \mathrm{C}$ due to the introduction of the micro-capsulated PCM with a phase change temperature of $18{ }^{\circ} \mathrm{C}$, and by about $5{ }^{\circ} \mathrm{C}$ if the micro-capsulated PCM with a $25^{\circ} \mathrm{C}$ threshold is taken into account. The latent additives were also shown to positively affect the dynamics of the heating process. In fact, the obtained temperature peaks were delayed by about 35 and 60 min thanks to the addition of the Microtek mPCM18D and the Micronal DS 5038X micro-capsules, respectively. Lastly, the time distance between the inflection points characterizing the monitored thermal waves increases from $5 \mathrm{~h}$ and $14 \mathrm{~min}$ to $5 \mathrm{~h}$ and $37 \mathrm{~min}$ and $5 \mathrm{~h}$ and $41 \mathrm{~min}$ if the micro-capsulated PCM with a melting temperature of $18{ }^{\circ} \mathrm{C}$ and the latent additives with a melting temperature of $25^{\circ} \mathrm{C}$ are considered, respectively.

The positive thermal effect of the PCM was also corroborated by the mechanical analyses carried on the samples. In particular, the PCM-doped pastes increased their characteristic compressive strength of about $7 \%$, despite the non-negligible reduction in density in the final composite, reaching an average characteristic strength of 27.77, and 29.18 MPa for the PCM18P and PCM25P sample, respectively, against the obtained value of $25.60 \mathrm{MPa}$ of the NP one. Additionally, the introduction of $1 \%$ in weight of PCM also allowed for obtaining lower coefficients of variation (COV), i.e., $0.079,0.075$ and 0.065 , for the NP, the PCM18P and PCM25P paste, respectively. According to the thermal analyses, cement pastes with $\mathrm{PCM}$ with a melting point at $25^{\circ} \mathrm{C}$ showed the best performance in terms of both resistance and reliability.

In conclusion, the thermo-mechanical investigations carried out in this work demonstrated the positive effect deriving from the introduction of a small amount of PCM, i.e., $1 \%$ of the total cement weight, in common cement-water composites. This innovative solution could represent an effective way to improve the final mechanical performance of concrete castings subjected to extensive thermal expansion phenomena, such as massive castings and castings at high temperatures. 
Author Contributions: Conceptualization, A.L.P. and F.U.; Methodology, A.L.P., F.U., C.F. and A.D.; Formal Analysis, C.F. and A.D.; Investigation, C.F. and A.D.; Data Curation, C.F. and A.D.; Writing-Original Draft Preparation, C.F. and A.D.; Writing-Review and Editing, A.L.P. and F.U.; Visualization, L.F.C. and F.C.; Supervision, L.F.C. and F.C.; Project Administration, L.F.C., A.L.P. and F.C.; Funding Acquisition, L.F.C., A.L.P., F.U. and F.C. In general, the authors declare to have equally contributed to the investigation and the publication of these key results.

Funding: The research leading to these results has received funding from the European Union's Horizon 2020 research and innovation program under Grant agreement No. 657466 (INPATH-TES) and No. 765057 (SaferUp!). The authors also thank the Microtek Laboratories, Inc. for providing the capsulated materials. The work is also partially funded by the Spanish government (ENE2015-64117-C5-1-R).

Acknowledgments: Acknowledgments are due to the "CIRIAF program for UNESCO" in the framework of the UNESCO Chair "Water Resources Management and Culture". Luisa F. Cabeza would like to thank the Catalan Government for the quality accreditation given to her research group (2017 SGR 1537).

Conflicts of Interest: The authors declare no conflict of interest.

\section{References}

1. Costa, C. Materials for Construction and Civil Engineering: Science, Processing, and Design; Springer International Publishing: Cham, Switzerland; Heidelberg, Germany; New York, NY, USA; Dordrecht, The Netherlands; London, UK, 2015; Chapter 1, pp. 1-50.

2. Huntzinger, D.N.; Eatmon, T.D. A life-cycle assessment of Portland cement manufacturing: Comparing the traditional process with alternative technologies. J. Clean. Product. 2009, 17, 668-675. [CrossRef]

3. Marcelino-Sadaba, S.; Kinuthia, J.; Oti, J.; Meneses, A.S. Challenges in Life Cycle Assessment (LCA) of stabilised clay-based construction materials. Appl. Clay Sci. 2017, 144, 121-130. [CrossRef]

4. De Sensale, G.R. Effect of rice-husk ash on durability of cementitious materials. Cem. Concr. Compos. 2010, 32, 718-725. [CrossRef]

5. Zain, M.; Islam, M.; Mahmud, F.; Jamil, M. Production of rice husk ash for use in concrete as a supplementary cementitious material. Constr. Build. Mater. 2011, 25, 798-805. [CrossRef]

6. D'Alessandro, A.; Fabiani, C.; Pisello, A.; Ubertini, F.; Luigi Materazzi, A.; Cotana, F. Innovative concretes for low-carbon constructions: A review. Int. J. Low-Carbon Technol. 2017, 12, 289-309. [CrossRef]

7. Sajedi, F.; Razak, H.A.; Mahmud, H.B.; Shafigh, P. Relationships between compressive strength of cement-slag mortars under air and water curing regimes. Constr. Build. Mater. 2012, 31, 188-196. [CrossRef]

8. Luo, R.; Cai, Y.; Wang, C.; Huang, X. Study of chloride binding and diffusion in GGBS concrete. Cem. Concr. Res. 2003, 33, 1-7. [CrossRef]

9. Bentz, D.P.; Stutzman, P.E. Evolution of porosity and calcium hydroxide in laboratory concretes containing silica fume. Cem. Concr. Res. 1994, 24, 1044-1050. [CrossRef]

10. Fic, S.; Szelag, M. Analysis of the development of cluster cracks caused by elevated temperatures in cement paste. Constr. Build. Mater. 2015, 83, 223-229. [CrossRef]

11. Land, G.; Stephan, D. Controlling cement hydration with nanoparticles. Cem. Concr. Compos. 2015, 57, 64-67. [CrossRef]

12. Sun, J.; Xu, K.; Shi, C.; Ma, J.; Li, W.; Shen, X. Influence of core/shell TiO2@SiO2 nanoparticles on cement hydration. Constr. Build. Mater. 2017, 156, 114-122. [CrossRef]

13. Marchon, D.; Flatt, R. 8-Mechanisms of cement hydration. In Science and Technology of Concrete Admixtures; Aïtcin, P.C., Flatt, R.J., Eds.; Woodhead Publishing: Sawston, UK, 2016; pp. 129-145,

14. Bullard, J.W.; Jennings, H.M.; Livingston, R.A.; Nonat, A.; Scherer, G.W.; Schweitzer, J.S.; Scrivener, K.L.; Thomas, J.J. Mechanisms of cement hydration. Cem. Concr. Res. 2011, 41, 1208-1223. [CrossRef]

15. Livesey, P.; Donnelly, A.; Tomlinson, C. Measurement of the heat of hydration of cement. Cem. Concr. Compos. 1991, 13, 177-185. [CrossRef]

16. Abbas, Z.H.; Majdi, H.S. Study of heat of hydration of Portland cement used in Iraq. Case Stud. Constr. Mater. 2017, 7, 154-162. [CrossRef] 
17. Bouasker, M.; Mounanga, P.; Turcry, P.; Loukili, A.; Khelidj, A. Chemical shrinkage of cement pastes and mortars at very early age: Effect of limestone filler and granular inclusions. Cem. Concr. Compos. 2008, 30, 13-22. [CrossRef]

18. Hu, X.; Shi, C.; Shi, Z.; Tong, B.; Wang, D. Early age shrinkage and heat of hydration of cement-fly ash-slag ternary blends. Constr. Build. Mater. 2017, 153, 857-865. [CrossRef]

19. Zreiki, J.; Bouchelaghem, F.; Chaouche, M. Early-age behaviour of concrete in massive structures, experimentation and modelling. Nucl. Eng. Des. 2010, 240, 2643-2654. [CrossRef]

20. Yuan, Y.; Wan, Z. Prediction of cracking within early-age concrete due to thermal, drying and creep behavior. Cem. Concr. Res. 2002, 32, 1053-1059. [CrossRef]

21. Bolander, J.E.; Berton, S. Simulation of shrinkage induced cracking in cement composite overlays. Cem. Concr. Compos. 2004, 26, 861-871. [CrossRef]

22. Lothenbach, B.; Winnefeld, F. Thermodynamic modelling of the hydration of Portland cement. Cem. Concr. Res. 2006, 36, 209-226. [CrossRef]

23. Cabeza, L.F.; Castellón, C.; Nogués, M.; Medrano, M.; Leppers, R.; Zubillaga, O. Use of microencapsulated PCM in concrete walls for energy savings. Energy Build. 2007, 39, 113-119. [CrossRef]

24. Lecompte, T.; Bideau, P.L.; Glouannec, P.; Nortershauser, D.; Masson, S.L. Mechanical and thermo-physical behaviour of concretes and mortars containing phase change material. Energy Build. 2015, 94, 52-60. [CrossRef]

25. D'Alessandro, A.; Pisello, A.; Fabiani, C.; Ubertini, F.; Cabeza, L.; Cotana, F. Multifunctional smart concretes with novel phase change materials: Mechanical and thermo-energy investigation. Appl. Energy 2018, 212, 1448-1461. [CrossRef]

26. Liu, H.; Bu, Y.; Guo, Q.; Sanjayan, J.G. Converting hydration heat to achieve cement mixture with early strength and low hydrating-thermal dissipation. Constr. Build. Mater. 2017, 151, 113-118. [CrossRef]

27. Cellat, K.; Beyhan, B.; Güngör, C.; Konuklu, Y.; Karahan, O.; Dündar, C.; Paksoy, H. Thermal enhancement of concrete by adding bio-based fatty acids as phase change materials. Energy Build. 2015, 106, 156-163. [CrossRef]

28. Šavija, B.; Schlangen, E. Use of phase change materials (PCMs) to mitigate early age thermal cracking in concrete: Theoretical considerations. Constr. Build. Mater. 2016, 126, 332-344. [CrossRef]

29. Aguayo, M.; Das, S.; Maroli, A.; Kabay, N.; Mertens, J.C.; Rajan, S.D.; Sant, G.; Chawla, N.; Neithalath, N. The influence of microencapsulated phase change material (PCM) characteristics on the microstructure and strength of cementitious composites: Experiments and finite element simulations. Cem. Concr. Compos. 2016, 73, 29-41. [CrossRef]

30. Fernandes, F.; Manari, S.; Aguayo, M.; Santos, K.; Oey, T.; Wei, Z.; Falzone, G.; Neithalath, N.; Sant, G. On the feasibility of using phase change materials (PCMs) to mitigate thermal cracking in cementitious materials. Cem. Concr. Compos. 2014, 51, 14-26. [CrossRef]

31. Microtek Labs. Available online: https://cdn2.hubspot.net/hubfs/4153344/MicrotekLaboratoriesDecember2017/ PDF/MPDS3300-0044Rev1.pdf?t=1516975227818 (accessed on 24 April 2018).

32. Microtek Labs. 2017. Available online: https://cdn2.hubspot.net/hubfs/4153344/MicrotekLaboratoriesDecember2017/ PDF/MPCM18DProductDataSheet.pdf?t=1516975227818 (accessed on 24 April 2018).

33. Gustafsson, S.E. Transient plane source techniques for thermal conductivity and thermal diffusivity measurements of solid materials. Rev. Sci. Instrum. 1991, 62, 797-804. [CrossRef]

34. International Organization for Standardization. Plastics Determination of Thermal Conductivity and Diffusivity Part 2: Transient Plane Source (Hot Disk) Method; SS-EN ISO 22007-2; ISO: Geneva, Switzerland, 2015.

35. Mihiretie, B.M.; Cederkrantz, D.; Sundin, M.; Rosén, A.; Otterberg, H.; Hinton, A.; Berg, B.; Karlsteen, M. Thermal depth profiling of materials for defect detection using hot disk technique. AIP Adv. 2016, 6, 085217. [CrossRef]

36. Norme Tecniche per le Costruzioni e Relativa Circolare Esplicativa. Available online: http:/ /www.consulentecasa. com/jdownloads/Miscellanea/NCT\%20-\%20Linee\%20Guida.pdf (accessed on 14 January 2008).

(c) 2018 by the authors. Licensee MDPI, Basel, Switzerland. This article is an open access article distributed under the terms and conditions of the Creative Commons Attribution (CC BY) license (http:/ / creativecommons.org/licenses/by/4.0/). 
Article

\title{
Study of the Thermal Properties and the Fire Performance of Flame Retardant-Organic PCM in Bulk Form
}

\author{
Anabel Palacios ${ }^{1}$, Alvaro De Gracia ${ }^{2}$, Laia Haurie ${ }^{3}$, Luisa F. Cabeza ${ }^{4}$, A. Inés Fernández ${ }^{1}$ and \\ Camila Barreneche ${ }^{1, *}$ \\ 1 Department of Materials Science \& Physical Chemistry, Universitat de Barcelona, Martí i Franqués 1, \\ 08028 Barcelona, Spain; apalacios@ub.edu (A.P.); ana_inesfernandez@ub.edu (A.I.F.) \\ 2 Departament d'Enginyeria Mecanica, Universitat Rovira i Virgili, Av. Paisos Catalans 26, \\ 43007 Tarragona, Spain; alvaro.degracia@urv.cat \\ 3 Departament de Tecnologia de l'Arquitectura, Universitat Politècnica de Catalunya, Av. Dr. Marañon 44-50, \\ 08028 Barcelona, Spain; laia.haurie@upc.edu \\ 4 GREA Innovació Concurrent, Universitat de Lleida, Edifici CREA, Pere de Cabrera s/n, 25001 Lleida, Spain; \\ lcabeza@diei.udl.cat \\ * Correspondence: c.barreneche@ub.edu
}

Received: 27 October 2017; Accepted: 29 December 2017; Published: 12 January 2018

\begin{abstract}
The implementation of organic phase change materials (PCMs) in several applications such as heating and cooling or building comfort is an important target in thermal energy storage (TES). However, one of the major drawbacks of organic PCMs implementation is flammability. The addition of flame retardants to PCMs or shape-stabilized PCMs is one of the approaches to address this problem and improve their final deployment in the building material sector. In this study, the most common organic PCM, Paraffin RT-21, and fatty acids mixtures of capric acid (CA), myristic acid (MA), and palmitic acid (PA) in bulk, were tested to improve their fire reaction. Several flame retardants, such as ammonium phosphate, melamine phosphate, hydromagnesite, magnesium hydroxide, and aluminum hydroxide, were tested. The properties of the improved PCM with flame retardants were characterized by thermogravimetric analyses (TGA), the dripping test, and differential scanning calorimetry (DSC). The results for the dripping test show that fire retardancy was considerably enhanced by the addition of hydromagnesite (50 wt \%) and magnesium hydroxide (50 wt \%) in fatty acids mixtures. This will help the final implementation of these enhanced PCMs in building sector. The influence of the addition of flame retardants on the melting enthalpy and temperatures of PCMs has been evaluated.
\end{abstract}

Keywords: phase change materials (PCMs); thermal energy storage (TES); flame retardants; dripping test; differential scanning calorimetry (DSC)

\section{Introduction}

In recent years, governments have started to be more aware of the urgent need to make better use of the world's energy resources. Total energy consumption in buildings (around 34\% [1]) has become a focus of studies and publications, with the aim of improving energy efficiency and reducing greenhouse gas emissions. Other than ameliorate the situation by reducing energy consumption and improving the efficiency of appliances, etc., the building envelopes must be more energy efficient as well, and thermal energy storage (TES) is one promising way to improve energy efficiency. Phase-change materials (PCMs) incorporated in the building walls can smooth temperature fluctuations and reduce electrical consumption by restricting the necessity to use electricity for heating/cooling during peak load periods $[2,3]$. 
Phase change materials (PCMs) have received much attention within the scientific community due to the fact that they present high-energy storage density when used in the required range of working temperatures. The implementation of organic PCMs in several applications such as heating and cooling or improvement of indoor building comfort is an important target in (TES) [4]. However, one of the major drawbacks of organic PCM implementation is flammability. The addition of flame retardants to PCMs or shape-stabilized PCMs is one of the approaches to address this problem and improve their final deployment as building materials.

The mechanism of action of PCMs is described as follows: when the temperature reaches the melting temperature they change their state from solid to liquid, being able to store large amounts of energy, and the same happens during the reverse process, but in this case the heat is released. As a result, PCMs in active systems provide high thermal energy storage capacity for the building component as a ventilated façade [5], or active slab, which might be used as storage in a building's active system [5] or to improve thermal inertia as passive systems inside buildings act as thermal regulators [6,7].

There are several ways to classify PCMs, according to their latent heat of fusion and melting point, according to temperature gradients [4] and, most commonly, according to the method used to exchange heat and to change the state, which was used by Abhat [8] in 1983. The most used PCM types are the one shown in Figure 1 within the temperatures range of this study (between 20 and $30^{\circ} \mathrm{C}$ ).

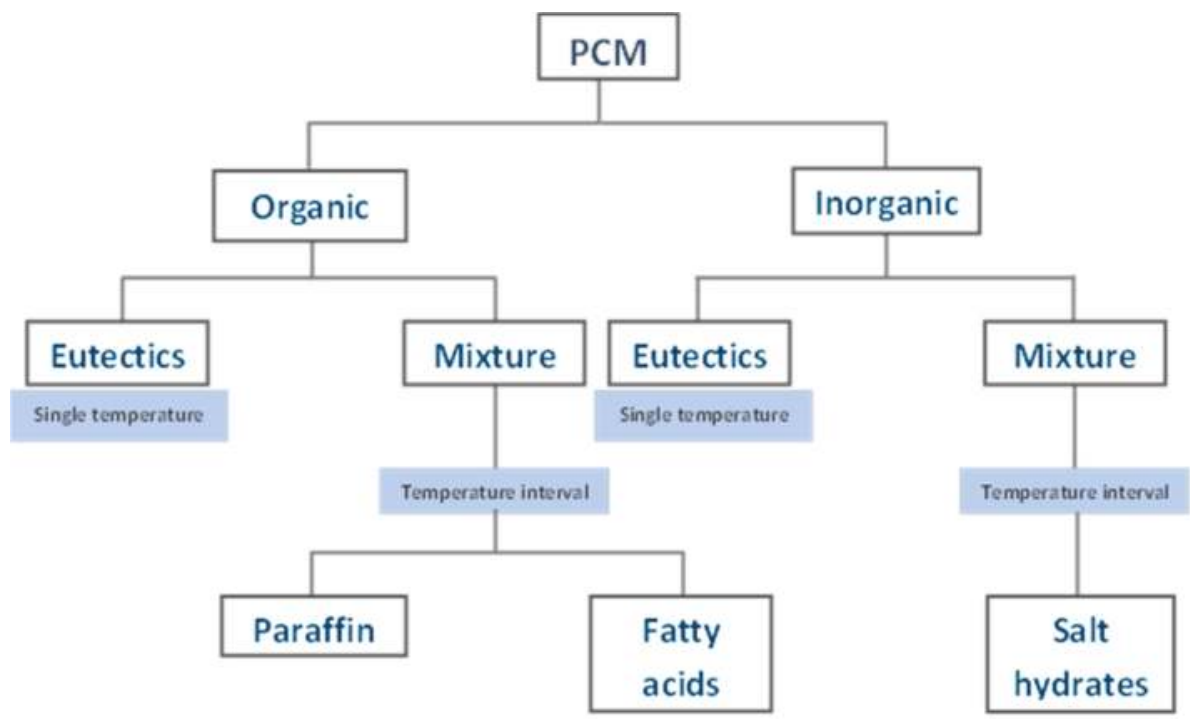

Figure 1. PCM classification adapted from Abhat [8] regarding the PCM type available within the temperature range of 20 to $30^{\circ} \mathrm{C}$.

The mainly studied organic PCMs are paraffin and fatty acids. As regards inorganic PCMs, salt hydrates are the most used. Cabeza et al. reported a comparison of advantages and disadvantages of organic and inorganic PCMs [4]. Inorganic PCMs present several drawbacks that complicate their use [9]. Drawbacks are sub-cooling, delay of the solidification process as well as capacity of store heat, corrosion and segregation. On the other hand, in general terms, organic PCMs represent an adequate option to be used in TES, accomplishing requirements such as chemical and thermal stability, no corrosion and low or no sub-cooling, which is an important topic in order to endure many cycles [10]. However, those substances also have problems such as lower phase change enthalpy, high flammability, and lower thermal conductivity than salt hydrates [11], but this can be minimized with the use of 
additives. Overall, the main drawbacks of inorganic PCMs are difficult to solve with the addition of additives. However, the addition of flame retardants or conductive particles could be a successful strategy to overcome the main drawbacks of organic PCMs.

In order to solve one of the major problems of organic PCM implementation in the buildings sector—flammability—several studies have been published [12-17]. Cai et al. [12] investigated the effect of adding expanded graphite (EG) and ammonium polyphosphate (APP) to PCM composites based on paraffin with a high-density polyethylene (HDPE) matrix. The results of thermogravimetric analysis (TGA) and cone calorimetry showed that EG and APP contributed to improving thermal stability properties and enhanced self-extinguishing properties of PCM composites. Moreover, thermal stability and flammability properties of palmitic acid/silicon dioxide $\left(\mathrm{SiO}_{2}\right)$ composites were studied by Fang et al. [13], where these composites were prepared by using sol-gel methods. Therefore, palmitic acid was used as PCM, silicon dioxide acted as the supporting material and melamine was incorporated as a flame retardant. The results reported that melamine and a multi-porous material $\left(\mathrm{SiO}_{2}\right)$ improve fire performance. In addition, Sittisart and Farid [14] studied a form-stable PCM consisting of paraffin (RT-21), high-density polyethylene (HDPE), and flame retardants such as magnesium hydroxide, aluminum hydroxide, expanded graphite (EG), ammonium polyphosphate (APP), pentaerythritol (PER), and treated montmorillonite (MMT), using a vertical burning test. They concluded that the addition of flame retardants improves thermal stability, and the best improvement in fire performance is showed by the mixture of $\mathrm{APP}+\mathrm{PER}+\mathrm{MMT}$ and $\mathrm{APP}+\mathrm{EG}$.

The present study is focused on how to reduce flammability of bulk-paraffin and bulk-fatty acid mixtures in liquid state without them being stabilized inside a matrix or encapsulated. To achieve this objective, organic PCMs were mixed with several flame retardants that act through different mechanisms to improve the fire performance of PCMs. The study of the effect of flame retardants directly on the bulk-PCM opens a new approach for improving fire performance of non-encapsulated PCMs. This will help the final implementation of these enhanced PCMs in the building sector. The main scope of the present work firmly connects fire-resistant performance with thermophysical properties.

\section{Materials and Methods}

\subsection{Phase Change Materials}

Different types of organic PCMs were tested in this study, namely paraffin and fatty acids.

Thermophysical properties of paraffin have been investigated by many researchers [18-21]. There are several commercial paraffins, which present different melting points and heat of fusion. Paraffin RT-21 meets desirable melting temperature $\left(21^{\circ} \mathrm{C}\right)$ in order to be implemented to improve thermal comfort in buildings. This temperature range is related to the building standards of major countries in the south of Europe where the comfortable indoor temperature of buildings are defined [22].

On the other hand, fatty acids show some differences compared with paraffin: narrow range of phase change, addition of antioxidants to reach chemical stability, high heat of fusion and less change in volume during phase change. Fatty acid melting points range from -7 to $71{ }^{\circ} \mathrm{C}$ depending on their chemical structure, while melting enthalpy varies from 45 to $210 \mathrm{~kJ} / \mathrm{kg}$ [18]. It is difficult to find a fatty acid that works in the thermal range required for building applications. Therefore, fatty acid eutectic mixtures provide an option to achieve proper phase change temperatures at close to $21^{\circ} \mathrm{C}$.

Paraffin RT-21 commercialized by Rubitherm [23] and fatty acid eutectic mixtures are described in Table 1 . The fatty acids used to prepare the eutectic mixtures were provided by Panreac (Barcelona, Spain) and their purities are $98 \%$ for capric acid and $98.5 \%$ for myristic acid and palmitic acid. The temperature ranges of these PCMs are adequate for the established comfort temperatures for building applications. Paraffin shows low thermal conductivity (around $0.2 \mathrm{~W} \mathrm{~m}^{-1}{ }^{\circ} \mathrm{C}^{-1}$ ), thermal and chemical stability, low volume changes during solidification process, and there are several different paraffinic PCMs suitable to be used to enhance thermal comfort in buildings. They are relatively cheap and are produced on a large scale [18]. 
Table 1. Properties of PCM used on this study (from literature).

\begin{tabular}{cccc}
\hline Compound & Melting Temperature $\left({ }^{\circ} \mathbf{C}\right)$ & Heat of Fusion $(\mathbf{k J} / \mathbf{k g})$ & Thermal Conductivity $\left(\mathbf{W} / \mathbf{m} \cdot{ }^{\circ} \mathbf{C}\right)$ \\
\hline Paraffin RT-21 & $21[23]$ & $100[23]$ & $0.2[23]$ \\
$73.5 \%$ Capric acid $+26.5 \%$ Myristic acid & $24.1[4]$ & $152[4]$ & n.a \\
$75.2 \%$ Capric acid $+24.8 \%$ Palmitic acid & $22.1[4]$ & $153[4]$ & n.a \\
\hline
\end{tabular}

\subsection{Flame Retardants}

Flame-retardant systems are proposed to delay or to inhibit combustion and there exist a large amount of flame retardants [24,25]. Nevertheless, the correct selection of the proper fire retardant is a key issue and not all of them will act properly for each PCM and application. Moreover, certain conditions are required in order to maintain the PCM properties. For example, the flame retardant has to be well dispersed and distributed in the PCM volume, and thermophysical PCM properties must remain constant.

Depending on their nature, flame retardants can act in the condensed or in the gas phase through a physical or chemical process [24,25]. Flame retardants can be classified according to their mechanism of action in three categories:

- Gas phase flame retardants: The flame retardant interferes with the free radical combustion reaction. Halogenated flame retardants are an example of this category.

- Endothermic flame retardants undergo an endothermic decomposition in the range of temperatures at which combustion takes place. This endothermic reaction helps to withdraw heat from the substrate. Furthermore, these compounds evolve non-flammable gases such as water or $\mathrm{CO}_{2}$ that have a dilution effect. The metal oxide formed during the decomposition of a metal hydroxide form an insulating protective coating on the condensed phase.

- Char-forming flame retardants: In this case, the flame retardant promotes the formation of a protective coating on the flammable material that hinders the heat and oxygen transfer. Polyphosphates and intumescent flame retardants (IFRs) are among this category. IFRs consist of a combination of a carbon source, an acid source and a foaming agent. Usually, ammonium polyphosphate, pentaerythritol and melamine are the main ingredients of an IFR.

In this study, five different flame retardants have been used: three endothermic flame retardants (commercial grades of alminum and magnesium hydroxide, and non-commercial synthetic hydromagnesite already used in previous studies [26]), as well as two commercial char-forming flame retardants (ammonium polyphosphate and an IFR). Halogenated flame retardants were deliberately not selected due to their toxicity and environmental impact $[25,27,28]$. Table 2 summarizes onset decomposition temperature and enthalpy of decomposition of flame retardants used in this study.

Table 2. Properties of tested flame retardant [24,29].

\begin{tabular}{|c|c|c|c|}
\hline Compound & Method & $\begin{array}{l}\text { Onset Decomposition } \\
\text { Temperature }\left({ }^{\circ} \mathrm{C}\right)\end{array}$ & $\begin{array}{c}\text { Enthalpy of Decomposition } \\
(\mathrm{kJ} / \mathrm{kg})\end{array}$ \\
\hline Aluminum hydroxide & Endothermic decomposition & 180 & 1300 \\
\hline Hydromagnesite & Endothermic decomposition & 200 & 800 \\
\hline APP & Char forming & 190 & - \\
\hline IFR & Char forming & 190 & - \\
\hline
\end{tabular}

\subsection{Formulations}

A matrix of formulations was tested in a preliminary study (see Table 3) in order to find the best performing flame retardant-PCM system. Several percentages of each flame retardant were tested to find the suitable flame-retardant load. The flame-retardant content required in order to reach a satisfactory fire performance depending on the nature and mechanism of action of the flame retardant 
was chosen and studied. According to the formulations used in the literature and recommended by the suppliers of polyolefin, it was decided to use at least $40 \%$ in weight for metal hydroxides and $15 \%$ for the char-forming flame retardants $[29,30]$.

Samples containing $40 \mathrm{wt} \%, 50 \mathrm{wt} \%$ and $60 \mathrm{wt} \%$ of hydromagnesite and magnesium hydroxide in the PCM were tested as well as samples with $15 \mathrm{wt} \%, 20 \mathrm{wt} \%$ and $25 \mathrm{wt} \%$ of ammonium phosphate (APP) and IFR systems (Table 3). In the case of the paraffin bulk-PCM, the amount of APP used will be up to $40 \mathrm{wt} \%$ in order to evaluate the performance of high loads of flame retardant. The final formulations selected and characterized, named optimum, were those that gave lowest flammability with the lowest amount of fire retardant addition. Note that the lower the fire-retardant percentage the lower the change of PCM thermophysical properties, following the mixtures law [31].

Table 3. Flame retardant formulations.

\begin{tabular}{l}
\hline \multicolumn{1}{c}{ Formulations } \\
\hline PCM $+(15 \%-20 \%-25 \%-40 \%)$ APP \\
PCM + (40\%-50\%-60\%) Hydromagnesite \\
PCM + (40\%-50\%-60\%) Magnesium hydroxide \\
PCM + (40\%-50\%-60\%) Aluminum hydroxide \\
PCM + (15\%-20\%-25\%) IFR \\
\hline
\end{tabular}

\section{Experimental Methods}

\subsection{Thermal Stability}

Thermogravimetrical analysis (TGA) technique was used to characterize the thermal stability of the samples under study. Analyses were performed on the pure PCMs between $50^{\circ} \mathrm{C}$ and $500{ }^{\circ} \mathrm{C}$ under $\mathrm{N}_{2}$ atmosphere with a flow of $80 \mathrm{~mL} / \mathrm{min}$ at a heating rate of $10^{\circ} \mathrm{C} / \mathrm{min}$ with a mass of approximately $30 \mathrm{mg}$ in a TA Instruments SDT Q600 (TA Instruments, Barcelona, Spain).

\subsection{Pyrolysis Combustion Flow Calorimeter (PCFC)}

A pyrolysis combustion flow calorimeter [32] from Fire Testing Technology standardized according to ASTM D7309 [33] was used to evaluate the flammability of the PCM. The equipment consists of a pyrolysis chamber, where small samples, around $5 \mathrm{mg}$, are heated under nitrogen atmosphere up to $750{ }^{\circ} \mathrm{C}$ at $1{ }^{\circ} \mathrm{C} / \mathrm{s}$. The evolved gases are then transported by an inert gas to the combustor that works at $900{ }^{\circ} \mathrm{C}$ in a flow of $20 \mathrm{~cm}^{3}$ of oxygen and $80 \mathrm{~cm}^{3}$ of nitrogen. The heat release rate is calculated from the oxygen consumed by the evolved gases during their combustion in the combustor.

\subsection{Dripping Test}

Flammability behavior was characterized by dripping test. The device is described in the Spanish standard UNE 23727 [34]. In this work the test was adapted to measure the properties of bulk PCM formulations. The dripping test is used to evaluate the flame and its propagation in a fire scenario.

PCM samples were weighted and introduced into a ceramic crucible, which was placed on a metallic grid $3 \mathrm{~cm}$ below a heat source of $500 \mathrm{~W}$. Under this test conditions the heat flux on the surface of the samples was $3 \mathrm{~W} / \mathrm{cm}^{2}$. The radiator was taken away and put back after each ignition and extinction. Three samples of each formulation were tested and the parameters determined were the time to ignition, the number of ignitions and the average time of flame persistence during the first $5 \mathrm{~min}$ of combustion. Temperature profiles reached during the dripping test were registered by a thermocouple.

Time to ignition $\left(t_{i}\right)$ is defined as the time at which the first flame appears on the surface of the sample. The average combustion time $\left(t_{c}\right)$ is related to the length of the combustion and, therefore, to the ability of the material to self-extinguish the flame once the heating source is removed. Finally, 
the number of ignitions is another parameter related to the fire reaction of the material. A combination of short combustion times together with numerous ignitions is a sign of a flammable material that is able to extinguish the flame if the heating source is removed.

\subsection{DSC}

Samples (PCMs with fire retardant) were evaluated by Differential Scanning Calorimetry (DSC), which is a well-known technique to analyze the latent heat of PCM. Therefore, DSC is able to determine the enthalpy of fusion and solidification, as well as the phase change temperature of the analyzed material. Furthermore, the analysis was performed between $T_{m} \pm 10{ }^{\circ} \mathrm{C}$ (being $T_{m}$ the PCM melting temperature) and $0.5^{\circ} \mathrm{C} / \mathrm{min}$ heating rate [35,36]. The amount of sample used was around $15 \mathrm{mg}$ and the sample was placed into $40 \mathrm{mg}$ aluminum crucibles, and the equipment used was a DSC 822e device supplied by Mettler Toledo (Barcelona, Spain). The experiment was tested under $50 \mathrm{~mL} \mathrm{~min}^{-1} \mathrm{~N}_{2}$ flow. In addition, the equipment precision is $\pm 0.3^{\circ} \mathrm{C}$ for temperature and $\pm 3 \mathrm{~kJ} \mathrm{~kg}^{-1}$ for enthalpy results.

\section{Results and Discussion}

\subsection{Thermal Stability}

TGA results describe the thermal decomposition of the PCMs. As can be observed in Figure 2, paraffin decomposes in one step between $190^{\circ} \mathrm{C}$ and $250{ }^{\circ} \mathrm{C}$. In the mixture of capric and myristic acids, a shoulder can be distinguished before the main peak and the derivate of the TGA curve of the eutectic mixture of capric and palmitic acids exhibits two peaks. This behaviour can be attributed to the different decomposition temperature of the fatty acids in accordance with the chain length.

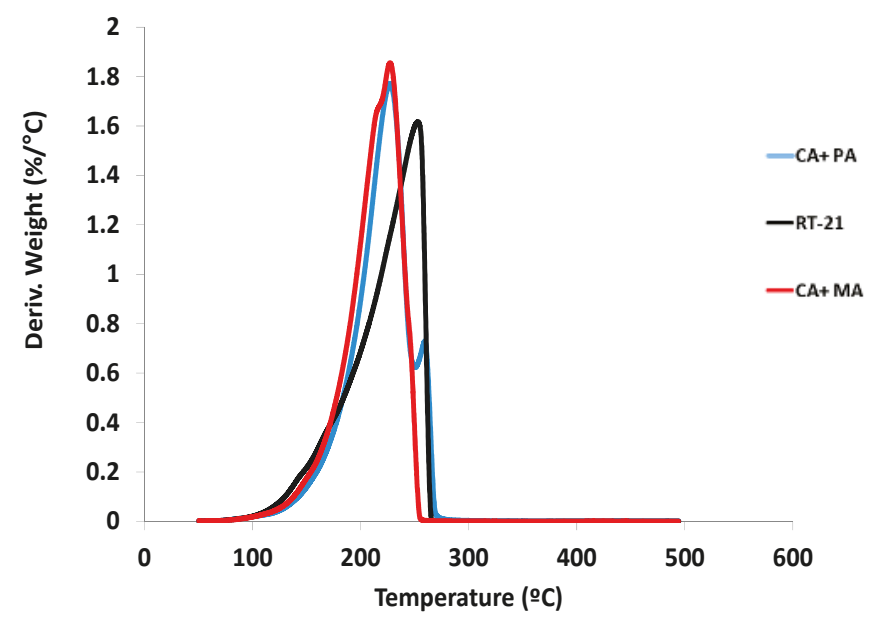

Figure 2. Derivative thermogravimetric curves of the PCM.

\section{2. $P C F C$}

The curves of heat release rate (HRR) vs. temperature are shown in Figure 3. As can be observed, decomposition of the three PCM starts at around $100{ }^{\circ} \mathrm{C}$. The peak of heat release rate (PHRR) corresponds to the maximum value of the HRR and the temperature of PHRR ( $\left.\mathrm{T}_{\mathrm{PHRR}}\right)$ is the temperature at which the PHRR takes place. High PHRR at low temperatures is usually related to a higher contribution in case of fire. In this case, all the samples have a similar $\mathrm{T}_{\mathrm{PHRR}}: 231^{\circ} \mathrm{C}$ for the paraffin, $230{ }^{\circ} \mathrm{C}$ for the eutectic mixture of caprilic and palmitic fatty acids, and $226^{\circ} \mathrm{C}$ for the eutectic mixture of caprilic and myristic acids. Regarding the PHRR, the paraffin PCM shows one unique peak 
of approx. $600 \mathrm{~W} / \mathrm{g}$. The CA + MA PCM shows one peak of $583 \mathrm{~W} / \mathrm{g}$ and a shoulder can be identified around $245^{\circ} \mathrm{C}$. The PHRR of the CA + PA mixture is significantly lower, at $466 \mathrm{~W} / \mathrm{g}$, and a smaller second peak $(292 \mathrm{~W} / \mathrm{g})$ takes place at $258^{\circ} \mathrm{C}$.

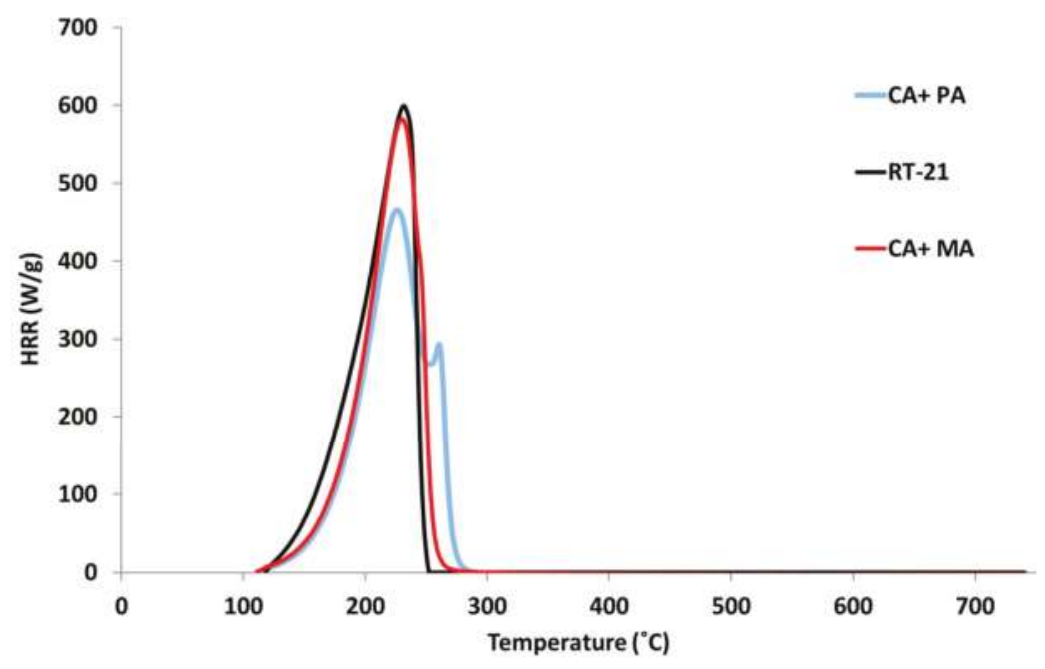

Figure 3. Heat released rate curves vs. temperature for organic PCM under study.

\subsection{Dripping Test}

As mentioned before, the preliminary study allowed us to select some of the percentages of flame retardant based on fire performance. Taking into account the formulations proposed in Table 3, the formulations with the lowest amount of each fire retardant that behaves with the best flammability performance are highlighted in Table 4.

Table 4. Dripping test results for PCM.

\begin{tabular}{cccc}
\hline Flame Retardant (wt $\%)$ & Ignition Time (s) & $\mathbf{N}^{\circ}$ of Ignitions & Average Combustion Time (s) \\
\hline Paraffin $\mathbf{R T}-21$ & 26 & 1 & 300 \\
$60 \%$ Paraffin $+40 \% \mathrm{APP}$ & 20 & 3 & 82 \\
$50 \%$ Paraffin $+50 \% \mathrm{HM}$ & 26 & 1 & 293 \\
$60 \%$ Paraffin $+20 \% \mathrm{IFR}$ & 50 & 2 & 289 \\
$50 \%$ Paraffin $+50 \% \mathrm{Al}(\mathrm{OH})_{3}$ & 27 & 1 & 286 \\
$50 \%$ Paraffin $+50 \% \mathrm{Mg}(\mathrm{OH})_{2}$ & 26 & 1 & 200 \\
CA + MA & 19 & 1 & 109 \\
$80 \%(\mathrm{CA}+\mathrm{MA})+20 \% \mathrm{APP}$ & 12 & 2 & 8 \\
$50 \%(\mathrm{CA}+\mathrm{MA})+50 \% \mathrm{HM}$ & 14 & 15 & 102 \\
$80 \%(\mathrm{CA}+\mathrm{MA})+20 \% \mathrm{IFR}$ & 21 & 2 & 117 \\
$50 \%(\mathrm{CA}+\mathrm{MA})+50 \% \mathrm{Al}(\mathrm{OH})_{3}$ & 19 & 2 & 224 \\
$50 \%(\mathrm{CA}+\mathrm{MA})+50 \% \mathrm{Mg}(\mathrm{OH})_{2}$ & 24 & 17 & 104 \\
\hline $\mathrm{CA}+\mathrm{PA}$ & 12 & 2 & 7 \\
$80 \%(\mathrm{CA}+\mathrm{PA})+20 \% \mathrm{APP}$ & 10 & 17 & 106 \\
$50 \%(\mathrm{CA}+\mathrm{PA})+50 \% \mathrm{HM}$ & 9 & 3 & 322 \\
$80 \%(\mathrm{CA}+\mathrm{PA})+20 \% \mathrm{IFR}$ & 16 & 1 & 4 \\
\hline $50 \%(\mathrm{CA}+\mathrm{PA})+50 \% \mathrm{Al}(\mathrm{OH})_{3}$ & 14 & 26 & \\
$50 \%(\mathrm{CA}+\mathrm{PA})+50 \% \mathrm{Mg}(\mathrm{OH})_{2}$ & 32 & & \\
\hline
\end{tabular}

Moreover, Table 4 provides the most important parameters in the dripping test: time to ignition $\left(t_{i}\right)$, the number of ignitions, and the average time of combustion $\left(t_{C}\right)$. 
An increase in the time to ignition is desirable, but it is not always obtained with the addition of flame retardants. This is due to the fact that many flame retardants act mainly on diminishing the heat release rate [37], which is believed to have more impact in case of a fire than ignitability [38].

The results for bulk paraffin PCMs demonstrate that the addition of $50 \%$ of endothermic flame retardants do not cause a significant improvement of the fire performance. The addition of char-forming flame retardants, APP and IFR, gives rise to a slight improvement. Paraffin PCM containing 20\% of IFR increases the time to ignition, and two ignitions take place, which means that after the first ignition the sample self-extinguished the flame and it is necessary to approach the heating source again to produce the second ignition. This behaviour slightly reduces the average combustion time. The maximum percentage initially established for APP (range between $15 \mathrm{wt} \%$ and $25 \mathrm{wt} \%$ ) was not enough to achieve fire inhibition in paraffin. For that reason, this percentage was increased up to $40 \mathrm{wt} \%$ in order to evaluate if higher loads could give rise to a better fire performance of paraffin. In the case of paraffin with $40 \mathrm{wt} \%$ of APP, ignitability is not improved, as can be noticed by the reduction in the time to ignition. However, the combination of PCMs and $40 \mathrm{wt} \%$ APP shows a clear ability to extinguish the flame, which is supported by the reduction in the average combustion time as well as the number of ignitions.

The self-extinguishing ability is much clear in some of the fatty acid flame retarded systems. Especially, magnesium hydroxide and hydromagnesite are remarkably good for reducing the combustion time and therefore also increase the number of ignitions. These two endothermic flame retardants exhibit their thermal decomposition at higher temperatures than aluminum hydroxide, which does not produce a remarkable effect on the fire performance. Probably in the dripping test the release of flammable gases is produced in a broad range of temperatures and therefore, flame retardants that act at higher temperatures or in a wider range of temperatures are favored. The char-forming flame retardants in the loads used ( $20 \%$ in weight) do not lead to a successful formation of protective char and, therefore, the fire performance of the PCMs is not improved.

In summary, the best performing formulation for each PCM-flame-retardant combination based on dripping test results that are highlighted in grey in Table 4 are: the addition of $50 \mathrm{wt} \%$ of hydromagnesite and magnesium hydroxide for fatty acid mixtures, and $40 \mathrm{wt} \%$ of APP slightly contributing to improve the fire reaction of paraffin. Aluminum hydroxide does not perform properly with the selected PCM.

Figure 4 illustrates ignition/extinction periods obtained for the dripping test for capric acid and myristic acid eutectic mixture, for example. The peaks and valleys show the time when the PCM ignites $\left(t_{i}\right)$ (up) and extinguishes $\left(t_{e}\right)$ (down). Furthermore, the period of time between each ignition and extinction is the combustion time $\left(t_{c}\right)$. Figure $4 \mathrm{~d}$ shows the case of the plain PCMs. It can be seen that once ignited, the PCM keeps on burning until the fuel is exhausted. The addition of APP as flame retardant does not introduce significant changes in the fire performance (Figure 4c), from which we can conclude that this flame retardant is not acting on the load and under the conditions applied. The elevated number of peaks in Figure $4 a$, b indicates that a high number of ignitions and extinctions occur during the test. This fact is related to the ability to extinguish the flame of the PCM formulations with magnesium hydroxide and hydromagnesite. In the case of magnesium hydroxide, a delay on the time to ignition is also observed.

\subsection{Thermal Characterization}

Thermophysical property results obtained by DSC are listed in Table 5. As expected, DSC results show how the PCM thermophysical properties are affected when fire retardants are added to the formulation.

Paraffin melting temperature and enthalpy were not affected by the addition of $40 \mathrm{wt} \%$ APP.

On the other hand, fatty acid mixtures showed a clear reduction of the melting enthalpy, by a factor of 3 when $50 \mathrm{wt} \%$ flame retardant is added, while the melting temperature remained almost equal to that of the single PCMs. 

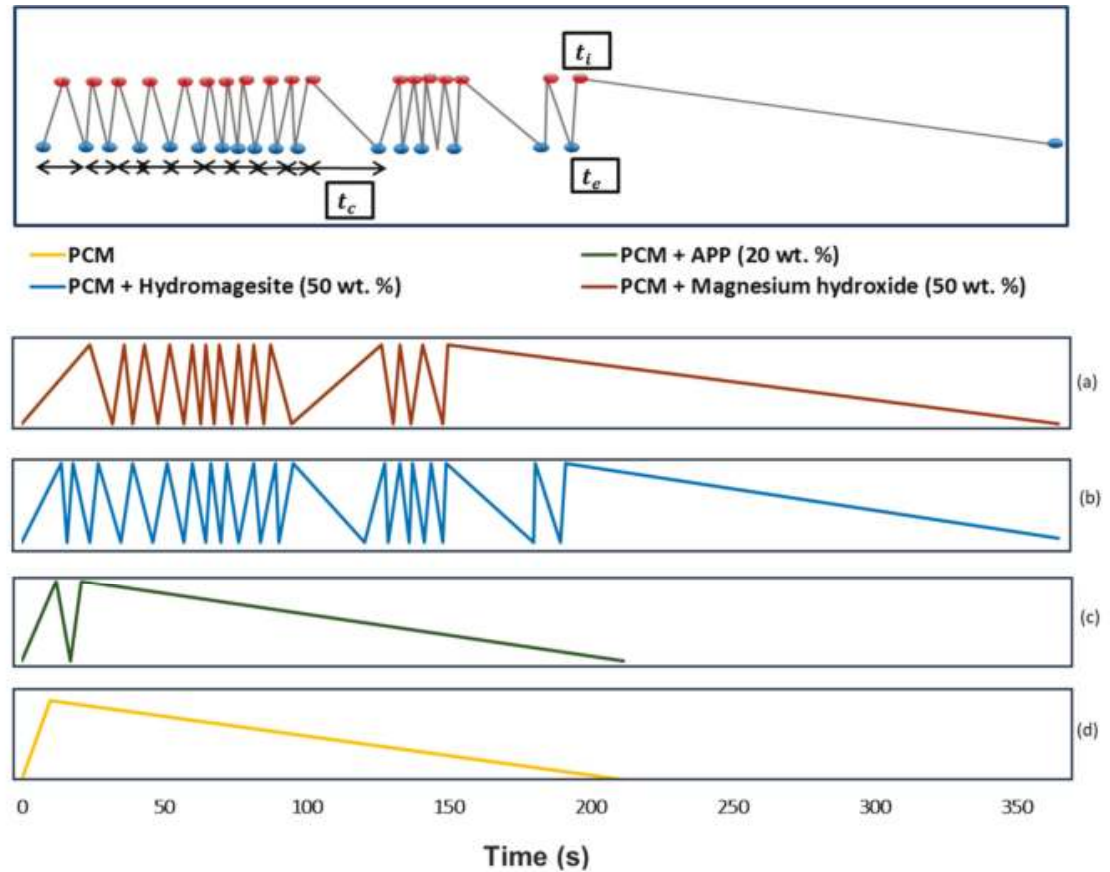

Figure 4. Legend of the parameters to comprehend the graph (above) and ignition-extinction periods for CA + MA mixture formulation with flame retardants (below). (a) Fire performance of capric and myristic eutectic with 50 wt \% magnesium hydroxide; (b) Fire performance of capric and myristic eutectic with $50 \mathrm{wt} \%$ hydromagnesite; (c) Fire performance of capric and myristic eutectic with $20 \mathrm{wt} \%$ APP; (d) Fire performance of capric and myristic eutectic mixture. $t_{i}$ : ignition time; $t_{e}$ : extinction time; $t_{c}$ : combustion time.

Table 5. DSC of PCM-fire retardant optimum combination results.

\begin{tabular}{cccc}
\hline & Compositions & Melting Enthalpy $(\mathbf{k J} / \mathbf{k g})$ & Peak Temperature $\left({ }^{\circ} \mathrm{C}\right)$ \\
\hline \multirow{3}{*}{ PCMs } & Paraffin RT-21 & $118 \pm 3$ & $22.3 \pm 0.2$ \\
& $73.5 \%$ Capric acid + 26.5\% Myristic acid & $143 \pm 3$ & $24.1 \pm 0.2$ \\
& $75.2 \%$ Capric acid + 24.8\% Palmitic acid & $141 \pm 3$ & $23.3 \pm 0.2$ \\
\hline & $60 \%$ Paraffin RT-21 + 40\% APP & $111 \pm 4$ & $22.6 \pm 0.2$ \\
PCM + Flame & $50 \%$ CA + MA + 50\% Hydromagnesite & $53 \pm 3$ & $22.0 \pm 0.2$ \\
Retardant & $50 \%$ CA + MA + 50\% Magnesium hydroxide & $55 \pm 3$ & $24.4 \pm 0.2$ \\
& $50 \%$ CA+PA + 50\% Hydromagnesite & $56 \pm 1$ & $19.0 \pm 0.6$ \\
& $50 \%$ CA+PA + 50\% Magnesium hydroxide & $55 \pm 2$ & $23.0 \pm 0.2$ \\
\hline
\end{tabular}

\subsection{Further Work}

In this paper, the thermal enthalpy of the different PCM systems has been determined. In order to obtain more information about the thermal performance of each PCM formulation, it would be interesting to evaluate their thermal inertia. In this case, the experiment developed must allow performing temperature/time curves of bulk PCMs with a control of the heat flow and measurements of the peak temperature under temperature oscillation. This kind of equipment is not commonly found [39] and therefore the authors have not been able to carry out these tests.

An alternative to direct measurement of thermal inertia is to determine an effective thermal inertia. A measurement procedure is described in the standard ASTM E 1321-Standard Test Method for 
determining material ignition and flame spread properties [40]. The dripping test used in this paper is not suitable for this kind of experiment, but some other small-scale radiant exposure tests that allow controlling and modifying the heat flux could be used to obtain effective thermal properties [41].

\section{Conclusions}

The effect of different types of flame retardants such as APP, MPP, hydromagnesite, magnesium hydroxide and aluminum hydroxide on PCMs was investigated. Fire performance of paraffin was not significantly improved by any of the flame retardants used. Nevertheless, $40 \mathrm{wt} \%$ APP slightly contributed to enhance the self-extinguishing ability of paraffin PCMs.

On the other hand, the considered fatty acid mixtures showed a remarkable improvement in the fire reaction when loadings of $50 \mathrm{wt} \%$ of hydromagnesite or magnesium hydroxide were added as flame retardants. Nevertheless, aluminum hydroxide, also an endothermic flame retardant, did not show any positive effect on the fatty acids. This fact could be explained due to the lower decomposition temperature of aluminum hydroxide compared with the other two endothermic flame retardants. The char-forming flame retardants did not improve the fire performance of the fatty acids.

Deeply analyzing the fire performance and thermal stability of organic PCM-flame-retardant mixture in bulk, it can be concluded that for fatty acids the enthalpy of fusion decreased following the mixtures law by the addition of flame retardants, but for paraffin melting enthalpy did not decrease. On the other hand, the phase change temperature remains equal.

In summary, magnesium hydroxide and hydromagnesite strongly affect the melting enthalpy and tend to decrease the melting temperature, while APP has a much lower influence on melting enthalpy and temperature when added to the bulk PCMs. The addition of flame retardants will decrease the flammability of bulk-organic PCMs, but more research is needed to perform a fire-retardant screening in order identify new fire retardants that would work with less quantity to keep the thermophysical properties of PCMs as high as possible to ensure their applicability.

Acknowledgments: The research leading to these results has received funding from the European Commission Seventh Framework Programme (FP/2007-2013) under grant agreement number PIRSES-GA-2013-610692 (INNOSTORAGE) and from the European Union's Horizon 2020 research and innovation program under grant agreement No. 657466 (INPATH-TES). The authors would like to thank the Catalan Government for the quality accreditation given to their research groups GREA (2014 SGR 123), DIOPMA (2014 SGR 1543) and GICITED (2014 SGR 1298), and also the MINECO for the project BIA2014-52688-R, ENE2015-64117-C5-1-R, ENE2015-64117-C5-2-R, and ENE2015-64117-C5-3-R. Alvaro de Gracia and Camila Barreneche would like to thank Ministerio de Economia y Competitividad de España for Grant Juan de la Cierva, FJCI-2014-19940 and FJCI-2014-22886, respectively.

Author Contributions: Camila Barreneche. and Anabel Palacios conceived and designed the experiments; Anabel Palacios performed the experiments; Camila Barreneche and Anabel Palacios analyzed the data; Alvaro de Gracia, Luisa F. Cabeza, A. Inés Fernández contributed reagents, materials and analysis tools; Anabel Palacios, Camila Barreneche and Laia Haurie wrote the paper. Alvaro de Gracia, Luisa F. Cabeza, and A. Inés Fernández review the main results of the experiments together with Anabel Palacios, Camila Barreneche and Laia Haurie.

Conflicts of Interest: The authors declare no conflict of interest.

\section{References}

1. International Energy Agency (IEA). Energy Technology Perspectives 2012 Pathways to a Clean Energy System. Energy Technol. Perspect. 2012, 1-12. [CrossRef]

2. Kundakçı, K.B.; Yılmaz, Z. The performance comparison of fan-assisted Trombe wall system. ITU J. Fac. Arch. 2013, 10, 198-211.

3. Özbalta, T.G.; Kartal, S. Heat gain through Trombe wall using solar energy in a cold region of Turkey. Sci. Res. Essays 2010, 5, 2768-2778.

4. Cabeza, L.F.; Castell, A.; Barreneche, C.; de Gracia, A.; Fernández, A.I. Materials used as PCM in thermal energy storage in buildings: A review. Renew. Sustain. Energy Rev. 2011, 15, 1675-1695. [CrossRef]

5. De Gracia, A.; Navarro, L.; Castell, A.; Ruiz-Pardo, Á.; Alvárez, S.; Cabeza, L.F. Experimental study of a ventilated facade with PCM during winter period. Energy Build. 2013, 58, 324-332. [CrossRef] 
6. De Gracia, A.; Oró, E.; Farid, M.M.; Cabeza, L.F. Thermal analysis of including phase change material in a domestic hot water cylinder. Appl. Therm. Eng. 2011, 31, 3938-3945. [CrossRef]

7. Cabeza, L.F.; Castellón, C.; Nogués, M.; Medrano, M.; Leppers, R.; Zubillaga, O. Use of microencapsulated PCM in concrete walls for energy savings. Energy Build. 2007, 39, 113-119. [CrossRef]

8. Abhat, A. Low temperature latent heat thermal energy storage: Heat storage materials. Sol. Energy 1983, 30, 313-332. [CrossRef]

9. Farid, M.M.; Khudhair, A.M.; Razack, S.A.K.; Al-Hallaj, S. A review on phase change energy storage: Materials and applications. Energy Convers. Manag. 2004, 45, 1597-1615. [CrossRef]

10. Sharma, A.; Tyagi, V.V.; Chen, C.R.; Buddhi, D. Review on thermal energy storage with phase change materials and applications. Renew. Sustain. Energy Rev. 2009, 13, 318-345. [CrossRef]

11. Khudhair, A.M.; Farid, M.M. A review on energy conservation in building applications with thermal storage by latent heat using phase change materials. Energy Convers. Manag. 2004, 45, 263-275. [CrossRef]

12. Cai, Y.; Wei, Q.; Huang, F.; Lin, S.; Chen, F.; Gao, W. Thermal stability, latent heat and flame retardant properties of the thermal energy storage phase change materials based on paraffin/high density polyethylene composites. Renew. Energy 2009, 34, 2117-2123. [CrossRef]

13. Fang, G.; Li, H.; Chen, Z.; Liu, X. Preparation and properties of palmitic acid $/ \mathrm{SiO}_{2}$ composites with flame retardant as thermal energy storage materials. Sol. Energy Mater. Sol. Cells 2011, 95, 1875-1881. [CrossRef]

14. Sittisart, P.; Farid, M.M. Fire retardants for phase change materials. Appl. Energy 2011, 88, 3140-3145. [CrossRef]

15. Zhang, P.; Hu, Y.; Song, L.; Ni, J.; Xing, W.; Wang, J. Effect of expanded graphite on properties of high-density polyethylene/paraffin composite with intumescent flame retardant as a shape-stabilized phase change material. Sol. Energy Mater. Sol. Cells 2010, 94, 360-365. [CrossRef]

16. Cai, Y.; Wei, Q.; Huang, F.; Gao, W. Preparation and properties studies of halogen-free flame retardant form-stable phase change materials based on paraffin/high density polyethylene composites. Appl. Energy 2008, 85, 765-775. [CrossRef]

17. Cai, Y.; Hu, Y.; Song, L.; Tang, Y.; Yang, R.; Zhang, Y.; Chen, Z.; Fan, W. Flammability and thermal properties of high density polyethylene/paraffin hybrid as a form-stable phase change material. J. Appl. Polym. Sci. 2006, 99, 1320-1327. [CrossRef]

18. Kenisarin, M.M. Thermophysical properties of some organic phase change materials for latent heat storage. A review. Sol. Energy 2014, 107, 553-575. [CrossRef]

19. Morikawa, J.; Hashimoto, T. Simultaneous measurement of heat capacity and thermal diffusivity in solid-solid and solid-liquid phase transitions of n-alkane. Thermochim. Acta 2000, 353, 291-296. [CrossRef]

20. Inaba, H.; Tu, P. Evaluation of thermophysical characteristics on shape-stabilized paraffin as a solid-liquid phase change material. Heat Mass Transf. 1997, 32, 307-312. [CrossRef]

21. Ukrainczyk, N.; Kurajica, S.; Šipušiæ, J. Thermophysical Comparison of Five Commercial Paraffin Waxes as Latent Heat Storage Materials. Chem. Biochem. Eng. Q. 2010, 24, 129-137.

22. Código Técnico de la Edificación (Spain). Available online: https:/ /www.codigotecnico.org/images/stories/ pdf/ahorroEnergia/DcmHE.pdf (accessed on 12 November 2017).

23. RT-21 Paraffin Datasheet. Available online: https://www.rubitherm.eu/media/products/datasheets/ Techdata_-RT21_EN_05112015.PDF (accessed on 12 November 2017).

24. Laoutid, F.; Bonnaud, L.; Alexandre, M.; Lopez-Cuesta, J.M.; Dubois, P. New prospects in flame retardant polymer materials: From fundamentals to nanocomposites. Mater. Sci. Eng. R Rep. 2009, 63, 100-125. [CrossRef]

25. Nguyen, N.P.M.Q. A review on fire protection for phase change materials in building applications. In From Materials to Structures: Advancement through Innovation; Attard, M.M., Samali, B., Song, C., Eds.; CRC Press: Boca Raton, FL, USA, 2012; pp. 561-566.

26. Haurie, L.; Fernández, A.I.; Velasco, J.I.; Chimenos, J.M.; Lopez Cuesta, J.-M.; Espiell, F. Synthetic hydromagnesite as flame retardant. Evaluation of the flame behaviour in a polyethylene matrix. Polym. Degrad. Stab. 2006, 91, 989-994. [CrossRef]

27. Morgan, A.B.; Gi, J.W. An overview of flame retardancy of polymeric materials: application, technology, and future directions. Fire Mater. 2013, 37, 259-279. [CrossRef]

28. Levchik, S.V.; Weil, E.D. Flame retardancy of thermoplastic polyesters-A review of the recent literature. Polym. Int. 2005, 54, 11-35. [CrossRef] 
29. Hapuarachchi, T.D.; Peijs, T. Aluminium trihydroxide in combination with ammonium polyphosphate as flame retardants for unsaturated polyester resin. Express Polym. Lett. 2009, 3, 743-751. [CrossRef]

30. Zhang, S.; Horrocks, A.R. A review of flame retardant polypropylene fibres. Prog. Polym. Sci. 2003, 28, 1517-1538. [CrossRef]

31. Fan, Z.; Tsakiropoulos, P.; Miodownik, A.P. A generalized law of mixtures. J. Mater. Sci. 1994, 29, 141-150. [CrossRef]

32. Lyon, R.E.; Walters, R.N. Pyrolysis combustion flow calorimetry. J. Anal. Appl. Pyrolysis 2004, 71, 27-46. [CrossRef]

33. ASTM D7309: Standard Test Method for Determining Flammability Characteristics of Plastics and Other Solid Materials Using Microscale Combustion Calorimetry; ASTM International: West Conshohocken, PA, USA, 2013.

34. UNE-23727:1990: Ensayos de Reacción al Fuego de los Materiales de Construcción. Clasificación de los Materiales Utilizados en la Construcción; Asociacion Espanola de Normalizacion: Madrid, Spain, 1990.

35. Lazaro, A.; Peñalosa, C.; Solé, A.; Diarce, G.; Haussmann, T.; Fois, M.; Zalba, B.; Gshwander, S.; Cabeza, L.F. Intercomparative tests on phase change materials characterisation with differential scanning calorimeter. Appl. Energy 2013, 109, 415-420. [CrossRef]

36. Barreneche, C.; Solé, A.; Miró, L.; Martorell, I.; Fernández, A.I.; Cabeza, L.F. Study on differential scanning calorimetry analysis with two operation modes and organic and inorganic phase change material (PCM). Thermochim. Acta 2013, 553, 23-26. [CrossRef]

37. Hirschler, M.M. Flame retardants and heat release: review of data on individual polymers. Fire Mater. 2015, 39, 232-258. [CrossRef]

38. Babrauskas, V.; Peacock, R.D. Heat Release Rate: The Single Most Important Variable in Fire Hazard. Fire Saf. J. 1992, 18, 255-272. [CrossRef]

39. De Gracia, A.; Barreneche, C.; Farid, M.M.; Cabeza, L.F. New equipment for testing steady and transient thermal performance of multilayered building envelopes with PCM. Energy Build. 2011, 43, 3704-3709. [CrossRef]

40. ASTM E 1321: Standard Test Method for Determining Material Ignition and Flame Spread Properties; ASTM International: West Conshohocken, PA, USA, 2009.

41. Mowrer, F.W. Analysis of Effective Thermal Properties of Thermall Thick Materials; Grant/Contract Reports (NISTGCR) 03-855; The National Institute of Standards and Technology (NIST): Gaithersburg, MD, USA, 2003 ; p. 25.

(C) 2018 by the authors. Licensee MDPI, Basel, Switzerland. This article is an open access article distributed under the terms and conditions of the Creative Commons Attribution (CC BY) license (http:/ / creativecommons.org/licenses/by/4.0/). 
Article

\title{
Mechanical and Thermal Performance of Macro-Encapsulated Phase Change Materials for Pavement Application
}

\author{
Xiangming Zhou ${ }^{1}$, Gediminas Kastiukas ${ }^{1, *}$, Claudio Lantieri ${ }^{2}$, Piergiorgio Tataranni ${ }^{2}$, \\ Rosolino Vaiana ${ }^{3}$ and Cesare Sangiorgi ${ }^{2}$ \\ 1 Department of Civil and Environmental Engineering, Brunel University London, London UB8 3PH, UK; \\ xiangming.zhou@brunel.ac.uk \\ 2 DICAM Department, University of Bologna, 40136 Bologna, Italy; claudio.lantieri2@unibo.it (C.L.); \\ piergiorg.tataranni2@unibo.it (P.T.); cesare.sangiorgi4@unibo.it (C.S.) \\ 3 DINCI Department of Civil Engineering, University of Calabria, Arcavàcata di Rende, 87036 Cosenza, Italy; \\ rosalino.vaiana@unical.it \\ * Correspondence: Gediminas.Kastiukas@brunel.ac.uk
}

Received: 30 June 2018; Accepted: 2 August 2018; Published: 10 August 2018

\begin{abstract}
Macro-encapsulated phase change material (PCM) lightweight aggregates (ME-LWA) were produced and evaluated for their mechanical and thermal properties in road engineering applications. The ME-LWAs were first characterised in terms of their physical and geometrical properties. Then, the ME-LWAs were investigated in detail by applying the European Standards of testing for the Bulk Crushing Test and the Polished Stone Value (PSV) coefficient as well as Micro-Deval and laboratory profilometry. In addition, the thermal performance for possible construction of smart pavements with the inclusion of ME-LWAs for anti-ice purposes was determined. The crushing resistance of the ME-LWAs was improved, while their resistance to polishing was reduced. Thermal analysis of the encapsulated PCM determined it to possess excellent thermal stability and a heat storage capacity of $30.43 \mathrm{~J} / \mathrm{g}$. Based on the research findings, the inclusion of ME-LWAs in surface pavement layers could be considered a viable solution for the control of surface temperatures in cold climates. Road safety and maintenance could benefit in terms of reduced ice periods and reduced treatments with salts and other anti-ice solutions.
\end{abstract}

Keywords: lightweight aggregates; phase change materials; macro-encapsulation; thermal performance; crushing test; road application

\section{Introduction}

Road surfaces, pavements and buildings all contribute to keeping urbanised environments three to four degrees warmer than surrounding non-urbanised areas, an issue that leads to the creation of so-called urban heat islands (UHIs). Dense dark surfaces such as bitumen on roads and building materials are the primary contributors since they accumulate and store heat during the day and then release it at night. A common strategy to reduce the UHI effect is to provide more shade with increased tree canopies. However, such passive methods have limitations due to the low availability of space in urban areas. One of the most effective active methods to reduce the heat accumulation is to incorporate a phase change material (PCM) as an active additive. PCMs have high latent heat storage densities and can, therefore, absorb thermal energy when transforming from solid to liquid or release it when turning back to a solid [1], thus maintaining constant temperature performance. PCMs have good insulation heat storage performance and have been used widely in the construction, renovation and energy-saving industries, especially for buildings [2,3]. However, their application in the road 
transport sector is still at the exploratory research stage and may introduce benefits from a road safety point of view. Here moisture control, in particular ice formation on the pavement surface, is addressed.

By means of an experimental analysis, Chen et al. [4] proved the feasibility of using PCMs in asphalt mixes and put forward the basic technical selection criteria used in asphalt mixes with PCMs. Ma et al. [5] conducted field simulation tests of asphalt mixtures with PCMs, concluding that they helped to decrease the highest temperature during the heating process and increase the lowest temperature during the cooling process, which reduced the temperature's adverse impact on asphalt mixtures. The test results indicated that an asphalt mixture with PCM actively influenced the pavement temperature with a damping effect, and also enhanced the temperature resistance capacity for asphalt layers, as well as improved the asphalt mixture adaptability to the changing environment.

The incorporation of PCMs into construction materials depends on the material properties and can be primarily achieved in the following three ways: (1) direct incorporation at the time of mixing, which has been successfully implemented in frame walls [6]; (2) immersion of the component in liquid PCM: a technique proven successful in concrete [7] and (3) micro-encapsulation [8] The method of encapsulation is the most advanced and popular because it allows for better dispersion, reduces the external volume changes and reduces the possibility of PCMs leaching into the surrounding material by eliminating the direct interaction between PCM and host material [9]. Micro-encapsulation can be achieved through physical processes such as spray cooling [10], spray drying [11], and the fluidized bed processes [12]. Chemical processes are also used and include in situ polymerization (interfacial polycondensation, suspension polymerization, and emulsion polymerization), complex coacervation, sol-gel method, and solvent extraction/evaporation method [13]. Physical methods of production are limited by their granulated sizes thus chemical methods are frequently employed to produce much smaller encapsulated PCM particles. Furthermore, Hawlader et al. [14] reported a substantial drop in heat storage capacity with the physical methods of production. Although PCM microcapsules have been produced on an industrial scale, the production process is very expensive, thus limiting their use to low volume incorporation applications such as textiles [15].

Alternatively, PCMs can also be encapsulated on a macro scale via a porous host material. In the study by Kastiukas et al. [16], liquid paraffin was forced into porous, lightweight expanded clay aggregates (LWA) using a vacuum impregnation system, similar to that used by Memon et al. [17]. This method proved to be simple to set up and allowed the impregnation to be precisely controlled.

Thus, the purpose of this study was to implement the authors' existing technological advancements of ME-LWAs for application in road pavements. ME-LWAs are evaluated for their mechanical and thermal suitability for pavements. Nonetheless, it is envisaged that the knowledge resulting from this study could be transferred to applications in concrete, mortar, grout, bituminous mixtures and surface treatments for unbound and hydraulically bound applications in construction works.

\section{Materials and Methods}

\subsection{Material Testing}

To produce the ME-LWAs, LWAs (Argex S.A, Bustos, Portugal) conforming to EN 13055-1 were used. The diameter of ME-LWA was determined using electronic callipers and was recorded as the average of three measurements per specimen, for a total of 10 specimens. The shell thickness was determined under the inspection of a light microscope and was taken as the average of 10 samples.

The average dry particle density of the ME-LWAs was determined in accordance with EN 1097-3 by sand pycnometry. 100 ME-LWAs were put into a flask and covered with a known amount of fine sand to measure the volume. The particle density was calculated by dividing the mass of the material by the measured volume.

The LWA water absorption was determined in accordance with EN 1097-6. 
The thermal conductivity of the raw LWA was measured in accordance to (EN 12664), using a Netzsch heat flow meter (HFM 436 Lambda) (Netzsch Gerätebau GmbH, Selb, Germany) heat flow meter with a hot plate and cold plate set at 35 and $15^{\circ} \mathrm{C}$, respectively.

Differential scanning calorimetry (DSC) analysis was adopted to evaluate phase changing behaviour, i.e., phase change temperature and thermal energy storage. The ME-LWA was crushed to a coarse powder, which was used to conduct the DSC test on a Q2000 (TA Instruments, New Castle, DE, USA). The DSC sample weight was approximately $10 \mathrm{mg}$. Several samples were tested, implementing two measuring cycles in a temperature range of $-20^{\circ} \mathrm{C}$ to $+80^{\circ} \mathrm{C}$, at a heating $/$ cooling rate of $5{ }^{\circ} \mathrm{C} / \mathrm{min}$. Calculations for the stored/released thermal energy in unit weight of the material, also known as specific phase change enthalpy $\left(\Delta \mathrm{H}_{\mathrm{m}} / \Delta \mathrm{H}_{\mathrm{c}}\right)$ were calculated by the DSC software TRIOS v4.3.1.

Other mechanical and physical tests encompassed the bulk crushing resistance, the Polished Stone Value, the Micro-Deval resistance to fragmentation, the laser profilometry and the thermal behaviour under relevant temperature external conditions and chamber controlled temperature drop. The latter was reproduced using existing data from a local climate station installed at the Bologna International Airport.

\subsection{PCM Impregnation and Coating}

The LWAs were sieved to obtain a maximum particle size of $8 \mathrm{~mm}$. This limit was chosen in consideration of a potential increase of $1 \mathrm{~mm}$ in particle diameter after coating, determined from previous research [16]. The LWAs were also blow-dried with compressed air to remove surface dust before impregnation. The absorption capacity of the LWA was determined by using the mass change of the PCM-impregnated LWA and the PCM density of $0.77 \mathrm{~g} / \mathrm{mL}$ and an average LWA intrusion volume of $0.82 \mathrm{~mL} / \mathrm{g}$ (determined using Mercury Intrusion Porosimetry (MIP)). For comparison, normal immersion of the LWA into PCM was also evaluated. The absorption capacity of normally immersed LWAs was only approximately $10 \%$ of that reached using vacuum impregnation.

Technical grade paraffin (Rubitherm GmbH, Berlin, Germany) was chosen as the PCM for the impregnation of LWAs. The thermophysical properties of the PCM are reported in Table 1.

Table 1. Thermophysical properties of technical grade paraffin PCM (obtained from manufacturer)

\begin{tabular}{cc}
\hline Properties & Values \\
\hline Phase change range temperature $\left[{ }^{\circ} \mathrm{C}\right]$ & $1-3$ \\
Thermal energy storage capacity $[\mathrm{kJ} / \mathrm{kg}]$ & $200( \pm 7.5 \%)$ \\
Specific heat capacity $[\mathrm{kJ} / \mathrm{kg} \cdot \mathrm{K}]$ & 2 \\
Liquid density at $15^{\circ} \mathrm{C}\left[\mathrm{kg} / \mathrm{m}^{3}\right]$ & 0.77 \\
Solid density at $-15^{\circ} \mathrm{C}\left[\mathrm{kg} / \mathrm{m}^{3}\right]$ & 0.88 \\
Thermal conductivity $[\mathrm{W} / \mathrm{m} \cdot \mathrm{K}]$ & 0.2 \\
Maximum operation temperature $\left[{ }^{\circ} \mathrm{C}\right]$ & 60 \\
\hline
\end{tabular}

To prevent the leakage of PCM from the pores of the LWA, the impregnated aggregates were coated using a commercial-grade polyester resin Palatal P4-01 (Aliancys AG, Schaffhausen, Switzerland), with a novel method developed in the previous research of [16]. Polyester resin was chosen due to its high surface hardness, stiffness, compressive and tensile strength. Some of the resin properties are reported in Table 2. The mixing ratio of adhesive:harder:catalyser, which would provide the most manageable working time, in this case, $15 \mathrm{~min}$, was determined to be 1:0.02:0.03 by mass. Due to the high viscosity of the resin, granite powder was used as the final step of the coating procedure to prevent agglomeration of the ME-LWAs. The granite powder was also intended to increase the roughness of the surface, helping the ME-LWA interlock with a potential matrix during hardening and provide better aggregate-paste bond strength. 
Table 2. Physical properties of Palatal P4-01 polyester resin.

\begin{tabular}{cc}
\hline Properties & Values \\
\hline Viscosity at $23^{\circ} \mathrm{C}[\mathrm{MPa} \cdot \mathrm{s}]$ & $540-610$ \\
Density at $20^{\circ} \mathrm{C}\left[\mathrm{kg} / \mathrm{m}^{3}\right]$ & 1100 \\
Cure time from $25^{\circ} \mathrm{C}$ to $35^{\circ} \mathrm{C}[\mathrm{min}]$ & $16-21$ \\
Maximum operation temperature $\left[{ }^{\circ} \mathrm{C}\right]$ & $135-155$ \\
\hline
\end{tabular}

PCM was introduced into the pores of the LWA using an in-house vacuum impregnation system (Figure 1). Weighed samples of LWA were placed into vacuum chambers and sealed using vacuum gel. Air entrapped within the pores of the LWA was removed under a vacuum pressure of $-860 \mathrm{mbar}$ for $30 \mathrm{~min}$. Liquid paraffin was then allowed to enter the chambers and completely submerge the LWAs. The air was then allowed to enter the chambers to help force the paraffin into the pores. After this, the sample was left to rest for a further $30 \mathrm{~min}$. Upon completion of the impregnation process, the PCM-LWAs were surface-dried using absorbent towels to remove excess paraffin and immediately placed into an environmental chamber maintained at a temperature below the phase change temperature to keep the PCM in a solid state.

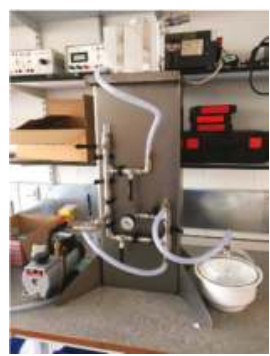

(a)

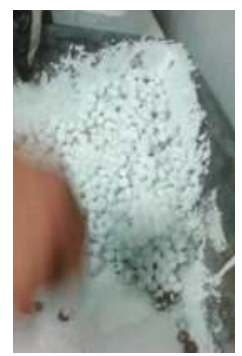

(b)

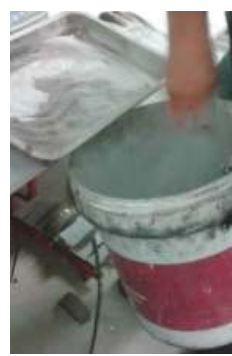

(c)

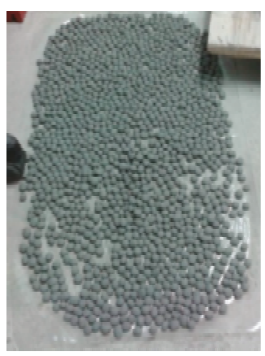

(d)

Figure 1. PCM impregnation and coating showing (a) the vacuum impregnation system (b) resin coating the PCM-LWA (c) introduction of granite powder, and (d) final ME-LWAs.

All the tests were conducted with ME-LWA samples that had been oven-dried in accordance with EN 1097-5 and subsequently conditioned at $23 \pm 5^{\circ} \mathrm{C}$ to allow the test specimen to cool to room temperature. Sampling of the ME-LWAs was conducted in accordance with EN 932-1.

\section{Results and Discussion}

\subsection{Geometric and Physical Characteristics of ME-LWAs}

Figure 2 shows a representative ME-LWA produced using the resin-granite powder coating described in the previous section. The ME-LWAs exhibit an almost spherical shape and homogeneous outer shell, which is the result of the constant rotation of the samples in the granite powder during the coating process. The minimum and maximum diameter were determined to be $8.00 \mathrm{~mm}$ and $11.50 \mathrm{~mm}$, respectively. The shell thickness was identified as $0.80 \mathrm{~mm}$ on average. Table 3 lists the main characteristics of the ME-LWA, while Figure 3 shows their internal pore size distribution before impregnation. 


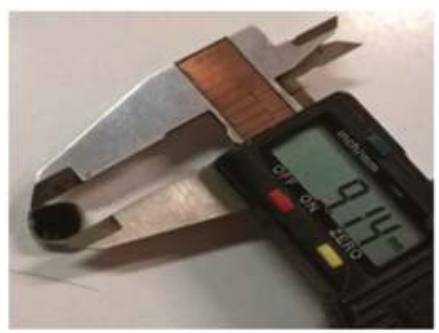

(a)

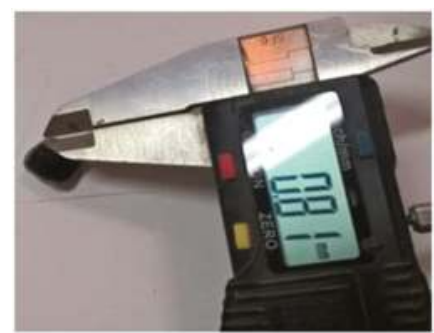

(b)

Figure 2. Measurement of (a) ME-LWA diameter and (b) ME-LWA coating thickness.

Table 3. Properties of the impregnated and coated LWA.

\begin{tabular}{cc}
\hline Properties & Values \\
\hline Loose bulk density $\left[\mathrm{kg} / \mathrm{m}^{3}\right]$ & $274( \pm 15 \%)$ \\
Thermal conductivity $\left[\mathrm{W} / \mathrm{m} \cdot{ }^{\circ} \mathrm{C}\right]$ & 0.10 \\
Water absorption capacity $[\mathrm{wt} \%]$ & 0.055 \\
PCM absorption capacity by vacuum impregnation $(1 \mathrm{~h})[\mathrm{wt} \%]$ & 95 \\
Average diameter $[\mathrm{mm}]$ & 8.00 \\
Average shell thickness $[\mathrm{mm}]$ & 0.80 \\
\hline
\end{tabular}

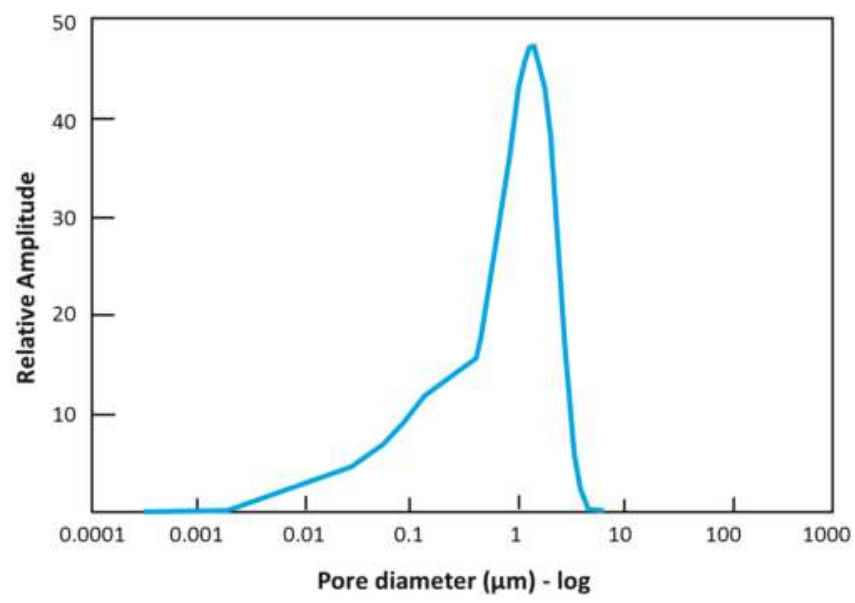

Figure 3. Pore size distribution of the LWA.

\subsection{Mechanical Strength}

The mechanical strength was evaluated by determining the bulk crushing resistance in accordance with EN 13055 [18]. The bulk crushing resistance can be carried out by means of two different procedures, depending on the dimension of the LWA and the weight of the sample. In the case of the manufactured ME-LWAs, Procedure 1 from the standard was chosen since the bulk density was above $150 \mathrm{~kg} / \mathrm{m}^{3}$. A sample of the ME-LWAs was placed in a steel cylinder of one-litre volume (Figure 4); a piston was then pressed into the cylinder to a depth of $20 \mathrm{~mm}$ in a $100 \mathrm{~s}$ time interval; the force was recorded in Newtons. This procedure was repeated on three test specimens. 


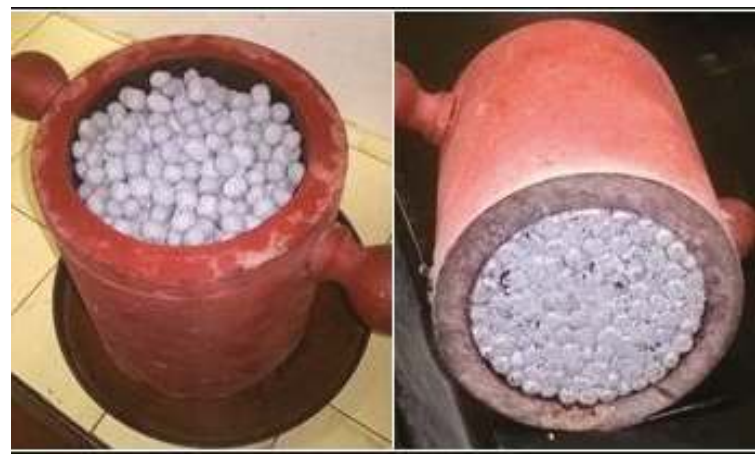

(a)

(b)

Figure 4. Bulk crushing resistance test of (a) before and (b) after testing.

The crushing resistance, $C_{b}$, of each sample was evaluated as follows:

$$
\mathrm{C}_{\mathrm{b}}=(\mathrm{L}+\mathrm{F}) / \mathrm{A}\left[\mathrm{N} / \mathrm{mm}^{2}\right],
$$

where $\mathrm{L}$ is the force exerted by the piston $[\mathrm{N}], \mathrm{F}$ is the compressive force $[\mathrm{N}]$, and $\mathrm{A}$ is the piston area $\left[\mathrm{mm}^{2}\right]$.

Table 4 presents the calculated average crushing resistance of the ME-LWAs and raw LWAs. The ME-LWAs were determined to have an average crushing resistance of $9.24 \mathrm{MPa}$, which, compared to raw LWAs, was more than 7 times higher. This demonstrates that the polyester resin coating considerably increased the resistance of the final ME-LWA product. The Lightweight Expanded Clay Aggregates handbook shows several paving solutions incorporating different types of expanded clay aggregates. For porous asphalt pavements, for instance, a $C_{b}$ value of $4.50 \mathrm{MPa}$ is required, which is approximately half that recorded for the ME-LWAs.

Table 4. ME-LWA crushing resistance.

\begin{tabular}{ccccc}
\hline Parameter & Sample 1 & Sample 2 & Average & Raw LWA \\
\hline Piston weight $[\mathrm{kN}]$ & 20.48 & 20.48 & 20.48 & \\
Piston area $\left[\mathrm{mm}^{2}\right]$ & $11,309.73$ & $11,309.73$ & $11,309.73$ & \\
Maximum load $[\mathrm{daN}]$ & 10,875 & 10,010 & 10,442 & \\
$\mathrm{C}_{\mathrm{b}}\left[\mathrm{N} / \mathrm{mm}^{2}\right]$ & 9.62 & 8.85 & 9.24 & 1.30 \\
\hline
\end{tabular}

\subsection{ME-LWA Thermal Stability}

To ensure the reliable and consistent performance of the ME-LWA, the PCM encapsulated within the pores of the host material should be thermally reliable over many melting and freezing cycles and show little or no change in thermal properties after a long period of service. Therefore, the ME-LWA were subjected to melting/solidifying cycles in a temperature- and humidity-controlled environmental chamber to detect if there was any change in the phase change behaviour of the PCM and further verify the encapsulation efficiency. Figure 5 a shows the typical two-day, time vs. temperature cycle, which lasted 5 weeks. The thermal properties, i.e., phase change temperature and latent heat of the PCM after repeated thermal cycling, were investigated by DSC. The DSC curve of the PCM before and after thermal cycling is shown in Figure $5 b$. When comparing the melting temperature of the PCM in the ME-LWA before and after thermal cycling, the melting temperature changes only by $0.7^{\circ} \mathrm{C}$ while the latent heat storage capacity at melting changes by $12.56 \mathrm{~kJ} / \mathrm{kg}$. The change in mass of the $200 \mathrm{~g}$ sample of ME-LWA was also only $0.9 \mathrm{~g}$. The changes observed in the thermal characteristics of the 
PCM contained in the LWA are very small; it can, therefore, be concluded that the prepared ME-LWA is thermally reliable.

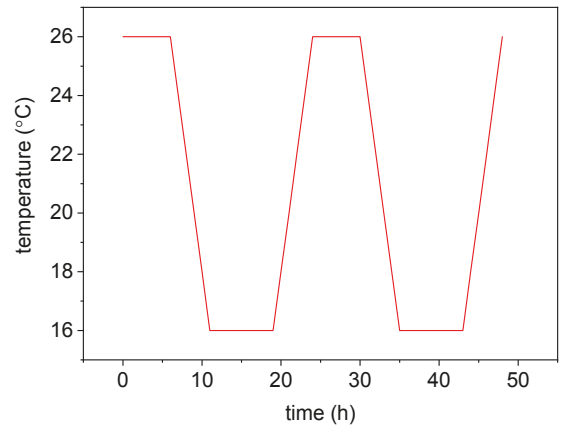

(a)

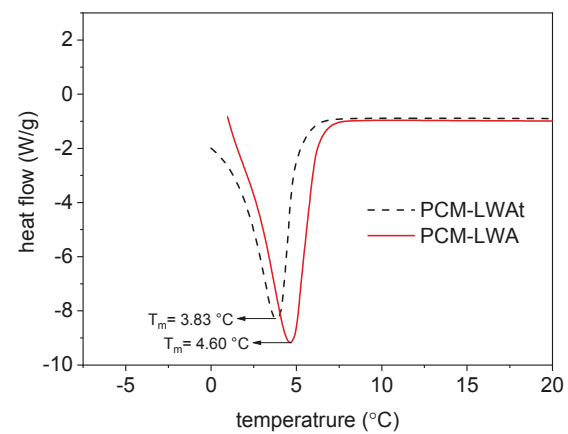

(b)

Figure 5. (a) Typical $48 \mathrm{~h}$ heating and cooling cycle and (b) DSC curves of ME-LWA before and after thermal cycling.

\subsection{Thermal Effectiveness of ME-LWA}

The differential scanning calorimetry (DSC) test was carried out with the aim of investigating the phase changing behaviour, particularly the phase change temperature and the thermal energy storage and release of ME-LWAs. The DSC curve of the ME-LWA in Figure 6 is constant between $+20{ }^{\circ} \mathrm{C}$ and $+80^{\circ} \mathrm{C}$, indicating that no significant variation of heat flow occurs and that the material remains stable without storing or releasing heat. However, in the range between $0{ }^{\circ} \mathrm{C}$ and $4{ }^{\circ} \mathrm{C}$, the phase change rises, as expected, during both the melting and solidification cycles. The maximum heat flow recorded during the melting cycle occurred at $3.41^{\circ} \mathrm{C}$. Meanwhile, during the solidification cycle, the maximum heat flow occurred at $0.99^{\circ} \mathrm{C}$. The specific enthalpies of the phase changes were recorded as $34.86 \mathrm{~J} / \mathrm{g}$ and $31.20 \mathrm{~J} / \mathrm{g}$ for the melting solidification cycles, respectively.

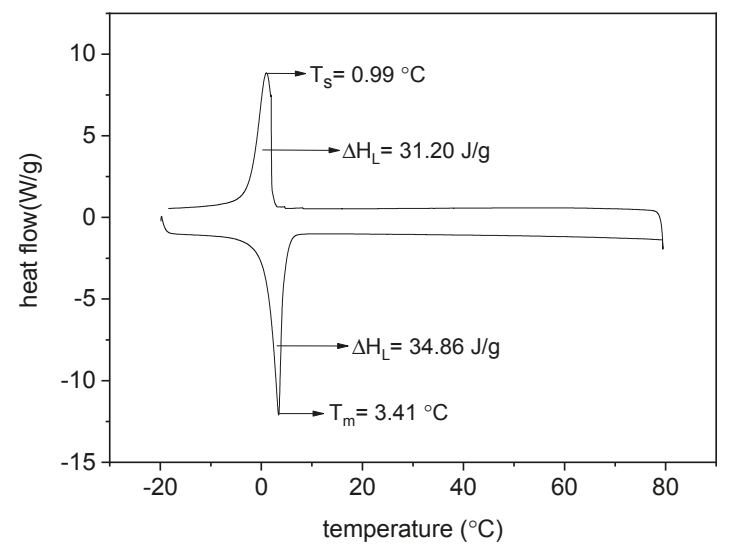

Figure 6. Thermal analysis test of the ME-LWA.

Figure 7 shows the results of DSC tests of the raw and PCM-impregnated LWA from two different samples of the coating layer, from which the melting temperature $\left(\mathrm{T}_{\mathrm{m}}\right)$ and solidification temperature 
$\left(T_{s}\right)$ were calculated. $T_{m}$ and $T_{s}$ represent the melting and solidification values, respectively, at the peak points of the DSC curve, i.e., the values at which the highest heat flow is reached. The resin-granite powder coating layer proved to be completely insensitive to the temperature variation. The raw LWA, being composed of clay previously treated at $1200^{\circ} \mathrm{C}$, also showed the same neutral thermal behaviour. A slightly different result was obtained from the sample composed of the internal coating layer in contact with the paraffin: very narrow peaks appeared during both the heating and cooling process, showing a $\mathrm{T}_{\mathrm{P}}=2.22^{\circ} \mathrm{C}$ and $\Delta \mathrm{H}_{\mathrm{m}}=0.9225 \mathrm{~J} / \mathrm{g}$ during the melting phase and $\mathrm{T}_{\mathrm{P}}=1.57^{\circ} \mathrm{C}$ and $\Delta \mathrm{H}_{\mathrm{C}}=1.045 \mathrm{~J} / \mathrm{g}$ during the solidification phase. These outcomes attest to the remarkable thermal capacity of PCM: just a very small amount of this material induces an enthalpy alteration in the system. Finally, very broad and sharp peaks were displayed by the PCM-impregnated LWA: concerning the solidification phase change, $\mathrm{T}_{\mathrm{S}}=2.04{ }^{\circ} \mathrm{C}, \mathrm{T}_{\mathrm{P}}=0.28^{\circ} \mathrm{C}$ and $\Delta \mathrm{H}_{\mathrm{C}}=62.44 \mathrm{~J} / \mathrm{g}$. Nonetheless, the high latent heat obtained is expected to decrease for the coated specimens.

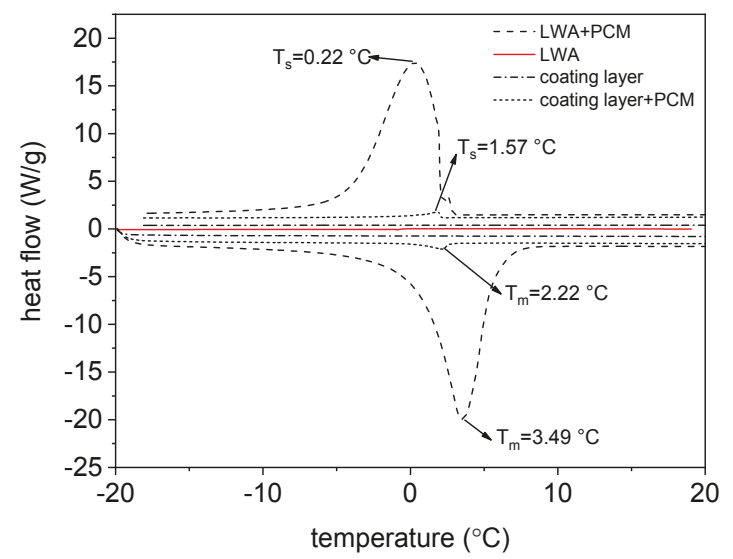

Figure 7. Thermal analysis tests of ME-LWA, the raw LWA and the resin coating.

DSC tests on ME-LWA aggregates were carried out for two sets of samples coated using different quantities of resin. Figure 8 a illustrates the results of two ME-LWA specimens coated with $10 \mathrm{wt} \%$ resin. Both specimens (taken from different ME-LWAs) revealed almost identical behaviour: the two curves are seen to be overlapped, with approximately the same amount of latent heat available at both melting and solidification, i.e., $31.61 \mathrm{~J} / \mathrm{g}$ and $31.02 \mathrm{~J} / \mathrm{g}$, respectively. The mean peak melting and solidification temperatures were also almost identical, averaging at $\mathrm{T}_{\mathrm{m} \text { (average) }}=3.3^{\circ} \mathrm{C}$ and $\mathrm{T}_{\mathrm{s} \text { (average) }}=1.1^{\circ} \mathrm{C}$, respectively. Figure $8 \mathrm{~b}$ illustrates the results of two ME-LWA specimens coated with $15 \mathrm{wt} \%$ resin. Comparing the two sets of curves, melting of the PCM occurred at a higher temperature than for the $10 \mathrm{wt} \%$ resin coated ME-LWA samples, whereas solidification occurred at a lower temperature. In fact, the mean values of the melting and solidification temperatures were $\mathrm{T}_{\mathrm{m}}$ (average) $=3.9{ }^{\circ} \mathrm{C}$ and $\mathrm{T}_{\mathrm{S} \text { (average) }}=0.0^{\circ} \mathrm{C}$, respectively. The latter offset in melting and solidification temperature is due to the increased resin content. Concerning the amount of latent heat, both specimens were characterized by sharp and broad bell-shaped curves, with the amount of latent heat available at melting and solidification equal to $31.70 \mathrm{~J} / \mathrm{g}$ and $33.76 \mathrm{~J} / \mathrm{g}$, respectively.

The transition in the solid-liquid phase change between $1{ }^{\circ} \mathrm{C}$ and $3{ }^{\circ} \mathrm{C}$ asserted by the PCM manufacturer's datasheet is in good agreement with the experimental results presented in Figure 8. The almost perfect overlap of the curves for both the $10 \mathrm{wt} \%$ and $15 \mathrm{wt} \%$ samples attests to the excellent reproducibility and repetitiveness of the recorded melting and solidification phases obtained using the DSC technique. These figures indicate that both sets of ME-LWAs exhibit similar thermal 
characteristics. This is because there is no chemical reaction between the paraffin PCM and the resin coating during the preparation of the ME-LWAs.

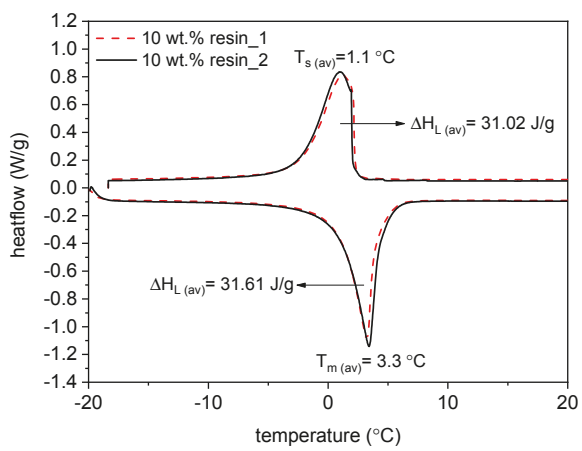

(a)

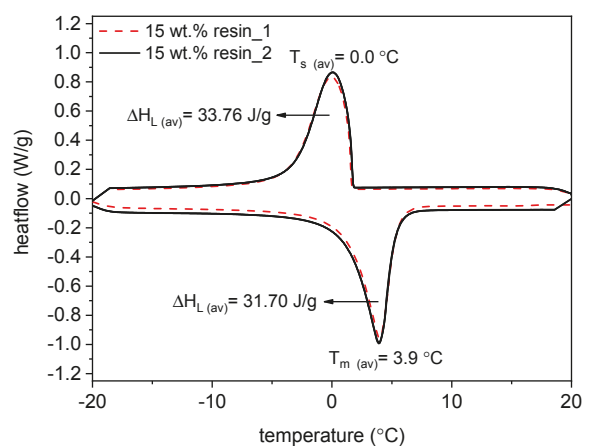

(b)

Figure 8. DSC tests of ME-LWAs samples from $-20{ }^{\circ} \mathrm{C}$ to $+20^{\circ} \mathrm{C}$ coated with (a) $10 \mathrm{wt} \%$ resin and (b) $15 \mathrm{wt} \%$ resin.

The specific theoretical enthalpy, $\mathrm{H}$, can be calculated as $\mathrm{H}=\Delta \mathrm{H} \times \mathrm{P}[\mathrm{J} / \mathrm{g}]$, where $\Delta \mathrm{H}$ is the specific phase change enthalpy of PCM, obtained from DSC test or directly from the datasheet, and P is the percentage in weight of paraffin hosted in LWA. The latent heat of PCM is provided by the supplier with an inaccuracy of $7.5 \%$ : its value was estimated at $185 \mathrm{~J} / \mathrm{g}$ in the worst case. Considering that the PCM absorption capacity by vacuum impregnation was calculated as $95 \%$, the theoretical enthalpy of the PCM hosted in a specific amount of LWA should be $175.75 \mathrm{~J} / \mathrm{g}$. Comparing this result with the value of specific enthalpy of impregnated aggregates without coating calculated by DSC test, equal to $62.44 \mathrm{~J} / \mathrm{g}$, it is clear that the heat transfer efficiency of ME-LWA was reduced. The lower thermal conductivity of the ME-LWA, along with the complex dispersion of the pores where the PCM is collected, undesirably affected the energy storage and release. This reduction is even more evident considering that the presence of the coating: the mean latent heat of the samples discussed above, is $32 \mathrm{~J} / \mathrm{g}$. The presence of polyester resin and granite powder contribute to the reduction of the ME-LWA specific enthalpy by approximately $50 \%$.

\subsection{ME-LWAs' Resistance to Fragmentation}

Many aggregates are more susceptible to abrasion when wet than dry [19]. The Micro-Deval test incorporates the use of water, in contrast to some other tests that are conducted on dry aggregates. The test results are helpful for evaluating the toughness/abrasion resistance of coarse aggregate subject to abrasion. In the EN 1097-2 standard of the Micro-Deval test [20], a Micro-Deval coefficient, which is the percentage of the original sample reduced to a size smaller than $1.6 \mathrm{~mm}$ during abrasion, is determined. However, in this study, the factor to be determined was the percentage of the original sample that did not incur any damage during the test. Hence, the procedure adopted here can be considered a variation of the EN 1097-1 standard. In terms of sample preparation, a sample of ME-LWA was weighed to the nearest gram and recorded as ' $A$ '. The sample was first soaked in pure water for $1 \mathrm{~h}$, then transferred to the Micro-Deval container with the steel charges. The test was run for $120 \mathrm{~min}$ at $80 \mathrm{RPM}$, resulting in a total of 9600 revolutions. After the test was complete, the sample was separated from the steel balls and washed with water until the runoff was clear and all materials smaller than the 125-micron sieve had passed through. The steel charge was removed manually, and the ME-LWA was then air-dried. Damaged ME-LWAs were determined visually and subsequently removed, and the mass of the undamaged ME-LWAs was recorded as ' $\mathrm{B}$ '. The percentage mass of ME-LWA that was left undamaged from the Micro-Deval abrasion test was calculated to the nearest $0.1 \%$. 
Some typical types of damage can be seen in Figure 9. The ME-LWAs suffered different degrees of damage, ranging from small and large pits in the coating (Figure 9a,b, respectively) to complete fracture with significant loss of coating (Figure 9c,d).

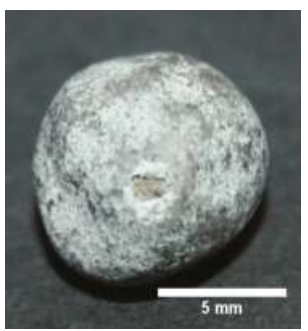

(a)

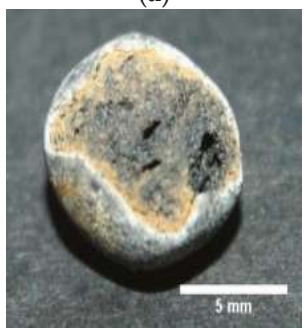

(c)

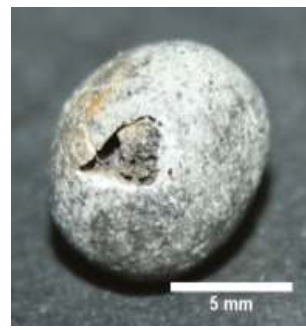

(b)

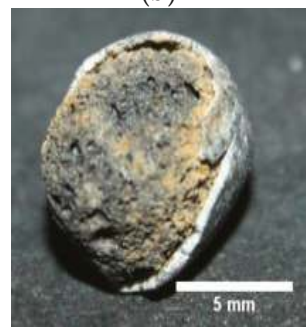

(d)

Figure 9. Fragmentation damage after Micro-Deval Showing (a) small pits (b) large pits (c) intermediate loss of coating and (d) significant loss of coating.

The mass of sample that did not incur any damage was determined to be $600 \mathrm{~g}$, hence $\mathrm{A}=1000 \mathrm{~g}$ and $\mathrm{B}=600 \mathrm{~g}$, then $\%$ ME-LWA damaged $=40 \%$. The interpretation of this result is straightforward and indicates that under the most severe conditions of wet abrasion, $40 \%$ of the ME-LWAs suffered some form of damage that could lead to the leaking of PCM. Under more realistic conditions, e.g., during the mixing of a cementitious binder, the ME-LWAs would not undergo such severe conditions.

\subsection{Surface Polishing Characteristics of ME-LAWs (Micro-Roughness and Friction under Traffic)}

Surface micro-roughness parameters and the Polished Stone Value coefficient (PSV) were carried out on ME-LWAs specimens by means of a laser profilometer scanning [21] according to EN 1097-8 [22]. The polishing cycle involved two phases of $3 \mathrm{~h}$ duration. In the first phase, coarse abrasive sand (size 300/600 $\mu \mathrm{m}$ ) and water were used; in the second phase, fine abrasive sand (smaller than $53 \mu \mathrm{m}$ ) was used instead, together with water. Two different types of aggregates were produced: ME-LWAs with $10 \%$ resin coating (ID sample: $10 \% \mathrm{R}$ ) and ME-LWAs with a first coating of $10 \%$ resin and a second coating of $15 \%$ resin (ID sample: $1015 \%$ R). Results were compared with a control non-coated aggregate (basalt, ID sample: CA) with high performance according to common road construction specifications [23,24]. Three specimens were prepared for each type of aggregate $(10 \% R, 1015 \% R$ and CA), for a total of nine specimens (Figure 10a,b).

It is important to point out that a superficial fragmentation of some granules was observed for some of the $10 \% \mathrm{R}$ samples (with a single resin coating) because they did not resist the smoothing action during the polishing test. This fragmentation was estimated to be around $16 \%$ of the surface area; however, this condition allowed the experimentation to be carried out correctly (Figure 10c). 


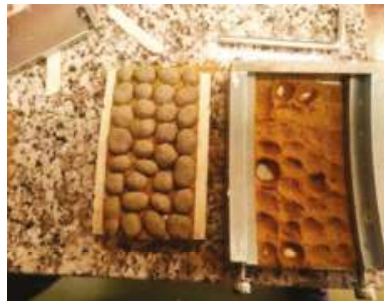

(a)

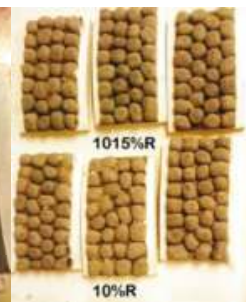

(b)
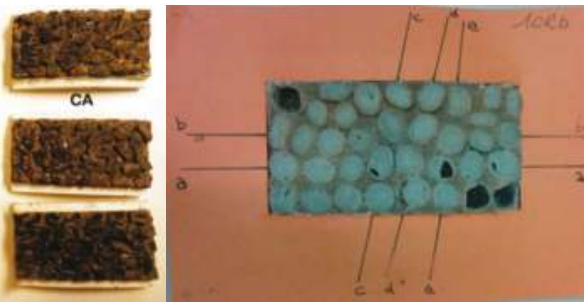

(c)

Figure 10. Specimens preparation for polishing test: (a) specimen demoulding; (b) sets of the experimentation specimens before polishing; (c) an example of superficial fragmentation of some granules after polishing test for a $10 \%$ R sample.

\subsubsection{Roughness Analysis}

As far as roughness analysis is concerned, a laser profilometer based on conoscopic holography was used [21,25,26] (Figure 11). Conoscopic holography is a non-contact digitizing technique used in submicrometric roughness measurements. This is in contrast to laser triangulation techniques, which are the most common solution for this kind of measurement, but there are fundamental limitations to their applicability when high precision, long standoffs or large apertures are needed, such as in this study [27]. For each aggregate sample, five profiles (a, b, c, d and e) were analysed on the top layer of the polished stone specimens. Two profiles along the same direction of polishing and three in the crosswise direction were carried out before and after the polishing test (Figure 12a). Micro-roughness parameters ( $\mathrm{Ra}, \mathrm{Rq}$ and $\mathrm{Rz}$ ) [28] were derived from the profile analysis, as reported in Table 5. In the literature it is also possible to calculate these parameters through laser measurements [29]. These technologies are also applicable on a different scale for measurements related to deterioration, road geometry and contact tire-surface [30-32].

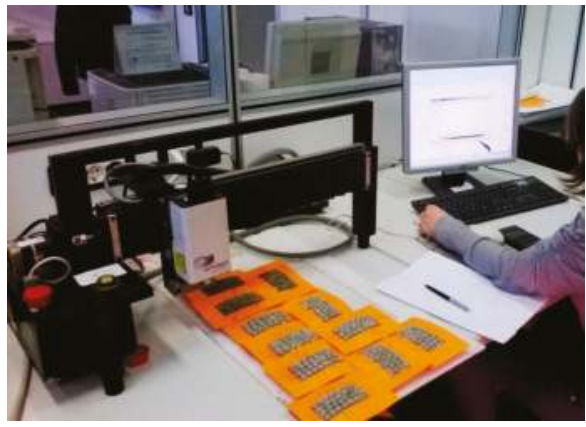

(a)

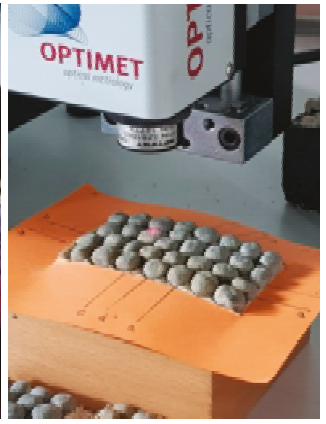

(b)

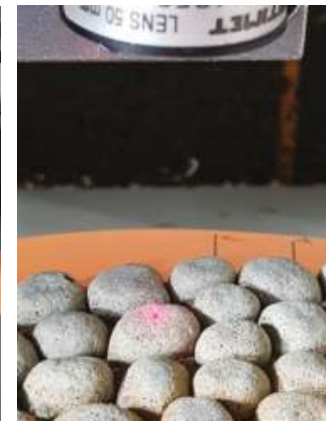

(c)

Figure 11. $(\mathbf{a}, \mathbf{b})$ Laser profilometer based on conoscopic holography used in the experimentation and (c) detail of the laser beam incidence on the surface of the granules. 


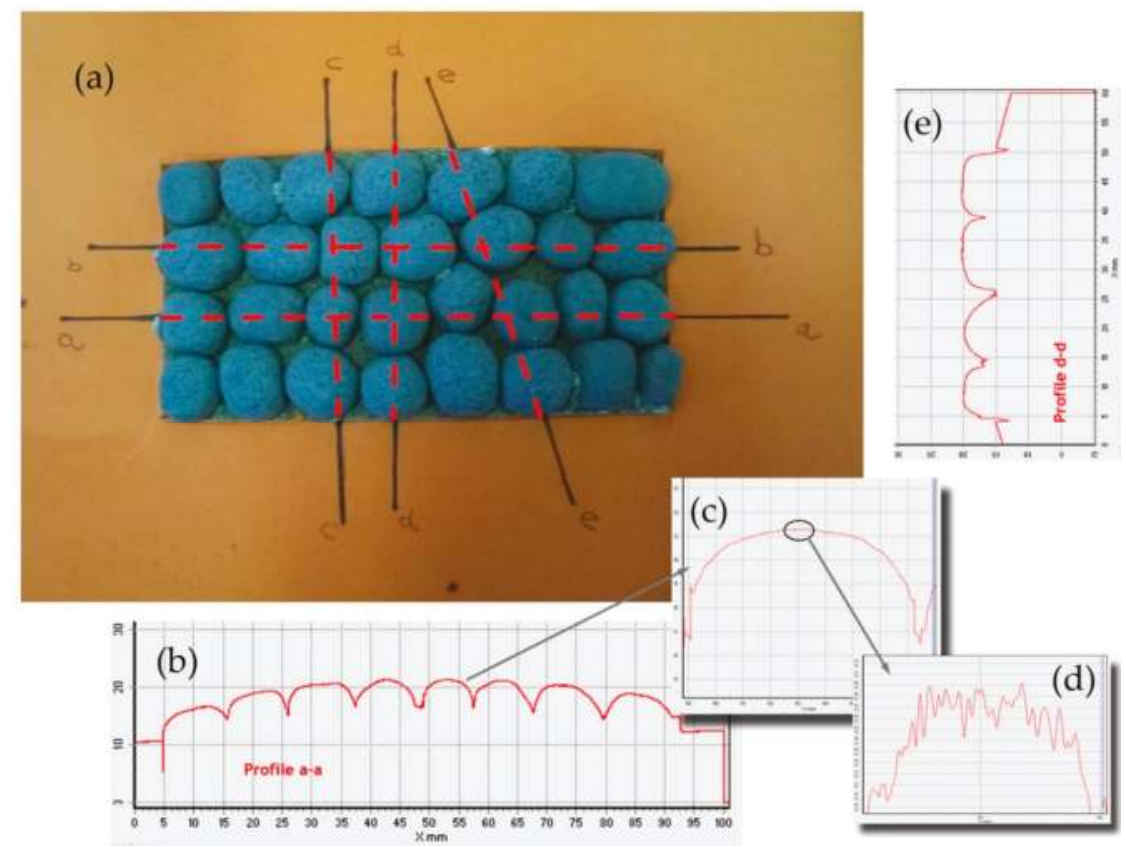

Figure 12. (a) Laser profilometer profile directions (b) profile along polishing direction a-a (c) zoom of granule surface (d) microprofile of granule surface (e) profile along crosswise direction d-d.

Table 5. Roughness analysis by laser profiles post-processing: descriptions and formulas of roughness parameters derived from microprofiles.

\begin{tabular}{l} 
Roughness \\
Parameters \\
\hline $\mathrm{Ra} \quad \begin{array}{c}\text { Arithmetical mean deviation } \\
\text { of the assessed profile }\end{array}$
\end{tabular}

Table 6 summarizes the roughness parameters before and after the polishing test. These values were derived from microprofiles sampled on the top of each aggregate with an average length 
(base-line) of 0.95-1.50 mm (Figure 12d). Furthermore, the percentage decreases between the two monitoring steps (before-after) were estimated.

Table 6. Roughness analysis by laser profiles post-processing: roughness parameter values before and after polishing test.

\begin{tabular}{|c|c|c|c|c|c|c|c|c|c|}
\hline \multirow{2}{*}{ ID Sample: CA } & \multicolumn{9}{|c|}{ Longitudinal and Cross Profiles } \\
\hline & \multicolumn{3}{|c|}{ Before } & \multicolumn{3}{|c|}{ After } & \multicolumn{3}{|c|}{ Depth Loss (-) or Depth Increase (+) } \\
\hline \multirow{4}{*}{$\begin{array}{l}\text { Profile roughness parameters } \\
\text { Average }[\mu \mathrm{m}] \\
\text { Number of microprofiles analysed } \\
\text { Length baseline of profile }[\mu \mathrm{m}]\end{array}$} & $\mathrm{Ra}$ & $\mathrm{Rg}$ & $\mathrm{Rz}$ & $\mathrm{Ra}$ & $\mathrm{Rq}$ & $\mathrm{Rz}$ & $\mathrm{Ra}$ & $\mathrm{Rg}$ & $\mathrm{Rz}$ \\
\hline & 15.3 & 19.7 & 42.8 & 14.5 & 18.7 & 41.5 & $-6 \%$ & $-5 \%$ & $-3 \%$ \\
\hline & \multicolumn{9}{|c|}{180} \\
\hline & \multicolumn{9}{|c|}{ Average $=1130 ; \operatorname{Max}=2800 ; \operatorname{Min}=300$} \\
\hline \multirow{2}{*}{ ID Sample: 10R\% } & \multicolumn{9}{|c|}{ Longitudinal and Cross Profiles } \\
\hline & \multicolumn{3}{|c|}{ Before } & \multicolumn{3}{|c|}{ After } & \multicolumn{3}{|c|}{ Depth Loss (-) or Depth Increase (+) } \\
\hline Profile roughness parameters & $\mathrm{Ra}$ & $\mathrm{Rq}$ & $\mathrm{Rz}$ & $\mathrm{Ra}$ & $\mathrm{Rq}$ & $\mathrm{Rz}$ & $\mathrm{Ra}$ & $\mathrm{Rq}$ & $\mathrm{Rz}$ \\
\hline Average $[\mu \mathrm{m}]$ & 13.8 & 17.5 & 40.9 & 11.1 & 13.9 & 32.0 & $-20 \%$ & $-20 \%$ & $-22 \%$ \\
\hline \multirow{2}{*}{$\begin{array}{l}\text { Number of microprofiles analysed } \\
\text { Length baseline of profile }[\mu \mathrm{m}]\end{array}$} & \multirow{2}{*}{\multicolumn{9}{|c|}{$\begin{array}{c}167 \\
\text { Average }=1506 ; \operatorname{Max}=2250 ; \operatorname{Min}=490\end{array}$}} \\
\hline & & & & & & & & & \\
\hline \multirow{2}{*}{ ID Sample: $1015 R \%$} & \multicolumn{9}{|c|}{ Longitudinal and Cross Profiles } \\
\hline & \multicolumn{3}{|c|}{ Before } & \multicolumn{3}{|c|}{ After } & \multicolumn{3}{|c|}{ Depth Loss (-) or Depth Increase (+) } \\
\hline Profile roughness parameters & $\mathrm{Ra}$ & $\mathrm{Rq}$ & $\mathrm{Rz}$ & $\mathrm{Ra}$ & $\mathrm{Rq}$ & $\mathrm{Rz}$ & $\mathrm{Ra}$ & $\mathrm{Rq}$ & $\mathrm{Rz}$ \\
\hline Average $[\mu \mathrm{m}]$ & 11.9 & 15.3 & 34.4 & 10.4 & 13.2 & 28.7 & $-12 \%$ & $-13 \%$ & $-17 \%$ \\
\hline \multirow{2}{*}{$\begin{array}{l}\text { Number of microprofiles analysed } \\
\text { Length baseline of profile }[\mu \mathrm{m}]\end{array}$} & \multicolumn{9}{|c|}{188} \\
\hline & \multicolumn{9}{|c|}{ Average $=948 ; \operatorname{Max}=1440 ; \operatorname{Min}=490$} \\
\hline Delta $X[\mu \mathrm{m}]$ & \multicolumn{9}{|c|}{10} \\
\hline
\end{tabular}

According to Table 6, for all samples (CA, 10\%R and 1015\%R), the depth parameters decrease after polishing, with the lowest percentage decrease (on average $-5 \%$ ) being recorded for sample CA. Also, samples with a double layer of resin $(1015 \% \mathrm{R})$ are more resistant to polishing (average decrease $-14 \%$ ) compared to $10 \% \mathrm{R}$ (on average $-20 \%$ ).

\subsubsection{Polished Stone Analysis}

The residual friction after the polishing test was also investigated in terms of PSV, for which the outcomes are shown in Table 7 and Figure 13. In order to better investigate the PSV variability, the performance of expanded clay (ID sample: EC) was considered. Usually, EC is used for the surface layer of anti-skid and anti-noise asphalt pavements. Table 7 shows the acceptance threshold values for the polishing test according to widely adopted Italian technical specifications, too.

Table 7. Average Polished Stone Values for samples tested (10\%R; $1015 \%$ R and CA) compared with PSVs of expanded clay and acceptance threshold values from Italian specifications (PSVs x 100).

\begin{tabular}{cccccc}
\hline ID & $\mathbf{1 0} \% \mathbf{R}$ & $\mathbf{1 0 1 5} \% \mathbf{R}$ & $\mathrm{CA}$ & EC & Specification \\
\hline PSV & 63 & 65 & 53 & 70 & $\geq 45-50$ \\
\hline
\end{tabular}

As shown in Table 7 and Figure 13, the average values of the PSV coefficient (multiplied by 100) for both types of ME-LWAs are higher than those registered for the control aggregate. More specifically, PSV is recorded to be 53 for CA versus 63 for 10\%R samples and 65 for the $1015 \%$ R sample. Finally, the average PSV registered for ME-LWAs is always higher than the specifications' acceptance thresholds. Based on the abovementioned findings, it is possible to highlight that the double surface-coating technique (1015\%R sample) allows for the attainment of a better performance than the aggregates made with a single-coating for road pavements in terms of both micro-roughness and friction development under traffic loadings. 


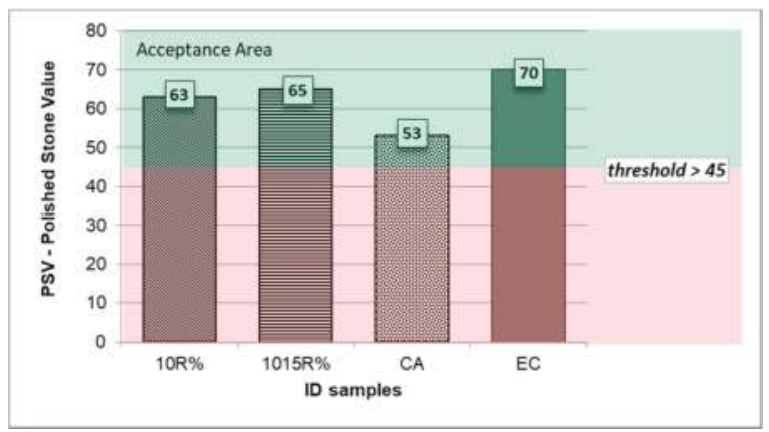

Figure 13. Histogram of average Polished Stone Values for samples 10\%R, 1015\%R and CA compared with PSVs of expanded clay and acceptance threshold values (green area) from common Italian technical specifications. (Note: in the figure PSVs are $\times 100$.).

\subsection{Thermal Analysis of Relevant Temperature Conditions}

Three further thermal measurement tests were carried out to obtain a more realistic evaluation of the ME-LWA effectiveness for their use as anti-icing solution in pavements. Two different samples of ME-LWAs and one sample of the raw LWA were prepared and tested in both a climatic chamber and a cold outdoor environment, monitoring their thermal behaviour as a result of the controlled and uncontrolled temperature reductions, respectively. Temperature measurements in the climatic chamber were performed using a FLIR thermal camera, infrared thermometer and thermocouples, while ambient temperature monitoring was conducted using a digital thermometer.

The measurements in both environments were performed for a sufficient time, during which sub-zero temperatures were reached. The FLIR thermal camera provided thermal pictures of the samples, from which the development of the temperature as a function of time was plotted (Figure 14). The two samples made of ME-LWA are placed on the left and right of the thermal pictures, respectively, while the natural porphyry aggregate is placed in the centre. Pictures are taken at 10-min intervals from 0 to $50 \mathrm{~min}$.

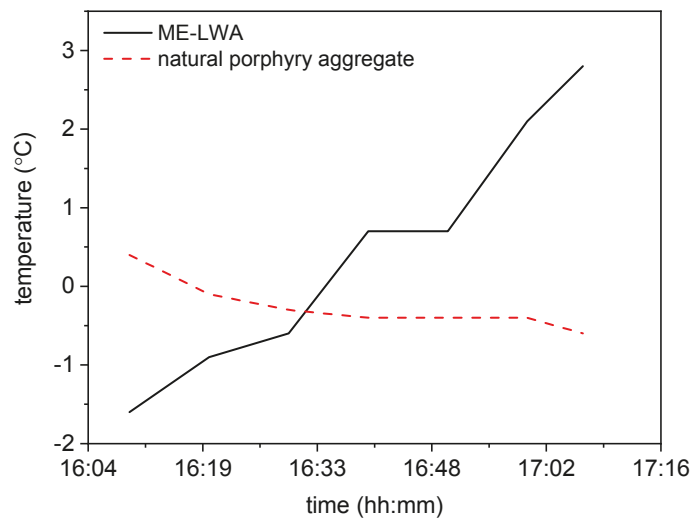

(a)

Figure 14. Cont. 


\begin{tabular}{|c|c|c|c|c|c|c|}
\hline $\begin{array}{c}\text { Time } \\
(\text { min) }\end{array}$ & 0 & 10 & 20 & 30 & 40 & 50 \\
\hline $\begin{array}{c}\text { Natural } \\
\text { porphyry } \\
\text { aggregate }\end{array}$ & & & & & & \\
\hline
\end{tabular}

(b)

Figure 14. (a) Temperature differences versus time and (b) thermal images of natural porphyry aggregate and ME-LWAs over a period of 50 minutes.

During the first $30 \mathrm{~min}$, the differences between the ME-LWA samples and the natural aggregate were negative, meaning that the ME-LWAs were colder. However, with the decrease in the temperature, between $30 \mathrm{~min}$ and the end of the test, the values became positive, showing that the ME-LWAs maintained higher temperatures than the natural aggregate, reaching a maximum value of $2.8{ }^{\circ} \mathrm{C}$. This trend was also observed from the thermal images presented in Figure 14b, which clearly show the remarkable effect of PCM heat flow, particularly in the last $30 \mathrm{~min}$, where the ME-LWAs can be seen to be glowing much brighter than the natural porphyry aggregates.

The measurement with the infrared thermometer (Figure 15) also shows a different evolution of the temperature: after 25-30 min, the ME-LWAs remained approximately constant and close to $2{ }^{\circ} \mathrm{C}$, while the natural porphyry aggregates continued to decrease, reaching a temperature of $0{ }^{\circ} \mathrm{C}$.

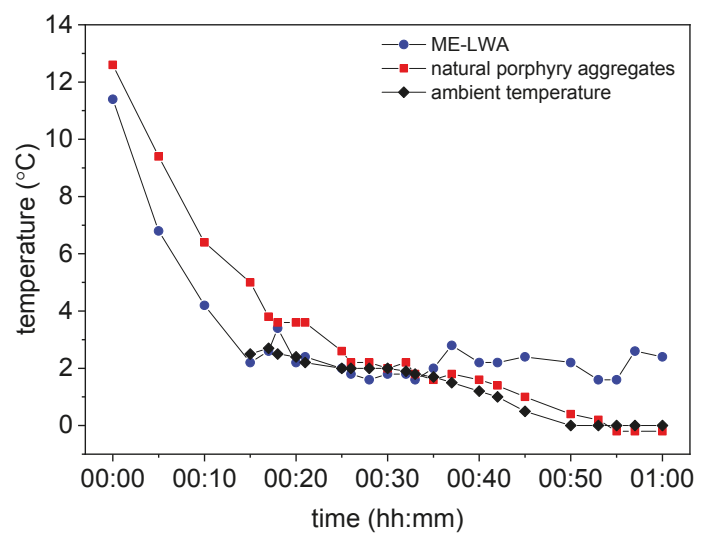

Figure 15. Development of temperature as a function of time using the infrared thermometer.

An additional test was carried out in a climatic chamber to evaluate how long ME-LWAs were able to maintain temperatures above $0{ }^{\circ} \mathrm{C}$. For this experiment, neither the thermal camera nor the infrared thermometer was used since the glass surfaces of the chamber would influence the measurements. Instead, digital thermocouples were embedded into samples of ME-LWAs and natural porphyry aggregates (Figure 16). This analysis was performed to simulate a typical local temperature drop 
during the night; the chamber temperature was manually controlled to reproduce a set of available field data from the nearby climate station.

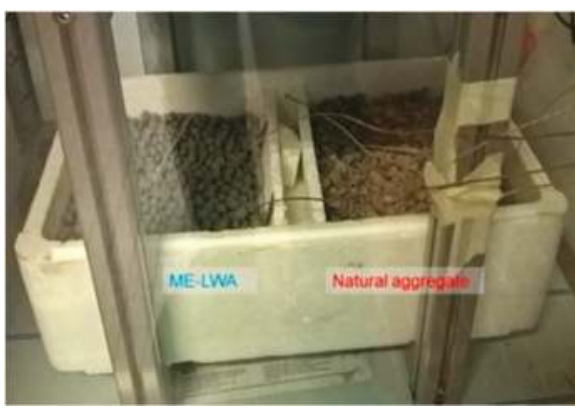

(a)

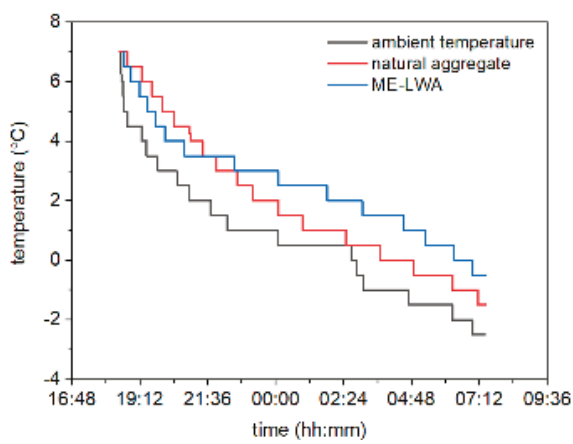

(b)

Figure 16. (a) Climatic chamber recording the temperature of ME-LWA and porphyry aggregates and (b) development of temperature versus time inside the climatic chamber.

Figure 16 shows the fitted time versus temperature curves of the chamber air, natural aggregates and ME-LWA. The curve for the ME-LWA shows attenuation in its slope starting from $3-4{ }^{\circ} \mathrm{C}$, while the natural aggregates continue to decrease in temperature, following almost the same trend as the chamber temperature. The meaningful difference in their behaviour was shown between approximately 04:30 and 07:00, when the sample temperature was below $0^{\circ} \mathrm{C}$. The ME-LWA, on the other hand, maintained a temperature above $0{ }^{\circ} \mathrm{C}$, more likely due to the PCM phase change from liquid to solid and subsequent exothermic heat flow.

The latter results are promising since this material has been demonstrated to have a remarkable delaying effect on the temperature drop as soon as $3{ }^{\circ} \mathrm{C}$ is reached. It is acceptable to assume that the presence of bitumen and other virgin aggregates in an asphalt mix would hinder PCM heat release; however, it is still likely that ME-LWA aggregates would slow down or prevent the formation of ice on road surfaces where the climate is not that harsh.

\section{Conclusions}

This preliminary work aims at the assessment of macro-encapsulated lightweight aggregates containing PCM for paving application purposes. In light of the above, the following concluding remarks can be made:

- $\quad$ The production of ME-LWA was proven feasible at a laboratory scale and could be implemented on an industrial level by taking into account the described variables;

- The geometrical and mechanical properties of the LWA are not affected by the macro-encapsulation process. The resin coating provides additional strength to the aggregates;

- The PCM is thermally stable, showing only a $0.7^{\circ} \mathrm{C}$ change in melting temperature after thermal cyclic testing. The resin coating undoubtedly reduces the thermal effects of the pure PCM. Nonetheless, an average latent heat of $32 \mathrm{~J} / \mathrm{g}$ can still be achieved.

- The strength of coating is a key point for the durability of the aggregates with traffic: a double coating of resin was proven effective against polishing, producing an aggregate with only a $9 \%$ lower polishing resistance than the reference basalt aggregate.

- The thermal effects on aggregate temperature were tested in a relevant climate using different sensors and temperature gradients with time: the ME-LWAs temperature was measured and was proven to be effective at maintaining a temperature above $0{ }^{\circ} \mathrm{C}$. 
Author Contributions: The research was conceived by all the authors and performed under the supervision of C.S. and X.Z. The experiments were conducted and data were primarily analysed by G.K., R.V. and P.T. The manuscript was prepared by G.K., R.V. and C.L.

Acknowledgments: Partial financial support from the European Commission Horizon 2020 MARIE Skłodowska-CURIE Research and Innovation Staff Exchange scheme through the grant 645696 (i.e., the REMINE project) is acknowledged.

Conflicts of Interest: The authors declare no conflict of interest. Also, the founding sponsors had no role in the design of the study; in the collection, analyses, or interpretation of data; in the writing of the manuscript, and in the decision to publish the results.

\section{References}

1. Pomianowski, M.; Heiselberg, P.; Zhang, Y.P. Review of thermal energy storage technologies based on PCM application in buildings. Energy Build. 2013, 67, 56-69. [CrossRef]

2. Castell, A.; Martorell, I.; Medrano, M.; Pérez, G.; Cabeza, L.F. Experimental study of using PCM in brick constructive solutions for passive cooling. Energy Build. 2010, 42, 534-540. [CrossRef]

3. Soares, N.; Costa, J.J.; Gaspar, A.R.; Santos, P. Review of passive PCM latent heat thermal energy storage systems towards buildings' energy efficiency. Energy Build. 2013, 59, 82-103. [CrossRef]

4. Chen, M.Z.; Hong, J.; Wu, S.P.; Lu, W.; Xu, G.J. Optimization of phase change materials used in asphalt pavement to prevent rutting. Adv. Mater. Res. 2011, 219, 1375-1378. [CrossRef]

5. Ma, B.; Wang, S.S.; Li, J. Study on application of PCM in asphalt mixture. Adv. Mater. Res. 2011, 168, 2625-2630. [CrossRef]

6. Evers, A.C.; Medina, M.A.; Fang, Y. Evaluation of the thermal performance of frame walls enhanced with paraffin and hydrated salt phase change materials using a dynamic wall simulator. Build. Environ. 2010, 45, 1762-1768. [CrossRef]

7. Hawes, D.W.; Banu, D.; Feldman, D. The stability of phase change materials in concrete. Sol. Energy Mater. Sol. Cells 1992, 27, 103-118. [CrossRef]

8. Ushak, S.; Cruz, J.M.; Cabeza, F.L.; Grágeda, M. Preparation and characterization of inorganic PCM microcapsules by fluidized bed method. Materials 2016, 9, 24. [CrossRef] [PubMed]

9. Hunger, M.; Entrop, A.G.; Mandilaras, I.; Brouwers, H.J.H.; Founti, M. The behavior of self-compacting concrete containing micro-encapsulated phase change materials. Cem. Concr. Compos. 2009, 31, 731-743. [CrossRef]

10. Özonur, Y.; Mazman, M.; Paksoy, H.Ö.; Evliya, H. Microencapsulation of coco fatty acid mixture for thermal energy storage with phase change material. Int. J. Energy Res. 2006, 30, 741-749. [CrossRef]

11. Borreguero, A.M.; Valverde, J.L.; Rodríguez, J.F.; Barber, A.H.; Cubillo, J.J.; Carmona, M. Synthesis and characterization of microcapsules containing Rubitherm ${ }^{\circledR} \mathrm{RT} 27$ obtained by spray drying. Chem. Eng. J. 2011, 166, 384-390. [CrossRef]

12. Izquierdo-Barrientos, M.A.; Sobrino, C.; Almendros-Ibáñez, J.A. Thermal energy storage in a fluidized bed of PCM. Chem. Eng. J. 2013, 230, 573-583. [CrossRef]

13. Jamekhorshid, A.; Sadrameli, S.M.; Farid, M. A review of microencapsulation methods of phase change materials (PCMs) as a thermal energy storage (TES) medium. Renew. Sustain. Energy Rev. 2014, 31, 531-542. [CrossRef]

14. Hawlader, M.N.A.; Uddin, M.S.; Khin, M.M. Microencapsulated PCM thermal-energy storage system. Appl. Energy 2003, 74, 195-202. [CrossRef]

15. Mondal, S. Phase change materials for smart textiles-An overview. Appl. Therm. Eng. 2008, 28, 1536-1550. [CrossRef]

16. Kastiukas, G.; Zhou, X.M.; Castro-Gomes, J. Development and optimisation of phase change material-impregnated lightweight aggregates for geopolymer composites made from aluminosilicate rich mud and milled glass powder. Constr. Build. Mater. 2016, 110, 201-210. [CrossRef]

17. Memon, S.A.; Cui, H.Z.; Lo, T.Y.; Li, Q.S. Development of structural-functional integrated concrete with macro-encapsulated PCM for thermal energy storage. Appl. Energy 2015, 150, 245-257. [CrossRef]

18. Lightweight Aggregates; BS EN 13055:2016; British Standards Institution: London, UK, 2016.

19. Test Method for Resistance of Fine Aggregate to Degradation by Abrasion in the Micro-Deval Apparatus; ASTM D7428-15; ASTM International Standard: West Conshohocken, PA, USA, 2015. 
20. Tests for Mechanical and Physical Properties of Aggregates. Methods for the Determination of Resistance to Fragmentation; BS EN 1097-2:2010; British Standards Institution: London, UK, 2010.

21. Characterization of Pavement Texture by Use of Surface Profiles-Part 3: Specification and Classification of Profilometers; ISO 13473-3:2002; International Organization for Standardization: Geneva, Switzerland, 2002.

22. Tests for Mechanical and Physical Properties of Aggregates. Determination of the Polished Stone Value; BS EN 1097-8:2009; British Standards Institution: London, UK, 2009.

23. Vaiana, R.; Praticò, F. Pavement surface properties and their impact on performance-related pay adjustments. In Proceedings of the 3rd International Conference on Tranportation Infrastructure, Pisa, Italy, 22-25 April 2014; pp. 579-587.

24. Iuele, T.; Vaiana, R.; Gallelli, V.; De Masi, F. The influence of aggregate lithological nature on pavement texture polishing: A comparative investigation on a test site in southern Italy. Adv. Civ. Eng. Mater. 2016, 5, 337-352. [CrossRef]

25. Praticò, F.G.; Vaiana, R. A study on the relationship between mean texture depth and mean profile depth of asphalt pavements. Constr. Build. Mater. 2015, 101, 72-79. [CrossRef]

26. Praticò, F.G.; Vaiana, R.; Iuele, T. Macrotexture modeling and experimental validation for pavement surface treatments. Constr. Build. Mater. 2015, 95, 658-666. [CrossRef]

27. Álvarez, I.; Enguita, J.M.; Frade, M.; Marina, J.; Ojea, G. On-line metrology with conoscopic holography: Beyond triangulation. Sensors 2009, 9, 7021-7037. [CrossRef] [PubMed]

28. Boscaino, G.; Pratico, F.G. A classification of surface texture indices of pavement surfaces. Bull. Lab. Ponts Chaussees 2001, 234, 17-34. (In French)

29. Bitelli, G.; Simone, A.; Girardi, F.; Lantieri, C. Laser scanning on road pavements: A new approach for characterizing surface texture. Sensors 2012, 12, 9110-9128. [CrossRef] [PubMed]

30. Lantieri, C.; Lamperti, R.; Simone, A.; Vignali, V.; Sangiorgi, C.; Dondi, G. Mobile laser scanning system for assessment of the rainwater runoff and drainage conditions on road pavements. Int. J. Pavement Res. Technol. 2015, 8, 1-9.

31. Dondi, G.; Barbarella, M.; Sangiorgi, C.; Lantieri, C.; De Marco, L. A semi-automatic method for identifying defects on road surfaces. In Proceedings of the International Conference on Sustainable Design and Construction (ICSDC), Kansas City, MO, USA, 23-25 March 2012.

32. Woodward, D.; Millar, P.; Lantieri, C.; Sangiorgi, C.; Vignali, V. The wear of stone mastic asphalt due to slow speed high stress simulated laboratory trafficking. Constr. Build. Mater. 2016, 110, 270-277. [CrossRef]

(C) 2018 by the authors. Licensee MDPI, Basel, Switzerland. This article is an open access article distributed under the terms and conditions of the Creative Commons Attribution (CC BY) license (http:/ / creativecommons.org/licenses/by/4.0/). 
Article

\title{
Preliminary Research on the Physical and Mechanical Properties of Alternative Lightweight Aggregates Produced by Alkali-Activation of Waste Powders
}

\author{
Piergiorgio Tataranni *, Giulia Maria Besemer, Villiam Bortolotti and Cesare Sangiorgi \\ DICAM Department, University of Bologna, 40136 Bologna, Italy; giuliamaria.besemer@studio.unibo.it (G.M.B.); \\ villiam.bortolotti@unibo.it (V.B.); cesare.sangiorgi4@unibo.it (C.S.) \\ * Correspondence: piergiorg.tataranni2@unibo.it
}

Received: 28 June 2018; Accepted: 18 July 2018; Published: 21 July 2018

\begin{abstract}
There is growing interest in construction field issues related to environmental protection, energy saving and raw materials. Therefore, the interest in recycling waste materials to produce new construction ones is constantly increasing. This study proposes a new methodology to produce lightweight aggregates (LWAs) by alkali-activation of two different waste powders: a digested spent bentonite clay and a basalt powder. Metakaolin, as secondary precursor, was added to the mixtures according to mix-design proportions, to improve the mechanical properties of the final materials, while a specific activators mix of Sodium Silicate and Sodium Hydroxide enabled the alkali-activation. The expansion process, on the other hand, was obtained using Peroxide within the liquid mix. The experimental LWAs were analyzed and tested in compliance with the EN 13055-1 standard. A more in-depth analysis on LWAs' air voids content and porosity was also carried out by the means of Mercury Intrusion Porosimetry and Nuclear Magnetic Resonance. The results were compared with those obtained from commercial Lightweight Expanded Clay Aggregate, which represents one of the most common LWAs in the construction field. According to the presented preliminary results, the use of alkali-activated waste powders seems to be a suitable solution for the production of eco-friendly LWAs by allowing the recycling of waste materials and energy saving for their production.
\end{abstract}

Keywords: alkali-activated materials; lightweight aggregates; nuclear magnetic resonance; expanded clay

\section{Introduction and Background}

Light Weight Aggregates (LWAs) are granular materials characterized by high porosity and low density. The European Standard EN 13055-1 [1] refers to LWAs as construction materials which present bulk density values lower than $1.2 \mathrm{Mg} / \mathrm{m}^{3}$ and particle density values lower than $2.0 \mathrm{Mg} / \mathrm{m}^{3}$. When used in the construction industry, LWAs offer functional and economic advantages. The pores of these materials determine favorable thermal and acoustical insulation properties and permit an overall reduction of the self-weight of the final products. LWAs can be found in nature, but most commonly they are artificially produced, either from natural raw materials or from industrial by-products [2]. In the latter case, source materials are subjected to specific treatments, which can be set to control the physical and microstructural properties of the resulting artificial LWA. The most widely used artificial LWAs are based on the processing of clay, slate, perlite, vermiculite, waste glass and ash. Among these, the use of Lightweight Expanded Clay Aggregate (LECA) is probably the most popular. LECA is a widely available and normed material that can be used in various applications including civil engineering projects. The production process of clay LWA comprises the pre-treatment of the raw dried clay and its consequent firing inside a rotary kiln. During the first stages of the process, the raw clay is finely grouted and formed in granules through the addition of water. The pelletized 
clay consequently undergoes drying and sintering at temperatures typically between $1050{ }^{\circ} \mathrm{C}$ and $1250{ }^{\circ} \mathrm{C}$. The heating and rotating action of the rotary kiln leads to decomposition processes and the generation of gases. These are mainly released by the decomposition and reduction of ferric oxides, by the combustion of organic particles, the blowing of entrapped water molecules and the decomposition of carbonates [3]. The formed gases act like blowing agents and expand the clay matter, which swells forming granules with volumes up to five times the initial pellets' dimensions. The granules are characterized by a porous inner structure and an outer hardened shell and result in an optimized weight-to-strength relationship. The physical and mechanical characteristics of LECA vary depending on the raw materials' composition, the firing temperature, and the rotation speed. Materials used in construction industry can present a compressive strength up to $4.5 \mathrm{~N} / \mathrm{mm}^{2}$ and a density of approximately $0.6 \mathrm{Mg} / \mathrm{m}^{3}$, with figures varying slightly according to the different producers.

Notations are listed in Table 1.

Table 1. Table of notations.

\begin{tabular}{cc}
\hline Notation & Description \\
\hline LWA & Light weight aggregate \\
LECA & Lightweight expanded clay aggregate \\
AAM & Alkali-activated material \\
L/S & Liquid-solid ratio \\
NMR & Nuclear Magnetic Resonance \\
TD-MRR & Time Domain Nuclear Magnetic Resonance Relaxometry \\
PSD & Distribution of pore sizes \\
MIP & Mercury Intrusion Porosimetry \\
Ud & Digested spent bentonite clay \\
B & Basalt powder \\
MK & Metakaolin \\
SS & Sodium silicate \\
SH & Sodium hydroxide \\
HP & Hydrogen peroxide \\
A $/ P$ & Activator-precursor ratio \\
IR & Standard Inversion-Recovery \\
CPMG & Carl-Purcell-Meiboom-Gill sequence \\
SSD & Saturated and surface-dried \\
\hline
\end{tabular}

In recent years, increasing effort has been invested into finding new ways to reuse waste materials [4-6] to produce LWAs. The main benefits are represented by the reduction of the production impacts, due to the replacement of natural raw materials, and by the reuse of considerable volumes of wastes. LWAs produced from industrial by-products, such as fly-ash, sludge, and other more unconventional raw materials, can be obtained from similar production processes that are fundamentally based on the sintering of materials at high temperatures between $1000{ }^{\circ} \mathrm{C}$ and $1200{ }^{\circ} \mathrm{C}$. Despite the beneficial incorporation and reuse of waste materials in LWAs, the energy demand of traditional production processes still represents a detrimental fact. To meet the deeper ecological goals, the cold-bonding agglomeration process is a valuable technique to produce artificial LWAs without the need of high-temperature sintering. The process is based on the use of a sloping rotary disc, where the wetting agent is added by drops and the material is pelletized by the action of scrapping blades $[7,8]$.

In the context of an increasing sustainability, the possibilities offered by alkali-activation (AA) are gaining relevance. Alkali-activated materials (AAMs) are alternative cementitious materials that result from the chemical reaction between silica and alumina-rich sources and strong alkali solutions [9-12]. The alkali solutions are composed of hydroxides or silicates, or by a combination of both. The most commonly used activators are potassium or sodium hydroxide and sodium silicate. The reaction can conceptually be split into three main steps, which take place almost contemporarily: dissolution, condensation and reorganization $[13,14]$. During the first stage, the alkali media induces the leaching of the aluminum and silicon ions contained in the precursors. These ions then interact and condensate 
resulting in the formation of an intermediate complex, which is often defined as a gel. Finally, with the increase in connectivity of the gel, a condensed reorganized structure is formed, typically composed of highly connected silica and alumina tetrahedra [15]. The reaction products are mainly amorphous binders, which show remarkable features in terms of mechanical strength, chemical stability, and fire resistance. A crucial fact in the reaction kinetics is determined by the precursors' composition, and secondarily, by the type and concentration of the alkali-activators. It is proved that a higher alkali content leads to a higher reactivity of the compounds. Nonetheless, strict control needs to be exercised on the liquid to solid ratio (L/S) of the mixture, to prevent the formation of a weak structure. A reasonably low $\mathrm{L} / \mathrm{S}$ ratio, combined with a curing treatment at temperatures lower than $100{ }^{\circ} \mathrm{C}$, is likely to give the most favorable results $[16,17]$. AA is quite a recent research field and because many industrial by-products present chemical compositions that match the requirements of AA, a wide range of materials are currently investigated as possible precursors.

The development of lightweight AAMs is drawn from the knowledge of cellular cement. Voids inside the cementitious binder can either be produced by adding a pre-foamed foam to the paste or by using chemical blowing agents, which release gas during the mixing phase. The addition of reactive metal powders, such as aluminum powder, liberates hydrogen gas, while a blowing agent such as hydrogen peroxide exhibits the expansion through the release of oxygen gas. The foaming technique and the mixture composition, as well as the curing conditions, differently influence the porosity and strength of the foamed materials. The combination of the alkali concentration and the $\mathrm{L} / \mathrm{S}$ ratio determine both the reaction rate and the viscosity of the AA paste, which are fundamental for a stable foaming. Experience on the expansion through hydrogen peroxide showed that a correct manipulation of the AA mix permits the generation of a controlled and stable foaming. Attempts in the production of AA LWAs comprise techniques such as the granulation of hardened AAMs [18] or the cold-bonding pelletization process [19]. More recently, favorable results were achieved using a high-shear granulator, where the AA mass is mixed inside the rotating device resulting in porous granules of different grain sizes [20,21].

In this study, hydrogen peroxide is used to produce AA LWAs, which are obtained from the alkali-activation of two different waste powders, originated from different industrial processes, combined to a certain amount of metakaolin. The activator is a specific solution of sodium hydroxide and sodium silicate.

Furthermore, and likely for the first time, LWAs materials were also studied with the Time Domain (TD) Nuclear Magnetic Resonance (NMR) Relaxometry of protons $\left({ }^{1} \mathrm{H}\right.$ TD-MRR). This technique is an important non-destructive and non-invasive tool for analyzing the structure of porous media, ranging from biological systems [22] to cement [23]. TD-MRR is characterized by two relaxation parameters, the longitudinal relaxation time $\left(\mathrm{T}_{1}\right)$, the transverse relaxation time $\left(\mathrm{T}_{2}\right)$ and by a magnetization vector (the sum of the polarized nuclear magnetic moment associated to the ${ }^{1} \mathrm{H}$ spins). Since a permeable porous medium is made of a network of interconnected pores of different shapes and sizes, when the TD-MRR experiment is performed on porous media fully saturated with water (or other fluid containing ${ }^{1} \mathrm{H}$ as for example oil) the relaxation times will be influenced by the presence of the pore walls. Because of the auto-diffusion, a molecule of the saturating fluid continuously moves in the pore network and hits the pore walls, and so the NMR magnetization intensity relaxes back to the equilibrium over a region that is larger than a single pore (the so-called diffusion cell) [24]. TD-MRR data will therefore be averaged over the diffusion cell giving a local average information on pores. If the molecular diffusion is fast enough to maintain the magnetization uniform within the diffusion cell, then $T_{1}$ and $T_{2}$ show distributions of relaxation times that could be related to the distribution of pore sizes (PSD).

To obtain the PSD of a sample via TD-MRR, the surface relaxivity of the sample should be known. Many techniques can be used to obtain it, each one with its own advantages and disadvantages. Here we used the comparison with the porosity analysis carried out with the classical Mercury Intrusion Porosimetry (MIP) method. Numerous experiments have shown that in many cases the $\mathrm{T}_{1}$ 
or $\mathrm{T}_{2}$ relaxation time distribution curves could be very similar to the pore size distribution curves obtained by MIP [25].

Results demonstrate a significant correlation between the data obtained with NMR and the outcomes from MIP analysis.

This study proposes a new procedure to produce LWAs by AA of two different waste powders. An in-depth analysis on LWAs' air voids content and porosity was also carried out by the means of MIP and NMR, providing practitioners and researchers with an alternative methodology to assess these characteristics.

\section{Materials and Experimental Program}

The AA process involves specific precursors and activators in a chemical reaction that leads to the development of cementing materials. In the present work, two different waste materials were used as precursors: a digested spent bentonite clay (named Ud) and a basalt powder (named B). These waste compounds were mixed with metakaolin $(\mathrm{M})$ to ensure adequate chemical properties and mechanical performances to the final mix. The liquid compounds needed for the AA process were sodium silicate (SS) and sodium hydroxide (SH), according to a specific mix-design. A commercial LWA (named LECA) was used as reference material through the research.

\subsection{Precursors}

\subsubsection{Digested Spent Bentonite Clay}

Ud used in the presented research is a waste from the food industry. The original material is a bleaching clay, which, thanks to its properties of capturing impurities from oils, is normally used for the decoloring process of oils. As a result of anaerobic digestion for biogas production, the waste materials is disposed of in a dump, with a residual oil content up to $1 \%$ by the weight of particles. After some preliminary experimental applications made by the authors for the production of AAMs with $\mathrm{Ud}$, it was found that initial calcination was helping the AA reactions. In fact, the heat treatment allows the material to better interact with the activators for the AA process.

\subsubsection{Basalt Powder}

The basalt powder is a residual from the extractions and productions processes in basalt quarries. Basalt deposits are present in almost every country and this material is widely used in the constructions field for its mineralogical, chemical, and physical properties. The extensive use of this material for bituminous mixtures and concretes leads to the production of huge quantities of powder during the preparation of aggregates. Different studies and researches have been done for the recycling and reuse of this waste powder in replacement of raw aggregates for the production of construction materials [26]. Some researchers have also verified the AA of the basalt powder, with positive outcomes [27].

\subsubsection{Metakaolin}

The use of metakaolin (MK) to produce AAMs is common. The chemical properties of this material makes it extremely suitable for the alkali reaction process, as verified by several applications. Metakaolin is a dehydroxylated form of the clay mineral kaolinite and it is obtained by heat treatment (around $700{ }^{\circ} \mathrm{C}$ ) of a natural kaolin [28].

Table 2 summarizes the precursors' physical properties, while Table 3 shows their chemical composition. 
Table 2. Ud, B and M physical properties.

\begin{tabular}{ccccc}
\hline Test & Unit & Ud & B & MK \\
\hline Size distribution (EN 13043) & $\%$ & $100 \mathrm{P}_{50 \mathrm{um}}$ & $100 \mathrm{P}_{50 \mathrm{um}}$ & $95 \mathrm{P}_{80 \mathrm{um}}$ \\
Water content (EN 1097-5) & $\%$ & 1.12 & 0.04 & 0.12 \\
Particle density (EN 1097-7) & $\mathrm{Mg} / \mathrm{m}^{3}$ & 1.86 & 2.70 & 2.40 \\
\hline
\end{tabular}

Table 3. Precursors' chemical composition.

\begin{tabular}{ccccc}
\hline Compound & Unit & Ud & B & MK \\
\hline $\mathrm{SiO}_{2}$ & $\% \mathrm{p} / \mathrm{p}$ & 43.9 & 45.3 & 55.2 \\
$\mathrm{CaO}$ & $\% \mathrm{p} / \mathrm{p}$ & 2.2 & 8.8 & 0.2 \\
$\mathrm{Na}_{2} \mathrm{O}$ & $\% \mathrm{p} / \mathrm{p}$ & 1.2 & 1.7 & 0.6 \\
$\mathrm{Al}_{2} \mathrm{O}_{3}$ & $\% \mathrm{p} / \mathrm{p}$ & 9.7 & 21.6 & 40.3 \\
$\mathrm{Fe}_{2} \mathrm{O}_{3}$ & $\% \mathrm{p} / \mathrm{p}$ & 5.4 & 8.5 & 1.4 \\
$\mathrm{SO}_{3}$ & $\% \mathrm{p} / \mathrm{p}$ & 1.4 & $<0.1$ & 0.2 \\
$\mathrm{MgO} \mathrm{P}_{2}$ & $\% \mathrm{p} / \mathrm{p}$ & 5.7 & 2.0 & 0.1 \\
$\mathrm{TiO}_{2}$ & $\% \mathrm{p} / \mathrm{p}$ & 0.7 & 0.7 & $<0.1$ \\
$\mathrm{ZnO}$ & $\% \mathrm{p} / \mathrm{p}$ & 0.8 & 0.2 & 1.5 \\
$\mathrm{~K}_{2} \mathrm{O}$ & $\% \mathrm{p} / \mathrm{p}$ & $<0.1$ & $<0.1$ & $<0.1$ \\
\hline
\end{tabular}

According to scientific bibliography, the chemical composition of basalt powder is particularly suitable for its alkali-activation, as verified during the mechanical characterization of the AAMs.

\subsection{Activators}

\subsubsection{Sodium Silicate}

$\mathrm{SS}\left(\mathrm{Na}_{2} \mathrm{SiO}_{3}\right)$ also commonly named as waterglass, is an aqueous solution of sodium oxide $\left(\mathrm{Na}_{2} \mathrm{O}\right)$ and silicon dioxide $\left(\mathrm{SiO}_{2}\right)$ mixed according to specific proportions. Modifying the ratio between $\mathrm{SiO}_{2}$ and $\mathrm{Na}_{2} \mathrm{O}$ one can obtain a solution with different properties, which is suitable for several applications, from the construction to the food field. The SS employed in this experimentation is a commercial product, with a $\mathrm{SiO}_{2} / \mathrm{Na}_{2} \mathrm{O}$ ratio of 1.99 and a viscosity of $150-250 \mathrm{MPa} \cdot \mathrm{s}$ at $20^{\circ} \mathrm{C}$.

\subsubsection{Sodium Hydroxide}

$\mathrm{SH}(\mathrm{NaOH})$ is a solution employed to dissolve aluminosilicate, increase the $\mathrm{pH}$ and to compensate the electric charge of the aluminates in the mixture. It is an inorganic compound that is a highly caustic base and highly soluble in water. A $10 \mathrm{M} \mathrm{SH}$ was used in the present work. According to scientific bibliography, the SH suggested molarity for AAMs, ranges between 8 and 12 M [29,30].

\subsection{Foaming Agent}

Hydrogen peroxide (HP) was used as chemical foaming agent in this research. The HP reacts to produce oxygen gas, and the expansion process of the AAMs' paste is due to the bubbles of $\mathrm{O}_{2}$ that are trapped into the mixture. It can be directly added to the AA mixture before curing, immediately developing the foaming process due to its sudden decomposition into water and oxygen gas. In this research, an HP solution 30\% w/w (110 vol.) was adopted.

\subsection{Experimental Program and Methods}

The experimental program was divided into three main steps. The first one was related to the alkali-activation synthesis and to the mechanical characterization of the AAMs. In this step, no foaming agent was added to the mixtures, which were characterized by their workability and compressive strength resistance [31]. The second phase of the research program was related to the 
production of LWAs. The correct amount of HP needed for the expansion process was evaluated and at the same time the mixing, casting, and curing procedures of the LWAs were defined. The third step focused on the LWAs characterization. The EN 13055-1 standard [1] specifies the properties of light aggregates either obtained through natural processes or artificially produced from natural or recycled materials, used in concrete, mortar and grout in buildings, roads, and other civil engineering applications. A set of tests was selected to investigate the most relevant properties of the LWAs, with the aim of evaluating their suitability to be used as construction materials. Different tests were carried out to characterize the alkali-activated LWAs. The geometrical properties were assessed based on the particle size distribution [32]. The physical characteristics were evaluated according to the loose bulk density [33], the water content [34] and the particle density and water absorption tests [35]. According to each reference standard, a specific number of samples was tested to have significant results.

A detailed analysis of porosity and pore size distribution of LWAs were also conducted by means of both MIP and NMR techniques.

The preparation of samples measured with TD-MRR was conducted as follows. The LWAs were dried in an oven at $60^{\circ} \mathrm{C}$ for $8 \mathrm{~h}$ and weighted (dry weight). Then the dried samples were saturated under vacuum with fresh water and weighted (saturated weight). To remove liquid from the outer surface of the aggregates (extrapellet liquid), shortly before TD-MRR analysis the LWA were removed from the liquid and rolled over a pre-soaked filter paper that ensures the liquid is not removed from the pores during the process. With the saturated and dry weights, it is possible to classically evaluate the quantity of absorbed water and therefore roughly check the quality of TD-NMR analysis. In fact, the intensity of the acquired TD-MRR signal is proportional to the amount of saturating water.

A few $20 \mathrm{~mm}$ internal-diameter test tubes were filled to a height of $30 \mathrm{~mm}$ (sensitive volume of the NMR probe) with the saturated LWAs and sealed with parafilm. TD-MRR relaxation signal curves were acquired with a console and a probe (equipped with a $25 \mathrm{~mm}$ internal-diameter coil) both manufactured by Stelar (Mede, PV, Italy), and using a permanent $0.18 \mathrm{~T}$ magnet (ESAOTE SpA, Genova, Italy).

Standard Inversion-Recovery (IR) and Carl-Purcell-Meiboom-Gill (CPMG) sequences [36] were used to acquire the $\mathrm{T}_{1}$ and $\mathrm{T}_{2}$ relaxation decay curves, respectively.

$\mathrm{T}_{1}$ and $\mathrm{T}_{2}$ relaxation decay curves were inverted to produce quasi-continuous relaxation time distributions by means of UpenWin (http://software.dicam.unibo.it/upenwin) a software that implements the 1D version of the inversion algorithm Upen [37]. Upen was specifically designed not to provide distribution details that are not supported by data, which might be misinterpreted, for example, as physically meaningful resolved pore compartments. To synthesize a complex distribution of values with only one single more manageable value, various scalar parameters (as for example, different kind of averages) could be computed from the relaxation time distribution itself. Distribution peaks position $\left(\mathrm{T}_{(1,2) \mathrm{pk}}\right)$ and geometric weighted average $\left(\mathrm{T}_{(1,2) \mathrm{g}}\right)$ are among these.

MIP experiments were performed by means of the mercury porosimeters PASCAL 140, measuring range $3.8 \mu \mathrm{m}-116 \mu \mathrm{m}$, and PASCAL 240, measuring range $7.4 \mathrm{~nm}-15 \mu \mathrm{m}$ (ThermoFisher Scientific, Waltham, Massachusetts, USA), where $5 \mathrm{~g}$ of the samples were measured using a mercury pressure range from 0 to 200 bar. The MIP data were analyzed by means of the SOL.I.D software (1.3.3, ThermoFisher Scientific, Waltham, Massachusetts, USA).

The results obtained during the characterization of the experimental aggregates, were always compared to values refer to traditional LECA. 


\section{Lightweight Aggregate Characterization}

\subsection{Alkali-Activated Material Characterization}

Two different mixtures were studied using the waste materials as precursors, combined with MK according to several proportions, to achieve adequate mechanical properties. In the mix-design step, different mixes were prepared varying the following variables:

- $\quad \mathrm{Ud} / \mathrm{MK}$ and B/MK;

- $\mathrm{SS} / \mathrm{SH}$;

- Activators/Precursors;

- Curing method.

The correct mix designs and curing method were chosen according to the workability of the alkali-activated pastes and the compressive strength of the cured mixtures. In the light of the above, the optimized mix designs for the two AAM are presented in Table 4 (precursors are in percentages on the weight of the mixtures).

Table 4. Mix design for AAM_Ud and AAM_B.

\begin{tabular}{cccccc}
\hline Mixture & Ud (\%) & B (\%) & MK (\%) & SS/SH & A/P \\
\hline AAM_Ud & 50 & 0 & 50 & 3 & 1 \\
AAM_B & 0 & 70 & 30 & 4 & 0.45 \\
\hline
\end{tabular}

The higher amount of MK in the AAM_Ud is adopted to achieve consistent compressive strengths. As mentioned before, the chemical composition of B makes this waste material suitable for the alkali activation process. The presence of MK is needed to optimize the mechanical properties of the AAM_B. Both mixtures had the same workability and the different $\mathrm{A} / \mathrm{P}$ ratio is mainly due the different absorption power of the mix of precursors. The performances in terms of compressive strength of both mixtures are shown in Figure 1 (avg. values of three samples). The tests were carried out on cubic samples $(40 \times 40 \mathrm{~mm})$ initially cured for $12 \mathrm{~h}$ in the oven at $70{ }^{\circ} \mathrm{C}$ and then kept at ambient temperature and humidity in the laboratory.

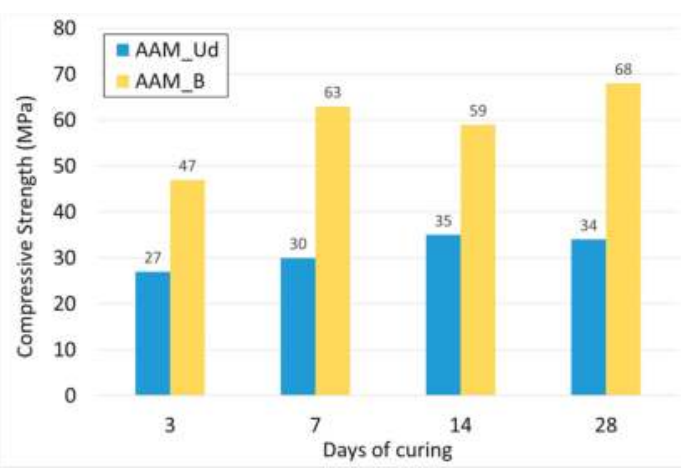

Figure 1. Compressive strength average results for AAM_Ud and AAM_B after 3, 7, 14 and 28 days of curing.

The different mechanical resistance of the two AAMs is clear. Despite the higher presence of MK into the AAM_Ud, the compressive strength for this mixture is almost half of the AAM_B one. This is mainly due to the chemical composition of $\mathrm{Ud}$, which has an $\mathrm{Al}_{2} \mathrm{O}_{3}$ content lower than 
basalt. According to scientific literature, a considerable amount of $\mathrm{Al}_{2} \mathrm{O}_{3}$ and $\mathrm{SiO}_{2}$ is favorable for the formation of a resistant microstructure during the AA process. As an overall result, it is worth noting that both mixtures reached significant compressive strength levels, if compared to other AAMs tested in several experimental applications [38-42] or traditional construction materials (cement concretes).

\subsection{LWA Synthesis and Production}

LWA synthesis starts from the addition of the expansion agent to the mixture after the mixing of precursors and activators for $10 \mathrm{~min}$. The quantity of $\mathrm{HP}$ to be added to the AAMs was chosen to obtain a high workability and a good level of expansion. A specific amount of HP was added to each mixture, due to the different composition of the two experimental AAMs. Several LWAs were produced with a HP concentration between $2 \%$ and $10 \%$ by the weight of the activators. As soon as the expansion process starts, the alkali-activated samples are produced by extruding the paste from a syringe and rolling the material to form the aggregate. After being placed at $70^{\circ} \mathrm{C}$ for $12 \mathrm{~h}$, the samples were weighted and crushed for a preliminary analysis of their mechanical resistance.

Table 5 shows the final mix design of both mixtures, in which the amount of HP is expressed in percentage on the weight of the activators. For the LWA_Ud, the quantity of MK was also increased from the original mix design to achieve a higher mechanical strength.

Table 5. Mix design for LWA_Ud and LWA_B.

\begin{tabular}{ccccccc}
\hline Mixture & Ud (\%) & B (\%) & MK (\%) & SS/SH & A/P & HP (\%) \\
\hline LWA_Ud & 40 & 0 & 60 & 3 & 1 & 5 \\
LWA_B & 0 & 70 & 30 & 4 & 0.45 & 7 \\
\hline
\end{tabular}

Figures 2 and 3 show the external and internal structure of the experimental LWAs and of the LECA one.

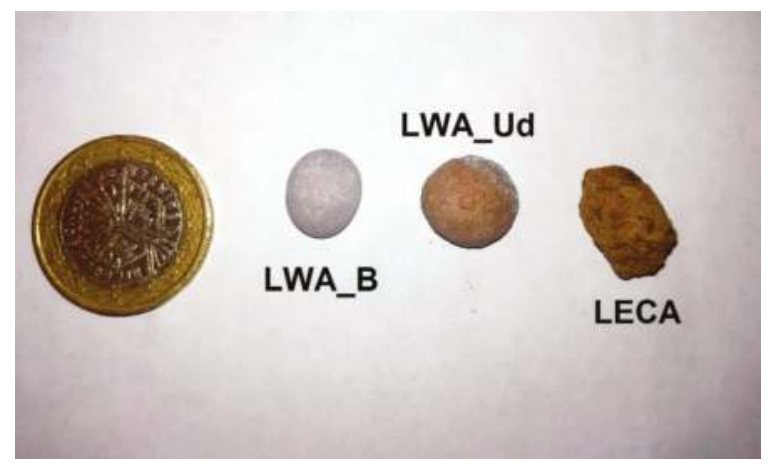

Figure 2. External shape of LWA_B (left), LWA_Ud (center) and LECA (right). 

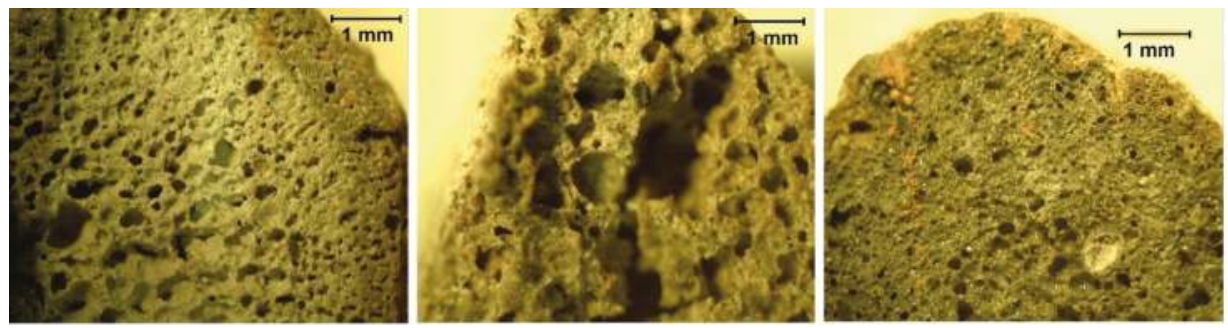

Figure 3. Inner structure of LWA_B (left), LWA_Ud (center) and LECA (right) by optical microscope.

The difference in terms of external shapes between the three samples is due to the different production methods: while the experimental LWAs are handcrafted in this preliminary research stage, the LECA is produced by heat treatment. This makes the external shape irregular and the inner structure rich in small voids. From the comparison of the pictures in Figure 3, it is almost clear the difference in terms of voids between the three different samples. The LWA_Ud seems to have the largest and irregular pores, while the LECA sample shows small and well distributed voids, as confirmed by the porosity analysis shown below.

\subsection{LWAs' Geometrical and Physical Properties}

The third stage of the research program is based on the LWA characterization. To evaluate the geometrical, physical, and mechanical characteristics of the experimental materials, laboratory tests were performed on samples in compliance with the EN 13055-1 standard [1]. The physical properties of the experimental LWAs and the reference one were assessed through the following tests:

- $\quad$ Particle size distribution [32];

- Loose bulk density and air voids [33];

- Water content [34];

- Particle density and Water absorption [35].

The physical analysis of LWAs was corroborated by means of NMR and MIP tests.

\subsubsection{Particle Size Distribution}

The determination of the particle size distribution of aggregates follows the EN 933-1 standard [32]. The test consists of dividing and separating the material into several particle size classifications of decreasing sizes by means of a series of sieves. The particle size distribution test is applied to every aggregate, including lightweight ones. Figure 4 shows the grading distributions of the three LWAs. 


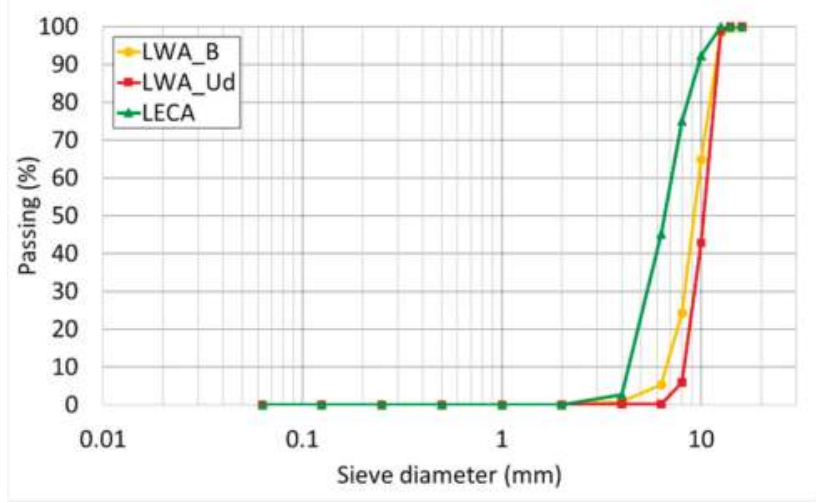

Figure 4. LWAs size distributions.

The particle sizes of the experimental aggregates are similar and can be classified as a $4 / 12.5 \mathrm{~mm}$ designation. The LECA grading distribution, even if classified according to the same class, is characterized by the presence of finer particles (Table 6).

Table 6. Passing material for LWA_B, LWA_Ud and LECA.

\begin{tabular}{cccc}
\hline Sieve $(\mathbf{m m})$ & Passing LWA_B (\%) & Passing LWA_Ud (\%) & Passing LECA (\%) \\
\hline 16 & 100.00 & 100.00 & 100.00 \\
14 & 99.71 & 100.00 & 100.00 \\
12.5 & 98.57 & 98.99 & 100.00 \\
10 & 64.90 & 42.84 & 92.39 \\
8 & 24.40 & 5.88 & 75.04 \\
6.3 & 5.39 & 0.17 & 45.11 \\
4 & 0.86 & 0.06 & 2.64 \\
1 & 0.00 & 0.00 & 0.00 \\
0.5 & 0.00 & 0.00 & 0.00 \\
0.063 & 0.00 & 0.00 & 0.00 \\
\hline
\end{tabular}

\subsubsection{Loose Bulk Density and Air Voids}

According to the EN 1097-3 standard [33], the loose bulk density calculated as the ratio between the mass of dry aggregates filling a specific container without compaction, and the capacity of that container. From the calculation of the loose bulk density, the air voids content is evaluated as the air-filled space between the aggregates filling the container. In compliance with the standard, a $5 \mathrm{~L}$ container was used considering the particle size distributions of the LWAs. Results are presented in Table 7.

Table 7. Loose bulk densities and voids content for the LWAs.

\begin{tabular}{cccc}
\hline & LWA_B & LWA_Ud & LECA \\
\hline L.b.d._Sample $1\left(\mathrm{Mg} / \mathrm{m}^{3}\right)$ & 0.701 & 0.473 & 0.422 \\
L.b.d.-Sample $2\left(\mathrm{Mg} / \mathrm{m}^{3}\right)$ & 0.703 & 0.476 & 0.420 \\
L.b.d.-Sample 3 $\left(\mathrm{Mg} / \mathrm{m}^{3}\right)$ & 0.702 & 0.475 & 0.424 \\
Avg. L.b.d. $\left(\mathrm{Mg} / \mathrm{m}^{3}\right)$ & 0.702 & 0.475 & 0.422 \\
Avg. Air voids $(\%)$ & 44.3 & 36.0 & 43.5 \\
\hline
\end{tabular}


The loose bulk density for the LWA produced with basalt is higher if compared to the other LWAs, and this is due to the higher mass of the samples. It is worth noting that the EN 13055-1 standard [1] classifies as lightweight the aggregates with a loose bulk density not exceeding $1.20 \mathrm{Mg} / \mathrm{m}^{3}$. There is not significant difference between LWA_Ud and LECA. If the air voids are taken into account, the different results are mainly related to the grading curve of each material and the related particles shape. The standard does not specify any limits or range of values for this parameter.

\subsubsection{Water Content}

According to the EN 1097-5 standard [34], the water content of aggregates is evaluated by successive weighing of samples placed in a ventilated oven $\left(110 \pm 5^{\circ} \mathrm{C}\right)$ until a constant mass is reached. The water content is determined as the difference between the wet and the dry mass and it is expressed as a percentage of the dry mass of the test portion. Table 8 shows the results.

Table 8. Water content for the LWAs.

\begin{tabular}{cccc}
\hline & LWA_B & LWA_Ud & LECA \\
\hline Water content (\%)—Sample 1 & 1.27 & 4.10 & 0.19 \\
Water content (\%)—Sample 2 & 1.27 & 4.44 & 0.17 \\
Avg. Water content (\%) & 1.27 & 4.27 & 0.18 \\
\hline
\end{tabular}

The water content varies from $4.27 \%$ of LWA_Ud to $0.18 \%$ measured for the LECA samples. The results are highly influenced by the storing condition of the samples. All the material were kept at ambient temperature inside the laboratory, stored in plastic bags. Both tests on basalt and expanded clay aggregates were performed during a dry season, whereas the tests on Ud specimens were performed when climate conditions were more humid. In the light of the above, further test are needed to verify the water content of LWAs on similar storing conditions. It shall be mentioned that EN 13055-1 [1] does not specify any water content limit for the LWAs.

\subsubsection{Particle Density and Water Absorption}

The EN 1097-6 standard [35] describes the pycnometer method for the evaluation of three different density parameters and the water absorption values for LWAs. According to the standard, the particle density is given by the ratio of mass to volume. Based on the conditions of weighting the test portion, the density is considered in saturated, saturated, and surface-dried (SSD) and in the oven-dried condition. The water absorption is then calculated using a soaking time of $24 \mathrm{~h}$. Average results are presented in Table 9.

The densities of the three LWAs are different because of the inner structure given by the expansion process. LECA is the lightest material while LWA_B is the heaviest one. It is worth noting that according to the EN 13055-1 standard [1] the aggregates with a particle density not exceeding $2.00 \mathrm{Mg} / \mathrm{m}^{3}$ are classified as lightweight. The water absorption values are strictly connected to the pores dimensions. Further observations on particle densities and porosity are shown in the porosity analysis section of this paper.

Table 9. Apparent, oven-dried, SSD particle densities and water absorption values for LWAs.

\begin{tabular}{cccc}
\hline & LWA_B & LWA_Ud & LECA \\
\hline Apparent particles density $\left(\mathrm{Mg} / \mathrm{m}^{3}\right)$ & 1.69 & 1.18 & 0.87 \\
Oven-dried particles density $\left(\mathrm{Mg} / \mathrm{m}^{3}\right)$ & 1.26 & 0.74 & 0.75 \\
SSD particles density $\left(\mathrm{Mg} / \mathrm{m}^{3}\right)$ & 1.52 & 1.11 & 0.85 \\
Water absorption after $24 \mathrm{~h}(\%)$ & 20 & 50 & 17 \\
\hline
\end{tabular}




\subsubsection{Analysis of Porosity}

To obtain the relaxation time distributions, the relaxation decay curves were inverted by means of UpenWin.

At first sight, the $T_{2}$ distributions (not shown) encoded at different echo time, allow us to state that there are not evident diffusion effects, more precisely, the $T_{2}$ distributions do not change varying the echo time. Therefore, the relaxation time distributions are strictly related to the local diffusion cell and consequently they are representative of the PSD. In Figure 5 the $T_{1}$ distributions of samples are shown. All the distributions show a well-defined and narrow peak at long times (around $1 \mathrm{~s}$ ) followed by long tail with a hump at shorter times. The areas under the relaxation curves are proportional to the NMR signals which, in turn, are proportional to the amount of saturating water.

The $\mathrm{T}_{1 \mathrm{pk}}$ of the three distributions are respectively: 1108, 1417 and 1519 milliseconds. Table 10 summarizes the weights and the NMR signal intensity computed on the distributions. The good proportionality of column 4 and 5, the maximum percentage discrepancy of their ratio (column 6) is approximately 7\%, allows us to state that the TD-MRR measurements were significant and reliable.

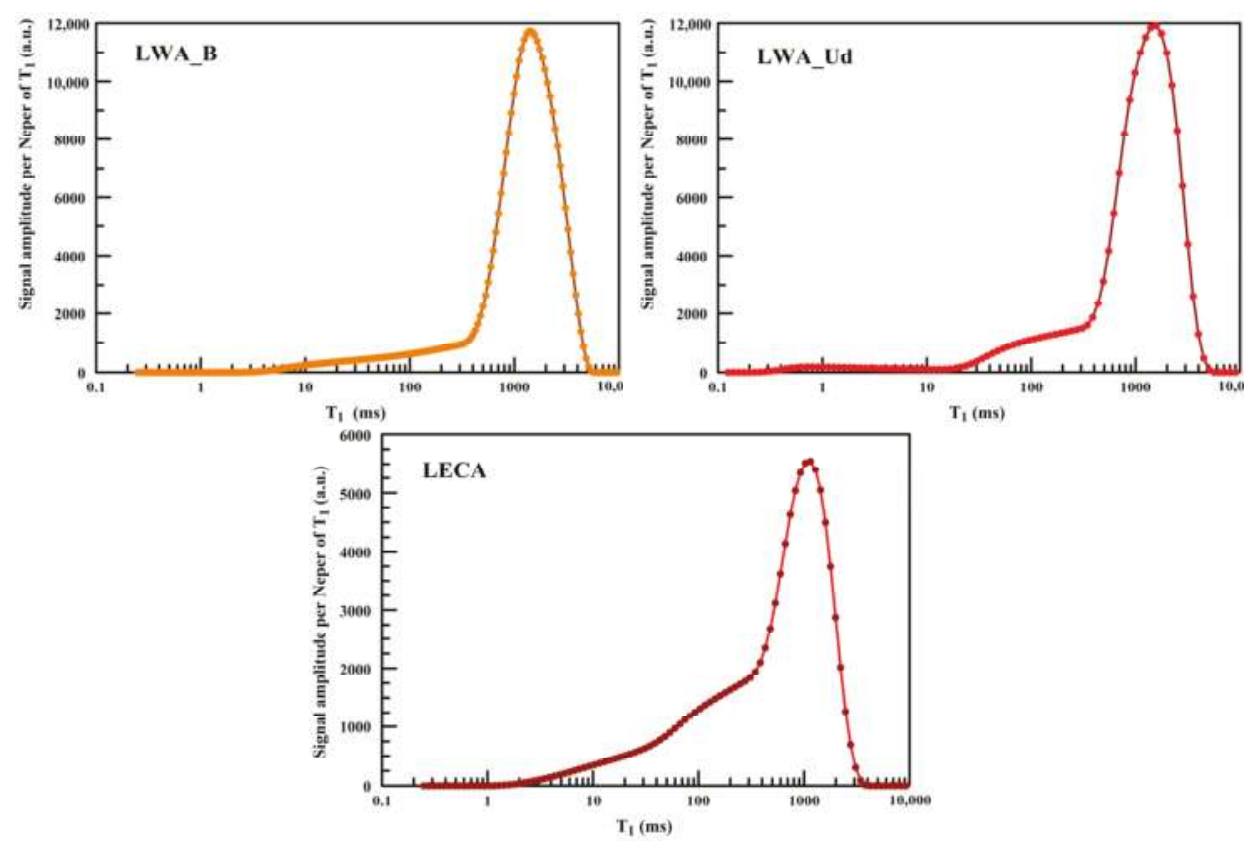

Figure 5. T 1 distributions for LWA_B, LWA_Ud and LECA.

Table 10. Weight of the samples used for the NMR measurements and the total NMR signal of the $\mathrm{T}_{1}$ distributions.

\begin{tabular}{cccccc}
\hline Sample & $\begin{array}{c}\text { Dry Weight } \\
(\mathbf{g})\end{array}$ & $\begin{array}{c}\text { Saturated Weight } \\
\mathbf{( g )}\end{array}$ & $\begin{array}{c}\text { Absorbed Water } \\
\mathbf{( g )}\end{array}$ & $\begin{array}{c}\text { T1 Total Signal } \\
\text { (Arbitrary Unit) }\end{array}$ & Ratio [-] \\
\hline LWA_B & 5.7 & 8.9 & 3.2 & 19,050 & 5953 \\
LWA_Ud & 4.1 & 7.7 & 3.6 & 21,290 & 5914 \\
LECA & 4.4 & 6.5 & 2.1 & 11,690 & 5567 \\
\hline
\end{tabular}


The TD-MRR PSDs obtained by calibrating the TD-MRR relaxation time distributions with MIP results are shown in Figure 6. The samples have significantly different PSDs. In particular, small pores in the range from few nanometers to some micrometers characterize the LECA aggregates. LWA_B and LWA_Ud have larger pores in the range of $0.1-200 \mathrm{~mm}$. If the two experimental aggregates are compared, the LWA_Ud has the larger pores, with a consistent density of pores whose size ranges between 100 and $200 \mathrm{~mm}$. Moreover, form the analysis of the area under the distribution curves, both experimental aggregates are characterized by a more homogeneous pore size distribution. These results are in line with that verified from the optical microscope images shown in Figure 3. The inner structure of LECA consists of small pores if compared to the experimental aggregates, while the Ud one has the largest diameter voids.
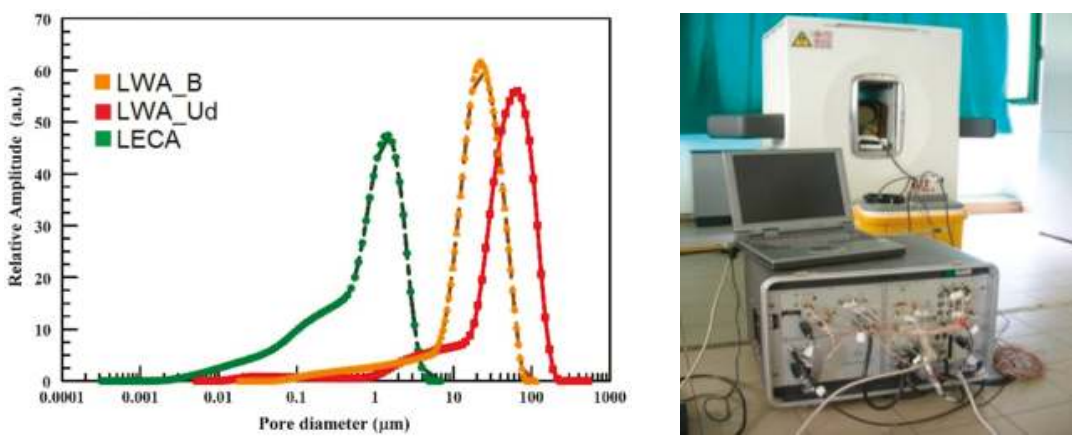

Figure 6. TD-MRR PSDs for LWA_B, LWA_Ud and LECA (left) and NMR equipment (right).

\subsection{LWAs' Mechanical Properties}

The mechanical characterization of LWAs is made through the evaluation of their crushing resistance. The EN 13055-1 standard [1] specifies the apparatus and two different test procedures related the loose bulk density of the LWAs. Based on previous results, the procedure n. 2 was adopted. According to the standard, three test specimens were prepared by filling a specific steel cylinder with aggregates. When compacted, the LWAs surface was leveled to the top rim of the container, which was then subject to a force given by a piston set to reach a compression of $20 \mathrm{~mm}$ in approximately $100 \mathrm{~s}$. The crushing resistance for each test specimen was than calculated using an equation that considers the load exerted by the piston, its area and the compression force and the area. Average results are shown in Table 11.

Table 11. Compression force and crushing resistance for LWA_B, LWA_Ud and LECA.

\begin{tabular}{cccc}
\hline & LWA_B & LWA_Ud & LECA \\
\hline Compression force $(\mathrm{N})$ & 51,100 & 12,150 & 38,050 \\
Crushing resistance $\left(\mathrm{N} / \mathrm{mm}^{2}\right)$ & 4.44 & 1.07 & 3.31 \\
\hline
\end{tabular}

Comparing the results, LWA_B shows the highest mechanical performance: its crushing resistance is three times that of LWA_Ud. If LECA is taken into account, the increase in crushing resistance for LWA_B is about $34 \%$. As general remark, it must be noted that the standard does not specify any limits or range of results in terms of crushing resistance for LWAs. However, considering the wide field of applications of these materials, a minimum value of crushing resistance is required for LWAs to be used as construction materials. Generally, according to the different uses in the civil engineering field, the threshold limit is set at $0.7 \mathrm{~N} / \mathrm{mm}^{2}$. As an example, for foundation layers between the floor and the finishing flooring, for the thermal and acoustic insulation of a building, a crushing resistance between 
0.7 and 1.5 is generally required. For structural lightweight concrete, this range is raised to a minimum of $4.5 \mathrm{~N} / \mathrm{mm}^{2}$ while for lightweight asphalt concretes the Italian technical specification requires a crushing resistance higher than $2.7 \mathrm{~N} / \mathrm{mm}^{2}$. In the light of the above, both the experimental LWAs meet the required minimum mechanical properties for their applications in the civil engineering field.

\section{Conclusions}

The study presented in the paper shows a new method to produce alternative LWAs by AA of two different waste powders. Two experimental alkali-activated LWAs have been developed using a basalt powder and a digested spent bentonite clay as main precursors. Based on the data presented in this work, several conclusions can be drawn which corroborate the validity and the smartness of the proposed solution for the production of alternative LWAs:

- The adopted waste materials were suitable for the AA process. The addition of HP to the pastes allowed the production of lightweight aggregates in the laboratory. The optimum quantity of the foaming agent was determined to obtain a workable paste and final aggregates with a good porosity-to-strength ratio.

- Because of the physical characterization of the LWAs, both the experimental materials showed appropriate properties to be classified as lightweight aggregates according to the EN 13055-1 standard [1]. LWA_B and LWA_Ud have densities lower than $2.00 \mathrm{Mg} / \mathrm{m}^{3}$ and a loose bulk density not exceeding $1.20 \mathrm{Mg} / \mathrm{m}^{3}$.

- Mechanical characterization was performed through the crushing resistance test. Even if the EN 13055-1 [1] does not specify any limits for LWAs, a specific crushing resistance value is required for their application as construction material. LWA_Ud has a crushing resistance $\left(1.07 \mathrm{~N} / \mathrm{mm}^{2}\right)$ higher than the lower limit usually specified for the application of LWAs in civil engineering projects. LWA_B has the highest mechanical resistance $\left(4.44 \mathrm{~N} / \mathrm{mm}^{2}\right)$ even if compared to the structural expanded clay, LECA, used in this research as reference lightweight construction material. These results are in line with the values recorded during the preliminary mechanical characterization of the alkali-activated paste materials and are mainly dependent on the chemical composition of the wastes used as precursors.

- The voids of the LWAs materials were studied by means of the Time Domain NMR Relaxometry of protons ( ${ }^{1} \mathrm{H}$ TD-MRR). The TD-MRR results were then compared with the porosity analysis carried out with the MIP method. In this work, the relaxation time distributions are strictly related to the local diffusion cell and effectively represent the distribution of pore sizes (PSD). From the correlation with MIP results, the TD-MRR PSD shows a structure rich in small voids for LECA samples, while larger and more size-homogeneous pores characterize the experimental aggregates. These results are corroborated by the visual analysis of the optical microscope images, which confirm the presence of larger voids for LWA_Ud if compared to LWA_B and LECA. Small pores instead characterize the latter.

In light of the above, the expansion of these AA waste materials seems to be a viable solution for the production of lightweight aggregates, allowing the recycling of materials otherwise dumped or sent to landfill. Moreover, the results confirm and validate the analysis of pore sizes and their distribution in lightweight aggregates according to TD-MRR and MIP methodologies.

Further investigations and tests are planned to back up these preliminary conclusions and to confirm the use of AA LWAs as construction materials. Additional research will focus on the Life Cycle Analysis of the proposed solution to justify their development on an industrial scale.

Author Contributions: Conceptualization, P.T. and C.S.; Methodology, C.S., P.T. and V.B.; Software, V.B.; Validation, C.S. and V.B.; Formal Analysis, C.S. and V.B.; Investigation, P.T. and G.M.B.; Resources, C.S.; Data Curation, P.T. and V.B.; Writing-Original Draft Preparation, P.T., G.M.B. and V.B.; Writing-Review \& Editing, C.S., P.T. and V.B.; Visualization, P.T. and V.B.; Supervision, C.S.; Project Administration, P.T. In general, the authors declare to have equally contributed to the investigation and the publication of these key results. 
Funding: This research received no external funding.

Acknowledgments: The authors wish to thank E. Cappellari, A. Lodi, B. Ravaglia and F. Peddis (DICAM, University of Bologna, Italy) for their technical assistance.

Conflicts of Interest: The authors declare no conflict of interest.

\section{References}

1. EN 13055-1. Lightweight aggregates. Lightweight aggregates for concrete, mortar and grout, 2002. Available online: https://standards.globalspec.com/std/873109/cen-en-13055-1 (accessed on 20 July 2018).

2. Arioz, O.; Kilinc, K.; Karasu, B.; Kaya, G.; Arslan, G.; Tuncan, M.; Korkut, M.; Kivrak, S. A preliminary research on the properties of lightweight expanded clay aggregate. J. Aust. Ceram. Soc. 2008, 44, 23-30.

3. Ayati, B.; Ferrándiz-Mas, V.; Newport, D.; Cheeseman, C. Use of clay in the manufacture of lightweight aggregate. Constr. Build. Mat. 2018, 162, 124-131. [CrossRef]

4. Sangiorgi, C.; Tataranni, P.; Mazzotta, F.; Simone, A.; Vignali, V.; Claudio, L. Alternative Fillers for the Production of Bituminous Mixtures: A Screening Investigation on Waste Powders. Coatings 2017, 7, 76. [CrossRef]

5. Tataranni, P.; Sangiorgi, C.; Simone, A.; Vignali, V.; Claudio, L.; Dondi, G. A laboratory and field study on 100\% Recycled Cement Bound Mixture for base layers. Int. J. Pavement Res. Technol. 2017. [CrossRef]

6. Sangiorgi, C.; Tataranni, P.; Simone, A.; Vignali, V.; Claudio, L.; Dondi, G. Stone mastic asphalt (SMA) with crumb rubber according to a new dry-hybrid technology: A laboratory and trial field evaluation. Constr. Build. Mat. 2018, 182, 200-209. [CrossRef]

7. Narattha, C.; Chaipanich, A. Phase characterizations, physical properties and strength of environment-friendly cold-bonded fly ash lightweight aggregates. J. Clean. Prod. 2018, 171, 1094-1100. [CrossRef]

8. Gesoğlu, M.; Özturan, T.; Güneyisi, E. Effects of fly ash properties on characteristics of cold-bonded fly ash lightweight aggregates. Constr. Build. Mat. 2007, 21, 1869-1878. [CrossRef]

9. Walkley, B.; Rees, G.J.; San Nicolas, R.; van Deventer, J.S.J.; Hanna, J.V.; Provis, J.L. New structural model of hydrous sodium aluminosilicate gels and the role of charge-balancing extra-framework Al. J. Phys. Chem. C 2018, 122, 5673-5685. [CrossRef]

10. Provis, J.L. Alkali-activated materials. Cem. Concr. Res. 2017, in press. [CrossRef]

11. Park, S.M.; Jang, J.G.; Lee, N.K.; Lee, H.K. Physicochemical properties of binder gel in alkali-activated fly ash/slag exposed to high temperatures. Cem. Concr. Res. 2016, 89, 72-79. [CrossRef]

12. Park, S.M.; Jang, J.G.; Lee, N.K. Unlocking the role of $\mathrm{MgO}$ in the carbonation of alkali-activated slag cement. Inorg. Chem. Front. 2018, 5, 1661-1670. [CrossRef]

13. Pacheco-Torgal, F.; Castro-Gomes, J.; Jalali, S. Alkali-activated binders: A review: Part 1. Historical background, terminology, reaction mechanisms and hydration products. Constr. Build. Mat. 2008, 22, 1305-1314. [CrossRef]

14. Fernández-Jiménez, A.; Palomo, A. Nanostructure/microstructure of fly ash geopolymers. In Geopolymers; Civil and Structural Engineering; Woodhead Publishing: Sawston, UK, 2009; pp. 89-117.

15. Provis, J.L.; Van Deventer, J.S.J. Introduction to geopolymers. In Geopolymers; Woodhead Publishing: Sawston, UK, 2009; pp. 1-11.

16. Khale, D.; Chaudhary, R. Mechanism of geopolymerization and factors influencing its development: A review. J. Mat. Sci. 2007, 42, 729-746. [CrossRef]

17. Part, W.K.; Ramli, M.; Cheah, C.B. An overview on the influence of various factors on the properties of geopolymer concrete derived from industrial by-products. Constr. Build. Mat. 2015, 77, 370-395. [CrossRef]

18. Jo, B.W.; Park, S.K.; Park, J.B. Properties of concrete made with alkali-activated fly ash lightweight aggregate (AFLA). Cem. Concr. Compos. 2007, 29, 128-135. [CrossRef]

19. Bui, L.A.T.; Hwang, C.L.; Chen, C.T.; Lin, K.L.; Hsieh, M.Y. Manufacture and performance of cold bonded lightweight aggregate using alkaline activators for high performance concrete. Constr. Build. Mat. 2012, 35, 1056-1062. [CrossRef] 
20. Yliniemi, J.; Nugteren, H.; Illikainen, M.; Tiainen, M.; Weststrate, R.; Niinimäki, J. Lightweight aggregates produced by granulation of peat-wood fly ash with alkali activator. Int. J. Miner. Process. 2016, 149, 42-49. [CrossRef]

21. Yliniemi, J.; Tiainen, M.; Illikainen, M. Microstructure and physical properties of lightweight aggregates produced by alkali activation-high shear granulation of FBC recovered fuel-biofuel fly ash. Waste Biomass Valoriz. 2016, 7, 1235-1244. [CrossRef]

22. Brizi, L.; Barbieri, M.; Baruffaldi, F.; Bortolotti, V.; Fersini, C.; Liu, H.; Nogueira d’Eurydice, M.; Obruchkov, S.; Zong, F.; Galvosas, P.; et al. Bone volume-to-total volume ratio measured in trabecular bone by single-sided NMR devices. Magn. Reson. Med. 2018, 79, 501-510. [CrossRef] [PubMed]

23. Bortolotti, V.; Fantazzini, P.; Mongiorgi, R.; Sauro, S.; Zanna, S. Hydration kinetics of cements by Time-Domain Nuclear Magnetic Resonance: Application to Portland-cement-derived endodontic pastes. Cem. Concr. Res. 2012, 42, 577-582. [CrossRef]

24. Halperin, W.P.; D'Orazio, F.; Bhattacharija, S.; Tarczon, J.C. Magnetic resonance relaxatio analysis of porous media. In Molecular Dynamics in Restricted Geometries; Klafter, K., Drake, J.M., Eds.; Wiley: New York, NY, USA, 1989.

25. Kenyon, W.E. Petrophysical Principles of Applications of NMR Logging. Log Anal. 1997, 38, 21-43.

26. Dobiszewska, M.; Beycioğlu, A. Investigating the Influence of Waste Basalt Powder on Selected Properties of Cement Paste and Mortar. In IOP Conference Series: Materials Science and Engineering; IOP Publishing: Bristol, UK, 2017; Volume 245.

27. Saraya, M.E.-S.I.; El-Fadaly, E. Preliminary Study of Alkali Activation of Basalt: Effect of NaOH Concentration on Geopolymerization of Basalt. J. Mat. Sci. Chem. Eng. 2017, 5, 58-76.

28. Elimbi, A.; Tchakoute, H.K.; Njopwouo, D. Effects of calcination temperature of kaolinite clays on the properties of geopolymer cements. Constr. Build. Mat. 2011, 25, 2805-2812. [CrossRef]

29. Somna, K.; Jaturapitakkul, C.; Kajitvichyanukul, P.; Chindaprasirt, P. NaOH-activated ground fly ash geopolymer cured at ambient temperature. Fuel 2011, 90, 2118-2124. [CrossRef]

30. Hamidi, R.M.; Man, Z.; Azizli, K.A. Concentration of NaOH and the Effect on the Properties of Fly Ash Based Geopolymer. Proc. Eng. 2016, 148, 189-193. [CrossRef]

31. EN 1015-11. Methods of Test for Mortar for Masonry. Determination of Flexural and Compressive Strength of Hardened Mortar, 2007. Available online: https://standards.globalspec.com/standards/detail?docid= 10180975 (accessed on 20 July 2018).

32. EN 933-1. Tests for Geometrical Properties of Aggregates. Determination of Particle Size Distribution. Sieving Method, 2013. Available online: https://standards.globalspec.com/standards/detail?docid= 1664473\&familyid=FDYOHBAAAAAAAAAA (accessed on 20 July 2018).

33. EN 1097-3. Tests for Mechanical and Physical Properties of Aggregates. Determination of Loose Bulk Density and Voids, 1998. Available online: https://standards.globalspec.com/standards/detail?docid=853630\& familyid=MFANHBAAAAAAAAAA (accessed on 20 July 2018).

34. EN 1097-5. Tests for Mechanical and Physical Properties of Aggregates. Determination of the Water Content by Drying in a Ventilated Oven, 2013. Available online: https://standards.globalspec.com/standards / detail?docid=1664391\&familyid=RCRPHBAAAAAAAAAA (accessed on 20 July 2018).

35. EN 1097-6. Tests for Mechanical and Physical Properties of Aggregates. Determination of Particle Density and Water Absorption, 2013. Available online: https://standards.globalspec.com/standards/detail?docid= 1618572\&familyid=KFANHBAAAAAAAAAA (accessed on 20 July 2018).

36. Farrar, T.; Becker, E. Pulse and Fourier Transform NMR_Introduction to Theory and Methods; Academic Press: New York, NY, USA, 1971.

37. Bortolotti, V.; Brown, R.J.S.; Fantazzini, P.; Landi, G.; Zama, F. Uniform Penalty inversion of two-dimensional NMR relaxation data. Inverse Prob. 2016, 33, 015003. [CrossRef]

38. Thakur, R.N.; Ghosh, S. Effect of mix composition on compressive strength and microstructure of fly ash based geopolymer composites. ARPN J. Eng. Appl. Sci. 2009, 4, 68-74.

39. Dassekpo, J.B.M.; Zha, J.; Zhan, J. Compressive strength performance of geopolymer paste derived from Completely Decomposed Granite (CDG) and partial fly ash replacement. Constr. Build. Mat. 2017, 138, 195-203. [CrossRef]

40. Kastiukas, G.; Zhou, X. Effects of waste glass on alkali-activated tungsten mining waste: Composition and mechanical properties. Mat. Struct. 2017, 50, 194. [CrossRef] 
41. Li, Y.-L.; Zhao, X.-L.; Raman, R.K.S.; Al-Saddi, S. Thermal and mechanical properties of alkali-activated slag paste, mortar and concrete utilising seawater and sea sand. Constr. Build. Mat. 2018, 159, 704-724. [CrossRef]

42. Tho-In, T.; Sata, V.; Boonserm, K.; Chindaprasirt, P. Compressive strength and microstructure analysis of geopolymer paste using waste glass powder and fly ash. J. Clean. Prod. 2018, 172, 2892-2898. [CrossRef]

(c) (1)

(C) 2018 by the authors. Licensee MDPI, Basel, Switzerland. This article is an open access article distributed under the terms and conditions of the Creative Commons Attribution (CC BY) license (http:/ / creativecommons.org/licenses/by/4.0/). 
Article

\title{
Self-Healing Capacity of Asphalt Mixtures Including By-Products Both as Aggregates and Heating Inductors
}

\author{
Marta Vila-Cortavitarte ${ }^{1, *}$, Daniel Jato-Espino ${ }^{1}$, Daniel Castro-Fresno ${ }^{1}$ and \\ Miguel Á. Calzada-Pérez ${ }^{2}$ \\ 1 GITECO Research Group, Universidad de Cantabria, 39005 Santander, Spain; jatod@unican.es (D.J.-E.); \\ castrod@unican.es (D.C.-F.) \\ 2 GCS Research Group, Universidad de Cantabria, 39005 Santander, Spain; calzadam@unican.es \\ * Correspondence: vilam@unican.es; Tel.: +34-942-201-703
}

Received: 13 April 2018; Accepted: 11 May 2018; Published: 15 May 2018

\begin{abstract}
Major advances have been achieved in the field of self-healing by magnetic induction in which the addition of metallic particles into asphalt mixtures enables repairing their own cracks. This technology has already been proven to increase the life expectancy of roads. Nevertheless, its higher costs in comparison with conventional maintenance caused by the price of virgin metallic particles still makes it unattractive for investment. This research aimed at making this process economically accessible as well as environmentally efficient. To this end, an intense search for suitable industrial by-products to substitute both the virgin metal particles and the natural aggregates forming asphalt mixtures was conducted. The set of by-products used included sand blasting wastes, stainless shot wastes, and polished wastes as metallic particles and other inert by-products as aggregates. The results demonstrated that the by-products were adequately heated, which leads to satisfactory healing ratios in comparison with the reference mixture.
\end{abstract}

Keywords: self-healing; asphalt mixtures; induction heating; magnetic induction; metallic by-products

\section{Introduction}

Self-healing is the process whereby asphalt can partially recover from damage and, therefore, produce an increase in the lifetime of roads and can limit the need for their maintenance. In other words, self-healing provides an opportunity to reduce road disruptions and attenuate the economic and environmental impacts stemming from the natural resources required to undertake traditional maintenance practices [1]. However, this process only happens innately in the absence of traffic loads and under ideal conditions in terms of temperature, which enables bitumen flowing to fill small cracks caused by aging phenomena [2]. The incorporation of different additives into asphalt mixtures contributes toward overcoming these limitations and improving their healing capacity. These catalyzers include encapsulated rejuvenators, nanoparticles, and metallic particles heated via either electromagnetic induction or microwaves [3]. Among them, induction heating has been found to be the most widely used approach for healing asphalt mixtures [4]. This technique consists of the resistance of conductive materials against cross Eddy currents induced by a magnetic field. In the context of asphalt mixtures, the process of magnetic induction only leads to the heating of the metallic particles performing as additives and having no effect on bitumen or aggregates [5].

The healing capacity of asphalt mixtures has become a widely researched topic over the last decade especially during the last three years. Liu et al. [6] examined the capacity of porous asphalt mixtures with steel fibers and wool to be heated via induction. Long steel wool with a small diameter 
was found to be more effective than short steel fibers with a large diameter to enhance the electrical conductivity of the mixtures. In contrast, the results obtained by García et al. [7] from 25 dense asphalt concrete mixtures with different lengths, quantities, and amounts of steel wool fibers suggested that the induction heating of the mixtures was enhanced using short fibers with large diameters. One year later, García et al. [8] extended the addition of steel wool fibers to porous asphalt. The healing rates of the mixtures were limited to $78 \%$ due to their deformation as a result of the heating process. Menozzi et al. [9] studied the reparation of microcracks caused by fatigue damage in dense asphalt mixtures including cast steel particles. The results demonstrated that the healing of the samples via induction heating enabled the extension of the lifetime of the samples up to $31 \%$. Bueno et al. [10] subjected a series of up-scaled asphalt slabs with cast iron particles to different loading cycles, which proves that the application of induction heating at the initiation of the cracks produced and enabled the recovery of the original performance of the specimens. Norambuena-Contreras and Garcia [11] compared the healing degree of dense asphalt mixtures with steel wool fibers by microwave and induction heating, which means that the former resulted in a better crack closure due to the higher temperature of the binder. Zhu et al. [12] applied micro-wave heating to a Stone Mastic Asphalt (SMA-13) mixture containing Ni-Zn ferrite powder, which was proven to significantly improve the healing ability of the specimens tested. Franesqui et al. [13] found that the cracks of different asphalt mixtures, such as Asphalt Concrete (AC), Béton Bitumineux Minces (BBTM) and Porous Asphalt (PA), were completely closed when heating several industrial wastes (steel wool, steel filing, and metallic powder) via microwaves.

In light of the target of these studies and the costs and emissions involved by using virgin metallic particles, this research aimed at assessing the healing capacity of asphalt mixtures containing different industrial by-products. This proves that these materials could be used as a substitute for raw materials when heated under magnetic induction. Therefore, the underlying goal sought was the valorization of a series of potentially conductive by-products including metallic particles in order to improve the viability of the healing process of asphalt mixtures in comparison with conventional maintenance practices by increasing its investment attractiveness and environmental efficiency.

\section{Materials and Methods}

The accomplishment of the objectives established in this research was approached by following the six steps shown in Figure 1. The first step consisted of contacting local metal industries to ask about their processes, their by-products, and potential collaboration by giving some samples for testing. Afterward, the collected by-products were fully characterized in laboratory including a heating under induction test over the metallic-based by-products to check the temperature they could achieve. The selection of the most suitable materials and the dosage of mixtures formed the fourth step, which was followed by the specimen preparation. Lastly, the calculation of a healing ratio by comparing the fracture resistance before and after healing illustrated the suitability of each experimental mixture. Steps three to six could be repeated at different times to optimize mixture designs in order to reach better healing ratios.

The mixture designed for the study was a dense asphalt concrete (AC-16) made of ophite as a coarse aggregate, limestone as a fine aggregate, and ferromagnetic particles to make the induction process possible. Both aggregates and ferromagnetic particles could be changed by one or more of the tested by-products.

The industrial by-products used for the replacement of raw materials are included in Figure 2. Nine different local industries provided samples of their by-products. As a result, 17 different by-products were provided to the laboratory, which were divided into four groups for simplification purposes. This includes by-products coming from sandblasting processes (SBx), slags from different processes (Sx), demolding sands (DSx), and by-products from machining processes (MBx). In addition, virgin metal particles for shooting (Virgin Metal Particles, REF) were used as a reference for the metallic by-products. 


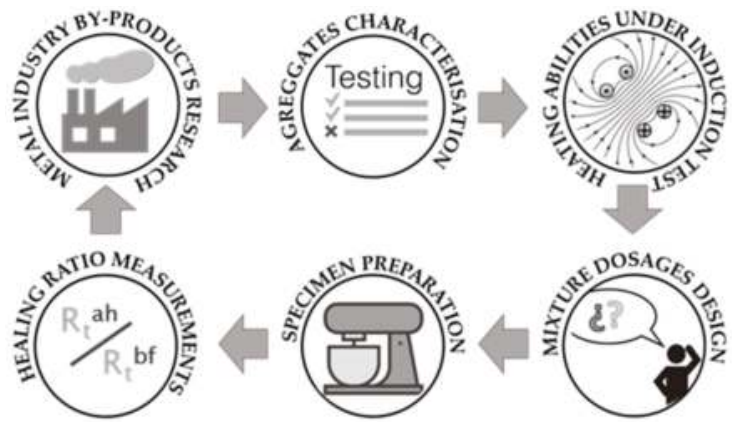

Figure 1. Flowchart of the six stages forming the proposed methodology.

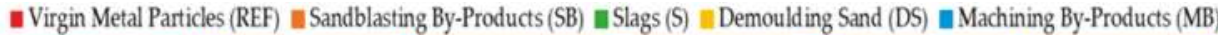
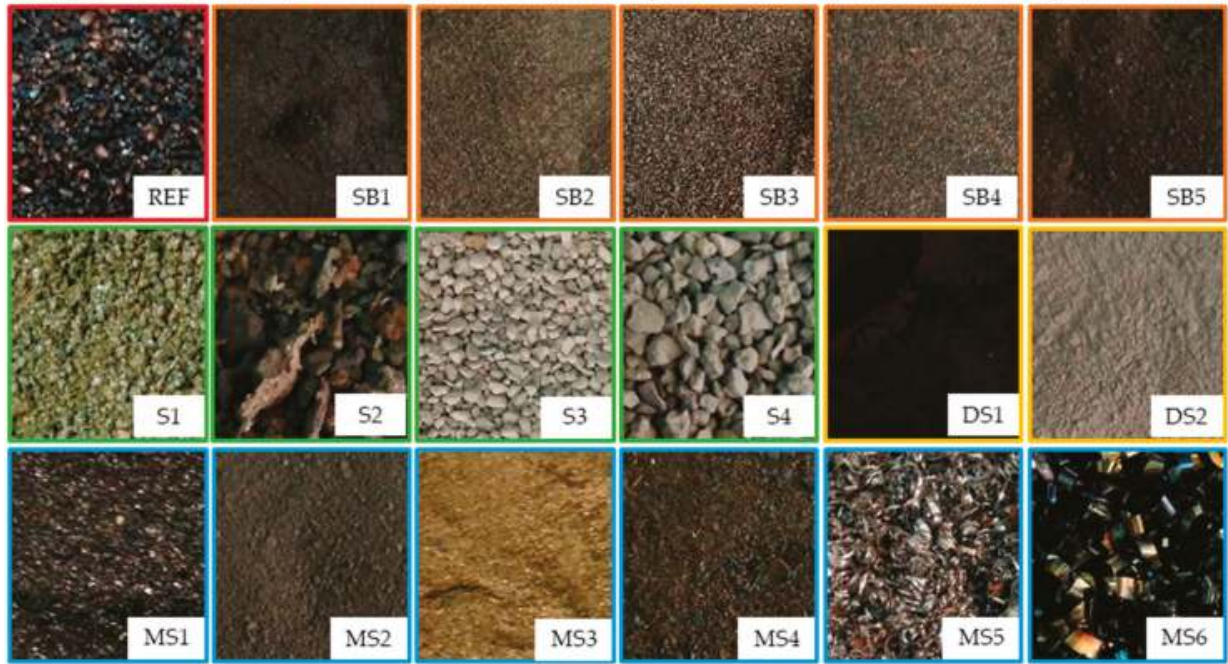

Figure 2. Textures of the set of by-products collected from local metal industries.

In particular, the by-products shown in Figure 2 were blasting shoot wastes (SB1 and SB4), steel grit wastes (SB2 and SB3), sand wastes from sandblasting processes (SB5), green foundry slags (S1), bronze foundry slags (S2), arc-electric foundry slags (S3 and S4), demolding sand from different foundries (DS1 and DS2), unburnt foundry wastes (MB1), foundry ashes (MB2), bronze powder from polishing (MB3), deburring wastes (MB4), and machining shavings (MB5 and MB6). It is worth mentioning that the by-products were used in a straightforward manner from the industry as long as possible without treating them in any way to prevent overshadowing their potential economic and/or environmental benefits derived from their valorization.

\subsection{Laboratory Tests}

Once in the laboratory, the materials were characterized according to the Particles Size Gradation (EN 933-1) standard [14] in order to determine the particle size distributions required for dosage mixtures and in order to obtain a fraction that could be substituted by the by-products. The specific weight $\left(\mathrm{g} / \mathrm{cm}^{3}\right)$ of the materials was also calculated using the pycnometer method. Given the metallic nature of many of the by-products used, this test was developed with methanol instead of water in order to avoid their oxidation and the potential distortion of results. 
In the case of the metallic by-products, an ad-hoc experiment was designed to check their suitability to be used as heating inductors in the healing process. To this end, a round wooden box was filled with the by-product and placed at $2 \mathrm{~cm}$ from the coil (see Figure 3a). An induction of $100 \mathrm{~A}$ intensity was applied over $3 \mathrm{~min}$ using the EASYHEAT 3542 system [15]. Both the heating time and the cooling time (15 extra minutes) were registered by an Optris PI Connect infrared thermometer-based camera [16] (see Figure 3b).

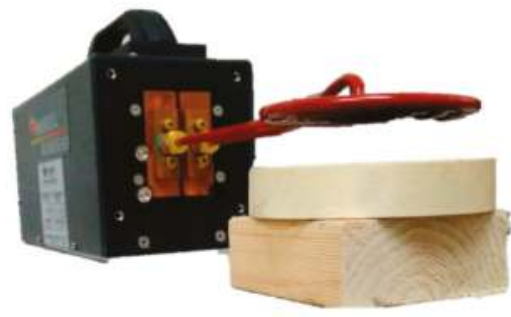

(a)

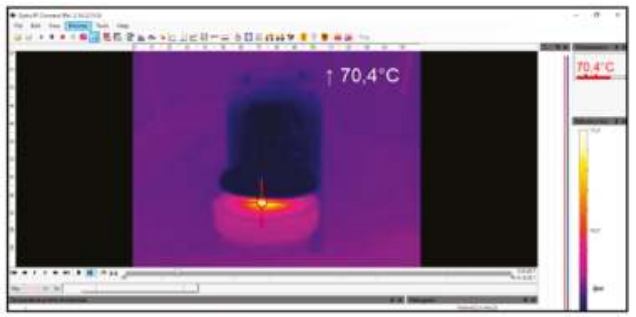

(b)

Figure 3. (a) By-products heating test; (b) Thermographic image recorded with the infrared camera.

Based on the results of the particle size distribution and the heating performance tests, the most suitable materials were selected to compound the experimental mixtures. The temperature they reached in comparison with that achieved by the REF particles helped support this choice.

\subsection{Mixture Dosage Design and Specimen Preparation}

Taking into account the huge differences among the by-products used, the dosage methods for the replacement of raw materials by the by-products made by weight or volume were discarded $[17,18]$. Instead, dosages were undertaken seeking a continue particle size distribution of an asphalt concrete (AC-16) (see Table 1). This premise implies that the amount of material the mixture could accept without relevant deviations of the spindle was determined by the particle size distribution itself. Therefore, each mixture was comparable with the others although the amount of materials substituted was always different. The minimum bitumen content was calculated based on the Spanish Standard EN 13108-1 [19], which proposes a minimum of $4.5 \%$ in mixtures with an aggregate density of $2.65 \mathrm{~g} / \mathrm{cm}^{3}$. According to the density $\rho$ of the aggregates of the experimental mixtures, the reduced bitumen quantity $(r b q)$ was determined by applying Equation (1).

$$
r b q(\%)=4.5 \cdot \frac{2.65 \mathrm{~g} / \mathrm{cm}^{3}}{\rho \mathrm{g} / \mathrm{cm}^{3}}
$$

Table 1. Particle size distribution of the reference mixture.

\begin{tabular}{ccccccccccc}
\hline Sieve Size $(\mathrm{mm})$ & 22 & 16 & 8.0 & 4.0 & 2.0 & 1.0 & 0.5 & 0.25 & 0.13 & 0.063 \\
\hline Spindle Center & 100.0 & 95.0 & 67.5 & 42.5 & 31.0 & 23.5 & 16.0 & 11.0 & 8.0 & 5.0 \\
Top Limit & 100.0 & 100.0 & 75.0 & 50.0 & 38.0 & 39.5 & 21.0 & 15.0 & 11.0 & 7.0 \\
Bottom Limit & 100.0 & 90.0 & 60.0 & 35.0 & 24.0 & 17.5 & 11.0 & 7.0 & 5.0 & 3.0 \\
\hline
\end{tabular}

Regarding the specimen preparation, the materials were combined in a laboratory mixer. Aggregates and bitumen were heated to $170{ }^{\circ} \mathrm{C}$ and $155^{\circ} \mathrm{C}$, respectively. The materials were added to the drum in the following order: coarse aggregate, fine aggregate plus metal particles, and bitumen. After a minute of mixing, the filler was added and all the materials were mixed together over 4 extra 
minutes. Metallic particles were mixed after heating with the fine aggregates in order to ensure a better distribution in the mixtures.

The samples prepared were half-height Marshall specimens $(101.6 \mathrm{~mm}$ diameter and $33 \mathrm{~mm}$ height) compacted with 40 blows on each side. This reduction in the size of the samples was carried out with the objective of saving materials. In order to facilitate the compaction of these half-height specimens, a wooden wedge was introduced at the bottom of the mould to compensate for the reduced amount of material and keep the height from which the impact was produced. The specimens were pre-notched to a depth and width of $0.5 \mathrm{~cm}$ in the laboratory in order to facilitate obtaining a straight crack when the specimens were broken without compromising the healing surface, which was large enough to enable their subsequent testing. Figure 4 shows a group of demoulded specimens with the dimensions mentioned above and the details of a pre-notched specimen.

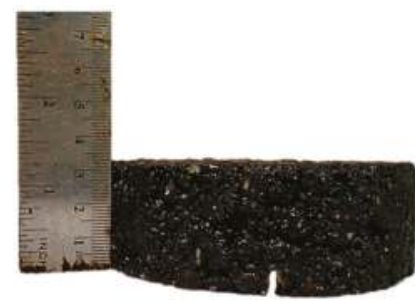

(a)

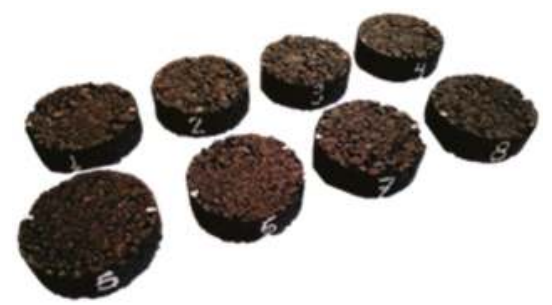

(b)

Figure 4. (a) Pre-notched sample; (b) Group of demolded specimens.

\subsection{Healing Measurements Using a Break-Heal-Break Test}

This test consisted of breaking the pre-notched specimens both before and after subjecting them to a magnetic induction field. The result of the test was the healing ratio $(H R)$, which is a percentage of remaining resistance in the specimens after being completely broken and healed by magnetic induction.

The test started by freezing the specimens. The fact that they were frozen made the break more fragile. Therefore, the specimen was broken into two pieces. In this particular case, the specimens were kept in the freezer for at least $24 \mathrm{~h}$ to ensure that the specimen core was also frozen. Once frozen, the specimens were broken through a three-point bending test in an ad-hoc manufactured cradle with $7 \mathrm{~cm}$ between supports (see Figure 5a). The specimens were placed for testing, which is illustrated in Figure $5 \mathrm{~b}$. This corresponds to the most suitable configuration, according to the type of support provided by the radial saw used. Although this setup differs from those used in previous studies $[8,11]$, the comparative nature of the test guaranteed its validity since the specimens were subject to the same conditions before and after breaking and healing. The speed of the test was set at $10 \mathrm{~mm} / \mathrm{min}$ and the fracture resistance of each sample was recorded with the load cell inserted in the compression machine $\left(R t^{b h}\right)$. After breaking the specimens, they were stored in a room at $20^{\circ} \mathrm{C}$ for two hours.

After tempering, the specimens were subjected to magnetic induction at a distance of $1 \mathrm{~cm}$ from the coil and with a frequency of $329 \mathrm{KHz}$, an intensity of induction between $200 \mathrm{~A}$ and $600 \mathrm{~A}$, and times between $90 \mathrm{~s}$ and $300 \mathrm{~s}$ (see Figure 6). The specimens rested $24 \mathrm{~h}$ after induction and then were frozen once more for $24 \mathrm{~h}$. Lastly, the specimens were broken again with the same parameters used before, which enabled us to determine the fracture resistance after healing $\left(R t^{a h}\right)$ and the Healing Ratio $(H R)$ (see Equation (2)). Considering that the geometry of the mixtures did not change before and after healing, $H R$ could also be calculated by relating the load applied by the laboratory press before $\left(L^{b h}\right)$ and after $\left(L^{a h}\right)$ the process.

$$
H R=\frac{R t^{a h}}{R t^{b h}}=\frac{L^{a h}}{L^{b h}}
$$




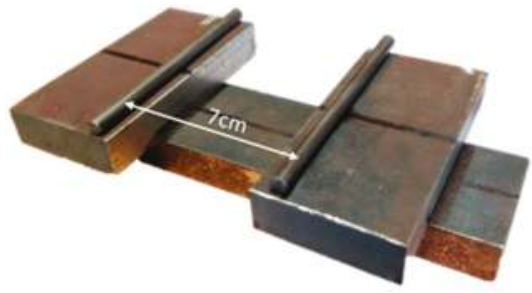

(a)

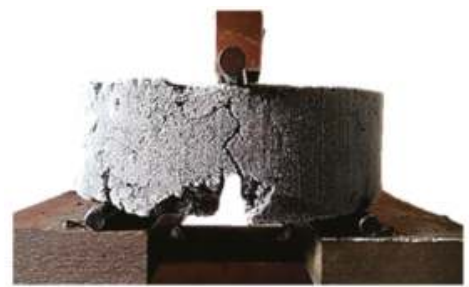

(b)

Figure 5. Details of the (a) Cradle with $7 \mathrm{~cm}$ between supports; (b) Three point bending test.

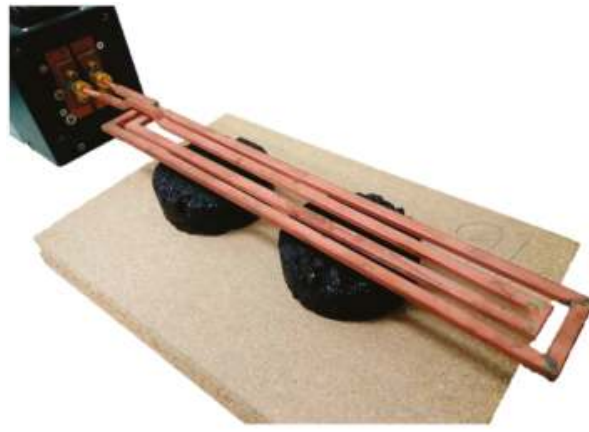

(a)

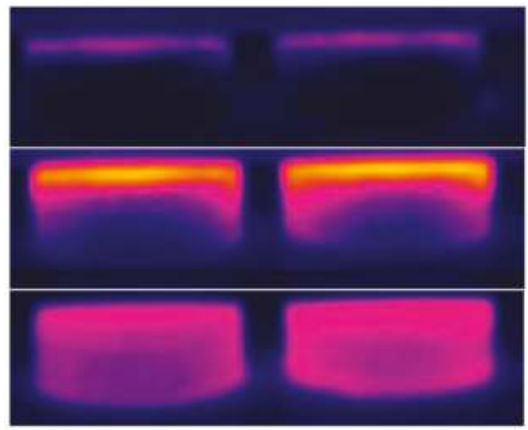

(b)

Figure 6. (a) Healing of specimens after magnetic induction; (b) Thermal images of the specimens.

\subsection{Statistical Analysis}

A sequential combination of inferential and descriptive statistics was undertaken to support the interpretation of the results achieved by investigating and validating the conclusions drawn from them. The implications of the statistical tests considered, which are summarized in Table 2, were analyzed through the $p$-value of these results, according to a significance level of 0.05 [20].

Table 2. Summary of the inferential and descriptive statistical significance tests used.

\begin{tabular}{ccc}
\hline Statistics & Type & Test \\
\hline inferential & parametric & student's $t$ test (2 groups) \\
& & one-way Analysis of Variance (ANOVA) ( $>2$ groups) \\
& nonparametric & Mann-Whitney U test (2 groups) \\
descriptive & Kruskal-Wallis test ( $>2$ groups) \\
& dependence & Pearson correlation coefficient \\
\hline
\end{tabular}

Inferential statistics were applied to compare the performance of the different types of asphalt mixtures tested across the main parameters involved in the healing process. This branch of statistics was approached using either parametric or nonparametric tests depending on whether the values of the samples for the parameters implicated were normal and homoscedastic or not. The normality and homoscedasticity of the samples were checked through the Shapiro-Wilk [21] and Levene's [22] tests, respectively. 
The use of descriptive statistics enabled the exploration of the relationships between the variables under analysis based on the results yielded by the inferential statistical tests, which could produce the grouping of some mixtures depending on their similarity. Since these variables were continuous, the Pearson correlation coefficient was applied to measure the strength and statistical significance of the linear relationships between parameters.

\section{Results and Discussion}

This section presents and discusses the results achieved after conducting the experimental steps included in the methodology outlined in Figure 1. Therefore, determining the particle size distribution and temperature achieved by the by-products is listed in Figure 2. Those by-products prove to be suitable in terms of induction heating and were considered for the preparation of the asphalt mixture specimens in order to be healed in subsequent steps. The nomenclature used in this section for the by-products is consistent with that presented in Figure 2 such that asphalt mixtures were named by adding "_M" to the abbreviation of the by-product(s) compounding them, e.g., a mixture including SB3 and SB5 was SB3_SB5_M.

\subsection{Laboratory Tests}

Table 3 gathers the particle size distribution (UNE 933-1) of all the by-products represented in Figure 2 except MB5 and MB6 whose curves were not calculated due to their shape. This could be dangerous for the grinders. These particular by-products were characterized only with the support of a measurement gauge, which yielded average diameters of $2 \mathrm{~mm}$ and $6 \mathrm{~mm}$, respectively.

Table 3. Particle size distribution of the by-products considered.

\begin{tabular}{cccccccccc}
\hline \multirow{2}{*}{ By-Product } & \multicolumn{7}{c}{ Sieve Size $(\mathbf{m m})$} \\
\cline { 2 - 10 } & $\mathbf{1 6}$ & $\mathbf{8}$ & $\mathbf{4}$ & $\mathbf{2}$ & $\mathbf{1}$ & $\mathbf{0 . 5}$ & $\mathbf{0 . 2 5}$ & $\mathbf{0 . 1 3}$ & $\mathbf{0 . 0 6 3}$ \\
\hline REF & 100.0 & 100.0 & 100.0 & 100.0 & 0.0 & 0.0 & 0.0 & 0.0 & 0.0 \\
SB1 & 100.0 & 100.0 & 100.0 & 100.0 & 100 & 99.8 & 94.2 & 70.9 & 43.8 \\
SB2 & 100.0 & 100.0 & 100.0 & 100.0 & 99.9 & 99.5 & 57.8 & 31.1 & 15.1 \\
SB3 & 100.0 & 100.0 & 100.0 & 100.0 & 92.5 & 71.3 & 37.15 & 12.3 & 1.2 \\
SB4 & 100.0 & 100.0 & 99.8 & 37.1 & 5.3 & 3.0 & 1.4 & 0.8 & 0.0 \\
SB5 & 100.0 & 100.0 & 100.0 & 100.0 & 100.0 & 100.0 & 95.2 & 82.8 & 58.0 \\
S1 & 100.0 & 100.0 & 98.2 & 71.7 & 28.6 & 6.7 & 1.8 & 0.7 & 0.0 \\
S2 & 96.2 & 89.0 & 75.8 & 53.0 & 36.2 & 5.9 & 2.5 & 0.9 & 0.0 \\
S3 & 100.0 & 100.0 & 100.0 & 0.0 & 0.0 & 0.0 & 0.0 & 0.0 & 0.0 \\
S4 & 100.0 & 100.0 & 100.0 & 100.0 & 0.0 & 0.0 & 0.0 & 0.0 & 0.0 \\
DS1 & 100.0 & 100.0 & 100.0 & 100.0 & 100.0 & 100.0 & 99.5 & 90.9 & 67.2 \\
DS2 & 100.0 & 100.0 & 100.0 & 96.0 & 95.6 & 86.7 & 9.7 & 1.4 & 0.0 \\
MB1 & 100.0 & 100.0 & 100.0 & 100.0 & 99.3 & 88.5 & 42.1 & 8.4 & 0.9 \\
MB2 & 100.0 & 100.0 & 100.0 & 99.7 & 98.1 & 89.3 & 78.5 & 62.3 & 44.0 \\
MB3 & 100.0 & 100.0 & 100.0 & 100.0 & 99.5 & 96.0 & 84.3 & 62.6 & 34.3 \\
MB4 & 94.63 & 88.2 & 81.5 & 67.7 & 53.3 & 43.1 & 19.6 & 10.3 & 5.7 \\
\hline
\end{tabular}

One of the main premises of magnetic induction is that the smaller the ferromagnetic particle is, the more effective both their spread is and the achievement of homogenous temperatures across the mixtures. Nevertheless, the importance of the filler show that bitumen interaction limits the amount of filler that can be added to asphalt mixtures in order to not exceed the threshold that bitumen can accept.

In addition to the particle size distribution, the other vital premise to facilitate healing by magnetic induction is the ease of the by-products to be heated, which depends on their ferromagnetic characteristics. The quantification of the thermal capacity of the materials enabled separating those by-products that were unsuitable to perform as heating inductors from those demonstrating to be valid for substituting virgin metal particles. The very first sift consisted of passing an ordinary magnet over the by-products in order to check their ferromagnetic potential aprioristically. This simple test allowed 
discarding some of them for their subsequent use as heating inductors such as S1, DS1, DS2, MS1, MS2, and MS3, which proved to lack enough magnetic attractiveness. Moreover, machining shavings MS5 and MS6 were not considered either due to their shape, which made them considerably difficult to mix with the other components and produced notable irregularities when the mixtures were compacted.

The remaining materials were tested, which was illustrated in Figure 3a. This yielded the results shown in Figure 7, which demonstrate that the magnetic induction barely affected some of the by-products such as S3 and S4. Their almost null influence by Eddy currents demonstrated they lacked enough metallic particles and/or they were too impure to be successfully heated. Similarly, SB1 and MS4 exhibited both a weak heat capacity and an unsuitable particle size distribution for manufacturing the asphalt mixtures. Although the bronze foundry slags reached the highest temperature, this peak was concentrated only in a few particles. Consequently, S2 was discarded because of its lack of homogeneity and the reduced number of metallic particles spread across their samples.

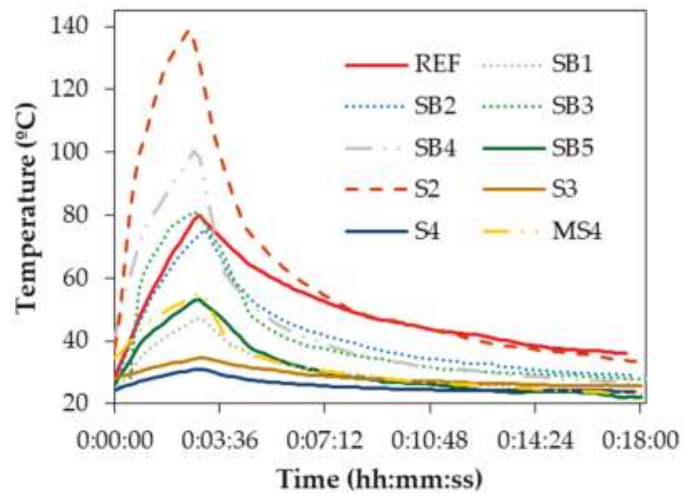

Figure 7. Temperature achieved by the by-products tested as potential heating inductors.

\subsection{Mixture Dosage Design and Specimen Preparation}

The design of the mixture dosage in the volume was preceded by the determination of the specific weight of the by-products selected in the previous step, which resulted in values of $7.850 \mathrm{~g} / \mathrm{cm}^{3}$, $7.639 \mathrm{~g} / \mathrm{cm}^{3}, 7.465 \mathrm{~g} / \mathrm{cm}^{3}, 3.585 \mathrm{~g} / \mathrm{cm}^{3}$ and $2.875 \mathrm{~g} / \mathrm{cm}^{3}$ for REF, SB3, SB4, SB5 and S1, respectively. According to the nomenclature adopted for the by-products, the experimental mixtures were REF_M, SB3_M,SB3_SB5_M,SB4_SB5_M, and S1_SB3_M. Their bitumen contents and particle size distributions are shown in Table 4.

Table 4. Bitumen content and particle size distribution of the mixtures.

\begin{tabular}{ccccccccccc}
\hline \multirow{2}{*}{ Mixture } & \multirow{2}{*}{$\begin{array}{c}\text { Bitumen in } \\
\text { Mixture (\%) }\end{array}$} & \multicolumn{8}{c}{ Sieve Mize (mm) } \\
\cline { 3 - 11 } & & $\mathbf{1 6}$ & $\mathbf{8}$ & $\mathbf{4}$ & $\mathbf{2}$ & $\mathbf{1}$ & $\mathbf{0 . 5}$ & $\mathbf{0 . 2 5}$ & $\mathbf{0 . 1 3}$ & $\mathbf{0 . 0 6 3}$ \\
\hline REF_M & 3.8 & 100.0 & 70.3 & 44.3 & 34.1 & 21.6 & 14.3 & 10.4 & 8.5 & 6.6 \\
SB3_M & 3.9 & 100.0 & 70.4 & 44.4 & 32.3 & 24.8 & 16.7 & 11.3 & 8.4 & 6.0 \\
SB3_SB5_M & 3.9 & 100.0 & 69.9 & 44.0 & 32.1 & 24.6 & 16.6 & 11.3 & 8.3 & 5.5 \\
SB4_SB5_M & 3.8 & 100.0 & 69.2 & 43.6 & 31.0 & 22.3 & 15.0 & 11.3 & 8.7 & 6.1 \\
S1_SB3_M & 3.8 & 100.0 & 70.9 & 44.6 & 32.3 & 24.9 & 16.8 & 10.6 & 8.4 & 6.9 \\
\hline
\end{tabular}

Another important data point was the percentage of metal particles or by-products that compounded the mixtures. In REF_M, 5.0\% of the mixture consisted of virgin steel grits (REF) while SB3_M, SB3_SB5, and S1_SB3_M contained 4.4\%, 3.7\% and 6.0\% of sand blasting steel wastes (SB3). The latter two mixtures were also formed of $1.8 \%$ and $5.3 \%$ of sand blasting products (SB5) and 
green foundry slags (S1), respectively, which amounts to a total of $5.5 \%$ and $11.5 \%$ of by-products in the mixture. Lastly, SB4_SB5_M included 5.0\% and 2.9\% of sandblasting by-products (SB4 and SB5). Therefore, although the amount of inductors contained by each experimental mixture was different, their particle size distribution resembled that of REF_M, which enabled comparing the healing potential of virgin metallic particles and by-products in purpose-designed mixtures.

\subsection{Healing Measurements Using a Break-Heal-Break Test}

The break-heal-break test was applied to all the specimens of the five asphalt mixtures presented in Table 4, according to the methodology explained through Figures 5 and 6 and Equation (2). The charts in Figure 8 illustrate the most relevant data collected from this test including the healing ratio $(H R)$ achieved by the mixtures, the time $(t)$ that the specimens were under induction (s), and the intensity (I) applied during this time (A). A time threshold of $300 \mathrm{~s}$ was established to prevent overextending the duration of the test in order not to compromise the efficiency of the healing process.

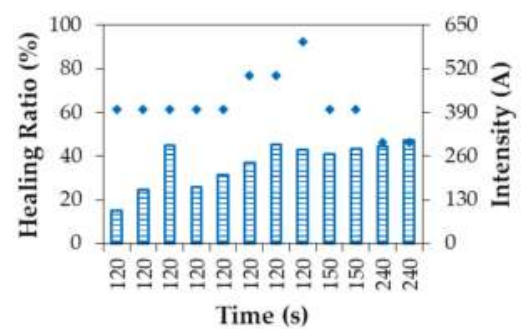

(a)

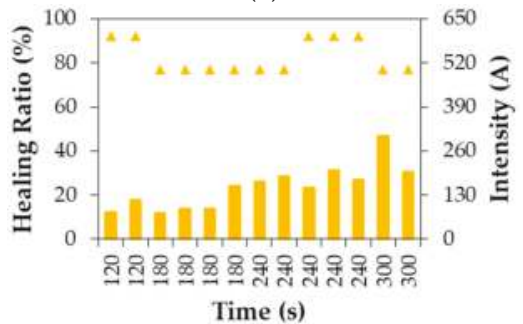

(c)

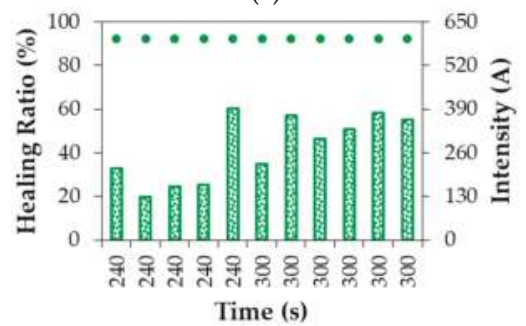

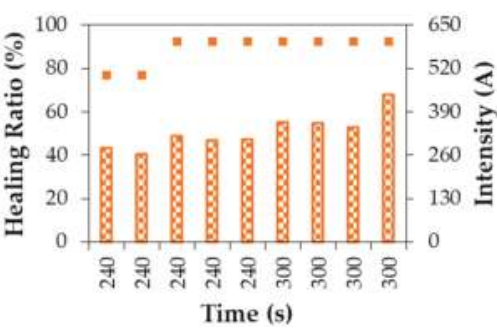

(b)

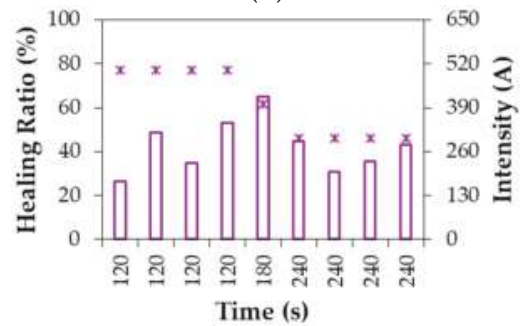

(d)

(e)

Figure 8. Healing ratios (\%) achieved in relation to the time (s) and intensity (A) applied to test the different mixtures studied (a) REF_M; (b) SB3_M; (c) SB3_SB5_M; (d) SB4_SB5_M; (e) S1_SB3_M. 
In the case of the REF_M (see Figure 8a), there were some specimens tested over $120 \mathrm{~s}$ with intensities ranging between $400 \mathrm{~A}$ and $600 \mathrm{~A}$. The trend in these specimens was to achieve a better healing ratio for a greater intensity. However, the surface temperature achieved by the samples under $500 \mathrm{~A}$ or $600 \mathrm{~A}$ exceeded $150^{\circ} \mathrm{C}$, which is a value that could be dangerous for the bitumen properties. This fact led us to test other specimens using less intensity and more time such as those tested for $240 \mathrm{~s}$ and $300 \mathrm{~A}$, which reached only $94{ }^{\circ} \mathrm{C}$. However, this reached the highest $H R$ for this mixture $(47 \%)$. A notable increase in the healing ratio was also produced when keeping intensity at $400 \mathrm{~A}$ and changing time from $120 \mathrm{~s}$ to $150 \mathrm{~s}$.

In contrast, the application of intensities of $300 \mathrm{~A}$ and $400 \mathrm{~A}$ did not heat enough the specimens of SB3_M, which led the values of intensity used with this mixture to increase. Therefore, significant values were obtained when applying $500 \mathrm{~A}$ during $240 \mathrm{~s}$, which reached a healing ratio of about $42 \%$ (see Figure $8 \mathrm{~b}$ ). Surface temperature was around $90^{\circ} \mathrm{C}$, which enabled bitumen to flow (flow starts above $60^{\circ} \mathrm{C}$ ) without being burnt. Another group of specimens was tested by increasing intensity to $600 \mathrm{~A}$ and yielding a value of $H R$ of $47 \%$. Lastly, a third group of specimens was subject to the experiment during 300 seconds with an intensity of $600 \mathrm{~A}$, which produced the best healing ratios with average values of $55 \%$. In this case, the results suggested a clear and direct relationship between time and intensity with $H R$. Overall, it is important to mention that despite of the time invested on the test, the temperature reached for these samples was always under $130^{\circ} \mathrm{C}$.

Figure 8c indicated that the influence of time on the healing ratio for SB3_SB5_M was higher than in previous cases, according to the differences between the results achieved with $180 \mathrm{~s} / 500 \mathrm{~A}$, $240 \mathrm{~s} / 500 \mathrm{~A}$, and $240 \mathrm{~s} / 600 \mathrm{~A}$. The best values of $H R$ in this mixture were reached for $300 \mathrm{~s}$ and $500 \mathrm{~A}$. However, they were far from those obtained for the remaining mixtures. The answer to this poor performance was found in the initial dosages where this mixture contained the lowest percentage of inductor particles, which were not enough to be properly spread over the mixture and made the healing process possible.

SB4_SB5_M is highlighted by the variability of results achieved by the specimens tested under the same conditions (see Figure 8d). The healing ratios of the samples healed for $120 \mathrm{~s}$ and $500 \mathrm{~A}$ and ranged from 26 to $53 \%$ while those that healed for $120 \mathrm{~s}$ and $500 \mathrm{~A}$ resulted in values of $H R$ from 30 to $45 \%$. Therefore, the identification of which parameter was more influential on the healing of this mixture was difficult. Instead, the properties of these specimens were found to hinder the correct heat distribution over the mixture and the flowing of bitumen along the cracks. According to the values compiled in Figure 8d, the most effective combo for this mixture was $180 \mathrm{~s}$ and $400 \mathrm{~A}$.

Finally, S1_SB3_M (see Figure 8d) was tested using times between $240 \mathrm{~s}$ and $300 \mathrm{~s}$ and an intensity of $600 \mathrm{~A}$. These parameters were used due to its similarities with SB3_SB5_M in terms of magnetic capacity and since both mixtures included sand blasting steel wastes as their main inductor. The general trend identified in the performance of these specimens suggested that the more time spent under induction, the higher the healing ratio is. The best results of $H R$ for this mixture were about $60 \%$.

To further explore the variables affecting the healing ratios achieved in Figure 8 by the mixtures listed in Table 4, Figure 9 shows the relationship of $H R$ with both the surface temperature achieved during the test $(T)$ and the product of time and intensity $(t \cdot I)$. Both aspects were merged because the combined effect of exposure and magnitude was expected to be proportionally related to the healing ratios obtained, which is demonstrated in Figure 9a. On the contrary, the dispersion of the heating inductors across the mixtures was the reason behind the weak link between $T$ and $H R$ revealed in Figure 9 b. Figure $9 \mathrm{c}$ shows the loads needed to break each sample before healing such that the average values rounded $460 \mathrm{kgf}$ for the reference mixture and exceeded $500 \mathrm{kgf}$ for the experimental mixtures with SB3_SB5_M providing the highest breaking resistance. 


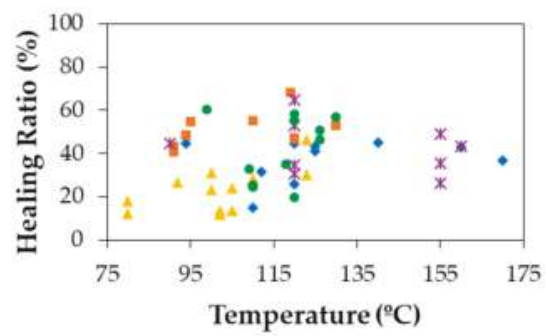

(a)

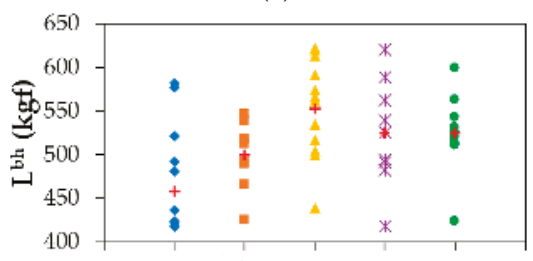

Asphalt mixture

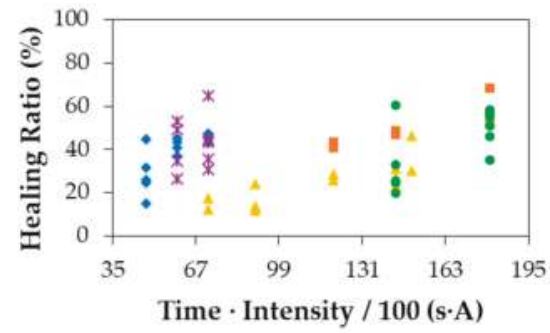

(b)

- REF

- SB3_M

\SB3_SB5_M

* SB4_SB5_M

- S1 SB3 M

+ Average $L^{\text {th }}$

(c)

Figure 9. Healing ratios (\%) achieved by the different mixtures in relation to (a) Temperature $\left({ }^{\circ} \mathrm{C}\right)$ and (b) The product of time by intensity (s.A); (c) Breaking loads applied to the mixtures before healing $\left(L^{b h}\right)$.

\subsection{Statistical Analysis}

The results represented in Figures 8 and 9 were analysed in statistical terms by starting with checking the normality of the data associated with the parameters $(t, I, t \cdot I, T, H R)$ used to evaluate the five types of mixtures considered in Table 4. The application of the Shapiro-Wilk test demonstrated that only $T$ was normally distributed for all the mixtures ( $p$-value $>0.05$ in all cases). However, its variance was heterogeneous according to the Levene's test ( $p$-value $<0.05)$. Therefore, the inferential analysis was carried out through nonparametric tests.

The Kruskal-Wallis test was used to evaluate all the mixtures at once, which resulted in the $p$-values listed in Table 5. This provided evidence of the statistically significant differences between the five groups $(<0.05)$. In view of these results, the Mann-Whitney $U$ test was applied to determine the absence or presence of significant differences for each pair of groups. The $p$-values compiled in Table 5 demonstrated that only two duos of mixtures (REF vs. SB4_SB5 and SB3 vs. S1_SB3) were statistically similar across all the variables involved $(>0.05)$. On the one hand, the metallic purity of the steel shot wastes in SB4_SB5 along with the particle size distribution of this mixture explained its resemblance to REF. On the other hand, the affinity between SB3 and S1_SB3 was caused by the presence of steel grit wastes as main inductors in both mixtures since S1 lacked magnetic attractiveness. The remaining results indicated that the values of $t, I, t \cdot I, T$, and $H R$ reached by the other pairwise comparisons were significantly different in the majority of cases. 
Table 5. Comparative evaluation of the asphalt mixtures using nonparametric inferential tests.

\begin{tabular}{cccccc}
\hline Comparison & \multicolumn{5}{c}{$\boldsymbol{p}$-Value } \\
\cline { 2 - 6 } & $\boldsymbol{t}$ & $\boldsymbol{I}$ & $\boldsymbol{t} \cdot \boldsymbol{I}$ & $\boldsymbol{T}$ & $\boldsymbol{H} \boldsymbol{R}$ \\
\hline REF vs. SB3 vs. SB3_SB5 vs. SB4_SB5 vs. S1_SB3 & 0.000 & 0.000 & 0.000 & 0.011 & 0.000 \\
REF vs. SB3 & 0.000 & 0.000 & 0.000 & 0.129 & 0.002 \\
REF vs. SB3_SB5 & 0.007 & 0.001 & 0.000 & 0.011 & 0.007 \\
REF vs. SB4_SB5 & 0.277 & 0.754 & 0.058 & 0.464 & 0.422 \\
REF vs. S1_SB3 & 0.000 & 0.000 & 0.000 & 0.740 & 0.316 \\
SB3 vs. SB3_SB5 & 0.030 & 0.126 & 0.014 & 0.647 & 0.000 \\
SB3 vs. SB4_SB5 & 0.006 & 0.000 & 0.000 & 0.040 & 0.113 \\
SB3 vs. S1_SB3 & 0.710 & 0.412 & 0.456 & 0.175 & 0.412 \\
SB3_SB5 vs. SB4_SB5 & 0.292 & 0.003 & 0.000 & 0.007 & 0.001 \\
SB3_SB5 vs. S1_SB3 & 0.011 & 0.009 & 0.001 & 0.011 & 0.004 \\
SB4_SB5 vs. S1_SB3 & 0.002 & 0.000 & 0.000 & 0.131 & 1.000 \\
\hline
\end{tabular}

In light of the outputs of the inferential analysis, both REF and SB3_M were combined with SB4_SB5_M and S1_SB3_M due to their respective similarity such that the final number of groups to evaluate using descriptive statistics was three (REF + SB4_SB5_M, SB3_M + S1_SB3_M, and SB3_SB5_M). The quantification of the relationships between the variables involved in the healing process for these groups resulted in the Pearson correlation coefficients shown in Table 6.

Table 6. Pearson correlation coefficients between the variables involved in the healing process.

\begin{tabular}{cccc}
\hline \multirow{2}{*}{ Interaction } & \multicolumn{3}{c}{ Group } \\
\cline { 2 - 4 } & REF + SB4_SB5_M & SB3_M + S1_SB3_M & SB3_SB5_M \\
\hline$t * T$ & -0.264 & $0.521^{*}$ & $0.825^{*}$ \\
$t * H R$ & 0.244 & $0.567^{*}$ & $0.826^{*}$ \\
$I * T$ & $0.504^{*}$ & $0.585^{*}$ & $-0.750^{*}$ \\
$I * H R$ & 0.032 & 0.109 & -0.105 \\
$(t \cdot I) * T$ & 0.104 & $0.632^{*}$ & $0.597^{*}$ \\
$(t \cdot I) * H R$ & $0.531^{*}$ & $0.512 *$ & $0.800^{*}$ \\
$T * H R$ & 0.006 & 0.109 & $0.628^{*}$ \\
\hline
\end{tabular}

These values suggested that the combined effect of time and intensity $(t \cdot I)$ provided the most solid positive correlation to $H R$. This showed that the longer and more intense the heating process is, the higher the healing ratio of the asphalt mixtures is. This coupled variable also explained the negative relationship between $I$ and $T$ for SB3_SB5_M, i.e., high intensities might not result in high temperatures unless they are applied for extended time periods. Therefore, heating time emerged as the most decisive parameter to ensure the healing of asphalt mixtures. The results in Table 6 also confirmed the uncertainty associated with the temperature achieved by the mixtures since this variable strongly depends on the distribution of the heating inductors across them, which hinders the existence of significant correlations for $H R$.

\section{Conclusions}

The outputs yielded by this investigation provided evidence of the potential for by-products to perform as heating inductors in asphalt mixtures. This was demonstrated through the application of a methodology consisting of the complete characterization in laboratory of a collection of diverse by-products supplied by metal industries, which enables their subsequent incorporation into asphalt mixtures that were tested in terms of healing capacity via magnetic induction. The set of by-products tested included slags, demolding sands, and materials from sandblasting and machining processes, 
which resulted in a varied sample of alternatives to compare with the virgin metallic particles contained in the reference mixture.

The results achieved in the different laboratory experiments revealed the limitations of some of the by-products used, which were unsuitable to be part of asphalt mixtures due to either their particle size distribution, their filler-bitumen interaction, or their heating capacity. According to these premises, the by-products selected due to their capacity as heating inductors were materials derived from sandblasting processes while green foundry slags were also added as a replacement of natural aggregates because of their lightness and particle size compatibility. The experimental mixtures required higher values of time and intensity than the reference one to reach satisfactory healing ratios except for the ones formed of steel shot wastes derived from sandblasting due to their similarity to the particle sizes of the virgin metals and the purity provided by this process. The relationships between the variables involved in the laboratory tests demonstrated that the greatest contributor for improving the healing ratios obtained was the time throughout which the samples were subject to magnetic induction.

However, time is also the variable with the greatest influence when determining the feasibility of the healing of asphalt mixtures through magnetic induction since the process needs to be both fast and effective. Consequently, further work needs to be undertaken in the search for optimal combinations of time and intensity to boost the healing of mixtures containing different by-products. Another course of action to explore in the future relates to the improvement in replicating the characteristics of the cracks to be healed in real conditions since those tested in thelaboratory were larger and more concentrated. Nonetheless, the results presented in this research pave the way towards a more sustainable healing of asphalt mixtures by showing that the addition of by-products provides a viable solution for overcoming the economic and environmental drawbacks associated with the use of virgin metallic particles.

Author Contributions: M.V.-C. conceived the research. M.V.-C. and M.Á.C.-P. planned the laboratory experiments. M.V.-C. designed the mixtures and carried out the laboratory tests. M.V.-C., D.J.-E., D.C.-F. and M.Á.C.-P. discussed the results. M.V.-C. and D.J.-E. wrote the paper.

Funding: This paper was financed by the Spanish Ministry of Economy and Competitiveness with funds from the State General Budget (PGE) and the European Regional Development Fund (ERDF) through the research project SIMA+ (Ref. BIA2016-77372-R).

Conflicts of Interest: The authors declare no conflict of interest. The founding sponsors had no role in the design of the study, in the collection, analyses, or interpretation of data, in the writing of the manuscript, or in the decision to publish the results.

\section{References}

1. Liu, Q.; García, A.; Schlangen, E.; Ven, M.V.D. Induction healing of asphalt mastic and porous asphalt concrete. Constr. Build. Mater. 2011, 25, 3746-3752. [CrossRef]

2. Barrasa, R.C.; López, V.B.; Montoliu, C.M.; Ibáñez, V.C.; Pedrajas, F.; Santarén, J. Addressing durability of asphalt concrete by self-healing mechanism. Procedia Soc. Behav. Sci. 2014, 162, 188-197. [CrossRef]

3. Tabaković, A.; Schlangen, E. Self-healing technology for asphalt pavements. In Advances in Polymer Science; Hager, M., van der Zwaag, S., Schubert, U., Eds.; Springer: Cham, Switzerland, 2016; Volume 273, pp. 285-306.

4. Ayar, P.; Moreno-Navarro, F.; Rubio-Gámez, M.C. The healing capability of asphalt pavements: A state of the art review. J. Clean. Prod. 2016, 113, 28-40. [CrossRef]

5. García, A.; Schlangen, E.; van de Ven, M.; Liu, Q. A simple model to define induction heating in asphalt mastic. Constr. Build. Mater. 2012, 31, 38-46. [CrossRef]

6. Liu, Q.; Schlangen, E.; García, A.; van de Ven, M. Induction heating of electrically conductive porous asphalt concrete. Constr. Build. Mater. 2010, 24, 1207-1213. [CrossRef]

7. García, A.; Norambuena-Contreras, J.; Partl, M.N. Experimental evaluation of dense asphalt concrete properties for induction heating purposes. Constr. Build. Mater. 2013, 46, 48-54. [CrossRef]

8. García, A.; Norambuena-Contreras, J.; Bueno, M.; Partl, M.N. Single and multiple healing of porous and dense asphalt concrete. J. Intell. Mater. Syst. Struct. 2015, 26, 425-433. [CrossRef] 
9. Menozzi, A.; Garcia, A.; Partl, M.N.; Tebaldi, G.; Schuetz, P. Induction healing of fatigue damage in asphalt test samples. Constr. Build. Mater. 2015, 74, 162-168. [CrossRef]

10. Bueno, M.; Arraigada, M.; Partl, M.N. Damage detection and artificial healing of asphalt concrete after trafficking with a load simulator. Mech. Time-Dependent Mater. 2016, 20, 265-279. [CrossRef]

11. Norambuena-Contreras, J.; Garcia, A. Self-healing of asphalt mixture by microwave and induction heating. Mater. Des. 2016, 106, 404-414. [CrossRef]

12. Zhu, X.; Cai, Y.; Zhong, S.; Zhu, J.; Zhao, H. Self-healing efficiency of ferrite-filled asphalt mixture after microwave irradiation. Constr. Build. Mater. 2017, 141, 12-22. [CrossRef]

13. Franesqui, M.A.; Yepes, J.; García-González, C. Top-down cracking self-healing of asphalt pavements with steel filler from industrial waste applying microwaves. Constr. Build. Mater. 2017, 149, 612-620. [CrossRef]

14. AENOR Norma UNE-EN 933-1:2012. Ensayos Para Determinar Las Propiedades Geométricas de los Áridos. Parte 1: Determinación de la Granulometría de Las Partículas. Método del Tamizado. Available online: http://www.aenor.es/aenor/normas/normas/fichanorma.asp?tipo=N\& codigo=N0049638\#.Wsd1i4hubcs (accessed on 6 April 2018).

15. Ambrell Solutions EASYHEAT ${ }^{\circledR} 4.2$ to $10 \mathrm{~kW}$ Induction Heating Systems. Available online: https:/ / www. ambrell.com/hubfs / Ambrell_PDFs/411-0075-10.pdf?t=1522932055039 (accessed on 6 April 2018).

16. OPTRIS Software. Optris PI Connect. Available online: https://www.optris.es/optris-pi-connect (accessed on 6 April 2018).

17. Pasetto, M.; Baldo, N. Resistance to permanent deformation of road and airport high performance asphalt concrete base courses. In Advanced Materials Research; Chang, J.-R., Yang, S.-R., Eds.; Scitec Publications: Uetikon am See, Switzerland, 2013; Volume 723, pp. 494-502.

18. Pasetto, M.; Baldo, N. Influence of the aggregate skeleton design method on the permanent deformation resistance of stone mastic asphalt. Mater. Res. Innov. 2014, 18, S3. [CrossRef]

19. AENOR Norma UNE-EN 13108-1:2008. Mezclas Bituminosas. Especificaciones de Materiales. Parte 1: Hormigón Bituminoso. Available online: http://www.aenor.es/aenor/normas/normas/fichanorma.asp? tipo=N\&codigo=N0042341\#.WurjWIiFPct (accessed on 3 May 2018).

20. Fisher, R.A. Statistical Methods for Research Workers; Oliver \& Boyd: Edinburgh, Scotland, 1925.

21. Shapiro, S.S.; Wilk, M.B.; Chen, H.J. A comparative study of various tests for normality. J. Am. Stat. Assoc. 1968, 63, 1343-1372. [CrossRef]

22. Brown, M.B.; Forsythe, A.B. Robust tests for the equality of variances. J. Am. Stat. Assoc. 1974, 69, $364-367$. [CrossRef]

(C) 2018 by the authors. Licensee MDPI, Basel, Switzerland. This article is an open access article distributed under the terms and conditions of the Creative Commons Attribution (CC BY) license (http:/ / creativecommons.org/licenses/by/4.0/). 
Article

\title{
An Innovative Approach to Control Steel Reinforcement Corrosion by Self-Healing
}

\author{
Dessi A. Koleva \\ Faculty of Civil Engineering and Geosciences, Department 3MD, Materials and Environment, Delft University \\ of Technology, Stevinweg 1, 2628 CN, Delft, The Netherlands; d.a.koleva@tudelft.nl; Tel.: +31-1527-87451
}

Received: 29 January 2018; Accepted: 12 February 2018; Published: 20 February 2018

\begin{abstract}
The corrosion of reinforced steel, and subsequent reinforced concrete degradation, is a major concern for infrastructure durability. New materials with specific, tailor-made properties or the establishment of optimum construction regimes are among the many approaches to improving civil structure performance. Ideally, novel materials would carry self-repairing or self-healing capacities, triggered in the event of detrimental influence and/or damage. Controlling or altering a material's behavior at the nano-level would result in traditional materials with radically enhanced properties. Nevertheless, nanotechnology applications are still rare in construction, and would break new ground in engineering practice. An approach to controlling the corrosion-related degradation of reinforced concrete was designed as a synergetic action of electrochemistry, cement chemistry and nanotechnology. This contribution presents the concept of the approach, namely to simultaneously achieve steel corrosion resistance and improved bulk matrix properties. The technical background and challenges for the application of polymeric nanomaterials in the field are briefly outlined in view of this concept, which has the added value of self-healing. The credibility of the approach is discussed with reference to previously reported outcomes, and is illustrated via the results of the steel electrochemical responses and microscopic evaluations of the discussed materials.
\end{abstract}

Keywords: corrosion; reinforced concrete; polymeric nanomaterials; self-healing

\section{Introduction}

Reinforced concrete is a durable material, capable of withstanding a variety of adverse environmental conditions. It is a highly alkaline composite, where the $\mathrm{pH}$ of the pore solution ranges between 12.7 and 13.5. The cementitious matrix in reinforced concrete acts as a physicochemical barrier and assures the passivity of the steel reinforcement, hence it usually provides corrosion protection for the steel surface. However, the open pore structure of the concrete cover (and matrix), allows aggressive substances to penetrate into the bulk material, initiating steel corrosion. The onset of steel corrosion is due to carbonation (a pH drop $<9$ in the bulk matrix) or chloride contamination (resulting in a corrosion-related local $\mathrm{pH}$ drop $<5$ on the steel surface) [1-7]. Upon steel de-passivation, corrosion initiation and propagation take place, increasing in rate, ultimately causing concrete cracking as a result of rust expansion. Concurrently, the steel cross-section is reduced, increasing the potential risks of a rapid structural failure.

So far, various mitigation and protection techniques have been investigated and applied, e.g., corrosion inhibitors, [8-10], protective (including polymer) coatings, surface sealers, etc. [11,12]. Among the electrochemical techniques for corrosion control, impressed current cathodic protection (ICCP) is known to be applied to structures in highly aggressive environments [5]. However, ICCP only targets the steel reinforcement and is well known to result in side effects for the bulk matrix or the steel/cement-paste interface, for instance an alkali aggregate reaction and/or bond-strength degradation [13-18]. Together with the risks of hydrogen embrittlement for the protected steel in 
pre-stressed concrete [14-16], ICCP has drawbacks and limitations for certain classes of material. Other electrochemical techniques, e.g., desalination and re-alkalization, conversely only target the cement-based material, restoring the chemical composition of the concrete pore water [16]. The result is a favorable medium for potential steel re-passivation. In other words, the available methods for corrosion control target either the steel alone, or only the concrete material.

Novel solutions for new cement blends, steel grades, novel coatings, self-healing approaches, etc., have been reported to show great potential [19-27]. Nevertheless, these methods, although claiming corrosion delay as a final outcome, aim only at the quality of the cement-based material. They do not consider the overall complex electrochemistry that governs the corrosion process itself or the phenomena within corrosion control. For instance, the involvement of nanomaterials (e.g., polymeric nanoparticles, inorganic nanoparticles, etc.) in cement-based systems was reported to result in increased compressive strength, matrix densification, resistance to the penetration of aggressive substances, etc. [28-30]. A comprehensive review of nanotechnology in concrete material science [31] gives a detailed overview of the application of nanomaterials for altered properties of cement-based systems. The majority of the literature reports, however, deal separately with either only the cement-based bulk matrix or the steel surface.

In view of the application of polymeric additives, including polymeric nanoparticles, in (reinforced) cement-based materials, admixed polyethylene oxide (PEO)-based polymers were reported to result in the re-distribution and improved dispersion of hydration products, an increase of electrostatic repulsive forces, and improved microstructural properties [32,33]. Here again, the target is only the cement-based material. Additionally, these admixtures are used in comparatively large quantities, e.g., between $3 \mathrm{wt} \%$ and $30 \mathrm{wt} \%$ per dry cement weight, as opposed to the possibility of employing significantly lower amounts of, for instance, polymeric nanomaterials. The commercially available "XSeed", a polymer-coated calcium-silicate-hydrate (CSH), is reported to be used in a concentration of $0.3 \mathrm{wt} \%$ per cement weight, improving microstructural properties [24]. All the above admixtures are used in significantly higher amounts if compared to the potential of employing tailor-made polymeric nanomaterials, e.g., at a minimum ten times lower concentration, as with the nanoparticles suggested in this work.

Besides the uses mentioned above, the application of polymeric nanoparticles for simultaneously achieved corrosion protection and improved bulk matrix properties in reinforced concrete has, to the author's best knowledge, not been reported by other authors or research groups. This is where this work aims at raising awareness regarding a feasible approach, which targets the reinforced concrete system as a whole, emphasizing the application of polymeric nanomaterials for proactive corrosion control in civil structures.

A brief background on steel passivity, passivity breakdown and cement-matrix-related degradation mechanisms are outlined first in view of the subject of this work. Next, the detailed concept of the above approach is communicated. The credibility of the approach is discussed with reference to previously reported outcomes. As concept-supporting information, the results for the steel electrochemical response, together with microscopic observations regarding the discussed materials and interfaces, are also presented.

\section{Steel Passivity, Passivity Breakdown and Matrix Carbonation-Background}

\subsection{Steel Passivity in Reinforced Concrete}

Corrosion of the steel reinforcement in reinforced concrete is an electrochemical corrosion process. Although concrete is referred to as a solid material, the pore water in the concrete bulk matrix is an aqueous medium. Hence, the pore water in contact with the steel reinforcement allows for oxidation and reduction reactions on the steel surface to be initiated and to progress over time. In a simplified way, the corrosion of steel in reinforced concrete is governed by two electrochemical reactions, presented by Equations (1) and (2), i.e., oxidation, or the dissolution of iron at anodic sites (Equation (1)) and 
the reduction of oxygen at local cathodes, consuming electrons generated by the metal dissolution (Equation (2)). The products of these reactions interact and in a final stage transform into a stable protective film on the steel surface. This protective (passive) film remains intact if not disturbed by aggressive substances (e.g., chloride ions) and/or changes in the $\mathrm{pH}$ of the concrete pore water (e.g., due to matrix carbonation). In the simplest case, a two layer structure of the passive film can be assumed, where the inner layer of $\mathrm{Fe} 3 \mathrm{O} 4$ adheres well to the steel substrate, restricts subsequent film growth and, therefore, limits further oxidation [34].

Passivity is often assigned to the outer, gelatinous layer of hydrous $\mathrm{Fe}_{2} \mathrm{O}_{3}$, however, both oxides constitute the protective ability and properties of the passive film on the steel surface. In other words, the oxidation and reduction processes (Equations (1) and (2)) are followed by film precipitation and development, for instance, the chemical reaction mechanisms as given in Equations (3) and (4), would initially take place. This film is a combination and/or a predominant occurrence of ferrous, $\mathrm{Fe}^{2+}$ and/or ferric, $\mathrm{Fe}^{3+}$ based compounds.

$$
\begin{gathered}
2 \mathrm{Fe} \rightarrow 2 \mathrm{Fe}^{2+}+4 \mathrm{e}^{-} \\
\mathrm{O}_{2}+2 \mathrm{H}_{2} \mathrm{O}+4 \mathrm{e}^{-} \rightarrow 4 \mathrm{OH}^{-} \\
\mathrm{Fe}^{2+}+2 \mathrm{OH}^{-} \rightarrow \mathrm{Fe}(\mathrm{OH})_{2} \\
\mathrm{Fe}(\mathrm{OH})_{2}+\frac{1}{4} \mathrm{O}_{2} \rightarrow \gamma-\mathrm{FeOOH}+\frac{1}{2} \mathrm{H}_{2} \mathrm{O}
\end{gathered}
$$

In the absence of chlorides and with the sustained $\mathrm{pH}$ of the medium, both compounds are chemically stable in the alkaline medium of the concrete matrix. With the increase in the maturity of the system overall, and depending on oxygen availability, the ferrous oxides tend to convert to more stable ferric oxides.

\subsection{Passivity Breakdown}

In conditions when chlorides are present in the pore water, the cement layers adjacent to the steel surface, or in the case of matrix carbonation $\left(\mathrm{CO}_{2}\right.$ penetration, resulting in a $\mathrm{pH}$ drop of the pore water), soluble complexes are formed from the originally available ferrous oxides. For example, intermediate compounds such as green rusts of type I and II (Equations (5) and (6)) will be formed. These are not-protective and do not constitute an adherent layer. Additionally, green rusts, as well as $\mathrm{Fe}(\mathrm{OH})_{2}$, are known to contain both hexagonal and cubic layers of close-packed oxygen [35]. These compounds exist prior to the formation of the purely cubic close-packed structure of the stable end products, i.e., $\mathrm{Fe}_{3} \mathrm{O}_{4}\left(\mathrm{FeO}+\mathrm{Fe}_{2} \mathrm{O}_{3}\right), \gamma$ - $\mathrm{FeOOH}$ or $\gamma-\mathrm{Fe}_{2} \mathrm{O}_{3}$.

$$
\begin{gathered}
6 \mathrm{Fe}(\mathrm{OH})_{2}+\mathrm{CO}_{3}{ }^{2-}+2 \mathrm{H}_{2} \mathrm{O} \rightarrow\left[\mathrm{Fe}_{4}{ }^{2+} \mathrm{Fe}_{2}{ }^{3+}(\mathrm{OH})_{12}\right]\left[\mathrm{CO}_{3} \cdot 2 \mathrm{H}_{2} \mathrm{O}\right]+2 \mathrm{e}^{-} \\
4 \mathrm{Fe}(\mathrm{OH})_{2}+\mathrm{Cl}^{-} \rightarrow\left[\mathrm{Fe}_{3}{ }^{2+} \mathrm{Fe}^{3+}(\mathrm{OH})_{8} \mathrm{Cl}\right]+\mathrm{e}^{-}
\end{gathered}
$$

In the presence of chloride ions, the stability of a close-packed arrangement is higher [36]. Consequently, a steel surface covered by a ferric-oxide-based film $\left(\mathrm{Fe}^{3+}\right.$-based) will exhibit a higher resistance to (electro) chemical reaction mechanisms involving carbonate or chloride ions, and subsequently, higher resistance to corrosion initiation and propagation [37].

According to existing models for chloride-induced passivity breakdown, an initial adsorption of chloride anions on the oxide (passive) film takes place [38], resulting in its chemical dissolution. This mechanism is very local in character [39] and initiates predominantly on structural defects or inhomogeneities on the steel surface [40]. The adsorption of chloride anions enables new pathways for charge exchange, which allows for steel corrosion initiation and/or propagation on the steel surface [39]. This mechanism of passivity breakdown, and the further increase in corrosion rate due to chloride ions in the medium, is similarly relevant for reinforced concrete. Free chloride ions 
only, or those present in the pore solution, would initiate or participate in the passivity breakdown and subsequent steel corrosion process. The ability of the concrete matrix to chemically bound chlorides (or the chloride-binding capacity of cementitious materials, and the formation of, e.g., calcium-chloro-aluminates) is, in fact, one of the reasons for the initial "delay" of chloride-induced corrosion in reinforced concrete, even when the penetration of a substantial amount of chloride ions from, e.g., the external environment, does exist. Therefore, the term "chloride threshold" has been accepted in the field, linking the chloride content at the time of corrosion initiation [41-45]. This aspect is not subject to further elaboration in this contribution, but mentioned for the clarity of the discussed mechanisms.

\subsection{Matrix Carbonation-Steel-Corrosion-Related Aspects}

Chemically bound chlorides can participate in the corrosion process if a $\mathrm{pH}$ drop in the pore solution occurs, as in the case of carbonation, for example. Carbonation is the process by which atmospheric $\mathrm{CO} 2$ slowly propagates in the depth of the bulk concrete. According to the simplified sequence of a carbonation process, $\mathrm{CO}_{2}$ penetrates the concrete cover, dissolves in the pore solution and reacts with Ca-bearing phases, e.g., $\mathrm{Ca}(\mathrm{OH})_{2}$, silicates and aluminates, which are all constituents of the cementitious bulk matrix. Chemical reactions, as the ones presented by Equations (7)-(10), would take place:

$$
\begin{gathered}
\mathrm{Ca}(\mathrm{OH})_{2}+\mathrm{CO}_{2} \rightarrow \mathrm{CaCO}_{3}+\mathrm{H}_{2} \mathrm{O} \\
2 \mathrm{SiO}_{2} \cdot 2 \mathrm{CaO} \cdot 2 \mathrm{H}_{2} \mathrm{O}+3 \mathrm{CO}_{2} \rightarrow 2 \mathrm{SiO}_{2}+3 \mathrm{CaCO}_{3}+3 \mathrm{H}_{2} \mathrm{O} \\
4 \mathrm{CaO} \cdot \mathrm{Al}_{2} \mathrm{O}_{3} \cdot 13 \mathrm{H}_{2} \mathrm{O}+4 \mathrm{CO}_{2} \rightarrow 2 \mathrm{Al}(\mathrm{OH})_{3}+4 \mathrm{CaCO}_{3}+10 \mathrm{H}_{2} \mathrm{O} \\
\mathrm{CaCO}_{3}+\mathrm{H}_{2} \mathrm{O}+2 \mathrm{CO}_{2} \rightarrow \mathrm{Ca}\left(\mathrm{HCO}_{3}\right)_{2}
\end{gathered}
$$

These reactions, (Equations (7)-(10)), lead to a $\mathrm{pH}$ drop in the pore solution from the original $\mathrm{pH}$ of $\geq 12.7$ to $\mathrm{pH}$ of ca. $8[37,46]$. Excess $\mathrm{CO}_{2}$ in the pore water results in the formation of bicarbonate (Equation (10)), additionally lowering the $\mathrm{pH}$ of the pore solution. This leads to the dissolution of Ca-bearing phases (Equations (7)-(9)), and consequently, also to the dissolution of calcium-chloro-aluminate complexes, which otherwise chemically bind chloride ions. Along with local acidification, the result is an increase in the free chloride ion concentration.

Besides resulting in changes of the $\mathrm{pH}$ of the pore water, the dissolution of Ca-bearing phases due to carbonation exerts alterations in the original matrix composition. Uniform (general) corrosion on the steel surface will be the result of lowering the $\mathrm{pH}$ of the pore water. In cases when both carbonation and chloride ingress are at hand, general corrosion would co-exist with chloride-induced, localized corrosion on the steel surface. Here again, the initially chemically-bound chlorides could be released, increasing the chloride threshold level for corrosion initiation [47,48], and/or contributing to an already ongoing corrosion process.

Carbonation of the matrix would also affect the properties of the overall product layer on the steel surface. In reinforced concrete, the protective ability of the passive film is additionally supported by Ca-rich phases, adhering well to the (steel) substrate or accumulated at the steel/cement matrix interface. Some reports discuss only a limited protection efficiency of the adhered calcium-rich layer [49], while others claim an increased protection ability of the Ca-substituted $\mathrm{Fe}_{3} \mathrm{O}_{4}$ and/or $\mathrm{Fe}_{2} \mathrm{O}_{3}[34,50]$. This layer is already different from the purely passive film, and is, therefore, to be considered as a product layer on the steel surface. The increased protective ability of a Ca-rich, iron oxide/hydroxide layer on the steel surface is due to the incorporation of $\mathrm{Ca}(\mathrm{OH})_{2}$ in the outer atom layers of the inner passive film, a consequence of the adsorption of $\mathrm{Ca}^{2+}$ ions in this (inner $\mathrm{Fe}_{3} \mathrm{O}_{4}$ ) film. Consequently, altered Ca-bearing phases in the system overall, or a reduced amount of Ca-containing compounds in the vicinity of the steel surface, as in conditions of carbonation, will result in reduced protective ability of the Ca-substituted iron oxide/hydroxide product layer. 
The above considerations are important in view of the concept discussed in this work and its approach to corrosion control, regarding two aspects. First, appreciation of the composition of the passive (and product) layer on the steel surface, when no passivity breakdown is at hand, was considered a useful reminder in view of the concept of corrosion control discussed in this paper. More importantly, the potential for a sustained or improved protective ability of the passive film, or the overall product layer on the steel surface, by, e.g., maintaining the Ca substitution of iron oxides/hydroxides, is of particular interest. Secondly, by considering the structure, composition and protective abilities of the product layer, together with relevant microstructural alterations in the bulk matrix, one can account for the presence of a (potential) excess of certain substances e.g., Ca-bearing compounds.

\section{Experimental Program-Introduction to Sequence and Approach}

The approach to corrosion control by utilizing specifically chosen nanomaterials was studied in sequential steps and a series of experiments, as normally employed in corrosion and corrosion control studies for reinforced concrete [51-58]. This is especially relevant to cases, as in this approach, where a modified (concrete) mix design and/or admixtures are to be evaluated for their effect on both the corrosion state of the steel reinforcement and the properties of the cementitious matrix. For instance, prior to tests in a steel-reinforced, cement-based system, corrosion studies in model aqueous medium, resembling the concrete pore water, were performed in parallel to studies of plain (non-reinforced) cement-based specimens. This allowed a preliminary evaluation of the effect of, e.g., the admixed nanoparticles in systems with lower heterogeneity levels. Hence, the electrochemical state of the steel surface could be more precisely evaluated, while the micromechanical, microstructural, etc., properties of the cementitious bulk material could be derived without an additional contribution of interfaces (e.g., steel/cement-paste interface). Positive outcomes would justify the next steps of studying the new additives or mix design in a reinforced mortar or concrete system, already allowing for evaluation of the material properties of both the steel and cementitious matrix together. The above considerations were followed with regard to the hereby discussed approach to corrosion control in reinforced concrete using nanomaterials. Consequently, a large number of experimental materials and methods were involved.

This paper examines only the concept of the approach to corrosion-induced damage control with the added value of self-healing. The experimental results, therefore, are limited to those supporting the credibility of the approach. Hence, details on the materials and methods are outlined at the end of this contribution as supporting information, narrowed down to the necessary minimum, where reference is also made to the full details of each method or experiment.

\section{The Concept of Nanoparticle Application in Reinforced Concrete}

\subsection{The Approach to Corrosion Control via Nanoparticles}

The approach to corrosion control in reinforced concrete using nanoparticles simultaneously targets the electrochemical response of the steel reinforcement, the material properties of the bulk cementitious matrix, as well as the properties at the steel/cement-paste interface and cement/aggregate (sand or gravel) interfaces. This synergetic approach (involving the fields of cement chemistry, micromechanics, electrochemistry and nanopolymer interactions) aims to reduce bulk matrix and interface permeability and to reduce pore network connectivity, and consequently to minimize diffusivity in the presence of polymeric nanoparticles. These would in turn result in a decreased penetration of aggressive substances in the bulk (cement-based) material. Improved properties of the passive film on the steel reinforcement were expected to be simultaneously achieved, as a result of the presence of the nanoparticles in the vicinity of the steel surface (barrier effects at the very least were expected). Next, only in the event of external influences (such as $\mathrm{CO}_{2}$ penetration, hence carbonation in the matrix, or Cl-penetration, followed by localized corrosion on the steel surface) 
would the nanoparticles participate in a self-healing mechanism. This would be triggered by release of a chosen chemical substance initially incorporated in their interior (e.g., $\mathrm{CaO}, \mathrm{Ca}(\mathrm{OH})_{2}$ ). The result would be at least a partially compensated calcium content in the bulk matrix and an improved (or ideally repaired) product layer on the steel surface.

The above mechanisms are not inhibitive-action-related, e.g., $\mathrm{CaO}\left(\right.$ or $\left.\mathrm{Ca}(\mathrm{OH})_{2}\right)$, and do not have inhibitive properties. The Ca-based "self-healing agent", trapped in the inner volume of the nanoparticles, was chosen as such due to the natural predominance of $\mathrm{Ca}$ in cement-based systems, with the following motivation: (i) the cementitious hydration products are Ca-based and possible reduction of the calcium content (e.g., due to carbonation-related phenomena or leaching-out in the presence of concentration gradients) negatively impacts microstructural and mechanical properties [59-62]; (ii) Ca-substituted iron oxide/hydroxide layers on the steel surface, as previously discussed, are known to be more corrosion resistant ([63], and references therein).

\subsection{The Concept with the Added Value of the Self-Healing of Corrosion Damage}

The concept of degradation control, with the added value of self-healing in the case of carbonation or chloride-induced corrosion, is schematically presented in Figure 1, from the event of damage initiation to the self-healing mechanism.

Figure 1a,b include sections (1) to (7), visualizing the main aspects with regard to reinforced concrete as a system, and outlining the approach to the degradation control employing nanoparticles. In Figure 1a, sections (1) to (3) present the reinforced concrete system, zones and interfaces of interest; section (4) includes a schematic presentation of the considered nanoparticles, i.e., polymeric micelles and vesicles. In Figure 1b, sections (5) to (7) schematically present the degradation events, related mechanisms and final outcome. The interfaces of major interest in the system "reinforced concrete" (Figure 1a, section (1)) are depicted in more detail in section (2), Figure 1a, visualizing the interface of the concrete cover/environment (left), the bulk cement-based matrix (middle) and the steel/cement-paste interface (right). The bulk concrete matrix, in the middle of section (2), is presented zoomed-in as section (3) (Figure 1a), presenting a portion of an aggregate particle (e.g., sand) in the cement matrix, the interfacial transition zone (ITZ) of the aggregate/cement paste, including a pore pathway (a connected pore and pore solution), both in the ITZ and the pore.

Figure 1 also schematically presents the expected uniform distribution of nanoparticles in each zone and at relevant interfaces (Figure 1a, sections (2), (3) and (4)). The nanoparticles, section (4), were expected to exert positive effects on both the cement-based material, as well as on the electrochemical state of the steel reinforcement. For instance, in their presence, reductions in porosity and pore size (section (3) (left), Figure 1a) are targeted for the cementitious material and relevant interfaces, on the one hand. On the other hand, the electrical double layer—see Figure 1a, section (3) (right)—and the overall electrochemical state of the steel surface, were expected to contribute to steel corrosion control. These alterations in material properties would be at hand prior to any degradation-related occurrences (e.g., $\mathrm{pH}$ reduction of the pore solution due to matrix carbonation or corrosion initiation, following chloride ion penetration).

Section (4), Figure 1a, schematically presents the particles chosen for this approach, based on polyethylene-oxide-polystyrene co-block polymers i.e., PEOx-b-PSy. Micelles and vesicles were intended for use, both being formations of a hydrophilic outer portion (corona or shell) and a hydrophobic counterpart (the "core" or enclosed volume). The micelles would affect the material properties mainly due to the presence of the polymer, whereas the effect of the vesicles was intended to be both due to the polymer itself and due to release of the loaded compound. In order to distinguish between these two effects, both "empty" vesicles (i.e., just water reservoirs) or "loaded" ones, i.e., with Ca-based compounds entrapped in their interior $\left(\mathrm{Ca}(\mathrm{OH})_{2}\right.$ in this case) were tested later in the experimental program. 


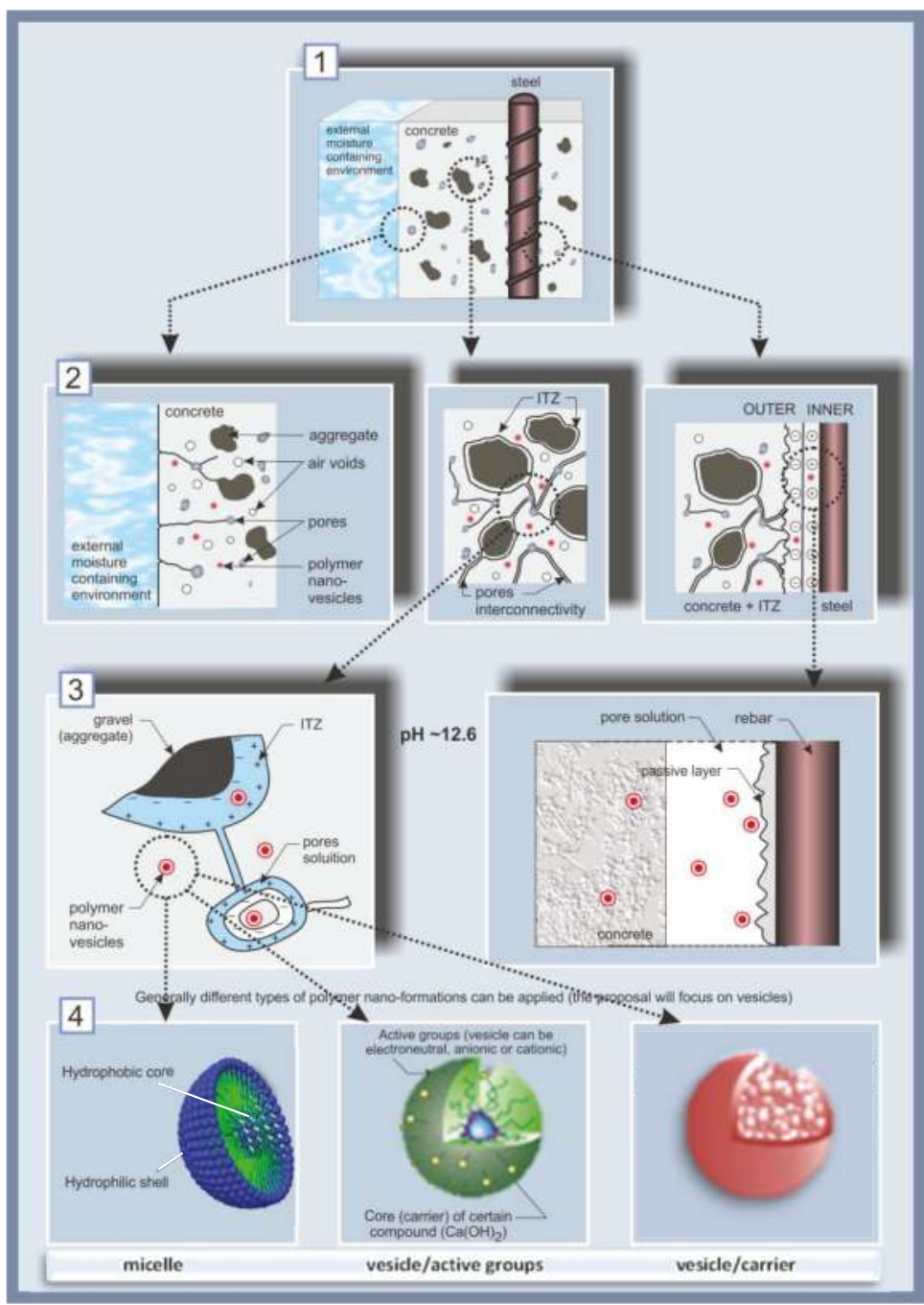

(a)

Figure 1. Cont. 


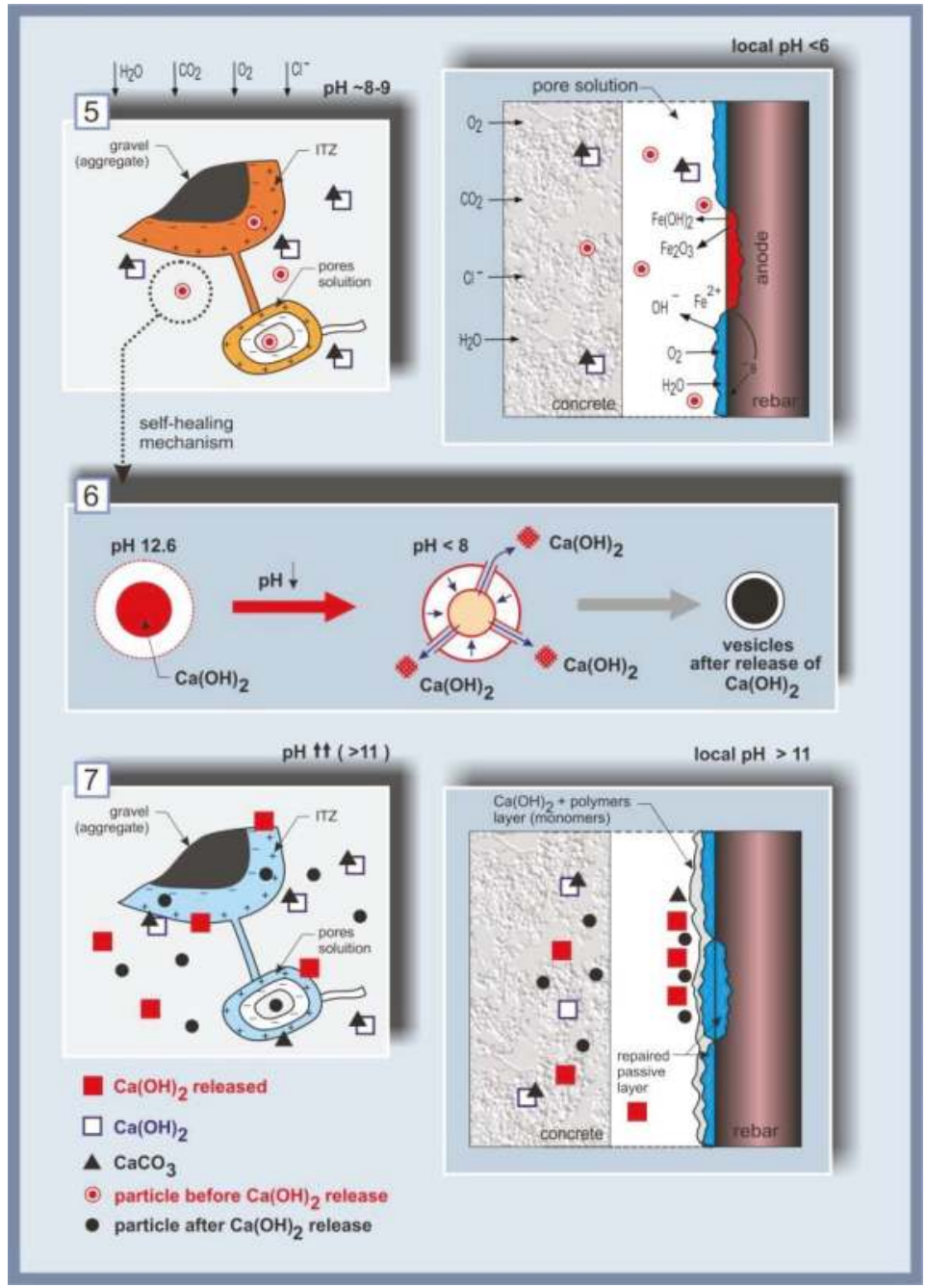

(b)

Figure 1. (a) The reinforced concrete system, (1), with relevant zones of interest, (2) and (3), i.e., the cementitious bulk matrix, the steel/cement-paste interface, and admixed nanoparticle distribution, (3), together with the relevant types of nanoparticles,(4); left-the system prior to changes from external factors, sections (1) to (4); (b) The reinforced concrete system after degradation, (5), followed by self-healing, (7), due to the effect of nanoparticles, (6).

The interfaces schematically depicted in Figure $1 \mathrm{a}, \mathrm{b}$ are also shown as experimental results in the Electron Microscopy (ESEM) micrographs in Figure 2 further below. For instance, the interfaces in the cementitious matrix (aggregate/cement paste) as given in Figure 1a, sections (2) and (3) (left and middle) are presented in Figure 2a,b, while the steel/cement-paste interface as sketched in Figure 1a, sections (2) and (3) (right) are depicted in Figure 2c,d, including the product layer on the steel surface (inlet in Figure 2c,d). 

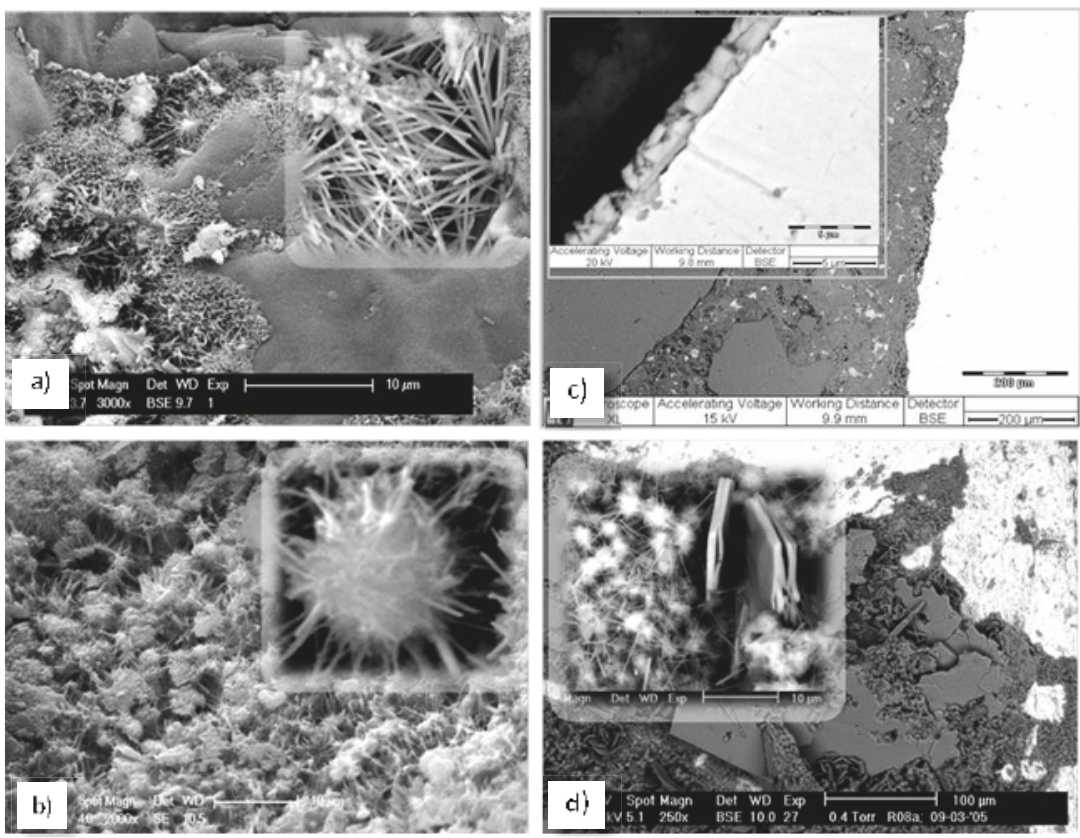

Figure 2. Electron microscopy (ESEM) micrographs, depicting the cement-based bulk matrix $(\mathbf{a}, \mathbf{b})$ and embedded steel (c,d) as follows: (a) a fracture of the interface aggregate (sand particle)/cement matrix, where the inlet depicts a hydration product (ettringite) of needle-shape morphology; (b) cement paste bulk matrix only-morphological observations on a fracture surface, depicting calcium-silicate hydrate (CSH) nucleation and growth; (c) the steel/cement-paste interface in reinforced mortar (a polished cross section), where the following are well visible: the aggregate (sand particles) in the matrix, the cement paste bulk (in the proximity of the steel surface) and a well-adhered product layer (inlet) on the steel surface (the steel reinforcement appears on the right side of the image and in the inlet); (d) longitudinal section (top surface) of a steel reinforcement, partially covered with cement relicts and Ca-based compounds of platy morphology $\left(\mathrm{CaO}, \mathrm{Ca}(\mathrm{OH})_{2}\right)$, together with $\mathrm{CSH}$ particles (inlet).

When no changes in the environment are relevant, the high alkalinity of the pore solution ( $\mathrm{pH} ~ 12.7-13.5)$ maintains steel passivity, i.e., the steel surface is covered by a passive layer (Figure 1a, section (3) (right)), including a well-adhering product layer (Figure 2c) and Ca-based surface products-Figure $2 \mathrm{~d}$ ). Together with improved bulk matrix characteristics, the nanoparticles, present in the vicinity of the steel surface, were expected to improve the properties of the passive layer. For instance, enhanced barrier effects would be expected due to physical or chemical interactions of the particles and cement hydration products. Additionally, the nanoparticles would interact with the iron oxide/hydroxide layer on the steel surface, hence also affecting the steel/cement-paste interface (electron microscopy of this interface is illustrated in Figure 2c,d). In other words, the admixed nanoparticles would result in superior material properties when no degradation mechanisms are yet involved.

Section (5) in Figure $1 \mathrm{~b}$ presents the effect of carbonation (left) or chloride-induced corrosion (right). As previously discussed, carbonation is the process of the reaction of $\mathrm{CO}_{2}$ with Ca-bearing compounds in the matrix, i.e., calcium-silica-hydrate (C-S-H) and $\mathrm{Ca}(\mathrm{OH})_{2}$, formation of $\mathrm{CaCO}_{3}$, which deposits in the bulk matrix, blocking the $\mathrm{Ca}(\mathrm{OH})_{2}$ (Figure $1 \mathrm{~b}$, section (5) (left)). This leads to a reduction of $\mathrm{pH}$ to ca. 8-9 in the pore solution and ultimately to uniform corrosion of the steel reinforcement. On the other hand, chlorides present at the steel/cement-paste interface will 
induce localized corrosion of the steel reinforcement and break down the passive layer (a local drop of $\mathrm{pH}$ to $<6$ would be relevant on the steel surface and within the localized damage (section (5), Figure $1 \mathrm{~b}$, (right), showing the formation of corrosion cells, ionic and electron flow, corrosion product formation, and the re-distribution of anodic and cathodic locations on the steel surface). Typical chloride-induced corrosion damage on reinforcing steel is presented by the ESEM micrographs in Figure 3c,d in comparison to control, non-corroding reinforcement (Figure 3a,b). Both cases in Figure 3 depict the steel/cement-paste interface in reinforced mortar (left) and the product layer on the steel surface (right). These will be discussed further below with respect to the outcomes when employing nanoparticles for corrosion control (Section 5).
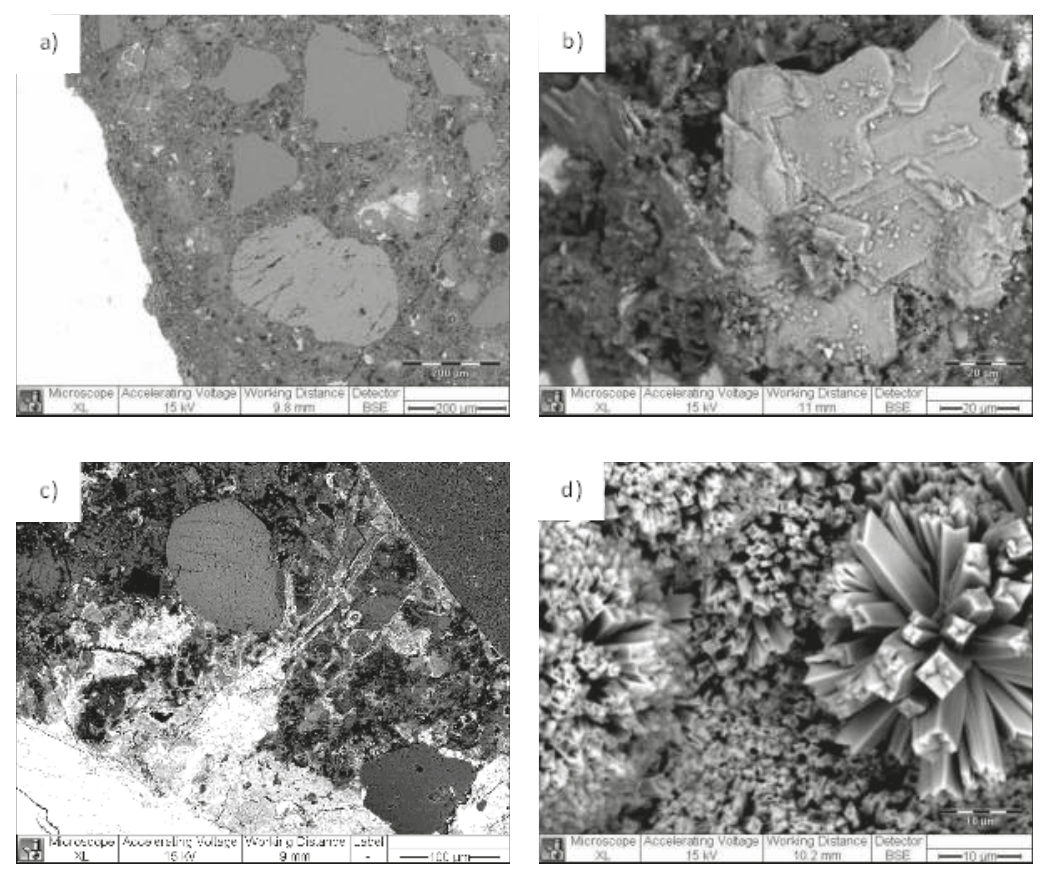

Figure 3. ESEM micrographs: steel/cement-paste interface in reinforced mortar $(\mathbf{a}, \mathbf{c})$ and product layer on the steel surface $(\mathbf{b}, \mathbf{d})$ for a control, non-corroding $(\mathbf{a}, \mathbf{b})$ and a corroding specimen $(\mathbf{c}, \mathbf{d})$.

Following the concept of the approach as given in Figure 1, the $\mathrm{pH}$ alterations in the pore solution, or those at the steel surface, were to trigger re-structuring of the initially admixed nanoparticles, section (6) in Figure 1b. A nanoparticle that releases an entrapped compound using a change in $\mathrm{pH}$ as a trigger was the original idea of the approach. However, the employed micelles and vesicles, being Polystyrene (PS)-based, were in fact stable in the event of $\mathrm{pH}$ changes. Therefore, the mechanisms upon which the nanoparticles exerted positive effects and improved material properties, finally leading to the self-healing of the previously-induced damage, section (7) in Figure 1b, were mainly mechanisms such as (i) salting-out effects of their PEO portion; (ii) the re-structuring and release of the core-containing compound due to osmotic pressure and micromechanical stress within hydration and corrosion product alterations and/or growth; and (iii) "nucleation site" effects1 prior to degradation phenomena. Additionally the hydrophobic PS-portion of the nanoparticles, which has a high glass transition temperature ( $\mathrm{Tg}$ ), results in the stability of the micelle or vesicle formation in conditions of no internal transformations and, consequently, to a gradual release of any hydrophobic load [64], which would be directed towards a higher release rate in the presence of micromechanical stress. The 
release of Ca-based compounds from the vesicles, induced by the above triggers, or by de-hydration in the presence of chlorides, will result in partial recovery of the calcium content in the pore solution, while the hydrophobic corona (or vesicle shell) will collapse over the hydrophobic PS counterpart (or the core). The "empty" nanoparticles would still contribute to reducing porosity in the system and bridging gaps or micro-cracks (the concept of which will be illustrated in Section 5), while the released compounds would stabilize the pore solution composition and/or improve the protective ability of the passive and product layers on the steel surface.

\section{Credibility of the Concept and Approach}

\subsection{PEO-b-PS Performance in Contact with Cementitious Materials-Preliminary Studies}

Prior to the production of PEO-b-PS-based micelles and vesicles for the purposes of the above discussed approach, the performance of this block co-polymer was evaluated when in contact with a cementitious material. Calcium-sulfoaluminate-based expansive additive (CSA) was used for this purpose, where the CSA particles (as dry powder) were "embedded" in a PEO-b-PS film. CSA was chosen for this demonstrative test, since the compound is a highly expansive agent, forming well distinguishable hydration products (ettringite) upon contact with the aqueous medium. This means that upon damage of the PEO-b-PS film, the CSA particles will immediately react to form well-visible hydration products.

The aim of this test was to observe the overall performance of PEO-b-PS in contact with cementitious hydration products. This was important in view of the performance of PEO-b-PS-based micelles or vesicles, when added to a cement-based mixture for the purposes of the approach described above. Next, the aim was to illustrate a designed release of cementitious hydration products when the CSA particles, initially "coated" with PEO-b-PS, would come in contact with alkaline solutions (like the pore water in a cement-based matrix). This process would occur in the event of a mechanical trigger (e.g., a micro-crack in the cementitious matrix) or damage of the PEO-b-PS "coating". Further, the produced hydration products would fill in gaps or bridge cracks, while the polymer itself would remain within the hydration products. This section only provides a visual illustration of the observed interactions.

A procedure of a "reversed micelle" formation was performed to coat single CSA particles (generally of the size between 10 and 100 micrometers) with a PEO-b-PS film: (i) an organic solvent that would allow the formation of a film but would prevent the initial formation of hydration products was used; (ii) the "coated" CSA was dried-out and produced as a film of individually coated CSA particles. The microscopic investigation in Figure 4 presents the uniform, non-treated film of the CSA+PEO-b-PS composite. The composite was broken into flakes and was water treated for $24 \mathrm{~h}$.
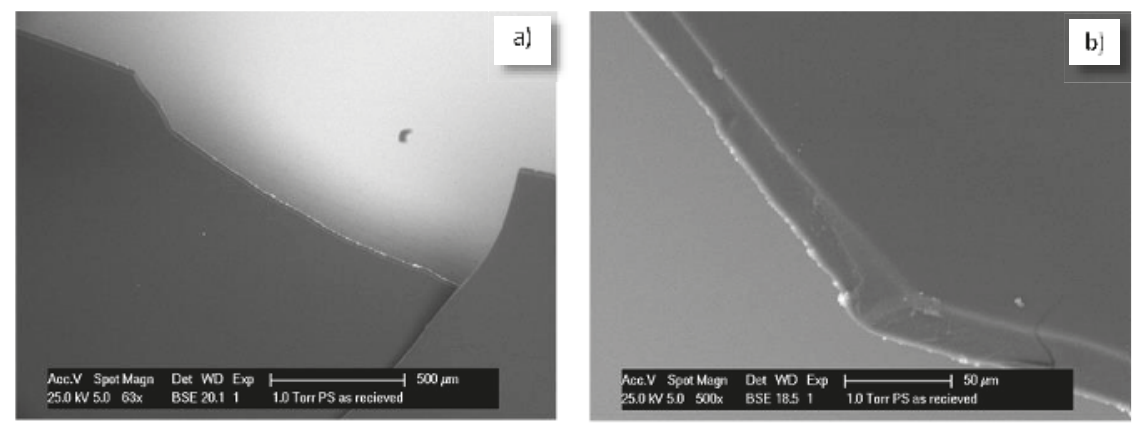

Figure 4. Polyethylene oxide-b-polystyrene coated Calcium sulfoaluminate (PEO-b-PS + CSA) composite-as produced (non-treated) film at magnification 63x (a) and 500x (b). 
Figure 5a depicts the film, containing CSA particles, where no reaction products were yet observed. Figure $5 \mathrm{~b}$ presents another portion of the film, where due to film rupture and the exposure of a CSA particle to the environment, a reaction product had already formed at the edge of the flake (marked area). In this case, after water treatment of the broken composite film, the CSA particles already had access to the medium and formed ettringite. The product growth is well visible at this edge location, as well as within a crack in the film (Figure 5c), together with the "left over" from the polymer, embedded in crystallites of the hydration product (Figure $5 c, d$, marked locations). It is interesting to note the observation of cracks bridged by the reaction products as well (Figure 5c). The Energy dispersive X-ray (EDX) patterns for the film only (mark 1 in Figure 5c) and the hydration product (mark 2 in Figure 5d) are also given in Figure 5, bottom row, confirming the expected composition of the investigated formations, i.e., carbon only for the polymer film and calcium sulfoaluminate together with carbon content (from the surrounding film) in the case of the hydration product.
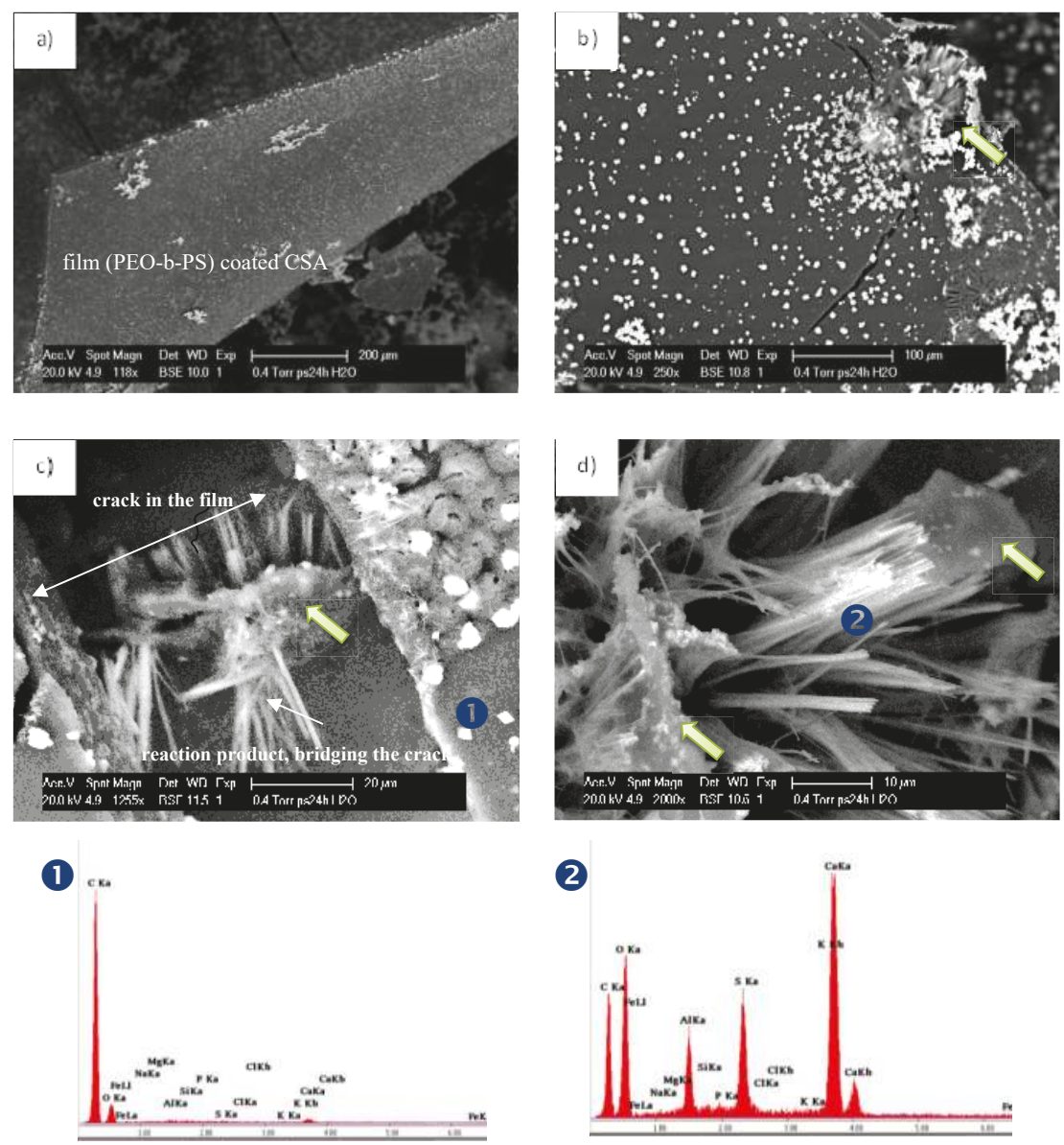

Figure 5. PEO-b-PS + CSA composite after $24 \mathrm{~h}$ treatment in aqueous medium (a, b); higher magnification of the reacted CSA, bridging micro-cracks (c) and a remaining PEO-b-PS on the hydration product (d). The EDX patterns (bottom of figure) present the result from local spot analysis, corresponding to the indicated locations in c) and d). 
The observations discussed in this section confirm the possibility that PEO-b-PS can "interact" with cementitious hydration products in a desired direction and were for purely illustrative purposes, rather than being elaborated with tests, which is not the subject of this contribution. What was also concluded is that a PEO-b-PS composite would be sufficiently brittle to "release" the embodied load, on the one hand. On the other hand, although the PEO-b-PS composite film cannot be directly compared to nano-sized, self-assembled formations such as micelles and vesicles, the results also show that PEO-b-PS would be stable in a cement-based material and react to external and designed triggers or damage.

\subsection{Micelles and Vesicles in (Reinforced) Cementitious Materials-Brief Review of the Main Outcomes}

The concept of the approach presented in Section 4.2 was studied in sequential steps of tests in model medium, plain (not reinforced) and reinforced cement-based materials. As mentioned above, PEO-b-PS micelles and vesicles were studied. The choice of using these architectures was initially based on previous studies of nanocomposite galvanic coatings, where PEO-based nanoparticles, added to electrolytes for $\mathrm{Zn}$ and $\mathrm{ZnCo}$ electrodeposition, were found to significantly increase the corrosion resistance of the coatings $[65,66]$. Later on, and in view of the increasingly expressed safety and health concerns regarding the application of nanomaterials in general [67], but also in view of the concepts for built-in recyclability in the construction industry, PEO-based nanoparticles were chosen, which are otherwise considered for medical and bio-medical applications, [64,68-70] (i.e., no health hazards related). PEO-b-PS micelles and vesicles were used for the purpose of the above discussed concept and approach. It is well known that polymeric micelles and vesicles present numerous possibilities to alter the properties of various material classes and are used in various applications. A thorough and indeed comprehensive review on polymeric vesicles, their preparation and responsive behavior can be found in a recently reported work [71], where the application of vesicles was discussed to reach beyond the traditional field of application, biomedicine, extending to nanoreactors [70,72], perfume containers [73], catalysis [74], water remediation [75], etc. This is due to the stimuli-responsive behavior of polymer vesicles, considered as smart materials. Traditional external triggers for targeted responsive behavior are $\mathrm{pH}$, temperature, light, electrical or magnetic fields, but also oxi-redox reactions (electron and ionic flows), micromechanical stress, etc. [76-79]. Hence, the applications for vesicles, together with the many possibilities to functionalize their characteristics so that the designed properties and response of the materials are achieved, are constantly increasing.

For the purpose of the concept and approach as discussed in Section 4, and in order to investigate the effects of the polymer itself on the material properties of the steel and cement-based matrix, the studies employed micelles first. Next, "loaded" nanoparticles, i.e., vesicles, were used to study degradation control with the added value of self-healing due to active substance release, which was the original aim of the study. The full scale of each experiment, together with detailed reports on the observed behavior and results are reported as separate works in specialized (corrosion or civil engineering) journals [56-58,80-83]. The significant effect of a very low concentration of nanoparticles (0.024 wt \% per cement weight) on the global bulk matrix properties-twice lower porosity and three orders of magnitude lower permeability of the micelle-modified matrix-is also reported $[80,81,84]$. Compared to the (generally) employed amounts of polymers (between $0.3 \%$ and $30 \mathrm{wt} \%$ ) and inorganic nanoparticles (e.g., Fe-, Ti-, Si-based oxides), the nanoparticles for the above discussed approach were used in extremely low concentrations (in the range of $2.4 \times 10^{-3} \mathrm{wt} \%$ for micelles and vesicles in model aqueous medium and $6 \times 10^{-3}$ wt \% per mortar weight for micelles/vesicles in cement-based, solid systems). Therefore, a "self-repair" or "self-healing" of the product layer on steel solely due to the released Ca-component is not realistic in view of these minimal concentrations. The most plausible mechanism(s) would be linked to enhanced chloride binding effects, on the one hand. On the other hand, the nature of the incorporation of the nanoparticles in the product layer on the steel surface, adsorption on the active (anodic) areas, altered oxi/redox reactions, and/or subsequently alerted composition of the product layer on the steel surface, would be of equally large significance. The former 
effects on the bulk matrix properties and enhanced chloride binding are related to the increased chloride threshold, i.e., delayed corrosion initiation or unsustained corrosion propagation. These were found to be related to micro-mechanical and microstructural alterations, e.g., a more uniform distribution of low density C-S-H, hence superior global bulk matrix properties were achieved $[80,81,84]$. The latter effects, related to enhanced corrosion resistance, were justified by electrochemical tests in model medium $[56,57,83]$, in cement paste and in reinforced mortar $[56,58,80,84]$, together with results from the steel surface analysis and the steel/cement-paste interface $[81,82]$. The next section contains some of the latest results for the electrochemical performance of steel, together with microscopic observations. These are well in line with the above outcomes and constitute the evidence for the concept and approach presented in this work.

\subsection{The Effect of Vesicles on the Corrosion Performance of Steel and on Bulk Matrix Properties}

The utilization of nanoparticles targeted a simultaneous improvement of the electrochemical performance of the steel and the bulk matrix microstructure. Further, a possible self-healing mechanism was aimed at, specifically when Ca-based vesicles were used. The results of the application of both "empty" and Ca-bearing vesicles ( $\mathrm{PEO}_{113}-\mathrm{b}-\mathrm{PS}_{760}$-based) are presented here as supporting evidence for the already discussed concept and approach.

Electrochemical measurements of steel electrodes, treated in both nanoparticle-free medium and model medium containing nanoparticles, were performed at defined time intervals after open circuit potential (OCP) stabilization. The model medium was also chloride-free (to represent control cases) and chloride-contaminated (for corroding cases). The OCP records are an indication of the active or passive state of the steel reinforcement. Figure 6a) depicts the recorded OCP values for all specimen groups in the model medium of cement extract (CE) (specimen designation and details on medium composition are as given in the supporting information). Figure $6 \mathrm{~b}$ presents the calculated polarization resistance values $\left(R_{p}\right)$ for steel electrodes treated in all investigated solutions after $24 \mathrm{~h}$, four days and seven days. As can be seen from the plots, a trend to OCP ennoblement (Figure 6a) and increasing $R_{p}$ (increased corrosion resistance), (Figure $6 b$ ), was observed within treatment for all control cases, irrespective of the presence or absence of nanoparticles in the medium (CE, CEV and CEVC, where $C E$ is nanoparticle-free; $C E V$ contains empty vesicles in the medium and CEVC contains calcium-containing vesicles in the medium). This is a general outcome within the stabilization of the steel passive layer in a chloride-free, alkaline medium such as CE.

For the corroding cases (CEn, CEVn and CEVCn), the cathodic shift of the OCP values (increased corrosion activity), specifically after $24 \mathrm{~h}$ (Figure 6a), together with significantly lower $R_{p}$ values (Figure 6b) were an expected outcome. An exception, however, was the CEVCn specimen, which represents steel treated in chloride-contaminated $\mathrm{CE}$ in the presence of $\mathrm{Ca}$-bearing vesicles. Prior to $24 \mathrm{~h}$, the OCP values for this specimen maintained levels similar to the active specimens CEn and CEVn (ca. $-200 \mathrm{mV}$ ), after which an anodic shift was observed and the OCP of the CEVCn specimens ended up being even more anodic than the control case CE at the end of the seven day test (168 h). This result clearly indicates the effect of Ca-bearing vesicles towards restored steel passivity. A similar effect was not observed when "empty" vesicles were present (see OCP records for the CEVCn and CEVn specimens, Figure 6a), although the OCP for the CEVn specimens (empty vesicles) was more anodic when compared to steel treated in vesicle-free solution (CEn specimen). The above accounts for a "barrier" effect only of the polymer (vesicles themselves) for CEVn specimens, rather than altered oxidation/reduction reactions towards improved steel surface layer properties, and repair, as obviously related to the CEVCn specimens. In support of the above, the following more specific points can be noted regarding the results in Figure 6a:

(i) After $1 \mathrm{~h}$ and $3 \mathrm{~h}$ of treatment, the corroding CEn specimens (vesicle-free medium) presented OCP values in the range of those for the control CE (ca. $-180 \mathrm{mV}$ ). For CEn specimens, corrosion initiation occurred between $3 \mathrm{~h}$ and $24 \mathrm{~h}$ and was sustained (and propagated) towards $96 \mathrm{~h}$ and $168 \mathrm{~h}$. This is proven by the recorded cathodic OCP shift after $24 \mathrm{~h}$, reaching ca. $-400 \mathrm{mV}$ towards 
the end of the test. For CEn at the initial time intervals ( $1 \mathrm{~h}$ and $3 \mathrm{~h}$ ), corrosion initiation and propagation compete with passive layer formation in the alkaline medium, while with treatment, the reaction mechanisms as previously described in Section 2.2., were determined by the rate of chloride ion adherence, surface layer dissolution and passivity breakdown;

(ii) For the time intervals of $1 \mathrm{~h}$ and $3 \mathrm{~h}$, the specimens in both the corroding and control conditions, where vesicles were present (i.e., $\mathrm{CEV}, \mathrm{CEVn}$ and $\mathrm{CEVC}$ and CEVCn), initially exhibited more cathodic OCP values (between -200 and $-230 \mathrm{mV}$ ). This is due to the competitive mechanisms of passive layer formation in the alkaline medium and the effect of vesicles and/or chloride ions. In other words, when vesicles were present, these acted as a barrier towards both passive layer formation and chloride-induced corrosion. The vesicles induced a resistance polarization for the oxidation and reduction reactions on the steel surface;

(iii) For the case of steel treated in chloride-free, empty-vesicle-containing solution (CEV specimens), surface stabilization was gradually achieved towards $168 \mathrm{~h}$ of treatment. However, the final OCP values were not as noble as those for the control (vesicle-free) CE case (ca. $-30 \mathrm{mV}$ for $\mathrm{CE}$ and ca. $-70 \mathrm{mV}$ for CEV). This result is due to the abovementioned limitations, which are not relevant for $\mathrm{CE}$ specimens. There was only supportive evidence for barrier effects and resistance polarization for the CEV specimens; the CEV specimens also had the highest global Rp value, recorded at the end of the test (Figure 6b). The empty vesicles in the corroding CEVn specimens initially induced the same barrier effect regarding passive layer formation, but also exerted a delay in corrosion initiation. This is evident from the ennoblement of OCP from $1 \mathrm{~h}$ to $24 \mathrm{~h}$ (ca. $-200 \mathrm{mV}$ to ca. $-180 \mathrm{mV}$ ). After $24 \mathrm{~h}$, however, the OCP for CEVn specimens shifted in the cathodic direction, reaching approx. $-240 \mathrm{mV}$ after $96 \mathrm{~h}$;

(iv) In contrast to all above cases, the steel electrodes in the medium with Ca-bearing vesicles (CEVC and CEVCn specimens) present similar values at $1 \mathrm{~h}$ and $3 \mathrm{~h}$, irrespective of the presence of chloride ions in the medium. These values are more cathodic, accounting for limitations regarding passive layer formation. For the control case (CEVC) an anodic shift was observed around 168 days, similar to the CE and CEV groups. Contrary to the corroding cases (CEn and CEVn) discussed above, the corroding specimens (CEVCn) show ennoblement only at all time intervals, with the most noble OCP at the end of the test. This accounts for a restructuring of the passive film on the steel surface for CEVCn, most likely the formation of a Ca-substituted product layer with higher corrosion resistance (phenomena previously discussed in Section 2). The superior corrosion resistance for CEVCn specimens was obviously triggered by the Ca-bearing vesicles in this case.

The OCP evolution discussed above for all specimens is well in line with the derived Rp values (Figure 6b). For instance, the Rp values for the CEVCn specimens clearly show an increasing trend, i.e., improved corrosion resistance towards the end of the test (Figure $6 \mathrm{~b}$ ), which was not observed for CEVn specimens, where "empty" vesicles were employed. Here again the difference between CEVn and CEVCn specimens is to be attributed to the vesicle type in the medium- "empty" for the former and Ca-bearing vesicles for the latter case-where the Ca-bearing vesicles introduce superior product layer properties to the steel surface.

The higher corrosion resistance and improved properties of the product layer in CEVCn specimens, potentially due to the "repair" of the initial corrosion damage, is evident from the potentio-dynamic PDP response for all cases at the end of the test, Figure $6 \mathrm{c}$.

After seven days of treatment, the corrosion and anodic currents for the corroding CEVCn specimens were comparable to those of the control (non-corroding) CE and CEVC cases, where the corrosion current for CEVC was the lowest recorded. In contrast to the altered electrochemical state of the steel surface in the presence of Ca-bearing vesicles, barrier effects alone are the most plausible for the steel specimens treated in "empty" vesicle-containing medium. This is evident from the similarly higher corrosion and anodic currents for both control (CEV) and corroding (CEVn) specimens (Figure 6c, CEV and CEVn). 

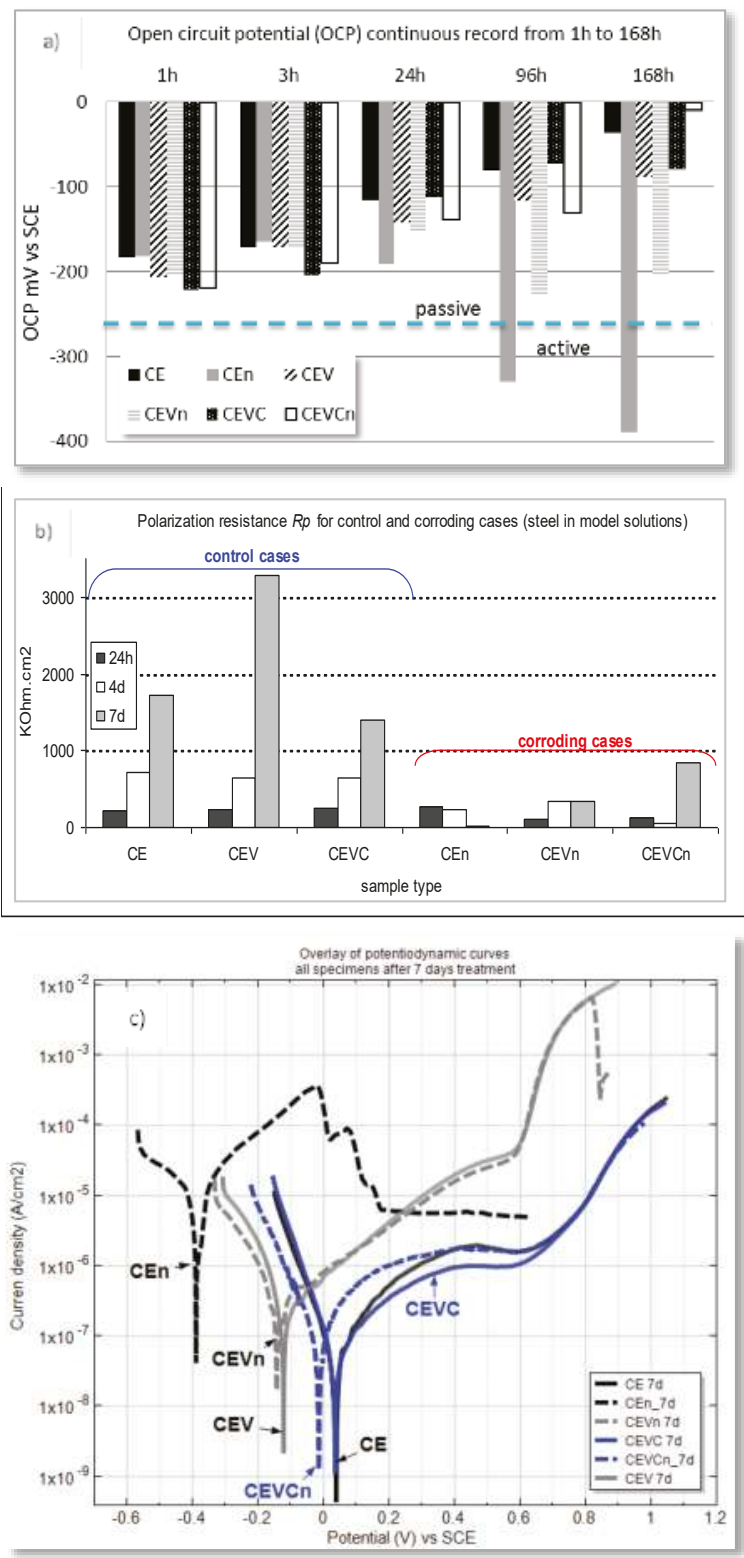

Figure 6. Electrochemical parameters derived for steel electrodes in model solutions: (a) Open circuit potential OCP records; (b) Polarization resistance Rp values and (c) Potentio-dynamic PDP response (specimens designation: $\mathrm{CE}$-control, non-corroding; $\mathrm{CEn}$-corroding: CEV—control, empty vesicles; CEVn—corroding, empty vesicles; CEVC—control, Ca-bearing vesicles; CEVCn—corroding, Ca-bearing vesicles).

In other words, corrosion propagation for the case of CEVn was impeded, while the establishment of a corrosion-resistant passive film for CEV specimens was also inhibited. The resulting currents were approximately one order of magnitude higher for these cases, compared to the control CE and 
the corroding CEVCn specimens. The highest corrosion and anodic currents were recorded for the corroding CEn specimens (Figure 6c), which was as expected for steel treated in nanoparticle-free, chloride-containing medium.

In view of the corrosion resistance and electrochemical-method-derived performance for steel in a cementitious matrix, it would be expected that the steel/cement-paste interface would have different properties in the presence of admixed nanoparticles. This was as previously discussed in relation to the concept and approach of nanoparticle application and with regard to Figure 1, where the relevant interfaces were schematically presented. Figure 3, as previously discussed, depicts micrographs of the steel/cement-paste interface in control and corrosion-reinforced mortar specimens, where the matrix was not modified with nanoparticles. As can be observed, an intact interface is relevant for the control case (Figure 3a), while corrosion products are clearly visible for the corroding case (Figure 3c), already penetrating the bulk matrix at a distance of more than 200 micrometers away from the steel surface. Figure 3 is an illustration of the reason for the steel/cement-paste interface degradation, and ultimately the reinforced concrete degradation, when chloride-induced corrosion takes place. For the corroding specimen (Figure 3c), corrosion products such as $\mathrm{Cl}$-containing iron oxi/hydroxides formed on the steel surface (e.g., akaganeite, Figure 3d). These were volume-expanding and caused continuous micro-cracking in the restricted bulk volume of the cement paste material. In contrast, for the control and non-corroding conditions, the steel surface was generally covered by $\mathrm{CaO} / \mathrm{Ca}(\mathrm{OH})_{2}$-substituted product layers, see Figure $3 \mathrm{~b}$, which except for maintaining steel passivity, did not cause microstructural alterations of the bulk matrix.

In the presence of vesicles and upon corrosion initiation, a re-structuring of the product layer on the steel surface was expected, following the mechanisms described in Figure 1. A product layer rich in Ca-substituted iron oxi/hydroxides is relevant for a matrix modified with Ca-bearing vesicles (as indirectly determined by electrochemical tests). In contrast, at minimum, barrier effects and reduced corrosion product formation should be observable for the matrix modified with "empty" vesicles. These outcomes were in fact recorded for the steel/cement-paste interface in the presence of both vesicle types and this was supported by $\mathrm{X}$-ray analysis on the steel surface. A detailed analysis of all conditions and the complete investigation of the reinforced mortar is reported in detail in [82]. In this contribution, the following supportive evidence (Figure 7) is briefly discussed based on light microscopy on the steel surface and the adjacent cement paste, illustrating the main objective of this work, i.e., presenting the concept of the approach utilizing nanoparticles for reinforced-cement-based materials.

As can be observed in Figure 7a,b for the control condition, there were no corrosion products on the steel surface and no corrosion products deposited in adjacent to the steel cement paste. Similarly, no corrosion products were observed for the specimens where the mortar contained Ca-bearing vesicles (Figure $7 \mathrm{~g}, \mathrm{~h}$ ).

In contrast, corrosion product accumulation on the steel surface and penetration into the bulk matrix were observed for the corroding, vesicle-free specimen (Figure 7c,d). When "empty" vesicles were admixed in the mortar matrix, the previously discussed limitations towards both passive layer formation, but also impeded corrosion propagation, resulted in reduced corrosion product formation, however, with evidence of corrosion product penetration into the bulk matrix (Figure 7e,f). Hence, the previously discussed corrosion resistance of the steel electrodes in simulated medium, recorded via electrochemical measurements (Figure 6), and the hypothesized performance in view of the steel/cement-paste interface, are hereby visualized (Figure 7) and evidence is provided of the "self-healing" or "self-repair" of the product layer on the steel surface in the presence of Ca-bearing vesicles. 

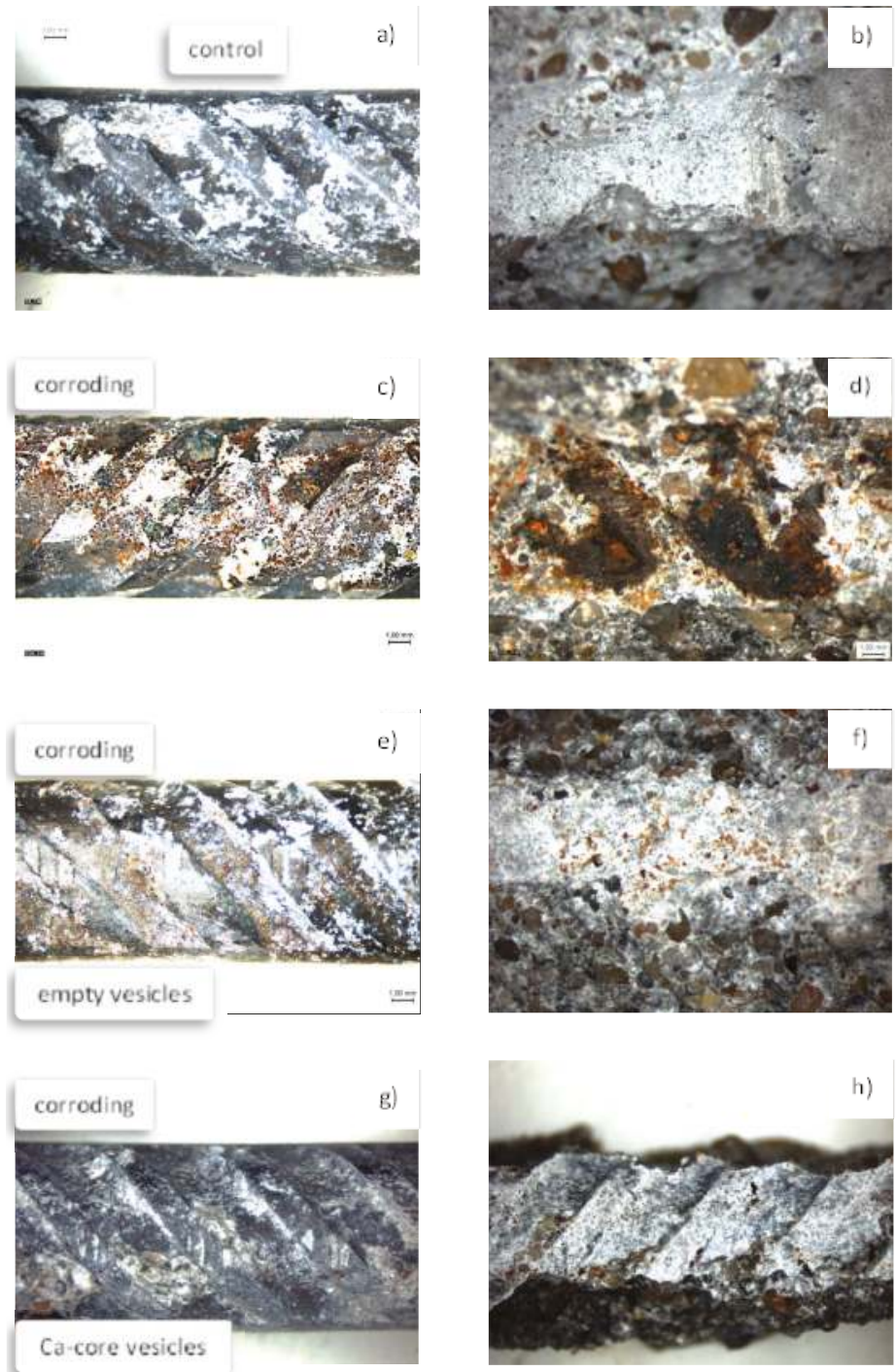

Figure 7. Light microscopy of the steel reinforcement (left column) and the corresponding "print" in the cement paste (i.e., adjacent bulk matrix) for control and corroding, vesicle-free specimens (a-d) and corroding, vesicle-containing specimens $(\mathbf{e}-\mathbf{h})$. The observations were recorded after the specimens were broken open after 300 days of conditioning.

The concept of nanoparticle application for reinforced concrete, as discussed in Section 4, clearly emphasized the importance of the approach in view of simultaneously affecting the steel reinforcement and the bulk matrix in a reinforced concrete system. The effect of nanoparticles on the bulk matrix properties was thoroughly investigated, starting from less heterogeneous systems (cement paste, plain mortar) and later studying reinforced-cement-based materials. Microstructure (e.g., porosity, pore size), micromechanics (e.g., elastic modulus of low and high density CSH), compressive strength, permeability, etc., were recorded for micelle- and vesicle-free mixtures versus modified mixtures. 
Some of the these results were briefly mentioned in Section 5 and reported in detail in specialized journals [80-84].

As supporting evidence of the concept and approach of nanoparticle application, the subject of this work (Figure 1), Figure 8 depicts the bulk matrix of mortar specimens at the hydration age of seven days as a comparison of a vesicle-free matrix (Figure 8 left) and Ca-based, vesicle-containing matrix (Figure 8 right). Of course, without quantification and detailed analysis of images such as those in Figure 8, no claims or scientific judgment can be made. Moreover, microstructural analysis is generally relevant to at least 35 locations (images of magnification $500 \times$, such as the ones in Figure 8). This is performed for statistical accuracy and following a known methodology that has been reported in detail $[18,63]$. Therefore, no further discussion will be included here, but rather pointing out only the clearly observable difference in the images in Figure 8.
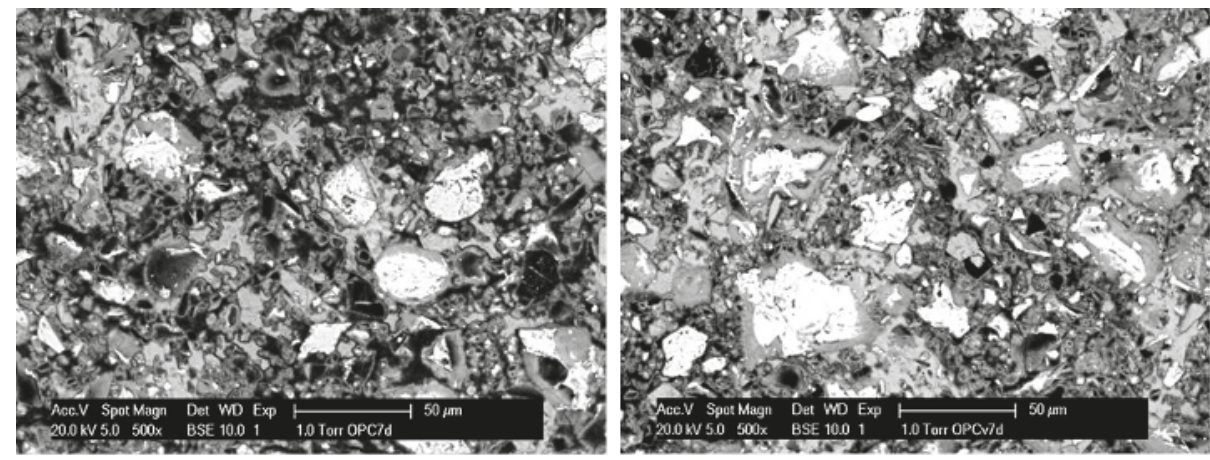

Figure 8. Cement-based bulk matrix (impregnated and polished cross sections of mortar specimens, as usually used for ESEM image analysis) without (left) and with (right) admixed nanoparticles (vesicles) at seven days of hydration age (black color represents pores and voids in the matrix, white color represents un-hydrated phases (cement grains), the grey colored features represent hydration products (e.g., C-S-H, $\mathrm{Ca}(\mathrm{OH})_{2}$; for image analysis of the bulk matrix, aggregates (as sand, gravel and relevant interfacial transition zones ITZs) are to be excluded, therefore the images do not present aggregates or interfaces).

As can be seen, the vesicle-free specimen (Figure 8, left) presents a relatively higher level of pore and void distribution, while the matrix in the vesicle-modified specimen (Figure 8, right) depicts well-visible high and low density CSHs (and less pores and voids). This illustrates the positive effect of admixed vesicles, as targeted and previously discussed within the concept of the approach in Section 4 . These outcomes are in line with what was reported for the effect of PEO-containing polymers on the properties of fresh cement paste, where reduced coagulation and sedimentation of the cement particles was discussed as being the result of a polymer-induced increase in electrostatic or repulsive forces during cement hydration $[85,86]$. The result is an altered distribution of the hydration products in the matrix, as also seen in Figure 8 and previously reported for both micelles and vesicles [81,84], where in addition, the aforementioned "nucleation site effect" [24] contributes further to the reduced porosity and permeability of the bulk cement matrix.

\section{Conclusions and Outlook}

The purpose of this contribution was to present the concept of an approach to employ polymeric nanoparticles to control reinforced concrete degradation.

The originality of the approach to control reinforced concrete degradation via specific nanoparticles is in the targeted simultaneous improvement of both steel and concrete properties, prior to any degradation, and to repair damage later on, in the event of impaired properties. The 
advantage of the utilization of nanoparticles in the discussed manner, is that these particles, initially introduced in the system to improve the mechanical and microstructural performance of the bulk cementitious material and the steel/cement-paste interface, will further participate in a self-healing process in terms of corrosion protection or product layer repair on the steel reinforcement.

Conclusive statements can be made on two main aspects: the implementation of tailor-made nanoparticles and autonomous self-healing. The former (tailored particles) have so far proven to be a feasible approach for corrosion control, evidenced by the significant influence of the minimal concentration of these on the material properties, namely achieved corrosion delay, superior steel product layer characteristics and contribution to increased passivity, rather than only enhanced barrier effects. The latter (self-healing aspects) are justified by (at the very least) the corrosion performance of steel when in contact with "empty" or Ca-bearing nanoparticles. For the former case, corrosion delay is only relevant, but no evidence of an improved electrochemical response was observed. For the latter case, corrosion propagation was not observed, which together with the improved stability of the product layer after an initially more active state, shows the possibility for self-healing or self-repair on the steel surface.

The next step in view of the above approach is to study the local electrochemical response on the steel surface (at the nano- to micro-levels), together with (changes in) the micromechanical properties of the product layer versus an altered chemical composition and morphology of the nanoparticles themselves and upon designed triggers. These results, when linked with the already available outcomes on global corrosion performance and the microstructural characteristics of the cement-based material and interfaces, are expected to substantiate the approach even further. For instance, the results will allow a simulation approach to provide evidence for this concept of corrosion control in view of the predictions regarding material performance versus optimum design by, e.g., varying the chemistry and concentration of the nanoparticles. Ultimately, this will support the feasibility of practical applications in reinforced concrete structures, with relevance to cost justifications and service-life predictions, compared to existing and more conventional methods and practices.

\section{Supporting Information: Materials and Methods}

\section{Model Medium and (Reinforced) Cement-Based Systems}

Model aqueous medium (cement extract) was used as a simulated pore solution for preliminary tests on the corrosion performance of steel in the presence of nanomaterials. Cement paste, mortar and reinforced mortar were studied as the solid, cement-based materials. The CE was prepared by mixing Ordinary Portland cement (OPC) CEM I $42.5 \mathrm{~N}$ and water in a ratio of 1:1, followed by stirring the suspension for $24 \mathrm{~h}$ and subsequent filtration. The $\mathrm{pH}$ of the filtrate (i.e., the $\mathrm{CE}$ ) was 12.7, and the chemical composition (derived by inductive coupled plasma spectrometry, ICP) was: $\mathrm{Ca}-201 \mathrm{mg} / \mathrm{L} ; \mathrm{K}-3.85 \mathrm{mg} / \mathrm{L} ; \mathrm{Na}-1.33 \mathrm{mg} / \mathrm{L} ; \mathrm{Al}-4 \mathrm{mg} / \mathrm{L}$ and $\mathrm{Fe}-<1 \mathrm{mg} / \mathrm{L}$. Both chloride-free and chloride-containing $\mathrm{CE}$ were used, and $\mathrm{NaCl}$ adjusted to $1 \% \mathrm{NaCl}$ in the $\mathrm{CE}$ was used as a corrosion-accelerating medium. These solutions were also additive-free or contained additives (i.e., polymeric nanomaterials and/or $\mathrm{NaCl}$ were added to the solution according the test requirements). More details on this generally employed methodology to test the corrosion performance of steel in CE, as a simulated environment, can be found in $[51,52,56,83]$.

Plain (non-reinforced) mortar cubes were cast to evaluate the properties of the cementitious bulk matrix, while reinforced mortar cylinders were used for tests of the steel corrosion state, cement-based matrix properties and steel/cementitious materials interface. Identical cement type (OPC), water-to-cement ratios (w/c 0.5) and cement-to-sand ratios (c/s 1:3) were used for all cement-based specimens. The non-reinforced-cement-based specimens were studied in sequential steps, e.g., after three, seven, 14 and 28 days of cement hydration for deriving microstructural and mechanical properties. This is a general approach in concrete material science to evaluate properties 
and performance with time of cement hydration. Details on all these types of experimental series are reported in [53-58,80-82,84].

After casting, the reinforced mortar cylinders with centrally-embedded reinforcement were cured in a fog room $\left(20^{\circ} \mathrm{C}, 98 \%\right.$ relative humidity) for 28 days. The specimens were lab conditioned as $1 / 3$ immersed in water or $5 \% \mathrm{NaCl}$ solution as external medium for the full test duration ( $>250$ days). This cylindrical geometry and specimen handling and conditioning is also as usually employed in corrosion studies on reinforced mortar and concrete, as reported in detail in the references cited above.

\section{Steel Electrodes and Steel Reinforcement}

For the tests in $\mathrm{CE}$, steel electrodes (St37) with a surface area of $4 \mathrm{~cm}^{2}$ were used, while construction steel FeB500 HKN (with a surface area of $16 \mathrm{~cm}^{2}$ ), centrally embedded in mortar specimens, were evaluated in reinforced mortar. All steel bars and St37 electrodes were equally treated prior to conditioning in the relevant solutions and/or casting in the mortar specimens (grinding, polishing, acetone cleaning and water rinsing for St37, and acetone cleaning and water rinsing for FeB500 HKN prior to casting). Three replicates per environment and condition were tested. The sample designation, relevant to the results discussed in this work, was as follows: group CE-control cases (chloride-free and nanoparticle-free); $\mathrm{CEn}$-corroding cases (NaCl-containing medium); $\mathrm{CEV}$ and $\mathrm{CEVC}$ stand for the non-corroding cases of steel in CE and mortar, where "empty" or Ca-loaded nanoparticles were present (either in the external CE medium or admixed in the mortar mixture), whereas CEVn and CEVCn stand for the corroding, nanoparticle-containing cases, respectively.

\section{Nanoparticles (Micelles and Vesicles)}

The nanoparticles in this work-micelles and vesicles—were polyethylene oxide polystyrene (PEO-b-PS)-based, where the copolymer was synthesized by atom transfer radical polymerization (ATRP) employing the macroinitiator technique [87]. $\mathrm{PEO}_{113}$-b-PS 70 di-block copolymer was used for the micelles, while $\mathrm{PEO}_{113}-\mathrm{b}-\mathrm{PS}_{760}$ was used for vesicle preparation. Both the micelles and vesicles were obtained by the dialysis method. The aqueous solution of micelles (or vesicles) in a concentration of $0.5 \mathrm{~g} / \mathrm{L}$, was added to the aqueous model medium (CE) or used directly as mixing water for the cement-based systems, resulting in $0.0024 \mathrm{wt} \%$ of the nanoparticles in aqueous medium or 0.024 wt \% per cement weight in the solid (cement-based) systems. Micelles and empty vesicles were employed first, in order to study material performance when no self-healing-related mechanisms were involved. In order to distinguish between "barrier" effects and "self-healing" due to Ca-release, both "empty" and Ca-containing vesicles were further used. Dynamic light scattering (DLS) (Malvern zeta-sizer, Nano ZS90, NL) and transmission electron microscopy (TEM) (Jeol JEM 1400 TEM) were performed, confirming a hydrodynamic radius of $50 \mathrm{~nm}$ for the micelles and 220 to $250 \mathrm{~nm}$ for the vesicles. Full details on the micelle and vesicle preparation and characterization are as previously reported in [80-83].

\section{Electrochemical Methods and Microscopy}

A Potentiostat PGSTAT 302N (Metrohm, NL) was used for all electrochemical tests for both steel in CE and for reinforced mortar. A common three-electrode cell arrangement was used for $\mathrm{CE}$, where a saturated calomel electrode (SCE) was used as the reference electrode, $\mathrm{Pt}$ was used as the counter electrode, and a steel electrode was used as the working electrode. The geometry of the reinforced mortar cylinders was also designed to resemble a three-electrode cell, where external Ti mesh served as the counter electrode, the centrally embedded reinforcement was the working electrode and an external SCE was the reference electrode (full details on this generally employed cell arrangement can be found in $[53,54,82])$. Linear polarization resistance (LPR) was performed in the range of $\pm 20 \mathrm{mV}$ vs OCP to derive polarization resistance $\left(R_{\mathrm{p}}\right)$ values, using linear regression and well-known considerations [88-90]. Potentio-dynamic polarization (PDP) was employed in the range of $-0.2 \mathrm{~V}$ to $+1.0 \mathrm{~V}$ vs open circuit potential $(\mathrm{OCP})$ at a scan rate of $0.5 \mathrm{mV} / \mathrm{s}$. The PDP curves 
allowed comparison of the electrochemical response with external and prolonged polarisation in view of the resistance to the anodic polarisation of the product layer on the steel surface (all electrochemical measurements were as generally employed for systems such as those in this study and as reported in the works referenced above).

Scanning electron microscopy (SEM) was used for morphological and microstructural studies, using an environmental ESEM Philips XL30, equipped with an energy dispersive X-ray (EDX) detector. SEM studies were relevant to the electrodes treated in CE steel, the steel reinforcement, the cementitious bulk matrix and the steel/mortar interface (experimental details and results for the complete test series can be found in the previous reports cited above). Light microscopy was performed as well and included in this work for visualization purposes, supporting the concept and feasibility of the discussed approach.

Acknowledgments: The author would like to thank Jie Hu from South China University of Technology, Guangzhou, China for the fruitful collaboration during his post-doctoral training in TU Delft on the relevant topic and project SHM08743 (2010-2012). Arch. Vesselin Kolev, Glavbolgarstroy Holding AD, is gratefully acknowledged for the graphical presentation of the self-healing concept in Figure 1. The financial support from the Netherlands Enterprise Agency (IOP grants SHM08743, SHM01041 and SHM012032 (2010-2017)) and the Dutch Technology Foundation STW, project number VENI 08104 (2007-2010), are gratefully acknowledged. The costs for the publication were covered by Delft University of Technology, The Netherlands.

Conflicts of Interest: The author declares no conflict of interest.

\section{References}

1. Bertolini, L.; Elsener, B.; Pedeferri, P.; Polder, R. Corrosion of Steel in Concrete: Prevention, Diagnosis, Repair, 1st ed.; Wiley: Weinheim, Germany, 2004.

2. Elsener, B. Macrocell corrosion of steel in concrete-Implications for corrosion monitoring. Cem. Concr. Compos. 2002, 24, 65-72. [CrossRef]

3. Garces, A.; Andrade, M.C.; Saez, A.; Alonso, M.C. Corrosion of reinforcing steel in neutral and acid solutions simulating the electrolytic environments in the micropores of concrete in the propagation period. Corros. Sci. 2005, 47, 289-306. [CrossRef]

4. Petterson, K. Corrosion and Corrosion Protection of Steel in Concrete; Swamy, R.N., Ed.; Academic Press: Sheffield, UK, 1994; p. 461.

5. Pedeferri, P. Cathodic protection and cathodic prevention. Constr. Build. Mater. 1996, 10, 391-402. [CrossRef]

6. Castle, J.E. Cathodic disbondment. AIP Conf. Proc. 1996, 354, 165-175.

7. Andrade, C.; Keddam, M.; Nóvoa, X.R.; Pérez, M.C. Electrochemical behaviour of steel rebars in concrete: Influence of environmental factors and cement chemistry. Electrochim. Acta 2001, 46, 3905-3912. [CrossRef]

8. Gaidis, J.M. Chemistry of corrosion inhibitors. Cem. Concr. Comp. 2004, 26, 181-189. [CrossRef]

9. Wombacher, F.; Maeder, U.; Marazzani, B. Aminoalcohol based mixed corrosion inhibitors. Cem. Concr. Compos. 2004, 26, 209-216. [CrossRef]

10. Volpi, E.; Foiadelli, C.; Trasatti, S.; Koleva, D.A. Development of smart corrosion inhibitors for reinforced concrete structures exposed to a microbial environment. Ind. Eng. Chem. Res. 2017, 20, 5778-5794. [CrossRef]

11. Bassi, R.; Davies, H. Testing Anti-Carbonation Coatings for Concrete. BRE Information paper 7/96, 1996, ISBN 9781860810930. Available online: http://www.ihsti.com/CIS/document/87656.

12. Buenfeld, N.; Zhang, J.Z. Chloride diffusion through surface-treated mortar specimens. Cem. Concr. Res. 1998, 28, 665-674. [CrossRef]

13. Obada, K.; Yeomans, S.R. Bond of ribbed galvanized reinforcing steel in concrete. Cem. Concr. Compos. 2000, 22, 459-467.

14. The Concrete Society. Diagnosis of Deterioration in Concrete; Concrete Society TR No56; The Concrete Society: Camberley, UK, 2000.

15. Andrade, C.; Holst, J.D. Coating Protection for Reinforcement; State of the Art Report; Comite Euro Intemational Du Beton, Ed.; Thomas Telford Publication: London, UK, 1995; p. 51.

16. Mietz, J. Electrochemical Rehabilitation Methods for Reinforced Concrete Structures: A State of the Art Report; Institute of Materials: London, UK, 1998. 
17. Koleva, D.A.; Hu, J.; Fraaij, A.L.A.; Stroeven, P.; Boshkov, N.; van Breugel, K. Cathodic protection revisited: Impact on structural morphology sheds new light on its efficiency. Cem. Concr. Compos. 2006, 28, 696-706. [CrossRef]

18. Koleva, D.A.; Copuroglu, O.; van Breugel, K.; Ye, G.; de Wit, J.H.W. Electrical resistivity and microstructural properties of concrete materials in conditions of current flow. Cem. Concr. Compos. 2008, 30, 731-744. [CrossRef]

19. Corradi, M.; Khurana, R.; Magarotto, R. Controlling performance in ready mixed concrete. Concr. Int. 2004, $26,123-126$.

20. Li, G. Properties of high-volume fly ash concrete incorporating nano-SiO 2 . Cem. Concr. Res. 2004, 34, 1043-1049.

21. Dalton, A.B.; Collins, S.; Muñoz, E.; Razal, J.M.; von Howard, E.; Ferraris, J.P.; Coleman, J.N.; Kim, G.B.; Baughman, R.H. Super-tough carbon nanotube fibres. Nature 2003, 423, 703. [CrossRef] [PubMed]

22. Silva, D.; Monteiro, P. Hydration evolution of C3S-EVA composite analyzed by soft X-ray microscopy. Cem. Concr. Res. 2005, 35, 351-357. [CrossRef]

23. Li, C.Z.; Feng, N.Q.; Li, Y.D.; Chen, R.J. Effects of polyethlene oxide chains on the performance of poly-carboxylate-type water-reducers. Cem. Concr. Res. 2005, 35, 867-873. [CrossRef]

24. Nicoleau, L. New Calcium Silicate Hydrate Network. Transp. Res. Rec. J. Transp. Res. Board 2010, 2142, 42-51. [CrossRef]

25. Dennis, R.V.; Viyannalage, L.T.; Gaikwad, A.V.; Rout, T.K.; Banerjee, S. Graphene nanocomposite coatings for protecting low-alloy steels from corrosion. ACS Bull. 2013, 92, 18-24.

26. Kirkland, N.T.; Schiller, T.; Medhekar, N.; Birbilis, N. Exploring graphene as a corrosion protection barrier. Corros. Sci. 2012, 56, 1-4. [CrossRef]

27. Lv, S.; Ma, Y.; Qiu, C.; Zhou, Q. Regulation of GO on cement hydration crystals and its toughening effect. Mag. Concr. Res. 2013, 65, 1246-1254. [CrossRef]

28. Collepardi, S.; Borsoi, A.; Ogoumah Olagot, J.J.; Troli, R.; Collepardi, M.; Cursio, A.Q. Influence of nano-sized mineral additions on performance of SCC. In Proceedings of the 6th International Congress Global Construction, Ultimate Concrete Opportunities, Dundee, UK, 5-7 July 2005.

29. Zhang, M.H.; Li, H. Pore structure and chloride permeability of concrete containing nano-particles for pavement. Constr Build.. Mater. 2011, 25, 608-616. [CrossRef]

30. Hui, L.; Xiao, H.; Yuan, J.; Ou, J. Microstructure of cement mortar with nano-particles. Compos. B 2004, 35, 185-189.

31. Sanchez, F.; Sobolev, K. Nanotechnology in concrete-A review. Constr. Build. Mater. 2010, 24, $2060-2071$. [CrossRef]

32. Plank, J.; Sachsenhauser, B. Experimental determination of the effective anionic charge density of polycarboxylate superplasticizers in cement pore solution. Cem. Concr. Res. 2009, 39, 1-5. [CrossRef]

33. Bassioni, G. A study towards greener construction. Appl. Energy 2012, 93, 132-137. [CrossRef]

34. Albani, O.A.; Zerbino, J.O.; Vilche, J.R.; Arvia, A.J. A comparative electrochemical and ellipsometric study of the iron electrodes in different alkaline electrolytes. Electrochim. Acta 1986, 31, 1403-1411. [CrossRef]

35. Misawa, T. The thermodynamic consideration for $\mathrm{Fe}-\mathrm{H}_{2} \mathrm{O}$ system at $25^{\circ} \mathrm{C}$. Corros. Sci. 1973, 13, $659-676$. [CrossRef]

36. Sudakar, C.; Subbanna, G.N.; Kutty, T.R.N. Effect of anions on the phase stability of $\gamma$-FeOOH nanoparticles and the magnetic properties of gamma-ferric oxide derived from lepidocrocite. J. Phys. Chem. Solids 2004, 64, 2337-2349. [CrossRef]

37. Bentur, A.; Diamond, S.; Berke, N.S. Steel Corrosion in Concrete; E \& FN: London, UK, 1997; p. 27.

38. MacDougall, B.; Graham, M.J. Corrosion Mechanisms in Theory and Practice, 2nd ed.; Marcus, P., Oudar, J., Eds.; Marcel Dekker Inc.: New York, NY, USA, 2002; p. 143.

39. Diéz-Perez, I.; Vericat, C.; Gorostiza, P.; Sanz, F. The iron passive film breakdown in chloride media may be mediated by transient chloride-induced surface states located within the band gap. Electrochem. Commun. 2006, 8, 627-632. [CrossRef]

40. Sato, N. An overview on the passivity of metals. Corros. Sci. 1990, 31, 1-19. [CrossRef]

41. Angst, U.M.; Boschmann, C.; Wagner, M.; Elsener, B. Experimental protocol to determine the chloride threshold value for corrosion in samples taken from reinforced concrete structures. J. Vis. Exp. 2017, 126, 56229. [CrossRef] [PubMed] 
42. Page, C.L. Advances in understanding and techniques for controlling reinforcement corrosion. In Proceedings of the 15th International Corrosion Congress, Granada, Spain, 22-27 September 2002.

43. Vassie, P. Reinforcement corrosion and the durability of concrete bridges. PRW Proc. Inst. Civ. Eng. 1984, 76, 713-723. [CrossRef]

44. Glass, G.K.; Buenfeld, N.R. The presentation of the chloride threshold level for corrosion of steel in concrete. Corros. Sci. 1997, 39, 1001-1013. [CrossRef]

45. Gaal, G.C.M. Prediction of Deterioration of Concrete Bridges. Ph.D. Thesis, Delft University of Technology, Delft, The Netherlands, 2004.

46. Poursaee, A. Corrosion of Steel in Concrete; Tuutti, K., Ed.; Swedish Cement and Concrete Research Institute: Stockholm, Sweden, 1982.

47. Reddy, B.; Glass, G.K.; Lim, P.J.; Buenfeld, N.R. On the corrosion risk presented by chloride bound in concrete. Cem. Concr. Compos. 2002, 24, 1-5. [CrossRef]

48. Hussain, S.E.; Rasheeduzzafar, S.; Al-Gahtani, A.S. Influence of sulfates on chloride binding in cements. Cem. Concr. Res. 1994, 24, 8-24. [CrossRef]

49. Leek, D.S.; Poole, A.B. The breakdown of the passive film on high yield mild steel by chloride ions. In Proceedings of the Third International Symposium on Corrosion of Reinforcement in Concrete, Wishaw, UK, 21-24 May 1990; Elsevier Applied Science: London, UK, 1990; pp. 65-73.

50. Oranowska, H.; Szklarska-Smialowska, Z. An electrochemical and ellipsometric investigation of surface films grown on iron in saturated calcium hydroxide solutions with or without chloride ions. Corros. Sci. 1981, 21, 735-747. [CrossRef]

51. Koleva, D.A.; Boshkov, N.; van Breugel, K.; De Wit, J.H.W. Steel corrosion resistance in model solutions, containing waste materials. Electrochim. Acta 2011, 58, 628-646. [CrossRef]

52. Koleva, D.A. Electrochemical behavior of corroded and protected construction steel in cement extract. Mater. Corros. 2011, 62, 240-251. [CrossRef]

53. Koleva, D.A.; Hu, J.; Fraaij, A.L.A.; Stroeven, P.; Boshkov, N.; de Wit, J.H.W. Quantitative characterisation of steel/cement paste interface microstructure and corrosion phenomena in mortars suffering from chloride attack. Corros. Sci. 2006, 48, 4001-4019. [CrossRef]

54. Koleva, D.A.; van Breugel, K.; De Wit, J.H.W.; van Westing, E.; Boshkov, N.; Fraaij, A.L.A. Electrochemical behavior, microstructural analysis, and morphological observations in reinforced mortar subjected to chloride ingress. J. Electrochem. Soc. 2007, 154, E45-E56. [CrossRef]

55. Koleva, D.A.; Hu, J.; Fraaij, A.L.A.; van Breugel, K.; de Wit, J.H.W. Microstructural analysis of plain and reinforced mortars under chloride-induced deterioration. Cem. Concr. Res. 2007, 37, 604-617. [CrossRef]

56. Koleva, D.A.; Denkova, A.G.; Boshkov, N.; van Breugel, K. Electrochemical performance of steel in cement extract and bulk matrix properties of cement paste in the presence of Pluronic 123 micelles. J. Mater. Sci. 2013, 48, 2490-2503. [CrossRef]

57. Koleva, D.A.; Hu, J.; Milkova, V.; van Breugel, K. Hybrid nano/micro-particles for increased steel corrosion resistance: Particles' alterations with $\mathrm{pH}$ change and steel behavior in cement extract and mortar. J. Mech. 2013, 1612, 3. [CrossRef]

58. Koleva, D.A.; van Breugel, K.; Ye, G.; Zhou, J.; Chamululu, G.; Koenders, E.A.B. Porosity and permeability of mortar specimens incorporating PEO113-b-PS218 micelles. Am. Concr. Inst. 2009, 267, 101-110.

59. Gawin, D.; Pesavento, F.; Schrefler, B. Modeling deterioration of cementitious materials exposed to calcium leaching in non-isothermal conditions. Comput. Methods Appl. Mech. Eng. 2009, 198, 3051-3083. [CrossRef]

60. Kamali, S.; Gerard, B.; Moranville, M. Modelling the leaching kinetics of cement-based materials-Influence of materials and environment. Cem. Concr. Compos. 2003, 25, 451-458. [CrossRef]

61. Marchand, J.; Bentz, D.P.; Samson, E.; Maltais, Y. Influence of calcium hydroxide dissolution on the transport properties of hydrated cement systems. In Workshop on the Role of Calcium Hydroxide in Concrete; Anna Maria Island: Holmes Beach, FL, USA, 2000; pp. 113-129.

62. Carde, C.; Francois, R.; Torrenti, J.M. Leaching of both calcium hydroxide and C-S-H from cement paste: Modeling the mechanical behaviour. Cem. Concr. Res. 1996, 26, 1257-1268. [CrossRef]

63. Koleva, D.A. Corrosion and Protection in Reinforced Concrete. Ph.D. Thesis, TU Delft, Delft, The Netherlands, 2007. 
64. Wang, G.; de Kruijff, R.; Stuart, M.C.A.; Mendes, E.; Wolterbeek, H.T.; Denkova, A.G. Polymersomes as radionuclide carriers loaded via active ion transport through the hydrophobic bilayer. Soft Matter. 2013, 9 , 727-734. [CrossRef]

65. Koleva, D.; Boshkov, N.; Raichevski, G.; Veleva, L. Electrochemical corrosion behaviour and surface morphology of electrodeposited zinc, zinc-cobalt and their composite coatings. Trans. Inst. Metal Finish. 2005, 83, 188-193. [CrossRef]

66. Koleva, D.A.; Boshkov, N.; Bachvarov, V.; Zhan, H.; de Wit, J.H.W.; van Breugel, K. Application of PEO113-b-PS218 nano-aggregates for improved protective characteristics of composite zinc coatings in chloride-containing environment. Surf. Coat. Technol. 2010, 204, 3760-3772. [CrossRef]

67. Lee, J.; Mahendra, S.; Alvarez, P.J.J. Nanomaterials in the construction industry: A review of their applications and environmental health and safety considerations. ACS Nano 2010, 4, 3580-3590. [CrossRef] [PubMed]

68. Chiappetta, D.A.; Sosnik, A. Poly(ethylene oxide)-poly(propylene oxide) block copolymer micelles as drug delivery agents: Improved hydrosolubility, stability and bioavailability of drugs. Eur. J. Pharmac. Biopharmac. 2007, 66, 303-317. [CrossRef] [PubMed]

69. Pitto-Barry, A.; Barry, N.P.E. Pluronic ${ }^{\circledR}$ block-copolymers in medicine: From chemical and biological versatility to rationalisation and clinical advances. Polym. Chem. 2014, 5, 3291-3297. [CrossRef]

70. Zhu, Y.Q.; Wang, F.Y.K.; Zhang, C.; Du, J.Z. Preparation and mechanism insight of nuclear envelope-like polymer vesicles for facile loading of biomacromolecules and enhanced biocatalytic activity. ACS Nano 2014, 8, 6644-6654. [CrossRef] [PubMed]

71. Zhua, Y.; Bo, Y.; Shuai, C.; Dua, J. Polymer vesicles: Mechanism, preparation, application, and responsive behaviour. Prog. Polym. Sci. 2017, 64, 1-22. [CrossRef]

72. Meeuwissen, S.A.; Rioz-Martinez, A.; de Gonzalo, G.; Fraaije, M.W.; Gotor, V.; van Hest, J.C.M. Cofactor regeneration in polymersome nano-reactors: Enzymatically catalysed Baeyer-Villiger reactions. J. Mater. Chem. 2011, 21, 18923-18926. [CrossRef]

73. Berthier, D.L.; Schmidt, I.; Fieber, W.; Schatz, C.; Furrer, A.; Wong, K.; Lecom-mandoux, S. Controlled release of volatile fragrance molecules from PEO-b-PPO-b-PEO block copolymer micelles in ethanol-water mixtures. Langmuir 2010, 26, 7953-7961. [CrossRef] [PubMed]

74. Geng, Q.R.; Du, J.Z. Reduction of 4-nitrophenol catalyzed by silver nanoparticles supported on polymer micelles and vesicles. RSC Adv. 2014, 4, 16425-16428. [CrossRef]

75. Zhu, Y.Q.; Fan, L.; Yang, B.; Du, J.Z. Multifunctional homopolymer vesicles for facile immobilization of gold nanoparticles and effective water remediation. ACS Nano 2014, 8, 5022-5031. [CrossRef] [PubMed]

76. Du, J.Z.; Fan, L.; Liu, Q.M. pH-sensitive block copolymer vesicles with variable trigger points for drug delivery. Macromolecules 2012, 45, 8275-8283. [CrossRef]

77. Qin, S.H.; Geng, Y.; Discher, D.E.; Yang, S. Temperature-controlled assembly and release from polymer vesicles of poly(ethylene oxide)-block-poly( $N$-isopropylacrylamide). Adv. Mater. 2006, 18, 2905-2909. [CrossRef]

78. Cerritelli, S.; Velluto, D.; Hubbell, J.A. PEG-SS-PPS: Reduction-sensitive disulfide block copolymer vesicles for intracellular drug delivery. Biomacromolecules 2007, 8, 1966-1972. [CrossRef] [PubMed]

79. Babin, J.; Pelletier, M.; Lepage, M.; Allard, J.F.; Morris, D.; Zhao, Y. A new two-photon-sensitive block copolymer nanocarrier. Angew. Chem. Int. Ed. 2009, 48, 3329-3332. [CrossRef] [PubMed]

80. Hu, J.; Koleva, D.A.; Ma, Y.; Schlangen, E.; Petrov, P.; van Breugel, K. The influence of admixed micelles on the microstructural properties and global performance of cement-based materials. Cem. Concr. Res. 2012, 42, 1122-1133. [CrossRef]

81. Hu, J.; Koleva, D.A.; van Breugel, K. Corrosion performance of reinforced mortar in the presence of polymeric nano-aggregates: Electrochemical behavior, surface analysis, and properties of the steel/cement paste interface. J. Mater. Sci. 2012, 47, 4981-4995. [CrossRef]

82. Hu, J.; Koleva, D.A.; Petrov, P.; van Breugel, K. Polymeric vesicles for corrosion control in reinforced mortar: Electrochemical behavior, steel surface analysis and bulk matrix properties. Corros. Sci. 2012, 65, 414-430. [CrossRef]

83. Hu, J.; Koleva, D.A.; De Wit, J.H.W.; Kolev, H.; van Breugel, K. Corrosion performance of carbon steel in simulated pore solution in the presence of micelles. J. Electrochem. Soc. 2011, 158, C76-C87. [CrossRef] 
84. Hu, J.; Koleva, D.A.; Ma, Y.; Schlangen, E.; van Breugel, K. Early age hydration, microstructure and micromechanical properties of cement paste modified with polymeric vesicles. J. Adv. Concr. Technol. 2013, 11, 291-300. [CrossRef]

85. Jansen, D.; Neubauer, J.; Goetz-Neunhoeffer, F.; Haerzschel, R.; Hergeth, W.D. Change in reaction kinetics of Portland cement caused by a superplasticizer-Calculation of heat flow curves from XRD data. Cem. Concr. Res. 2012, 42, 327-332. [CrossRef]

86. Mollah, M.; Adams, W.; Schennach, R.; Cocke, D. A review of cement-superplasticizer interactions and their models. Adv. Cem. Res. 2000, 12, 153-161. [CrossRef]

87. Matyjaszewski, K.; Xia, J. Atom transfer radical polymerization. Chem. Rev. 2001, 101, 2921-2990. [CrossRef] [PubMed]

88. Stern, M.; Geary, A.L. Electrochemical Polarization I. A Theoretical Analysis of the Shape of Polarization Curves. J. Electrochem. Soc. 1957, 104, 56-63. [CrossRef]

89. Andrade, C.; Alonso, C. Corrosion rate monitoring in the laboratory and on-site. Constr. Build. Mater. 1996, 10, 315-328. [CrossRef]

90. Zornoza, E.; Paya, J.; Garces, P. Chloride-Induced corrosion of steel embedded in mortars containing fly ash and spent cracking catalyst. Corros. Sci. 2008, 50, 1567-1575. [CrossRef]

(C) 2018 by the author. Licensee MDPI, Basel, Switzerland. This article is an open access article distributed under the terms and conditions of the Creative Commons Attribution (CC BY) license (http:/ / creativecommons.org/licenses/by/4.0/). 


\title{
Article \\ Self-Sealing Cementitious Materials by Using Water-Swelling Rubber Particles
}

\author{
Leyang $\mathrm{Lv}^{1,2, *}$, Erik Schlangen ${ }^{1}$ and Feng Xing ${ }^{2}$ \\ 1 Micromechanics Laboratory (MICROLAB), Faculty of Civil Engineering and Geosciences, \\ Delft University of Technology, Stevinweg 1, 2628 CN Delft, The Netherlands; Erik.Schlangen@tudelft.nl \\ 2 Guangdong Province Key Laboratory of Durability for Marine Civil Engineering, \\ School of Civil Engineering, Shenzhen University, Shenzhen 518060, China; xingf@szu.edu.cn \\ * Correspondence: L.Lu-2@tudelft.nl; Tel.: +31-616-775-996
}

Received: 25 July 2017; Accepted: 18 August 2017; Published: 22 August 2017

\begin{abstract}
Water ingress into cracked concrete structures is a serious problem, as it can cause leakage and reinforcement corrosion and thus reduce functionality and safety of the structures. In this study, the application of water-swelling rubber particles for providing the cracked concrete a self-sealing function was developed. The feasibility of applying water-swelling rubber particles and the influence of incorporating water-swelling rubber particles on the mechanical properties of concrete was investigated. The self-sealing efficiency of water-swelling rubber particles with different content and particle size was quantified through a permeability test. The sealing effect of the water swelling rubber particles was monitored by $\mathrm{X}$-ray computed tomography. The experimental results show that, by using $6 \%$ of these water swelling rubber particles as a replacement of aggregates in concrete, up to $64 \%$ and $61 \%$ decrease of water permeability was realized for $0.7 \mathrm{~mm}$ and $1.0 \mathrm{~mm}$ cracks. Furthermore, when the concrete cracks, the water swelling rubber particles can act as a crack bridging filler, preventing the crack from fully separating the specimens in two pieces.
\end{abstract}

Keywords: water swelling rubber; self-sealing; cementitious materials; bridging effect

\section{Introduction}

Cracking related deterioration seriously influences the integrity and durability of concrete. Cracks cause water leakage, therefore reducing the functionality of civil structures such as dams, retaining walls, tunnels. Cracks also induce aggressive agents by water transport through the crack, which is often detrimental to the service life of reinforced concrete structures. Furthermore, when the ingress of aggressive agents leads to corrosion of the reinforcement, it can even pose a threat to the safety of the structure. Although better structural design and content proportion of components can help to decrease the probability of cracking, cracks, as an unavoidable feature of concrete, need a more efficient and automatic way to reduce the hazardous effect it brings.

In recent years, inspired from biological self-healing phenomenon, the concept of self-sealing concrete began to show its beneficial and great application potential in the field of civil engineering [1-3]. Up to now, several solutions were studied targeting to reduce the permeability of concrete automatically. Among these, autogenous self-healing is one of the main promising mechanisms in cementitious materials. This oldest self-healing mechanism has already been observed in concrete structures that can be tracked back to the nineteenth century [4]. The advantage of this method is that no additives are needed to mix into concrete. Therefore, the chemical and mechanical properties of concrete will not be influenced. When the cracked concrete is exposed to water, the cracks may autogenous heal under a certain environmental condition. However, the limitations are also obvious. The autogenous self-healing can only occurs for a narrow crack with crack width typically less than $0.3 \mathrm{~mm}$ [5]. In addition, there are several specific requirements to the healing conditions such as temperature, wet/dry 
cycle and the ions in the water which cannot be easily achieved in practice. Autonomic healing is another self-healing mechanism that is often applied to compensate the drawbacks of autogenous self-healing in cementitious materials. By mixing healing agent such as bacteria, expansive powder mineral, superabsorbent polymer etc., the cracks can be healed more efficiently, whenever the healing agent is triggered upon crack formation or other stimuli. The microcapsule based self-healing concept has shown its application potential in cementitious materials [3,6-10]. While the healing effect can be hindered due to the weak bonding strength between the polymeric microcaspules and inorganic cementitious matrix. The feasibility of using Bacterial spores as healing agent has been also found and developed [2,3,5,11]. However, similar to autogenous self-healing, the efficiency of bacteria-based self-healing is restricted to the duration of immersion and the composition of the incubation solution, which brings a challenge in real situations. More recently, water-swelling materials such as superabsorbent polymer (SAP) were found to be a new type of self-healing materials that holds a potential in crack self-sealing [12-15]. The result shows that the application of SAP can provide up to $85 \%$ and $98 \%$ decrease of the peak flow and cumulative flow, leading to the complete sealing of a $0.3 \mathrm{~mm}$ crack. However, practical application of SAP in civil engineering structures was rather limited due to its detrimental effect on compressive strength. Recent results show that the addition of $5 \%$ SAP by weight of cement will lead to a reduction of $80 \%$ of compressive strength of concrete [12].

To find a material which is practically applicable of providing a long-term sealing capacity of concrete structure, three requirements should be satisfied: (1) the materials can survive in and have a good bonding with concrete structure; (2) the sealing function of the selected material can be triggered automatically upon water ingress or other stimuli without or with less human intervention and (3) the incorporation of the material should have only limited negative effect on the mechanical properties of concrete structure, and if possible, even enhance it. Previously, the possibility of applying rubber phases as partial replacement of sand in concrete has been investigated. This method attempted to make the concrete, a traditional building material, embrace more versatile performances such as higher energy dissipation, ductility, durability, damping ratio, impact resistance and toughness [16-19]. The results of these studies show that the waste tire rubber reinforced concrete (RRC) could be an ideal ecological component of concrete when it was applied as a replacement of aggregate and subjected to dynamic loading conditions. While, to our knowledge, no research can be found on the application of rubber with water swelling function to provide the concrete with a combined self-sealing and self-bridge function. Water swelling rubber is a new type of construction material which has been widely used for sealing of precast concrete elements (e.g., man holes), shaft rings and pipe lead-throughs etc. The matrix of water swelling rubber is butyl rubber, a copolymer of isobutylene with isoprene, also known as Isoprene Isobutylene Rubber (IIR). The expanding property results from the irrevocable bonding of polyurethane-based water-expanding polymer resin which has been pre-mixed in the IIR during the fabrication process. The physical characteristics of water swelling rubber is similar to the ordinary IIR. While, comparing with SAP, the benefit of this type rubber is, only limited volume of can swell up within $24 \mathrm{~h}$. This $24 \mathrm{~h}$ swelling delay provide a precious time for the mortar to be harden. The reason for this delay can be attribute to the IIR rubber prolonged the time for those pre-mixed polyurethane-based water-expanding polymer resin in it from being swell by the moisture in the water. After 7 days on contact with water, the water swelling rubber can finally swell up to $250 \%$ of its original volume. The benefit of this rubber is, there will be a swelling delay when it first contact with water. Meanwhile, due to the sticky texture and strong surface bonding strength of water swelling rubber, it is expected that the expanded rubber could have a good adhesion to the crack faces, provide some crack bridging effect and keep the cracked pieces together.

Accordingly, the core concept of this work is to utilize water swelling rubber particles (WSRPs) as a new ingredient of cementitious materials to enable the ability for the cracked concrete structure to reduce its permeability and partially regain its deteriorated mechanical properties. Once cracks have formed in the concrete structure and water penetrates, the rubber may swell gradually, expand along the crack and partly seal the crack. Subsequently, the embedded rubber can also act as reinforcing 
particles that provide an extra bond strength and prolong the serviceability of cracked concrete. In this study, the feasibility of applying WSRPs in crack self-sealing was proven by both a simulated crack and X-ray Computed Tomography (XCT) technology. The influence of incorporation of WSRPs on the mechanical properties was investigated. Water permeability tests were carried on a series of WSRPS embedded mortar specimens to study the influence of WSRPs size and content, and crack width on the self-sealing effect. Finally, the capability of crack bridging to the cracked mortar, provided by the embedded WSRPs, was characterized.

\section{Experimental}

\subsection{Materials}

Water swelling rubber (AQUA TACKSEAL) was provided by TPH Bausysteme GmbH, Norderstedt, Germany. To obtain the water swelling rubber particles (WSRPs), the as received materials were first frozen by liquid nitrogen and then crushed immediately in a grinder by hand. Before the mass water swelling rubber began to be crushed into smaller particles, the cement powder will be added in the grinder. These cement powder will be act as desiccants, sticking on the new formed surface of WSRPs to prevent the new formed particles from being stick together. After that, the crushed WSRPs were sieved by a screen with mesh diameter of $0.5 \mathrm{~mm}, 1 \mathrm{~mm}, 2 \mathrm{~mm}$ and $4 \mathrm{~mm}$ respectively. Figure 1 shows the images of granular WSRPs used in this study highlighting the difference in particle size, (a) S size (b) M size and (c) L size.

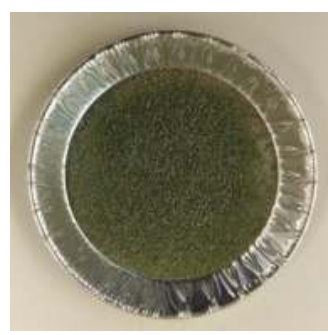

(a)

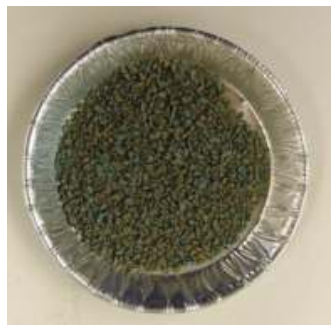

(b)

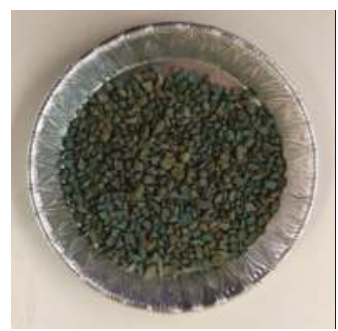

(c)

Figure 1. Images of WSRP with size range of (a) $0.5-1 \mathrm{~mm}(\mathrm{~S})$; (b) 1-2 mm (M) and (c) 2-4 mm (L).

Ordinary Portland cement, CEM I $52.5 \mathrm{~N}$ and CEN standard RILEM sand was used for the preparation of mortar samples. The grading of the sand is given in Table 1, which complies with the requirements of EN 196-1 (§ 5) and ISO 679: 2009 (§ 5). Deionised water was used as batch water.

Table 1. The Grading of Standard CEN Sand.

\begin{tabular}{ccccccc}
\hline Square Mesh Size (mm) & 2 & 1.6 & 1 & 0.5 & 0.16 & 0.08 \\
\hline Cumulative (\%) Retained & 0 & 7 & 33 & 67 & 87 & 99 \\
\hline
\end{tabular}

\subsection{Sample Preparation}

According to the EU standard EN 196-1 [20], the amount of cement was kept constant at $450 \mathrm{~g}$, water to cement ratio was selected as 0.5 , and $1350 \mathrm{~g}$ filler (Rilem sand + WSRPs) was used as fine aggregate. Mortar was chosen as a representative material for concrete. The mixture contents of cement mortar are shown in Table 2. Four series of specimens including S, M, L, and B were made. For those WSRPs incorporated cement mortar, series S, M and L represent the size of incorporated WSRPS, ranging $0.5-1 \mathrm{~mm}, 1-2 \mathrm{~mm}$ and 2-4 $\mathrm{mm}$. The following numbers 1,3 and 6 stand for the volume ratio of which the sand were replaced by WSRPs. Series B, as reference, is pure mortar without WSRPs. 
To avoid any interference and complication in the results, superplasticizer was not used. Mortar mixtures were mixed in a Hobart mixer by a certain order. In the first step, cement, sand and WSRPs were put in the mixer and mixed thoroughly at low gear $(60 \mathrm{rpm})$ for $60 \mathrm{~s}$. Water was then added in the mixer and mixed at low gear (60 rpm) for another $60 \mathrm{~s}$. After the mixing, the mortar mixtures were cast into a standard prismatic moulds $\left(40 \times 40 \times 160 \mathrm{~mm}^{3}\right)$ and cylinder moulds $(60 \mathrm{~mm}$ long and with a diameter of $33.5 \mathrm{~mm}$ ). Following the work of Palin et al. [21], the cylinder mould has two opposite notches on both long side of the height ( $2 \mathrm{~mm}$ wide and $3 \mathrm{~mm}$ deep). The casted moulds were then vibrated on a shaking table to remove the voids. All the specimens were de-moulded after $24 \mathrm{~h}$ and then cured in a fog room at normal curing condition $\left(20^{\circ} \mathrm{C},>95 \% \mathrm{RH}\right)$ for 28 days. For each mix (with different ID), 6 standard prisms and 9 cylinders were casted. Figure 2 illustrates images of the mixture prepared for mixing procedure (a); the prisms for mechanical test (b) and the cylinder with notches for water permeability test (c).

Table 2. Mix content of cement mortar.

\begin{tabular}{ccccccc}
\hline Series & ID & Water (g) & Cement (g) & Sand (g) & WSRPs (g) & WSRPs/Sand (vol \%) \\
\hline \multirow{2}{*}{ S } & S1 & 225 & 450 & 1338.9 & 11.1 & 1 \\
& S3 & 225 & 450 & 1309.5 & 33.0 & 3 \\
& S6 & 225 & 450 & 1285.7 & 64.3 & 6 \\
\hline \multirow{3}{*}{ M } & M1 & 225 & 450 & 1338.9 & 11.1 & 3 \\
& M3 & 225 & 450 & 1309.5 & 33.0 & 6 \\
\hline \multirow{2}{*}{ L } & M6 & 225 & 450 & 1285.7 & 64.3 & 1 \\
& L1 & 225 & 450 & 1338.9 & 11.1 & 3 \\
\hline B 3 & 225 & 450 & 1309.5 & 33.0 & 0 \\
\hline
\end{tabular}

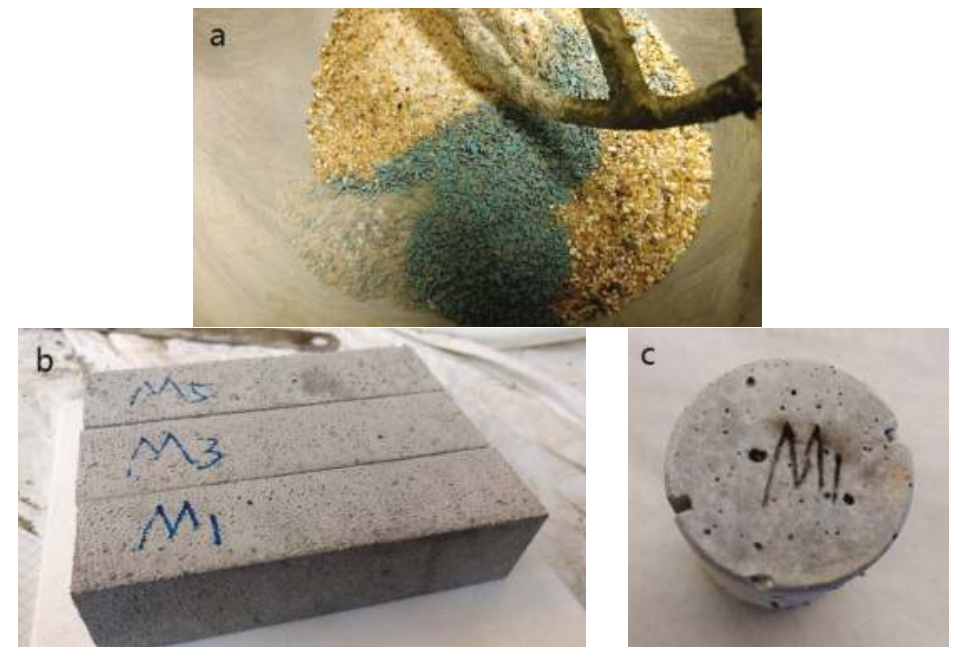

Figure 2. Images of (a) the mixture prepared for mixing procedure; (b) prisms for mechanical test and (c) cylinder with double notches for water permeability test.

\subsection{Morphology of WSRPs in Mortar}

The morphology of cement mortar containing L and M sized WSRPs was imaged by a digital camera. The size of specimens that was used for the observations is a $40 \times 40 \times 5 \mathrm{~mm}^{3}$ slice. 
The microstructure of the WSRPs embedded mortar was characterized by an environmental scanning electron microscope (ESEM) under low vacuum mode (XL30, Philips, Amsterdam, The Netherlands). All imaging was performed in low vacuum. Before the test, the surface of the sample is ground by four different grinding papers $(500 \#, 800 \#, 1200 \#, 4000 \#)$. Then, the sample was polished by hand on a lapping table using diamond paste with particle diameter of $6 \mu \mathrm{m}, 3 \mu \mathrm{m}, 1 \mu \mathrm{m}$ and $0.25 \mu \mathrm{m}$. The polishing time is $5 \mathrm{~min}$ for each step.

\subsection{Feasibility of Applying WSRPs in Concrete—Proof of Concept}

\subsubsection{Swelling Effect of WSRPs in Simulated Crack}

The swelling effect of WSRPs in concrete was first investigated using a simulated crack. To clearly see the swelling effect, a glass sheet was used to cover the cross section of WSRPs containing mortar sample. In order to ensure the crack width, a double sided plastic tape with a thickness of $0.8 \mathrm{~mm}$ was inserted in between the glass sheet and the section surface of the sample to control the simulated crack width to $0.8 \mathrm{~mm}$. The swelling effect of the WSRPs was recorded by acquiring images with a digital camera after $0,3,7$ days of immersion in deionized water.

\subsubsection{Sealing Effect of WSRPs in Mortar}

X-ray computed tomography (XCT) is a versatile, non-destructive inspection method, which has been widely applied in various fields of research [22,23]. Recently, the application of this technique has also been extended to the area of civil engineering [10,24-26]. As a non-destructive imaging technique, $\mathrm{XCT}$ provides an approach to study the internal information of a cementitious structure based on the principle of different X-ray absorption between phases or elements [27]. In this study, X-ray computed tomography (XCT) scanning technology (Nanotom, GE Inspection Technologies, Lewistown, PA, USA) was applied to proof the swelling and sealing function of WSRPs within hardened mortar. To visualize the sealing effect of WSRPs in mortar, the WSRPs embedded mortar cylinder before and after the sealing process was subjected to XCT. In between, the cylinder was immersed in deionised water for 7 days. In this study, the raw XCT images were acquired at an acceleration voltage of $120 \mathrm{kV}$ with an exposure time of $4 \mathrm{~s}$ and X-ray power of $8 \mathrm{~W}$. The resolution of CT scans was set to $20 \mu \mathrm{m}$. The final data set of XCT consisted of 1440 radiographs of which each image was acquired with a $0.25^{\circ}$ rotation. Then, phase retrieval and tomographic reconstruction were performed to improve the boundaries and signals using the software supplied by the manufacturer. A series of reconstructed tomographic images (X-Z plane) were consequently imported into a commercial software (VGStudio MAX 3.0, Volume Graphics $\mathrm{GmbH}$, Heidelberg, Germany) for segmentation and 3D visualization. Section images of the reconstructed 3D volume containing the information of the crack and WSRPs were compared. The crack self-sealing effect was quantified via an additional analysis of the 3D volume by comparing the volume fraction of WSRPs and crack in regions around the healed crack, before and after the healing process.

\subsection{Influence of the WSRPs on the Mechanical Properties of Mortar}

The flexural and compression strength of the WSRPs embedded cement mortar was measured according to TS EN 1015-11 (2000) [28]. In order to obtain the flexural strength of the mortars, $40 \times 40 \times 160 \mathrm{~mm}^{3}$ specimens were used. The specimens were tested after 28 days curing for flexural strength under three-point loading with the span between supports being of $100 \mathrm{~mm}$. The average of results obtained from three prismatic specimens was reported as flexural strength. The two broken parts of the $40 \times 40$ $\times 160 \mathrm{~mm}^{3}$ retained after the flexural strength test were used for compressive strength. The loading rate was $500 \mathrm{~N} / \mathrm{s}$. The loading area was $40 \times 40 \mathrm{~mm}^{2}$. The average of results obtained from six broken pieces was reported as compressive strength. To investigate the influence of ageing on the mechanical properties, same test was conducted again on the remaining three specimens which were stored in lab environment for 28 days after the first 28 days of hydration. 


\subsection{Evaluation of Self-Sealing Ability}

\subsubsection{Crack Calibration}

A crack with a certain width was induced on the cylinder specimens for the evaluation of the water sealing effect. Before the test, two steel rods were placed at each side of the notch. Then an Instron 8872 servohydraulic testing machine (Instron Corp., Canton, MA, USA) was used to apply a compressive load on the steel rods until the cylinder split diametrically. Spacers with a width of $2.4,2.7$ and $3.0 \mathrm{~mm}$ were placed thoroughly in the notches. Since the width of notches is $2 \mathrm{~mm}$, therefore by placing spacers with a width of 2.4, 2.7 and $3.0 \mathrm{~mm}$, cracks widths of $0.4,0.7$ and $1.0 \mathrm{~mm}$ can be achieved. Then the spacers were moved to half way of the notches, and a two-component adhesive, Plex 7742 and Pleximon 801 (Evonik Rohm GmbH, Darmstadt, Germany), was mixed and applied on the space of notches. After the adhesive was harden, the spacers were removed and the rest space of notches were completely filled by adhesive. A watertight permeability cell was used to seal the cylinder specimens and to connect them with the permeability test setup. To prevent the water leakage from pores or defects of cylinders during the permeability test, before the cylinder was placed into the cell, rubber rings were attached on both ends of the cylinder specimen. In the end, stereomicroscope analysis was applied to measure the actual widths of cracks. A tolerance variation was set for those sample with a crack variable over a certain criteria to be screened out from being permeability test. For the $0.4 \mathrm{~mm}$ sample, the tolerable variation is $0.05 \mathrm{~mm}$, for $0.7 \mathrm{~mm}$ sample, the tolerable variation is 0.07 and for $1.0 \mathrm{~mm}$ sample, the tolerable variation is $0.1 \mathrm{~mm}$. This means the variable of those tested sample within the tolerable variation was regarded as and reflected on the standard deviation of water permeability.

\subsubsection{Water Permeability}

The water permeability was tested according to the work of Palin et al. [29]. Briefly, the permeability cells were first attached to the bottom of permeability setup. Water was then poured into reservoirs at the top of each setup. To provide an almost constant water pressure of $0.1 \mathrm{bar}$, the water level of each permeability setup was manually controlled to a height of $1 \mathrm{~m}$ from the top of the water surface to the bottom of the permeability cell. To start the test, taps of the reservoirs were released. Water flowing through the cracks and was collected separately by buckets beneath the testing setups. The weight of the water in the buckets was weighted and recorded two times at two continuous $5 \mathrm{~min}$. The permeability test was performed after 0 and 7 days submersion of the crack-induced specimens in tap water. The permeability $\left(\mathrm{cm}^{3} \cdot \mathrm{s}^{-1}\right)$ is simply defined as the volume of flowed out water from the crack $\left(\mathrm{cm}^{3}\right)$ divides the recorded time (s). For each data point, 3 replicate samples were tested.

\subsection{Visual Assessment of Crack Bridging Function of WSRPs}

The crack bridging function is a unique characteristic of WSRPs which can prevent the crack from fully separating the specimens in two pieces. To visualize the function, a mortar prism with WSRPs embedded was fractured by tensile force using Axial Tension-Compression Systems (8872, Instron Corp., Canton, MA, USA). Then the tensile force was removed, the cracked area of WSRPs embedded mortar was recorded by a digital camera.

\section{Results and Discussion}

\subsection{Morphology of WSRP in Mortar}

The morphology of WSRPs embedded mortar is shown in Figure 3, highlighting the dispersibility, size and shape of the embedded WSRPs. As can be seen from the figure that, both large size and medium size WSRPs (blue) are well dispersed in mortar. No obvious voids can be found around the WSRP, demonstrating that the quartz shape WSRPs have a good connection with the mortar matrix, reminiscent of aggregates. For better understanding the influence of WSRPs on the surrounding microstructure of mortar. The interface zone between WSRPs and mortar matrix at the age of 28 days 
of the sample was investigated by ESEM. It can be clearly seen from Figure 4 that no macro or micro-cracks were found due to the WSRP. Meanwhile, the magnified image in Figure $4 \mathrm{~b}$ tends to show that WSRPs has an effective adhesion with cement paste. All the above-mentioned phenomena suggest that the embedded WSRPs do not change evidently during the process of hydration and the existence of WSRPs will not influence the microstructure of the surrounding mortar.

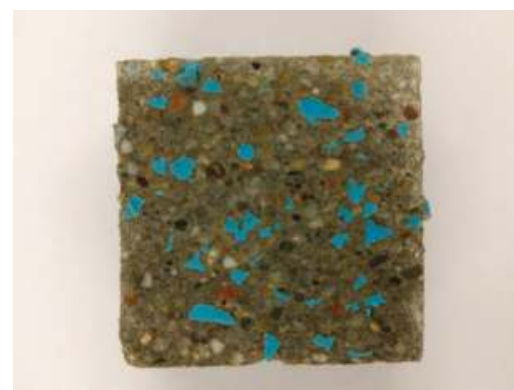

(a)

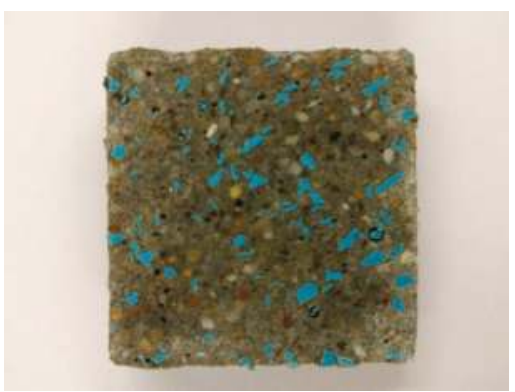

(b)

Figure 3. Images of the cross section of mortar specimen with WSRPs embedded (a) L series (particle size: 2-4 mm); (b) M series (particle size: 1-2 mm). The length of scale bar in images is $10 \mathrm{~mm}$.

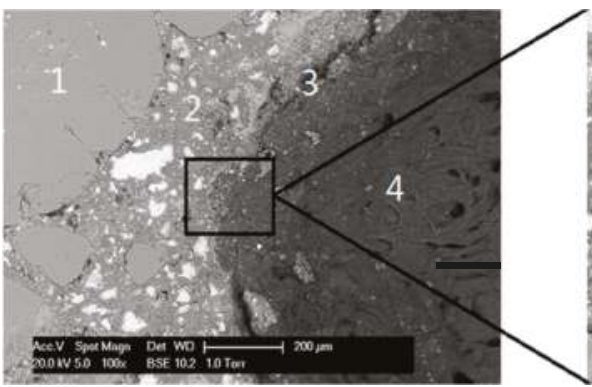

(a)

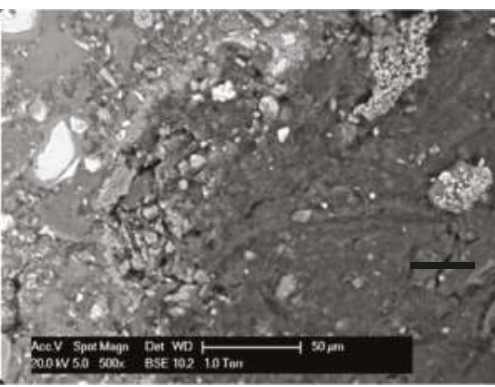

(b)

Figure 4. ESEM images showing the microstructure of interface zone between WSRP and mortar matrix at the age of 28 days. (a) at left side highlights (1) the aggregate particles, (2) hydrated and unhydrated cement paste, (3) WSRPs and cement paste interface and (4) embedded WSRPs; (b) at the right side shows an enlarged view of the microstructure of the interface.

\subsection{Proof of Concept}

\subsubsection{Swelling Effect of WSRPs at Simulated Crack}

The self-sealing function of the WSRPs in cementitious materials is realized through swelling of WSRPs and expanding into the cracks. To proof the feasibility of applying WSRPs in mortar to reduce the volume of crack and further decrease the permeability, a preliminary test was carried out. In order to have a direct view of the swelling process of WSRPs, a glass sheet was used as one side of the crack, covering on a slice of WSRPs embedded mortar. Figure 5 shows the swelling process of WSRP in the simulated crack. As can be seen from the figure, at the initial stage (0 days), the WSRPs (blue) keep their original shape. After 3 day of immersion in water, the embedded WSRPs begin to swell and expand in the crack space in which some of them join together which partly blocks the crack space. As the immersion time goes to 7 days, the WSRPs expand only a little bit. The results 
demonstrate that the self-sealing action of WSRPs can be activated and reach its maximum function within 7 days.

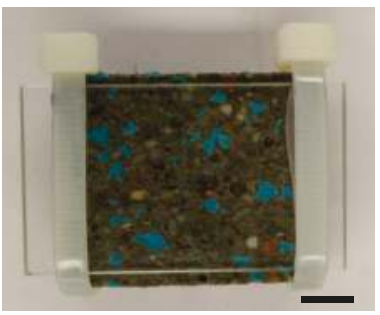

(a)

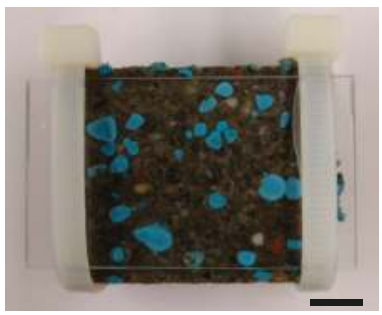

(b)

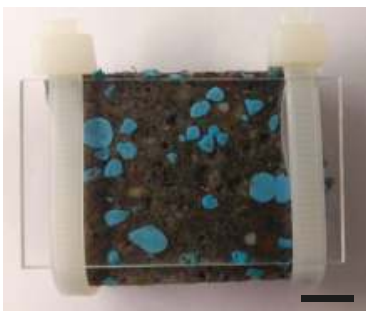

(c)

Figure 5. Swelling effect of WSRPs in a simulated crack with a crack width of $0.8 \mathrm{~mm}$ at (a) 0 day; (b) 3 days and (c) 7 days of the specimens being immersed in water. The length of scale bar in images is $10 \mathrm{~mm}$.

\subsubsection{Self-Sealing Effect of WSRPs in Mortar}

In this study, XCT was used to study the self-sealing effect of WSRPs on cracks in the hardened mortar specimens. In the first step, the reconstructed 3D volumes of the cracked mortar samples, before and after the water healing process, were coupled by selecting the same rotation angle (Figure 6a). In the second step, tomographic slices of a longitudinal cross-section of the reconstructed 3D volumes were selected and compared in detail (Figure 6b). Finally, various phases of composite material is differentiated and colored base on the principle of different grey value (result from different density of material) under the X-ray. Therefore, only those material with the same density can be selected out. Since the rubber is basically a polymer which has much less density than cementitious material, all grey value fall into a certain band of grey value chart should be the material of rubber. So the material within this grey value was selected and then labeled with a blue color. It can be clearly seen that, before the water healing process, the crack runs through the entire specimen. This means that the water can permeate through the crack directly without any obstacle. While, after the healing process, WSRPs (blue) which are located along the crack were swollen in response to water absorption and partially blocked the crack, as can be seen in the white boxed areas. This result revealed that the WSRPs hold a potential to be applied for self-sealing cracks in mortar with the water swelling function.

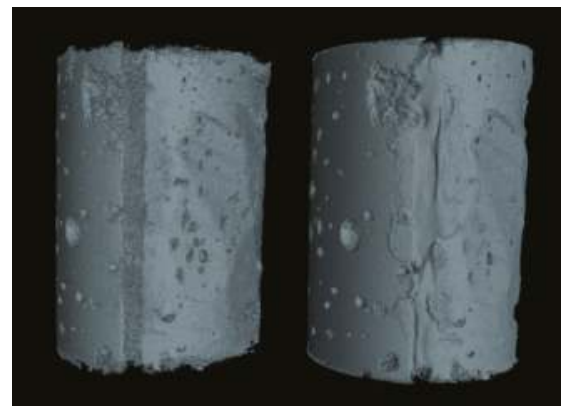

(a)

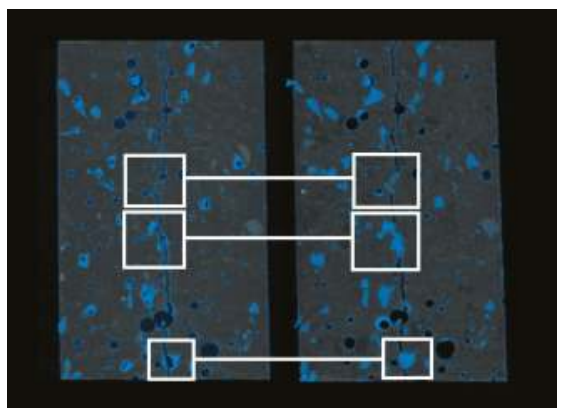

(b)

Figure 6. Reconstructed XCT images of (a) samples with WSRPs embedded cement mortar and (b) samples before and after sealing of the crack by WSRPs (blue particles). The length of scale bar in images is $10 \mathrm{~mm}$. 


\subsection{Influence of WSRPs on the Mechanical Properties of Mortar}

It is known that the use of $1 \%$ superabsorbent polymer (SAP) will results in an around $18 \%$ decrease in strength $[12,30]$. Similarly, a significate decrease in concrete compressive strength with increasing amount of rubber phase in the mixture can always be detected regardless the different nature, size and composition of tyre rubber $[16,31]$. The result shows that the compressive strength of rubberised concrete decreased $20 \%, 28 \%$ and $64 \%$ by adding $2 \%, 3 \%$ and $6 \%$ of tyre rubber in concrete [32]. In this study, the influence of incorporating $1 \%, 3 \%$ and $6 \%$ of WSRPs on the mechanical properties of mortar was evaluated systematically. Section 2.2 explains the specimen preparation for the mechanical tests. For each mix 3 replicate prisms were cast. 10\% of WSRPs replacement has also tried, while since it detrimentally influence on the mechanical properties of mortar, so the results are taken into our system consideration in this paper.

Figure 7a shows the relationship between the flexural strength and content of WSRPs. The error bar represents the standard deviation of the test results. It is clear that the addition of WSRPs has a negative effect of the flexural strength of the specimens. For the $\mathrm{L}$ series specimens, the flexural strength was decreased by values ranging from 10.5 to $20 \%$ with increasing the content of WSRPs from 1 to $6 \%$. The flexural strength of $\mathrm{M}$ series specimens decreased up to $18 \%$ for those specimens containing $6 \%$ WSRP. The addition of S size WSRPs has the least influence on the flexural strength. It only decreased $6 \%$ of its flexural strength for a dosage of $6 \%$ WSRPs. As expected, the incorporation of WSRPs also had a negative effect on the compressive strength of the specimens. As shown in Figure $7 \mathrm{~b}$, by incorporation of $1 \%, 3 \%$ and $6 \%$ of WSRPs, the compressive strength for $\mathrm{S}, \mathrm{M}$ and $\mathrm{L}$ series specimens reduced by $0-10 \%, 8-18 \%$ and $20-26 \%$ respectively.

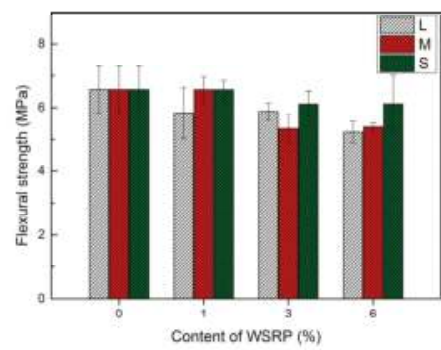

(a)

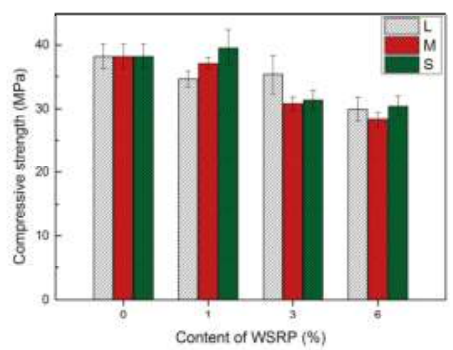

(b)

Figure 7. Influence of WSRPs incorporation on the (a) flexural strength and (b) compressive strength of specimens (with different additions of WSRPs, $0 \%(\mathrm{R}), 1 \%, 3 \%, 6 \%$ of sand by volume percentage, respectively) at the age of 28 days of hydration.

As it is expected that the addition of WSRPs will have a long-term effect on the internal strength and humidity, the mechanical properties of the specimens at a later age (28 days of hydration and then 28 days stored in laboratory environment) were investigated on the $L$ series specimens. The results are shown in Figure 8. According to the results, a significant increase was observed for both flexural and compressive strength in each series of specimens. For the flexural strength, a maximum of $38.7 \%$ increase was found for specimens containing $6 \%$ of large size WSRPs at age of 56 days compared to 28 days. Regarding the compressive strength, an obvious increase was noticed for the R and L1 specimens while only slight increase was found on L3 and L6. It can be seen that longer storage time can enhance the mechanical properties for both of the specimens with and without WSRPs. The main reason for this can be attribute to the further hydration of those unhydrated cement powder. Meanwhile, it is noteworthy that after a 28 days storage in laboratory environment, both flexural strength and compressive strength of WSRPs containing specimens were even higher or similar to the reference specimens with $0 \%$ of WSRP at 28 days of hydration. The possible reason for this 
enhancement can be partly attributed to the fact that, after a 28 days storage in laboratory environment, the WSRPs in specimens has less swelling pressure than the specimens which were just taken out from the fog room at 28 days of hydration, which will result in a lower stress concentration in the specimens and, therefore, a higher mechanical strength of specimens. Another explanation for this is that the absorbed water in WSRPs during the hydration process promoted a further hydration of the cement matrix by yielding their absorbed water into the surrounding cement matrix for the formation of new C-S-H crystals after 28 days of hydration. This can be deduced from the obviously slightly higher increase of flexural strength in the specimens with WSRPs than the reference specimen with $0 \%$ of WSRPs after a 28 days storage in laboratory environment. Similar describe can be found for superabsorbent polymer. While the details of further hydration function is beyond the scope of this paper and will be analyzed and described in further publications.

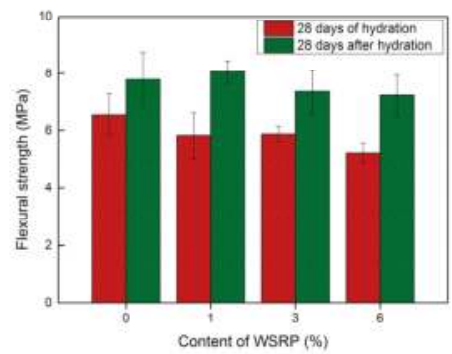

(a)

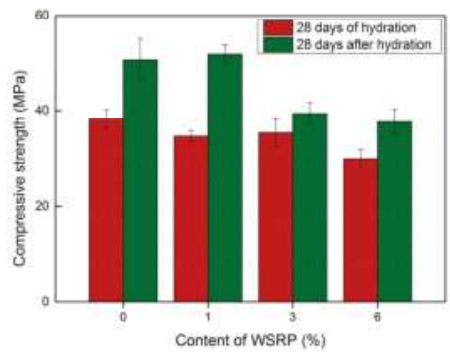

(b)

Figure 8. Influence of WSRPs incorporation on the (a) flexural strength and (b) compressive strength of specimens at different ages ( 28 days of hydration and 28 days storage at lab environment after 28 days of hydration).

\subsection{Self-Sealing Effect}

The permeability of specimens before and after the healing process was measured and plotted. Figure 9 shows the data comparing the permeability of samples after 0 and 7 days of immersion with different content of WSRPs ( $0 \%(\mathrm{R}), 1 \%, 3 \%, 6 \%$ of sand by volume percentage) and different crack width in the sample $(0.4 \mathrm{~mm}, 0.7 \mathrm{~mm}$ and $1.0 \mathrm{~mm})$.

For $0.4 \mathrm{~mm}$ crack width samples, the permeability decreased within the range of $13-27 \%$ with the addition of $1-6 \%$ of small size rubber filler ( $\mathrm{S}$ series). In this particular experimental setup, reductions caused by $1 \%$ concentration of medium size $(\mathrm{M})$ and large size $(\mathrm{L})$ rubber fillers were negligible. However, the performance of specimens containing 3-6\% of both medium (M) and large (L) size rubber fillers were more advanced compared to the small size particles (S) series with $18-42 \%$ and $38-45 \%$ respectively. Despite the permeability reductions due to the addition of WSRPs, only few percentage points of self-sealing capability can be found on most of the $0.4 \mathrm{~mm}$ crack width samples by comparing the permeability before and after healing process. For $0.7 \mathrm{~mm}$ crack width samples, the $\mathrm{S}$ series still show limited effect with only maximum $18 \%$ decrease of permeability. While medium series $(\mathrm{M})$ and large series (L) rubber systems showed much better performance in permeability reductions, ranging of $17-56 \%$ and $16-64 \%$ consecutively for WSRPs concentration of $1-6 \%$. Although the value of the flow rate is much high than for samples with 0.4 and $0.7 \mathrm{~mm}$ crack, samples with $1.0 \mathrm{~mm}$ crack width had a similar trend of permeability reductions. A permeability reduction of $22-58 \%$ and $23-61 \%$ can be found for the medium size (M) and large size (L) rubber systems with $1.0 \mathrm{~mm}$ crack width. While there is no significant decrease of permeability for small size (S) rubber system.

Moreover, a noticeable improvement could be found by comparing the permeability decreases before and after the rubber was allowed to swell after 7 days of immersion (self-sealing process). This self-sealing effect became more prominent for the samples subjected to crack widths of $0.7 \mathrm{~mm}$ 
and $1 \mathrm{~mm}$ as they showed relatively steeper decrease of permeability. In general, samples which were prepared with higher concentration and larger particles size seemed to show more significant further permeability reduction by the self-sealing effect of WSRPs. For $0.7 \mathrm{~mm}$ crack width sample, the swollen rubber for medium series (M) sample could lower the permeability to about $6-12 \%$ compared to the sample before immersion. While, the swollen rubber of large series (L) rubber could generate further reduction of about $3-20 \%$ with the rubber concentration of $1-6 \%$. In addition, in $1.0 \mathrm{~mm}$ crack width samples the permeability can be further lowered in the range of 3-22\% and $12-24 \%$ respectively for both medium (M) and large (L) samples.

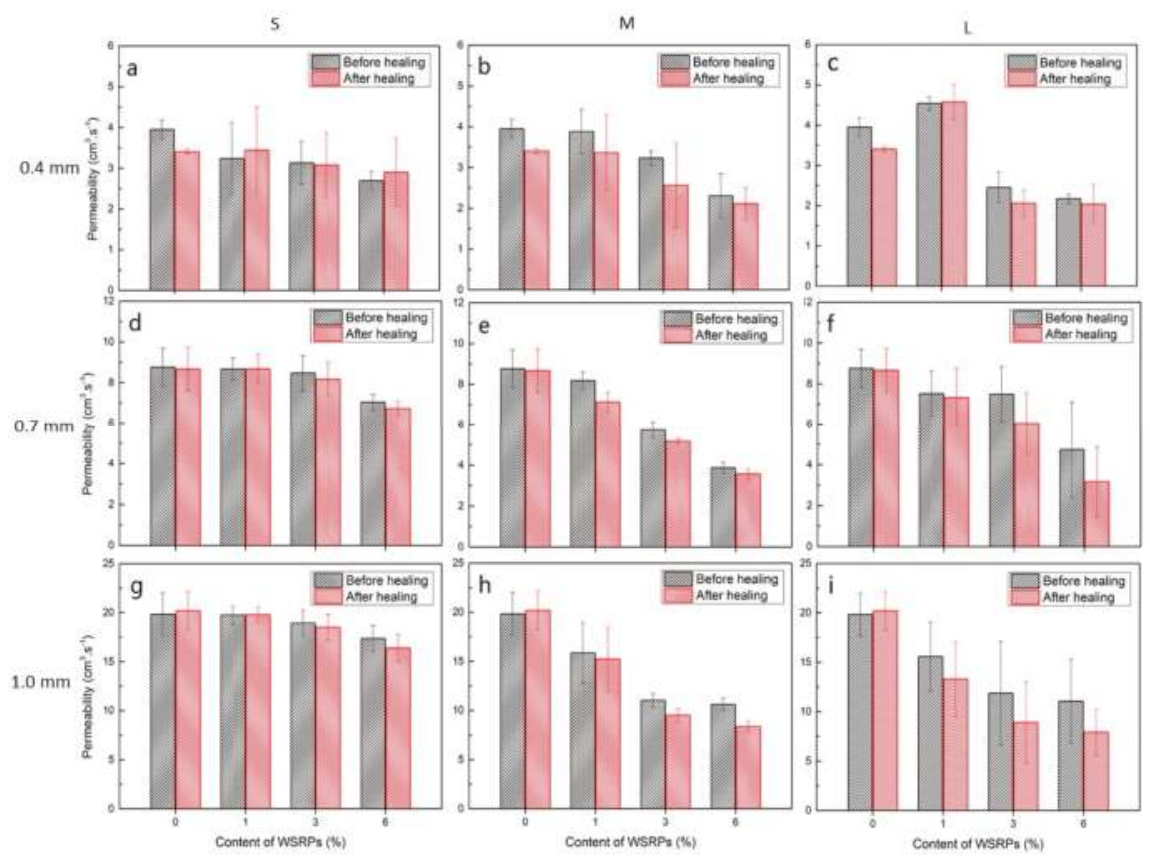

Figure 9. Data of permeability test of series S, M, L and B comparing the permeability at 0 day and after 7 days of water healing process in the form of 9 bar chart graphs. Graphs $(\mathbf{a}, \mathbf{d}, \mathbf{g})$ show the permeability data of S series samples with different crack width $(0.4,0.7$ and $1.0 \mathrm{~mm})$; Graphs (b,e,h) show the permeability data of M series samples with different crack width (0.4, 0.7 and $1.0 \mathrm{~mm}$ ); Graphs $(\mathbf{c}, \mathbf{f}, \mathbf{i})$ show the permeability data of L series samples with different crack width $(0.4,0.7$ and $1.0 \mathrm{~mm})$.

In general, based on the results of reducing water permeability of the mortar specimens, the reduction of water permeability in this study can be attribute to two reasons. The first reason is the exist of WSRPs on the crack partly block the path of water flow. This can be reflected form the immediately decrease of permeability for those sample with WSRPs embedded, even for those "before healing" series. The second reason is the swelling effect of WSRPs further enlarge the volume of WSRPs which blocking the crack that enable the permeability decrease further. This can be reflected by the further decrease of permeability after 7 days healing. Meanwhile, the large series (L) samples performed almost similar to the medium series (M) samples for both $0.7 \mathrm{~mm}$ and $1 \mathrm{~mm}$ crack width. These occurrences can be attribute to the balance between limitations of rubber expansion and the spatial availability within the cracked sample. This means that swelling of large size particles is more difficult since the dimensions of the spaces in which it has to squeeze through are smaller in comparison. Another matter to consider is the significantly higher scatter in the data collected for the large specimen series (L) compared to the medium size samples $(\mathrm{M})$ due to the lower probability of particle homogenisation. 
Since, in order to occupy the same volumetric ratio, less large particles are needed while more grains of small and medium size are required which causes uneven particles distribution within the mortar samples. Then it ultimately causes $\mathrm{L}$ series sample to have a lower probability to be homogenously present in the crack path. Therefore, although L and M size particles may seem to produce the same average permeability reduction in this study, there are chances in which the self-sealing function of $\mathrm{L}$ series samples may underestimated because of the inferior particle distribution.

\subsection{Visual Assessment of the Crack Bridging Function of WSRPs}

The crack bridging function is a unique characteristic of WSRPs. The embedded WSRPs can provide the concrete with a bridging effect to prevent the crack from fully separating the specimen. Typical images of the crack area of the WSRPs embedded sample were examined by digital camera and are shown in Figure 10. As can be seen from the Figure 10a, after the tensile load was first imposed and then removed from the cracked sample loaded with $4 \%$ of WSRPs, the blue rubber particles become visible inside the crack. It is clear that the cracked specimen halves were kept together by the WSRPs and the specimen is not separated into two parts. This phenomenon indicates that the embedded rubber has the ability to bridge the cracked faces of the specimen. Figure 10b,c shows the internal crack surface of sample with only $1 \%$ of WSRPs. It can be seen that, even if the specimen was completely broken, the remnant of rubber particles were still binding to the mortar and the original grainy rubber was stretched into a fiber-like structure. This observation demonstrates that the rubber has a good bonding strength with mortar matrix, which corresponds well with the result of the microstructure study by ESEM in Section 3.1.

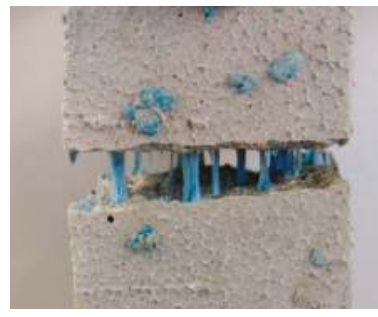

(a)

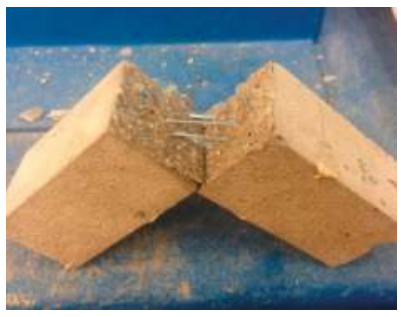

(b)

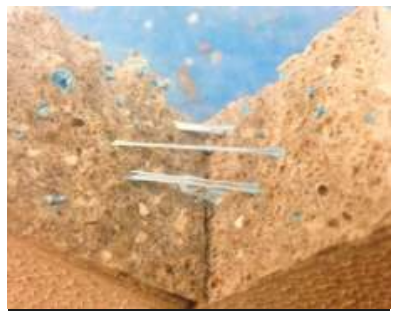

(c)

Figure 10. Typical images showing the crack bridging function of mortar with (a) $4 \%$ and (b,c) $1 \%$ of WSRPs as a replacement of sand.

\section{Conclusions}

In this study, granulated water swelling rubber (WSRPs) was first used for reducing permeability of large cracks (width: 0.4 to $1 \mathrm{~mm}$ ) through volume blocking and volume expansion triggered by water absorption. The XCT result shows that the WSRPs have an obvious self-sealing effect in the cracked mortar. The incorporation of MSRPs into the system lowered the compressive strength by maximum of $19 \%$ with addition of $6 \%$ of large size WSRPs, and the flexural strength by maximum of $20 \%$ with incorporation of $6 \%$ large size WSRPs. The sealing function of WSRPs in mortar was studied for cracks of $0.4 \mathrm{~mm}, 0.7 \mathrm{~mm}$ and $1.0 \mathrm{~mm}$ wide. It was found that the addition of WSRPs in mortar was able to partially lower the permeability, which was decreased even further after the samples were immersed in water for 7 days. In general, degree of permeability reductions increases with higher concentration of WSRPs. Larger particles shows higher effect of the self-sealing function. The data collected from permeability test suggests that medium (M) and large (L) particles showed more or less similar impact in permeability decrease. A maximum permeability reduction of $58 \%$ and $64 \%$ can be found for the medium size (M) and large size (L) rubber systems with WSRPs concentration of $6 \%$. 
In addition, a crack bridging function of WSRPs that can bridge the cracked mortar and prevent the two halves from completely separating was discovered.

This work contributes to the search for an effective material to completely or partially decrease the permeability of cementitious materials with less influence on mechanical properties. Comparing with previous study, the novel preparation and application of WSRPs give the cement mortar, a traditional construction material, a self-sealing ability with less sacrificing the mechanical properties. However, as a new self-sealing agent, some improvements to the proposed study are still needed. Firstly, water swelling rubber particles which are particularly applicable to our application with less initial swelling at the hydration stage and with higher swelling pressure at the self-sealing period should be developed. Meanwhile it was found that the swelling capacity of WSRPs is highly influenced by the crack size and particle sizes. Therefore optimization procedures of size and concentration of WSRPs to increase the distribution probabilities and improve self-sealing performance should be developed. In addition, in preparing for practical application, further focus will be given to an accurate measurement of the potential of the crack bridging function of the WSRPs in cementitious materials.

Acknowledgments: This project was financial supported by the National Natural Science Foundation of China (No. 51120185002/U1301241). The author received funds from TU Delft Open Access Fund for covering the costs to publish in open access.

Author Contributions: All authors conceived and designed the experiments. Leyang Lv performed the experiments. Leyang Lv and Erik Schlangen analyzed and discussed the data. All authors wrote the paper.

Conflicts of Interest: The authors declare no conflict of interest.

\section{References}

1. Schlangen, E.; Joseph, C. Self-Healing Processes in Concrete. In Self-Healing Materials; Wiley-VCH Verlag GmbH \& Co., KGaA: Weinheim, Germany, 2009; pp. 141-182.

2. Wiktor, V.; Jonkers, H.M. Quantification of crack-healing in novel bacteria-based self-healing concrete. Cem. Concr. Compos. 2011, 33, 763-770. [CrossRef]

3. Wang, J.Y.; Van Tittelboom, K.; De Belie, N.; Verstraete, W. Use of silica gel or polyurethane immobilized bacteria for self-healing concrete. Constr. Build. Mater. 2012, 26, 532-540. [CrossRef]

4. Hearn, N. Self-sealing, autogenous healing and continued hydration: What is the difference? Mater. Struct. 1998, 31, 563-567. [CrossRef]

5. Van Tittelboom, K.; De Belie, N. Self-healing in cementitious materials-A review. Materials 2013, 6, 2182-2217. [CrossRef] [PubMed]

6. Yang, Z.X.; Hollar, J.; He, X.D.; Shi, X.M. A self-healing cementitious composite using oil core/silica gel shell microcapsules. Cem. Concr. Compos. 2011, 33, 506-512. [CrossRef]

7. Dong, B.Q.; Wang, Y.S.; Fang, G.H.; Han, N.X.; Xing, F.; Lu, Y.Y. Smart releasing behavior of a chemical self-healing microcapsule in the stimulated concrete pore solution. Cem. Concr. Compos. 2015, 56, 46-50. [CrossRef]

8. Ge, S.G.; Wan, F.W.; Lu, J.J; Yu, J.H. Molecular Self-Assembled Microcapsules Prepared by In Situ Polymerization Technology for Self-Healing Cement Materials. J. Inorg. Organomet. Polym. Mater. 2011, 21, 841-845. [CrossRef]

9. Maes, M.; Van Tittelboom, K.; De Belie, N. The efficiency of self-healing cementitious materials by means of encapsulated polyurethane in chloride containing environments. Constr. Build. Mater. 2014, 71, 528-537. [CrossRef]

10. Van Tittelboom, K.; De Belie, N.; Van Loo, D.; Jacobs, P. Self-healing efficiency of cementitious materials containing tubular capsules filled with healing agent. Cem. Concr. Compos. 2011, 33, 497-505. [CrossRef]

11. Wang, J.Y.; Soens, H.; Verstraete, W.; De Belie, N. Self-healing concrete by use of microencapsulated bacterial spores. Cem. Concr. Res. 2014, 56, 139-152. [CrossRef]

12. Lee, H.X.D.; Wong, H.S.; Buenfeld, N.R. Self-sealing of cracks in concrete using superabsorbent polymers. Cem. Concr. Res. 2016, 79, 194-208. [CrossRef]

13. Song, C.; Choi, Y.C.; Choi, S. Effect of internal curing by superabsorbent polymers-Internal relative humidity and autogenous shrinkage of alkali-activated slag mortars. Constr. Build. Mater. 2016, 123, 198-206. [CrossRef] 
14. Gruyaert, E.; Debbaut, B.; Snoeck, D.; Diaz, P.; Arizo, A.; Tziviloglou, E.; Schlangen, E.; De Belie, N. Self-healing mortar with $\mathrm{pH}$-sensitive superabsorbent polymers: Testing of the sealing efficiency by water flow tests. Smart Mater. Struct. 2016, 25, 084007. [CrossRef]

15. Snoeck, D.; Van Tittelboom, K.; Steuperaert, S.; Dubruel, P.; De Belie, N. Self-healing cementitious materials by the combination of microfibres and superabsorbent polymers. J. Intell. Mater. Syst. Struct. 2014, 25, 13-24. [CrossRef]

16. Bignozzi, M.C.; Sandrolini, F. Tyre rubber waste recycling in self-compacting concrete. Cem. Concr. Res. 2006, 36, 735-739. [CrossRef]

17. Hesami, S.; Hikouei, I.S.; Emadi, S.A.A. Mechanical behavior of self-compacting concrete pavements incorporating recycled tire rubber crumb and reinforced with polypropylene fiber. J. Clean. Prod. 2016, 133, 228-234. [CrossRef]

18. Xie, J.H.; Guo, Y.C.; Liu, L.S.; Xie, Z.H. Compressive and flexural behaviours of a new steel-fibre-reinforced recycled aggregate concrete with crumb rubber. Constr. Build. Mater. 2015, 79, 263-272. [CrossRef]

19. Youssf, O.; ElGawady, M.A.; Mills, J.E.; Ma, X. An experimental investigation of crumb rubber concrete confined by fibre reinforced polymer tubes. Constr. Build. Mater. 2014, 53, 522-532. [CrossRef]

20. Methods of Testing Cement-Part 1: Determination of Strength; European Committee for Standardization: Brussels, Belgium, 2005; EN, T. 196-1: 2016.

21. Palin, D. A Rapid and Repeatable Method for Establishing the Water Permeability of Cracked Mortar Specimens. In Proceedings of the Fib Concrete-Innovation and Design, Copenhagen, Denmark, 18-24 May 2015.

22. Astolfo, A.; Qie, F.X.; Kibleur, A.; Hao, X.J.; Menk, R.H.; Arfelli, F.; Rigon, L.; Hinton, T.M.; Wickramaratna, M.; Tan, T.W.; et al. A simple way to track single gold-loaded alginate microcapsules using X-ray CT in small animal longitudinal studies. Nanomed. Nanotechnol. Biol. Med. 2014, 10, 1821-1828. [CrossRef] [PubMed]

23. Mookhoek, S.D.; Mayo, S.C.; Hughes, A.E.; Furman, S.A.; Fischer, H.R.; van der Zwaag, S. Applying SEM-Based X-ray Microtomography to Observe Self-Healing in Solvent Encapsulated Thermoplastic Materials. Adv. Eng. Mater. 2010, 12, 228-234. [CrossRef]

24. Lv, L.; Schlangen, E.; Yang, Z.; Xing, F. Micromechanical Properties of a New Polymeric Microcapsule for Self-Healing Cementitious Materials. Materials 2016, 9, 1025. [CrossRef] [PubMed]

25. Lv, L.; Yang, Z.; Chen, G.; Zhu, G.; Han, N.; Schlangen, E.; Xing, F. Synthesis and characterization of a new polymeric microcapsule and feasibility investigation in self-healing cementitious materials. Constr. Build. Mater. 2016, 105, 487-495. [CrossRef]

26. Gallucci, E.; Scrivener, K.; Groso, A.; Stampanoni, M.; Margaritondo, G. 3D experimental investigation of the microstructure of cement pastes using synchrotron X-ray microtomography $(\mu \mathrm{CT})$. Cem. Concr. Res 2007, 37, 360-368. [CrossRef]

27. Landis, E.N.; Keane, D.T. X-ray microtomography. Mater. Charact. 2010, 61, 1305-1316. [CrossRef]

28. Methods of Test for Mortar for Masonry_Part 11: Determination of Flexural and Compressive of Harddened Mortar; European Committee for Standardization: Brussels, Belgium, 1999; EN, T. 1015-11.

29. Palin, D.; Jonkers, H.; Wiktor, V. Autogenous healing of sea-water exposed mortar: Quantification through a simple and rapid permeability test. Cem. Concr. Res. 2016, 84, 1-7. [CrossRef]

30. Klemm, A.J.; Sikora, K.S. The effect of Superabsorbent Polymers (SAP) on microstructure and mechanical properties of fly ash cementitious mortars. Constr. Build. Mater. 2013, 49, 134-143. [CrossRef]

31. Son, K.S.; Hajirasouliha, I.; Pilakoutas, K. Strength and deformability of waste tyre rubber-filled reinforced concrete columns. Constr. Build. Mater. 2011, 25, 218-226. [CrossRef]

32. Khatib, Z.K.; Bayomy, F.M. Rubberized portland cement concrete. J. Mater. Civ. Eng. 1999, 11. [CrossRef]

(C) 2017 by the authors. Licensee MDPI, Basel, Switzerland. This article is an open access article distributed under the terms and conditions of the Creative Commons Attribution (CC BY) license (http:/ / creativecommons.org/licenses/by/4.0/). 
Article

\title{
Development of Self-Sensing Textile Strengthening System Based on High-Strength Carbon Fiber
}

\author{
Marcin Górski *, Rafał Krzywoń and Magdalena Borodeńko \\ Department of Structural Engineering, Silesian University of Technology, ul. Akademicka 5, 44-100 Gliwice, \\ Poland; rafal.krzywon@polsl.pl (R.K.); m.borodenko@onet.eu (M.B.) \\ * Correspondence: marcin.gorski@polsl.pl; Tel.: +48-608-018-778
}

Received: 26 September 2018; Accepted: 20 October 2018; Published: 22 October 2018

\begin{abstract}
The monitoring of structures is one of the most difficult challenges of engineering in the 21 st century. As a result of changes in conditions of use, as well as design errors, many building structures require strengthening. This article presents research on the development of an externally strengthening carbon-fiber textile with a self-sensing option, which is an idea is based on the pattern of resistive strain gauges, where thread is presented in the form of zig-zagging parallel lines. The first laboratory tests showed the system's high efficiency in the measurement of strains, but also revealed its sensitivity to environmental conditions. This article also presents studies on the influence of temperature and humidity on the measurement, and to separate the two effects, resistance changes were tested on unloaded concrete and wooden samples. The models were then placed in a climatic chamber, and the daily cycle of temperature and humidity changes was simulated. The research results confirmed preliminary observations of resistivity growths along with temperature. This effect is more visible on concrete samples, presumably due to its greater amount of natural humidity. The strain measurement with carbon fibers is very sensitive to temperature changes, and applications of this method in practice require compensation.
\end{abstract}

Keywords: CFRP strengthening; textile sensor; strain gauge errors compensation

\section{Introduction}

About 50 years ago, Concor and Owston [1] noted that most carbon fibers behaved in a simple manner of linear rises in resistance with strain. They also defined some of their important characteristics for measurements, such as the initial stress of fibers, time-dependency, and high strain rate or contact resistance [2]. Since these discoveries, the idea of self-sensing carbon fibers has been developed and is still being improved upon today. Currently, the development of two independent technologies using conductivity of carbon-based materials for strain measurement can be observed: printed gauges based on graphite carbon nanotubes [3-7], and those based on continuous carbon fiber [8-17]. It should be emphasized that only the latter allows for the use of an essential feature of carbon fibers, which is their high strength. In recent years, a form of technology enabling the 3D printing of carbon fiber sensors has also appeared $[17,18]$, which should help in avoiding a quite laborious assembly process and should also improve sensor quality. Carbon fibers can also be used as light sensors [19].

Measurements performed using resistive strain gauges allow for investigation of changes in strain expressed by the change in resistance. Unfortunately, the measurement of the actual mechanical deformation overlaps with various effects which disturb the measurement signal. These effects mainly consist of environmental influences, such as thermal elongation of the measured object, temperature-dependent changes in strain gauge resistance, thermal shrinkage of the strain gauge measuring film, the thermal response of connecting wires, and surface conductivity with increasing humidity. Carbon-based materials are much more temperature-sensitive than conventional strain 
sensors made of constantan (copper-nickel alloy) [20]. The above factors may be influenced by the method of laying the wire, the material from which the strain gauge was made, and the ground material, as well as the type of adhesive that fixed the strain gauge. In the case of carbon fibers, their straightness may also be important [2,12], as well as the contact resistance [13] which may be limited by the matrix material, and the method of assembling the electrodes.

Typically, the effects of temperature and humidity is compensated for in the measurements by combining several strain gauges to form a half or full bridge. Through the skillful combination of strain gauges of the most commonly used Wheatstone bridge circuit [21], where strains from the measuring strain gauges and compensation strain gauges are reflected in the measurement signal with opposite signs (positive and negative), the bridge voltage only represents mechanical strains, and the temperature/humidity-dependent effects abolish each other.

Because identical, or even similar working conditions of measuring and compensating strain gauges may not always be possible, modern computational compensation systems which have been adapted to the thermal and moisture responses of strain gauges are increasingly being used today. In these types of measurements, a quarter-bridge circuit is sufficient, but knowledge of the temperate and humidity response functions is necessary.

This paper describes preliminary studies on the temperature-resistant dependence of externally strengthening carbon-fiber textile sensors which have been developed. These tests may be used in the future to build temperature-change compensation functions necessary for use in regard to future devices in engineering practice. They may also be used for development by the authors' strengthening system, but it is believed that such self-monitoring solutions with reliable information about its deformation in various environmental conditions may be used for future smart structures made out of conductive fibers.

\section{The Idea of a Self-Sensing Strengthening System}

Controlling the deformation of FRP (fiber reinforced polymer) composite fibers is much more important than the steels themselves traditionally used in constructions. This is due to their linear elasticity until destruction, which is usually sudden. For this reason, it is advisable to control the strains of the composite fibers to indicate excessive elongation, which may threaten their breaking or delamination.

As will be described in this paper, the system takes advantage of two features of carbon fibers, which are their superb strength and electrical conductivity. The idea of measuring strains by recording changes in the resistance is based on operation principles of strain gauges invented in 1938 by Edward E. Simmons and Arthur C. Ruge [22]. Such strain gauges contain a long, thin conductive wire arranged in a zig-zag pattern of parallel lines. The parallel orientation of wires multiplies the reading, which allows for an increase in the accuracy of the measurement of small strains. The same concept was used to create smart textiles. Carbon fiber tow, commonly used as a reinforcement for FRP strengthening systems, can also act as an electrical conductor. Deformation leads to changes in electrical resistance, allowing for self-monitoring of the fabric. The first tests have shown that changes in resistance for a single tow is too small compared to the technical abilities of the recording equipment used (e.g., the Wheatstone bridge). Increasing the length of the tow by its wiggly lead (a copy of the zig-zag pattern of the strain gauge) significantly improved the sensitivity of the system.

In this way, the first prototype was tested on small models of concrete slabs. To verify the accuracy of the measurement, the device was accompanied by paper strain gauges adhered along its length (Figure 1). A more detailed description of these studies is described in [23]. These initial trials confirmed the capabilities of developed smart textiles. However, the sensitivity of the device still proved to be insufficient, especially for measuring small changes in the strain. A modification allowing for an increase in the accuracy of the measurement was the introduction of two parallel threads of carbon fibers placed end to end. When the measuring bridge was connected to the two ends of independent threads, the measured resistance increased, because besides the previous volume resistance (growing 
with the elongation of carbon fiber), the phenomenon of contact resistance appeared at the interface of parallel fiber threads. Despite the controversy, which was mainly related to the accuracy of the measurement of large deformations at which micro-cracks may appear, and with them, rapid increases in contact resistance, the results of the first tests turned out to be quite promising. The developed textile sensor was checked in regard to whether it could act as an independent external reinforcement and as a local strengthener of existing reinforcements. The strengthening effectiveness which was achieved can also be assessed by using a single carbon sheet layer while maintaining the ability to measure strains until their failure. The results of these studies are presented in Reference [24].

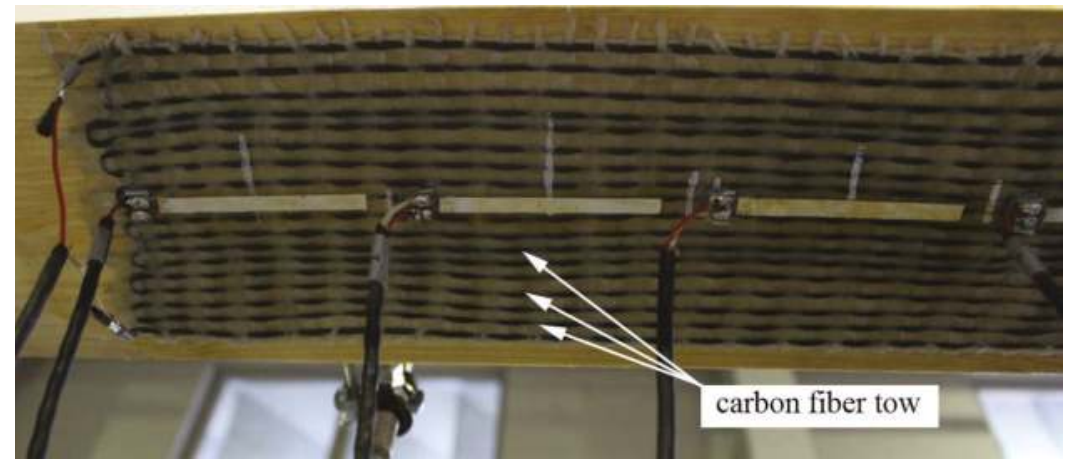

Figure 1. The second generation of the textile sensor, adhered to a specimen of timber.

The production method described was based on traditional plain weaving, where alternating carbon and acrylic threads were arranged as a warp and stabilized by the weft made of cotton. However, this technology has several significant disadvantages. The carbon fiber must be continuous and well-stressed during weaving, which may hinder the automation of production, and only the classic weaving loom can be used. The fiber types used also makes the textile sensor soft, and although this facilitates its application, after adhering, the carbon fibers may not be sufficiently stressed, which can worsen the strengthening efficiency and change the gauge factor. Specifying the gauge factor value is also one of the biggest drawbacks of the developed solution, which is why paper strain gauges were used for the trial tests. We also observed that resistance not only depended largely on the total length of the carbon fiber thread, but also on the lamination conditions. This is most likely related to the change in contact resistance conditions.

The third generation of the intelligent textile is currently being tested. The concept of the parallel arrangement of a double-fiber thread was abandoned, which means that the volumetric resistance is now directly responsible for the measurement. However, the main modification is in regard to the production method. Weaving was replaced by fiber stabilization by fastening to the composite mesh matrix, and for this purpose, a glass fiber plaster-reinforcing mesh was used. The dry carbon thread was stitched to the mesh strands, as shown in Figure 2. This stabilization method reduces the risk of accidental short-circuiting during the assembly of the sensor, and there is no need for additional separating acrylic threads. The sensor can be manufactured even in construction-site conditions, and no machines are needed, such as a weaving loom. The change in the resistance of this type of sensor should depend mainly on the total length and cross-section of the carbon fibers; therefore, its gauge factor should be easier to determine. 


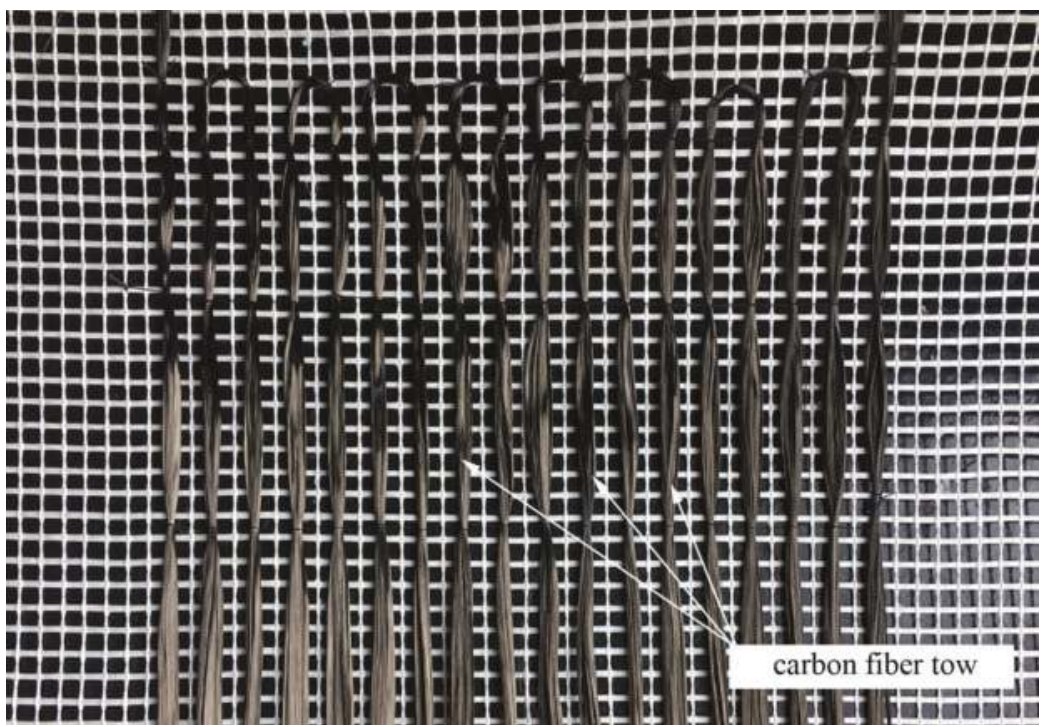

Figure 2. The third generation of the textile sensor during fabrication.

\section{Tests of the Influence of Temperature on the Resistance}

The research described in this chapter is concerned with two types of sensors: the woven sensor, which was the second generation, adhered to the wooden substrate; and stabilized mesh, the latest generation, adhered to the concrete substrate. In our tests, two-component epoxy S\&P Resin 55HP was used, which is an adhesive intended for fixing carbon sheets onto various types of substrate. Wet lay-up technology was also applied according to the following method: firstly, the substrate was impregnated, then the sensor was placed, and the carbon fibers were aligned and saturated with resin. The adhesive layer was leveled out with a roller.

Table 1 presents the basic parameters of both types of sensors. The wooden sample was made of pinewood, and dimensions of the prism were $1200 \times 158 \times 78 \mathrm{~mm}$. The wood fibers and sensor thread were parallel. The thermal expansion coefficient along the fibers of pinewood was equal at around $3 \times 10^{-6} /{ }^{\circ} \mathrm{C}$. The precast concrete sample was made of plain concrete, and was $1000 \mathrm{~mm}$ long and $200 \times 60 \mathrm{~mm}$ for the cross-section. The coefficient of the thermal expansion of concrete was equal to $12 \times 10^{-6} /{ }^{\circ} \mathrm{C}$.

The models were tested after more than a month of curing the adhesive. They were stored in a stable temperature of about $20^{\circ} \mathrm{C}$ and $60 \%$ humidity two hours before the test samples were placed in a climatic chamber (Figure 3).

Due to the preliminary nature of the research, tests for both samples were provided in four different cycles of temperature change with a small variation in humidity, which are the following:

- heating from $+20{ }^{\circ} \mathrm{C}$ to $+40{ }^{\circ} \mathrm{C}$ with a simultaneous humidity change from $60 \%$ to $65 \%$,

- heating from $+20{ }^{\circ} \mathrm{C}$ to $+60{ }^{\circ} \mathrm{C}$ with a simultaneous change in humidity from $60 \%$ to $65 \%$,

- cooling from $+20^{\circ} \mathrm{C}$ to $0{ }^{\circ} \mathrm{C}$ with a simultaneous reduction of humidity from $60 \%$ to $55 \%$,

- cooling from $+20{ }^{\circ} \mathrm{C}$ to $-20{ }^{\circ} \mathrm{C}$ with a simultaneous reduction of humidity from $60 \%$ to $55 \%$.

Concrete and wooden samples were tested separately. Before the next test cycle, they were stored for at least two days in the above-mentioned stable conditions to regain their natural humidity and temperature. 
After being placed in a climatic chamber, a control measurement was carried out using a simple ohmmeter to check initial resistance and to exclude accidental short circuits. Then, the textile sensor was connected to the Wheatstone bridge, the chamber was closed, and the test commenced.

Table 1. Properties of the textile sensor.

\begin{tabular}{|c|c|c|}
\hline Sensor Generation & Woven (2nd) & Mesh Stabilized (3rd) \\
\hline construction & $\begin{array}{l}\text { Two parallel threads of conductive } \\
\text { carbon fiber, separated by a thread of } \\
\text { acrylic fiber }\left(1.17 \mathrm{~g} / \mathrm{cm}^{3}\right) \text { and } \\
\text { stabilized by cotton weft }\left(1.54 \mathrm{~g} / \mathrm{cm}^{3}\right)\end{array}$ & $\begin{array}{l}\text { A single thread of conductive } \\
\text { carbon fiber fastened to the glass } \\
\text { fiber plaster-reinforcing mesh } \\
\quad\left(8 \times 8 \mathrm{~mm} ; 145 \mathrm{~g} / \mathrm{cm}^{3}\right)\end{array}$ \\
\hline number of loops & 18 & 18 \\
\hline length of the sensor & $1000 \mathrm{~mm}$ & $1000 \mathrm{~mm}$ \\
\hline rowing & $\begin{array}{l}2 \times 24,000 \text { filaments/thread } \\
2 \times 1600 \text { tex }\end{array}$ & $\begin{array}{l}24,000 \text { filaments/thread } \\
1600 \text { tex }\end{array}$ \\
\hline strength of carbon fiber & \multicolumn{2}{|c|}{$5000 \mathrm{MPa}$} \\
\hline modulus of elasticity & \multicolumn{2}{|c|}{$270 \mathrm{GPa}$} \\
\hline elongation at break & \multicolumn{2}{|c|}{$1.9 \%$} \\
\hline filament resistivity & \multicolumn{2}{|c|}{$14 \mu \Omega \mathrm{m}$} \\
\hline initial resistance & $174.8 \Omega^{1}$ & $212 \Omega$ \\
\hline gauge factor & 1.24 & 1.19 \\
\hline
\end{tabular}

${ }^{1}$ Before lamination of fibers.

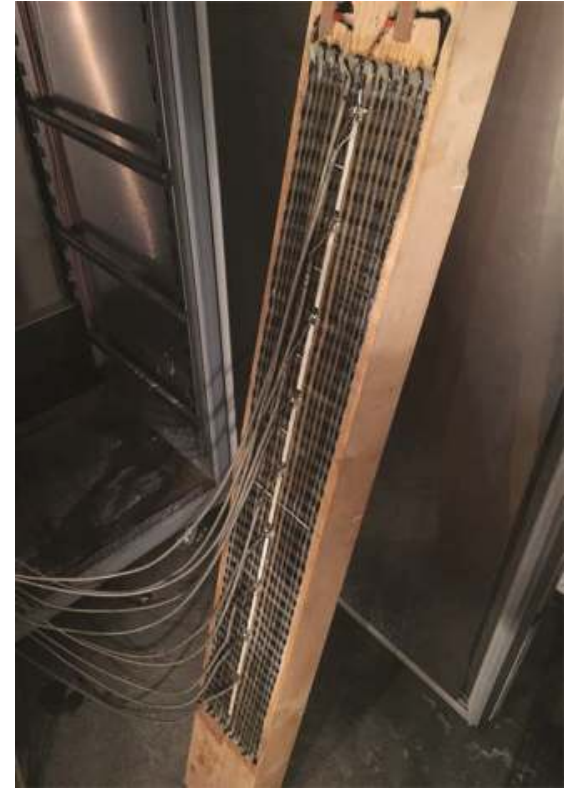

(a)

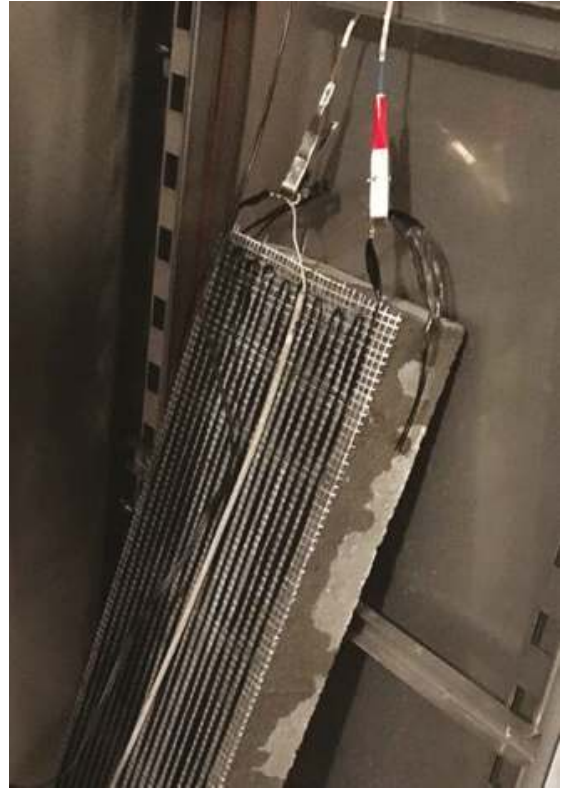

(b)

Figure 3. Samples inside the climate chamber during the test: (a) second generation of the sensor and timber sample; (b) third generation of the sensor and concrete sample. 
Although the climatic chamber allows for simultaneous changes in temperature and humidity, the temperature remains the priority. It should be also emphasised that increases and decreases in temperature are not linear; in the first phase there is an intense change, followed by a phase of slow adjustment to the expected temperature. This property causes the measurement paths in similar temperature ranges to vary in cycles with different limit values.

During the test, the actual temperature and humidity were saved by the chamber controller, and strains simultaneously expressed by resistance changes were recorded every $60 \mathrm{~s}$ through a computer connected to the Wheatstone bridge. This measurement corresponded to the sum of the actual thermal deformation of the sample and apparent deformation, which expresses the wanted change in the sensor's resistance under the influence of temperature and humidity.

\section{Test Results}

The influence of temperature and relative humidity on the resistance measurement must be considered simultaneously, and it should also be noted that the relative humidity itself is temperature-dependent. To simplify the interpretation of the results, measures were provided for relatively small changes in relative humidity and the results are presented only as a function of temperature. They are shown in diagrams as having a form of dependence on temperature changes on the apparent deformation measured. Figures 4 and 5 show the test results for the sensor on a wooden sample, and Figures 6 and 7 on a concrete sample. The situation of the rising temperature and the decreasing temperature is shown independently in separate diagrams.

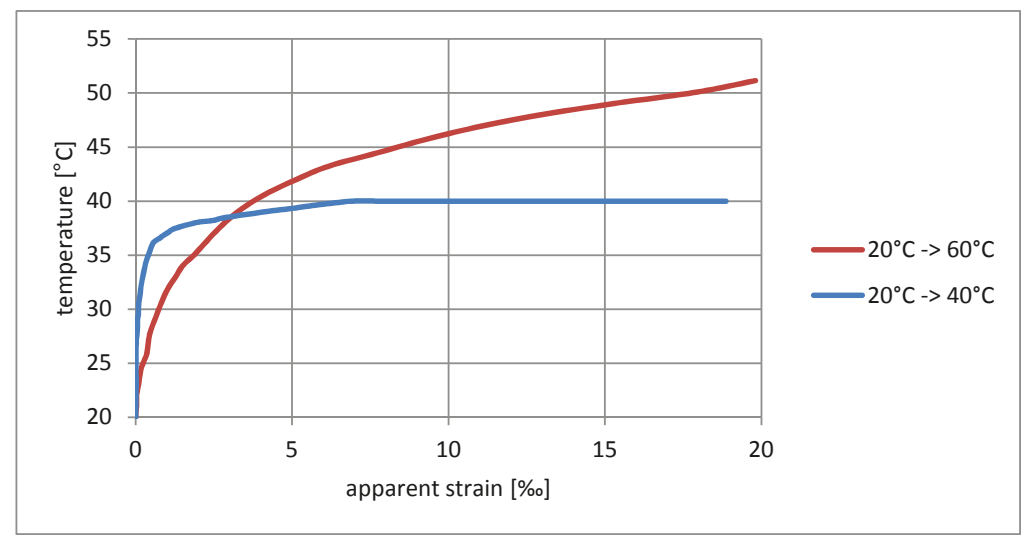

Figure 4. Dependence of apparent strain on rising temperature for the wooden sample. 


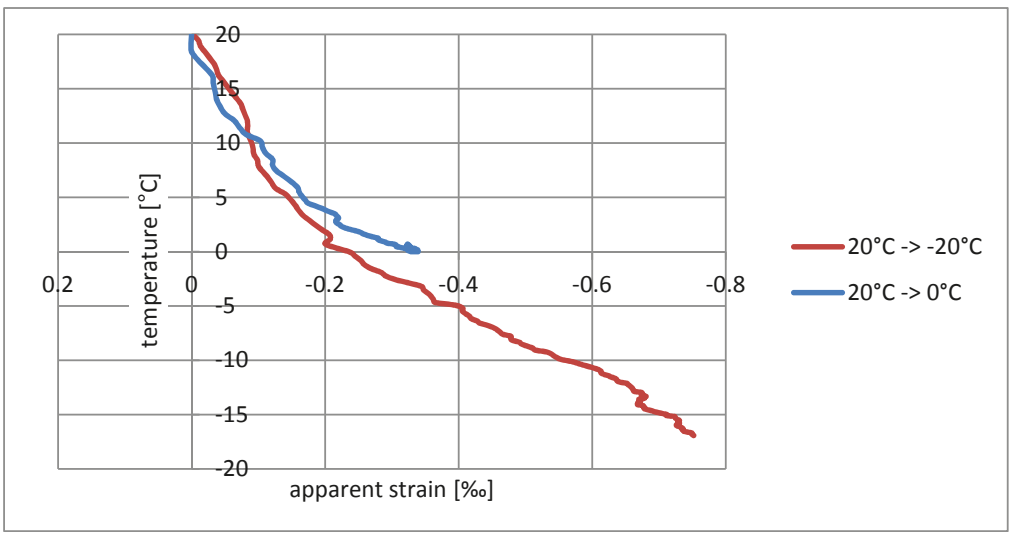

Figure 5. Dependence of apparent strain on decreasing temperature for the wooden sample.

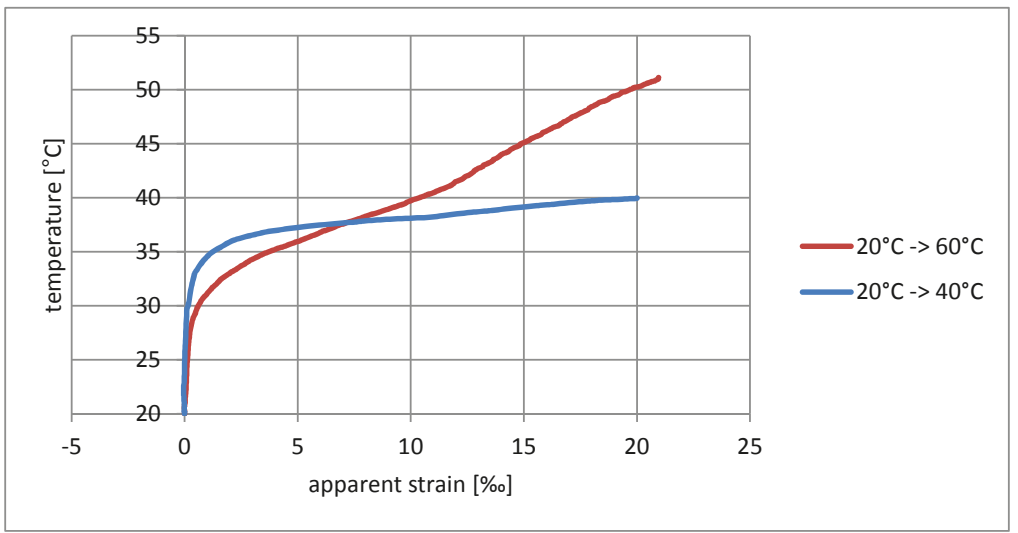

Figure 6. Dependence of apparent strain on rising temperature for the concrete sample.

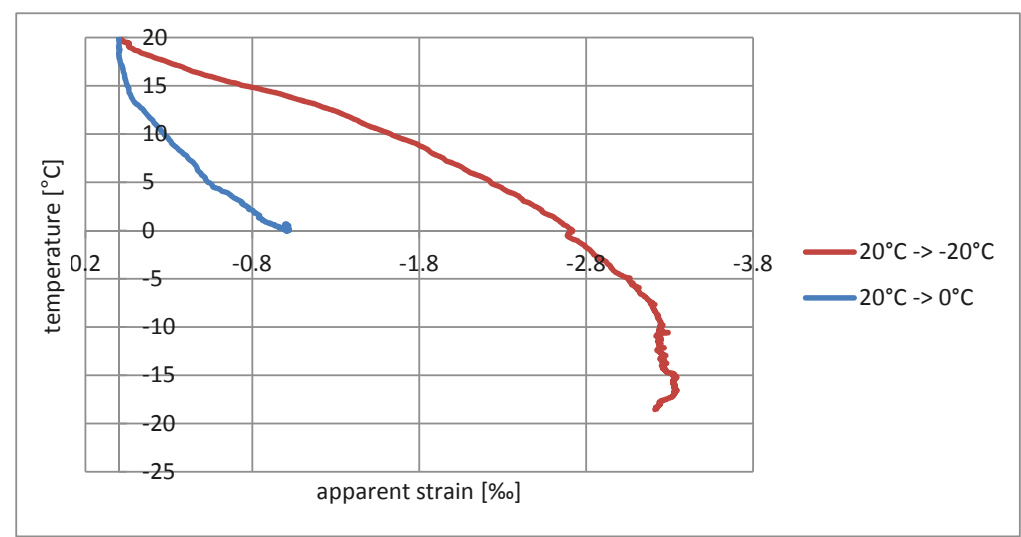

Figure 7. Dependence of apparent strain on decreasing temperature for the concrete sample. 


\section{Discussion}

The measured strain consists of a real elongation of the sample as a result of thermal expansion and apparent strain resulting from the temperature sensitivity of the textile sensor. The direction of observed resistance changes is correct. The increase in temperature is indicated as elongation, while the temperature decrease is indicated as shortening. If the changes shown were purely the result of thermal deformability, then for wood the measured deformation should not exceed $0.003 \% 0 /{ }^{\circ} \mathrm{C}$, whereas for concrete it should be $0.012 \% /{ }^{\circ} \mathrm{C}$; however, the obtained strains are much greater, and the temperature-strain relationship is also not linear.

The sensor's tendency to indicate apparent strain is particularly visible for rising temperatures. Considering the elasticity coefficient of carbon fibers, the measured strain $>20 \%$ (Figures 4 and 6) theoretically corresponds to a stress of $5.4 \mathrm{GPa}$, thus breaking the carbon fibers in practice. The situation is slightly better in the case of decreasing temperatures' $\%$, where the error for wood does not exceed $0.75 \%$ (Figure 5), while for concrete it is 3.3\% (Figure 7). This difference can also result from a slightly different type of textile sensor used in both cases. However, a faster increase in the conductivity of concrete samples may also result from humidity changes on their surface-due to the better thermal conductivity, the concrete base can promote condensation of water vapor. The indicated value of apparent strain shows the impact of compensation due to temperature changes on the result. The tendency for there to be such a high inflation of the result when the temperature rises means that the system is completely unusable, even for approximate estimations of stress changes.

Another interesting observation is related to the speed of temperature changes. Heating tests from $20{ }^{\circ} \mathrm{C}$ to $40{ }^{\circ} \mathrm{C}$ and $20{ }^{\circ} \mathrm{C}$ to $60{ }^{\circ} \mathrm{C}$, as well as cooling tests from $20{ }^{\circ} \mathrm{C}$ to $0{ }^{\circ} \mathrm{C}$ and $20{ }^{\circ} \mathrm{C}$ to $-20{ }^{\circ} \mathrm{C}$ were running at different rates (shown in Figure 8). The paths of strain increments in the same temperature ranges shown in the diagrams (Figures 4-7) are totally different. At the current level of research, it is difficult to explain the reason for this phenomenon. It may be associated with the evaporation of water from the surface of the material, which would explain the fastest increase in resistance in the first phase of heating. To confirm this theory, further research is needed for different rates of temperature change in various ranges.

The problem of the influence of humidity, including its condensation, also needs to be solved. The physical mechanisms of the sensor's high sensitivity to temperature changes also require clarification. Perhaps it will be necessary to modify the concept of a developed textile sensor, such as by introducing an additional isolating layer that cuts off carbon fibers from moisture.

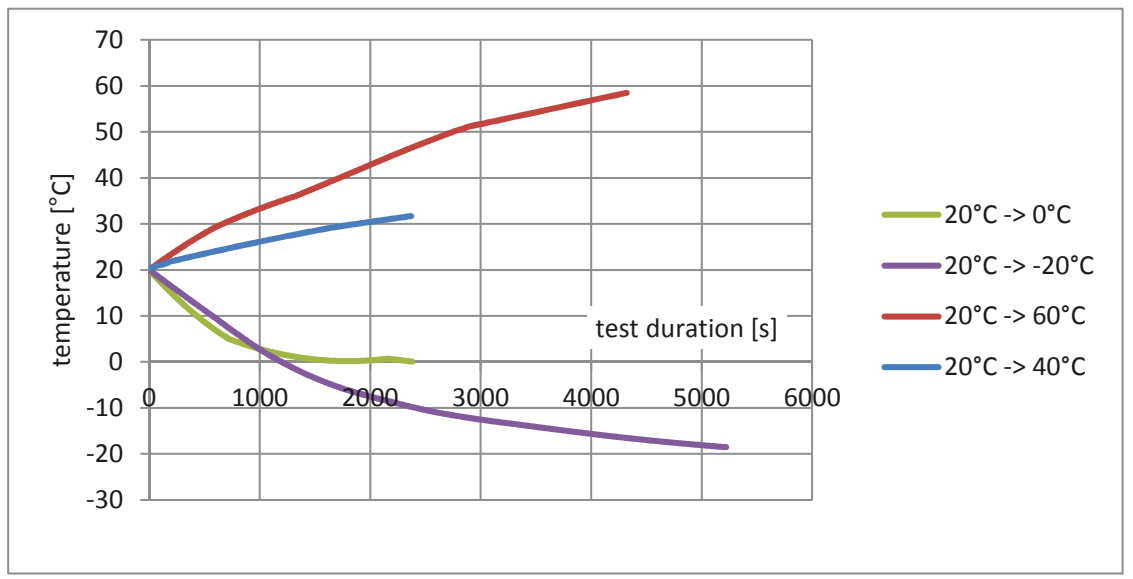

Figure 8. Paths of the temperature change during heating or cooling inside the climate chamber. 


\section{Conclusions}

Previous research on self-monitoring smart textiles made out of carbon fiber [23,24] demonstrated the problem of the effects of the environment on the observed results. Former tests were conducted in stable environmental conditions, while the self-monitoring strengthening system is expected to be built into existing building structures as bridges, which will be affected by various levels of temperature and moisture during their performance.

It is thus crucial to define the relation between the readings of the structure's deformation under the load with changing environmental conditions.

Due to very few studies on this issue, the research team undertook steps to describe this phenomenon and to provide reliable tools for the determination of real strain situations in different temperatures and humidity levels.

The presented tests are only preliminary and do not allow a full determination of the relationship between temperature and resistance change. However, even such limited research shows the importance and complexity of the problem. Resistance changes are not only a function of temperature changes, but also the speed of these changes or the type of substrate. Caused by increasing temperature, an indication error exceeding 5.4 GPa clearly illustrates the importance of the development of measurement methods using carbon fibers, and the development of effective methods to compensate for the changes in temperature.

Authors believe that this research path may be important not only for developed smart strengthening systems, but also for other conductive fiber-based smart structures for various branches of the industry.

Author Contributions: Conceptualization, M.G., R.K. and M.B.; Methodology, M.G. and R.K.; Validation, M.G., and R.K.; Investigation, M.G., R.K. and M.B.; Resources, R.K.; Writing-Original Draft Preparation, R.K. and M.G.; Editing, M.G. and R.K.; Project Administration, M.G.

Funding: This research was funded by Silesian University of Technology grant number BK235/RB6/2017.

Acknowledgments: The authors acknowledge the European Union for financing the development of intelligent textile sensor (Project 251373 FP7-PEOPLE-2009-IAPP-Intelligent Systems for Structures Strengthening and Monitoring INSYSM) and by the Polish Ministry of Science and Higher Education (Grant No. BK-235/RB6/2017 and BK-237/RB6/2018). Authors acknowledge also FISIPE Synthetic Fiber SA, Portugal and S\&P Reinforcement Poland for the supply of carbon fibers.

Conflicts of Interest: The authors declare no conflict of interest. The funders had no role in the design of the study; in the collection, analyses, or interpretation of data; in the writing of the manuscript, and in the decision to publish the results.

\section{References}

1. Conor, P.C.; Owston, C.N. Electrical resistance of single carbon fibres. Nature 1969, 223, 1146-1147. [CrossRef]

2. Owston, C.N. Electrical properties of single carbon fibres. J. Phys. D Appl. Phys. 1970, 3, 1615-1626. [CrossRef]

3. Sebastian, J.; Schehl, N.; Bouchard, M.; Boehle, M.; Li, L.; Lafdi, K. Health monitoring of structural composites with embedded carbon nanotube coated glass fiber sensors. Carbon 2014, 66, 191-200. [CrossRef]

4. Pecora, A.; Maiolo, L.; Minotti, A.; De Francesco, R.; De Francesco, E.; Leccese, F.; Cagnetti, M.; Ferrone, A. Strain gauge sensors based on thermoplastic nanocomposite for monitoring inflatable structures. In Proceedings of the IEEE International Workshop on Metrology for Aerospace, MetroAeroSpace, Benevento, Italy, 29-30 May 2014; pp. 84-88.

5. Goffredo, R.; Ferrone, A.; Maiolo, L.; Pecora, A.; Accoto, D. A miniaturized electrolytic pump sensorized with a strain gauge based on thermoplastic nanocomposite for drug delivery systems. In Proceedings of the 37th Annual International Conference of the IEEE Engineering in Medicine and Biology Society (EMBC), Milan, Italy, 25-29 August 2015; IEEE: Piscataway, NJ, USA, 2015; pp. 3205-3208. 
6. Choi, G.; Lee, J.W.; Cha, J.Y.; Kim, Y.J.; Choi, Y.S.; Schulz, M.J.; Moon, C.K.; Lim, K.T.; Kim, S.Y.; Kang, I. A spray-on carbon nanotube artificial neuron strain sensor for composite structural health monitoring. Sensors 2016, 16. [CrossRef] [PubMed]

7. Maita, F.; Bruno, S.A.; Castiello, A.; Ruggeri, M.; Pecora, A.; Maiolo, L. Integrated steering wheel system based on nanostructured elastomeric sensors for real time detection of driver drowsiness status. In Proceedings of the 2017 IEEE Sensors, Glasgow, UK, 29 October-1 November 2017; IEEE: Piscataway, NJ, USA, 2017; pp. 1-3.

8. Schulte, K.; Baron, C. Load and failure analyses of CFRP laminates by means of electrical resistivity measurements. Compos. Sci. Technol. 1989, 36, 63-76. [CrossRef]

9. Kaddour, A.S.; Al-Salehi, F.A.R.; Al-Hassani, S.T.S.; Hinton, M.J. Electrical resistance measurement technique for detecting failure in CFRP materials at high strain rates. Compos. Sci. Technol. 1994, 51, 377-385. [CrossRef]

10. Wang, X.; Chung, D.D.L. Continuous carbon fibre epoxy-matrix composite as a sensor of its own strain. Smart Mater. Struct. 1996, 5, 796-800. [CrossRef]

11. Park, J.B.; Okabe, T.; Takeda, N.; Curtin, W.A. Electromechanical modelling of unidirectional CFRP composites under tensile loading condition. Compos. Part A Appl. Sci. Manuf. 2002, 33, 267-275. [CrossRef]

12. Angelidis, N.; Wei, C.Y.; Irving, P.E. The electrical resistance response of continuous carbon fibre composite laminates to mechanical strain. Compos. Part A Appl. Sci. Manuf. 2004, 35, 1135-1147. [CrossRef]

13. Todoroki, A.; Samejima, Y.; Yoshiyasu Hirano, Y.; Matsuzaki, R. Piezoresistivity of unidirectional carbon/epoxy composites for multiaxial loading. Compos. Sci. Technol. 2009, 69, 1841-1846. [CrossRef]

14. Wen, J.; Xia, Z.; Choy, F. Damage detection of carbon fiber reinforced polymer composites via electrical resistance measurement. Compos. Part B Eng. 2011, 42, 77-86. [CrossRef]

15. Yeh, F.Y.; Chang, K.C.; Liao, W.C. Experimental investigation of self-sensing carbon fiber reinforced cementitious composite for strain measurement of an RC portal frame. Int. J. Distrib. Sens. Netw. 2015, 11, 531069. [CrossRef]

16. Goldfeld, Y.; Ben-Aarosh, S.; Rabinovitch, O.; Quadflieg, T.; Gries, T. Integrated self-monitoring of carbon based textile reinforced concrete beams under repeated loading in the un-cracked region. Carbon 2016, 98 , 238-249. [CrossRef]

17. Yao, X.; Luan, C.; Zhang, D.; Lan, L.; Fu, J. Evaluation of carbon fiber-embedded 3D printed structures for strengthening and structural-health monitoring. Mater. Des. 2017, 114, 424-432. [CrossRef]

18. Luan, C.; Yao, X.; Shen, H.; Fu, J. Self-sensing of position-related loads in continuous carbon fibers-embedded 3d-printed polymer structures using electrical resistance measurement. Sensors 2018, 18. [CrossRef] [PubMed]

19. Wang, S.; Chung, D.D.L. Temperature/light sensing using carbon fiber polymer-matrix composite. Compos. Part B Eng. 1999, 30, 591-601. [CrossRef]

20. Zymelka, D.; Yamashita, T.; Takamatsu, S.; Itoh, T.; Kobayashi, T. Printed strain sensor with temperature compensation and its evaluation with an example of applications in structural health monitoring. Jpn. J. Appl. Phys. 2017, 56, 05EC02. [CrossRef]

21. Wheatstone, C. An account of several new instruments and processes for determining the constants of a voltaic circuit. Phils. Trans. R. Soc. Lond. 1843, 4, 469-471.

22. Stein, P. Strain Gage history and the end of the twentieth century. Exp. Tech. 2001, 25, 15-16. [CrossRef]

23. Salvado, R.; Lopes, K.; Szojda, L.; Araujo, P.; Gorski, M.; Jose Velez, F.; Castro Gomes, J.; Krzywon, R. Carbon fiber epoxy composites for both strengthening and health monitoring of structures. Sensors 2015, 15, 10753-10770. [CrossRef] [PubMed]

24. Krzywon, R.; Gorski, M.; Dawczynski, S.; Szojda, L.; Castro Gomes, J.; Salvado, R. Self-monitoring strengthening system based on carbon fiber laminate. J. Sens. 2016, 2016. [CrossRef]

(C) 2018 by the authors. Licensee MDPI, Basel, Switzerland. This article is an open access article distributed under the terms and conditions of the Creative Commons Attribution (CC BY) license (http:/ / creativecommons.org/licenses/by/4.0/). 
Article

\title{
Poly(hydroxyalkanoate)s-Based Hydrophobic Coatings for the Protection of Stone in Cultural Heritage
}

\author{
Serena Andreotti ${ }^{1,2}$, Elisa Franzoni ${ }^{1,2, *}$, Micaela Degli Esposti ${ }^{1,2}$ and Paola Fabbri ${ }^{1,2}$ \\ 1 Department of Civil, Chemical, Environmental and Materials Engineering, University of Bologna, \\ 40131 Bologna, Italy; serena.andreotti2@unibo.it (S.A.); micaela.degliesposti@unibo.it (M.D.E.); \\ p.fabbri@unibo.it (P.F.) \\ 2 Consorzio Interuniversitario di Scienza e Tecnologia dei Materiali (INSTM), 50121 Firenze, Italy \\ * Correspondence: elisa.franzoni@unibo.it; Tel.: +39-051-209-0339
}

Received: 4 December 2017; Accepted: 17 January 2018; Published: 20 January 2018

\begin{abstract}
Reversibility is a mandatory requirement for materials used in heritage conservation, including hydrophobic protectives. Nevertheless, current protectives for stone are not actually reversible as they remain on the surfaces for a long time after their hydrophobicity is lost and can hardly be removed. Ineffective and aged coatings may jeopardise the stone re-treatability and further conservation interventions. This paper aims at investigating the performance of PHAs-based coatings for stone protection, their main potential being the 'reversibility by biodegradation' once water repellency ended. The biopolymer coatings were applied to three different kinds of stone, representative of lithotypes used in historic architecture: sandstone, limestone and marble. Spray, poultice and dip-coating were tested as coating techniques. The effectiveness and compatibility of the protectives were evaluated in terms of capillary water absorption, static and dynamic contact angles, water vapour diffusion, colour alteration and surface morphology. The stones' wettability after application of two commercial protectives was investigated too, for comparison. Finally, samples were subjected to artificial ageing to investigate their solar light stability. Promising results in terms of efficacy and compatibility were obtained, although the PHAs-based formulations developed here still need improvement for increased durability and on-site applicability.
\end{abstract}

Keywords: protectives; water repellency; bio-based biodegradable polymers; conservation; PHA; marble; limestone; sandstone

\section{Introduction}

The protection of architectural elements against water is one of the main challenges for the conservation of cultural heritage, as water is a major cause of material degradation, which may be physical, mechanical or chemical [1-8]. In this context, water may have different origins, such as rain, relative humidity (condensation), or capillary rise from soil. Protection requires an accurate analysis of the water source and paths and a subsequent design of drain systems to control the water run-off from the top to the bottom of the construction. However, this is often not possible or not sufficient in heritage buildings, due to several existing restraints. For this reason, an approach combining chemical and physical protection is often needed to mitigate the problem, and the application of hydrophobic coatings over the exposed surface, preventing water penetration through the material porosity without hindering water vapour transport, has been demonstrated to be an effective solution [4,9-11].

The most common organic-based hydrophobic protectives that are currently available, such as silanes and siloxanes, waxes, acrylic resins or fluorinated polymers, have shown some limitations [5], such as short-term water repellency [12] due to polymer ageing [1], or undesired effects such as 
yellowing or coating detachment (due to poor compatibility with inorganic substrates $[3,4,13]$ ). Many studies were targeted to improve organic-based formulations by means of inorganic nanoparticle addition $[3,4,10,13-15]$, for increasing the coating hydrophobicity $[3,4,10,14]$ or providing the surface with self-cleaning properties [10,15-17]. A different approach, based on inorganic treatments, was proposed by other authors $[12,13,18]$, mostly for the protection of calcitic materials from acid or clean rain, hence specifically targeted to preventing stone dissolution rather than hindering water ingress into stone. These treatments provide good compatibility and durability [14], but may exhibit limited penetration into the substrate [3] or the formation of cracks, which limit their protective performance [13].

However, in the development of protective formulations, besides achieving good performance, the requirements imposed by the Restoration Charters $[19,20]$ must be fulfilled. In fact, any protective coating should be not only effective, but also compatible with the substrate (without altering the water vapour permeability and aesthetic appearance-such as colour and reflectance-too much, or causing unwanted damage) and durable, but also reversible, i.e., removable at some future date, should that prove necessary $[3,8,9,21]$. However, in the context of stone conservation, reversibility is "more idealistic than realistic" [21], as even the most soluble of treatments can be extremely difficult to remove. For this reason, any treatment is required to be at least retreatable. Reversibility and retreatability are becoming more and more crucial in conservation, because the number of interventions in buildings already restored in the past is increasing. Notably, incompatible or simply ineffective protectives that cannot be removed from the substrate may jeopardise the subsequent repair treatments. Organic protectives, although they were considered reversible in the 1970s and 1980s by means of suitable solvent application, are actually very hard to remove after decades of exposure to outdoor conditions, which irreversibly altered their composition and properties [22,23]. Hence, after losing their water repellency effectiveness (after 5-10 years according to some authors [24,25]), these protectives remain on the surface of building materials for a long time, subject to continuous ageing. As a result, the dictum of reversibility and retreatability of water repellents represents a great challenge for the scientific community, as it is necessary to ensure that there are no unforeseen consequences of multiple applications of maintenance coatings [21].

In recent years, biopolymers have attracted interest as protective materials for stones [26,27], due to their potential compliance with the reversibility/retreatability requirement. Although biodegradable polymers cannot be properly defined as 'reversible' (they do not exhibit any improved solubility with respect to current protectives) or 'retreatable' (they do not exhibit any particular compatibility with new coatings of a different nature), they are expected to completely disappear from the stone once their water repellency action has ceased, without jeopardising or influencing further treatments. For this reason, biopolymers might be considered 'intrinsically reversible', as they do not leave any permanent residue in the stone and do not cause any unforeseen consequences in subsequent conservation work.

So far, zein, chitosan, poly(3-hydroxybutyrate) (PHB) and poly(lactic acid) (PLA) have been tested as marble coatings against sulphation [25], although the results are only preliminary. PLA has been studied in association with fluorine, by means of the synthesis of fluorinated PLA copolymers $[27,28]$ with nanoparticles [29] or with both fluorine and nanoparticles [30] to enhance the water repellency of the coatings, but the evaluation of their effectiveness is still at a preliminary stage.

Poly(hydroxyalkanoate)s (PHAs) are a class of naturally occurring thermoplastic linear polyesters that are synthesised as high molecular weight polymer chains by several species of bacterial strains [31-34], fed with renewable carbon sources such as sugars and agricultural wastes. PHAs have been well known since the beginning of the last century, but only recently have their peculiar features been taken into consideration for the development of functional and advanced solutions for different fields. At present, PHAs can be used in many applications such as medical implant materials [33,34], drug delivery carriers [31,34], packaging [31,33,34], moulded goods [33], paper coatings [33] and non-woven fabrics [33]. Poly(3-hydroxybutyrate) (PHB) is the most widespread polymer in the PHAs class. It is a highly crystalline linear homopolymer with chemical structure 
$-\left[\mathrm{O}-\mathrm{CH}\left(\mathrm{CH}_{3}\right)-\mathrm{CH}_{2}-(\mathrm{C}=\mathrm{O})\right]_{n}-$. The physical and mechanical properties of PHB are similar to those of polypropylene [30,32,34], even if PHB is less ductile [31,33,35].

Poly(3-hydroxybutyrate-co-3-hydroxyvalerate-co-4-hydroxyvalerate) (PHBVV) is a statistical copolymer composed of 3-hydroxybutyrate, 3-hydroxyvalerate and 4-hydroxyvalerate repetitive units. Due to its structure and to the presence of hydroxyvalerate moieties, PHBVV is less crystalline, more flexible and tougher than PHB.

The application of PHAs for the protection of stones in cultural heritage is strongly supported by several peculiarities of these biopolymers: intrinsic hydrophobic nature, which avoids the impellent need to include fluorine and inorganic nanofillers in the protective coating; low acidity, which avoids unfavourable interactions with the stone surface; and biodegradability in environmental conditions, which provides the surface treatment with an intrinsic reversibility (after tailoring the surface treatment duration) once the water-repellent action finishes.

On this basis, the present research is aimed at developing formulations based on PHAs to be used as protective treatments and evaluating their performance on three different types of stones, namely limestone, sandstone and marble (Figure 1). These stones were selected in order to investigate the protective effectiveness of the treatments on substrates differing in colour, microstructure and chemical composition. PHB and PHBVV solutions were coated onto the stones by different techniques (i.e., spray, poultice and dip coating), as the application method is known to have a strong effect on the amount of material penetrating in the stone or deposited on the surface and in order to assess the influence of the application method onto the overall performance of the treatments. Spray represents the most widely used method for the application of protectives on-site and poultice is an application method commonly used in conservation practice, for both cleaning and consolidation; dip coating, despite being basically not applicable on-site, was adopted to produce a uniform deposition of the protectives on the sample's surface, hence to investigate the behaviour of the treatment in 'ideal' application conditions.

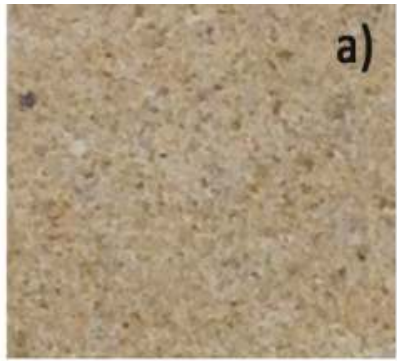

sandstone

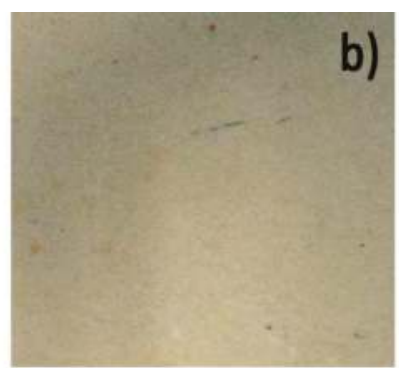

limestone

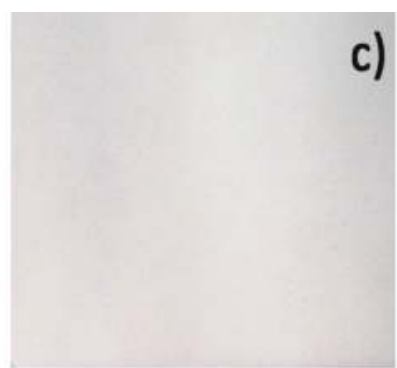

marble

Figure 1. Substrates selected: (a) sandstone (Siena stone); (b) limestone (Lecce stone); (c) marble (Carrara marble).

The performance and compatibility of the protective hydrophobic treatments were investigated in terms of capillary water absorption, static and dynamic contact angles, surface tension, water vapour diffusion, colour alteration and surface morphology.

The stone wettability after the application of the biopolymers was compared with that achieved using two commercial water-repellents widely used for stone conservation, i.e., a silane and siloxane solution (labelled 'Sol-SIL') and a mixture of silane and siloxane emulsified in water (labelled 'Emul-SIL'). 


\section{Results}

\subsection{Stone Characterisation}

Porosity was determined in untreated and coated stones because it plays a major role in all the degradation phenomena that are related to water absorption and also in the effectiveness of protective treatments. The results of mercury intrusion porosimetry (MIP) analysis of the stones are reported in Figure 2. Sandstone, limestone and marble exhibit very different microstructures in terms of total open porosity $(O P)$, mean pore radius $\left(r_{\mathrm{a}}\right)$ and pore size distribution. Limestone is characterised by the highest porosity $(O P=37.7 \%)$, the broadest pore size distribution (significant amount of pores can be noticed between $0.1 \mu \mathrm{m}$ and $4 \mu \mathrm{m}$ ) and an average pore radius equal to $2.2 \mu \mathrm{m}$. Sandstone exhibits a medium-high porosity $(O P=18.6 \%)$, mean pore radius $3.3 \mu \mathrm{m}$ and most of the pores are in the radius range $1-5 \mu \mathrm{m}$. Finally, marble exhibits the lowest porosity $(O P=2.3 \%)$ and the largest mean pore radius $\left(r_{\mathrm{a}}=6.9 \mu \mathrm{m}\right)$.

The high porosity of sandstone and limestone, together with their pore size mostly in the range 0.1-10 $\mu \mathrm{m}$, makes them vulnerable to salt and ice deterioration [36], both of them made possible by the presence of moisture in the stone, hence the need for protecting these stones by water-repellents arises, in order to avoid the stone powdering, crumbling and flaking that cause a significant loss of heritage material.

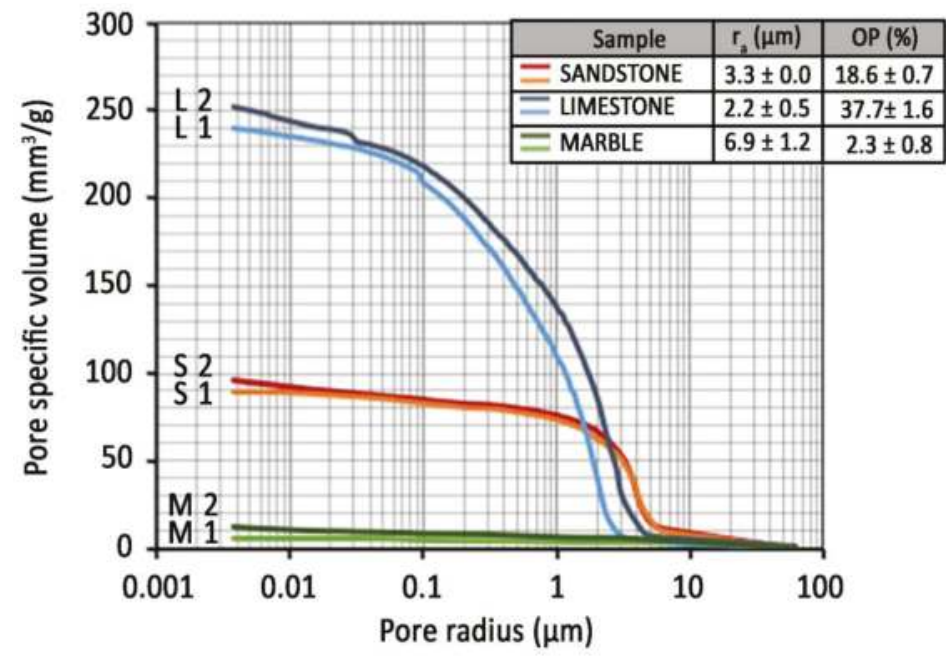

Figure 2. Pore size distribution curves of two samples of sandstone, limestone and marble, obtained by MIP. Open porosity $(O P)$ and average pore radius $\left(r_{\mathrm{a}}\right)$ in the table were averaged for two samples (L1 and L2 indicate the two limestone samples, S1 and S2 the two sandstone samples, M1 and M2 the two marble samples).

The results of XRD (X-ray Diffraction) analysis and the calcite and dolomite percentages, determined by the Dietrich-Frühling method, are reported in Table 1.

The three stones are mainly composed of calcite: $88 \%$ in sandstone, $86 \%$ in limestone and $98 \%$ in marble, with the latter also containing $2 \%$ dolomite. Furthermore, sandstone contains quartz, while in limestone traces of fluoroapatite were detected. The mostly calcitic composition of these stones makes them susceptible to chemical attack in polluted atmospheres, with consequent formation of black crusts at the expense of the original materials-hence, again, the opportunity for protecting these stones from water (whose presence boosts chemical attack) arises. 
Table 1. Results of XRD analysis (+++: dominantly present, ++: present, + : traces) and calcite and dolomite content (\%) measured by the Dietrich-Frühling gas volumetric method.

\begin{tabular}{ccccccc}
\hline \multirow{2}{*}{ Substrate } & \multicolumn{3}{c}{ XRD } & \multicolumn{3}{c}{ Dietrich-Frühling Method } \\
\cline { 2 - 7 } & Calcite & Quartz & Fluorapatite & Dolomite & Calcite (\%) & Dolomite (\%) \\
\hline sandstone & +++ & ++ & - & - & 88 & - \\
limestone & +++ & - & + & - & 86 & - \\
marble & +++ & - & - & + & 98 & 2 \\
\hline
\end{tabular}

\subsection{Hydrophobicity of the Coated Stones}

\subsubsection{Water Absorption by Capillarity}

The ability of a treatment to reduce the capillary water absorption of stone represents the main goal of any protective; hence, this test can be considered one of the most significant parameters for predicting the real on-site performance of the treatment. The results are presented in the following using the labelling code reported in Table 2.

The water absorption curves of sandstone treated with PHB and PHBVV are reported in Figure 3, together with the curves of the untreated samples (label "UT-"). The slope of the first part of the curve, which is approximately linear, represents the so-called 'sorptivity' (capillary absorption rate), while the horizontal part indicates that sample saturation has occurred. The time for the calculation of the ratio of protection $(R p)$ was set at $1 \mathrm{~h}$ and at $48 \mathrm{~h}$, as explained in Section 4.5.1, and the results are reported in Table 3. In fact, after $1 \mathrm{~h}$ the slope of the first part of the curves notably decreases even if the untreated samples of sandstone do not reach a plateau, but continue to absorb water even after $24 \mathrm{~h}$. The fast absorption of water by sandstone is consistent with its pore size distribution shown in Figure 2. PHB shows a good protective performance, as its $R p$ moves from $87 \%$ to $100 \%$ after $1 \mathrm{~h}$ in contact with water and from $68 \%$ to $91 \%$ after $48 \mathrm{~h}$. PHBVV shows an even better performance, strongly reducing the absorption of water during all the $48 \mathrm{~h}(R p \sim 90 \%)$. In both cases, the poultice application gives the best results, probably due to the higher quantity of biopolymer retained on the stone with this method.

Table 2. Sample codes.

\begin{tabular}{ccccc}
\hline Stone & Polymer & Dip Coating & Poultice Coating & Spray Coating \\
\hline \multirow{5}{*}{ sandstone } & PHB & D-SANDs-PHB & P-SANDs-PHB & S-SANDs-PHB \\
& PHBVV & D-SANDs-PHBVV & P-SANDs-PHBVV & S-SANDs-PHBVV \\
& Sol-SIL & D-SANDs-Sol-SIL & P-SANDs-Sol-SIL & S-SANDs-Sol-SIL \\
& Emul-SIL & D-SANDs-Emul-SIL & P-SANDs-Emul-SIL & S-SANDs-Emul-SIL \\
\hline \multirow{5}{*}{ limestone } & PHB & D-LIMEs-PHB & P-LIMEs-PHB & S-LIMEs-PHB \\
& PHBVV & D-LIMEs-PHBVV & P-LIMEs-PHBVV & S-LIMEs-PHBVV \\
& Sol-SIL & D-LIMEs-Sol-SIL & P-LIMEs-Sol-SIL & S-LIMEs-Sol-SIL \\
& Emul-SIL & D-LIMEs-Emul-SIL & P-LIMEs-Emul-SIL & S-LIMEs-Emul-SIL \\
\hline \multirow{5}{*}{ marble } & PHB & D-MARBLE-PHB & P-MARBLE-PHB & S-MARBLE-PHB \\
& PHBVV & D-MARBLE-PHBVV & P-MARBLE-PHBVV & S-MARBLE-PHBVV \\
& Sol-SIL & D-MARBLE-Sol-SIL & P-MARBLE-Sol-SIL & S-MARBLE-Sol-SIL \\
& Emul-SIL & D-MARBLE-Emul-SIL & P-MARBLE-Emul-SIL & S-MARBLE-Emul-SIL \\
\hline
\end{tabular}

The water absorption curves and ratio of protection values $(R p)$ of the commercial protectives are reported in Figure 4 and Table 3, respectively. There are no significant differences between the performance of Sol-SIL and Emul-SIL, as they contain similar polymeric compounds. Both products show high protection ( $R p$ varying from $98 \%$ to $90 \%$ after $1 \mathrm{~h}$ and from $89 \%$ to $97 \%$ after $48 \mathrm{~h}$ ). The application of the products by dip coating seems to increase their efficacy; in particular, Emul-SIL applied by dip coating can reach a $97 \%$ protection ratio after $48 \mathrm{~h}$ of testing. The performance of the 
PHBVV solution, regardless the application method, and the PHB solution applied by poultice, is comparable to that obtained by the two commercial products.

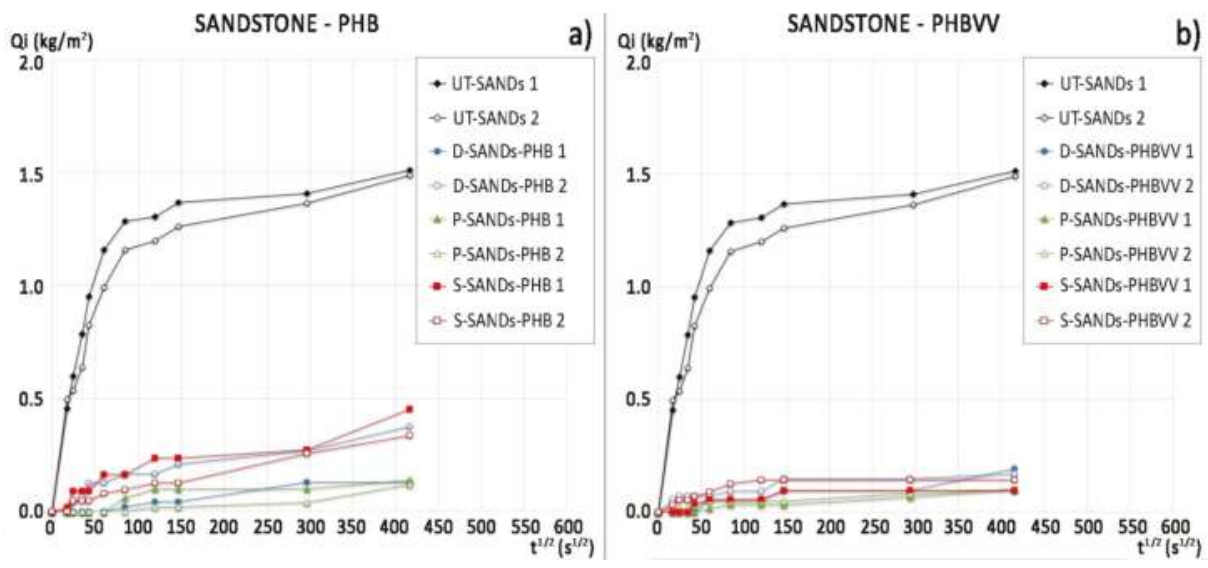

Figure 3. Water absorption curves of sandstone samples treated with: (a) PHB-based formulation by dip coating, poultice and spray and of untreated samples (duplicate samples for each condition); (b) PHBVV-based formulation by dip coating, poultice and spray and of untreated samples (duplicate samples for each condition).

Table 3. Determination of the mean ratio of protection $(R p, \%)$ for treated samples of sandstone after $1 \mathrm{~h}$ and after $48 \mathrm{~h}$ of capillary absorption test.

\begin{tabular}{|c|c|c|c|c|c|c|c|c|}
\hline \multirow{3}{*}{$\begin{array}{l}\text { Application } \\
\text { Method }\end{array}$} & \multicolumn{8}{|c|}{ Sandstone } \\
\hline & \multicolumn{2}{|c|}{ РНB } & \multicolumn{2}{|c|}{ PHBVV } & \multicolumn{2}{|c|}{ Sol-SIL } & \multicolumn{2}{|c|}{ Emul-SIL } \\
\hline & $\begin{array}{c}\text { Rp (\%) } \\
\text { after } 1 \mathrm{~h}\end{array}$ & $\begin{array}{c}\operatorname{Rp}(\%) \\
\text { after } 48 \mathrm{~h}\end{array}$ & $\begin{array}{c}\text { Rp (\%) } \\
\text { after } 1 \mathrm{~h}\end{array}$ & $\begin{array}{c}\text { Rp }(\%) \\
\text { after } 48 \mathrm{~h}\end{array}$ & $\begin{array}{c}\text { Rp (\%) } \\
\text { after } 1 \mathrm{~h}\end{array}$ & $\begin{array}{c}\operatorname{Rp}(\%) \\
\text { after } 48 \mathrm{~h}\end{array}$ & $\begin{array}{c}\text { Rp (\%) } \\
\text { after } 1 \mathrm{~h}\end{array}$ & $\begin{array}{c}\text { Rp (\%) } \\
\text { after } 48 \mathrm{~h}\end{array}$ \\
\hline dip coating & 94 & 84 & 92 & 86 & 98 & 92 & 96 & 97 \\
\hline poultice & 100 & 90 & 96 & 92 & n.a. & n.a. & n.a. & n.a. \\
\hline spray & 85 & 75 & 92 & 90 & 90 & 89 & 91 & 90 \\
\hline
\end{tabular}

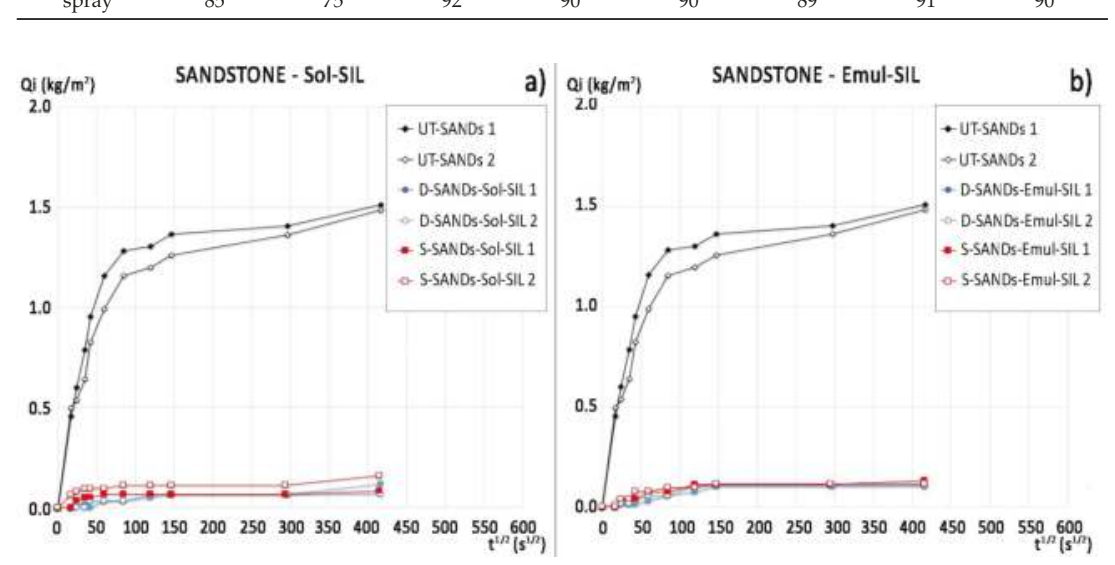

Figure 4. Water absorption curves of sandstone samples treated with: (a) Sol-SIL by dip coating and spray and of untreated samples (replicate samples for each condition); (b) Emul-SIL by dip coating and spray and of untreated samples (replicate samples for each condition). 
Figure 5 shows the water absorption curves of limestone samples, while the mean ratio of protection values, calculated at $30 \mathrm{~min}$ (where the slope of the untreated curves suddenly changes) and at $48 \mathrm{~h}$ for all the samples, are reported in Table 4 . Limestone shows a relatively high water absorption (final water uptake $\sim 305 \mathrm{~kg} / \mathrm{m}^{3}$, Figure 5), compared to sandstone (final water uptake $\sim 93 \mathrm{~kg} / \mathrm{m}^{3}$, Figure 3), in consequence of the significantly higher open porosity.

In this case, the performance of PHB-based protectives is significantly different from that of PHBVV-based. While PHBVV shows excellent capacity to reduce the water absorption regardless the application method used, PHB does not provide significant protection ( $R p=0 \%$ for PHB applied by dip coating) or protects only in the short term: $R p$ is equal to $98 \%$ and $68 \%$ after $30 \mathrm{~min}$ for PHB applied by poultice or spraying, respectively, but $R p$ is equal to only $43 \%$ and $28 \%$ after $48 \mathrm{~h}$. The higher effectiveness of the spray application with respect to the dip coating seems due to its more abundant deposition on the surface rather than deeper penetration into the sample. The performance of the two commercial protective products in limestone seems independent from the application method used, as shown by the water absorption curves in Figure 6 and the $R p$ values in Table 4 . The protection provided by Sol-SIL is higher than the one given by Emul-SIL, as Emul-SIL strongly reduces the sorptivity in the first $6 \mathrm{~h}$ but then its efficacy decreases, while Sol-SIL provides the same protective performance until the end of the test ( $R p$ equal to $95 \%$ after $48 \mathrm{~h}$ ). The performance of PHBVV solution applied by dip coating and poultice is comparable to that provided by Sol-SIL.

Capillary absorption test was not performed on marble samples, as their extremely low porosity causes insignificant water absorption, even for the untreated samples.

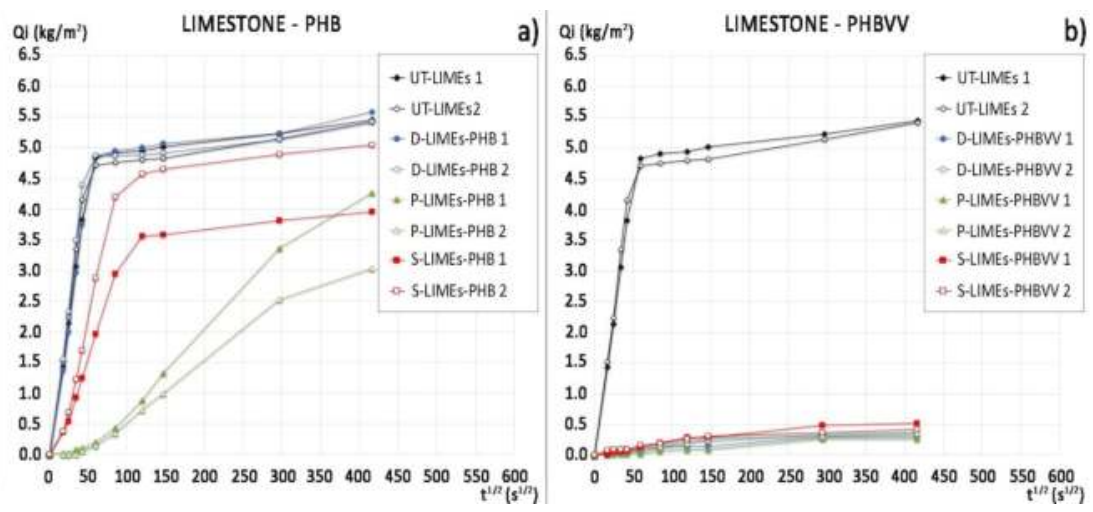

Figure 5. Water absorption curves of limestone samples treated with: (a) PHB-based formulation by dip coating, poultice and spray and of untreated samples (duplicate samples for each condition); (b) PHBVV-based formulation by dip coating, poultice and spray and of untreated samples (duplicate samples for each condition).

Table 4. Determination of the mean ratio of protection (Rp, \%) for treated samples of sandstone after $1 \mathrm{~h}$ and after $48 \mathrm{~h}$ of capillary absorption test.

\begin{tabular}{|c|c|c|c|c|c|c|c|c|}
\hline \multirow{3}{*}{$\begin{array}{l}\text { Application } \\
\text { Method }\end{array}$} & \multicolumn{8}{|c|}{ Limestone } \\
\hline & \multicolumn{2}{|c|}{ РHB } & \multicolumn{2}{|c|}{ PHBVV } & \multicolumn{2}{|c|}{ Sol-SIL } & \multicolumn{2}{|c|}{ Emul-SIL } \\
\hline & $\begin{array}{c}\mathrm{Rp}(\%) \\
\text { after } 1 \mathrm{~h}\end{array}$ & $\begin{array}{c}\text { Rp }(\%) \\
\text { after } 48 \mathrm{~h}\end{array}$ & $\begin{array}{c}\text { Rp (\%) } \\
\text { after } 1 \mathrm{~h}\end{array}$ & $\begin{array}{c}\operatorname{Rp}(\%) \\
\text { after } 48 \mathrm{~h}\end{array}$ & $\begin{array}{c}\text { Rp (\%) } \\
\text { after } 1 \mathrm{~h}\end{array}$ & $\begin{array}{c}\text { Rp }(\%) \\
\text { after } 48 \mathrm{~h}\end{array}$ & $\begin{array}{c}\mathrm{Rp}(\%) \\
\text { after } 1 \mathrm{~h}\end{array}$ & $\begin{array}{c}\operatorname{Rp}(\%) \\
\text { after } 48 \mathrm{~h}\end{array}$ \\
\hline dip coating & 0 & 0 & 99 & 96 & 99 & 95 & 99 & 87 \\
\hline poultice & 98 & 43 & 98 & 94 & n.a. & n.a. & n.a. & n.a. \\
\hline spray & 68 & 28 & 76 & 91 & 98 & 95 & 98 & 88 \\
\hline
\end{tabular}




\subsubsection{Contact Angle Measurements}

The hydrophobicity induced on the stone surface was evaluated by means of both static and dynamic contact angle measurement, to obtain a reliable evaluation of samples water wettability. The contact angles of PHB and PHBVV alone had been previously determined on glass slides immersed in the polymer solution and let to evaporate (solvent casting), obtaining the following values:

- $\quad$ PHB: static contact angle $88^{\circ} \pm 1^{\circ}$; dynamic contact angle (advancing) $90^{\circ} \pm 1^{\circ}$; dynamic contact angle (receding) $56^{\circ} \pm 3^{\circ}$;

- $\quad$ PHBVV: static contact angle $88^{\circ} \pm 1^{\circ}$; dynamic contact angle (advancing) $92^{\circ} \pm 1^{\circ}$; dynamic contact angle (receding) $63^{\circ} \pm 2^{\circ}$.

Tables 5-7 report the contact angle values for sandstone, limestone and marble, respectively. Static contact angle values measured on the untreated samples show huge differences in the three substrates: marble exhibits the highest contact angle $\left(\theta=41^{\circ} \pm 7^{\circ}\right)$, followed by sandstone $\left(\theta=15^{\circ} \pm 4^{\circ}\right)$ and limestone, for which an immediate and complete absorption of the drop occurs $\left(\theta=0^{\circ} \pm 0^{\circ}\right)$. These differences are related to both the chemical composition of the three stones and their surface roughness and porosity. It is actually not straightforward to characterise non-ideal solid surfaces (i.e., chemically heterogeneous and porous) through static contact angle measurements, because on such surfaces the only measurable value is the apparent contact angle, which can be largely different from the ideal contact angle $[2,9,37,38]$. However, for the purposes of this study, the effects of porosity and chemical non-homogeneity on the contact angle were not addressed in detail.

Sandstone treated with PHB exhibits static contact angles slightly above $90^{\circ}$, which is considered the borderline value between a hydrophobic $\left(\theta>90^{\circ}\right)$ and a hydrophilic behaviour $\left(\theta<90^{\circ}\right)$, hence its performance is satisfactory even if not outstanding. Conversely, PHBVV-based protective shows a static contact angle between $90^{\circ}$ and $125^{\circ}$ (Table 5), hence markedly hydrophobic behaviour. The best improvement was given by PHBVV applied by poultice, but in all the other samples treated by PHB and PHBVV the application method was not found to play a key role. Sol-SIL induces the highest hydrophobicity $\left(\theta=140^{\circ}\right)$, while the performance of Emul-SIL is comparable to that of PHBVV. Standard deviation values are higher for PHA formulations than for the two commercial products, suggesting that the latter more homogeneously distribute on the stone's surface.
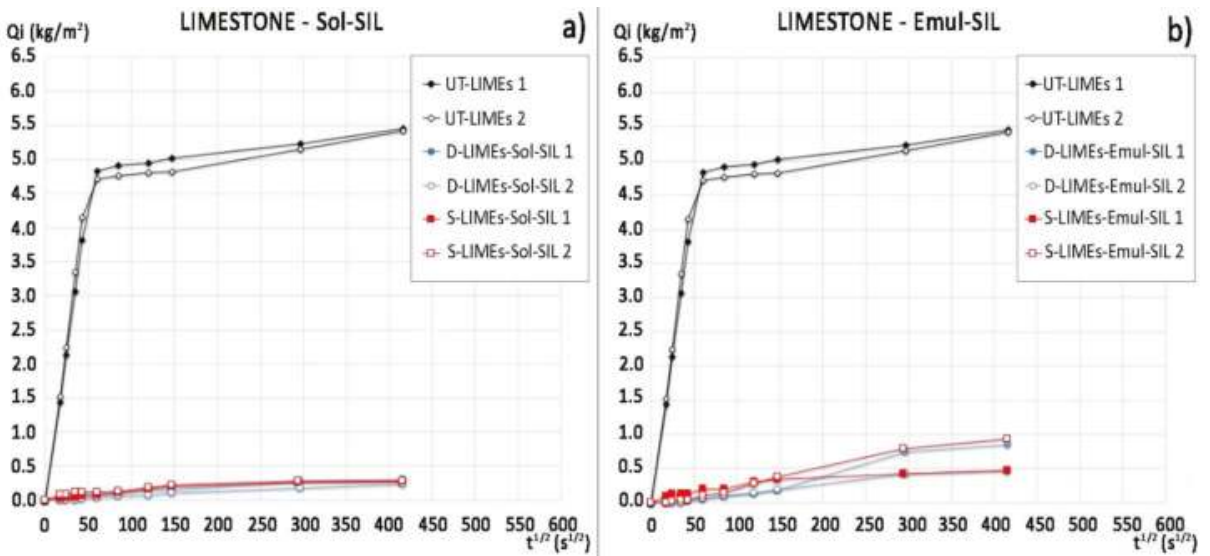

Figure 6. Water absorption curves of limestone samples treated with: (a) Sol-SIL by dip coating and spray and of untreated samples (replicate samples for each condition); (b) Emul-SIL by dip coating and spray and of untreated samples (replicate samples for each condition). 
Table 5. Static contact angle $(\theta)$ and dynamic contact angles referred to the first immersion cycle $\left(\theta_{\text {adv } 1}\right.$, $\left.\theta_{\text {rec1 }}\right)$ for untreated and treated sandstone.

\begin{tabular}{cccc}
\hline \multirow{2}{*}{ SAMPLE } & Static Contact Angle & \multicolumn{2}{c}{ Dynamic Contact Angle } \\
\cline { 2 - 4 } & $\boldsymbol{\theta}\left(^{\circ}\right)$ & $\boldsymbol{\theta}_{\text {adv1 }}\left(^{\circ}\right)$ & $\boldsymbol{\theta}_{\text {rec1 }}\left(^{\circ}\right)$ \\
\hline UT-SANDs & $15 \pm 4$ & 40 & 0 \\
D-SANDs-PHB & $95 \pm 8$ & 104 & 0 \\
P-SANDs-PHB & $93 \pm 9$ & 113 & 19 \\
S-SANDs-PHB & $97 \pm 11$ & 102 & 8 \\
D-SANDs-PHBVV & $104 \pm 12$ & 113 & 0 \\
P-SANDs-PHBVV & $123 \pm 9$ & 126 & 0 \\
S-SANDs-PHBVV & $101 \pm 8$ & 107 & 33 \\
D-SANDs-Sol-SIL & $140 \pm 1$ & 146 & 25 \\
S-SANDs-Sol-SIL & $142 \pm 4$ & 134 & 28 \\
D-SANDs-Emul-SIL & $125 \pm 4$ & 132 & 26 \\
S-SANDs-Emul-SIL & $124 \pm 1$ & 138 & \\
\hline
\end{tabular}

Table 6. Static contact angle $(\theta)$ and dynamic contact angles referred to the first immersion cycle ( $\theta_{\text {adv } 1}$, $\theta_{\text {rec1 }}$ ) for untreated and treated limestone.

\begin{tabular}{cccc}
\hline \multirow{2}{*}{ SAMPLE } & Static Contact Angle & \multicolumn{2}{c}{ Dynamic Contact Angle } \\
\cline { 2 - 4 } & $\boldsymbol{\theta}\left(^{\circ}\right)$ & $\boldsymbol{\theta}_{\text {adv1 }}\left(^{\circ}\right)$ & $\boldsymbol{\theta}_{\text {rec1 }}\left(^{\circ}\right)$ \\
\hline UT-LIMEs & $0 \pm 0$ & 16 & 0 \\
D-LIMEs-PHB & $108 \pm 7$ & 119 & 0 \\
P-LIMEs-PHB & $112 \pm 5$ & 110 & 0 \\
S-LIMEs-PHB & $113 \pm 6$ & 124 & 0 \\
D-LIMEs-PHBVV & $119 \pm 4$ & 128 & 26 \\
P-LIMEs-PHBVV & $126 \pm 7$ & 117 & 0 \\
S-LIMEs-PHBVV & $120 \pm 6$ & 122 & 38 \\
D-LIMEs-Sol-SIL & $143 \pm 2$ & 143 & 48 \\
S-LIMEs-Sol-SIL & $146 \pm 1$ & 166 & 0 \\
D-LIMEs-Emul-SIL & $118 \pm 1$ & 150 & 0 \\
S-LIMEs-Emul-SIL & $124 \pm 1$ & 141 & \\
\hline
\end{tabular}

Table 7. Static contact angle $(\theta)$ and dynamic contact angles referred to the first immersion cycle ( $\theta_{\text {adv } 1 \text {, }}$ $\left.\theta_{\text {rec1 }}\right)$ for untreated and treated marble.

\begin{tabular}{cccc}
\hline \multirow{2}{*}{ SAMPLE } & Static Contact Angle & \multicolumn{2}{c}{ Dynamic Contact Angle } \\
\cline { 2 - 4 } & $\boldsymbol{\theta}\left(^{\circ}\right)$ & $\boldsymbol{\theta}_{\text {adv1 }}\left(^{\circ}\right)$ & $\boldsymbol{\theta}_{\text {rec1 }}\left(^{\circ}\right)$ \\
\hline UT-Marble & $41 \pm 7$ & 60 & 19 \\
D-Marble-PHB & $80 \pm 6$ & 78 & 29 \\
P-Marble-PHB & $80 \pm 9$ & 92 & 26 \\
S-Marble-PHB & $79 \pm 6$ & 102 & 8 \\
D-Marble-PHBVV & $84 \pm 4$ & 85 & 30 \\
P-Marble-PHBVV & $109 \pm 10$ & 104 & 24 \\
S-Marble-PHBVV & $84 \pm 4$ & 92 & 41 \\
D-Marble-Sol-SIL & $120 \pm 6$ & 133 & 17 \\
D-Marble-Emul-SIL & $119 \pm 4$ & 108 & 34 \\
\hline
\end{tabular}

For limestone (Table 6) the PHAs formulations produce the highest improvement of static contact angle with respect to sandstone and marble, starting from the condition of complete absorption of the untreated samples $\left(\theta=0^{\circ}\right)$ and reaching values between $110^{\circ}$ and $125^{\circ}$. As for sandstone, Sol-SIL gives the highest values of contact angle $\left(\theta>140^{\circ}\right)$, while the performance of Emul-SIL is comparable to that of PHBVV. Again, standard deviations for PHA formulations exceed those of the two commercial products. 
The static contact angles measured on marble samples treated by PHB and PHBVV are doubled with respect to the untreated stone (Table 7), but they do not reach $90^{\circ}$, hence not showing proper water-repellent behaviour. The only exception is PHBVV applied by poultice $\left(\theta=109^{\circ} \pm 10^{\circ}\right)$. Instead, both Sol-SIL and Emul-SIL make static contact angle reach values around $120^{\circ}$, although their standard deviation is here comparable to those of untreated stone and of stones treated with PHB and PHBVV. This quite high standard deviation can be due to a lower homogeneous coverage of the marble surfaces by means of commercial protective treatments with respect to sandstone and especially limestone, possibly correlated to the low roughness of the marble, which notably reduces the presence of anchorage points useful for coating adhesion and to the full calcitic composition of marble, which does not promote the chemical bonding with the silicon-based protectives.

Tables 5-7 also report the values of advancing contact angles, determined by dynamic measurement. As expected for rough and non-homogenous surfaces, for the untreated stones the values of static and advancing contact angles are quite different. Conversely, the advancing and static contact angles are in fairly good agreement in all samples treated with PHB- and PHBVV-based formulations, being the advancing contact angle very close to the static one or slightly higher (difference less than $10^{\circ}$ ). This is representative of the capability of the PHAs-based formulation to enter into the surface porosity and modify the surface chemistry of the stones. The same consideration can be done for sandstone and marble samples treated by Sol-SIL and Emul-SIL, while limestone exhibited very high advancing contact angles, in the range between $140^{\circ}$ and $166^{\circ}$, and generally higher than the respective static contact angle.

Results clearly show that all the protectives applied on marble lead to poor improvements, due the very low porosity of the starting substrate.

As regards to receding contact angles measured for sandstone and limestone, only few samples treated with PHB or PHBVV exhibited receding contact angles higher than $0^{\circ}$, but significantly lower than $90^{\circ}$ (being equal or lower than $26^{\circ}$, Tables 5 and 6). The commercial product Sol-SIL reached receding contact angles included between $25^{\circ}$ and $48^{\circ}$, higher than those given by the PHAs formulations (Tables 5 and 6). The commercial product Emul-SIL gives similar results of Sol-SIL if applied to sandstone, while $0^{\circ}$ of receding contact angle if applied to limestone (Tables 5 and 6 ).

Marble is the only stone that recorded, as untreated stone, a receding contact angle higher than $0^{\circ}$ (being equal to $19^{\circ}$, Table 7). Due to that, samples treated with PHB-, PHBVV-formulation and commercial products exhibited contact angles higher than $0^{\circ}$ and included between $8^{\circ}$ and $40^{\circ}$. However, a significant improvement of the receding contact angle for the treated stones is not evident, with the receding contact angle being in some cases lower than the one obtained for the untreated sample.

Advancing contact angle represents the upper limit of every possible contact angle configuration; hence, it is expected to be influenced by the presence of any protective treatment. For this reason, a high increase of advancing contact angle with respect to the untreated samples confirms the presence and action of the protective on the stone's surface $[2,9,37]$. Instead, receding contact angle is considerably influenced by the presence of defects and heterogeneity, which are correlated both to the stone mineralogical composition and to incomplete coverage of the stone substrate by the polymer $[2,9,37]$. However, the roughness of stone inevitably causes a certain amount of hysteresis between advancing and receding contact angles. Hence, although a good protective should theoretically provide the stone with high dynamic contact angles (both advancing and receding angles $>90^{\circ}$ ), a certain amount of water may be absorbed by the stone by capillarity (possible, despite the treatment application) or may be retained in the stone roughness during the measurements of dynamic contact angles. For this reason, the argument of the arccosine function $F_{0} / L \gamma$ in Equation (3) (Section 4.5.2) may happen to exceed 1 , as the presence of water increases the sample mass and so the value of $F_{0}$. In this case, the contact angle calculation leads to a value equal to $0^{\circ}$ even if, from a trigonometric point of view, the equation cannot be solved. This tricky aspect of contact angle calculation is due to the fact that the Wilhelmy theory used for contact angle measurements with the force tensiometer does not take into 
account water absorption or entrapment during the test. As a result, the $0^{\circ}$ receding contact angles reported in Tables 5 and 6 actually derive from values of $F_{0} / L \gamma>1$ and it should be concluded that these stone samples, due to their porosity, heterogeneity and roughness, are not suitable for receding contact angle measurement by means of force tensiometer.

The comparison between results obtained by static and dynamic contact angle measurement and by capillary water absorption test can be useful to clarify the entire performance of protective treatments applied on stone substrates.

Sandstone samples treated by PHB generally exhibit a relatively good performance in terms of dynamic contact angle (advancing angle $>100^{\circ}$, Table 5), water absorption (with the lowest $R p$ equal to $75 \%$ after $48 \mathrm{~h}$, Table 3 ) and static contact angle (slightly higher than $90^{\circ}$, Table 5). PHB applied by dip coating gave very good results in terms of water absorption, with $R p$ equal to $94 \%$ after $1 \mathrm{~h}$ and $84 \%$ after $48 \mathrm{~h}$ (Table 3). However, PHBVV gave even better results in terms of capillary water absorption (Figure $3 \mathrm{~b}$ ) and advancing and static contact angles when applied by dip coating and poultice (both angles $>100^{\circ}$, Table 5). Static and dynamic contact angles after treatment by Sol-SIL and Emul-SIL are comparable and maximum for this type of substrate $\left(\theta\right.$ equal to $140^{\circ}$ for Sol-SIL and $125^{\circ}$ for Emul-SIL, $\theta_{\text {adv }}$ between $135^{\circ}$ and $145^{\circ}$, Table 5). The same consideration can be made on their performance in terms of water absorption by capillarity (with $R p$ comprised between $89 \%$ and $97 \%$, Table 3).

For limestone, the performance of PHB is good in terms of static and advancing contact angles (comprised between $110^{\circ}$ and $125^{\circ}$, Table 6), but not fully satisfactory in terms of capillary water absorption $(R p<40 \%$, Table 4$)$. In the case of PHBVV, there is a good agreement between the performance evaluated in terms of capillary water absorption and wettability: the great reduction in water absorption $(R p>90 \%$, Table 4$)$ is accompanied by high contact angles (with the static and advancing ones between $120^{\circ}-125^{\circ}$, Table 6 ). Sol-SIL gave the best results both in terms of wettability (static and advancing contact angles $>140^{\circ}$, Table 6) and reduction of capillary water absorption ( $R p$ equal to $95 \%$ after $48 \mathrm{~h}$, Table 4). Also Emul-SIL gave very good results on this stone (Tables 4 and 6 ).

Thus, in light of the present results, a good performance in terms of wettability does not always correspond to a good performance in terms of capillary water absorption and vice versa. Moreover, low $\left(\theta_{\text {rec }}<25^{\circ}\right)$ or zero receding contact angles are generally not correlated with high water absorption, as explained above. This highlights the importance of analysing different aspects concerning protective performances.

\subsection{Colour Measurement}

Colour alteration values, determined by spectrophotometer and calculated on the basis of the coordinates in the CIELAB space $\left(\Delta E^{*}\right)$, are reported in Figure 7 for stone treated with PHB and PHBVV, with respect to untreated ones. The CIELAB colour space was established by the "Commission Internationale de L'Eclairage" (CIE) in 1976 and allows to represent each colour by the three coordinates $L^{*}$ (axis black-white), $a^{*}$ (axis green-red) and $b^{*}$ (axis yellow-blue). The difference between two colours can be determined by the formula $\Delta E^{*}=\left(\Delta L^{* 2}+\Delta a^{* 2}+\Delta b^{* 2}\right)^{1 / 2}$.

For colour compatibility in the conservation field, any consolidating or protective treatment is required to produce a $\Delta E^{*}$ lower than 5 , considering that the human eye cannot detect colour alterations with $\Delta E^{*}<2-3$.

Results show that PHB formulation (Figure 7a), regardless the application method, gives acceptable values of $\Delta E^{*}$ both for sandstone (values between 2 and 3.5) and limestone (values about 4), while colour variations for marble are imperceptible to the human eye ( $\Delta E^{*}$ lower than 1$)$. After the application of PHBVV, the colour alteration is acceptable for sandstone samples $\left(\Delta E^{*}\right.$ between 2 and 4.5 ) and marble (undetectable by human eye) (Figure $7 \mathrm{~b}$ ), while for limestone, $\Delta E^{*}$ is higher than the threshold for dip coating application $\left(\Delta E^{*} \approx 6\right)$.

Based on these results, PHB- and PHBVV-based treatments can be considered compatible from an aesthetic point of view with all the stones considered. Dip coating application seems to give systematically higher colour changes with respect to the other methods, but only in one case (PHBVV) 
did it produce excessive colour alteration. This higher colour impact of dip coating cannot be ascribed to a higher amount of protective on the surface, because this is not the case; hence, a deeper investigation into the surface distribution of the polymer will be necessary to find an explanation of this aspect.

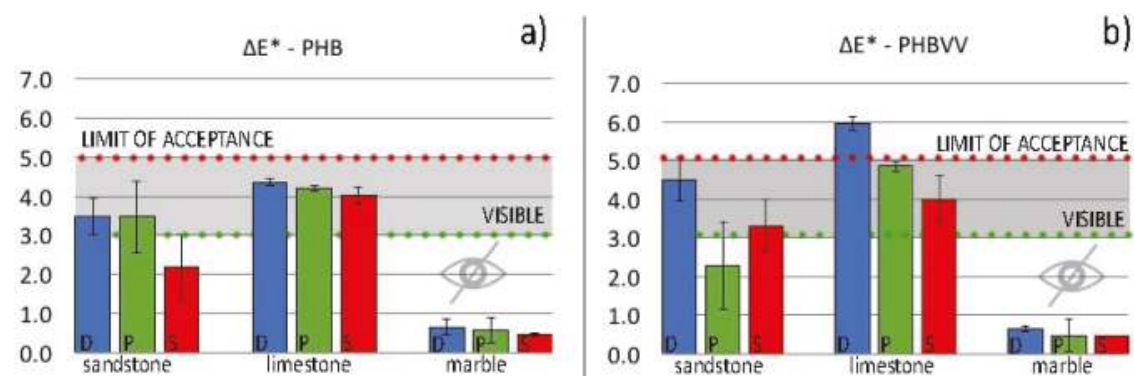

Figure 7. Colour alterations $\left(\Delta E^{*}\right)$ determined by spectrophotometry on sandstone, limestone and marble: (a) before and after the application of PHB-based treatment; (b) and before and after the application of PHBVV-based treatment. The three application methods are represented as D (dipcoating, blue bars), P (poultice, green bars) and S (spray, red bars).

In Table 8 the average variations $\Delta L^{*}, \Delta a^{*}$ and $\Delta b^{*}$ are reported, for a better understanding of colour alterations. The values that mostly influence $\Delta E^{*}$ are $\Delta b^{*}$, indicating a yellowing of the surface, and $\Delta L^{*}$, indicating a darkening.

Table 8. Average $\Delta L^{*}, \Delta a^{*}$ and $\Delta b^{*}$ values of sandstone, limestone and marble treated with PHB and PHBVV with respect to untreated conditions.

\begin{tabular}{ccccccc}
\hline \multirow{2}{*}{ Colour Coordinate } & \multicolumn{2}{c}{ Sandstone } & \multicolumn{2}{c}{ Limestone } & \multicolumn{2}{c}{ Marble } \\
\cline { 2 - 7 } & PHB & PHBVV & PHB & PHBVV & PHB & PHBVV \\
\hline$\Delta L^{*}$ & $2.5 \pm 1.5$ & $2.5 \pm 1.5$ & $1.7 \pm 2.5$ & $4.1 \pm 2.7$ & $0.8 \pm 0.4$ & $0.8 \pm 0.6$ \\
$\Delta a^{*}$ & $-0.4 \pm 0.2$ & $-0.4 \pm 0.4$ & $-0.6 \pm 0.3$ & $-0.5 \pm 0.2$ & $0.1 \pm 0.1$ & $0.1 \pm 0.1$ \\
$\Delta b^{*}$ & $-2 \pm 1.6$ & $-2.3 \pm 2.5$ & $-3.6 \pm 0.9$ & $-3.2 \pm 2.3$ & $0.3 \pm 0.4$ & $-0.4 \pm 0.2$ \\
\hline
\end{tabular}

\subsection{Water Vapour Diffusion Test}

Results of the water vapour diffusion test for stone treated with PHB and PHBVV are reported in Tables 9-11, where the water vapour transmission rate of the untreated substrate $\left(V_{\mathrm{S}}\right)$, of the treated substrate (substrate plus coating, $V_{\mathrm{CS}}$ ), of the coating $(V)$, the water vapour diffusion-equivalent air layer thickness $\left(S_{\mathrm{d}}\right)$ and the corresponding water vapour transmission rate class are collected. In order to obtain good compatibility between the protective treatment and the stone substrate, it is essential not to significantly alter the water vapour diffusion of the stone. Due to the different microstructure, untreated sandstone, limestone and marble have notably different water vapour diffusion rates $(V)$. In particular, limestone exhibits the highest $\mathrm{V}\left(278 \mathrm{~g} / \mathrm{m}^{2}\right.$. day, Table 10$)$, which is more than three times that of sandstone $\left(86 \mathrm{~g} / \mathrm{m}^{2}\right.$.day, Table 9$)$ and more than 13 times higher than that of marble $\left(21 \mathrm{~g} / \mathrm{m}^{2}\right.$.day, Table 11).

In sandstone, the results of water vapour diffusion testing show good compatibility between the coatings and the stone, as every coating applied on sandstone can be classified in the high water vapour diffusion rate class, except for PHB and PHBVV applied by poultice. These give a medium water vapour diffusion rate class, probably related to the high quantity of polymer retained in the stone after poultice (Table 9). Nevertheless, even in the case of poultice application of PHB and PHBVV, the water vapour transmission rate of the stone is reduced by less than $30 \%$ (being $V_{\mathrm{S}}=86 \mathrm{~g} / \mathrm{m}^{2}$. day and $V_{\mathrm{CS}}=59-60 \mathrm{~g} / \mathrm{m}^{2}$. day for samples treated with PHB and PHBVV by poultice, Table 9). 
PHB and PHBVV treatments applied on limestone are classified in the high water vapour diffusion rate class (Table 10). Nevertheless, two samples, namely S-LIMEs-PHB and D-LIMEs-PHBVV, gave values of water vapour transmission rate $\left(V_{\mathrm{CS}}\right)$ that are notably lower with respect to the other samples (being, respectively, $V_{\mathrm{CS}}=163 \mathrm{~g} / \mathrm{m}^{2}$. day and $V_{\mathrm{CS}}=126 \mathrm{~g} / \mathrm{m}^{2}$. day versus $V_{\mathrm{CS}}>260 \mathrm{~g} / \mathrm{m}^{2} \cdot$ day for the other samples). This cannot be due to a higher amount of protective applied, but seems related to the considerable heterogeneity that characterises stone samples and, in particular, limestone [39].

Table 9. Results of water vapour diffusion test for sandstone treated with PHB and PHBVV and untreated.

\begin{tabular}{ccccc}
\hline Sample & $\begin{array}{c}V_{\mathrm{S}} \text { or } V_{\mathrm{CS}} \\
\left(\mathrm{g} / \mathrm{m}^{2} \cdot \text { Day }\right)\end{array}$ & $\begin{array}{c}V \\
\left(\mathrm{~g} / \mathbf{m}^{2} \cdot \text { Day }\right)\end{array}$ & $\begin{array}{c}S_{\mathrm{d}} \\
(\mathbf{m})\end{array}$ & Water Vapour Transmission Rate Class \\
\hline UT-SANDs & $V_{\mathrm{S}}=86$ & - & - & - \\
D-SANDs-PHB & $V_{\mathrm{CS}}=67$ & 302 & 0.10 & high \\
P-SANDs-PHB & $V_{\mathrm{CS}}=93$ & $>680$ & 0.03 & high \\
S-SANDs-PHB & $V_{\mathrm{CS}}=59$ & 190 & 0.16 & medium \\
D-SANDs-PHBVV & $V_{\mathrm{CS}}=60$ & 194 & 0.16 & high \\
P-SANDs-PHBVV & $V_{\mathrm{CS}}=76$ & $>680$ & 0.05 & high \\
S-SANDs-PHBVV & $V_{\mathrm{CS}}=80$ & $>680$ & 0.03 & \\
\hline
\end{tabular}

Table 10. Results of water vapour diffusion test for limestone treated with PHB and PHBVV and untreated.

\begin{tabular}{ccccc}
\hline Sample & $\begin{array}{c}V_{\mathrm{S}} \text { or } V_{\mathrm{CS}} \\
\left(\mathbf{g} / \mathbf{m}^{2} \cdot \text { Day }\right)\end{array}$ & $\begin{array}{c}V \\
\left(\mathbf{g} / \mathbf{m}^{2} \text {-Day }\right)\end{array}$ & $\begin{array}{c}S_{\mathrm{d}} \\
(\mathbf{m})\end{array}$ & Water Vapour Transmission Rate Class \\
\hline UT-LIMEs & $V_{\mathrm{S}}=278$ & - & - & - \\
D-LIMEs-PHB & $V_{\mathrm{CS}}=306$ & $>680$ & 0.01 & high \\
P-LIMEs-PHB & $V_{\mathrm{CS}}=266$ & $>680$ & 0.01 & high \\
S-LIMEs-PHB & $V_{\mathrm{CS}}=163$ & 391 & 0.08 & high \\
D-LIMEs-PHBVV & $V_{\mathrm{CS}}=126$ & 231 & 0.14 & high \\
P-LIMEs-PHBVV & $V_{\mathrm{CS}}=326$ & $>680$ & 0.02 & high \\
S-LIMEs-PHBVV & $V_{\mathrm{CS}}=295$ & $>680$ & 0.01 & high \\
\hline
\end{tabular}

Table 11. Results of water vapour diffusion test for marble treated with PHB and PHBVV and untreated.

\begin{tabular}{ccccc}
\hline Sample & $\begin{array}{c}V_{\mathrm{S}} \text { or } V_{\mathrm{CS}} \\
\left(\mathrm{g} / \mathrm{m}^{2} \cdot \text { Day }\right)\end{array}$ & $\begin{array}{c}V \\
\left(\mathrm{~g} / \mathrm{m}^{2} \cdot \text { Day }\right)\end{array}$ & $\begin{array}{c}S_{\mathrm{d}} \\
(\mathbf{m})\end{array}$ & Water Vapour Transmission Rate Class \\
\hline UT-MARBLE & $V_{\mathrm{S}}=21$ & - & - & - \\
D-MARBLE-PHB & $V_{\mathrm{CS}}=24$ & 171 & 0.18 & medium \\
P-MARBLE-PHB & $V_{\mathrm{CS}}=15$ & 49 & 0.64 & medium \\
S-MARBLE-PHB & $V_{\mathrm{CS}}=11$ & 24 & 1.32 & medium \\
D-MARBLE-PHBVV & $V_{\mathrm{CS}}=25$ & 124 & 0.25 & medium \\
P-MARBLE-PHBVV & $V_{\mathrm{CS}}=23$ & 199 & 0.16 & medium \\
S-MARBLE-PHBVV & $V_{\mathrm{CS}}=18$ & 130 & 0.24 & \\
\hline
\end{tabular}

All the coatings applied on marble belong to the medium class of water vapour diffusion (Table 11), but given the extremely low water vapour diffusivity of marble, the significance of this parameter is quite limited and even a medium water vapour transmission rate can be considered compatible. For the same reason, the fact that the values of water vapour transmission rate after PHBVV application are comparable or even higher with respect to the untreated stone appears simply due to the difficulty of determining accurately the water vapour diffusion in this very compact stone.

Based on the results, the compatibility from the point of view of water vapour transmission capacity is ensured for both the PHB and PHBVV formulations and for all the stones investigated.

\subsection{Coating Morphology Analysis}

Treated samples showing the best performance in terms of water repellency were analysed by scanning electron microscopy in order to evaluate the interfacial adhesion between the protective 
coating and the stone, and the coating homogeneity. Of course, the poultice method gives rise to the thickest coatings because it allows us to deposit a greater amount of protective solution onto the surface; therefore, samples produced by the poultice method were used for the purposes of this specific analysis.

Some images obtained by FEG-SEM (Field Emission Gun Scanning Electron Microscopy) for sandstone, limestone and marble samples treated with PHB and PHBVV by poultice are reported in Figures 8-10. In sandstone, no significant morphological differences between the PHB and PHBVV coatings can be noticed. In both cases the polymer tends to penetrate the capillary pores of the stone, assuming a shape similar to a cobweb. Pores are not totally filled by the polymer, suggesting that the treatment does not give a pore blocking effect. The polymer is present at all the observed depths (approximately $650 \mu \mathrm{m}$ ) and as a thin layer over the top of the surface (dark layer in Figure 8b). Images of treated cross sections of limestone (Figure 9) suggest a distribution of the polymer similar to that observed in sandstone, but in this case the morphology of the polymer in the pores is sheet-like. Due to the very low porosity of marble, both PHB and PHBVV accumulate in layers of various thickness $(1-4 \mu \mathrm{m})$ over the stone's surface (Figure 10).
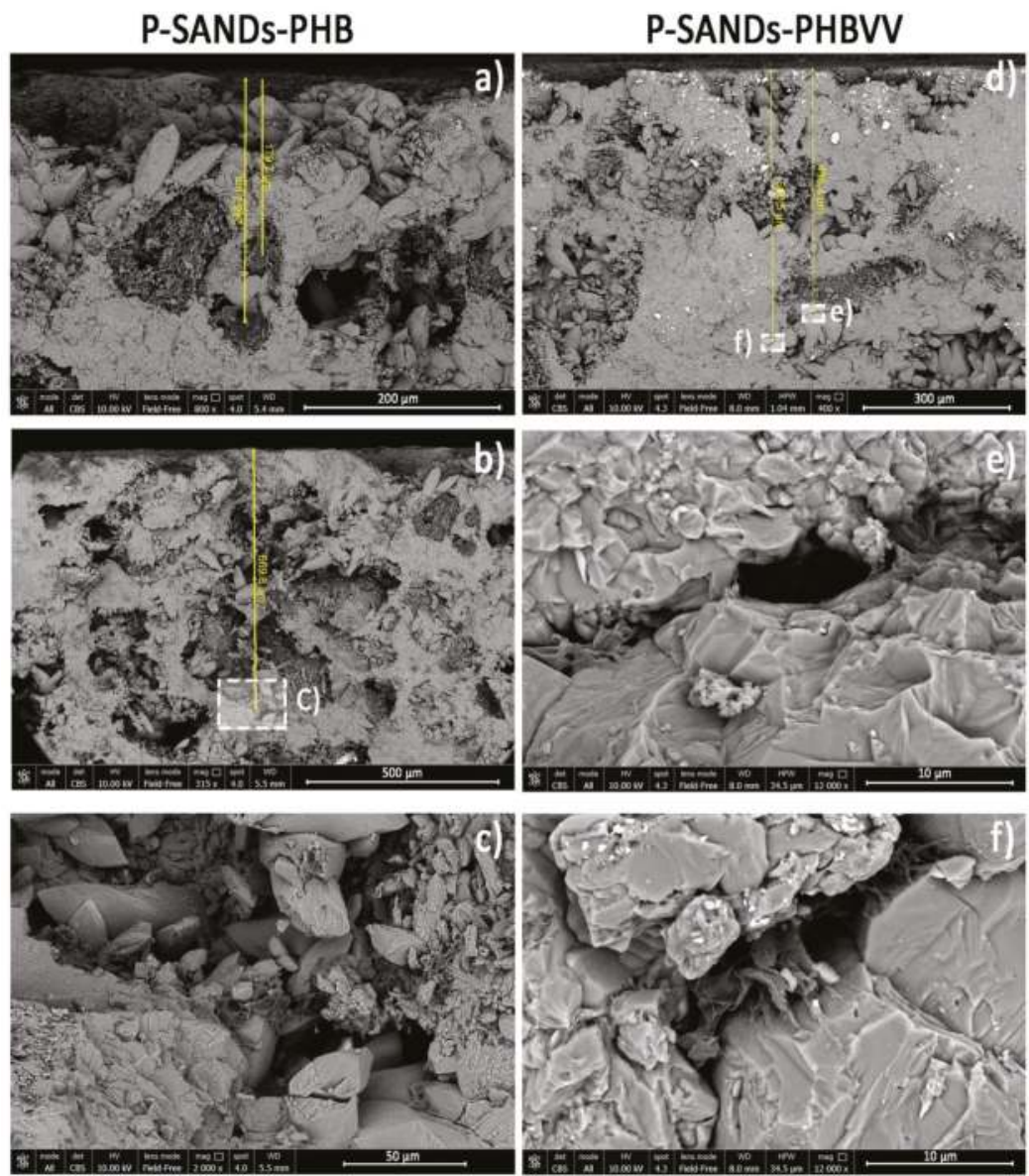

Figure 8. FEG-SEM images of sandstone treated: (a-c) with PHB-based formulation by poultice; (d-f) with PHBVV-based formulation by poultice. 

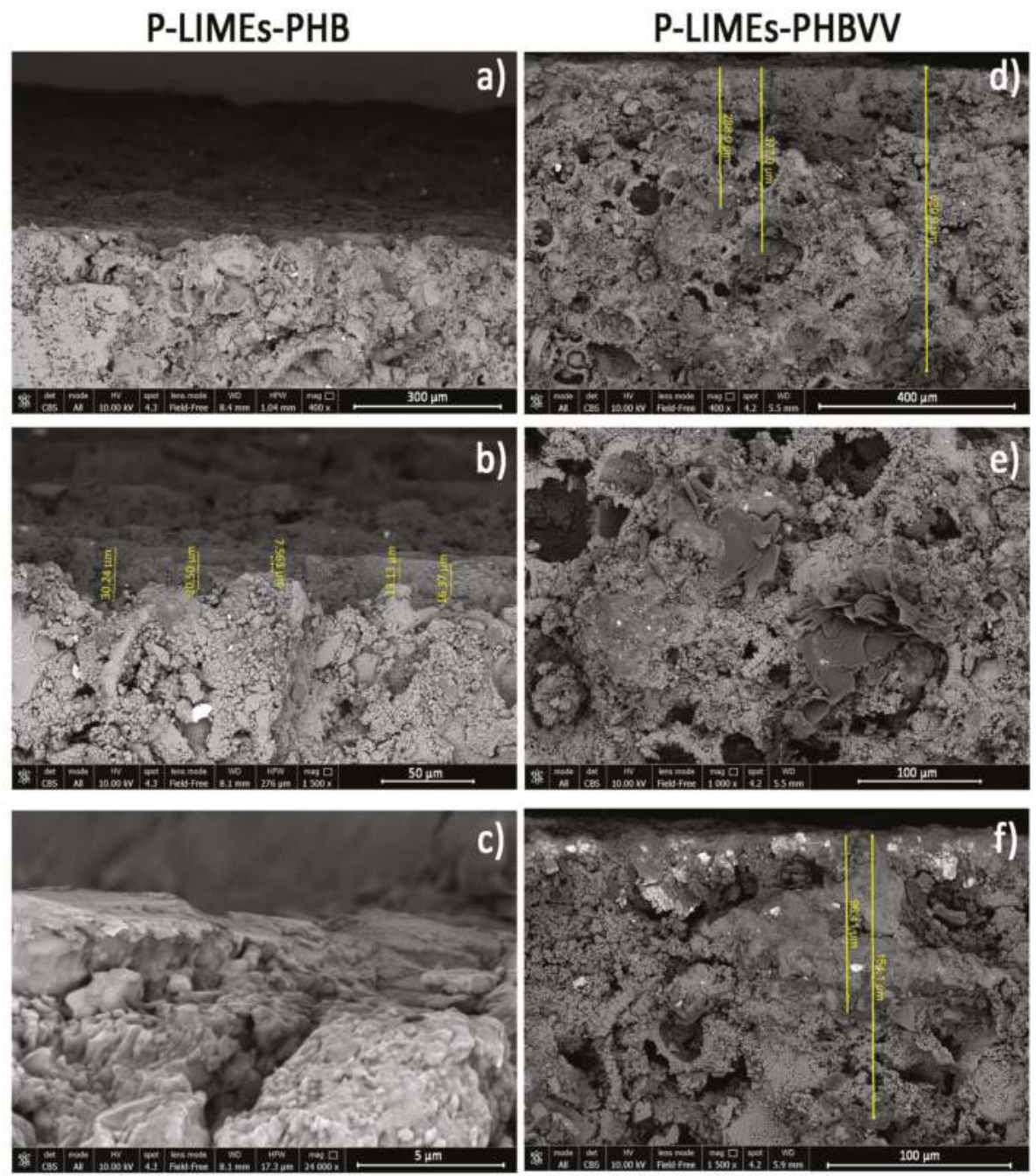

Figure 9. FEG-SEM images of limestone treated: $(\mathbf{a}-\mathbf{c})$ with PHB-based formulation by poultice; (d-f) with PHBVV-based formulation by poultice. 

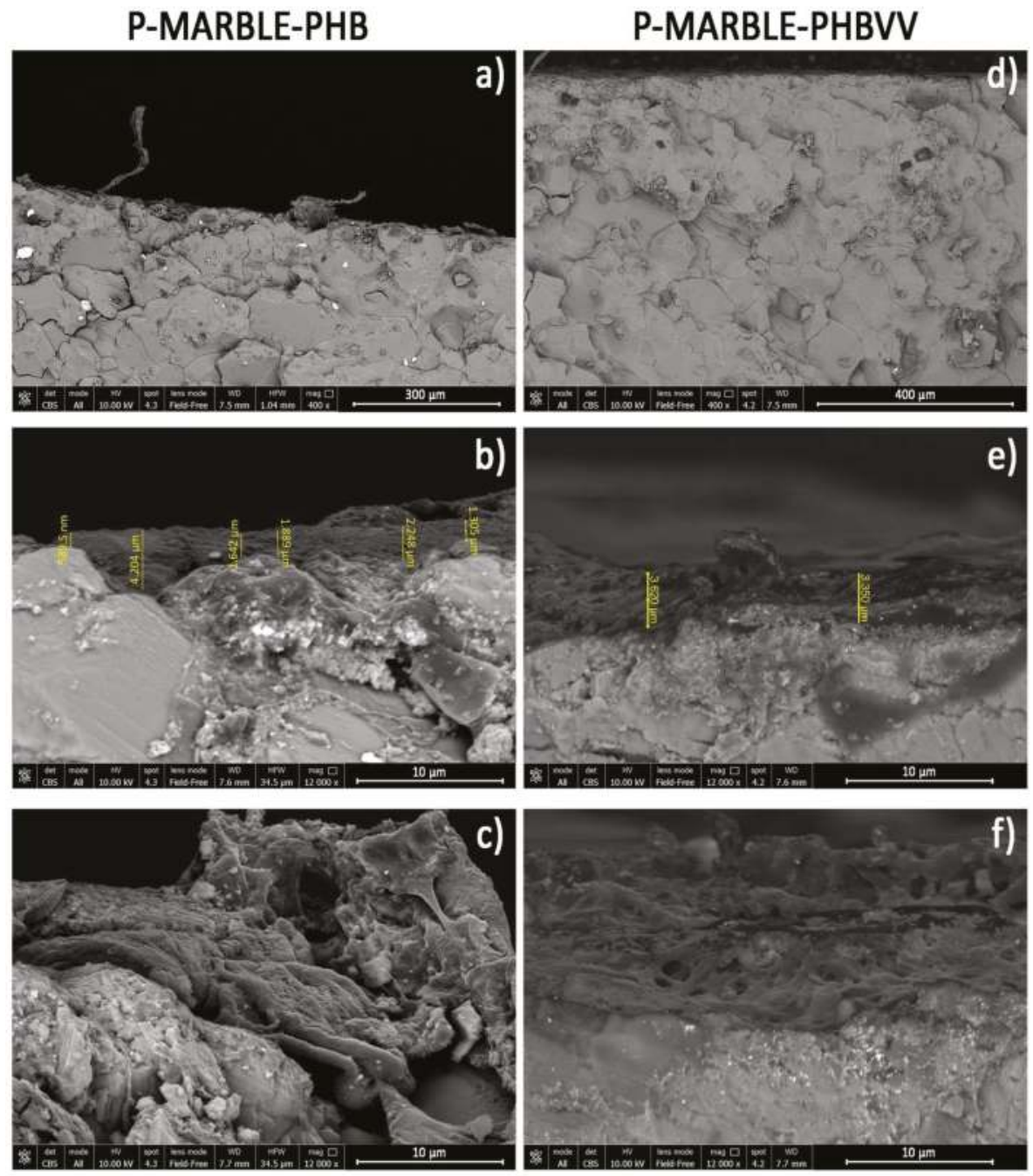

Figure 10. FEG-SEM images of marble treated: (a-c) with PHB-based formulation by poultice; (d-f) with PHBVV-based formulation by poultice.

\subsection{Artificial Ageing}

The results of static contact angle measurement after seven days of artificial ageing in the climatic chamber are reported in Table 12, where a drastic decrease of water repellency can be observed for PHB and PHBVV, while the commercial products experience a limited decrease in the contact angle (especially Sol-SIL). However, considering the water absorption by capillarity, PHB and PHBVV seem to provide some residual effectiveness on sandstone even after the artificial ageing (Figure 11 and Table 13), while for limestone the loss of hydrophobicity is confirmed (Figure 12 and Table 14).

These preliminary results suggest that measures must be taken for improving the durability of these PHB and PHVV formulations for the application targeted in this study (for example, adding additives and stabilisers). 
Table 12. Static contact angle values determined after the artificial ageing (c.a.: complete absorption; n.d.: not determined).

\begin{tabular}{ccccc}
\hline \multirow{2}{*}{ Protective } & Application & Sandstone & Limestone & Marble \\
\cline { 3 - 5 } & Method & $\boldsymbol{\theta}\left(^{\circ}\right)$ & $\boldsymbol{\theta}\left(^{\circ}\right)$ & $\boldsymbol{\theta}\left(^{\circ}\right)$ \\
\hline \multirow{3}{*}{ PHB } & dip coating & $6 \pm 6$ & c.a. & $21 \pm 6$ \\
& poultice & $21 \pm 7$ & c.a. & $21 \pm 7$ \\
& spray & $17 \pm 0$ & c.a. & $17 \pm 5$ \\
\hline \multirow{2}{*}{ PHBVV } & dip coating & $14 \pm 3$ & c.a. & $39 \pm 8$ \\
& poultice & $14 \pm 4$ & c.a. & $21 \pm 12$ \\
& spray & $16 \pm 3$ & c.a. & $26 \pm 8$ \\
\hline \multirow{2}{*}{ Sol-SIL } & dip coating & $123 \pm 5$ & $128 \pm 3$ & $112 \pm 8$ \\
& spray & $126 \pm 7$ & $125 \pm 4$ & n.d. \\
\hline \multirow{2}{*}{ Emul-SIL } & dip coating & $123 \pm 6$ & $127 \pm 4$ & $110 \pm 9$ \\
& spray & $124 \pm 6$ & $129 \pm 5$ & n.d. \\
\hline \multirow{2}{*}{} & & & &
\end{tabular}

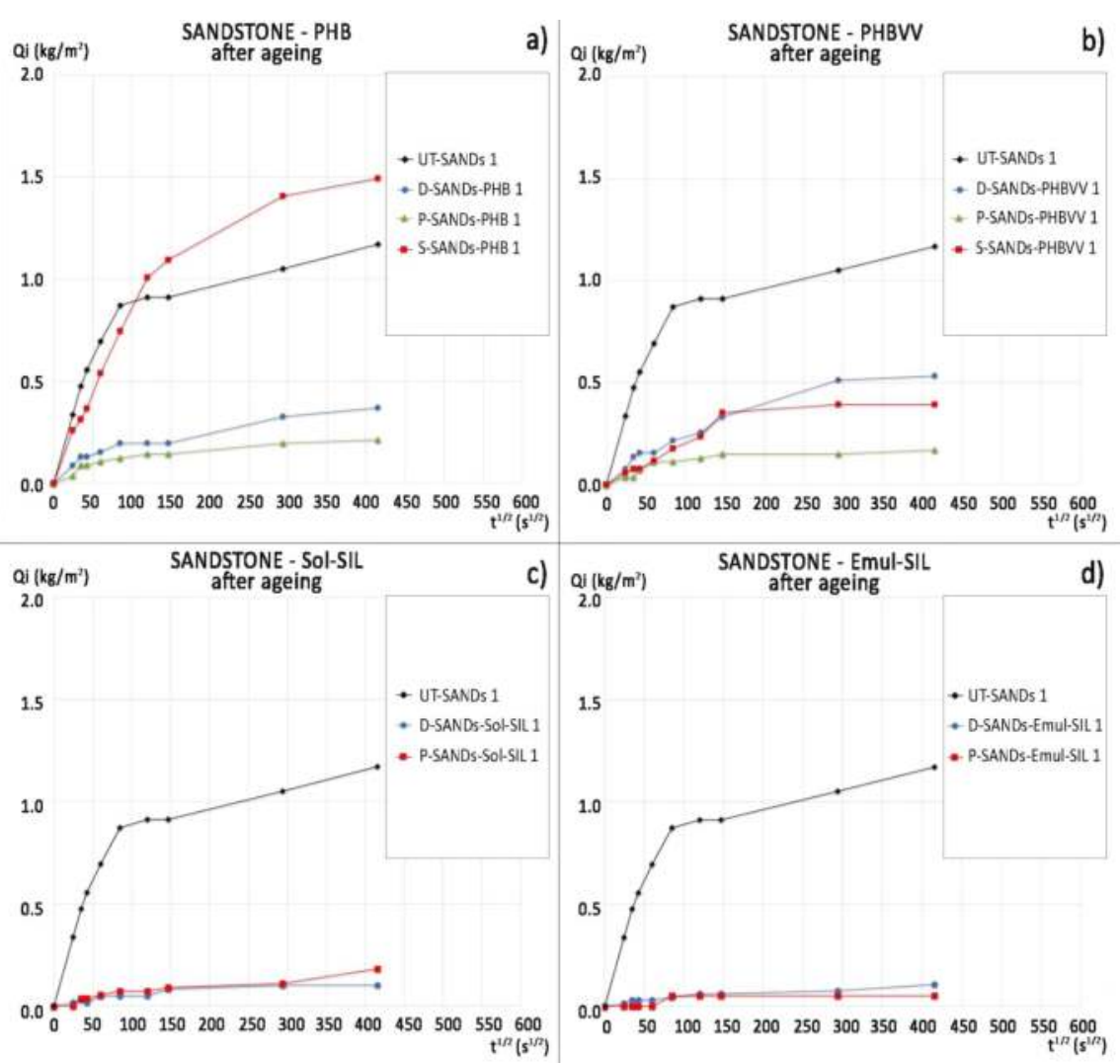

Figure 11. Capillary water absorption curves of sandstone after artificial ageing referred to: (a) samples treated with PHB-based formulation; (b) samples treated with PHBVV-based formulation; (c) samples treated with Sol-SIL; (d) samples treated with Emul-SIL. 
Table 13. Mean ratio of protection of the treatments on sandstone after the artificial ageing (n.d.: not determined).

\begin{tabular}{ccccccccc}
\hline \multirow{2}{*}{$\begin{array}{c}\text { Application } \\
\text { Method }\end{array}$} & \multicolumn{2}{c}{ PHB } & \multicolumn{2}{c}{ PHBVV } & \multicolumn{2}{c}{ Sol-SIL } & \multicolumn{2}{c}{ Emul-SIL } \\
\cline { 2 - 8 } & $\begin{array}{c}\text { Rp (\%) } \\
\text { after } \mathbf{~ h}\end{array}$ & $\begin{array}{c}\text { Rp (\%) } \\
\text { after 48 h }\end{array}$ & $\begin{array}{c}\text { Rp (\%) } \\
\text { after 1 h }\end{array}$ & $\begin{array}{c}\text { Rp (\%) } \\
\text { after 48 h }\end{array}$ & $\begin{array}{c}\text { Rp (\%) } \\
\text { after 1 h }\end{array}$ & $\begin{array}{c}\text { Rp (\%) } \\
\text { after 48 h }\end{array}$ & $\begin{array}{c}\text { Rp (\%) } \\
\text { after 1 h }\end{array}$ & $\begin{array}{c}\text { Rp (\%) } \\
\text { after 48 h }\end{array}$ \\
\hline dip coating & 80 & 71 & 77 & 54 & 91 & 90 & 94 & 88 \\
poultice & 83 & 80 & 83 & 85 & n.d. & n.d. & n.d. & n.d. \\
spray & 0 & 0 & 74 & 66 & 91 & 83 & 100 & 95 \\
\hline
\end{tabular}
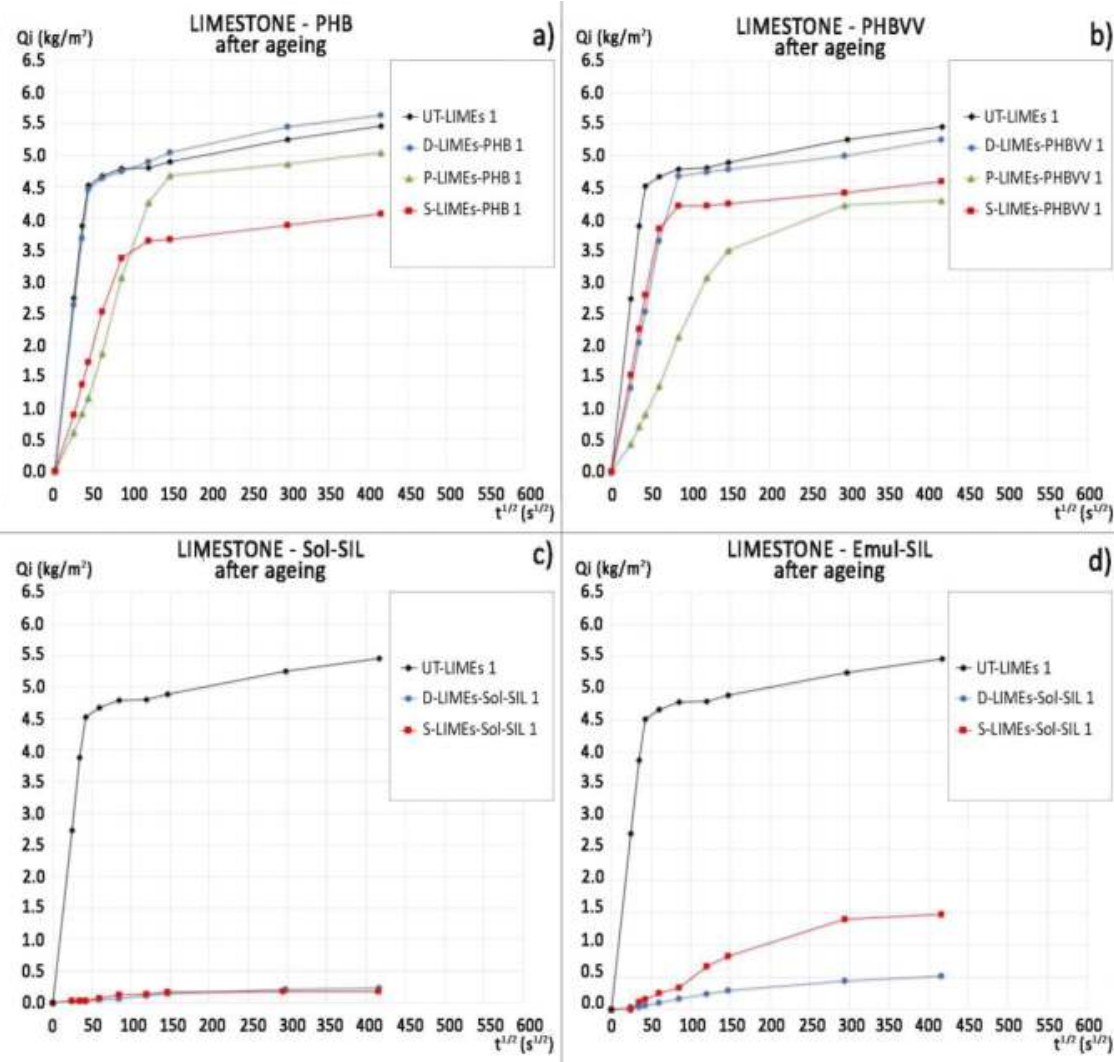

Figure 12. Capillary water absorption curves of limestone after artificial ageing referred to: (a) samples treated with PHB-based formulation; (b) samples treated with PHBVV-based formulation; (c) samples treated with Sol-SIL; (d) samples treated with Emul-SIL.

It is noteworthy that one of the most important features of PHAs is their spontaneous degradation under environmental conditions; therefore, the results obtained by the accelerated ageing should not come as any surprise, but on the contrary demonstrate that spontaneously reversible surface treatments for stones can be successfully developed using bioplastics. This actually represents a very important target for the protection of stones in cultural heritage, where tailoring of the duration of the treatment can be addressed by a proper selection of the molecular features of the biopolymer chains, because they directly influence the environmental duration of the coating. 
Table 14. Mean ratio of protection of the treatments on limestone after the artificial ageing (n.d.: not determined).

\begin{tabular}{ccccccccc}
\hline \multirow{2}{*}{$\begin{array}{c}\text { Application } \\
\text { Method }\end{array}$} & \multicolumn{2}{c}{ PHB } & \multicolumn{2}{c}{ PHBVV } & \multicolumn{2}{c}{ Sol-SIL } & \multicolumn{2}{c}{ Emul-SIL } \\
\cline { 2 - 9 } & $\begin{array}{c}\text { Rf (\%) } \\
\text { after } \mathbf{~ h}\end{array}$ & $\begin{array}{c}\text { Rp (\%) } \\
\text { after 48 h }\end{array}$ & $\begin{array}{c}\text { Rp (\%) } \\
\text { after 1 h }\end{array}$ & $\begin{array}{c}\text { Rp (\%) } \\
\text { after 48 h }\end{array}$ & $\begin{array}{c}\text { Rp (\%) } \\
\text { after 1 h }\end{array}$ & $\begin{array}{c}\text { Rp (\%) } \\
\text { after 48 h }\end{array}$ & $\begin{array}{c}\text { Rp (\%) } \\
\text { after 1 h }\end{array}$ & $\begin{array}{c}\text { Rp (\%) } \\
\text { after 48 h }\end{array}$ \\
\hline dip coating & 0 & 6 & 0 & 34 & 98 & 96 & 96 & 90 \\
poultice & 45 & 32 & 41 & 27 & n.d. & n.d. & n.d. & n.d. \\
spray & 25 & 46 & 82 & 19 & 98 & 97 & 92 & 80 \\
\hline
\end{tabular}

\section{Discussion}

A preliminary investigation into the possible use of PHAs for the protection of stone in cultural heritage was carried out. Results demonstrated that PHAs can be purposely used as a polymer basis for the development of protective coatings for different kinds of stone (sandstone, limestone and marble were tested), and their intrinsic biodegradability in environmental conditions can be purposely exploited to generate temporary treatments that do not need any removal, which is an important target for the protection of cultural heritage. Results showed that the molecular structure of the PHAs does not play a fundamental role, even if PHBVV usually gives rise to slightly better results than PHB.

Experiments demonstrate that the application method, together with the porosity of the stone, strongly influences the amount of polymer deposited on the stone, and the effectiveness of the protective treatment as a consequence. Limestone, given its higher porosity, retains a higher amount of protective treatment than sandstone; therefore, more significant improvement in hydrophobization is reached. As far as the deposition method is concerned, poultice causes a much higher protective uptake with respect to dip coating and spray, in porous stones as in sandstone and limestone, with the uptake being maximum for the latter. In the case of marble, given its extremely low porosity, a very limited uptake was observed for any protective and any application method, so improvements induced by the presence of the protective are limited.

The PHAs-based protective formulations generally give good results in terms of colour change and water vapour diffusion. Only in one case (PHBVV applied by dip coating to limestone) was the colour change slightly higher than the threshold accepted in the conservation field. The water vapour transmission rate class was generally 'high' in sandstone and limestone.

Investigating the performance of protectives on real stone samples is very challenging, as each of the testing methods used provides only partial insight into the expected performance on site. For this reason, it is very important to develop a testing procedure that is actually able to reproduce in the lab the protective performance that is expected in real on-site exposure. In particular, the use of a force tensiometer might be too severe in relation to the real condition of stone on-site, which does not involve a complete immersion in water. From this point of view, the capillary absorption test can be considered more representative, although the water in the test is supplied by interposition of a wet layer of filter papers rather than by plain water or rainfall. For these reasons, it would be useful to develop new test methods targeted to investigate in a more realistic way the performance of protective treatments for stone, for example by simulated artificial rain tests.

Further optimisations of the biopolymer-based formulations, mainly looking for 'greener' solvents in substitution of chloroform and adding stabilisers for tailoring the polymer durability, are currently in progress.

\section{Materials and Methods}

\subsection{Stone Samples}

The three lithotypes used for the purposes of this study were:

- Sandstone: a medium-porosity calcitic sandstone, Siena stone, was selected (provided by Il Casone S.p.A., Firenzuola, Italy). It is mainly composed of calcareous grains and low amounts of quartz, 
bound by calcitic cement. This stone is typical of Tuscany architecture, but representative of a class of stones widely used in historical architecture.

- Limestone: a high porosity organogenic calcareous stone, Lecce stone, quarried in the Lecce area in Italy (Cursi-Zollino-Melpignano quarry) and provided by Décor s.r.l., San Giovanni in Fiore, Italy), was selected. It is mainly composed of calcite, with traces of phosphatic minerals. This limestone was widely used in Baroque architecture in the Puglia region and is similar to several other porous limestones widespread in the Mediterranean basin.

- Marble: Carrara marble, a very low porosity stone (supplied by Imbellone Michelangelo, s.a.s. Bologna, Italy), quarried in the Apuan Alps in Tuscany and widely used in historical architecture and statues, was selected. It is mainly composed of calcite, with small traces of dolomite.

Stone samples were obtained by wet sawing of quarried slabs. Sample size and geometry were different according to the type of test to be performed. Before the application of any protective treatments, the samples were gently brushed under water, kept in an oven at $40^{\circ} \mathrm{C}$ for $24 \mathrm{~h}$ and then in laboratory conditions until constant weight.

\subsection{Stone Characterisation}

The microstructure of the substrates was investigated in terms of pore size distribution, total open porosity $(O P)$ and average pore radius $\left(r_{\mathrm{a}}\right)$, by mercury intrusion porosimetry (MIP) on duplicate fragments (about $1 \mathrm{~g}$ mass) per stone type. For this purpose, a Fisons Macropore Unit 120 and a Porosimeter 2000 Carlo Erba (Tecmat, Como, Italy) were used.

The mineralogical composition of each stone was determined by powder X-ray diffraction (XRD), in a Philips Diffractometer PW 1840 (Panalytical, Almelo, The Netherlands), 40 kV/20 mA, Cu K $\alpha$ radiation. The carbonate amount, expressed as $\mathrm{CaCO}_{3}(\mathrm{wt} \%)$, was determined on duplicate samples by the Dietrich-Frühling gas volumetric method. This method is based on the quantification of the $\mathrm{CO}_{2}$ volume released by reacting the powdered sample with $\mathrm{HCl}$. The method also allows for dolomite quantification [40], as the reaction velocity between $\mathrm{HCl}$ and dolomite is lower than that between $\mathrm{HCl}$ and calcite, so it is possible to distinguish between them.

For any stone type and condition, all the tests (described here and in Section 4.5) were carried out on two duplicate samples.

\subsection{Protective Formulations}

PHB and PHBVV were kindly provided as experimental grades by Bio-on SpA (Bologna, Italy) with an average ponderal molecular weight of $\mathrm{Mw}=122,500$ and $\mathrm{Mw}=279,500$, respectively; the ponderal molecular weight was determined by gel permeation chromatography (GPC) analysis dissolving $10 \mathrm{mg}$ of sample (powder) in $2 \mathrm{~mL}$ of chloroform and using toluene as a flow marker (Chromatographyc system Agilent 1260 Infinity System by Agilent, Santa Clara, CA, USA, based on two columns: PLgel MiniMIX-A for the separation of molecules with molecular weight up to $4 \times 10^{7} \mathrm{~g} / \mathrm{mol}$ and TOSOH TSKgel SuperMultipore HZ-M for the separation of molecules with molecular weight in the range $10^{2}-2 \times 10^{6} \mathrm{~g} / \mathrm{mol}$ ); both were used as received without any further purification. The molar content of 3-hydroxyvaleric acid (3HV) and 4-hydroxyvaleric acid (4HV) units of PHBVV was determined by means of Bruker NMR Avance400 spectrometer (Bruker, Billerica, MA, USA) and the software Bruker TopSpi (version 3.2, Bruker, Billerica, MA, USA), using 10-15 mg of sample (powder) dissolved in $1 \mathrm{~mL}$ of $\mathrm{CDCl}_{3}$. PHBVV has a $3 \mathrm{HV}$ units molar content of $11 \%$ and a 4 -HV units molar content of $2 \%$. The melting temperature of the PHB used for this study was $173{ }^{\circ} \mathrm{C}$, and $146^{\circ} \mathrm{C}$ for PHBVV, hence both polymers can be considered suitable for exterior applications; glass transition temperature was not detectable by DSC for PHB due to its high crystallinity, while it was $-2{ }^{\circ} \mathrm{C}$ for PHBVV. Homogeneous solutions of PHAs were obtained by dissolving the polymers in boiling $\mathrm{CHCl}_{3}$ at a concentration of $3 \mathrm{wt} / \mathrm{vol} \%$; cold solutions were strained with a syringe filter $(0.45 \mu \mathrm{m})$ before use, in order to eliminate any possible insoluble traces. At this preliminary stage of 
the research, aimed at evaluating the potential of PHB and PHBVV as protectives, chloroform was used as the solvent (despite not being usable in the workplace), as it is known to be effective for this class of biopolymers, exhibiting a low solubility in many classical polymer solvents.

Two commercial protectives widely used for stone conservation were tested too, for comparison's sake. The first one, labelled Sol-SIL, is constituted by silane and siloxane dissolved in white spirit (active content of $7 \mathrm{~m} / \mathrm{m} \%$, commercial name Idrosil ${ }^{\circledR}$ Pronto CA-WS, by Antares, San Lazzaro di Savena, Italy); the second, labelled Emul-SIL, is constituted by silane and siloxane emulsified in water (active content of $8 \mathrm{~m} / \mathrm{m} \%$, commercial name Antipluviol ${ }^{\circledR} \mathrm{W}$, Mapei, Milano, Italy).

\subsection{Application Methods}

The PHB- and PHBVV-based treatments were applied by:

- Dip coating (sample coding: "D-"): samples were completely immersed in the solution for $10 \mathrm{~min}$. The samples destined to capillary water absorption test and water vapour permeability determination were only partially immersed in the solution for $10 \mathrm{~min}$ (keeping them suspended from the top), in order to obtain just one treated surface.

- Poultice (sample coding: "P-"): the sample surface to be treated was covered with a 1.5-thick layer of cotton wool, then the formulation was spilled over the cotton layer $(0.16 \mathrm{~L}$ of solution per $1 \mathrm{dm}^{2}$ of treated surface) and the samples were immediately covered with an aluminium sheet to prevent the solvent from evaporating. The poultice was left wrapped for $24 \mathrm{~h}$, to allow the absorption of the protective in the stone. Thereafter, the aluminium sheet was removed and the cotton layer was left over the samples until complete drying. Only one surface was treated per each sample, except for samples prepared for the measurement of static and dynamic contact angle, which were completely covered by a poultice.

- Spray (sample coding: "S-"). A low-pressure spray nebuliser (FPM gaskets industrial sprayer, Volpitech 2, Volpi, Casalromano, Italy) was used for this purpose. The surfaces of the samples to be treated were put in vertical position and subjected to 15 sprays, corresponding to about $0.02 \mathrm{~L}$ of solution per $1 \mathrm{dm}^{2}$ of surface. The distance between the nozzle and the sample was about $40 \mathrm{~cm}$. Only one surface per each sample was treated, except for samples prepared for the measurement of static and dynamic contact angle, which were completely sprayed.

- Sol-SIL and Emul-SIL were applied by spray, as recommended by the manufacturers, but also by dip coating, for comparison's sake, using the procedures previously described.

\subsection{Characterisation of the Coated Stones}

\subsubsection{Capillary Water Absorption}

The water absorption by capillarity was determined according to EN 15801 [41] on two replicate samples $\left(25 \times 25 \times 19 \mathrm{~mm}^{3}\right)$ for each combination of formulation (PHB-based or PHBVV-based) and application method (dip coating, poultice and spray). Two untreated samples were tested for reference. Samples were put in contact with a $1 \mathrm{~cm}$-thick layer of filter paper immersed in deionised water up to the half of its thickness, then weighed at fixed intervals of time, until $48 \mathrm{~h}$. The ratio of protection by capillarity $(R p \%)$ was calculated as:

$$
R p \%=\frac{Q_{\mathrm{UT}}-Q_{\mathrm{T}}}{Q_{\mathrm{UT}}} \times 100,
$$

where $Q_{\mathrm{UT}}$ and $Q_{\mathrm{T}}$ are, respectively, the mean mass of water absorbed by the untreated and the treated sample at the time when, according to [42], the plateau of absorption is reached. The ratio of protection by capillarity ( $R p \%$ ) was also calculated referring to $48 \mathrm{~h}$ of test. As $R p$ is calculated from the mean mass of water absorbed by the untreated and treated samples (two duplicate samples for each condition), the standard deviation is not reported for this parameter in Tables 3, 4, 13 and 14. 


\subsubsection{Contact Angle Measurement}

Samples used for the static and dynamic contact angle measurements were slabs sized $30 \times 25 \times 2.5 \mathrm{~mm}^{3}$, in which all the faces were treated. To eliminate powder or non-adherent particles from the surfaces, samples were gently sprayed with clean compressed air before testing.

The static contact angle measurement was performed by using water as the drop phase; the sessile drop method was used, and drop profiles were analysed by means of a OCA system (Dataphysics Contact angle system, software SCA20, Filderstadt, Germany); a drop volume of $3 \mu \mathrm{L}$ was used. Results are the mean of at least 10 measurements carried out on different points of the stones' surfaces.

Dynamic contact angle measurement was performed at room temperature using a force tensiometer Sigma 700 (Biolin Scientific, Västra Frölunda, Sweden) and the results were elaborated by One Attension software (Biolin Scientific) for the calculation of the advancing $\left(\theta_{\text {adv }}\right)$ and receding $\left(\theta_{\text {rec }}\right)$ contact angles, considering the steady-state conditions.

The tensiometer measures the variations of force that occur during the sample immersion and emersion from water. These variations are correlated to buoyancy and to the surface tension, as represented in Figure 13 [37]. The water surface tension acts along the immersed perimeter of the stone samples and it is tilted with respect to the $\mathrm{z}$ axis of $\theta_{\mathrm{adv}}$, during immersion, and $\theta_{\text {rec }}$, during emersion. In correspondence of the zero depth of immersion the buoyancy is equal to zero. The instrument returns a graph that has on the $x$ axis the depth of the immersion of the sample in water and, on the $y$ axis, the value of the force $(F)$ recorded during the test divided for the wet perimeter of the sample $(L)$. Carrying only the linear trend of the force variations to the zero depth of immersion, the extrapolated value of force $(F)$ depends only on the surface tension of water $(\gamma)$ acting along the wet perimeter $(L)$ and projected in the direction of the force measurement:

$$
F_{0}=L \gamma \cos \theta
$$

The contact angle is the only unknown parameter and can be calculated by applying the reverse equation:

$$
\theta=\arccos \frac{F_{0}}{L \gamma} \text {. }
$$

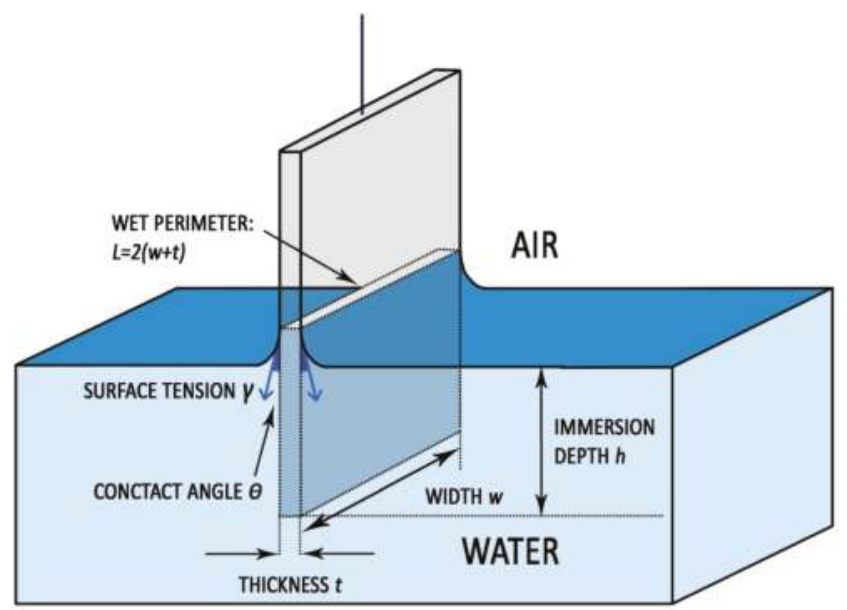

Figure 13. Schematic representation of surface tension acting during the sample immersion and emersion in water by means of force tensiometer when $\theta<90^{\circ}$. For clarity's sake, the surface tension effect was represented only for the longer sides of the wet perimeter. 
The immersion and emersion speed was set at $10 \mathrm{~mm} / \mathrm{min}$, while the immersion depth was fixed at $10 \mathrm{~mm}$, in order to obtain sufficiently averaged results (and for this reason, no standard deviation values are reported in Tables 5-7). Although some researchers recommend a lower speed of immersion/emersion $[2,9,37,43]$, the value of $10 \mathrm{~mm} / \mathrm{min}$ was selected here to reduce the effect connected to some possible water absorption during the test.

Differently from the static contact angle measurement, the dynamic one gives actually a range, where the advancing and the receding contact angles represent, respectively, the maximum and minimum values that apparent contact angle may assume, and this provides a more complete understanding of sample wettability. Moreover, the results are averaged along the entire surface immersed in water; hence, they are expected to overcome some of the problems connected to punctual measurement.

\subsubsection{Colour Measurement}

Colour measurements were performed on slabs $\left(30 \times 20 \times 2.5 \mathrm{~mm}^{3}\right)$, before and after the application of the treatments. For this purpose, a portable spectrophotometer with sphere geometry (model SP62, X-rite, Grand Rapids, MI, USA) with an aperture of $8 \mathrm{~mm}$ was used. The colour alteration $\left(\Delta E^{*}\right)$ produced on each stone by the treatments was determined using the $L^{*}, a^{*}$ and $b^{*}$ coordinates in the CIELAB space [44].

For each sample, two measurements of $L^{*}, a^{*}$ and $b^{*}$ were collected both before and after the treatment, and the mean values of $L^{*}, a^{*}$ and $b^{*}$ were calculated. From these latter values the colour alteration $\left(\Delta E^{*}\right)$ was calculated. The same procedure was applied to the sample duplicate and the average colour alteration was then calculated.

\subsubsection{Water Vapour Diffusion Test}

Water vapour diffusion test was performed using the "wet-cup method" according to ISO 7783 [45]. For each combination of formulation and application method, the test was performed on one prismatic sample $\left(50 \times 50 \times 20 \mathrm{~mm}^{3}\right)$. Given the large area under testing, one sample was considered representative for investigating the water vapour diffusion of the untreated and treated stones. The water vapour transmission rate of each coating, $V\left(\mathrm{~g} / \mathrm{m}^{2}\right.$.day), was calculated as a function of the water vapour transmission rate of the coating plus substrate $\left(V_{\mathrm{CS}}\right)$ and of the water vapour transmission rate of the untreated substrate $\left(V_{S}\right)$, following the procedure proposed for non-self-supporting coatings in the cited standard:

$$
V=\frac{V_{\mathrm{CS}} \times V_{\mathrm{S}}}{V_{\mathrm{S}}-V_{\mathrm{CS}}}
$$

Moreover, the water vapour diffusion-equivalent air layer thickness, $S_{\mathrm{d}}(\mathrm{m})$, was calculated for each sample according to the equation:

$$
S_{\mathrm{d}}=\frac{\delta_{\mathrm{a}} \times \Delta_{\mathrm{PV}}}{V}
$$

where $\delta_{\mathrm{a}}$ is the water vapour permeation coefficient of air at standard temperature and pressure and $\Delta_{\mathrm{PV}}$ is the difference between the partial water vapour pressure in the test cup and that in the test enclosure. After determining $V$ and $S_{\mathrm{d}}$, it is possible to classify the transmission rate of water vapour according to EN 1062-1 [46] in:

- $\quad$ high water vapour transmission rate class $(V 1)$, if $V>150 \mathrm{~g} / \mathrm{m}^{2}$. day and $S_{\mathrm{d}}<0.14 \mathrm{~m}$;

- medium water vapour transmission rate class $(V 2)$, if $15 \mathrm{~g} / \mathrm{m}^{2}$.day $<V \leq 150 \mathrm{~g} / \mathrm{m}^{2}$.day and $0.14 \mathrm{~m} \leq S_{\mathrm{d}}<1.4 \mathrm{~m}$;

- low water vapour transmission rate class $(V 3)$ if $V \leq 15 \mathrm{~g} / \mathrm{m}^{2}$. day and $S_{\mathrm{d}} \geq 1.4 \mathrm{~m}$. 


\subsubsection{Scanning Electron Microscopy}

The morphology of the cross-section of surface treated prismatic stone samples $\left(10 \times 15 \times 10 \mathrm{~mm}^{3}\right)$ was observed by FEG-SEM (FEI Nova NanoSEM 450, Thermo Fisher Scientific, Waltham, MA, USA). Stones treated with PHB- and PHBVV-based formulations applied by poultice on one surface were observed. The cone for back-scattered electrons was set to the widest opening in order to obtain morphological images and, at the same time, the definition of the contrast in function of the chemical composition given by the back-scattered electrons (BSE) mode. The main purpose of the observation of the cross sections was to investigate not only the morphology of the coatings, but also their possible penetration in the porosity of the samples.

\subsubsection{Accelerated Ageing}

Treated samples were kept for seven days in a climatic chamber (Discovery chamber DY340, by Angelantoni Industrie S.p.A., Cimacolle, Italy, ACS Environmental testing division) at $40{ }^{\circ} \mathrm{C}$ and $60 \%$ relative humidity and subjected to solar light radiation $\left(1.2 \mathrm{~W} / \mathrm{m}^{2}\right)$ emitted by a lamp supplied with the chamber. Samples were then subjected to static contact angle measurement and to capillary water absorption testing in order to evaluate the performance of treatments after artificial ageing.

Acknowledgments: Bio-on S.p.A. is gratefully acknowledged for materials and economic support given to this research. Thanks to Micaela Degli Esposti and Alessandro Sinisi for GPC and NMR characterisation of PHAs.

Author Contributions: Elisa Franzoni and Paola Fabbri conceived the study and designed the experiments; Serena Andreotti and Micaela Degli Esposti performed the experiments; Serena Andreotti, Elisa Franzoni, Micaela Degli Esposti and Paola Fabbri analyzed the data; Serena Andreotti and Elisa Franzoni collaborated on the writing of the paper; Paola Fabbri revised the paper.

Conflicts of Interest: The authors declare no conflict of interest. The founding sponsors had no role in the design of the study; in the collection, analyses, or interpretation of data; in the writing of the manuscript, and in the decision to publish the results.

\section{References}

1. Toniolo, L.; Poli, T.; Castelvetro, V.; Manariti, A.; Chiantore, O.; Lazzari, M. Tailoring new fluorinated acrylic copolymers as protective coatings for marble. J. Cult. Herit. 2002, 3, 309-316. [CrossRef]

2. Della Volpe, C.; Penati, A.; Peruzzi, R.; Siboni, S.; Toniolo, L.; Colombo, C. The combined effect of roughness and heterogeneity on contact angles: The case of polymer coating for stone protection. J. Adhes. Sci. Technol. 2000, 14, 273-299. [CrossRef]

3. Esposito Corcione, C.; Manno, R.; Frigione, M. Sunlight curable boehmite/siloxane-modified mathacrlylic nano composites: An innovative solution for the protection of carbonate stone. Prog. Org. Coat. 2016, 97, 222-232. [CrossRef]

4. Esposito Corcione, C.; Manno, R.; Frigione, M. Novel hydrophobic free solvent UV-cured hybrid organic-inorganic mathacrylic-based coatings for porous stones. Prog. Org. Coat. 2014, 77, 803-812. [CrossRef]

5. Manodius, P.N.; Tsakalof, A.; Karapanagiotis, I.; Zuburtikudis, I.; Panayiotou, C. Fabrication of super-hydrophobic surfaces for enhanced stone protection. Surf. Coat. Technol. 2009, 203, 1322-1328. [CrossRef]

6. Manodius, P.N.; Karapanagiotis, I.; Tsakalof, A.; Zuburtikudis, I.; Kolinkeová, B.; Panayiotou, C. Super-hydrophobic films for the protection of outdoor cultural heritage assets. Appl. Phys. A-Mater. 2009, 97, 351-360. [CrossRef]

7. Stefanidou, M.; Karozou, A. Testing the effectiveness of protective coatings on traditional bricks. Constr. Build. Mater. 2016, 111, 482-487. [CrossRef]

8. Alessandrini, G.; Aglietto, M.; Castelvetro, V.; Ciardelli, F.; Peruzzi, R.; Toniolo, L. Comparative evaluation of fluorinated and un fluorinated acrylic copolymers as water-repellent coating materials for stone. J. Appl. Polym. Sci. 2000, 76, 962-977. [CrossRef] 
9. Brugnara, M.; Degasperi, E.; Della Volpe, C.; Maniglio, D.; Penati, A.; Siboni, S.; Toniolo, L.; Poli, T.; Invernizzi, S.; Castelvetro, V. The application of the contact angle in monument protection: New materials and methods. Colloid Surf. A 2004, 241, 299-312. [CrossRef]

10. Kapridaki, C.; Maravelaki-Kalaitzaki, P. $\mathrm{TiO}_{2}-\mathrm{SiO}_{2}-\mathrm{PDMS}$ nano-composite hydrophobic coating with self-cleaning properties for marble protection. Prog. Org. Coat. 2013, 76, 400-410. [CrossRef]

11. Licchelli, M.; Malagodi, M.; Weththimuni, M.L.; Zanchi, C. Water-repellent properties of fluoroelastomers on a very porous stone: Effect of the application procedure. Prog. Org. Coat. 2013, 76, 495-503. [CrossRef]

12. Matteini, M. Inorganic treatments for the consolidation and protection of stone artifacts. Conserv. Sci. Cult. Herit. 2008, 8, 13-27.

13. Graziani, G.; Sassoni, E.; Franzoni, E.; Scherer, G.W. Hydroxyapatite coatings for marble protection: Optimization of calcite covering and acid resistance. Appl. Surf. Sci. 2016, 368, 241-257. [CrossRef]

14. Manodius, P.N.; Karapanagiotis, I. Modification of the wettability of polymer surfaces using nanoparticles. Prog. Org. Coat. 2014, 77, 331-338. [CrossRef]

15. Munafò, P.; Goffredo, G.B.; Quagliarini, E. $\mathrm{TiO}_{2}$-based nanocoatings for preserving architectural stone surfaces: An overview. Constr. Build. Mater. 2015, 84, 201-218. [CrossRef]

16. Quagliarini, E.; Bondioli, F.; Goffredo, G.B.; Licciulli, A.; Munafò, P. Self-cleaning materials on architectural heritage: Compatibility of photo-induced hydrophilicity of $\mathrm{TiO}_{2}$ coatings on stone surfaces. J. Cult. Herit. 2013, 14, 1-7. [CrossRef]

17. Colangiuli, D.; Calia, A.; Bianco, N. Novel multifunctional coatings with photocatalytic and hydrophobic properties for the preservation of stone building heritage. Constr. Build. Mater. 2015, 93, 189-196. [CrossRef]

18. Conti, C.; Colombo, C.; Dellasega, D.; Matteini, M.; Realini, M.; Zerbi, G. Ammonium oxalate treatment: Evaluation by $\mu$ Raman mapping of the penetration depth in different plasters. J. Cult. Herit. 2012, 12, 346-355. [CrossRef]

19. Ministero della Pubblica Istruzione. Carta Italiana del Restauro; Circolare No. 117; Ministero della Pubblica Istruzione: Rome, Italy, 6 April 1972. (In Italian)

20. De Naeyer, A.; Arroyo, S.P.; Blanco, J.R. Krakow Charter 2000: Principles for Conservation and Restoration of Built Heritage; Bureau Krakow 2000: Krakow, Poland, 2000.

21. Doehne, E.; Price, C.A. Stone Conservation: An Overview of Current Research, 2nd ed.; The Getty Conservation Institute: Los Angeles, CA, USA, 2010.

22. Favaro, M.; Mendichi, R.; Ossola, F.; Russo, U.; Simon, S.; Tomasin, P.; Vigato, P.A. Evaluation of polymers for conservation treatments of outdoor exposed stone monuments. Part I: Photo-oxidative weathering. Polym. Degrad. Stab. 2006, 91, 3083-3096. [CrossRef]

23. Favaro, M.; Mendichi, R.; Ossola, F.; Russo, U.; Simon, S.; Tomasin, P.; Vigato, P.A. Evaluation of polymers for conservation treatments of outdoor exposed stone monuments. Part II: Photo-oxidative and salt-induced weathering of acrylic-silicone mixtures. Polym. Degrad. Stab. 2007, 92, 335-351. [CrossRef]

24. Lazzarini, L.; Laurenzi Tabasso, M. Il Restauro Della Pietra; Utet Scienze Tecniche: Torino, Italy, 2010. (In Italian)

25. Moreau, C.; Leroux, L.; Vergès-Belmin, V.; Fronteau, G.; Barbin, V. Which Factors Influence Most the Durability of Water Repellent Treatments: Stone Properties, Climate or Atmospheric Pollution? In Proceedings of the Hydrophobe V, 5th International Conference on Water Repellent Treatment of Building Materials, Brussels, Belgium, 15-16 April 2008; pp. 129-142.

26. Ockack, Y.; Sofuoglu, A.; Tihminlioglu, F.; Böke, H. Protection of marble surfaces by using biodegradable polymers as coating agent. Prog. Org. Coat. 2009, 66, 213-220. [CrossRef]

27. Frediani, M.; Rosi, L.; Camaiti, M.; Berti, D.; Mariotti, A.; Comucci, A.; Vannucci, C.; Malesci, I. Polylactide/Perfluoropolyether Block Copolymers: Potential Candidates for Protective and Surface Modifiers. Macromol. Chem. Phys. 2010, 211, 988-995. [CrossRef]

28. Giuntoli, G.; Rosi, L.; Frediani, M.; Sacchi, B.; Frediani, P. Fluoro-Functionalized PLA Polymers as Potential Water-Repellent Coating Materials for Protection of Stone. J. Appl. Polym. Sci. 2012, 125, 3125-3133. [CrossRef]

29. Ockack, Y.; Sofuoglu, A.; Tihminlioglu, F.; Böke, H. Suistanable bio-nano composite coatings for the protection of marble surfaces. J. Cult. Herit. 2015, 16, 299-306. [CrossRef]

30. Pedna, A.; Pinho, L.; Frediani, P.; Mosquera, M.J. Obtaining $\mathrm{SiO}_{2}$-fluorinated PLA bionanocomposites with application as reversible and highly-hydrophobic coatings of buildings. Prog. Org. Coat. 2016, 90, 91-100. [CrossRef] 
31. Khanna, S.; Srivastava, A.K. Recent advances in microbial polyhydroxyalkanoates. Process Biochem. 2005, 40, 607-619. [CrossRef]

32. Fiorese, M.L.; Freitas, F.; Pais, J.; Ramos, A.M.; de Aragão, G.M.F. Recovery of polyhydroxybutyrate (PHB) from Cupriavidus necator biomass by solvent extraction with 1,2-propylene carbonate. Eng. Life Sci. 2009, 9, 454-461. [CrossRef]

33. Bugnicourt, E.; Cinelli, P.; Lazzeri, A.; Alvarez, V. Polyhydroxyalkanoate (PHA): Review of synthesis, characteristics, processing and potential application in packaging. Express Polym. Lett. 2014, 8, 791-808. [CrossRef]

34. Chen, G.-Q. Plastics Completely Synthesized by Bacteria: Polyhydroxyalkanoates. Microbiol. Monogr. 2010, 14, 17-37.

35. El-Hadi, A.; Schnabel, R.; Straube, E.; Müller, G.; Henning, S. Correlation between degree of crystallinity, morphology, glass temperature, mechanical properties and biodegradation of poly (3-hydroxyalkanoate) PHAs and their blends. Polym. Test. 2002, 21, 665-674. [CrossRef]

36. Benavente, D. Why Pore Size Is Important in the Deterioration of Porous Stones Used in the Built Heritage. Macla 2011, 15, 41-42.

37. Brugnara, M.; Della Volpe, C.; Penati, A.; Siboni, S.; Poli, T.; Toniolo, L. Correct use of the contact angle in the evaluation of the protective action induced from polymer coating on the stone. Annali di Chimica-Società Chimica Italiana 2003, 93, 881-888.

38. Meiron, T.S.; Marmur, A.; Saguy, I.S. Contact angle measurement on rough surfaces. J. Colloid Interface Sci. 2004, 274, 637-644. [CrossRef] [PubMed]

39. Graziani, G.; Sassoni, E.; Scherer, G.W.; Franzoni, E. The application of hydroxyapatite-based treatments to salt-bearing porous limestones: A study on sodium sulphate-contaminated Lecce stone. In Proceedings of the SWBSS 2017, 4rd International Conference on Salt Weathering of Buildings and Stone Sculptures, Potsdam, Germany, 20-22 September 2017; pp. 176-186.

40. Leone, G.; Leoni, L.; Sartori, F. Revisione di un metodo gasometrico per la determinazione di calcite e dolomite. Atti Soc. Toscana Sci. Nat. Mem. Ser. A 1988, 95, 7-20.

41. European Norm EN 15801:2009. Conservation of Cultural Property, Test Methods, Determination of Water Absorption by Capillarity; CEN (European Committee for Standardization): Brussels, Belgium, 2009.

42. Italian Standard UNI 10921:2001. Beni Culturali-Materiali Lapidei Naturali ed Artificiali-Prodotti Idrorepellenti-Applicazione su Provini e Determinazione in Laboratorio Delle Loro Caratteristiche; UNI (Ente Italiano di Normazione): Milan/Italy, taly, 2001.

43. Wang, C.; Nair, S.; Wynne, K.J. Wilhelmy balance characterization beyond contact angles: Differentiating leaching from nanosurface reorganization and optimizing surface modification. Polymer 2017, 116, 565-571. [CrossRef]

44. Gómez-Polo, C.; Portillo Muñoz, M.; Cruz Lorenzo Luengo, M.; Vicente, P.; Galindo, P.; Martín Casado, A.M. Comparison of the CIELab and CIEDE2000 color difference formulas. J. Prosthet. Dent. 2016, 115, 65-70. [CrossRef] [PubMed]

45. International Standard. Standard ISO 7783:2011. Paints and Varnishes-Determination of Water-Vapour Transmission Properties-Cup Method; ISO (International Organization for Standardization): Geneva, Switzerland, 2011.

46. European Norm EN 1062-1:2015. Paints and Varnishes-Coating Materials and Coating Systems for Exterior Masonry and Concrete-Part 1: Classification; CEN (European Committee for Standardization): Brussels, Belgium, 2015.

(C) 2018 by the authors. Licensee MDPI, Basel, Switzerland. This article is an open access article distributed under the terms and conditions of the Creative Commons Attribution (CC BY) license (http:/ / creativecommons.org/licenses/by/4.0/). 


\title{
Article \\ Smart Metamaterial Based on the Simplex Tensegrity Pattern
}

\author{
Anna Al Sabouni-Zawadzka * and Wojciech Gilewski \\ Faculty of Civil Engineering, Warsaw University of Technology, 00-661 Warsaw, Poland; w.gilewski@il.pw.edu.pl \\ * Correspondence: a.sabouni@il.pw.edu.pl; Tel.: +48-22-234-5753
}

Received: 20 February 2018; Accepted: 23 April 2018; Published: 26 April 2018

\begin{abstract}
In the present paper, a novel cellular metamaterial that was based on a tensegrity pattern is presented. The material is constructed from supercells, each of which consists of eight 4-strut simplex modules. The proposed metamaterial exhibits some unusual properties, which are typical for smart structures. It is possible to control its mechanical characteristics by adjusting the level of self-stress or by changing the properties of structural members. A continuum model is used to identify the qualitative properties of the considered metamaterial, and to estimate how the applied self-stress and the characteristics of cables and struts affect the whole structure. The performed analyses proved that the proposed structure can be regarded as a smart metamaterial with orthotropic properties. One of its most important features are unique values of Poisson's ratio, which can be either positive or negative, depending on the applied control parameters. Moreover, all of the mechanical characteristics of the proposed metamaterial are prone to structural control.
\end{abstract}

Keywords: metamaterial; smart material; smart structure; tensegrity

\section{Introduction}

Metamaterials are usually defined as man-designed and man-made, which are not observed in nature, composite structures with unusual or non-typical properties [1-3]. Features of metamaterials are determined mainly by morphology of the structure in the scale bigger than molecular, and in smaller degree by chemical or phase composition. This area has been under considerable and important scientific research in recent years. Many state-of the-art applications refer to electromagnetic waves and phenomena [4-6], solar photovoltaic cells and panels [7,8], energy absorption, including seismic [9] and acoustic waves [10,11], and mechanical metamaterials [12-16] (for example, with unusual dynamic properties, negative Poisson's ratio, non-typical modulus of extension and volumetric changes, ultra-light, and ultra-stiff materials).

Smart materials are the materials that are able to convert one form of energy (mechanical, magnetic, electrical, etc.) into another in a reversible and repeatable process [17,18]. They are capable of sensing changes in the environmental conditions, responding to them in a predetermined manner, in an appropriate time and returning to their original shape as soon as the stimulus is removed.

Smart structures are the structures with the ability to sense and respond adaptively to changes in their environment $[19,20]$. This feature distinguishes them from the conventional ones. Whereas, the main purpose of the traditional structures is to provide strength and carry loads acting on them, the smart ones adapt in a pre-designed manner to a functional need, modifying their shape, stiffness, or damping characteristics in order to minimize the deflection and possible damage.

In the context of the above definitions, the metamaterial with smart features is more close the term smart structure than smart material. 
Standard engineering materials when compressed along a particular direction are the most commonly observed to expand in the directions orthogonal to the applied load. This property is measured by a Poisson's ratio, which is a good example to characterize the mechanical metamaterial. Positive Poisson's ratio in the range of 0.0 to 0.5 is observed for the majority of engineering materials, which means that it is a typical material property. However, theory of elasticity permits negative values and for anisotropic materials also the coefficients bigger than 0.5. Negative Poisson's ratios are not observed in nature and this is why metamaterials with such a property are being looked for. An interesting group of up to date efforts in this area are metamaterials that are based on the origami patterns [21-26], inspired by the art of paper folding. The most efficient is the Miura-Ori origami pattern.

Another interesting type of structures that allow for building materials with negative Poisson's ratios are tensegrities. The term "tensegrity" was first introduced by Buckminster Fuller (see [27] for historical details). Several definitions of this concept can be found in the literature [27-29]. For the purpose of this paper, a tensegrity structure is defined as a pin-jointed system with a particular configuration of cables and struts that form a statically indeterminate structure in a stable equilibrium. Tensegrities consist of a discontinuous set of compressed elements inside a continuous net of tensioned members, which have no compressive stiffness. Infinitesimal mechanisms, which occur in tensegrity structures, are balanced with self-stress states [30,31]. Occurrence of a self-stress state in a structure indicates that there is a certain set of internal forces in structural members, which are independent from external loading and boundary conditions because they are in self-equilibrium.

To major advantages of tensegrity systems belong: large stiffness-to-mass ratio, deployability, reliability, controllability $[27,28]$, as well as programmable deployment [32]. Moreover, tensegrities have some unique features that result from the infinitesimal mechanisms, which are stabilized by self-stress forces. It is possible to control their static and dynamic properties by adjusting the pre-stressing forces [33-36].

As it was presented in [37], there are some particular features of tensegrity structures, following which one can classify them as smart structures. There are: self-control, self-diagnosis, self-repair, and self-adjustment (active control).

Self-control of tensegrity systems consists in self-stiffening of the structures under the applied load that causes displacements consistent with the infinitesimal mechanism mode. External loading acts similarly to the self-stress-it eliminates singularity of the problem, additionally, pre-stresses the structure and stiffens it. Self-diagnosis relates to the possibility of damage detection and identification by measuring the internal forces in active members. Damage of one structural element affects the distribution and level of self-stress in the whole structure. Self-repair of tensegrity structures is realized by adjusting self-stress forces. A proper change of pre-stressing level can compensate the damaged element and restore the values of structural displacements from before damage. Self-adjustment (active control) in regard to tensegrity systems is related with the ability of self-adjustment through self-stress forces. Both the pre-stressing of the whole structure and its part causes a stiffening of the system and the reduction of its displacements. Therefore, active control of tensegrities can be realized by adjusting the level of self-stress in only one selected part of the structure.

The objective of the present paper is to develop a metamaterial based on the 4-strut simplex tensegrity module [38], which was exhibiting the smart structure features. A continuum model [39,40] is applied to identify the qualitative properties of the proposed metamaterial. Its mechanical characteristics can be controlled with the self-stress state and cable to strut properties ratio, including positive or negative values of Poisson's ratio. According to the best knowledge of the authors, there are almost no papers in this field in the available literature, with the first valuable attempt on the mechanical response of three-dimensional (3D) tensegrity lattices by [41]. 


\section{Unit Cell}

The proposed metamaterial is based on one of the best known tensegrity modules-a 4-strut simplex (Figure 1). As all typical tensegrity structures, it is a pin-jointed system consisting of isolated compressed elements (four struts) inside a continuous net of tensioned members (twelve cables) [27-29]. The 4-strut simplex module is obtained from a regular prism by rotating one of its bases 135 degrees clockwise or counter clockwise.
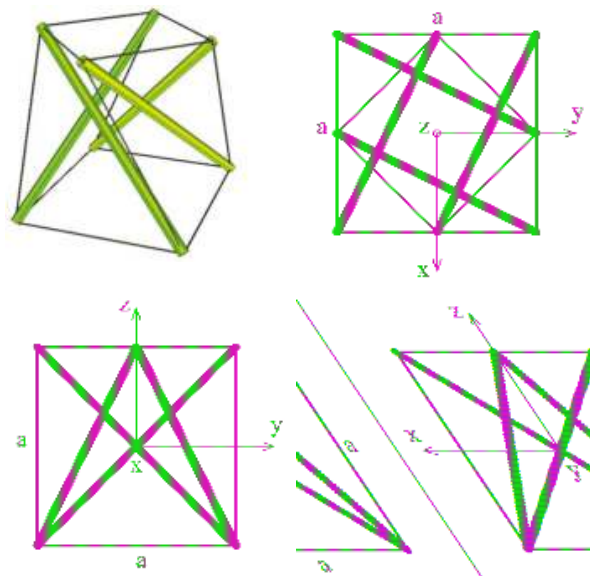

Figure 1. Geometry of a unit cell—a 4-strut simplex module-axonometry and three views.

One of the unique features of tensegrities are infinitesimal mechanisms that are balanced with self-stress states [30,38]. The considered simplex module has one infinitesimal mechanism (Figure 2a) and one corresponding self-stress state (Figure 2b) - self-stress is expressed by the relative forces in struts and cables with a multiplier $S_{0}$, which can be any positive real number.

(a)

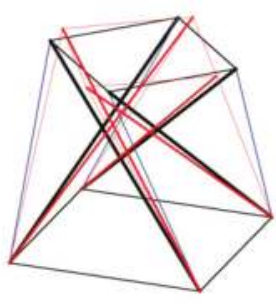

(b)

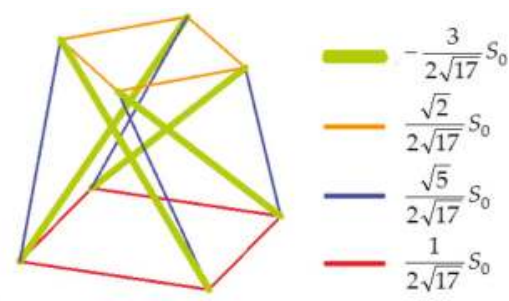

Figure 2. 4-strut simplex: (a) Infinitesimal mechanism; and (b) self-stress state.

Any material or system that is based on tensegrity is complicated regarding both its geometry and mechanics. Therefore, it is often difficult or even impossible to determine and understand its properties using typical tools. Standard methods, such as the finite element method, enable analysing any kind of structure, but they do not explain if the analysed system exhibits anisotropic, orthotropic properties, or is characterized by some other type of elastic symmetry. Using such methods, it is also hard to describe unusual properties in the sense of a metamaterial.

In order to analyse all of the untypical, unique features of tensegrity systems and to identify their properties, a continuum model $[39,40]$ is suggested. The aim of the proposed model is to facilitate the identification and the understanding of mechanical characteristics of tensegrities through the 
qualitative comparison with a continuum body $[42,43]$ with equivalent features. The model is built by assuming that the strain energy of an unsupported tensegrity structure is equivalent to the strain energy of a solid $[39,40]$.

The strain energy of a tensegrity truss, according to the finite element method (FEM), is a quadratic form of nodal displacements q:

$$
E_{S}^{F E M}=\frac{1}{2} \mathbf{q}^{T} \mathbf{K} \mathbf{q}
$$

where $\mathbf{K}=\mathbf{K}_{L}+\mathbf{K}_{G}, \mathbf{K}_{L}$-global linear stiffness matrix, $\mathbf{K}_{G}$-global geometric stiffness matrix. The self-stress of the structure is represented by the geometric stiffness matrix. The strain energy of a solid, according to the symmetric linear 3D elasticity theory (LTE), can be expressed as:

$$
E_{s}^{L T E}=\frac{1}{2} \int_{V} \varepsilon^{T} \mathbf{E} \varepsilon d V,
$$

where $\varepsilon$-vector of strain components (containing normal strains and shear strains), $\mathrm{E}-6 \times 6$ elasticity matrix in Voight's notation [42] (including 21 independent coefficients). In order to analyse mechanical properties of the structure, such as Young's moduli, shear moduli, Poisson's ratios, etc., the strain energy of an unsupported tensegrity structure is compared to the strain energy of a cube of edge length a. It is assumed that the strain energy of the cube is constant in its whole volume. To compare the energies and to build the equivalent elasticity matrix, the nodal displacements $\mathbf{q}$ of the structure are expressed by the average mid-values of displacements and their derivatives in the centre of the cube of edge length $a$, with the use of Taylor series expansion. In case of small values of $a$, terms with the factor $a^{n}(n>1)$ can be regarded as higher order terms and are omitted. This assumption leads, in a quantitative sense, to the infinitesimal model. Mechanical characteristics can be determined from the inverse elasticity matrix $\mathbf{H}=\mathbf{E}^{-1}[42]$ :

$$
\mathbf{H}=\left[\begin{array}{cccccc}
\frac{1}{E_{1}} & -\frac{V_{21}}{E_{2}} & -\frac{V_{31}}{E_{3}} & \frac{\lambda_{11}}{G_{1}} & \frac{\lambda_{21}}{G_{2}} & \frac{\lambda_{31}}{G_{3}} \\
-\frac{V_{12}}{E_{1}} & \frac{1}{E_{2}} & -\frac{V_{32}}{E_{3}} & \frac{\lambda_{12}}{G_{1}} & \frac{\lambda_{22}}{G_{2}} & \frac{\lambda_{32}}{G_{3}} \\
-\frac{V_{13}}{E_{1}} & -\frac{V_{23}}{E_{2}} & \frac{1}{E_{3}} & \frac{\lambda_{13}}{G_{1}} & \frac{\lambda_{23}}{G_{2}} & \frac{\lambda_{33}}{G_{3}} \\
\frac{\kappa_{11}}{E_{1}} & \frac{\kappa_{21}}{E_{2}} & \frac{K_{31}}{E_{3}} & \frac{1}{G_{1}} & \frac{\mu_{21}}{G_{2}} & \frac{\mu_{31}}{G_{3}} \\
\frac{\kappa_{12}}{E_{1}} & \frac{\mathcal{2 n}_{22}}{E_{2}} & \frac{\mathcal{K}_{32}}{E_{3}} & \frac{\mu_{12}}{G_{1}} & \frac{1}{G_{2}} & \frac{\mu_{32}}{G_{3}} \\
\frac{K_{13}}{E_{1}} & \frac{K_{23}}{E_{2}} & \frac{\kappa_{33}}{E_{3}} & \frac{\mu_{13}}{G_{1}} & \frac{\mu_{23}}{G_{2}} & \frac{1}{G_{3}}
\end{array}\right] .
$$

The following technical coefficients can be defined for anisotropic body: Young's moduli $(E)$, shear moduli $(G)$, Poisson's ratios $(v)$, coefficients $(\mu)$-relations between shear strains in perpendicular directions, coefficients $(\lambda)$-relations between normal strains in three directions and shear strains in one direction, and coefficients $(\kappa)$-relations between shear strains in three directions and normal strains in one direction.

Moreover, limiting conditions for the values of mechanical characteristics can be found using the assumption that both matrices $\mathbf{E}$ and $\mathbf{H}$ have to be positive definite (see $[44,45]$ for details).

The continuum analysis of tensegrities allows for: estimate the influence of self-stress on the behaviour of the system, assess how the characteristics of cables and struts affect the whole structure and determine the mechanical characteristics of materials or structures that are based on tensegrity modules.

In the continuum analysis of a unit cell, a 4-strut simplex module inscribed into a cube of edge length $a$ (Figure 1) was considered. The module itself is an anisotropic structure, but as proved in the following sections it can be arranged in such a way that the material based on a simplex tensegrity pattern has orthotropic properties. 
The elasticity matrix E obtained for the analysed module has the following form:

$$
\mathbf{E}=\left[\begin{array}{cccccc}
e_{11} & e_{12} & e_{13} & e_{14} & 0 & 0 \\
& e_{11} & e_{13} & -e_{14} & 0 & 0 \\
& & e_{33} & 0 & 0 & 0 \\
& & & e_{12} & 0 & 0 \\
& & & & e_{13} & 0 \\
\text { sym. } & & & & & e_{13}
\end{array}\right]
$$

where:

$$
\begin{aligned}
& e_{11}=\frac{2 E A}{a^{2}}(0.314815+1.39827 \cdot k-0.0794978 \cdot \sigma), \\
& e_{12}=\frac{E A}{a^{2}}(0.296296+0.707107 \cdot k-0.0134742 \cdot \sigma), \\
& e_{13}=\frac{E A}{a^{2}}(0.740741+0.357771 \cdot k+0.17247 \cdot \sigma), \\
& e_{14}=\frac{E A}{a^{2}}(-0.2222222-0.0808452 \cdot \sigma), \\
& e_{33}=\frac{2 E A}{a^{2}}(0.592593+1.43108 \cdot k-0.17247 \cdot \sigma), \\
& k=\frac{(E A)_{\text {cable }}}{(E A)_{\text {strut }}},(E A)_{\text {strut }}=E A, \sigma=\frac{S}{E A},
\end{aligned}
$$

$E$-Young's modulus of the strut, $A$-cross-sectional area of the strut.

Two characteristic parameters were used in the analysis: $k$ and $\sigma$. Parameter $k$ is defined as a ratio between the stiffness of cables and struts, parameter $\sigma$ determines the level of self-stress.

It should be noticed that the proposed unit cell is an anisotropic structure, as there are non-zero coefficients $e_{14}$ and $e_{24}=e_{14}$ in the determined elasticity matrix (4). However, it is proved in the following sections that the material that is based on such unit cells exhibits orthotropic properties.

\section{Tensegrity Cellular Material}

Simplex tensegrity modules that are described in the previous section can be arranged in different patterns to form a material with certain properties. Depending on the type of the module used (with the basis rotated clockwise or counter clockwise) and the way in which the modules are connected, a material with different mechanical characteristics can be obtained. In the present paper, a material with orthotropic properties is proposed, as it exhibits some special features, such as a negative Poisson's ratio.

Figure 3 presents a system consisting of four simplex modules that are connected through common cables of the lower bases and common nodes of the upper bases.

The following elasticity matrix $\mathbf{E}$ was determined for the analysed system using the continuum approach:

$$
\mathbf{E}=\left[\begin{array}{cccccc}
e_{11} & e_{12} & e_{13} & 0 & 0 & 0 \\
& e_{11} & e_{13} & 0 & 0 & 0 \\
& & e_{33} & 0 & 0 & 0 \\
& & & e_{12} & 0 & 0 \\
& & & & e_{13} & 0 \\
\text { sym. } & & & & & e_{13}
\end{array}\right]
$$

where:

$$
\begin{aligned}
& e_{11}=\frac{2 E A}{a^{2}}(0.314815+1.13709 \cdot k-0.0794978 \cdot \sigma), \\
& e_{12}=\frac{E A}{a^{2}}(0.296296+0.707107 \cdot k-0.0134742 \cdot \sigma), \\
& e_{13}=\frac{E A}{a^{2}}(0.740741+0.268328 \cdot k+0.17247 \cdot \sigma), \\
& e_{33}=\frac{2 E A}{a^{2}}(0.592593+1.07331 \cdot k-0.17247 \cdot \sigma) .
\end{aligned}
$$

The obtained elasticity matrix (5) indicates that the four-module layer, although based on anisotropic unit cells, exhibits orthotropic properties. 
Following this reasoning, an eight-module supercell (Figure 4), which was built from two four-module layers, was considered. The upper layer of the system was created by putting the four-module layer upside-down and connecting it with the bottom layer through common cables.
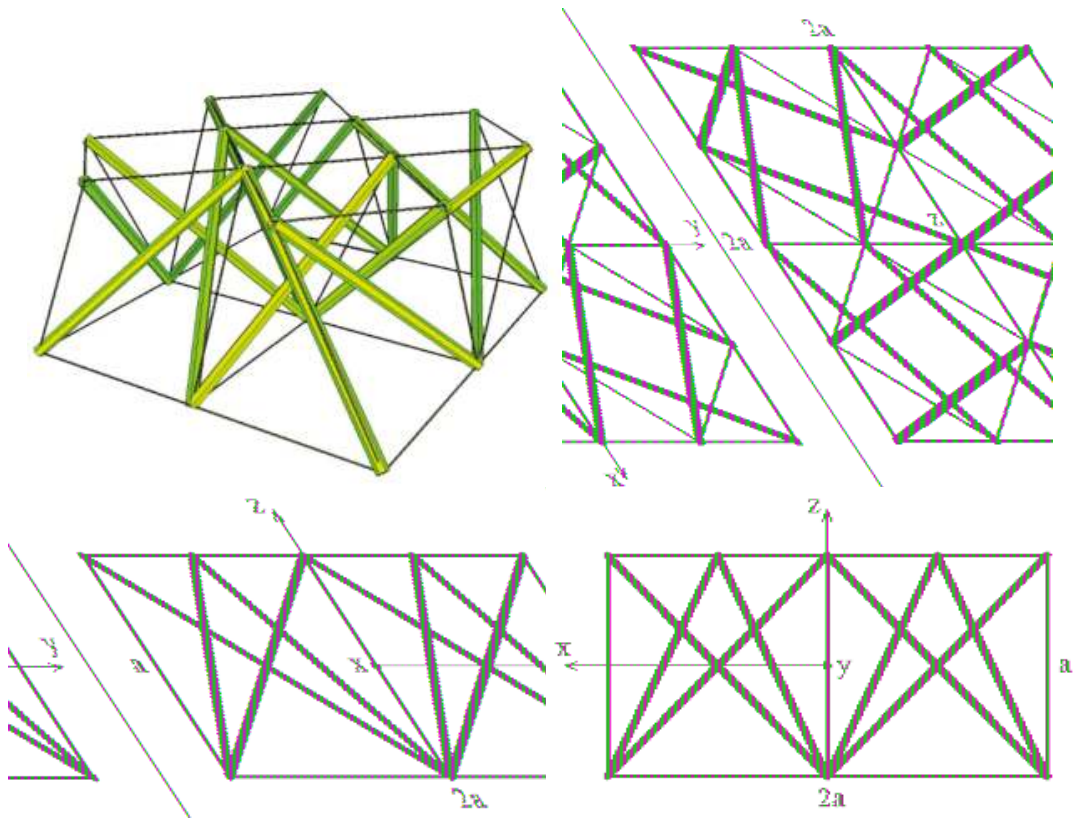

Figure 3. Geometry of a four-module layer-axonometry and three views.

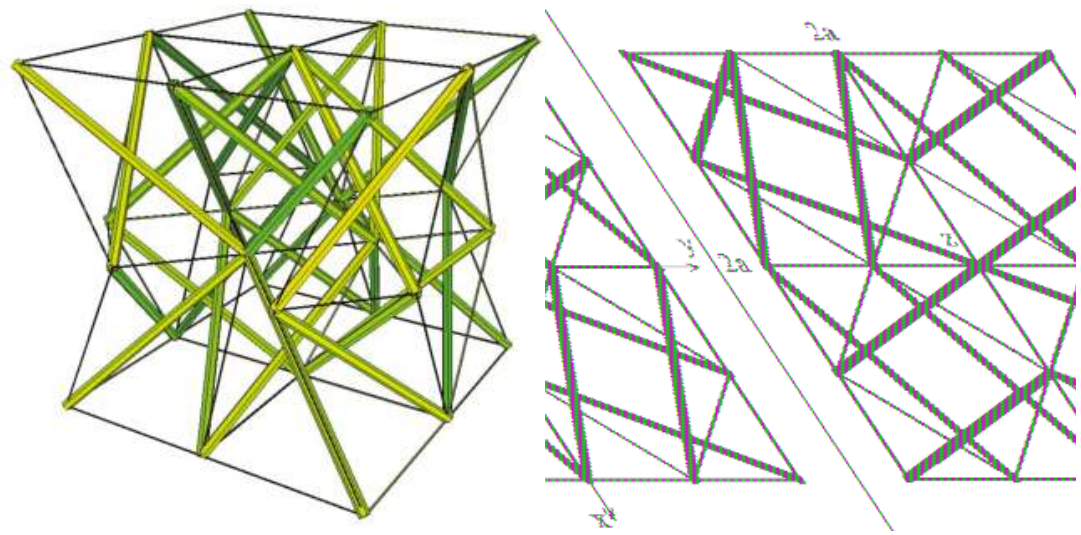

Figure 4. Cont. 

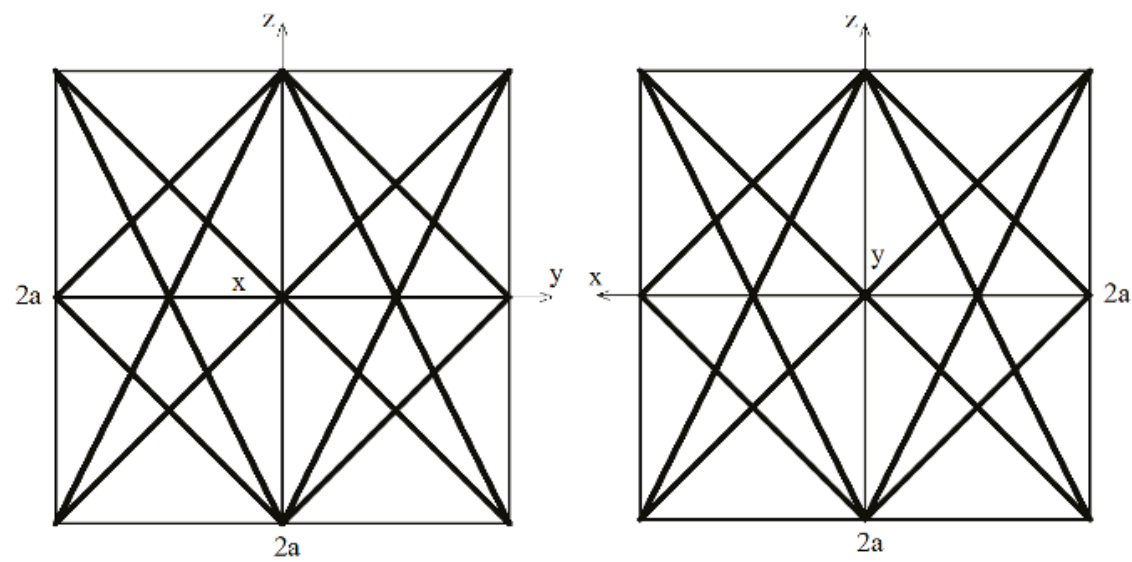

Figure 4. Geometry of an eight-module supercell-axonometry and three views.

The elasticity matrix $\mathbf{E}$ that was obtained from the continuum analysis of the considered supercell has the following form:

$$
\mathbf{E}=\left[\begin{array}{cccccc}
e_{11} & e_{12} & e_{13} & 0 & 0 & 0 \\
& e_{11} & e_{13} & 0 & 0 & 0 \\
& & e_{33} & 0 & 0 & 0 \\
& & & e_{12} & 0 & 0 \\
& & & & e_{13} & 0 \\
\text { sym. } & & & & & e_{13}
\end{array}\right]
$$

where:

$$
\begin{aligned}
& e_{11}=\frac{2 E A}{a^{2}}(0.314815+0.960318 \cdot k-0.0794978 \cdot \sigma), \\
& e_{12}=\frac{E A}{a^{2}}(0.2962963+0.353553 \cdot k-0.0134742 \cdot \sigma), \\
& e_{13}=\frac{E A}{a^{2}}(0.740741+0.268328 \cdot k+0.17247 \cdot \sigma), \\
& e_{33}=\frac{2 E A}{a^{2}}(0.592593+1.07331 \cdot k-0.17247 \cdot \sigma) .
\end{aligned}
$$

Similarly to the four-module layer, the analysed eight-module supercell has orthotropic properties. In order to find mechanical characteristics of the structure, an inverse matrix $\mathbf{H}=\mathbf{E}^{-1}$ with seven independent coefficients was determined:

$$
\mathbf{H}=\mathbf{E}^{-1}=\left[\begin{array}{cccccc}
\frac{1}{E_{1}} & -\frac{v_{12}}{E_{1}} & -\frac{v_{31}}{E_{3}} & 0 & 0 & 0 \\
-\frac{v_{12}}{E_{1}} & \frac{1}{E_{1}} & -\frac{v_{31}}{E_{3}} & 0 & 0 & 0 \\
-\frac{v_{13}}{E_{1}} & -\frac{v_{13}}{E_{1}} & \frac{1}{E_{3}} & 0 & 0 & 0 \\
0 & 0 & 0 & \frac{1}{G_{1}} & 0 & 0 \\
0 & 0 & 0 & 0 & \frac{1}{G_{2}} & 0 \\
0 & 0 & 0 & 0 & 0 & \frac{1}{G_{2}}
\end{array}\right]
$$

with the following values:

$$
\begin{aligned}
& E_{1}=E_{2}=\frac{\left(e_{11}-e_{12}\right)\left(2 e_{13}^{2}-\left(e_{11}+e_{12}\right) e_{33}\right)}{e_{13}^{2}-e_{11} e_{33}}, \\
& E_{3}=\frac{-2 e_{13}^{2}+\left(e_{11}+e_{12}\right) e_{33}}{e_{11}+e_{12}}, \\
& G_{1}=e_{12} \\
& G_{2}=G_{3}=e_{13},
\end{aligned}
$$




$$
\begin{aligned}
& v_{12}=v_{21}=\frac{e_{13}^{2}-e_{12} e_{33}}{e_{13}^{2}-e_{11} e_{33}}, \\
& v_{13}=v_{23}=\frac{-e_{13}\left(e_{11}-e_{12}\right)}{e_{13}^{2}-e_{11} e_{33}}, \\
& v_{31}=v_{32}=\frac{e_{13}}{e_{11}+e_{12}} .
\end{aligned}
$$

Symmetry of the matrix $\mathbf{H}$ generates the following condition: $v_{13} / E_{1}=v_{31} / E_{3}$. Moreover, the limiting conditions that are described in $[44,45]$ resulting from the positive definition of the matrices $\mathbf{E}$ and $\mathbf{H}$ have to be satisfied: $E_{1}>0, E_{2}>0, E_{3}>0, G_{1}>0, G_{2}>0, G_{3}>0$, and $v_{12} v_{21}<1, v_{13} v_{31}<1, v_{23} v_{32}<1, v_{12} v_{21}+v_{13} v_{31}+v_{23} v_{32}+v_{12} v_{31} v_{23}+v_{21} v_{13} v_{32}<1$.

Analysis of the above limiting conditions and the domains of the determined mechanical characteristics leads to conditions that limit the values of parameters $k$ and $\sigma$. All of the considered conditions are described by curves, which, for engineering purposes, can be approximated with the equations of straight lines (see [46] for details). A general condition that is a common domain for all of the mechanical characteristics can be written, as follows:

$$
\sigma>3.69983 k
$$

In addition to the above condition, in the analyses presented in this paper the values of $k$ and $\sigma$ are limited to: $k<1$ and $\sigma<1$. Figure 5 presents a range of possible values of the considered parameters. The boundary line of the region $\sigma>3.69983 k$ is also marked on the contour plots of the selected mechanical characteristics presented below.

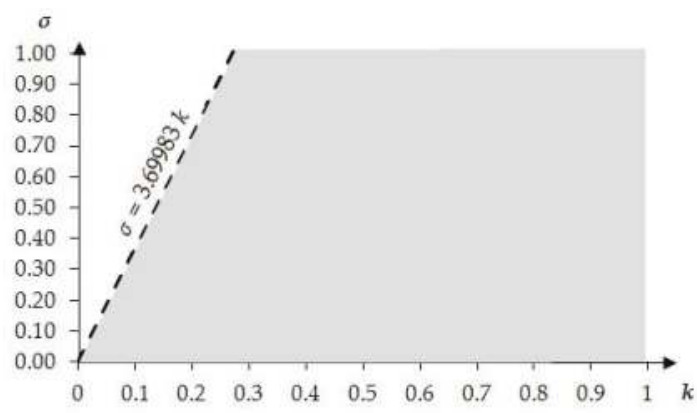

Figure 5. Range of possible values of the parameters $k$ and $\sigma$.

Using the results obtained from the presented continuum analysis, an influence of element properties and self-stress on the mechanical characteristics of the eight-module supercell can be determined. Figures 6-11 show how the selected mechanical characteristics depend on the defined parameters $k$ and $\sigma$. The values of these characteristics should be considered in the range that is shown in Figure 5. It should be noticed that all of the analysed mechanical characteristics are prone to structural control-their values might be controlled by adjusting either the properties of struts and cables or the values of prestressing forces in structural members. This feature distinguishes the proposed smart metamaterial from the traditional ones. Whereas, a typical material exhibits certain properties that are constant (assuming that the rheology is neglected), the characteristics of a smart material can be actively controlled.

Parameter $k$ depends on the physical and geometrical cable to strut ratio and can be fixed for the supercell at a certain level. Slightly different is the role of self-stress parameter $\sigma$, which can be adjusted during exploitation of the material to control the values of elastic coefficients. As it is seen in Figures 6 and 7, the influence of these parameters on the Young's and shear modulus is quite significant. 


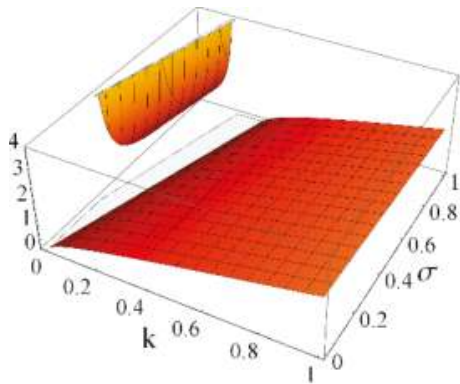

(a)

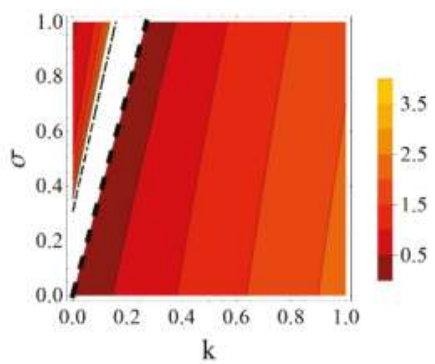

(b)

Figure 6. Young's modulus $E_{1}$ (divided by the factor $E A / a^{2}$ ) with the limiting condition $E_{1}>0$ : (a) three-dimensional (3D) plot; and (b) contour plot.

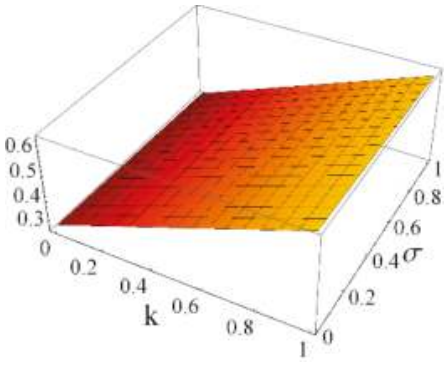

(a)

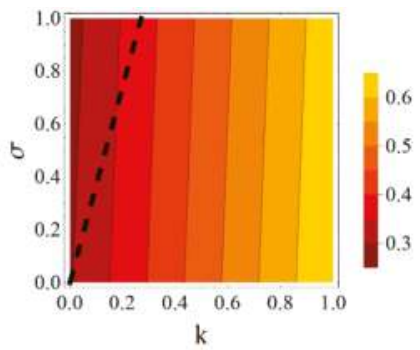

(b)

Figure 7. Shear modulus $G_{1}$ (divided by the factor $E A / a^{2}$ ) with the limiting condition $G_{1}>0$ : (a) 3D plot; and (b) contour plot.

The most important features that characterize mechanical metamaterials are negative values of Poisson's ratio. In the case of the proposed eight-module supercell Poisson's ratio $v_{12}$ (and $v_{21}=v_{12}$ ), can reach negative as well as positive values in the considered range of the parameters $k$ and $\sigma$ (Figures 8 and 9 , as an example for the fixed value of the parameter $k=0.4$ ). The influence of the self-stress parameter $\sigma$ on the value of Poisson's ratio $v_{12}$ is significant. The gaps between the positive and negative values of the Poisson's ratios $v_{12}$ and $v_{13}$ visible in Figures 8 and 10 result from the domains of these functions. However, they are not taken into account as they lie outside the considered range (Figure 5).

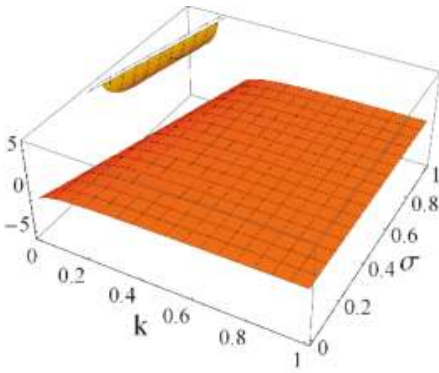

(a)

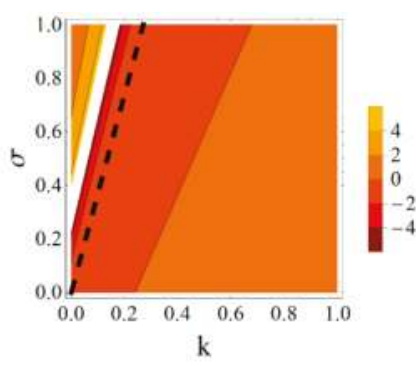

(b)

Figure 8. Poisson's ratio $v_{12}=v_{21}$ : (a) 3D plot; and, (b) contour plot. 


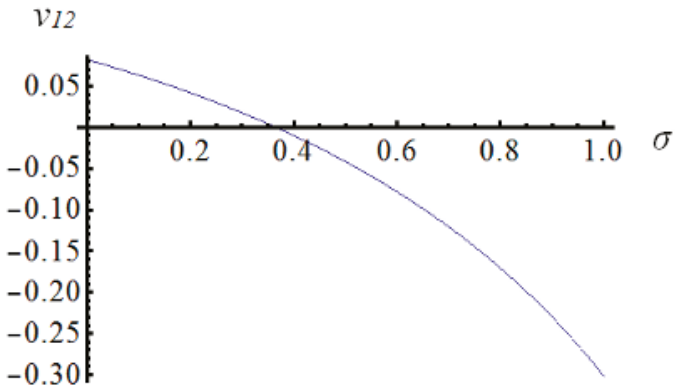

Figure 9. Poisson's ratio $v_{12}=v_{21}$ changing sign (the plot for $k=0.4$ ).

The other Poisson's ratios $v_{13}$ and $v_{31}$ are always positive (Figures 10 and 11) in the considered domain (Figure 5). While the coefficient $v_{13}$ is not very sensitive to $k$ and $\sigma$, the other one $v_{31}$ can be adjusted by changing these two parameters.

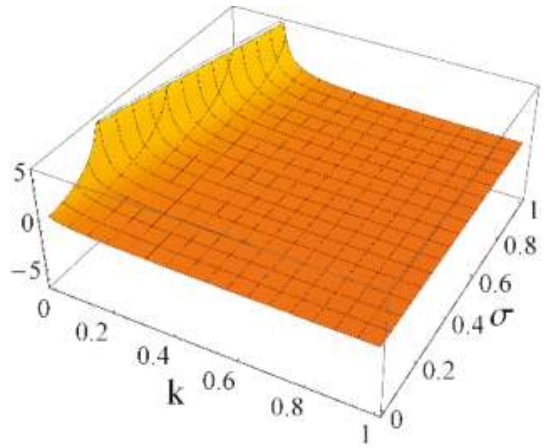

(a)

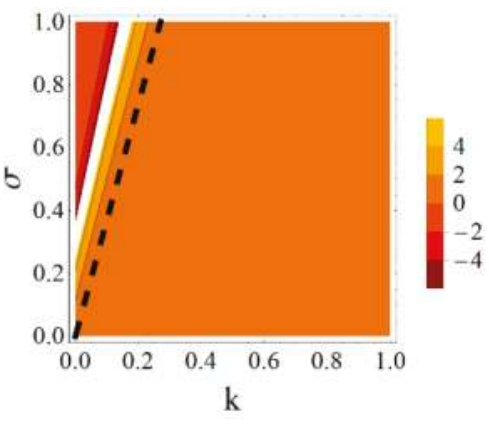

(b)

Figure 10. Poisson's ratio $v_{13}=v_{23}$ : (a) 3D plot; and (b) contour plot.

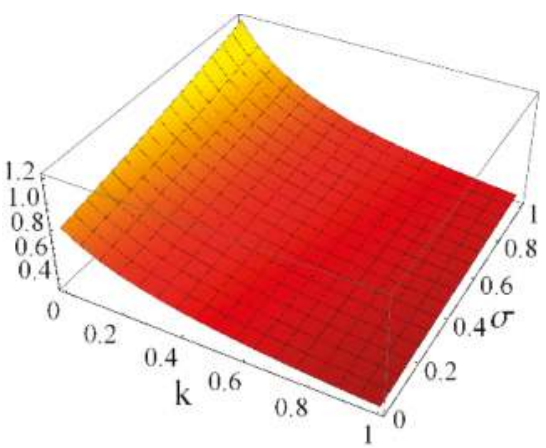

(a)

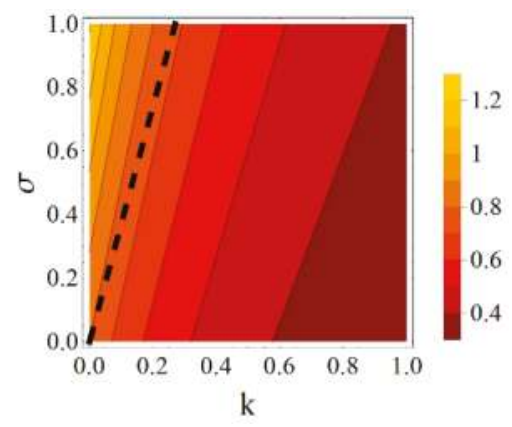

(b)

Figure 11. Poisson's ratio $v_{31}=v_{32}$ : (a) 3D plot; and (b) contour plot.

It should be highlighted that in the case of the analysed system, the selected Poisson's ratios can not only have negative values, but they can also change sign (Figure 9). It means that the proposed 
metamaterial behaves differently depending on the adopted parameters $k$ and $\sigma$. This is a unique feature of smart metamaterials. Such a material can act like a standard material with positive Poisson's ratios, and, in the same time, it can be changed into a metamaterial with negative values of these mechanical characteristics.

The considered eight-module supercells can be used to build a metamaterial of any volume. The properties of such a material are the same as the properties of the supercell. An example of a metamaterial that is built from the analysed eight-module supercells is presented in Figure 12.

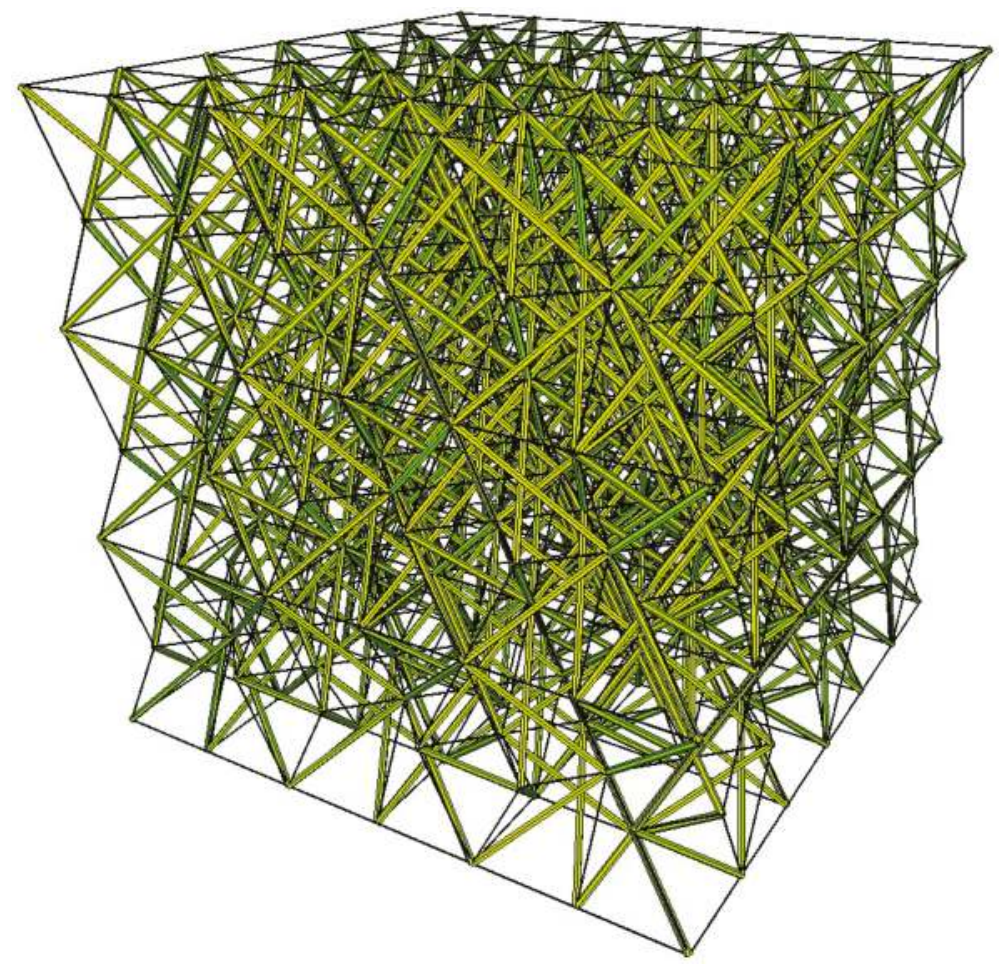

Figure 12. Metamaterial built from the eight-module supercells.

\section{Conclusions}

The present paper focuses on the development and analysis of a novel cellular metamaterial based on the simplex tensegrity pattern. The proposed material is constructed from supercells, each of which consists of eight 4-strut simplex modules. Using continuum model different structures are analyzed: a unit cell, a four-module layer, and an eight-module supercell. The continuum analysis of tensegrities allows for: estimating the influence of self-stress on the behaviour of the system, asses how the characteristics of cables and struts affect the whole structure and determine mechanical characteristics of materials or structures based on tensegrity patterns.

The proposed unit cell is an anisotropic structure. However, it can be arranged in such a way that that the material based on the simplex tensegrity pattern exhibits orthotropic properties. Such a material is considered in this paper. The developed metamaterial has some unusual properties, which are typical for smart structures. It is possible to control its mechanical characteristics by adjusting the level of self-stress or by changing the properties of structural members. 
One of the most important features of the proposed cellular metamaterial is a unique behaviour of some of its Poisson's ratios. Depending on the applied control parameters, they can be either positive or negative. This feature is of a great importance as far as the active control of the system is concerned.

Moreover, the proposed metamaterial exhibits features which characterize smart systems. Its mechanical properties, such as Young's moduli, shear moduli and Poisson's ratios, can be adjusted and controlled during the exploitation via the self-stress parameter $\sigma$ to satisfy the self-control, self-diagnosis, active-control, as well as self-repair conditions (see $[20,37,46]$ for details of smart tensegrity structures). It is possible due to the occurrence of infinitesimal mechanisms that are balanced with self-stress states.

The results that are presented in this paper indicate a great potential that lies in the proposed metamaterial. Thanks to its unique features, such as the negative Poisson's ratio, controllability, sensitivity to the self-stress, the material can be applied in smart structural systems. The members constructed from such a material can be designed to adapt to a functional need by modifying their shape, stiffness, or damping characteristics in order to minimize the deflection and possible damage.

In the future works, it is worth developing and analysing smart metamaterials based on different tensegrity patterns. Other modules are to be considered as unit cells in various arrangements-orthotropic and anisotropic ones. The proposed systems are to be considered from the point of view mechanical properties (using the continuum approach), as well as inherent smartness and the conditions that have to be satisfied in order to regard a structure as smart.

Author Contributions: Introduction was written by W.G. The continuum model of unit cell and cellular material was developed by A.A.S.-Z. and W.G. The elasticity matrices were determined by A.A.S.-Z. Analysis of the results and conclusions were written by A.A.S.-Z. and W.G.

Funding: This research received no external funding.

Conflicts of Interest: The authors declare no conflict of interest.

\section{References}

1. Cui, T.J.; Smith, D.R.; Liu, R. Metamaterials. Theory, Design and Applications; Springer: New York, NY, USA; Dordrecht, The Netherlands; Heidelberg, Germany; London, UK, 2010.

2. Engheta, N.; Ziolkowski, R.W. Metamaterials. Physics and Engineering Explorations; Wiley-Interscience: New York, NY, USA, 2006.

3. Singh, G.; Ni, R.; Marwaha, A. A review of metamaterials and its applications. Int. J. Eng. Trends Technol. 2015, 19, 305-310. [CrossRef]

4. Schurig, D.; Mock, J.J.; Justice, B.J.; Cummer, S.A.; Pendry, J.B.; Starr, A.F.; Smith, D.R. Metamaterial electromagnetic cloak at microwave frequencies. Science 2006, 314, 977-980. [CrossRef] [PubMed]

5. Soukoulis, C.M.; Linden, S.; Wegener, M. Negative refractive index at optical wavelengths. Science 2007, 315, 47-49. [CrossRef] [PubMed]

6. Soukoulis, C.M.; Wegener, M. Past achievements and future challenges in the development of three-dimensional photonic metamaterials. Natl. Photonics 2011, 5, 523-530. [CrossRef]

7. Vora, A.; Gwamuri, J.; Pala, N.; Kulkarni, A.; Pearce, J.M.; Guney, D.O. Exchenging ohmic losses in metamaterial absorbers with useful optical absorption for photovoltaics. Sci. Rep. 2014, 4901. [CrossRef]

8. Wu, C.; Neuner, B., III; John, J.; Milder, A.; Zollars, B.; Savoy, S.; Shvets, G. Metamaterial-based integrated plasmonic absorber/emitter for solar thermos-photovoltaic systems. J. Opt. 2012, 14, 024005. [CrossRef]

9. Brule, S.; Javelaud, E.H.; Enoch, S.; Guenneau, S. Experiments on seismic metamaterials: Molding surface waves. Phys. Rev. Lett. 2014, 112. [CrossRef] [PubMed]

10. Chen, H.; Chan, C.T. Acoustic cloaking in three dimensions using acoustic metamaterials. Appl. Phys. Lett. 2007, 91. [CrossRef]

11. Mei, J.; Ma, G.; Yang, M.; Yang, Z.; Wen, W.; Sheng, P. Dark acoustic metamaterials as super absorbers for low-frequency sound. Nat. Commun. 2012,3, 756. [CrossRef] [PubMed] 
12. Lee, J.B.; Peng, S.; Yang, D.; Roh, Y.H.; Funabashi, H.; Park, N.; Rice, E.J.; Chen, L.; Long, R.; Wu, M.; et al. A mechanical metamaterial made from a DNA hydrogel. Nat. Nanotechnol. 2012, 7, 816-820. [CrossRef] [PubMed]

13. Bertoldi, K.; Reis, P.M.; Willshaw, S.; Mullin, T. Negative poisson's ratio behavior by an elastic instability. Adv. Mater. 2010, 22, 361-366. [CrossRef] [PubMed]

14. Kadic, M.; Buckmann, T.; Stenger, N.; Thiel, M. On the practicability of pentamode mechanical metamaterials. Appl. Phys. Lett. 2012, 100, 191901. [CrossRef]

15. Lee, J.H.; Singer, J.P.; Thomas, E.L. Micro-/Nanostructured mechanical metamaterials. Adv. Mater. 2012, 24, 4782-4810. [CrossRef] [PubMed]

16. Zheng, X.; Lee, H.; Weisgraber, T.H.; Shusteff, M.; Deotte, J.; Duoss, E.B.; Kuntz, J.D.; Biener, M.M.; Ge, Q.; Jackson, J.A.; et al. Ultra-light, ultra-stiff mechanical metamaterials. Science 2014, 344, 1373-1377. [CrossRef] [PubMed]

17. Farshad, M. Intelligent materials and structures. Sci. Iran. 1995, 2, 65-87.

18. Lopes Junior, V.; Steffen, V., Jr.; Savi, M.A. Dynamics of Smart Systems and Structures; Springer: New York, NY, USA; Dordrecht, The Netherlands; Heidelberg, Germany; London, UK, 2016.

19. Schwartz, M. Smart Materials; Taylor and Francis: Abingdon, OX, USA, 2009.

20. Gilewski, W.; Al Sabouni-Zawadzka, A. On possible applications of smart structures controlled by self-stress. Arch. Civ. Mech. Eng. 2015, 15, 469-478. [CrossRef]

21. Schenk, M.; Guest, S.D. Geometry of Miura-folded metamaterials. Proc. Natl. Acad. Sci. USA 2013, 110, 3276-3281. [CrossRef] [PubMed]

22. Lv, C.; Krishnaraju, D.; Konjevod, G.; Yu, H.; Jiand, H. Origami based mechanical metamaterials. Sci. Rep. 2014, 4, 5979. [CrossRef] [PubMed]

23. Silverberg, J.L.; Evans, A.A.; McLeod, L.; Hayward, R.C.; Hull, T.; Santangelo, C.D.; Cohen, I. Using origami design principles to fold reprogrammable mechanical metamaterials. Science 2014, 345, 647-650. [CrossRef] [PubMed]

24. Eidini, M.; Paulino, G.H. Unraveling metamaterial properties in zigzag-base folded sheets. Sci. Adv. 2015, 1, e1500224. [CrossRef] [PubMed]

25. Filipov, E.T.; Tachi, T.; Paulino, G.H. Origami tubes assembled into stiff, yet reconfigurable structures and metamaterials. Proc. Natl. Acad. Sci. USA 2015, 112, 12321-12326. [CrossRef] [PubMed]

26. Waitukaitis, S.; Menaut, R.; Chen, B.G.G.; van Hecke, M. Origami multistability: from single vertices to metasheets. Phys. Rev. Lett. 2015, 114, 055503. [CrossRef] [PubMed]

27. Skelton, R.E.; de Oliveira, M.C. Tensegrity Systems; Springer: New York, NY, USA; Dordrecht, The Netherlands; Heidelberg, Germany; London, UK, 2009.

28. Motro, R. Tensegrity: Structural Systems for the Future; Kogan Page Science: London, UK, 2003.

29. Wroldsen, A.S. Modelling and Control of Tensegrity Structures. Ph.D. Thesis, Department of Marine Technology, Norwegian University of Science and Technology, Norwegian, Norway, 2007.

30. Calladine, C.R.; Pellegrino, S. First-order infinitesimal mechanisms. Int. J. Solids Struct. 1991, 27, 505-515. [CrossRef]

31. Adam, B.; Smith, I.F.C. Learning, self-diagnosis and multi-objective control of an active tensegrity structure. Adv. Eng. Struct. Mech. Constr. 2006, 439-448. [CrossRef]

32. Liu, K.; Wu, J.; Paulino, G.H.; Qi, H.J. Programable deployment of tensegrity structures by stimulus-responsive polymers. Sci. Rep. 2017, 7, 3511. [CrossRef] [PubMed]

33. Adam, B.; Smith, I.F.C. Self-diagnosis and self-repair of an active tensegrity structure. J. Struct. Eng. 2007, 133, 1752-1761. [CrossRef]

34. Bel Hadj Ali, N.; Smith, I.F.C. Dynamic behavior and vibration control of a tensegrity structure. Int. J. Solids Struct. 2010, 47, 1285-1296. [CrossRef]

35. Fest, E.; Shea, K.; Smith, I.F.C. Active tensegrity structure. J. Struct. Eng. 2004, 130, 1454-1465. [CrossRef]

36. Moored, K.W.; Kemp, T.H.; Houle, N.E.; Bart-Smith, H. Analytical predictions, optimization, and design of a tensegrity-based artificial pectorial fin. Int. J. Space Struct. 2011, 48, 3142-3159. [CrossRef]

37. Al Sabouni-Zawadzka, A.; Gilewski, W. Inherent smartness of tensegrity structures—Structural elements applications. In Proceedings of the International Association for Shell and Spatial Structures (IASS), Amsterdam, The Netherlands, 17-20 August 2015. 
38. Koohestani, K.; Guest, S.D. A new approach to the analytical and numerical form-finding of tensegrity structures. Int. J. Solids Struct. 2013, 50, 2995-3007. [CrossRef]

39. Gilewski, W.; Al Sabouni-Zawadzka, A. Continuum model of cable-strut structures with self-stress included. Int. J. Space Struct. 2018. in preparation.

40. Gilewski, W.; Kasprzak, A. Description of the influence of self-stress on the properties of tensegrity modules using a continuum approach. In Theroretical Foundations of Civil Engineering, Vol. IV. Technical Mechanics; Szcześniak, W., Ataman, M., Eds.; Warsaw University of Technology Publishing House: Warsaw, Poland, 2013; pp. 117-126. (In Polish)

41. Rimoli, J.J.; Pal, R.K. Mechanical response of 3-dimensional tensegrity lattices. Compos. Part B 2017, 115, 30-42. [CrossRef]

42. Green, A.E.; Zerna, W. Theoretical Elasticity; Oxford University Press: Oxford, UK, 1968.

43. Chadwick, P.; Vianello, M.; Cowin, S. A new proof that the number of linear elastic symmetries is eight. J. Mech. Phys. Solids 2001, 49, 2471-2492. [CrossRef]

44. Ting, T.C.T. Positive definiteness of anisotropic elastic constants. Math. Mech. Sloids 1996, 1, 301-314. [CrossRef]

45. Zheng, Q.S.; Chen, T. New perspective on Poisson's ratio of elastic solids. Acta Mech. 2001, 150, 191-195. [CrossRef]

46. Al Sabouni-Zawadzka, A. On Possible Applications of Smart Structures in Bridge Engineering. Ph.D. Thesis, Warsaw University of Technology, Warsaw, Poland, 2016.

(c) 2018 by the authors. Licensee MDPI, Basel, Switzerland. This article is an open access article distributed under the terms and conditions of the Creative Commons Attribution (CC BY) license (http:/ / creativecommons.org/licenses/by/4.0/). 

MDPI

St. Alban-Anlage 66

4052 Basel

Switzerland

Tel. +41616837734

Fax +41 613028918

www.mdpi.com

Materials Editorial Office

E-mail: materials@mdpi.com

www.mdpi.com/journal/materials

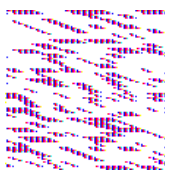



MDPI AG

St. Alban-Anlage 66

4052 Basel

Switzerland

Tel: +41 616837734

Fax: +41 613028918 